\title{
Let there be light : towards rational use of lasers in medicine
}

Citation for published version (APA):

Vondeling, H. (1998). Let there be light : towards rational use of lasers in medicine. [Doctoral Thesis, Maastricht University]. Datawyse / Universitaire Pers Maastricht. https://doi.org/10.26481/dis.19980220hv

Document status and date:

Published: 01/01/1998

DOI:

10.26481/dis.19980220hv

Document Version:

Publisher's PDF, also known as Version of record

\section{Please check the document version of this publication:}

- A submitted manuscript is the version of the article upon submission and before peer-review. There can be important differences between the submitted version and the official published version of record.

People interested in the research are advised to contact the author for the final version of the publication, or visit the DOI to the publisher's website.

- The final author version and the galley proof are versions of the publication after peer review.

- The final published version features the final layout of the paper including the volume, issue and page numbers.

Link to publication

\footnotetext{
General rights rights.

- You may freely distribute the URL identifying the publication in the public portal. please follow below link for the End User Agreement:

www.umlib.nl/taverne-license

Take down policy

If you believe that this document breaches copyright please contact us at:

repository@maastrichtuniversity.nl

providing details and we will investigate your claim.
}

Copyright and moral rights for the publications made accessible in the public portal are retained by the authors and/or other copyright owners and it is a condition of accessing publications that users recognise and abide by the legal requirements associated with these

- Users may download and print one copy of any publication from the public portal for the purpose of private study or research.

- You may not further distribute the material or use it for any profit-making activity or commercial gain

If the publication is distributed under the terms of Article $25 \mathrm{fa}$ of the Dutch Copyright Act, indicated by the "Taverne" license above, 
Cover design : ttilla Jeuken, "s Hertogenbosch and Theo Peters (Comma-s Ontwerpers), 's Hertogenbosch

Production : Datawyse I Universitaire Pers, Maastricht

Lay-out : Jan van Emmerik, Maastricht

Sponsors : Dutch Medical Laser Society, European Laser Association, Carl Zeiss B.V.

Copyright $1998 \mathrm{H}$. Vondeling, Amsterdam.

Parts of this book may be copled for educational purposes if the source is identified.

ISBN $90-9011383-5$ 


\title{
Let there be light \\ Towards rational use of lasers in medicine
}

\author{
PROEFSCHRIFT
}

ter verkrijging van de graad van doctor aan de Universiteit Maastricht op gezag van de Rector Magnificus,

Prof. Dr. A.C. Nieuwenhuijzen Kruseman, volgens het besluit van het College van Dekanen, in het openbaar te verdedigen op vrijdag 20 februari 1998 om 14.00 uur,

door

Hindrik Vondeling

geboren te Groningen op 4 juni 1960

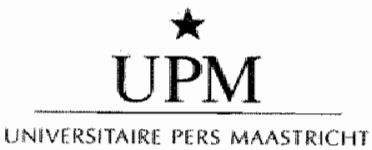


Promotores:

Prof. H.D. Banta (oud hoogleraar Universiteit Maastricht)

Prof. Dr. J.A.M. Maarse

\section{Beoordelingscommissie:}

Prof. Dr. J.A. Knottnerus (Voorzitter)

Prof. Dr. M.J.C. van Gemert (Universiteit van Amsterdam)

Prof. Dr. F. Hendrikse

Prof. Dr. Ph.E.V.A. Van Kerrebroeck

Prot. Dr. W. van Riossum (Rijksuniversiteit Groningen) 


\section{HOPE}

Wat ropt dy iene fuggel bliid,

Wat klinkt syn wille klear en wiid,

No tear en fyn,

Dan sterk en stoer,

De fjilden oer,

De loften yn;

Wat ropt dy iene fûgel bliid;

Hy sjongt in komstge tiid -

De himel bôget heech en klear,

It goudljocht sparket oer syn fear -

En duorjend giet

Yn golle klank

Sa fol en blank

Syn leauwend liet;

It goudljocht sparket oer syn fear -

Hy wit himsels net mear

Hy sjongt de jonge tûken grien -

Der is oan de alde beam net ien

Dy't by syn rop

Yn slomme bliuwt;

Syn sjongen driuwt

De knoppen op,

Hja boarste en bloele, lien foar ien,

Hy júblet alle tûken grien -

O hope, dy't yn 't kealste hout

Dyn nêsten fan gedichten bout,

Sjong lûd en blier

Trach dizze dei

Dyn profesij:

tt Is net te ier!!

Mei "t deade hout de lesste twivel stjert;

Myn Fryslân, "t ils dyn eigen sjongend hert -

Fedde Schurer

Oan heit en mem 

Part One $-\| n t r o d u c t i o n$ and General $\|$ ssues $\ldots \ldots \ldots \ldots \ldots \ldots \ldots \ldots \ldots$

1 Introduction $\ldots \ldots \ldots \ldots \ldots \ldots \ldots \ldots \ldots \ldots \ldots \ldots \ldots \ldots \ldots \ldots \ldots$

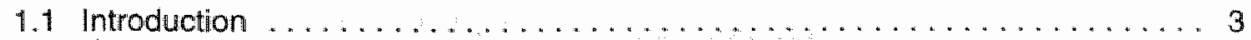

1.2 How to approach an assessment of lasers in medicine? . . . . . . . . . . . 4

1.3 What is the clinical context of the development of medical laser applications? . . 5

1.4 Medical lasers and technology assessment, a synchronic development? .... . 5

1.5 What can you expect to find in a thesis on medical lasers from a technology assessment point of view? . . . . . . . . . . . . . . . . . . . . . 5

1.6 References . . . . . . . . . . . . . . . . . . . . . . . . . . . 10

2 Medical technology assessment and medical lasers $\ldots \ldots \ldots \ldots \ldots \ldots \ldots \ldots$

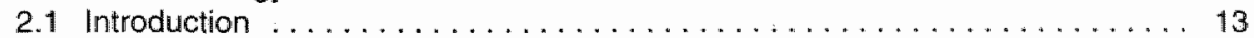

2.2 Some basic concepts . . . . . . . . . . . . . . . . . . . . . 13

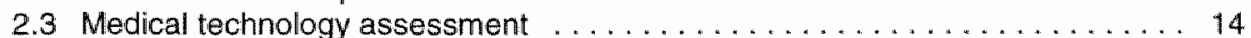

2.4 Lasers and medical applications of lasers $\ldots \ldots \ldots \ldots \ldots \ldots \ldots \ldots \ldots \ldots \ldots$

2.5 Assessing medical applications of lasers . . . . . . . . . . . . . . . 23

2.6 Assessment of medical lasers in the context of the development of minimally

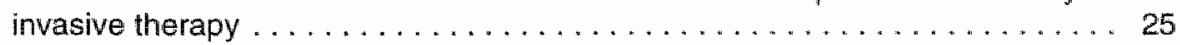

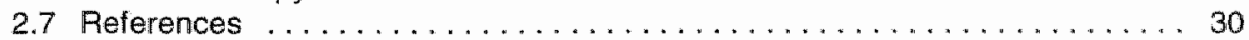

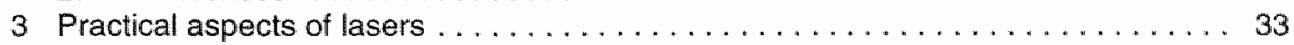

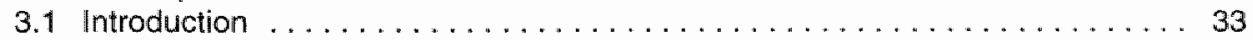

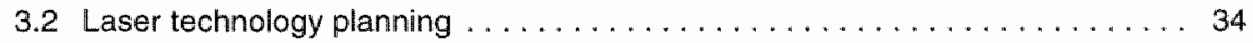

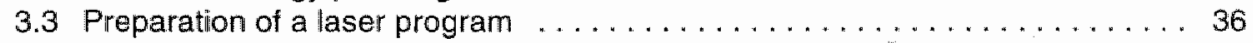

3.4 Implementation of a laser program $\ldots \ldots \ldots \ldots \ldots \ldots \ldots \ldots \ldots \ldots \ldots$

3.5 Laser satety management . . . . . . . . . . . . . . . . . . . . 40

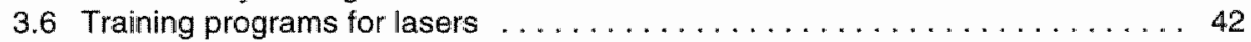

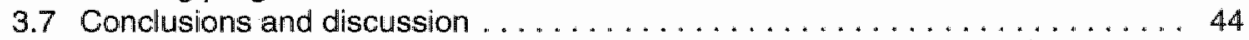

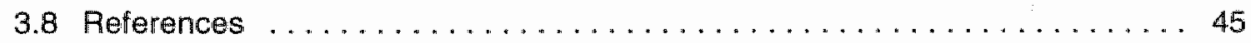

Part Two - Effectiveness and Cost-Effectiveness of Selected Laser Applications . . . 47

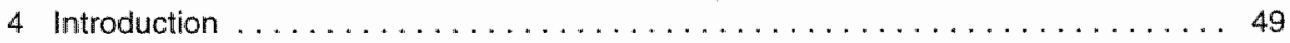

4.1 Contents and structure . . . . . . . . . . . . . . . . . . . . 49

4.2 Literature search strategy $\ldots \ldots \ldots \ldots \ldots \ldots \ldots \ldots \ldots \ldots \ldots \ldots . \ldots \ldots$

4.3 Evaluation of safety, effectiveness and cost-effectiveness . . . . . . . . . . . 50

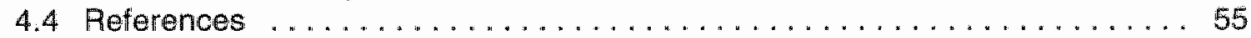

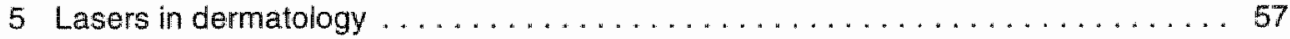

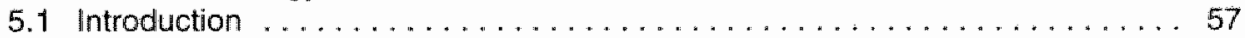

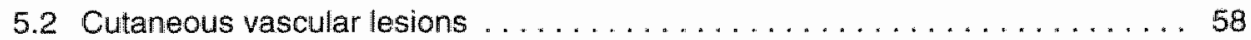

5.3 Cutaneous pigmented lesions . . . . . . . . . . . . . . . . . 66

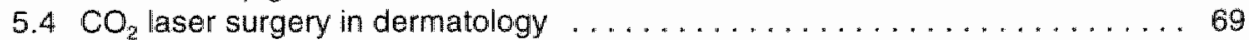

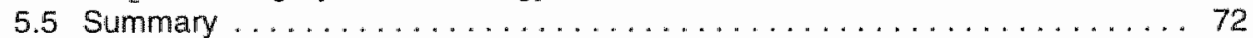

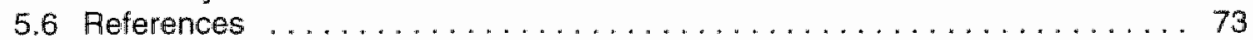

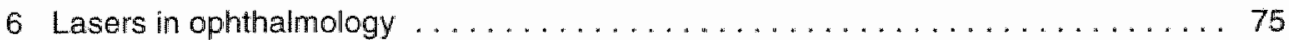

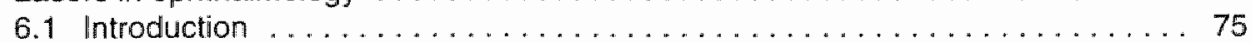

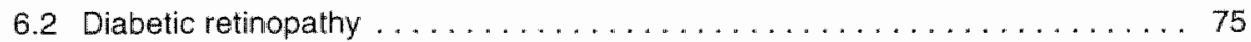

6.3 Age-related (senile) macular degeneration . . . . . . . . . . . . . . 77

6.4 Retinal vein occlusion $\ldots \ldots \ldots \ldots \ldots \ldots \ldots \ldots \ldots \ldots \ldots \ldots$

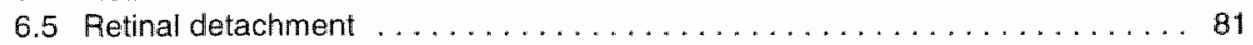

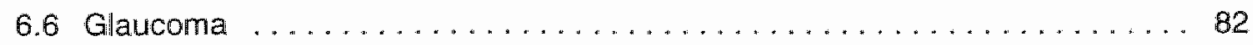




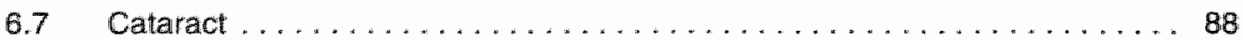

6.8 Photorefractive and phototherapeutic keratectomy ............. 90

6.9 The potential role of diode lasers . . . . . . . . . . . . . . . . . 90

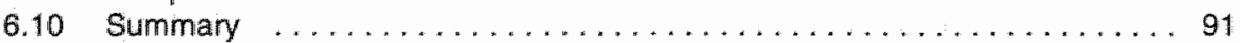

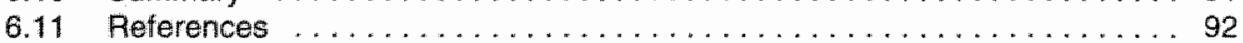

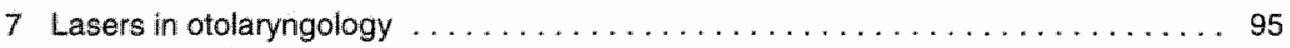

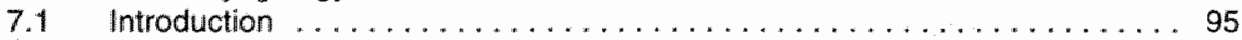

7.2 Safety and complications of $\mathrm{CO}_{2}$ laser microsurgery $\ldots \ldots \ldots \ldots \ldots . \ldots 5$

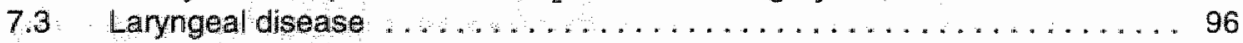

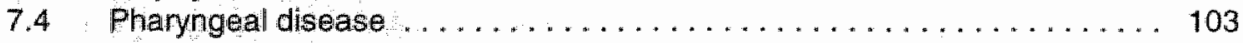

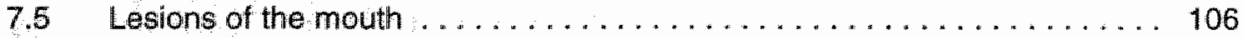

7.6 Tonsillitis . . . . . . . . . . . . . . . . . . . . . . . . 107

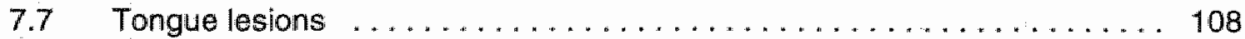

7.8 Otology . . . . . . . . . . . . . . . . . . . . . . . . . . . . 109

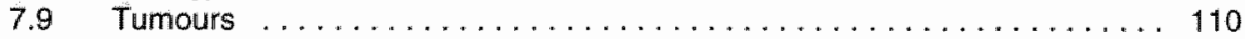

7.10 Nasal lesions . . . . . . . . . . . . . . . . . . . . . . . . 111

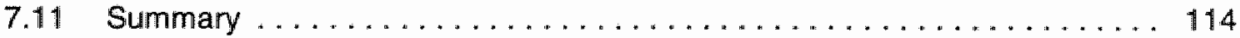

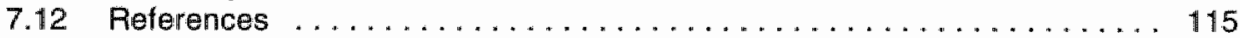

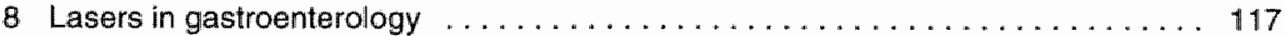

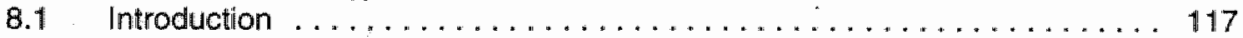

8.2 Upper gastrointestinal bleeding $\ldots \ldots \ldots \ldots \ldots \ldots \ldots \ldots \ldots \ldots \ldots \ldots 117$

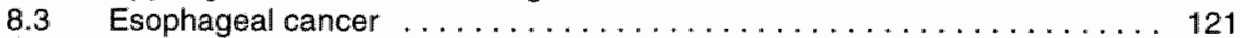

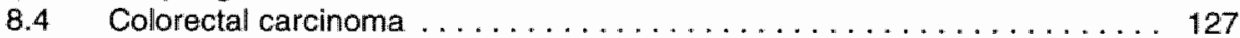

8.5 Colorectal adenomas . . . . . . . . . . . . . . . . . . . . . . 133

8.6 Hemorrhoids . . . . . . . . . . . . . . . . . . . . . . . 135

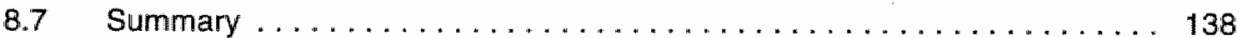

8.8 References . . . . . . . . . . . . . . . . . . . . . . . . . . . 139

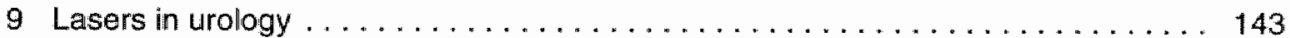

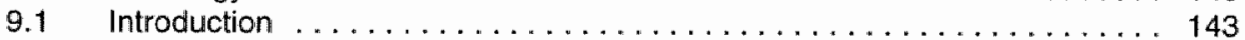

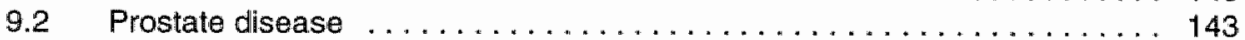

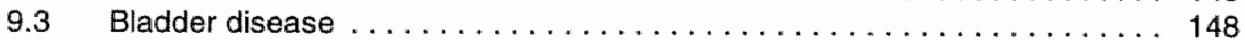

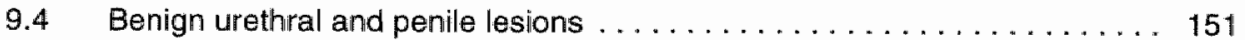

9.5 Penile precancerous and cancerous lesions . . . . . . . . . . . . 152

9.6 Upper urinary tract urothelial tumours $\ldots \ldots \ldots \ldots \ldots \ldots \ldots \ldots \ldots \ldots \ldots$

9.7 Hemangioma, cystitis and vasovasostomy . . . . . . . . . . . . . 153

9.8 Urolithiasis . . . . . . . . . . . . . . . . . . . . . . . . . . 154

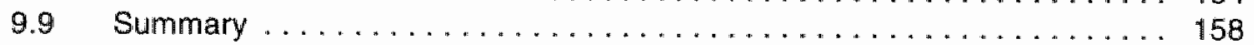

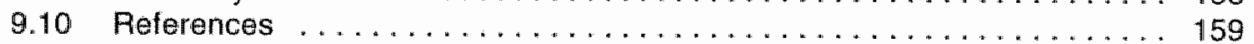

10 Lasers in gynecology . . . . . . . . . . . . . . . . . . . . . . . 163

10.1 Introduction . . . . . . . . . . . . . . . . . . . . . . 163

10.2 Cervical intraepithelial neoplasia ...................... 163

10.3 Vulvar, vaginal, anal and perianal neoplasia $\ldots \ldots \ldots \ldots \ldots \ldots \ldots \ldots$

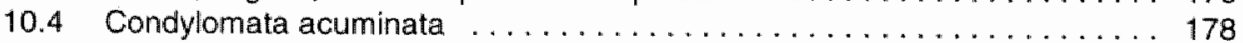

10.5 Infertility due to occluded Fallopian tubes $\ldots \ldots \ldots \ldots \ldots \ldots \ldots \ldots \ldots \ldots 1$

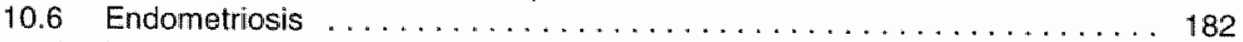

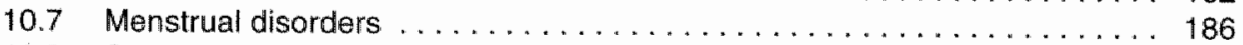

10.8 Summary . . . . . . . . . . . . . . . . . . . . . . . . . . 190

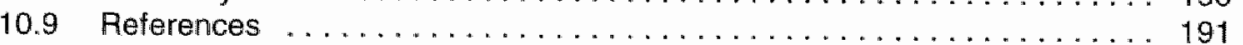

11 Summary and Discussion $\ldots \ldots \ldots \ldots \ldots \ldots \ldots \ldots \ldots \ldots \ldots \ldots \ldots$

11.1 Individual specialties $\ldots \ldots \ldots \ldots \ldots \ldots \ldots \ldots \ldots \ldots \ldots \ldots \ldots \ldots \ldots$

11.2 Combination of specialties $\ldots \ldots \ldots \ldots \ldots \ldots \ldots \ldots \ldots \ldots \ldots \ldots \ldots$

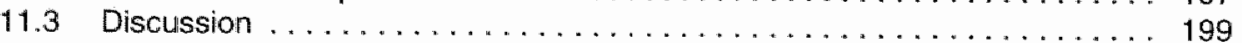

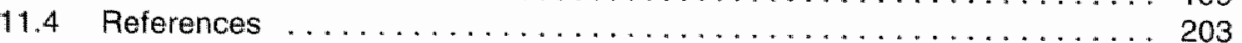


12 Diffusion of medical lasers in the Netherlands . . . . . . . . . . . . . . 207

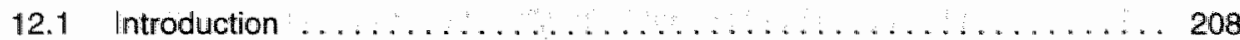

12.2 Methodology $\ldots \ldots \ldots \ldots \ldots \ldots \ldots \ldots \ldots \ldots \ldots \ldots \ldots \ldots \ldots \ldots \ldots$

12.3 Results .................................. 209

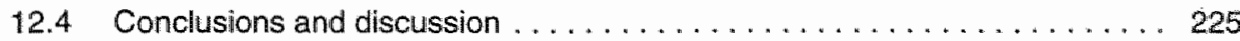

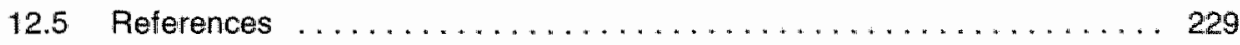

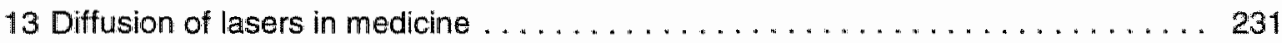

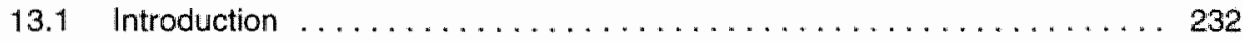

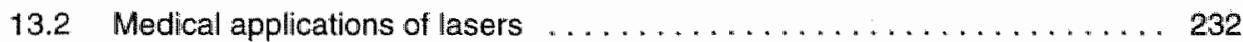

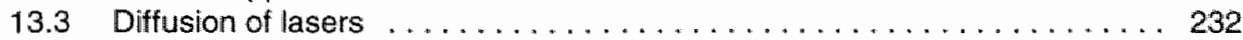

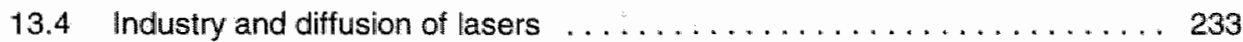

13.5 Safety of lasers . . . . . . . . . . . . . . . . . . . . . . . 234

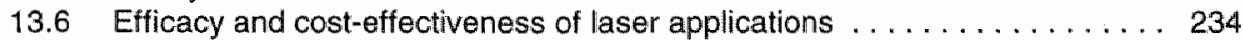

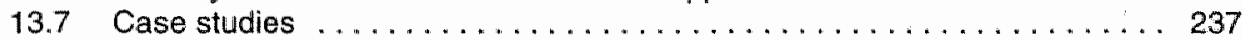

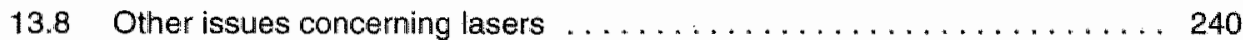

13.9 Pollicy implications of lasers in health care . . . . . . . . . . . . 240

13.10 References ................................. 242

14 Diffusion of minimally invasive therapy in the Netherlands $\ldots \ldots \ldots \ldots \ldots \ldots 247$

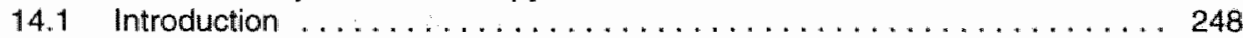

14.2 Control of health care technology in the Netherlands . . . . . . . . . 248

14.3 Methods . . . . . . . . . . . . . . . . . . . . . . . . . . . 249

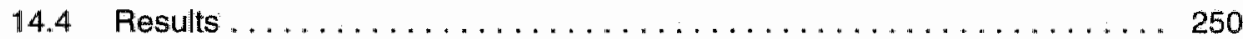

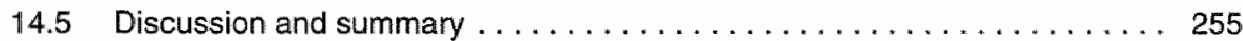

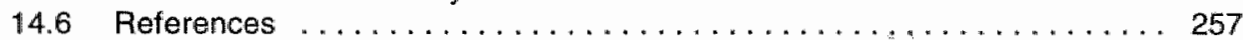

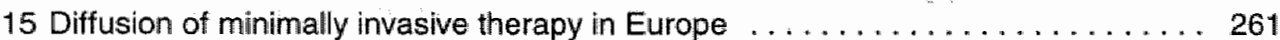

15.1 Diffusion of the specific cases . . . . . . . . . . . . . . . . 262

15.2 Diffusion in relation to effectiveness and cost-effectiveness . . . . . . . . 266

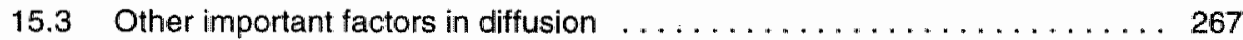

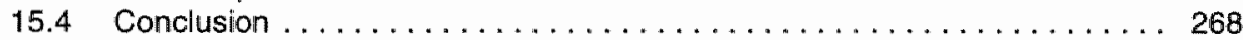

15.5 References ............................... 269

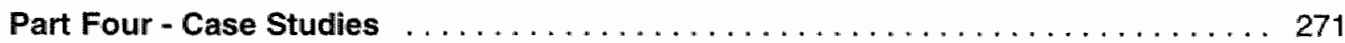

16 Evaluation of argon laser treatment of diabetic retinopathy and its diffusion in the Netherlands . . . . . . . . . . . . . . . . . . . . . . . . . . . 273

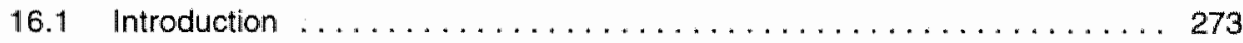

16.2 Evaluation of effectiveness of alternative photocoagulation procedures in treatment of diabetic retinopathy . . . . . . . . . . . . . . 274

16.3 Cost-studies and cost-effectiveness analyses of argon laser treatment of diabetic retinopathy . . . . . . . . . . . . . . . . . . 276

16.4 Rationalle for and cost-effectiveness of alternative screening strategles for diabetic retinopathy in the diabetic population ... . . . . . . . . . . 277

16.5 Diffusion of argon lasers in the Netherlands . . . . . . . . . . . . 280

16.6 Screening programs . . . . . . . . . . . . . . . . . . . . . . 282

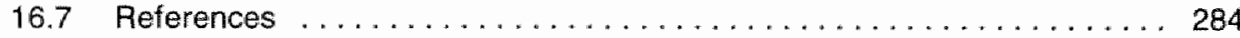


17 Evaluation and diffusion of Excimer laser treatment of myopia in the United States and in the Netherlands

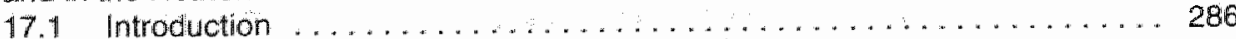

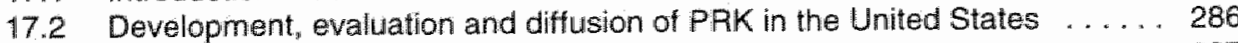

17.3 Early diffusion and evaluation of PRK in the Netherlands .......... 287

17.4 Political visibility of PRK and further evatuation $\ldots \ldots \ldots \ldots \ldots \ldots \ldots . \ldots \ldots$

17.5 Discussion .......................................... 290

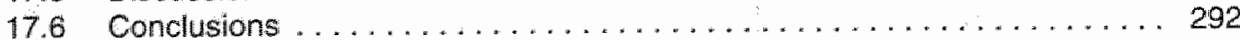

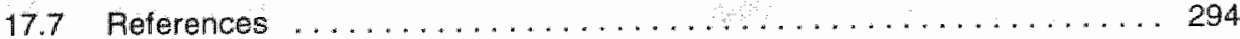

Part Five - Policy Implications . . . . . . . . . . . . . . . . . . . . . . . . . . . . 297

18 Policies towards the safe use of medical lasers in the Netherlands and in the

European Union . . . . . . . . . . . . . . . . . . . . . . . . . . . . . . . . . . 299

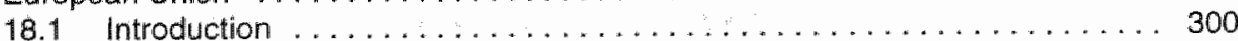

18.2 EU policy and standards bodies governed by private law. . . . . . . . . . 301

18.3 Dutch national legistation . . . . . . . . . . . . . . . . . . . . . 304

18.4 Liability for errors involving medical laser equipment . . . . . . . . . . . . 307

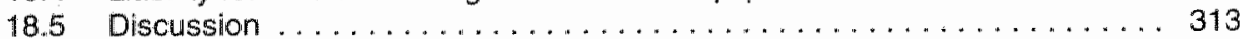

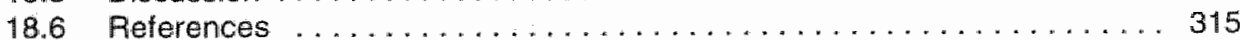

19 Strategies for successful evaluation and policy-making toward health care

technology on the move: the case of medical lasers . . . . . . . . . . . . . . 319

19.1 Introduction ....................................... 320

19.2 Identification and evaluation of medical lasers in the COMAC-HSR project . 322

19.3 Laser treatments that have been well-evaluated . . . . . . . . . . . . . . . 325

19.4 Argon laser treatment of diabetic retinopathy . . . . . . . . . . . . . 325

19.5. Excimer laser coronary angioplasty in the United States and

in the Netherlands .................................. 326

19.6 Discussion . . . . . . . . . . . . . . . . . . . . . . . . . . . 328

19.7 Towards a strategy for timely evaluation $\ldots \ldots \ldots \ldots \ldots \ldots \ldots \ldots \ldots \ldots$

19.8 Conclusions . . . . . . . . . . . . . . . . . . . . . . . . . . 3330

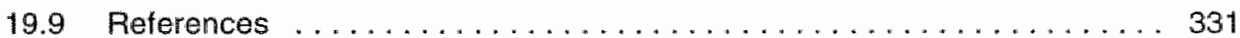

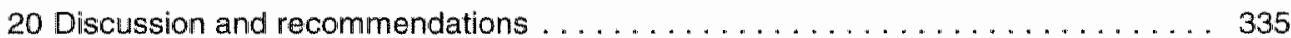

20.1 Introduction . . . . . . . . . . . . . . . . . . . . . . 335

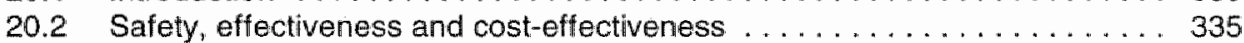

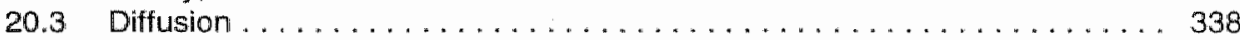

20.4 Implications of lasers \& minimally invasive therapy . . . . . . . . . . 34 1

20.5 Policies towards lasers and minimally invasive therapy . . . . . . . . . . 343

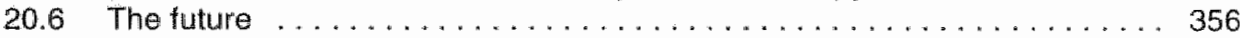

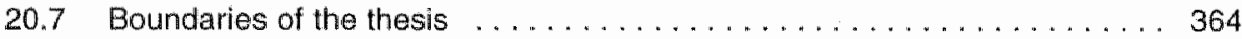

20.8 Final statement . . . . . . . . . . . . . . . . . . . . . . 364

20.9 Recommendations . . . . . . . . . . . . . . . . . . . . . 364

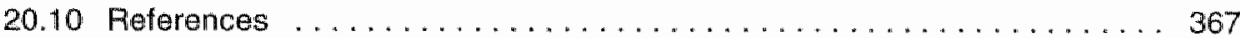

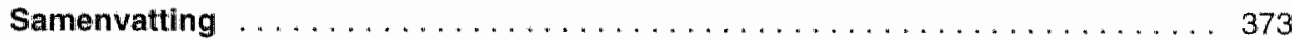

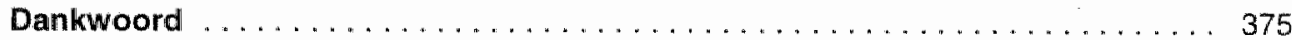

Curriculum Vitae $\ldots \ldots \ldots \ldots \ldots \ldots \ldots \ldots \ldots \ldots \ldots \ldots \ldots \ldots$ 
Part One - Introduction and General Issues 


\section{Introduction}

\subsection{Introduction}

Why a thesis on lasers? Because they are fascinating! In a speculative account, Hecht and Theresi ${ }^{[15]}$ trace the origins of this fascination back to the previous century. In HG Wells' 'The War of the Worlds', published in 1898, alien creatures terrorize our planet with a 'heat-torch', a bundle of energy which was so hot and so powerful that it destroyed everything that came in its way. Suddenly there was a flash of light, wrote Wells, it swept swiftly and accurately round, this flaming death, this invisible, unavoidable sword of heat. Wells' heat torch inspired generations of sciencefiction writers in creating waveguns and deadly rays of light. So when the first laser finally was made in 1960, movie directors and writers immediately used the literature-derived theme of its destructive power and replaced the word 'laser' by 'wavegun' or 'lasergun'.

The powerful image of lasers was fuelled by spectacular achievements as well. In 1962 it was demonstrated that laser light can be projected to the moon. At a distance of almost 400,000 kilometres, its reflection was clearly visible as a spot of an estimated 4 kilometres in diameter. A laser was one of the main features in the James Bond thriller Goldfinger in the early sixties. James Bond (played by Sean Connery at that time) was tied on a metal table. A laser placed above the table produced a bright red beam which easily cut through the table while moving slowly to Bond's body. Obviously, it had the power to cut Bond in half.

In 1969, the General Assembly of the United Nations for the first time expressed its concern of the potential military applications of laser technology (UN Resolution 2602D (XXIV), 1969, cited by WA Smit ${ }^{[27]}$. Despite the UN resolution, laser technology would play an important role in the US Governments Strategic Defense Initiative (SDI), as announced by the former US President Reagan in a 1983 speech (the 'Star Wars' speech), which evoked a controversy both among military experts, physicists and informed citizens ${ }^{[13 !}$. As a result in the late eighties this part of the SDI program was skipped ${ }^{[29]}$. However, other military applications were developed in the early nineties. In 1994, the International Committee of the Red Cross (ICRC) launched a public information campaign to mobilize public opinion against the further development of portable, blinding laser weapons ${ }^{[3]}$. To the lay public this continuous stream of information may have contributed to the mysterious, perhaps frightening image of lasers.

Although military applications are perhaps the most spectacular and therefore may have dominated our perception of lasers, there are many non-military laser applications as well of which some are quite impressive too. Laser light is used for entertainment in discotheques, to support life-performances of pop-acts and to celebrate special events. In everyday-life lasers have revolutionized listening to music as it allowed the development of cd players. In supermarkets lasers are employed as bar code scanners, changing the working conditions of employees while allowing faster and more accurate transactions with customers and improving stock control at the same time. In offices laser-printers have become a common phenomenon. Besides the applications listed here, laser technology has found a variety of applications in industry and communication. Overall, the scale of applications has become much greater than both the early science-fiction writers and the physicians who stood at the cradle of the development of lasers had ever thought of ${ }^{[13,15,19]}$.

But why a thesis on medical lasers? All lasers have in common that they represent an archetype of what we intuitively most easilly experience as technology; it is a highlly visible physical apparatus. But perhaps more than in any other category of applications, medical use of lasers has been associated with magic. Since 1962, when lasers were introduced in medicine, its proliferation has been accompanied with high expectations of its benefits, as expressed in articles in both the 
lay-press and well-respected medical and scientific joumals. Headlines of such articles strongly appeal to the curiosity of the reader: 'Laser - Science Fiction oder eine neue Dimension in der Medizin?"1:6], "The light fantastic - lasers brighten the future ${ }^{[177}$, "Lasers take a shine into medicine" ${ }^{|8|}$, are only three of many-lold used examples. Besides appealing to one"s curiosity, an appeal is made to one's believe in technological progress and, more specifically related to medical technology, to one's expectations of improvements in the capacity of the health care system to cure disease ${ }^{\text {[2]! }}$

A second source of motives to select medical lasers for a thesis markedly contrasts with the previous descriptions. The other side of the glorious coin of medical technology is that it is controversial. Expressions such as 'the technological imperative' referring to the virtual impossibility not to use a new technology when it has become available, and "technological fix', the inclination to exclusively look for technological solutions to problems generated by the application of technology, have often been applied to new forms of medicall technologies ${ }^{[23 ; 28,28]}$ " In addition, it is challenging to specify questions ellicited by developments characterized as "the medical arms race' (although this may pertain in particular to the US context) ${ }^{121}$, the 'technologization of medicine' and the 'medicalization of society' to the case under study. Thus, besides a fascination for lasers as a manifestation of the technological ability of mankind, the controversilal aspects of its advent are impoitant as well for its selection.

\subsection{How to approach an assessment of lasers in medicine?}

When the firs Professor in medical laser applications was appointed in the Netherlands, Dr.

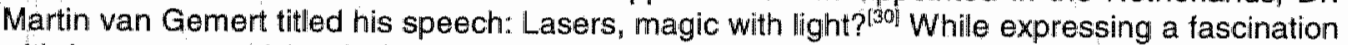
with lasers as a driving force to be active in the field, as a scientist at the same time Prof. wan Gemert pledged to look at lasers from a down-to-earth, demystified perspective. This seems a sound attitude not only for physicists in this particular field of laser applications but also for technology assessors who aim at evaluating medical lasers. Hopefully this attitude results in an approach which is helpfull in preventing from getting 'blinded' by the glittering laser light or from adopting a myopic perspective towards its benefits and risks. It is up to the reader to judge if we have managed to achieve this goal. We believe that in any serious researchers' view, lasers represent no more than a tool, one of the many tools making up the medical armamentarium. It is people who invent or design tools, people who use and modify them and people that can be subjected to policy-making in an attempt to influence the process of technological change ${ }^{[24,25]}$. A down-to-earth, demystified approach should also be applied to the distinction between science, technology and society (or 'culture'). We all know that technology in its widest manifestations has become fully integrated in our everyday lives. As a result many analysts nowadays agree that the classic borders between science, technology and society both in general terms and in medicine, can hardly be distinguished any more, which has implications for the analytical approach of a single 'technology $1(4,25)$. This notion and the belief or conviction that (medical) technology or, more general, processes of technological change can be influenced, are centrall for any researcher that aims at providing information that can be used to support decision-making processes by policymakers, as medical technology assessors are supposed to do.

In summary, even now that we have reduced the phenomenon of medical lasers to its right proportions and after having elucidlated some of the basic premises behind its assessment, the subject is still fascinating, because of its many manifestations, its broad variety of actual and potential applications and the rapid technological developments that characterize the field. 


\subsection{What is the clinical context of the development of medical laser applications?}

Lasers have allowed many new diagnostic and therapeutic applications in medicine. Most of the medical laser applications have in common that they have the potential to substitute for more invasive, conventional procedures. Today, many analysts agree that the development of imedical lasers is embedded in the development of an entirely new field that has emerged since the mideighties: the field of minimally inwasive therapy. The development of this rapidly growing area of medical treatment has often been called revolutionary, because it causes substantially reduced trauma to the person undergoing it, and because it has the (related) potential to stimulate dramatic change in the structure of hospital and health care facilities ${ }^{[4]}$. Development of techniques in minimally invasive therapy (MIT) has been stimulated not only by the development of new tools such as lasers and related technologies, but also, and this is more important, by the development of endoscopes, new imaging techniques (such as CT, PET and MRI) and vascular catheters ${ }^{[7]}$. It is these developments that allow exploration of the full clinical potential of lasers. Therefore, any assessment of lasers should be understood in the broader context of the development of the field of minimally invasive therapy.

\subsection{Medical lasers and medical technology assessment, a synchronic development?}

In 1975, the first medical technology assessment program was launched in the United States ${ }^{|4|}$. One year later the first interim-analysis of a randomized clinical trial evaluating lasers was published, also in the United States ${ }^{[0]}$. Since then medical technology assessment programs have been organized in many countries, among them the country where this thesis was produced, the Netheriands. In 1988, an Institute for Medical Technology Assessment was founded in the Netherlands, and lasers were one of the first subjects taken up by the Institute ${ }^{[9]}$. Since then, a variety of organizations and institutions have started medical technology assessment programs. In the same period, two Clinical Laser Research Centers were established in the Netherlands, one in the capital, Amsterdam, and one in Utrecht. These synchronic developments allowed for collaborative efforts, especially in an EC-project on medical lasers ${ }^{[5]}$, which is one of the most important sources this thesis builds upon.

\subsection{What can you expect to find in a thesis on medical lasers from a technology assessment point of view?}

In order to provide a view on the contents of an evaluation of medical lasers from a technology assessment perspective and to further explain the remark on the relationship between lasers and minimally invasive therapy it seems best to use an example. In the late eighties, early nimeties conventional gall-bladder removal (cholecystectomy), was rapidly substituted by a minimally invasive technique, laparoscopic gall-bladder removal ${ }^{\|4\|}$. In conjunction with this change, instead of ellectrocautery or a knife (scalpel), lasers were promoted to use for the actual cutting. This process was analysed in the US from a laser marketing perspective by Micheal Moretti in $1991^{[20]}$. The laser featuring in this story is a so-called KTP laser. This laser emits green light and is manufactured by a company called LaserScope. In the analysis, Moretti addressed an audience of laser manufacturers.

'At LaserScope, KTP has been their claim to fame and it's amazing what power this had. General surgeons are convinced that KTP is somehow better than anything else. It's a psychological phenomenon but we all know that in marketing psychology reigns supreme. 
So they're convinced that it's good, better so it is better. Until they're unconvinced of that everybody else has got to play catch-up with LaserScope who has this unique green laser. In fact, the surgeons come up to you or walk up to exhibitors at these shows and the first question they ask is do you have one of those green lasers? They have no idea what it is or why it's better, just they heard that a green laser is somehow better. So that's the position that LaserScope has put itself in and really manipulated and used that to their best advantage. I have a lot of respect for that company'.

Issues raised in this section of the account are:

- the role of industry;

- the rationality of the process underlying a physicians' judgment on the value of a laser treatment and the subsequent decision whether or not to adopt and use it.

'The unique KTP' technology keeps the price high. (...) Until Laser Photonics can get a working frequency doubled laser on the market, LaserScope has no competition. And it could be that they will saturate the market for these $\$ 140000-150000$ systems before anybody has the opportunity to get in there and do anything about it. I think what really must happen is an education process that is ongoing. It'll take a while but surgeons will realize that there's nothing godlike about the KTP (...) and that there are other advantages to other lasers. I think that other companies must just persevere and wait for the new phenomenon to die down a little bit and then I think surgleons who become more educated about lasers will understand that they're really looking for all kinds of parameters not just the color green. I think that 'surgeons just may be equating green with money' and so it's a very primitive process that's going on here'.

Following from this it seems appropriate to include issues such as:

- the structure of the laser industry;

- the market for lasers;

- the role of financial incentives for physicians in adopting a laser;

- the costs of lasers;

- the interrelationship of industry and physicians, e.g. in educational processes.

'Another potential problem that LaserScope has (and they"re really immersed in this controversy right now) is convincing surgeons that a laser, let's forget that it's green or blue or whatever, is better than electro-surgery. And l'll just briefly explain that surgeons have been using electro-surgery or electro-cautery or the bovie knife for years and there's a system in every operating room. There might be two or three in a hospital. These are low-cost, the laser can never price-compete in price with electro-surgery. They're low cost, they"re entrenched and every surgeon knows how to use it. A surgeon could begin doing gall-bladder removals tomorrow with electro-surgery. He doesn't have to take an expensive laser course. He doesn"t have to risk the liability with the laser. He can use electro-surgery. And that's the major problem here. It could be that the electro-surgery companies are going to pull the rug out from underneath the laser companies by just upgrading their equipment and their marketing efforts'.

Additional subjects to be studied are:

- the cost-effectiveness of laser applications versus conventional treatment or other minimallyinvasive treatment modalities;

- the integration of lasers in clinical care;

- the skills required to perform laser surgery;

- the content of educational courses for physicians who want to use lasers;

- legal aspects of the use of lasers. 
It think the answer to this is that nevitably the companies must cultivate new applications. Once they have their foot in the doot here with gall-bladder or laparoscopic procedures they have to quickly cultivate the opportunity they have to quickly push their way into other areas using the popularity using the momeritum that's now going. And I feel that companies like LaserScope, if they don't want to crash like a couple of companies have. if they don't want to go through a cycle like Trymedine has gone through in the last couple years, they"re going to have to diversify their produktline. They're going to have to add other solid state lasers you know. Especially when other people begin to introduce green lasers ${ }^{[20]}$.

Issues to be studied emerging from this information are:

- the profitability, market structure and competitiveness of laser manufacturers;

- marketing strategies of manufacturers and the diffusion of medical lasers;

- incentives for the development of new laser applications.

Another subject that warrants inclusion in the thesis is laser safety. This, and a number of related issues, can be nicely illustrated by a case-history, presented by ECRI in the Fall 1993 issue of its newsletter Accident Investigator ${ }^{[10]}$.

'A young obstetrician/gynecologist who had recently completed his residency had been granted privileges to practice at a hospital. His privileges included the surgical procedures generally associated with that specialty, such as caesarian sections. Medical lasers had become available for some gynecological procedures, and the hospital had purchased a carbon dioxide $\left(\mathrm{CO}_{2}\right)$ laser for use in procedures involving the external genitalia.

The physician was aware that neodymium:yttrium-aluminum-garnet lasers had been approved by the US Food and Drug Administration (FDA) for certain intrauterine endoscopic surgical procedures, such as uterine septal ablation. He proposed that the hospital acquire an $\mathrm{Nd}: \mathrm{YAG}^{\prime} \mathrm{A}$ laser to perform such procedures and remain competitive with other local hospitals. Nd:YAG laser capability was also in demand by other surgical departments'.

Issues to be included are:

- the system of accreditation or certification of physicians;

- the influence of physicians on hospitals to adopt lasers;

- the role of the FDA in evaluating medical devices;

- the possibility to share lasers across departments.

'The hospital purchased a used Nd:YAG laser from the original manufacturer. The laser was inspected by the manufacturer before delivery. Even though later versions were available, the operator's manual provided with the unit was the same manual that had been supplied when it was purchased new by another hospital several year's earlier.

The physician attended an Nd:YAG laser training course specific to obstetrical and gynecological procedures taught by a physician of repute in that field. The course addressed theory and use and prowided hands-on training using an animal model. After the laser was delivered to the hospital, a manufacturer's representative provided training to nurses on setting up the laser and operating its controls.

Shortly after the training, a young woman was scheduled for a hysteroscopic examination (endoscopy of the uterus) with a possibility for laser septal ablation. The hysteroscopic examination revealed a uterine septum. In preparing for the laser ablation, the doctor distended the uterus employing a commonly used liquid. Although his experience with the use of the technology on humans was limited to observation of some procedures and although no physician experienced with this procedure was present to offer guidance or assistance, the physician elected to perform the procedure. Just as the ablation was 
completed, the patient suffered an unexplained cardiac arrest and could not be resuscitated. An autopsy revealed that her cardiac arrest was caused by a massive air embolism originating from her uterus:

The case was analyzed as follows. The laser beam from an Nd:YAG laser is transmitted to the operative sitte through a fiberoptic cannula (fiber), of which there are many different types. When energy is emitted from the end of the fiber, the fiber tip can become hot and must be cooled. Some fibers are made with a sheath tube, which is purged with a gas or liquid to cool the fiber tip. Unsheathed fibers are cooled by immersion in solution or use special tips that minimize heat production.

In this case, the patient's air embolism resulted from inappropriate use of an air-cooled, sheathed fiber. The investigation revealed that both the training course attended by the physician and the operator's manual for the laser warned against the use of $\mathrm{CO}_{2}$ to distend the uterus during laser surgery. However, neither addressed the use of air-cooled fibers in the uterus. In fact, the physician remembered seeing bubbles emanate from some fibers and not from others during his training.

Training provided for the hospital nurses by the manufacturer's representative did not detail the differences between fibers or appropriate fiber sellection for different procedures. Although the nurses are not responsible for selecting the fiber, training might have enabled them to detect the physician's error.

The manufacturer offered to provide technical assistance for 'first-time' procedures. However, the patient was scheduled before that technical assistance could be arranged. Although the nurses operating the laser were uncomfortable in proceeding with the case, the physician was selfconfident and elected to proceed. (Subsequent to the incident; a manufacturer"s representative told the hospital that an air-cooled fiber was appropriate. Thus, the presence of a manufacturer's representative may not have prevented the incident).

Subsequently, a review of medical device databases and clinical papers on hysteroscopic laser surgery revealed that experts in the field had published papers describing air embolism and warning against the use of air-cooled fibers in the uterus. The search also revealed previous reports of deaths related to intrauterine use of an air-cooled Nd:YAG laser fiber ${ }^{\text {tol }}$.

Issues to include are:

- the responsibilities of the professionals involved in using a laser;

- more general: laser safety measures and laser safety organization;

- the role of industry in organizing medical laser courses;

- more general: the responsibility for organizing educational courses;

- the contents of educational courses for physicians and laser nurses;

- the role of laser safety literature in improving clinical practice.

Some of the policy-implications of lasers can be illusitrated by the questions that Dutch Members of Parliament asked the State Secretary of Health in early 1994 on a new ophthalmological laser procedure, which are reproduced here ${ }^{[2]}$.

'1 Are you aware that excimer lasers are being used to treat myopia (nearsightedness)?

2 How many patients are being treated in the Netherlands and where is this taking place?

3 What is your opinion on this form of treatment? Do you endorse the view that it can cause a number of side effects, that the results cannot be guaranteed and that the condition it is intended to ameliorate, often improves with age anyway? 
4 What is your opinion of the fact that doctors and clinics buy expensive laser equipiment and recoup the costs in a commercial and irresponsible manner by approaching the public and promoting the treatment? What are your views of the fact that this form of treatment is advertised, for example in a school magazime and that infiormation evenings are held to drum up customs?

5 Do you share the view of $\mathrm{P}$. de Jong, a Rotterdam ophthalmologist, who says that excimer laser treatment should take place only in one clinic and the results carefully recorded so that risks, costs and results can be evaluated?

6 Do you intend subjecting laser surgery for myopla and the way in which it is advertised to further regulations?"

One of the general concerns of health care policy-makers that has not yet been explicitly discussed but which is highly important for the content of the thesis, are increasing health care costs. In 1991, the average share of total expenditure on health in total domestic expenditure in the OECD-countries was $8.1 \%$. Medical technology has often been pointed at as one of the important contributors to rising health care costs. Lasers are an example of fairly expensive high technology medical tools, with capital costs varying from a few thousand to nearly half a million dollars. Therefore, the question that should be asked is: do lasers represent a good investment in health? Or, as economists say: 'is it value for money'? This question is becoming more and more of interest to all players in the field and has been recognized by important organizations such as the World Health Organization, the World Bank, and the European Union ${ }^{[4]}$. A 1993 presidential column of the American Society for Laser Medicine and Surgery demonstrates the growing importance of this issue for physicians as well. The former President of the Society, Dr. R.G. Wheeland, starts out in his column that the era of health care reform in the US creates significant challenges in the immediate future. He then continues: 'For those of us who use hightechnology equipment (read that as "expensive') like lasers, in our clinical activities, those challenges are certain to be very real indeed. Not only are we likely to have to justify the utilization of a laser to perform a given surgical procedure, but we will also likely have to show the costeffectiveness of a laser procedure over the conventional procedure. Minimally invasive surgical procedures, many of which are performed using lasers, are obviously effective in reducing health care costs by shortening the hospital or eliminating hospitalization entirely by performing the procedure in ambulatory setting. However, the ultimate way to document the benefits of utilizing laser technology is by the accumulation and publiciation of objective data ${ }^{[3 t]}$.

It is these 'objective' data that we will be focusing on. Anecdotal information of what has been done in the past is provided by a statement of Arthur L. Schawlow, Ph.D., and Nobel Prize winner in physics for developing the maser, a device closely related to the laser. During the 1991 Arnual Meeting of the American Society for Laser Medicine and Surgery (ASLMS) he observed that there has been no methodological approach to the field of medical laser research. Until recently, he states, most research was carried out by clinicians performing non-randomized trials, with different devices and wavelengths, using widely different study-designs. This disorganized approach has confused many physicians and made it impossible to compare results in a productive inanner ${ }^{(i)}$.

It should be noticed that medical laser technology in general is still early in its life-cycle, which on the one hand can be expected to create difficulties in organizing evaluation studies but on the other hand creates good opportunities for policy-making. Also for other reasons the field of medical lasers could be interesting for policy-makers. The medical laser market sized over US\$ 200 million in 1990 and was characterized by a strong (double digit) growth. The total number of units sold was estimated over 30,000 in that year ${ }^{[18,20,21 !}$.

The impressions that make up this introductory chapter are worked out in the individual chapters of the thesis. The contents organize in five parts. Part 1 consists of the first three chapters, discussing a number of general issues. Part 2, covering chapter 4 to 11, provides an assessment 
of the evidence of the safety, effectiveness and costeffectiveness of sefected medical laser applications in six specialties. Part 3, covering chapter 12 to 15 , focuses on diffusion of medical lasers and minimally invasive therapy. Part 4 comprises two chapters, 16 and 17 , presenting casestudies on the effectiveness, cost-effectiveness, diffusion and policy implications of ophthamological laser applications. Part 5 discusses policy implications of lasers in general and covers chapter 18 to 20 . Of these, chapter 20 is a discussion chapter, focusing on the overall implications of what has been presented.

\subsection{References}

1. American Soclety for Lasier Medicine and Surgery (ASLMS). Lasers today and tomorrow. Naws from the Annual Meeting of the Amorican Society for Laser Medicine and Surgery (ASLMS). American Hospital Associalion, A specia member brieting, 1991 .

2. Annex B. Questions in Parliament and the reply of the State Secretary. In: Health Council of the Nethenlands, Standing Commitee on Medicine. Lasers in sight. Laser correction of refractive errors. Publication no. 199319E. Health Councit. the Hague, the Netherlands, 1993.

3. Anomymous. Lasers - Let there be light. The Economist, December 24, 1994 - January 6th, 1995: 96-8.

4. Banta: HD, Luce BA. Medical technology and its assessment. An international perspective. Oxford University Press. Oxfordi, New York, Tokyo, 1993 .

5. Banta HD, Schou I (Eds. I. Lasers in Health Care. Effectiveness, Cost-Effectiveness and Palicy-Amphications. Academic Publishing, Frederiksberg, Denmark, 1991.

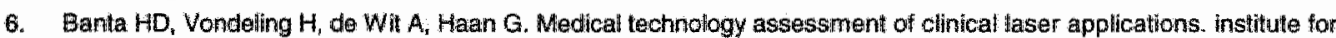
Medical Technology Assessment (iMTA). Report Number 90.04. IMTA, University of Limburg, Maastricht \& Erasmus University Fotterdam, Rotterdam, the Netherlands, 1990 (in Duich).

7. Banta HD (Ed), Minimaly invasive therapy in hve European countres. Diffusion, Effectiveness and Cost-Ettectiveness. Health Policy Monographs Vo: 3 . Elsewer, Amsterdam, London, New York, Tokyo, 1993.

B. Crosis F. Lasers take a shine into medicine. New Scientist 1986(20):38-43.

9. Diabetic Retinopathy Research Group (DRS). Preliminary report on the effects of photocoagulation therapy. American Journal of Ophthalmology $1976(81): 383-96$.

10. ECAI, Case history: the hazards of new technologies. In: Accident Investigator. The Newsletter of ECRI's Accident and Forensic Investigation Group $1993(2) 1: 1-2$

11. ECRI. Lasers. An: Heath Technology Checklist 1993/1994. Health Technology Trends 1993(9):Supplement.

12. ECAI. Towards ending the medical arms race. Health Technology Assessment News 1993, Nowember-December:1,4.

13. Foundation for Public Education on Science and Technology (PWT). Scientific intormation on Lasers. PWT. Feport Nr. 14, Utrecht, the Netheriands, 1990 (in Dutch).

44. Go PMNYH. Minimally inwasive surgery, Publication th the series: Proqgessin medicine; short and concise Amsterdam University Piress, Amsterdam, 1994 (in Dutch)

15. Hecht F. Teresi D. Laser, Supertool of the 1980s. Ticknor \& Fields Publlishers, New York, 1982. (Transiated as: Laser. Dodelik wapen, genezende straal. Elsevier Publishers, Amsterdam, Brussel , 1985).

16. Hofstetter $A_{\text {u }}$ Laser - Science Fiction oder eine neue Dimension in der Medizin? Urologe [A]1985(24):310-2.

17. Jacobs $M$. The light fantastic. Lasers brighten the future. The Futurist $1985(19) 6: 36-8$.

18. Lewilt MR. The kaser market in the 1990's. In: Forrest GT, Levitt MR (Eds.). The laser marketplace in 1991 . A semina: examining recent trends and directions in thie worldwide market for lasers. The Society of Optical instrumentation Engine ers (SPIE). SPIE Publication 1520, SPIE, Bellimgham, Washington, US, 1991, $p_{n} 1-36$.

19. Mekendale SC, KC Rustagi. The laser - a versatile tool. Impact of Science on Society 1988(149):47-59.

20. Moretti M. The Medical Marketplace. In: Forrest GT, Levitt MR (Ecis.). The laser marketplace in 1991. A seminar examining recent trends and directions in the worldwide market for lasers. The Society of Optical Instrumentation Engineers, (SPIE). SPIE Publication 1520 , SPIE, Bellinhgham, Washington, US, 1991, p. 118-31.

24. Müller GiJ, HP Berlien. The medical laser market in Germany. In: Banta HD, Schou I (Eds.). Lasers in Health Care. Effectiveness, Cost-Effectiveness and Policy-Implications. Academic Publishing, Frederiksberg, Denmark, 1991, p. 503.

22. Organization for Economic Cooperation and Development (OECD). OECD Health Systems. Facts and trends 19601991. Health Policy Studies No 3. Volume 1 and 2. OECD, Paris, France, 1993.

23. Reilnders L. Plaster. Is medical technology healthy enough? In; Schwartz M, Jansma $\mathbb{F}$ (Eds.). The technological culture. De Balie Publishers - S. Franke, Amsterdam, the Netherlands, 1989, p. $57-9$ (in Dutch). 


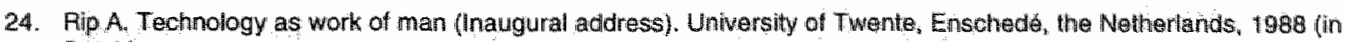
Dutch):

25. Schwarz M. Jansma $R$ (Eds.). The technological culture. De Balie Publishers - S. Franke, Amsterdam, the Netherlands, 1989 (in Dutch).

26. Schwarz M Entrance, Im: The technological culture. De Balie Publishers - S. Franke, Arnsterdam, the Netherlands" 1989, p $9-15$ (in Dutch).

27. Smit WA. Laser fusion. Interweavenenness of civil and military research. Farm Magazine 7801. Center for \|ssues on Science and Society, the Farm;" University of Twente, Enschede, the Netherlands 1978 (in Dutch).

28. Tymstra Tj. Should medical technology guide our lives? In: Beter worden door minder zorg. Ontwikkelingen in de Gezondheidszorg deel 14. Stichting Sympoz, Platform woor ontwikkelingen in de Gezondheidszorg, Amstelveen, 1992, p. 53-6 (in Dutch).

29. Wan Calmthout $M$. 'The laser is good for every normall human being". Interview with Prof. N. Bloembergen, Nobel Prize Winner for Physics in 1981. De Volkskrant, November 24, 1990 (daily newspaper, in Dutch).

30. wan Gemert MJC. Lasers, magic with light? (Inaugural address). University of Amsterdam, Amsterdam, the Netherlands, 1991 (in Dutch).

31. Wheeland RG. Presidential column (no title). American Society for Laser Medicine and Surgery Inc, News and Announcements. Lasers in Medical Science 1993(13):688-9. 
$\therefore$

3 


\section{Medical technology assessment and medical lasers}

\subsection{Introduction}

This chapter introduces medical technology assessment (MTA) and medical applications of lasers. Assessment of medical lasers is outtined and it is explained how the assessment of lasers has been carried out in the context of the development of the field of minimally invasive therapy. As a first example of this approach and to follow-up on Chapter 1, the use of lasers versus electrocautery as part of laparoscopic cholecystectomy is presented as a case.

\subsection{Some basic concepts}

In an influential publication in the Netherlands, the Public Health Future Forecast Repart of the National Institute for Health and Environmental Hygiene ${ }^{[45]}$ health is regarded as a dynamic process characterized by changes in equilibrium between the individual and its emwironment. The concept of health is defined in medical technology assessment as health-related quality of life or health status. A common definition of health-related quality of life is:" the value assigned to duration of life modified by the impaiments, functional states, perceptions, and social opportunities that are influenced by disease, injury, treatment, or policy ${ }^{\text {is0,62!! }}$. Basic information on the actual measurement of health-related quality of life (or health status), is provided in Chapter 4. Moving from the individual perspective to a more general perspective, publlc health is described as the health status of (parts of) the population. Health policy in its broadest sense can be thought of as the actions of governments and any other actors aimed at maintaining or improving the health status of a population ${ }^{[45]}$.

In order to serve as an input for health policy making the Public Health Future Forecast Report presents a basic model for public health ${ }^{[45]}$. The model consists of three parts: health policy, determinants of health and health status. Among the determinants of health are health care and health care technology. These determinants are, of course, subject to policy making. A widelyused definition of health care technology (or medical technology) is "the drugs, devices and medical and surgical procedures used in health care, and the organizational and supportive systems within which such care is provided"10! Thus, although this thesis focuses on the assessment of a device, the term 'medical technology' includes more than just devices' ${ }^{101}$. Health care technology is usually classified by distinguishing it on two dimensions: medical purpose and physical nature. The first dimension allows to distinguish six different purposes: diagnostic. preventive, therapeutic (including palliative), rehabilitative, organizational or adiministrative, and supportive. On the second dimension, copying the definition, three different types are distinguished: drugs, devices, and procedures ${ }^{[10]}$.

It is clear that health care and health care technology contribute significantly to a healthien population. Nevertheless, just as conventional technology "also health care technology has become controversial. This is partly due to its pervasiveness. Also, some health care technologies relate directly to existential issues, such as the nature of the mind or of life itself ${ }^{10 !}$. At the same time, the area of financial cost is certainly important. Societies pay for health care and health care technology, and the rising costs have become of growing concern ${ }^{19}$. As a result, and given the rapid technological change in health care, health care technology assessment (or medical technology assessment, in short MTA) has been developed. 


\subsection{Medical technology assessment}

\subsubsection{A general framework}

In the US, in 1975, the first health care technology assessment program was developed by the Office of Technology Assessment (OTA) of the US Congress ${ }^{[9.10 i}$. MTA is, of course, Just like health care and health care technology, also aimed at the overall goal of a healthier population. MTA involves a highly multidisciplinary approach to the evaluation of medical interventions in a very broad sense, aimed at supporting decision-making processes. In a narrow sense, MTA should serve as an aid in health policy decision making. In a broad sense (see the definition of health policy). MTA should be a source of information not only needed by policy-makers in developing policy and formulating legislation and regulations, but also a source of information needed by industry in developing products, by health professionals in treating and serving patients, and by consumers in making personal health decisions ${ }^{[9 !}$. MTA can be seen as an activity that contributes to the information that feeds back to health policy. To achieve this, four types of actions can be distinguished in a medical technology assessment program. These are: identlfication, testing, synthesis and dissemination.

Identification refers to the process of monitoring technologies and selecting those in need of study. A process of priority-setting is nowadays regarded as a separate step after identification. The selected technologies are tested, which means that appropriate data collection and analysis is conducted. The third step involves synthesizing existing information, combining the collection and interpretation of existing information and the results of the testing step. Usually, this step also includes making recommendations or judgments about appropriate use. The fourth step is the process of dissemination of this information to the appropriate persons who use or make decisions concerning the use of health care technology $y^{10]}$. In recent years, "dissemination' is seen as too passive a process, and methods of implementation are being sought. Implementation, which could be regarded as the fifth and final step, is intended to turn changes in knowledge and attitudes of physicians into changes in behaviour ${ }^{[11]}$. The results of synthesis, the dissemination- and the implementation step feed back to the step of identification (see Figure 2.1).

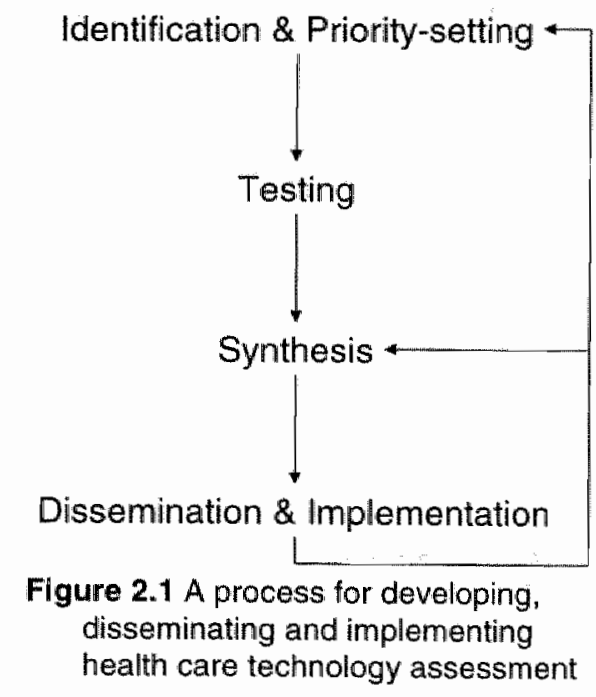


In practice, many national technology assessment agencies are not responsible for every stage in the process at the same time. For example, the Dutch Health Council identifies new technologies by monitoring technological developments and advises the Minister of Health on the scientific state-of the art of the safety and effectiveness of sefected medical technologies; covering the first and the third step in the process. The synthesizing activity of the Health Council is limited to existing information. Primary data collection is left to individual researchers.

Research in the field of MTA, covering the second step in the process, was stimulated when in 1988 a National Medical Technology Assessment Program was established by the Dutch Health Insurance Executive Council. This program, known as Investigational Medicine, is jointly funded by the Ministry of Education, Culture and Science (OC\&W) and the Ministry of Health, Welfare and Sport (WWS).

Priority setting in the program is achieved by assessing the scientific merits of the applications and, to some extent, by applying a number of societal criteria ${ }^{[39,47]}$. The results of the assessments in the context of the Investigational Medicine Program are presented to the relevant health care decision makers and disseminated in national and international journals in the field of medicine and, when appropriate, in health economics or other journals. To guarantee dissemination of assessments in the Netherlands, the results of each project will be published as part of a special scientific series of publications, which will be initiated by the Health Insurance Executive Council. Both INAHTA and the Cochrane Collaboration are anticipated to play a stimulating role in the international dissemination of the results of the Dutch assessment program ${ }^{[32]}$. The final step, implementation, is not yet well-developed.

As a variety of organizations is involved in either one or a combination of steps in the process, there is a need for coordination and integration ${ }^{[9-11]}$. This applies not only to the field of medical technology assessment, but to the entire field of clinical and health services research. How this should be achieved is a subject of debate in the Netherlands ${ }^{[3,40]}$. The need for coordination and integration of activities in the field: of medical technology assessment is also felt at the internationall level. Hopefully, the 1993 established International Network for Agencies in Health Care Technology Assessment (INAHTA), which unites HCTA agencies in Australia, the United States, Canada, France, the United Kingdom, Sweden, Spain, Switzerland and the Netherlands, will expand its role in this process in the coming years ${ }^{[14]}$.

\subsubsection{Technology assessment as an aid to decision-making}

The steps of a medical technology assessment program described above, should be made when the information is needed. This is a precondition for successful use of the information that can be obtained from an MTA program in decision-making processes. Thus, the timing of the assessment is important. Also, the right information should be available when it is needed, which refers to the content or the scope of the assessment.

In order to correctly decide when to assess a technology it is helpful to think of technology as going through a life-cycle. Usually, the life cycle of technology is subdivided in five stages. During the first stage one can think of technology which is not yet developed, so-called future technology. The second stage refers to technollogy prior to adoption. Technology in this stage is labelled as emerging technology. The third stage covers technologies which are actually in the phase of adoption. Technology in this stage is termed new technology. In the fourth stage, when the technology is widely used, the term accepted technology is applied. In the fifth and final stage technology has become old-fashioned and should be taken out of use. One speaks of obsolete technology in this stage. The concept of the life-cycle of a technology is closely related to elementary models for the development of medical technology, based on a distinction of basic 
hesearch, applied research, targeted development, and its diffusion in the health care system. The diffusion of a medical technology is described as its pattern of spread into the health care system, and consists of two stages: adoption, which refers to the purchase or acquisition of technology, and use. More information on diffusion can be found in Part Three.

Each stage in the life-cycle of technology requires its own decision-making processes, dependent on the type of technology and the actor. Restricting the discussion to medical devices here, in case of a concept for a future medical device, industry must decide whether or not to commit resources to develop it. Government agencies, e.g. the Ministry of Economic Affairs, may have to decide whether or not to subsidize the R\&D process. The selection of future medical devices for assessment and the scope of the assessment are based on their perceived importance, e.g. in terms of costs, impact on health, or impact on the health care system. Some authors, e.g. Hutton (1993), advocate an active role of biomedical engineers and potential users in assessment of technologies starting in this early stage in the life-cycle. This could be a way of testing the appropriateness of the initial design concept and could lead to a redirection of the research if the reaction of users is unfavourable. Also this approach, called constructive technology assessment, could help the developers to focus on the right type of solution for a particular problem or to identify the real problem that users would like to be solved ${ }^{\{36\}}$. To promote the role of biomedical engineers in medical technology assessment the International Federation for Medical \& Biological Engineering (IFMBE) established a special division for Health Care Technology Assessment in $1992^{1511}$. In general, the assessment of future devices is of a speculative nature and is rather uncommon.

If the development of the device proceeds, a point is reached where the term emerging technology can be applied. At some point the industry faces the decision if it wants to market and promote the device. Government agencies then have to decide if the device is allowed on the market and if yes on what conditions. Usually, contrary to new drugs and biologics, medical devices have to be tested for technical safety only before they are allowed on the market. This situation is characteristic for Europe (see Chapter 18). In the United States one can point at the role of the Food and Drug Administration (FDA) as a gate-keeper for marketing medical devices (since 1976). Manufacturers have to submit data on safety and effectiveness of selected (highrisk) technologies to the FDA, which then decides upon the allowance to market the technology.

In case of new technology, which has now by definition entered the market, decisions have to be made that affect its diffusion. More specifically, physicians and hospitals have to decide whether or not to purchase the technology. This is closely related to, among other factors, the anticipated annual number of patients eligible for treatment. In turn, patients have to give informed consent for treatment. Government agencies may have to decide whether or not to reimburse and/or to regulate its use. Sometimes these decisions have to be taken simultaneously because they interact. For example, a decision to reimburse the use of medical technology provides an important incentive for its diffusion. This can be anticipated by controlling the diffusion of the technology at this stage and have the technology evaluated in a limited number of centers. In general, it is necessary to assess efficacy and safety while the technology is in its early stages of its If e-cycle, in order to avoid the possibility that inefficacious or even harmful medical technologies rapidly diffuse in a health care system. It is perhaps for this reason that Goodman (1993) has characterized MTA as a 'look before you leap' form of inquiry is!! In selected cases, for example in case of high capital costs of the device or anticipated high volumes of treatment, economic evaluation is performed synchronically with the evaluation of safety and efficacy, preferably alongside randomized controlled trials. In general, cost-effectiveness or cost-utility analyses (see also Chapter 4 ) can be done at any stage in the life-cycle, but are probably most useful before widespread use ${ }^{[10]}$. Also, ethical implications that could only be speculated upon in the first two stages, may become clear. Furthermore, the technology may challenge certain 
Important societal beliefs. It might be that the time encompassed by this stage in the life-cycle of the technology is the most important time to collect data on its social effects ${ }^{[9]}$.

When a technology is in the fourth stage of its life-cycle, and has diffused widely (accepted technology): generally little attention is paid to it. The technology is considered mature, contrasting to its alleged immaturity in earlier stages in the life-cycle. However, despite this suggestive term, there are a number of reasons for examining a technology at this stage. Firstly, the costs of the technology tend to come down over time. Secondly, its users may have become more proficient. Thirdly, the device may become easier to handle because of manufacturer's modifications. Fourthly, the (combination of) indications for the technology may have changed after its introduction. Usually, indications broadem: the technology may be used with less severe cases of the disease or with older or younger people ${ }^{|10|}$. Fifthly, there may be a difference between the efficacy of a technology (as assessed under ideal circumstances, for example in the context of an RCT in centers of excellence) and its effectiveness (this refers to the benefits of the procedure assessed undier normal conditions of use in community seltings) ${ }^{[36 \|}$. If MTA research documents a difference between the optimum and the actual outcome of a technology-based intervention, methods and techniques from the field of quality assurance can be used to fill this $\operatorname{gap}^{[15]}$.

Whenever a new technology emerges for a specific disease, or when an existing technology has been demonstrated to be ineffective or harmful, it may or should, respectively, become obsollete. Assessment of efficacy, safety, costs and other aspects, depending on the classification of both the old and new technology, may provide information that can guide (partial) substitution processes $^{[10]}$. These processes are initiated by decisions such as to stop manufacturing, promoting, providing and paying for the technology.

Overall, three types of government policy making may result from an assessment in the last three stages of the life-cycle of a technology. A technology might be encouraged when the assessment shows that it is valuable enough to be diffused very rapidly. The technology might be discouragled if the opposite holds true, for example if the technology is not cost-effective (anymore). Thirdly, it might be left alone to diffuse or to be replaced without active policy intervention if the results of the assessment are interpreted as 'neutra| ${ }^{[10]}$. It has also been pointed at that assessment activities sometimes need to be repeated as the technology proceeds in its life-cycle. In theory, a technollogy can be assessed in each stage of its life-cycle, with a maximum of five times. In other words, MTA should be regarded as an iterative process ${ }^{[10,13,31]}$. The number and type of studies would be determined by the importance of technological decisions to be made and by the resources available for assessment. In practice, most assessments that have been done have dealt with technology in the later stages of its life-cycle. One reason is perhaps that, compared to early assessments; more and more accurate information is available at later stages in the lifecycle. This is not an argument for waiting, however. Decisions must be made on the technology, and they should be based on the best information that can be developed. If mistakes are made in early assessments, they can be corrected in assessments carried out in subsequent stages of the life-cycle ${ }^{[?]}$.

\subsection{Lasers and medical applications of lasers}

\subsubsection{Lasers}

Laser is an acronym for light amplification by stimulated emission of radiation. The active laser material is a collection of atoms, moleculles or ions of a solid, liquid or gas in which absorbed energy from an external source is converted to intense beams of light with specific characteristics that allow a variety of applications in society (see Chapter 1). 
The principles upon which the laser functions were established in the early twentieth century with Bohr's theory of quantum electron states and Einstein's theory of stimulated emission. As early as 1917, Einstein proposed that an atom in an excited state could be forced to produce a photon (a single unit of optical energy) of a specific wavelength, travelling in a specific direction, if it is struck by a photon possessing the same wavelength and direction. From 1917 to the 1950 s, quantum theory was researched extensively using techniques of atomic and molecular spectroscopy. In 1958, Schawlow and Townes described the physical principals of microwave amplification by stimulated emission of radiation (maser), a concept first shown to be feasible by Gordon and colleagues in 1955. In 1960, Theodore Maiman, at Hughes Aircraft Company, generated the first laser beam by exiting a ruby rod with intense pulses of light from a flash lamp "It was Maiman who first coined the acronym laser. In 1961 Javan et al. developed the first continuous wave (cW) laser using a helium/neon gas mixture, and Johnson developed the Nd:YAG laser, which emits in the near infrared portion of the spectrum. The argon laser, a laser that emits. in the blue-green part of the spectrum, was built in 1962 by Benett ${ }^{[28]}$. Also the first diode laser was built in $1962^{[55]}$ Various wavelengths in the visible and infrared area of the spectrum can be achieved with this type of laser ${ }^{[57]}$. The $\mathrm{CO}_{2}$ laser, which emits spectral energy in the far infrared area of the spectrum, was developed by Patel et al. in $1964^{[28,30]}$. In that year, the Russian researchers Basov and Prokhorov, and Townes (US) shared the Nobel prize in physics for their work $k^{[30 ; 611}$.

The name given to the laser (ruby, $\mathrm{HeNe}, \mathrm{Nd}: Y A G$, argon, $\mathrm{CO}_{2}$ ) is representing the lasing component of the laser medium. However, there are some exceptions. Diode lasers utilize semiconductor materials as laser medium. Dye lasers utilize a medium consisting of an organic dye in a liquid solvent. Common dyes are couramine and rhodamine. Free electron lasers utilize a stream of excited electrons passing through an undulator or wiggler as the active medium. A final category are the so-called Excimer lasers. Excimer is a contraction of excited dimer. A homopolar dimer is a molecule consisting of two identical atoms, such as $\mathrm{N}_{2}$ or $\mathrm{O}_{2}$. Basov first demonstrated in 1970 that the excited homopolar dimer $\mathrm{Xe}_{2}$ could form the basis of a laser. It was quickly found out that other homopolar dimers, but especially heteropolar dimers, consisting of mixtures of noble gases and halogens (e.g. krypton and fluoride or xenon and chloride) could form the basis of efficient pulsed systems, emitting at ultraviolet wavelengths ${ }^{[19 ; 56]}$.

All present-day lasers basically have the same structure. The active laser medium is enclosed in a resonator or optical cavity with two parallel mirrors at each end. The reflective axes of the mirrors are optically collinear with the axis of the cavity. The external source of energy that drives the process usually is an electric current or an intense optical light source, depending on the laser medium. This energy is often called the pumping energy because it pumps the atoms of the active material to higher energy levels. Atoms that are used for generating laser light all possess one or more higher energy levels which have an exceptionally long life-time, or a metastable state. This allows development of a 'population inversion'. In this situation there are more atoms in an excited energy state than in a lower energy state. A single photon of the correct energy, emitted by some other atom, can serve as a trigger to stimulate the excited atoms residing in the metastable state to emit their energy coherently with the trigger photon. This causes the process of stimulated emission, in which excited atoms are stimulated to emit their energy. This process would not continue long if the photons of light were allowed to completely exit the active medium. This is prevented, however, by a partially reflective mirror that lets only a predetermined fraction of the laser light 'leak' through, while reflecting the remaining light back into the active medium. This allows the process of stimulated emission to amplify the light as it passes the additional excited atoms. At the back end of the active medium is a second mirror, which is totally reflective. It returns the photons of light back into the active medium, where more excited atoms produce further amplification. Stimulated emission continues as long as the input energy from the pumping source is sufficient to maintain a population inversion ${ }^{|39|}$. 
The characteristics of laser light can be briefly summarized:

- it is monochromatic, meaning that the laser can be designed to generate only one colour (or one wavelength) at a time. Tuning an optical element within the laser allows the operator to change from one wavelength to another ${ }^{[3,56]}$. Both free ellectron tasers, which are still highly experimental, and dye lasers are especially well-suited for tuning.

- it is coherent, meaning that all light waves of one frequency emitted from a laser are in phase and parallel with one another ${ }^{[56,61\}}$

- it is collimated; due to spatial coherence, photons in a laser beam propagate in a single direction with very low divergence ${ }^{155,671}$.

These properties differ markedly from ordinary light, which is multichromatic and neither coherent nor collimated. Therefore, according to Absten and Joffe (1989), lasers is to light what music is to noise ${ }^{[2]}$

\subsubsection{Medical applications of lasers}

Laser applications in medicine promptly followed the development of lasers in the early 1960's. The first medical applications of the first laser; the ruby laser, were reported in 1962, in ophthaimology and dermatology ${ }^{[1,30,55]}$. Since then medical applications of lasers were increasingly described in the medical literature. New types of lasers and new types of laser beam delivery systems have determined the development of medical laser applications. Especially the development of endoscopes in the 60 s and the development of thin, flexible optical fibers in the 70 s (a spin-off of the communication industry ${ }^{1 / 3}$ ) largely expanded the potential use of lasers in medicine. Transmission of laser light allows the endoscopist to channel laser energy to internal body organs with non-invasive or minimally-invasive techniques. This offers laser treatment of a variety of conditions in specialities such as gastroenterology, urology, pulmonology, otolaryngology and gynecology. Likewise, optical fibers can be intraduced in catheter systems, allowing laser treatment of several cardiovascular conditions. As a result, lasers have gradually found applications in virtually every specialty of medicine.

Table 2.1 lists the different types of lasers that are presently used in medicine. Basically, in Table 2.1 three types of lasers are distinguished, based on the wavelength of the light emitted. Medical lasers are availlable in a wide range of the electromagnetic spectrum, ranging from the infrared (IR) (10 $600 \mathrm{~nm}$ for the $\mathrm{CO}_{2}$ laser) to the ultraviolet (UV) (193 nm for the argon fluoride (ArF) Excimer laser). Table 2.1 also shows that the majority of the different types of lasers are used by more than one specialty. Especially the $\mathrm{CO}_{2}$ laser and the Nd:YAG laser are used in wide variety of specialties. Theoretically, this offers the opportunity to share lasers across specialties. In contrast, the use of some other types of lasers is confined to only one specialty, usually ophthalmology. Of all medical llasers, the argon laser is used most frequently, especially due to its applications in ophthalmology.

As outlined in paragraph 2.4.1 lasers can also be categorized according to the properties of the laser medium, which can be a solid, a gas or a liquil.

Solid-state lasers include the crystal-lasers (Er:YAG, Ho:YAG, Nd:YAG, KTP) and diode lasers. In crystal lasers either erbium, holmium, neodymium or other atoms are embedded in a matrix of crystalline Yttrium-Aluminum-Garnet ${ }^{[57]}$. KTP refers to Potassium-Titanyl-Phosphate, which is a crystal that is used to halve the wavelength (double the frequency) of the $1064 \mathrm{~nm} \mathrm{Nd:YAG}$ (invisible) laser light to produce the $532 \mathrm{~nm}$ (green) light (see also Chapter 1). Modern publications usually refer to the frequency-doubled Nd:YAG laser, because the KTP crystal is not the only crystal that can accomplish Nd:YAG laser frequency doubling. The diode in diode lasers is made by wafers of positivelly and negatively charged semiconductor materials. Gas lasers include $\mathrm{CO}_{2}-$, 
argon-, krypton-, copper-vapour- (Cu), gold-vapour- (Au), excimer-and HeNe lasers. HeNe lasers; which emit red light, are used to aim the light of lasers operating in the invisible part of the spectrum. To achieve this the aining beam is transmitted coaxially with the main laser beam. in addition, HeNe lasers are used experimentally in selected clinical applications ${ }^{[57]}$. Dye lasers are the only lasers that use a liquid medium: One of the main advantages of dye lasers is that their emission can be tuned to a variety of wavelengths.

Table 2.1 Lasers in medicine

\begin{tabular}{|c|c|c|c|c|}
\hline \multicolumn{2}{|c|}{ Medium } & \multicolumn{2}{|c|}{ Wavelength (nm) } & Specialty \\
\hline \multicolumn{2}{|c|}{$\mathrm{CO}_{2}$} & 10600 & (IR) & 1356789101415 \\
\hline \multicolumn{2}{|c|}{ EnYAG (Erbium) } & 2940 & (IA) & $2,13,16$ \\
\hline \multicolumn{2}{|c|}{ Ho:YAG (Holmium) } & 2060 & (IR) & $2,13,16$ \\
\hline \multicolumn{2}{|c|}{ Nd:YAG cw/qs } & 1064 & $(\| \mathrm{A})$ & $1.23456781011 e+29$ \\
\hline \multicolumn{2}{|c|}{ Nd:YAG cw/qs } & 1318 & (IA) & \\
\hline \multicolumn{2}{|c|}{ GaAs (diode) } & 904 & $(\mathbb{R})$ & 11 \\
\hline \multicolumn{2}{|c|}{ GaAsP (diode) } & $630-800$ & (IR,VIS) & 11 \\
\hline \multicolumn{2}{|c|}{ HeNe (Helium-neon) } & 633 & (VIS) & aiming laser \\
\hline \multicolumn{2}{|l|}{ Dye } & $320-980$ & ,VIS,UV) & $1,3,7,8,11,12,15$ \\
\hline \multicolumn{2}{|c|}{ Krypton } & $\begin{array}{l}647 \\
568\end{array}$ & $\begin{array}{l}\text { (VIS) } \\
\text { (VIS) }\end{array}$ & $\begin{array}{l}11 \\
11\end{array}$ \\
\hline \multicolumn{2}{|c|}{ Gold-Vapour } & 628 & (VIS) & $1,3,7,8,12,15$ \\
\hline \multicolumn{2}{|c|}{ Copper-Vapour } & 511,578 & (VIS) & $3,6,7$ \\
\hline \multicolumn{2}{|l|}{ KTP } & 532 & (VIS) & $1,3,6,7,10,12$ \\
\hline \multicolumn{2}{|c|}{ Argon } & $\begin{array}{l}514,5 \\
488\end{array}$ & $\begin{array}{l}\text { (VIS) } \\
\text { (VIS) }\end{array}$ & $\begin{array}{l}11 \\
2,3,5,11,13,16\end{array}$ \\
\hline \multicolumn{2}{|c|}{ XeF (Xenon Fluoride; Excimer) } & 351 & (UV) & $2,13,16$ \\
\hline \multicolumn{2}{|c|}{$\mathrm{XeCl}$ (Xenon Chloride; Excimer) } & 308 & (UV) & $2,11,13,16$ \\
\hline \multicolumn{2}{|c|}{ KrF (Krypton Fluoride; Excimer) } & 248 & (UV) & 11 \\
\hline \multicolumn{2}{|c|}{ ArF (Argon Fluoride; Excimer) } & 193 & (UV) & 11 \\
\hline \multicolumn{5}{|c|}{ Specialty } \\
\hline \multicolumn{2}{|c|}{$\begin{array}{l}1 \text { Anaesthesia } \\
4 \text { Gastroenterology } \\
7 \text { ENT } \\
10 \text { Neurosurgery } \\
13 \text { Radiology } \\
16 \text { Vascular Surgery }\end{array}$} & $\begin{array}{l}2 \text { Cardio } \\
5 \text { Gyneo } \\
8 \text { Pulmo } \\
11 \text { Ophth } \\
14 \text { Dentis }\end{array}$ & $\begin{array}{l}9 y \\
\text { ogy } \\
\text { ology }\end{array}$ & $\begin{array}{l}3 \text { Dermatology } \\
6 \text { General Surgery } \\
9 \text { Oral Surgery } \\
12 \text { Oncology } \\
15 \text { Urology }\end{array}$ \\
\hline $\begin{array}{l}\text { VIS } \\
\text { UV }\end{array}$ & 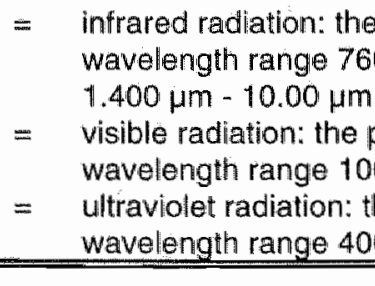 & $\begin{array}{l}\text { rtion of the } \\
-1 \mathrm{~mm} \text {, } \\
\text { tion of the } \\
400 \mathrm{~nm} \text {. } \\
\text { portion of } \\
760 \mathrm{~nm} .18\end{array}$ & $\begin{array}{l}\text { ectromagn } \\
\text { h the regio } \\
\text { lled the ne } \\
\text { ctromagne } \\
\text { electroma } \\
\text { rce: }{ }^{[26 \mid)} \text {. }\end{array}$ & $\begin{array}{l}\text { tic spectrum within the } \\
\text { is } 760 \mathrm{~nm}-1.400 \mu \mathrm{m} \text { and } \\
r \text { - and mid-IR regions. } \\
\text { c spectrum within the } \\
\text { netic spectrum within the }\end{array}$ \\
\hline
\end{tabular}


As noticed earlier, there is a variety of methods of delivering the laser beam from the resonator to the target tissue. The three basic methods are: direct irradiation, transmission through an articulated arm, and transmission through flexible optical fibers ${ }^{\left[a, w_{3}\right]}$. Nowadays all types of lasers can be transmitted through optical fibers with the exception of $\mathrm{CO}_{2}$ lasers and the $193 \mathrm{~nm}$ ArF Excimer laser. This is a result of gradual developments in fiberoptics. $\mathrm{CO}_{2}$ lasers have historically only been used on the surface of the body or in parts where an 'articulatied arm' can make the tissue accessible, such as in the abdomen. An 'articulated arm' or 'optical arm' consists of a hollow tube with several mechanically moveable joints. At each joint, a mirror directs the beam through the tube to the next mirror ${ }^{[39 !}$. In the late eighties (hollow) wave guides have become available to transmit $\mathrm{CO}_{2}$ laser light, which has expanded its use in for example ENT and gynecology ${ }^{2}$ :ab . Light of other lasers, for example argon and continuous wave Nd:YAG laser light can be transmitted through optical fibers since the early seventies ${ }^{[n]}$.

Lasers can be used in two temporal operation modes: either continuous wave (cw) or pullsed. The argon laser and almost all applications of the $\mathrm{CO}_{2}$ laser are continuous. The duration of the $\mathrm{cw}$ is usually determined by a foot switch on the laser. Commonly, the laser exposures are in the range of 0.1 to 100 seconds. Pulsed lasers, for example Excimer lasers, have more recently entered the clinic and deliver pulse durations from 100 femtoseconds to 500 microseconds ${ }^{[37]}$. By operating lasers in a pulsed mode, high peak powers can be obtained, and damage to surrounding tissue adjacent to the lasing site can be limited ${ }^{[57]}$. Q-switching, applied in particular in Nd:YAG lasers, is one of a variety of methods to obtain high power, short duration light pulses ${ }^{[57}$. In general, it is easier to transmit cw laser light through optical fibers than pulsed laser light. To illustrate this, as recently as the early nineties optical fibers have become available that can be used to transmit $Q$ switched Nd:YAG laser light ${ }^{[20]}$.

There is a variety of application systems for pulsed and $\mathrm{CW}$ lasers. $\mathrm{CO}_{2}$ laser light may be applied by directing it to a focusing lens in a hand piece. To use $\mathrm{CO}_{2}$ laser light as a tool for very precise operation a micromanipulator is used. This device, attached to the operating microscope, directs the beam by moveable mirrors, controlled by a joy-stick, to the center of the microscope's field of view. The viewing system of the microscope and the laser beam can be brought in focus at the same location ${ }^{[3]}$.

In ophthalmology, laser light is almost always applied through a slit lamp similar to a micromanipulator on an operating microscope ${ }^{[2]}$. In dermatology, laser light can be applied by means of a hand piece at the end of the fibre. This is sometimes combined with a scanner, e.g. in treatment of portwine stains. In case of fiberoptic delivery, tips may be positioned at the end of the fiber, allowing direct contact with the tissue. Frequently used are sapphire and metal-tips (or 'hot' tips), especially in angioplasty. A second technique that allows direct tissue contact is by using probes at the end of the fiber, for example in selected ophthalmological procedures (endocular probes $)^{1201}$.

\section{Laser-tissue interaction}

Laserlight can be reflected, transmitted, scattered or absorbed by tissue. Sometimes, one or more of these processes happen the same time, each representing a percentage of its energy. When laser light is absorbed, it is converted to other types of energy, which results in clinical effects. The process of conversion is usually termed laser-tissue interaction (LTI). This process is dependent on the properties of the laser and the properties of the tissue. One of the clearest descriptions of $L_{\text {LTI }}^{[37 \mid}$ is by Jacques (1993), who uses the picture of an interaction cube to classify LTI. The three axes $(x, y, z)$ of this interaction cube are the interaction mechanism $(x)$, the level of biologic organization $(y)$ and the time course of the tissue reaction $(z)$. The $x$-axis distinguishes photothermal, photochemical and photomechanical interactions. The $y$ axis distinguishes the level of organelles, cells and tissues, whereas the z-axis distinguishes immediate physical effects, delayed response and long-term healing. Using this interaction cube, LTI can be described in terms of a combination of one or a combination of 27 possibilities. Less specific but more common in

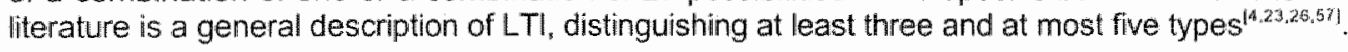


The most elaborate classification of interaction mechanisms includes: photothermal, photoacoustic, photoablation, photochemical and photobiostimulation.

In case of photohermal LTI the laser light is canverted to heat in the tissue. This is the mode of action of, for example; the $\mathrm{CO}_{2}$ laser, the argon- and the $\mathrm{CW}$ Nd:YAG laser. Therefore, these lasers are also called "thermal lasers'. Again, most authors distinguish at least 3 and at most 5 types of effects of heat on biologic material: hyperthermia, coagulation, carbonization, thermolysis and vaporization.

Generally, thyperthermia involves a long exposure to a mildly elevated temperature, and the effects are not immediately apparent to the eye but rather usually require at least 24 hours to develop into necrosis ${ }^{[27]}$. For example, hyperthermia treatment of liver tumor nodules has been performed using Nd:YAG lasers in combination with percutaneous interstitial probes ${ }^{[2.57]}$. The distinction between hyperthermia and photocoagulation is not strictly defined, but generally, photocoagulation requires only a short exposure to a high temperature and the effects are immediate and visible ${ }^{[37 !}$. Depending on the author, the principle of either hyperthermia or photocoagulation is used in hemostasis, tissue welding and induction of necrosis in tumors ${ }^{[2,5,56.60]}$.

Carbonization and thermolysis are terms for the processes that occur when biologic material is heated between 100 and 300 degrees Celsius. The $\mathrm{CO}_{2}$ laser can be used to vaporize tissue, which occurs when temperatures rise to over 300 degrees Celsius. When the vaporized zone is small (100-500 $\mu \mathrm{m})$, the effect of an incision is obtained. The $\mathrm{CO}_{2}$ laser is therefore often used as a surgical tool.

The argon laser falls between the $\mathrm{CO}_{2}$ and the Nd:YAG laser. Its main applications have historically been in dermatology and in ophthaimology, where the device is used, among other applications, to coagulate blood vessels e.g in treatment of diabetic retinopathy and portwine stains in adults.

Photoacoustic LTI is also known as photodisruptive and photomechanic LTI. The photomechanical effect is the result of plasma formation, which' expansion generates a spherical shock wave, propagating at sound velocity, which leads to localized mechanical rupture when the pressure rise is higher than the yield strength of the tissue. Important applications for photoacoustical applications can be found in ophthalmology and urology. The Q-switched Nd:YAG laser is used for photodisruption of secondary cataract (posterior capsulotomy) in ophthalmology. Pulsed dye lasers are used to fragment impacted ureter stones ${ }^{156 !}$.

Photoablation is the type of LTI when laser light induces a rupture of bonds between tissue molecules. The breaking of intramolecular bonds, also called photodissociation, occurs through conversion of photonic energy to kinetic energy and, to a small extent, heat. The photoablative effect can be used for very precise removal of tissue. For example, pulsed Excimer lasers are used to recanalize arteries by ablating plaque and in correcting myopia by ablating cell layers from the central part of the cornea. in contrast with thermal lasers, Excimer lasers are sometimes called 'cold lasers' because of the relatively small thermal effects produced ${ }^{\text {[3a! }}$. The applications listed here are discussed in more detail in Chapter 6, 17 and 19.

In general, photochemical LTI refers to the induction of chemical changes in tissue resulting from illumination by laser light. Although not completely correct the term is usually identified with the interaction of lasers with photo-sensitizers. This interaction is used in photodynamic therapy (PDT). In PDT, systemic injection of a photosensitizer with specificity for tumor cells is intravenously administered, followed by illumination of the tissue with light of a wavelength that is absorbed by the photosensitizer. This causes a phototoxic reaction, resulting in selective destruction of the tumor. Historically, the most commonlly used photosensitizer is hematoporfyrine derivative (HPD). Nowadays usually a purified form of HPD, Photofrin 11 , is used in most clinical applications ${ }^{|4|}$. To achieve absorption a laser is used that emits red light (in the range of 625-630 $\mathrm{nm}$ ). Usually a cw dye laser or, less common, a copper-vapour laser is applied for this purpose ${ }^{14.57 !}$.

Photobiostimulation refers to the induction of alterations in the biochemical system of mammalian cells, leading to changes in physiological functioning ${ }^{156 \mid}$. These alterations can both imply decreases 
or increases of, for example, hemoglobin synthesis, leucocyte-phagocytosis, immune response, fibroblast procollagen production, alteration of organ parenchymatous activity, etc but in general most emphasis is placed on the stimulation of synthesis processes. As the most probable mechanism of photobiostimulation is photochemical, some authors categorize biostimulation under this heading ${ }^{[23.56,50)}$. Some of the most frequent clinical applications of biostimulation are HeNe or diode laser treatment of acute and chronic pain and in stimulating wound healing ${ }^{|20,56|}$. The lasers that are used for biostimulation are often considered as a separate group called 'low power lasers' or 'soft lasers'. Low power lasers can be defined as lasers that deliver an output power in the milliwatt range, compared with the output power of 1-150 watt of other types of lasers, which are collectively called 'high power lasers ${ }^{i 56 !}$. This distinction is not always correct, as diode lasers can be used for both high power (e.g. in ophthalmology) and low power applications. This depends, in part, on its delivery mode (cw versus pulsed) ${ }^{[57]}$.

\subsection{Assessing medical applications of lasers}

\subsubsection{Identification and selection}

In 1985, the Dutch Steering Committee on Future Health Scenarios supported a study to identify future and emerging technology and to do prospective assessment on some selected technologies ${ }^{[s]}$. Medical lasers were chosen as one of the 6 highest priority technologies. It was recognized that medical lasers were a relatively new, rapidly changing technology which could have strong impacts on the health care system of individual countries. On the one hand, it was argued, lasers offer potential benefit to health, but on the other hand the capital investments were considered to be reasonably large for an individual laser, and quite large in the aggregate. Nonetheless, health care applications of lasers were, and to some extent still are, a growth industry. Companies with the capability of working with laser technology were moving into health care and started producing specialized instruments. Thus, lasers combined concerns about health benefits, limited resources in health care " and economic development for the Netherlands. Considering the potential of cardiovascular laser applications, a prospective assessment of lasers in cardiology was carried out to examine possible futures ${ }^{[\theta]}$. As far as we know this is the only study on future technology in the laser field. In all other countries identification and selection of lasers as a subject for assessment happened in a later stage of the life-cycle of the technology. In Australia, for example, the National Health and Medical Research Council examined the use of laser acupuncture devices (a low power laser application) in 1983. The Council concluded that there was no valid evidence of any beneficial therapeutic effect from such devices and expressed their concern about their safety. In September 1983 the Medical Advisory Committee of the Council noted the other applications of lasers in medicine and saw a need for a better understanding of their benefits and risks ${ }^{[6]}$.

\subsubsection{Timing and scope of the assessment}

The timing of the first assessment of any technology may be a problem. If the technology is assessed too early, the evaluation may be of little benefit both for the researchers and policymakers. The technology may not be mature, new uses may appear continually and, as a result of a too early evaluation, technological change may be impeded. If the technology is assessed too late, the evaluation may be of little use to decision-makers. This problem is embodied in Professor Martin Buxton's statement, "It's always too early to assess a technology, until, suddenly, it"s too late." Although iterative evaluation gives a partial answer to this dilemma, one may wonder how this has been dealt with in the case of lasers. Also, when it was decided to assess lasers, many times only specific applications were assessed. Of the selection of applications subsequently often a selection of aspects (effectiveness, cost-effectiveness, safety etc.) was made. 
In the United States, the country where lasers where first manufactured and marketed, a first assessment on $\mathrm{CO}_{2}$ lasers in surgery was published in 1982 by the Office of Health Technology Assessment of the Department of Health and Human Services ${ }^{[46]}$. This was followed by a Dlagnostic and Therapeutic Technology Assessment (DATTA) on $\mathrm{CO}_{2}$ lasers in gynecologic malignancies ${ }^{12 a}$. These assessments focused on the safety and efficacy of laser treatment, just as subsequent assessments of $\mathrm{CO}_{2}$ lasers in head and neck surgery ${ }^{\text {fa3 }}$, of argon lasers in trabeculoplasty (laser treatment of glaucoma, an ophthalmological condition) ${ }^{\text {[4t4 }}$, and of $Q$-switched Nd:YAG lasers in posterior capsulotomy ${ }^{42]}$ by the National Center for Health Services Research in $1984^{[22-4]}$. At the time of the assessment both the use of argon lasers in treatment of trabeculoplasty and the use of $\mathrm{CO}_{2}$ lasers in head and neck surgery was already widespread in the US $S^{143.44}$. In July 1984, two manufacturers received premarket approval for two models of $Q$ switched Nd:YAG lasers from the US Food and Drug Administration, based upon their submission of safety and effectiveness data from controlled clinical trials examining its potential for treatment of posterior capsulotomy. According to the US National Eye Institute, the procedure was becoming widespread at the time of the assessment ${ }^{[42]}$. Since then many assessments have followed on a variety of laser applications. Original data collection has been funded on a large scale in particular by the National Eye Institute (NEI) of the National Institutes of Health (NIH). Reviews and other synthesizing activities have, among others, been organized by ECRI and physician societies such as the American Medical Association (AMA) (the Diagnostic and Therapeutic Technology Assessment (DATTA) Program) and the American Society for Gastrointestinal Endoscopy (ASGE)).

In Australia, lasers were identified and selected for assessment in 1983. In that year the Medical Advisory Committee of the National Health and Medical Research Council asked the Australian National Health Technology Advisory Panel (NHTAP) to review the applications of lasers in medicine. A report of this first assessment was produced by the National Health Technology Advisory Panell in 1985, covering laser applications in ophthalmology, dermatology, neurosurgery, otolaryngology, gynecology , pulmonology, peripheral vascular surgery and gastroenterology. Also, some attention was paid to photodynamic therapy and the use of low power lasers ${ }^{[8]}$. These assessments were all timed early in the life-cycle of almost all laser applications discussed, illustrated by a number of no more than 10 lasers in use per specialty. The exception was an ophthalmological application, photocoagulation of the retina. For this indication more than 40 lasers were in use at the time of the assessment, representing nearly half of all lasers (83). The report covered issues of safety, training, efficacy; cost-effectiveness and diffusion of lasers ${ }^{(5)}$. A supplementary report, on the cost-effectiveness of laser applications in gynecology, was published in $1987^{[5]}$. The original Australian assessment (1985) was updated in $1993^{[4]}$. Medical lasers have developed rapidly and it is therefore highly desirable to iteratively review this subject, as is the case with many other technologies ${ }^{[15]}$.

At the level of the European Community, in 1988 the COMAC-BME reported on the results of a workshop on safety of medical laser applications and laser-tissue interaction ${ }^{[27]}$. Also in 1988 , the COMAC-HSR decided to support studies in economic appraisal of medical technology. One of the three studies funded focused on medical laser applications. The project (1988-1991) included, by selection, laser applications that were more or less in common use. Thus, exceptions were made for areas that were identified as clearly experimental, such as orthopedics and neurosurgery. As a result, 43 laser applications were covered in dermatology, ophthalmology, otolaryngology, pulmonology, cardiology and peripheral vascular surgery, gastroenterology, urology and gynecology. Photodynamic. Therapy and low power lasers were discussed as separate issues. In addition, attention, was paid to lasers in dentistry. The study focused on both effectiveness, costeffectiveness and policy implications of lasers and was published in $1991^{1121}$

In the Netherlands, the Dutch Working Group on Assessment of Medical Equipment in University Hospitals published a report on laser safety in $1984^{[16]}$. The report was limited to those specialties where lasers had actually been introduced. At that time about 80 lasers were in use in the Netherlands, predominantly in ophthalmology. The Dutch Health Council published a literature 
survey of the (cost)-effectiveness of the use of lasers in gynecology in 1990:5]. The study covered both mature and immature applications. Furthermore, the most important technology assessment program in the Netherlands, the Investigational Medicine Program; Iunded a number of studies evaluating laser applications in the early nineties. Examples are excimen laser coronary angioplasty, dye laser treatment of portwine stains and photodynamic therapy of non-small cell lung cancer (NSCLC).

In summary, although some countries have been earlier and more advanced in their approach than others the assessment of lasers has followed more or less a common paltern. Only one future assessment was performed. All other assessments focused on a selection of laser applications that were already in clinical use. Safety and efficacy of medical lasers were of major concern in most early assessments, although occasionally attention was paid to diffusion processes In later assessments the scope usually was broadened to include cost-effectiveness of lasers and policy implications. No ethical aspects were ever identified ${ }^{[4,12]}$.

Combining the theoretical framework of medical technology assessment with the data on lasers as presented here and in Chapter 1 , it is suggested that safety, efficacy, effectiveness, costeffectiveness, the organization of laser facilities, and the education, training, and certification of laser-users and assisting personnell are relevant aspects to include in an assessment of medical lasers. Also attention needs to be paid to the diffusion and policy implications of lasers.

\subsection{Assessment of medical lasers in the context of the development of minimally invasive therapy}

\subsubsection{Introduction}

As explained in Chapter 1, an assessment of medical lasers should be understood in the broader context of the development of the field of minimally invasive therapy (MIT). The field of MIT became more important in the late eighties. A proposal to assess this field was granted to the Netherlands Organization for Applied Scientific Research (TNO) by the FAST Program of the European Commission and the Swedish Council on Technology Assessment in Health Care (SBU) in 1991. The project was headed by Prof. Banta. The assessment was carried out in five countries: the United Kingdom, Denmark, France, Germany, and the Netherlands ${ }^{[34)}$. This project offered the possibility to understand the results of the EC-study on lasers and its update as presented in this thesis in the broader context of the development, effectiveness, cost-effectiveness and diffusion of selected cases of MIT. Ten cases of minimally invasive therapy were included in the study, including laser treatment of bladder tumours and palliation of colon cancer by endoscopic intervention. Lasers play a major role in this intervention. A third case where lasers play a role is endoscopic treatment of upper gastrointestinal bleeding. The remainder of cases included extracorporeal shock-wave lithotripsy and percutaneous nephrolitotomy, laparoscopic treatment of endometriosis, laparoscopic removal of ovarian cysts, laparoscopic cholecystectomy. laparoscopic appendectomy, catheter treatment of coronary artery disease, and arthroscopic knee surgery. The ten cases were selected based on the idea that they should represent a mixture of applications that were already in widespread use and applications that could potentially come into widespread use. Thus, some of these assessments were intended to take place relatively early in the life-cycle of each technology, whereas others were intended to evaluate more mature applications. Also, to set an example for evaluation of minimally invasive therapyn, an assessment was included of a mature, well-evaluated laser application, argon laser treatment of diabetic retinopathy (see Chapter 16 ) $^{1 / 43}$. More information on the diffusion of these cases can be found in Chapter 14 and Chapter 15. 


\subsubsection{Case: assessment of the use of lasers as part of laparoscopic cholecystectomy}

Of all minimally invasive therapies, laparoscopic cholecystectomy (LC) has diffused most rapidly on an international scale since its development in France in 1987. This process has been extremely well documented in the international literature ${ }^{(46)}$ and; as outlined above, this case was included in the EC-study on minimally invasive therapy.

At the start of the development of laparoscopic cholecystectomy lasers were preferred over electrocautery as the gallbladder dissection method of choice in the United States ${ }^{[24,29\}}$ : Explanations for this situation may have been that 1) lasers were excessively marketed by manufacturers both to surgeons and the general public ${ }^{35.41 !}$, 2) the fact that Dr. E.J. Reddick, the surgeon credited with introducing $L C$ to the US $S_{4}$ preferred the laser for gallbladder dissection ${ }^{[24,53,54\}}$ and 3) patient demand specifically for laser-assisted laparoscopic cholecystectomy in the US ${ }^{[35]}$. However, the status of lasers for this purpose was soon challenged. As early as November 1990. one year after introduction of LC to the US, a very critical editorial on this issue appeared in the American Journal of Surgery ${ }^{64 !}$. In a comparison of 25 cholecystectomies using a laser and another 25 using an electrocautery unit, the authors found no difference in patient outcome and no complications related to the devices. In fact, the electrocautery unit shaved off about 20 minutes of the hour-long procedure, largely because it produced less bleeding than the laser ${ }^{24 !}$. The use of the laser resulted in an increase of the patient's bill by US $\$ 546$. Besides these extra costs, the hospital where the procedure was carried out, the Mississippi Baptist Medical Center, Jackson, had to pay for extra nursing staff during LC using a laser, following the recommendations of the Association of Operating Room Nurses (Denver, Colorado). The annual salary of this nurse, which was hired specifically for laser-assisted LC, was US\$32 $000^{[24]}$. Furthermore, the presence of a more skillful first assistant was judged mandatory with the use of lasers, contrasting with the use of electrocautery which was judged easier to use and easier to learn. The authors base this judgment on the experience that the electrocautery hook provided tension and countertension for selective coagulation, whereas the laser made its operator a one-armed surgeon, assuming a more passive 'aim-and-fire' approach. Therefore, after having treated the first 70 patients using a laser, the remainder of their 350 cases underwent electrocautery for dissection. In a discussion of their findings, the authors judged that the capital costs of the laser, US\$120 000, could be more effectively utilized by fully furnishing three operating rooms with laparoscopic and video equipment, thus simplifying scheduling in any busy operating theatre. In other cases, the laser benefit that was promoted could actually be detrimental by limiting the availability of LC in hospitals with a progressively limited budget as well as complicating the accreditation of would-be laparoscopic surgeons. Overall, they regarded lasers for this purpose as a cost-ineffective technological fantasy $y^{\left[\mathbb{S}^{2}\right]}$.

In February 1991, partly based on the experience by Voyles et al. described above, ECRI recommended not to purchase a laser for use during $\mathrm{LC}^{[24]}$. The particular issue of ane of ECRI's journals, Health Technology Trends, included a statement of Dr. E.J. Reddick (the innowator), who expressed the opinion that for easy and routine LC cases there is no clinical difference between the laser and the electrocautery unit ${ }^{24 \mid}$. A few months earlier, in November 1990 , panelists of a Diagnostic and Therapeutic Technology Assessment (DATTA) on LC had concluded that dissection of the gallbladder from the liver bed can be performed with either electrocautery or laser. This DATTA was published in JAMA by the American Medical Association (AMA) in March 1991 ${ }^{[2 !}$. At the same time, a first attempt was made to collect and interpret the evidence of both procedure ${ }^{135]}$. Based on the data produced by Foyles et al. (1990), the author (Hunter) performed a retrospective study comparing 100 patients undergoing $L C$ using a KTP laser with 100 patients undergoing LC with monopolar electrocautery. Preliminary results of a prospective RCT comparing LC with Nd:YAG laser $(n=15)$ versus monopolar electrocautery $(n=15)$ were added to the analysis. Hunter (1991) concluded that there was a tendency for laser dissection to take more time than electrosurgery. No conclusions could be drawn on differences in safety and effectiveness of both procedures. Subjectively, easy gallbladder dissection could be easily performed with either instrument. Dissections in which hemostasis was difficult seemed to go more smoothly with 
electrosurgery. The author noted that besides the KTP and the Nd:YAG laser also the argon laser had been used in LC. In addition, a controversy was noted as to use contact or noncontact techniques.

In April 1991, the then largest study on LC was published in the New England Journal of Medicine. representing the experience of 20 surgical groups in the Southern United States ${ }^{[63 \mid}$. The 20 surgical groups, including 59 surgeons, participated in a prospective uncontrolled study including 1518 patients. Twelve of the 20 groups $(60 \%)$ initially used laser dissection as the principal mode of remowing the gall bladder from the hepatic bed. Seven of the 12 groups (60\%) switched to cautery alone as their members became more accomplished in the use of the laparoscopic procedure. The totai number of LCs performed with use of laser dissection was 441 (29.1\%); 314 of these were performed with a KTP laser and 127 with an Nd:YAG laser. The remaining patients were operated on with cautery as the principal excision method. In the discussion section of their article the authors stated that their study settled the important question of whether laser dissection or cautery is better for the removal of the gall bladder from the hepatic bed. Both were judged to be safe and effective, so long as they are used by trained physicians, although cautery was judged to be cheaper. Unfortunately, in the results section of their article, no specific data on safety, effectiveness and costs of either method was included. Nevertheless, this article may have been quite influentiall, as it included a large number of patients and because it was published in a highly rated journal.

In the same period as discussed here for the US, both clinical researchers and editors in other countries had published articles and editorials on the subject of $L C$, including the role of lasers. For example, as early as 1988 (that is even before the introduction of LC in the US) a randomized controlled trial was published in the United Kingdom in which contact Nd: YAG laser surgery $(n=10)$ was compared to surgery with knife and diathermy $(n=10)$ in patients who underwent LC. This small RCT suggested that the use of the Nd:YAG laser "which was associated with capital costs of BP 36000 at that time, did not confer any advantage over conventional surgery, while almost doubling operative time (from about half an hour to an hour) ${ }^{[59]}$ "A few years later, the authors of an article summarizing new trends in gallstone management concluded that laser therapy does not appear to have any advantage over the diathermy hook for meticulous hemostasis and ease of dissection ${ }^{[19 !}$. This article, just as the RCT discussed above, was published in the British Journal of Surgery.

In March 1991, results of a European multicenter study on LC were published ${ }^{20 \|}$. In this retrospective study, results of 7 European centers were reported, including 20 surgeons who operated upon 1,236 patients. The centers were located in Scotland (1), France (3), and Germany (3). Fourteen of the 20 surgical teams (70\%) used an electrosurgical hook knife on a routine basis for dissection of the gall bladder. Six teams (30\%) used a combination of scissors and graspers routinely for this purpose. Several teams used additional techniques on an ancillary basis. Five leams (25\%) used an Nd:YAG laser, while two teams used either pledget swab dissection, berci spatula or hydrodissection on this basis. Although no detailed results were provided the authors concluded that, despite differences in dissecling lechniques used in laparoscopic surgery, the outcome from the various centers was comparable.

Considering the preliminary evidence, it seems that the safety and effectiveness of laser dissection is not different from electrocautery. It also seems reasonable to assume that laser dissection is more costly than electrosurgery. Hunter (1981) suggested that to provide conclusive evidence RCTs should be organized, designed to test the hypothesis that there is no difference between both procedures ${ }^{\{35\}}$. Such studies would not be organized. Actually, one more RCT would be published on this subject, by Bordelon et al. in $1993^{\text {in! }}$. In the introduction to their sludy the authors characterize the debate whether to use the laser or electrocautery for dissection as most emotional, with little available data. At the same time, the authors write that this debate has become less intensified in the recent past. 
A total of 103 patients with symptomatic cholefithiasis were enrolled in the study by Bordelon et al. in the period from September 1990 through August 199 1 $^{17 /}$. Randomization was achieved using a number table. Fifty-one patients underwent electrosurgical dissection and 52 patients underwent (contact tip $15 \mathrm{~W}$ cW) Nd:YAG laser dissection. One patient in the electrosurgical group and two patients in the laser group required conversion to open cholecystectomy, leaving 50 patients in each group completing the study. The mean time (sd) required for dissection of the gallbladider from the liver bed was 23.6 (9.6) minutes in the laser group, compared to $19.2(8.5)$ minutes in the electrosurgery group (t-test, significant difference, $p<0.02$ ). These vallues exclude set-up and calibration time of the laser, taking 2 to 5 extra minutes. In six cases, due to either laser malfunctioning (4 cases) or fiber fragmentation (two cases), laser dissections were completed with electrosurgery. Blood loss was estimated by the operator and expressed on a scale ranging from 0 , indicating no blood loss, to 4 , indicating exsanguinating bleeding necessitating conversion to open cholecystectomy. The mean (sd) blood loss score in the laser-and electrosurgery treated groups were $0.78(0.85)$ and $0.34(0.66)$, respectively. Although this resulted in a significant difference in favour of electrodissection, the use of a t-test as employed by the authors seems inappropriate with these data. In contrast with the subjective data on blood loss, there was no difference in the decrease of the mean hematocrit value in both groups. A total of 15 gallbladders were perforated with the Nd:YAG laser, compared to eight perforations in the electrosurgical group. Superficial coagulation injury of the liver, requiring no specific treatment, occurred in 18 of the laser-dissected patients and 14 of the electrosurgically dissected patients (ns). The authors concluded that the use of laser dissection during LC is not associated with any advantages. Instead in most instances it appeared that electrosurgical dissection was superior. Despite a questionable method of randomization and partially inadequate statistical testing, the results of this study are more credible than those of other studies.

Bordelon et al. (1993) pointed to cost aspects as well in their study. They reported that most hospitall laser safety committees require a laser technician to stand at the machine during laser operation, representing an additilonal personnell cost. There was also a difference in material costs. Bare laser fibres costed about US\$125, rising to $\$ 150$ to $\$ 200$ for sculpted fibers. In comparison, a disposable electrosurgical handpiece with an ' $L$ ' hook and spatula electrodes costed US\$300. Furthermore, reusable electrosurgical electrodes were available on the market, contrasting with sculpted laser fibers, which could only be used once. Of course, appropriate depreciation of the capital costs of the laser would add considerably to the total cost of the laser procedure. The combination of data in this study with those of other studies suggest that the use of lasers for this purpose is not cost-effective. Formal cost-studies, however, have been lacking.

The use of lasers for this indication has gradually fallen out of use. In reviews of LC published after 1992 or so. whenever removal of the gallbladder from its bed in the liver is discussed, authors either indicate that lasers are onlly occasionally used ${ }^{[34]}$ or that laser devices are not used because of their costs and the perceived lack of any significant advantages of this device over electrocautery ${ }^{\mid 52\}}$. Thus, although lasers were preferred over electrocautery for gallbladder dissection at the start of the development of LC in the US this has gradually changed. Of course. either with or without lasers, the diffusion of LC has continued rapidly. One may wonder in how far lasers have actually been used for this purpose. Dr. E.J. Reddick estimated in early 1991 that in the United States as many as $30 \%$ of all LCs were completed with a laser ${ }^{|2 / 2|}$. This may have been an underestimation because, as shown in the study of the United States Southern Surgeons Club, 12 of the 20 teams $(60 \%)$ used a lase ${ }^{463}$. In Europe, where at least 6 different techniques for dissection of the gallbladder were employed, of which electrocautery was most common, the laser may never have reached a sitatus of routime use for this purpose ${ }^{[2]}$. In addition, this study showed that in Europe, whenever a laser was selected, the Nd:YAG laser may have been more frequently used than the KTP laser, which may have been the laser of choice in the US.

How does this information compare to the information on the role of various actors in the process as provided by Moretti in Chapter 1 ? Moretti (1991) expressed the opinion that surgeons were the primary actors in the process of adopting a (KTP) laser for use in $L C_{x}$ as a result of industry 
marketing strategies ${ }^{[4 !}$. To some extent, published literature confirms this view for example, Voyles et al. (1990) describe that the introduction of LC in the US was accompanied by a llarge inumber of (industry-sponsored) courses across the country, which to a large extent focused on the physics and application of laser energy (see also Chapter 20). As a result of this and other incentives, laser-assisted LC was espoused with great enthousiasm by surgeons ${ }^{164}$. But as we have seen, evaluation of the use of lasers by some of these surgeons resulted in a rapid change in practice.

Besides the interest of surgeons in laser-assisted LC, authors have highlighted the interest of hospitals to use LC and, perhaps even more, laser-assisted LC as a marketing tool. As Hunter (1991) points out: marketing managers have been very aware that the American population responds favourably to the mention of laser surgery, and, he adds, in the minds of many, it is the laser that has made LC a reality ${ }^{[35]}$. This does of course not automatically imply that every surgeon supported the use of lasers. To illustrate both this statement and the alleged power of the word 'laser' in marketing the author quotes reports of surgeons who advertised laser LC, and then talked patients into the use of ellectrosurgery when the patient agreed to the operation ${ }^{135 !}$.

According to ECRI, the case illustrates that the mere fact that a laser can be used for a certain procedure does not mean that it is the best instrument for that use. Hospitals in the US learned that lesson, according to ECRI, when they rushed to acquire lasers for LC anly to find out later that most surgeons preferred a far less expensive electrosurgical device ${ }^{[25]}$. This interpiretation now seems a bit unidimensional. One should, as illustrated above, take into account that many other factors have also played a role in the process of diffusion and abandonment of laser-assisted $L C$. The case shows as well that the development of laser applications is sometimes very closely linked to the development of minimally invasive procedures. Actually, this is the only example of a laser procedure that jumped on the bandwagon right at the start of the development of a minimally invasive procedure for a common condition. The pattern of evaluation, however, showing that the best studies are done when the technology has been availlable for some time, is quite common. The same applies to the lack of formal cost studies and quality of life studies. More assessments of selected laser applications, either representing exceptions to this rule or conforming to it, can be found in Part Two. 


\subsection{References}

1. Abela GS thistorical background, development, and hutute trends of lasers in cardiovascular surgery. In: Abela GS (Ed). Lasers in cardiovascular medicine and surgery: fundamentals and techniquas. Kluwer Academic Publishers. Boston Dordrecht, London, 1990, p. 9-17.

2. Absten GT, Joffe SN. Lasers in medicine. An introductory guide (second edition), Chapman and Hall Ltd. London, 1989.

3. Apfelberg DB. Blophysics, advantages, and installation of laser systems. In: Apfeiberg DB (Ed.). Evaluation and installation of surgicial laser systems. Springer Verlag, New York, Berlin, Heidelberg; London, Paris, Tokyo "1987, p. $1-17$.

4. Australian institute of Heatth and Weliare. Australian Center for Medical Laser Technology Inc Lasers in medicine. Australian institute of Health and Welfare, Health Care Technology Series Number 8. Canberra, 1993.

5. Australian Institufte of Health. Lasers in gymecology. A Report by the National Heallh Technology Advisory Panel. Australian institute of Health, Canberra, 1987.

6. Australiari National Health Technology Advisory Panel. Lasers in medicine. National Health Technology Advisory Panel, Caniberra, Australlia, 1985.

7. Banta HD (Ed). Minimally invasive therapy in five European countries. Diffusion, effectiveness and costeffectiveness. Health Policy Monographs Vol, 3. Elsewier. Amsterdam, London, New York, Tokyo,1993.

8. Banta HD, Gellins A. Anticipating and Assessing Health Care Technology, Volume 4. Health care applications of lasers: the fulure treatment of coronary artery disease. Report of the Scenario Commission on Future Health Care Technology. Martinus Nifhoff Publishers, a member of the Kluwer Academic Publishers Group, Dordrecht, Boston. Lancaster, 1988 .

9. Banta HD, Gelins A. Anticipating and Assessing Health Care Technology, Volume 1. General considerations and policy conclusions. Report of the Scenario Commission an Future Health Care Technology. Martinus Nijhoff Publishers, A member of the KJuwer Academic Publishers Group, Dordreacht, Boston, Lancaster, 1987.

10. Banta HD, Luce B. Health care technology and its assessment: an international pierspective. Oxford University Press, Oxford, New York, Tokyo, 1993.

11. Banta HD, Oortwijn WJ, wan Beekum WT. The organization of health care technology assessment in the Netheirlands. Rathenau Institute, the Hague, the Netherlanids, 1995.

12. Banta HD, Schou I (Eds.). Lasers in Health Care. Effectiveness "Cost-Effectiveness and Policy Implications. Academic Publishing "Frederiksberg, Denmark, 1991.

13. Banta HD, Thacker $S$. The case for reassessment of health care technology: once is not enough. Woumal of the American Medical Association 1990(264)2:235-40.

14. Banta HD. International Network for Agencies in Health Care Technology Assessment (INAHTA) established. International Society for Techmology Assessment in Health Care, Newsletter 1993(5)/4:4.

15. Banta HD. Pushing the limits: technology assessment in health care. (inaugural address) Universily of Limburg, Maastricht, 1990.

16. Boere $H$, Schoo $L$, Slot $A$, Vogel J. Safety measures for the use of lasers in medicine. Dutch Working Group on Assessment of Medicall Equipment (WIBAZ), 1984 (in Dutch).

17. Bordelon BM, Hobday KA, Hunter JG. Laser vs electrosurgery in laparoscopic cholecystectomy. A prospective randomlzed trial. Archives of Surgery 1993(128)February:233-6.

18. Cheslyn-Curtis S, Russell RCG. New trends in gallstone management. British Joumal of Surgery 1991(78)2*143-9.

19. Cross FW, Bowker T.J. The Excimer laser-tissue interactions and aarly clinical results. In: Abela GS (Ed.). Laser medicine and surgery: fundamentals and techniques. KIuwer Academic Publishers, Boston, Dordrecht, London. 1990, p. $45 \times 58$.

20. Cuschieri A, Dubois F, Mouie' J, Mouret P, Becker $H$, Buess $G$. Trede M. Troidl $H$. The European experience with laparoscopic cholecystectomy. The American Joumal of Surgery 1991(161)3:385-7.

21. Diagnostic and Therapeutic Technology Assessment (DATTA). Laparoscopic cholecystectomy. Journal of the Americian Medical Association 1991(265)12:1585-7.

22. Diagnostic and Therapeutic Technology Assessment (DATTA). CO lasier treatment in gynecologic malignancies. Journal of the American Medical Assaciation 1983(50):672.

23. Dutch National Committee Laser Safety. Laser safety in health care. The Netherlands Orglanization for Applied Scientific Research (TNO), Leiden, the Netherlands; 1993 (in Dutch).

24. ECRI. Does laser $=$ better? Another look. Health Technology Trends 1991(3)2:1,8.

25. ECRI. Lasers. In: Health Technology Checklist 1993/1994. Hea:th Techmology Trends 1993(9)Supplement $1: 20-1$.

26. Eggink GJ, Kaptein C, van Kempen RJ, van der Meulen FW, Teirlinck CJPM, Vaartjes SR (Eds.). Dutch National Committee on Laser Safeby. Laser safety in health care (second edition). The Netherlands Organization for Applied Scientific Research (TNO), Leiden, the Netherlands, 1993.

27. European Commurvity Concerted Action Program for the Development of Medical Laser Applications. Proceedings of the First Plenary Workshop on Safety and Laser-Tissue interaction, Berlin Nowember 1988. Publlshed as a supplement to the journal Lasers in Medical Science, Bailière Tindall, London, 1989. 
28. Fuller TA Fundamentals of lasers in surgery and medicine. In Dixon JA (Ed). Surgical applications of lasers: Year Book Medictal Pubulishers; Inc, Chicago, London, 1903, p. 11-28.

29. Gelijns AC, Fendrick. AM. The dynamics of innovation in minimally invasive therapy. In: Banka HD (Ed) . Minimally invasiwe therapy in five European countries. Diffusion, effectiveness and cost-effectiveness. Health Policy Monographs Vol. 3, Elsevier, Amsterdam, London, New York, Takyo, 1993, p. 153-60.

30. Goldman L. The histony and dewelopment of the medical laser. In GS Abela (Ed.). Laser medicine and surgery: fundamentals and techniques. Kluwer Academic Publishers, Boston, Dordrecht London; 1990, p, 3-7.

31. Goodman CS. Technology assessiment in healthcare: a means for pursting the goals of biomedical engineering. Health care technology assessment special feature. Medical and Biologlical Engineering and Computing $1993(31): H T A 3-10$.

32. Health Insurance Executive Councit. Annual Account. Investigational Medicine 1995. Health Insurance Executive Council, Amstelveen, the Netherlands, 1996 (in Dutch).

33. Health Insurance Executive Council. Governmental point of view on coordination and financing of research for health. Advise number 616. Health Insurance Executive Council, Amstelveen, the Netherlands, 1994 (in Dutch).

34. Hobbs KEF. Lapraroscopic cholecystectomy. Gut 1995(36):161-4.

35. Hunter JG. Laser or electrosurgery for laparoscopic cholecystectomy? The American Journal of Surgeny 1991 $(161) 3: 345-9$

36. Hutton J. Assessment of medical technology: the role of engineers. Health care technology assessment special feature. Medical and Biological Engineering and Computing 4993(31):HTA11-5.

37. Jacques SJ. Laser-tissue interactions. Photochemical, photothermal and photomechanical. In: Schwesinger WH, Hunter JG (Eds.). Lasers in general surgery. The Surgical Clnics of North America 1992(72)3:531-58.

38. Johnson J. Laser physics and its relevance to applications in medicine. In: GS Abela (Ed.), Laser medicine and surgery: fundamentals and technilques. Kluwer Acadernic Publishers, Boston, Dordrecht, London, 1990, p. 21-30.

39. Mastenbroek. CG. Applicalion of societal criteria in assessing investigational medicine research projects. Tijdschrift voor Sociale Gezondheidszorg 1994(72)2:105-6 (in Dutch).

40. Ministry of Health 1995. Public health policy 1995, Dutch Parliament. Meeting Year 1995*1996, 24126 nr 9, Sdu Publishers, the Hague, the Netherlands, 1995 (in Dutch).

41. Moretti M. The Medical Marketplace. In: Forrest GT, Levitt MR (Eds.). The laser marketplace in 1991. A seminar examining recent trends and directions in the worldwide market for lasers. The Society of Optical Instrumentation Engineers (SPIE). SPIE Publication 1520, SPIE, Bellingham, Washington, USA, 1991, p. 118-31.

42. National Center for Health Services Research. Assessment of Nd:YAG lasers for posterior capsulotomies. Report No. 21. In: Health Technology Assessment Reports 1984. Rockville, Maryland, DHHS Publication No PHS 85$3373: 317-25$.

43. National Center for Health Services Research. Assessment of carbon dioxide lasers in head and neck surgery. Report No. 10. in: Health Technology Assessment Reports 1984. Rockwille, Maryland, DHHS Publication No PHS 85-3373:135-42.

44. National Center for Hiealth Services Research. Assesisment of laser trabeculoplasty (LTP) for open angle glaucoma. Report No. 23. In: Health Technology Assessment Reports 1984. Rockwille, Maryland "DHHS Publication No PHS 85-3373:3711-90

45. National Institute for Publlic Health and Environmental Hygiene (RIVM). Public Health Future Forecast. Public health status and forecast: the health status of the Dutch population in the period 1950-2010. Sdu Publishers, the Hague, the Netherlands, 1993 (in Dutch).

46. Office of Health Technology Assessment. Carbon dioxide laser surgery. Assessment Report Series, Volume 2. Number 23. Washington, DC. Office of the Assistant Secrotary for Health. Department of Health and Human Services, 1982 .

47. Oortwijn W. Ament A. Wondeling H. Tools for use of societal criteria in priority setting in evaluation of medical technology in the Netherlands - development and testing of a checklist. In: Malek M (Ed.). Setting priorities in health care. John Willey \& Sonis "Chichester, New York, Brisbane, Toronto, Singapore "1994, p. 203-16.

48. Office of Technology Assessment, Health care technology and its assessment in eight countries, OTA.BP.H-140, Washington, DC: UIS Government Printing Office, February 1995.

49. Pass HI. Review. Photodynamic therapy in oncology: mechanisms and clinical use. Joumal of the National Cancer Institute 1993:(85)6:443-56.

50. Patrick OL. Erickson P. Health status and health policy. Allocatimg resources to health care. Oxford University Press, New York, Oxford, 1993.

51. Persson J. No title (editoriali,. Health care technology assessment special foature. Medical and Biological Engineering and Computing 1993(31):HTA 1-2.

52. Quilici PJ. Laparoscopic cholecystectomy. Gastrointestinal Endoscopy Clinics of North Airnerica 1993(3)2:221-37.

53. Reddick EJ, Olsen DO, Spaw A, Baird D, Asbun H, OrReilly Mi, Fisher K, Saye W. Safe performance of difficult. laparoscopic cholecystectomies. The American Journal of Surgery 1991(161):377-80.

54. Reddick EJ, Olsen DO. Laparosicopic laser cholecystectomy: a comparison with mini-laparotomy cholecystectomy. Surgical Endoscopy 1989(3):131-3. 
55. Fol P Leistungslaser und hire medizinischen anwendungen. Eine Infonmationsschrift des NFP 19 Biomedizinische technik des Schweizenischen Nationalfonds. Institult fur Biomedizinische Informatik, Uiviversitä tund ETH Zürich, Schwellz, 1991 .

56. Schou 1. Low power lasers. In Banta HD, Schou I (Eds). Lasers in Health Care Effectiveness, Cost-Effectiveness and Policy Implications. Academic Publisting, Frederiksberg, Denmark, 1991, p. $204-10$.

57. Schou 1, Sprangers FLH, van Gement MJC. Technical aspects of clinical laser applications. In: Banta HD, Schou 1 (Eds.) Lasers in Health Care. Effectiveness, Cost-Effectiveness and Pollicy Implications. Academic Publishing, Frederiksberg, Denmark, 1991, p. 6-23.

58. Soper $\mathrm{N}_{3}$ Brunt M Korhl K. Laparoscopic general surgery. New England Journal of Medicine 1994(330)6:409-19.

59. Steger AC, Moore KM, Hira N. Contact Haser or conventional cholecystectomy: a controlled triall. British Joumal of Surgery 1988(75)3:223-5.

60. Sterenborg HJCM, Müfler GJ (Eds.). Finall Report. Working Group Laser Safety. European Community concerted action program into the development of medical laser applicationis. Concept (1994).

64. Strikwerda S. Argon non laser ablation in obstructive atherosclerosis. A quantitative and histopathologic study of acute effects (thesis). University of Leiden, the Netherlands; 1988.

62. Walker SR, Rosser RM. Quality of life assessment. Key issues in the 1990s. Kluwer Academic Publishers, Dordrecht, Boston, London, 1993.

63. The Southem Surgeons Club. A prospective analysis of 1518 cholecystectomies. The New England Journal of Medicine $1991(3.24) 16: 1073-8$.

64. Voyles CR, Meena AL, Petro AB, Haick AJ, Koury AM. Electrocautery is superior to laser for laparoscopic cholecystectomy (editorial). The American Joumal of Surgery 1990(160)5:457.

65. de Wit GA, Vondeling H, Banta HD. Lasers in gynecology, a literature survey. Health Council, Report No A90/07, the Hague, the Netherlands, 1991 (in Dutch). 


\section{Practical aspects of lasers}

\subsection{Introduction}

A variety of practical aspects need to be addressed to ensure proper use of lasers in daily clinical practice. To start with, acquiring a laser is not necessarily a simple process. It is not just the laser that has to be bought, in most cases a laser system requires acquisition of a combination of ancillary equipment as well. As we have seen in Chapter 2, this may include endoscopes, fibers, tips and operation microscopes.

When the investment is well prepared the next step consists of addressing a number of rather practical questions relating to the installation requirements of the laser, its location and the (projected) staffing of the program. Examples of installation requirements of laser systems are the need for a cooling system or a fume extractor. In principle, a laser can be used in one or a combination of locations: an out-patient department, an operating room; a clinic, or a specially developed laser area. The steps of laser technology planning and the subsequent preparation of a laser program are discussed in paragraph 3.2 and 3.3 , respectively.

Implementation of a laser program requires a thorough understanding of the hazards of lasers and active laser safety management. The hazards of lasers fall into two major categories: those to the eye, skin and associated hazards, e.g. fire, x-rays, electrical high voltage, and hazards of mutagenic components and carcinogenic cells in 'laser smoke'. To assure the safety of personnel and patients a number of safety measures, for the most part based on laser standards and protocols, should be implemented. A brief overview of these subjects is presented in paragraph 3.4. Because of the complexity of the technology, its associated hazards and the involvement of various different professionals, organizational adjustments are required as well. The management context of the safe and efficient use of llasers is discussed in paragraph 3.5. Unfortunately, all hazard control measures are subject to human error. To minimize the incidence and impact of human error high quality education and training programs should be set up. These subjects are addressed in paragraph 3.6. The final paragraph draws some conclusions and briefly discusses some future trends. Readers who wish to be informed in more detail on the subjects covered in this chapter are kindly referred to the second edition of the report 'Laser safety in health care' by the Dutch National Committee on Laser Safety (in press).

This chapter is partly based on:

Teirlinck C, Vondeling H. Practical aspects of medical laser applications. In: Banta HD and Schou I (Eds.). Lasers in health care. Effectiweness, cost-effectiveness and policy implications. Academic. Publishing, Frederiksberg Denmark, 1991, p. 24-35.

Health Council'. Laser safety in health care. Assessment of the report 'Laser safety in hospitals' of the National Committee on Laser Safety. Health Councif. Report 1992/06. the Hague, the Netherlands, 1992 (in Dutch).

Teirlinck C.JPM, Vondeling H. Laser safety in health care. Assessment of the report 'Laser safety in hospitals' of the National Committee on: Laser Safety. Technologie in de Gezondheidszorg (TG) 1992(10):40.2 (in Dutch).

Vaartjes SR, Dignum PH, van Kempen $\mathbb{R} J_{n}$ wan der Meulem FW, Sterrenburg PGJ, Vondeling H. Structure of responsibility and the laser safety framework in hospitals. In: Eggink GJ, Kaptein C, van Kempen RJ, van der Meulen FW, Teirlinck C.JPM, Vaartjes SR (Eds.). National Commiltee on Laser Safety, Laser safety in heralth care (second edlition). The Nethertands Organization for Applied Scientific Research (TNO), Leiden, the Netherlands (in press): p. $109-20$.

4 The author served as external secretary of the Health Councill's Laser Safety Committee in the periad 1991-1992 and was invited to join the National Committee on Laser Safety in 1993. The other three articles cited here were jointly written witth Members of this Committee.

2. This report is also available in Dutch. 


\subsection{Laser technology planning}

Hospitals need to approach the acquisition of lasers cautiously to ensure that the appropriate equipment is obtained, that it will be well-utilized for procedures in which it offers a demonstrable improvement over alternative technologies, and that it will also conform to the overall strategic plan of the institution.

Poor planning in the past has caused many new lasers to collect dust from low utilization. Therefore to avoid this from happening as a first step in laser technology planning it has been recommended, e.g. by ECRI, to establish a Laser Technology Committee (LTC)idal. Similarly, the Dutch National Hospital Institute (NZ) (which changed its name to Dutch Hospital Federation; NZf) recommends establishing a laser investment council, especially when the hospital has not previously invested in laser equipment?

According to ECRI (1992), the following personnel would be appropriate for membership of the ad hoc LTC the chief of surgery, the chief of ophthalmology, a physician from each subspecialty that uses lasers, an anesthesiologist, an operating room supervisor, a risk manager, a clinical engineer, a facilities engineer, an administrator, a laser safety officer (LSO), a laser operating room technician and a patient advocate (optional) ${ }^{101}$. Although some professionals will be involved in every investment made in lasers, the Dutch Hospital Federation stresses that the combination of representatives in the $\mathrm{LTC}$ may to some extent differ per investment ${ }^{[7]}$. The Federation also lists nurses as potential candidates for the LTC ${ }^{[1}$. ECRI and NZf agree that the LTC should be headed by a coordinator, who serves as an intermediary between the manufacturer(s) and the hospital. In practice, this could be the Laser Safety Officer, a key professional of the Laser Safety Committee ${ }^{|25|}$ (see paragraph 3.4).

Before the actual purchase and development of the laser treatment program, certaim important data are needed. Firstly, it should be determined for which applications a laser could provide costeffective care. For this purpose, information on the cost-effectiveness of selected procedures, such as that presented in Part 2, can be collected. Then the case-load history of the applications in the specialties where the laser could be used in the future should be reviewed, for example for the last one to three years. This information serves to forecast the usage level of the laser. As a general rule, ECRI recommends not to purchase a laser, unless at least 50 procedures can be performed on an annual basis ${ }^{(10)}$. More detailed recommendations are provided in Intermezzo 3.1 .

Intermezzo 3. 1. ECRI recommendations for laser technology planning

As will be discussed in Chapter 12 , ECRl found in a 1991 survey of hospitals in the United States with generat punpose surgical lasers that, on average, the se devices were used in less than $5 \%$ of fotal surgical procedures ECRl accompanied this finding with tentative guidelines for the adoption of new medical la sers per specialty. Firstly. the key to adequate utilization of surgical lasers is multidisciplinary use. Many hospilals in the United States lacked the patient volume to support even one surgical laser, especially if its use was limited to only one or two specialties. Secondly, if adoption of a laser is considered, the choice could be determined by caseload and case-mix. Taking these factor's wito account ECRl states that in primary hospltals with less than 100 beds either a CO, Nd:YAGKTP. or an argon laser could be an appropriate investment for the combination of the specialties of gynecology and geneval surgery. In newrology, either a $\mathrm{CO}_{2}$ - or an $\mathrm{Nd}$ : YAG laser could be made available. $1 n$ urology, an $N$ d: YAG laser could be bought. In ophthalmology, an argon or a qs Nd:YAG lasier could be installed. For the combination of the speclalties of dermatology, plastic surgery and otolaryngology, a $\mathrm{CO}_{2}$ laser could be the most appropriate hvestment. At the same time ECRI notes that not all small primary hospital's will need lasers.

In primary hospitals with 100 to 300 beds, purchase of a Ho: YAG laser could be considered in general surgery. In netrology, a KTP or an argon laser could be invested in. In urology, either a KTP-, argon- or CO laser could be punchased. In gastroenterology, an Nd:YAG laser could be a good investment. In ophthalmology, this could appiy to a diode laser. In dermatology and plastic surgery, either a KTP. or an argon laser could be installed. in otolaryngology, either an No:YAG- a KTP- or an argon laser could be bought. Finally, in onthopedics a Ho:YAGor an Nd:YAG laser could be adopted.

In secondary hospitais, that is hospitals with 300 to 500 beds, adoption of a pulsed dye-laser could be considered in urology. In tertiary hospitals, that is hospitals with more than 500 beds, adoption of a copper-wapour-or a pulsed dye laser could be considered. Likewise, a pulsed dye laser could be an appropriate investment in plastic surgery. while investing in an excimer laser could be considered in cardiovascular surgery in large-sized institutions. In a 
comment on these recommendations if was stressed that hosplals should not purchase a laser for a single indication whess they are large, speciatly centers in that field and can draw on adequate numbers of patients.

Of course, a minimum usage level could vary with the complexity and costs of the application. The annual throughput co-determines not only the actual cost-effectiveness of laser procedures as delivered in the institution, but also the quality of care. Physicians go through a learning cune before their skills are optimal and it is generally accepted that a minimum annual case-load of patients is needed to maintain these skills (see paragraph 3.5).

The information on the expected annual case-load and case-mix is essential for a cost analysis of the proposed program as well. For this purpose start-up time and start-up costs need to be considered too. Further costs in addition to the cost of the laser itself include the costs of ancillary equipment, the cost of safety measures, and installation and maintenance costs. As outlined in Chapter 2, the need for accessories and supportive devices depends on the application. For example, an ophthalmological application of lasers requires a slit-lamp and a microscope adapter as a tool for very precise operation. A micromanipulator is used in microsurgical laser applications. Additional disposable equipment may be used such as gas cylinders for $\mathrm{CO}_{2}$ and special drapes. It should be noticed that especially disposable accessories can be key factors in the high cost of using a technology. For example, laser fibers in angioplasty can cost several hundred dollars per procedure. Other invesiments may include an instrument table, a chair or bed for the patient, cleaning and sterilization boxes, and calibration and maintenance instruments, such as equipment to refine or polish fibers. Finally "costs of personnel, which include physician costs and the occasional necessity of staffing changes, and costs of the education and training for the physician and other personnel who will operate the laser, such as nurses and engineers, need to be considered (further information on the cost of a laser facility is given in Chapter 4).

Outside ophthalmology, the probability that investing in a laser is justified is higher when the laser can be shared across specialities than when its use is restricted to only one specialty. For example, a Nd:YAG laser can be used by the departments of gastroenterology, pulmonology, urology and neurosurgery, whereas the $\mathrm{CO}_{2}$ laser can be used by the ENT and gynecology department (see Chapter 2).

Furthermore, the investment in lasers has to be weighted against investments in other technologies. For example, if a urology department considers purchasing a laser lithotripter but does not have up-to-date endoscopic equipment, it is preferable to put the lithotripter on hold until the endoscopic equipment has been replaced ${ }^{10]}$.

The LTC needs to have a thorough understanding of the (potential) cost-effectiveness ${ }_{4}$ costs, and alternatives for new laser procedures so that it can make the right choice for a particular application rather than succumb to marketing pressures or pressure from one, or even a few, physicians who are enamoured with lasers. A similar procedure can be followed to support decisions on replacement-investments. In this case the LTC should find out how the laser has been used, how often it has been used and whether the equipment is near the end of its useful life. Again, part of the work of the LTC should include an assessment (in this case a reassessment) of technological alternatives to the existing laser. This could either be a new type of laser (e.g. a diode laser replacing an argon laser in ophthalmology), or developments in minimally invasive therapy outside using lasers (e.g. injection sclerotherapy versus Nd:YAG laser treatment of upper gastrointestinal bleeding). This could lead to a change in therapy by redirecting investments. Furthermore, both new and replacement investments in laser technology have to be in line with the overall strategic plan of the institution, which defines what direction it will take with its services. Some institutions may want to become a 'center of excellence' or a referral hospital for certain clinical services, such as cardiovascular surgery and oncology. Investment in lasers may well fit in this picture and at the same time increase the competitiveness of the institution ${ }^{\mid 7.10 .21\}}$. However, there may be a tension between the strategic goals of an institution and providing cost-effective and high-quality care. If one particular institution is unable to sustain a particular investment, sometimes the investment and 
explaitation costs can be shared with other institutions by purchasing a laser that is transported from one institution to another at regular intervals.

If, based on available data and deliberations upon strategic goals, it is decided to purchase a new or replace an existing laser, the next step in the procedure is implemented. As laser technology usually represents a relatively large capital investment, competitive bids are requested and then evaluated. This step is useful in particular in a situation of many alternative systems, which is the case when a "commodity-laser", such as an Nd: $\mathrm{YAG}-\mathrm{CO}_{2}$ - or argion laser is bought or when the capital costs of the laser or of the ancillary equipment is exceptionally high, such as that of ophthalmological Excimer lasers (capital costs: US\$245 000).

In acquiring one or more laser systems, the most common types of capital equipment financing alternatives are: cash purchase, conventional bank financing, capital and operating leases, and per-use rental Occasionally, philanthropic donations are made specifically for the laser purchase. The financing alternative chosen will depend on many factors, e.g. the hospitals's present and projected cash position and financing philosophy, the prevailing interest rates, the hospital's creditworthiness, reimbursement considerations, and its profit or non-profit status ${ }^{100}$.

After the bids are received, each company that is competitive can be requested to bring the laser on-site so that physicians can work with it and try it on inanimate objects. An evaluation form should be developed so that each physician trying the laser can give his or her reactions. The laser coordinator can also develop a summary form of this and other information to help the LTC make its decision ${ }^{|1|}$. The summary form should include a price comparison, advantages and disadvantages, physician evaluation, and staff evaluation. Then it is decided both which type of laser should be purchased and which manufacturer should deliver it.

\subsection{Preparation of a laser program}

To continue the adoption process, the manufacturer's representative can be asked to meet with the LTC to pass on further information on the practical and safety aspects of setting-up the laser facility?

Three practical questions need to be addressed at this stage:

1 what are the installation requirements for the laser?

2 where should the laser be located?

3 what is the appropriate staff of the program?

\subsubsection{Installation requirements}

The most common and expensive installation requirements for a laser are related to the electric power supply, the cooling system and the need for smoke evacuators.

\section{Electrical power supply}

Historically, a $\mathrm{CW}$ Nd:YAG laser needed a three phase electrical power supply. $\mathrm{CO}_{2}$ lasers require no added electrical installation, although some laser systems have speciall plugs that call for an appropriate electrical outlet. A dedicated circuit is not necessary, but it can be useful if other electrical requirements for the procedure might overload the circuit. The outlet for the laser and the related circuit breaker in the electrical closet should be clearly identified ${ }^{16]}$.

\section{Cooling system}

The overall efficiency of the laser process is very low. A high percentage of the electrical energy supplied to the laser, ranging from 70 to $98 \%$, is converted into heat. Therefore, an efficient cooling system may be necessary. Cooling can be achieved either by using water or air ${ }^{[p]}$. Again "the 
requirements differ, largely depending on the type of laser. The cW NdYAG laser needs forced-flow water cooling from a wall faucet to maintain stable internal conditions. Leak-proof, quick disconnect hose connectors should be used for cooling water ${ }^{\text {t3i }}$. There are anecdotes about cooling water hose failures during a surgical procedure, such that surgical personnell had to stand in a pool of water while completing the procedure. Such incidents point out the additional need to have the instrument properly grounded and protected with ground fault protection circuits.

Most recent types of lasers use air cooling. The excess heat produced by operating the laser may exceed the capacity of the air-cooling system. In this case either the capacity of the system has to be adapted or the laser should be equipped with a stand-alone cooling system. A second consideration is that the heat may interfere with the laminar air-flow in the operation field, which interferes with sterile operating conditions. Therefore, the exit of the cooling system should never be directed towards the operation-table.

\section{Fume extraction}

Fume or 'smoke' is generated in many procedures with a $\mathrm{CO}_{2}$ or a Nd:YAG laser. This smoke or plume, resulting from volatized tissue components, causes air-contamination and may contain viable cancer cells if malignancies are treated. The odours may be highly unpleasant and noxious. A fume extractor, containing activated charcoal filters, solves this problem and helps to keep the surgical work-site clearly visible ${ }^{\| \text {isy }}$. in general, a fume extractor is essential for cases involving treatment of tumors, large excisions with the laser, and cases involving continuous lasing at high powers.

\subsubsection{Location}

The optimum location for a laser facility may not be easy to determine. Of course, nursing and anesthesia support systems need to be in close proximity to the laser treatment area ${ }^{[21]}$. In general, two considerations have been found to be important ${ }^{[t]}$ :

1 the treatment(s) to be offered, and

2 maximum safety and a minimum of restrictive rules.

Lasers that are used in one specific department are usually located in that department. For example, ophthalmological lasers are located in the ophthalmology department. There is no reason to locate this laser in the operating room. On the other hand, angioplasty lasers need to be located close to the diagnostic equipment, that is, in the operating room or the catheterization laboratory.

The best location for a non-ophthalmological laser, for reasons of safety, is often the operating room. In the out-patient department, the stream of patients and personnel is less disciplined and controllable. If an institution considers purchasing a second or a third laser and the choice of location is not primarily determined by arguments of specially function, a choice can be made based on safety arguments. In a clustered location, which means that the second laser is located in the same space as the first, safety procedures can be more easily maintained or modified.

A final but not unimportant consideration originates from the possibility that some types of lasers can be shared by different departments. For such multi-specialty use, easy mobility of the laser would be helpful. In practice, historically, this has proven to be virtually impossible. The $\mathrm{CO}_{2}$ laser, for example, cannot be easily moved because of its sensitive system of delicately positioned articulating mirrors which easily become misaligned. In case of the older types of Nd:YAG lasers mobility was impossible due to the fixed water cooling system and high voltage wiring. Despite the advent of new laser systems, mobility of physicians and patients may still be preferable, but such a choice puts a higher demand on the organizational structure of the laser program (see paragraph 3.4). In theory, the department that contributes mostly to the average annual case-load could be the best candidate to furmish the laser room. 


\subsubsection{Staffing of the program}

Staffing a laser program depends on the scope of the program. Initially, utilizing selected persons from existing staff will meet the laser program needs. At least two persons sharing the same professional background should be trained to work with the laser program to assure coverage during vacation, sick leave or other lost time. The selection criteria include education base, dependability, motivation, interest in the program, leadership qualities, and commitment. As the laser program expands a team concept may be considered ${ }^{116 !}$.

\subsection{Implementation of a laser program}

\subsubsection{Hazards of lasers}

Implementation of a laser program requires a thorough understanding of the hazards of lasers and active laser safety management. The hazards of lasers fall into two major categories: those to the eye, skin and associated hazards, e.g. fire, x-rays, electrical high voltage, and hazards of mutagenic components and carcinogenic cells in laser smoke ${ }^{[13]}$.

Direct or indirect exposure to laser energy may cause irreparable injury to the eye and thermal burns to the skin. Obviously, laser-caused fires in the operating room are a major safety concern. All kinds of materials in the laser room, such as drapes, towels, tapes and gauze pads can catch fire in case of direct or indirect laser radiation. Much attention has been given to fires associated with procedures using endotracheal tubes and anesthetic gases. X-rays may result from highvoltage discharges in some laser systems. This is of importance both in clinical applications and during maintenance or repair sessions. Electrical hazards from lasers can be life-threatening. Lasers frequently employ large capacitors and high-energy potentials that can cause fatal shock. There are also concerns that plumes from procedures involving malignant tissues may contain viable tumorigenic debris ${ }^{[13]}$ (see Intermezzo 3.2. A survey of laser safety measures).

In light of these hazards, the specific aim of laser safety management in hospitals is to maintain an adequate level of safety, first of all for the patient, but also for the personnel present in the laser room, without preventing effective and efficient treatment of the patient. To achieve this aim, in other words to achieve quality of care in laser use, a combination of standards and protocols thas been developed.

Internezzo 3.2. A survey of laser safety measures

In order to maintain an adequate level of safety for the patient and the personnel present in the laser room, without preventing effective and efficient treatment of the patient, a number of practical measures both inside and outside the laser room need to be implemented.

Outside the laser room

With respect to the entrance door of the laser room, one can differentiate between open and closed departments. Only personnel and accompanied patients have access to a closed department. Historicaly, it has been argued that in an open department the entrance doors have to be locked when the laser is used or that the laser has to be switched off when the door is opened. The latter situalion is judged undesirable because it may interrupt the laseir procedure at a critical stage. To avoid this situation, and at the same time promote safe use of lasers, it has been suggested to connect the laser with a warning light system outside the operating room. The system should operate when the laser is actually used. In addition, the entrance doors should be posted with warning signs stating "laser operating' or something like 'danger - kaser treatment area'.

inside the laser room

Inside the laser room a distinction can be made between the walls and windows of the room and the materials in the room. As a general rute, windows thave to be armored to prevent laser radiafion from leaving the room. An exception is the $\mathrm{CO}_{2}$ laser, which radiation is fully absorbed by glass.

in order to understand safety measures related to medical materials, basic knowledge on the interaction of laser light and materials is necessavy. Basically, one or a combination of three physical processes occur when laser light interacts with material: reflection, absorption or transmission. Laser light can be reffected on the surface of surgical tools in an unexpected direction. The reflection coefficient mainly depends on the wavelength of the laser. It is best to use special surgical tools that have ebonized, anodized or sandblasted surfaces. Use of refiective objects near 
the beam fireld should be awoided The use of shor focal fenses hejps to minhme the hazard from specular reflection because the beam will be strongly divergent after passing the focal point lowening the eneroy fower

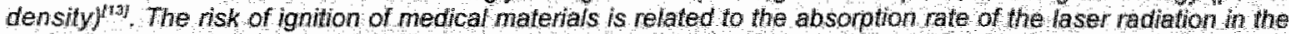
material the temperature rise on account of that absorption, and the whammation temperature of the material. The absorption of laser light depends both on the color of the material and he wawelength of the laser The inflammabifity of white material is lower than the inhammabifity of colored material when fisers ave used which ermit light at wavelengths in the wisible and nearinfrared spectrum. Also, the transmiting properties of the material can be important. One safety measure is thoroughy moistening white cotton drapes. This decreases the transmitting

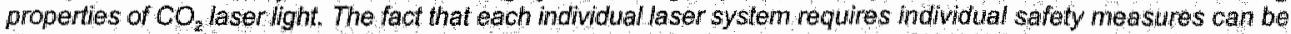
ithustrated by the opposite effect of this same measurement on the transmission of Nd: YAG Vaser light Another point to cansider is protection of vilnerable apparafus in the operating room. Anesthesia apparatus, infusion liquids containing proteins, and infusion sels have to be shielded from laser radiation.

When a procedure requires the use of high concentrations of oxygen or laughing gas or flammable anesthelic gases, the risk of ignition increases. The combination of a laser procedure in the (highy ainway using anesthetic gases and endotracheal tubes needs special safety measures ${ }^{\text {tas }}$. Use of matte finish, metallic endotracheal tubes seems to be the most effective prevention of fires. Also, specially designed laser-resistant disposable tubes have been marketef ${ }^{\text {s. }}$. During endotracheal procedures the patient's head may be covered with wet drapes to prevont

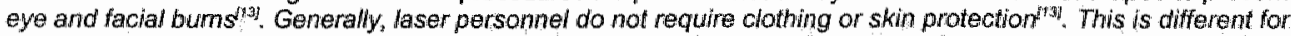
the patients who, when anesthesia is applied, will not react to radiation on their eyes or skin. Clearly, the patient is the most vuinerable party in leser treatment.

\subsubsection{The role of standards and protocols}

The International Standards Organization (ISO) is perhaps most important in the development and promulgation of standards with regard to medical devices and biomaterials, in particular its Technical Committees (TC)-194, Biological Evaluation of Medical Devices, and TC-150, Implants for Surgery ${ }^{[18 \mid}$. The most commonly used standard in Europe is the IEC 825 of the International Electrotechnical Commission ${ }^{[4 t]}$. This standard has been incorporated in the European standard NEN 60825. The standard which is most commonly used in the US is the ANSI Z-136-3 standard ${ }^{11\}}$. This standard, which has been revised in 1995, has a specific volume dedicated to the safe use of lasers in health care facilities ${ }^{|22|}$.

In these standards, based on the risks for the human eye and skin, values are provided for Maximum Permissible Exposures (MPE values) to laser light. Based on MPE's, Nominal Ocular Hazard Distance (NOHD) values have been calculated. Outside this area, the level of radiation is acceptable in terms of MPE values. The area within an NOHD is called the Nominall Hazard Zone (NHZ). Unless stated otherwise this $\mathrm{NHZ}$ encompasses the entire laser room, according to the Dutch National Committee on Laser Safety. To prevent injury to the eyes of personnel in the laser room either spectacles, wrap around lenses, goggles, or related forms of eye protection can be applied ${ }^{[22}$. Because of the importance of eye protection, specific standards have been developed for laser eye protectors. In case of goggles these standards, listed in the German DIN 58215 , include ${ }^{[i]}$.

- protection of the eyes against laser radiation from every direction;

- a reduction of the level of transmission of laser radiation that is compatible with MPE values:

- high transmission for wavelengths in the visible part of the spectrum;

- protection from laser radiation for a period of at least 10 seconds;

- eyewear must be clearly and properly matched to the kind of laser radiation for which the eyewear is intended and the optical density of the goggles should be indicated.

Standards for the use of lasers and other medical devices are in a continuous process of revision and updating. In particular the activities in the international standards are of special interest to the medical devices community because these standards will set acceptable levels of quality and performance of medical devices, promote understanding across national boundaries, establish requirements, and facilitate foreign trade ${ }^{119}$. More information on laser standards is provided in Chapter 18.

The standards are complemented with protocols that, according to the Dutch National Committee on Laser Safety, should at least include the type of laser, the (combinations of) room(s) where to 
use the laser, the specialty or specialties and (combination of) recommended clinical applications, protective measures for patients (e.g goggles, sheets), the allocation of responsibility of operating the laser, the duties of a laser assistant, operating instructions of the laser, how to prepare a clinical treatment session, the use of laser safety goggles for personnel, the control of additional safety provisions (both with respect to the device and the room). control of the technical properties of the working laser and accessories, control of the safe use of lasers during treatment, the procedure for calibrating the laser, control and cleaning of the laser after treatment, guidelines how to handle technical fallures, the availability and use of smoke-evacuators, and control of the entrance of the laser room (e.g. who holds the key of the room). Furthermore, the committee has drafted protocols for the use of $\mathrm{CO}_{2}-$ argon and Nd:YAG lasers as well as the use of lasers per specialty. These protacols have been realized in close cooperation of clinicians, anesthesiologists, biomedical engineers and industry representallves. Therefore, the Committee feels that these suggestions deserve attention of a broad audience ${ }^{[255}$.

All hazard control measures represented in standards and protocols are subject to human error. The most reliable protection systems against laser radiation injury are those protective features that are engineered into the laser design or that are designed into the facility. High quality laser safety management and education and training programs should minimize human error.

\subsection{Laser safety management}

It should be clear from the previous paragraphs that the administrative role does not end with appointment of an LTC or a laser task force that prepares the purchase of a laser. The complexity of laser technology, the involvement of various different professionals and the need to adhere to a variety of safety measures, standards and protocols requires structural organizational adjustments in order to assure quality of laser use. Therefore, a second committee that should be appointed by the hospital board is a laser safety committee (LSC), which should be a permanent committee, chaired by a Laser Safety Officer (LSO).

The Dutch National Committee on Laser Safety formulated a number of recommendations for the duties and membership of LSC and the duties and qualifications of the LSO, respectively ${ }^{\mid 25 \|}$.

\section{Duties of the LSC are:}

1 drafting rules for safety measures required in rooms where lasers are used;

2 drafting and if necessary modifying safety protocols for the use of the various laser types, in the various types of rooms and for each user discipline"

3 where appropriate, drafting specific safety rules for anesthesia and ventilation during the use of lasers;

4 assessing aspects of laser safety when laser equipment is procured and assessing the maintenance protocols to be used;

5 advising the hospital board on whether medical specialists who use lasers, and the personnel who assist them, are adequately trained in the use of new laser procedures;

6 drafting and keeping up to date a compulsory oral and written package of instructions for laser workers (physicians and assisting staff):

7 dealing with reported safety incidents involving lasers, drafting preventive measures and ensuring to the best of its ability that these measures are carried out;

8 preparing (annual) reports on its activities.

Recommended membership of the LSC is as follows:

1 a Laser Safety Officer (LSO);

2 a representative of the medical specialties that use lasers;

3 a representative of assisting staff from each department which uses lasers;

4 a representative of the medical instrumentation department;

5 an anesthesiologist if lasers are used on patients under anesthesia. 
It: is essential to have the relevant physicians and members of assisting staff from the various departments as members of the LSC in order to produce measures which are workable and acceptable. Meeting this demand aiso ensures reliable feedback to the committee from those working with lasers on a day-to-day basis. Without such feedback inadequate safety measures would just be poorly implemented rather then modified followed by proper implementation. Also the Laser Safety Officer can make important contributions here.

The recommended duties of the LSO are as follows:

1 assembling and studying relevant literature;

2 maintaining all relevant contacts inside and outside the hospital;

3 passing on all relevant knowledge to everyone in the institution involved with laser safety;

4 preparing discussions by the laser safety committee on the drafting of rules and protocols by drawing up concrete proposals in consultation with the relevant physicians and their assisting staff;

5 organizing and monitoring the proper conduct of measurements in regard to the choice of materials and safety rules;

6 giving users, assisting staff and technical staff, or ensuring that they receive, instruction on laser safety in general and related procedures, rules and protocols in particular;

7 maintaining and updating an administrative database including laser users and assisting staff, the lasers, laser rooms and the agreed safety rules and protocols;

8 helping to coordinate the activities of the LSC with other committees aimed at improving hospital safety;

9 investigating reported incidents involving laser safety (occurrences which resulted or might have resulted in accidents or unnecessary risks); in preparation for their consideration by the LSC;

10 reporting of accidents to the Labour Inspectorate;

11 arranging the option of an eye test following a laser accident potentially entailing eye damage.

It is apparent that there is a major risk of eye injury when lasers are not used safely. An establishment in which lasers are used must make provisions for eye damage following a laser incident to be assessed by an expert within 72 hours (see also Chapter 18). The relevant procedure must be decided on and published by the hospital board. Once the LSO has been notified of an accident involving eye dlamage it is part of his duties to ensure, unless the worker concerned declines an eye test, that a recommended ophthalmologist is contacted promptly. The victim should inform the ophthalmologist when the incident occurred, the type of laser used (wavelength), its settings (output, pulsewidth, pulse type, fibre diameter or distance between the site of the burn and the microscope and handpiece) and any other relevant facts. The results of the eye test are presented to the accident victim. The LSO asks permission to use this information for further processing into preventive measures.

To be fit for the job, the LSO should be educated at least at a school of higher vocational education (B.Sc.-level). The LSO should have a comprehensive grasp of the physical, technical and biological aspects of clinical llaser use. For this the LSO will need additional techmical study plus broad experience and extensive contacts within the hospital. He also needs to possess good communication skills and to be respected as an expert interlocutor by physicians, assisting staff and technical staff. Only then will these professionals be adequately motivated to abide by the safety rules. Reasons for inadequate motivation may be that the physical necessity of the safety measures is not understood, the importance of prevention is not recognized, or that the measures impede the smooth progress of patient treatment of the efficiency of the department. Thus, communication is essential at all stages of the development and implementation of safety measures. Bad measures are never accepted. Good measures require a lot of communication. Good communication is again necessary thereafter for instruction in the workings of the safety policy and for regular assessment of this policy. 
It goes without saying that both small and large hospitals as well as private clinics which use lasers must ensure that their use of lasers is safe. It may be to the advantage of smaller hospitals to draw on expert laser safely services serving a whole region. In this context one can envisage an LSO working regionally. Major considerations here are:

1 an LSO must hold an office in at least one of the hospitals concerned which should be sufficiently large to allow remaining fully abreast of all matlers concerned with the practical use of lasers and accessories;

2 in each of the hospitals concerned, responsiblity for all the duties to be carried out by the various parties concerned must be clearly defined and laser safety management must be fully integrated in the overall safety management in the hospital;

3 a tregional" LSO may be employed under a part-time contract or on a consultancy basis. whereby one LSO employed by a hospital may be consulted by another LSO who has lesS specific knowledge. In both cases it is necessary for the regional duties of the LSO(s) in the hospitals concerned to be clearly established;

4 the regional LSO must be enabled to become a member of a LSC in any hospital. A regional LSC is not recommended, because the members would not feel closely enough involved in each individual hospital and would not be sufficiently familiar with its circumstances. There must of course be an adequate level of technical support in every hospital where lasers are used;

5 concerning responsibilities, it makes sense that where an establishment employs a part-time LSO, this professional should be appointed as chairman of the LSC in that hospital.

In the Netherlands two different trends influence the need for regional LSO's. On the one hand, due to mergers and (occasionally) hospital closures, the need for regionall LSO's decreases: At the same time, due to the emergence and (slow) increase of the number of private clinics, the need for regional LSO's increases.

\subsection{Training programs for lasers}

A number of non-governmental organizations are involved in laser training programs: the European Laser Association national laser societies and safety boards, laser centers affiliated with hospitals and universities, laser specialty societies, manufacturers, hospitals and independent groups of laser surgeons. These training programs often differ in scope and are sometimes exclusively directed to physicians.

In 1988 the European Laser Association (ELA) developed standards for approval of a workshop for physicians, regarding content and quality ${ }^{120]}$. It has been suggested that workshops should be conducted by experimental professionals from institutes or institutions of a certain standard with proven longstanding experience in laser therapy within the specific discipline(s) that are addressed in the workshop. A workshop should last at least two days and at least one day should be reserved for hands-on training. The agenda of the workshop should include:

- a pretest in which the participants give a statement of their level of and demand for laser education;

- laser physics and laser-tissue interaction;

- indications for laser therapy;

- anesthetic techniques;

- safety regulations, standards and the corresponding safety measures;

- complications of laser procedures;

- cost-effectiveness of selected applications;

- key operator procedures as an introduction to hands-on training; 
- hands-on training to simulate the various tissue effects at a variety of power outpult;

- live operations, which should be performed at least half a day;

- presentation and discussion of case reports;

- a post-test and a diploma at the end of the workshop.

Even after going through such a workshop; participants will not be fully educated as laser surgeons, and the diploma surely does not mean that one is fully qualified in laser therapy ${ }^{1201}$. This would require some form of certification; which is controversial on a European level. Besides developing standards, the ELA frequently sponsors workshops, often in cooperation with the national laser society, individual laser centers and laser specialty societies.

Traditionally, manufacturers and distributors of medical lasers are an important source of laser information and education "even though the training they offer is limited in scope ${ }^{\mid \text {"! }}$. Generally, a manufacturer provides courses in laser physics and safety, as well as hands-on training and using specific equipment, but does not instruct surgeons in clinical techniques. However, some manufacturers and distributors are becoming increasingly involved in educational activities, to the extent that they organize programs in various surgical specialties and engage laser surgeons to lecture and instruct other surgeons in clinical techniques. Laser training for nurses is provided by manufacturers, hospitals, and university centers. Nurses staffing the areas where laser equipment is installed should become knowledgeable and skilled in laser application to develop care plans, to ensure that certain safety precautions are observed, to develop a post-treatment evaluation process, and to assure appropriate discharge instructions ${ }^{[17]}$.

Some hospitals organize in-house training programs ${ }^{11 !}$. Although these programs are expensive for the hospital to conduct, the tuition paid by physicians may offset the costs, especially if the program is opened to physicians from other institutions. The benefits are training for many staff members and personnel, increased enthusiasm and cooperation among departments, widespreard understanding of laser safety procedures, and an overall raising of the hospital's laserconsciousness'.

Hospital technicians, such as biomedical equipment technicians, may find it useful to attend laser programs for background information. However, as outlined in paragraph 3.5 , they should not be permitted to service a laser without first obtaining special training in this field.

Finally, an educational session should be offered to anesthesiologists who will provide anesthesia to patients having laser treatments ${ }^{[15]}$.

To solve the educational problems of each subgroup of professionals, it would be helpful if the ELA and the various professional organizations would join efforts to develop and encourage workshops focused on the entire spectrum of professionals involved in the use of lasers. Perhaps the outline of the German certificate program comes closest to fulfilment of these needs. This program is divided into two parts ${ }^{|A|}$. The first part provides information that should be of common interest to physicians, supporting medical staff and technicians. This part provides information on physics, laser-tissue interaction, principles of laser systems and accessories, and the safety regulations. In addition, the course provides information on the principles of laser applications and therapeutic guidelines. In the second part, training for different fields of medicine is offered to medical dactors only. Specific information is provided on indications, contra-indications, application rules, and problems of after-care. The inexperienced physicians assist in laser applications and perform a number of treatments defined for the special fields of medicine, supervised by an acknowledged expert. In the final stage of the course the attained knowledge is assessed and certified.

More information of the different forms of certification that usually follow training programs can be found in Chapter 20. 


\subsection{Conclusions and discussion}

Acquisition and installation of a laser is a complex and lengthy process, requiring a concerted effort of many professionals. The entire procedure, starting from the moment that a physician expresses the wish to use a laser until the laser can be actually used, usually takes 1 up to 1.5 years, especially in case of a first investment. After delivery it may take 6 months before the entire system is operational and 'user-friendly" Safe, effective and efficient use of lasers is facilitated by careful adherence to standards, protocols, and in particular by maintaining a well-trained staff. Most education and training in laser fechnology is fragmented, however.

Due to rapid technological developments, new investments may be judged necessary after a relatively short period of time, perhaps a few years. Several factors contribute to this situation. Firstly, there is a trend towards smaller, cheaper, multifunctional, more energy efficient and more mobile lasers which do not require extensive additional power supply and cooling facilities. Secondly, increasingly, two or more lasers are combined in one product by manufacturers. For example, Coherent has recently produced a combined Ho-YAG-and Nd:YAG laser ${ }^{[2]}$. Thirdly, due to rapid technological development in the field of minimally invasive therapy as a whole, the question whether a laser needs to be replaced by another laser or another minimally invasive procedure will become more important. In answering this question it should be taken into account that innovative optical fiber technology enables more lasers to be used in minimally invasive therapy ${ }^{[2]}$ (see also Chapter 20).

Besides complicating (replacement) investment decision-making, these trends increase opportunities for sharing and also make the choice of location of the laser less critical. Despite this increased flexibility, however, safety measures will still have to differ depending on the specific type of laser. Therefore, the individual locations for use of lasers are not interchangeable without modification. For example, a laser room well suited for $\mathrm{CO}_{2}$ laser procedures may have normal glass windows because the light of this laser is fully absorbed by glass. This is not adequate for near infrared radiation, as emitted by the Nd:YAG laser. In this case the use of shutters or nonflammable shades is recommended ${ }^{(13)}$. Thus, the technical developments create new opportunities which can only be optimally applied when at the same time appropriate attention is paid to aspects of safety.

Other technical developments specifically improve the safe use of lasers. For example, a technological improvement supporting the safe use of ophthalmological lasers is the development of a gyroscope that, fitted to a headband, switches off an eye-guided medical laser if the ophthalmologist makes a sudden, rapid movement while using the device, e.g. in performing retinal surgery ${ }^{[2]}$. Of course, the continuous development of new laser systems and accessories, including accessories such as the gyrascope described above, justifies vregular attention to the aspects of education and training as well.

In summary, the increasing number and the introduction of different types of lasers will allow the implementation of multi-laser interdisciplinary programs. The organizational structure of programs will become more complex and safety measures will also need to be more complex and more specific. This may be complemented by specific lechnical improvements in laser safety. These improvements warrant contínuous monitoring by laser safety committees, laser-using physicians and other professionals involved. 


\subsection{References}

1. American National Standards institute (ANSil). Safe use of lasers in health care facillities, Standard Z-136.3. American National Standards Instituite, Laser Institute of America. Orlando, Fil "United States, 1988.

2. Anonymous. Frost \& Sullivan Market Inteiligence Reports. World surgicial laser market. Journal of Mecical Engineering \& Tecthology 1995(19)5:187.

3. Anonymous. Head-mounted gyroscope acts as safety switch. Opto \& Laser Europe 1996(28):15.

4. Berlien HP. The German accreditation rules for laser medicine. In: European Comamunity Medical Laser Concerted Action Program. Proceedings of the first plenary workshop on safety and laser-tissue interaction. Balliere Tindall. London, 1989 , p.385-95.

5. Carruth JAS. Training in Great Britain. In.. European Community Medical Laser Concerted Action Program. Proceedings of the first plenary workshop on satety and laser-tissue interaction. Baillière Tindall, London, 1989, p. $373-4$.

6. Deutsche Industry Norm (DIN) 58125. Laserschutzfilter und Laserschutzbrillen, Sicherheitstechnische Anforderungen und Prufung. Berlin 1988.

7. Dutch National Hospital Institute. Lasers in the hospital. A preliminary surwey. Utrecht, the Netherlands 1989 (in Dutchy.

8. ECRl. Evaluation: laser-resistant endotracheal tubes and wraps. Health Devices 1990(19)4:112-39.

9. ECRl. Health Technology Checklist 1993/1994. In: Health Technology Trends 1993(Supplement 1).

10. ECRI. Laser technology planning. Health Devices 1992(21)9:297-305.

11. ECRI. An. Lasers: facilitating their acquisition and wider use. In." Moretti M (Ed.). Medical Lasers Buyers" Guide 1988. Littleton, MA,: PennWell Publishing Co., 1987, p. 118-23.

12. Gray $F$. Mittelman $H$. Implementation and management of a laser program. \|n: Apfelberg DB (Ed.), Evaluation and installation of surgical laser systems. Springer Verlag. New York, 1987, p. 54-68.

13. Holmes JA. A summary of safety considerations for the medical and surgical practitioner. In: Apfelberg DB (Ed.) Evaluation and instaliation of surgical laser systems. Springer Verlag, New York, 1987, p. 89-95.

14. International Electrolechnical Commission (IEC). Radiation safety of laser products, equipment classification, requirements and user's guide. $\| E C_{n}$ Publication 825 , Geneva, 1984.

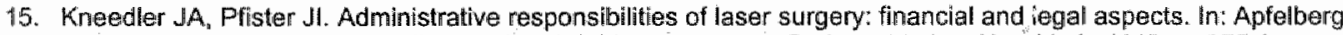
$\mathrm{DB}(\mathrm{Ed}$.). Evaluation and installation of surgical laser systems. Springer Verlag, New York, 1987, p. 375-9.

10. Mackety $\mathrm{CJ}$. Administrative responsibility for laser sungery: nursing and administrative roles. In: Moretti $M$ (Ed.). Medical Laser Buyers' Guide 1988. Littleton, MA: PennWell Publishing Co., 1987, p. 102-10.

17. Macular Photocoagulation Study Group. Argon laser photocoagullation for senile macullar degeneration. Archives of Ophthalmology 1982(100):912-8.

18. Mueller EP, Ciarkowski A, McDermott K. Regulation of biomaterials and medical devices. In: Bronzino JD (Ed.). CRC Press, Inc., United States, 1995, p. 2802-118.

19. Orfice of Technology Assessment. The quality of medical care: Information for consumers. OTA-H-386, Washington, D.C: US Government Printing Office, 1988.

20. Paulsen JW. Laser training and accreditation in Denmark. In: European Community Medical Laser Concerted Action Program. Proceedings of the first plenary workshop on safety and laser-tissue interaction. Bailliere Tindall, London. 4989, p. 375-9.

21. Pfister J. Purchasing a laser. In: Moretti M (Ed.). Medical laser buyers' guide 1988. Littleton, MA:PennWell Publishing Co., 1987, p. 111-2.

22. Sliney DH. Laser safety. Lasers in Surgery and Medicine 1995(16):215-25.

23. Snow JiC, Norton ML, Saluja TS, Estanislao AF. Fire hazard during $\mathrm{CO}_{2}$ laser microsurgery on the larynx and trachea. Anesthesia and Analgesia 1976(55)1:146-7

24. Sterrenburg PG., wan der Meulen PW, Oei-Lim VLE, Vaartjes SR, Zandbergen RA. Lasers in clinical medicine. In Eggink Gu, Kaptein C, wan Kempen RJ, van der Meulen FW, Tellinck C.JPM, Vaartjes SR (Eds.). National Committee on Laser Safety, Laser safety in health care (second edition). The Netherlands Organization for Applled Scientific Research (TNO), Division Prevention and Health, Leiden, the Netheirlands, p. 11-5 (in press).

25. Vaartjes SR, Dignum PH, van Kempen RJ, van der Meulen FW, Sterremburg PG., Vondeling H. Struchure of responsibillity and the laser safety framework in hospitals. In: Eggink GJ. Kaptein C, van Kempen RJ "van dier Meuten FW, Teirlinck C.JPM, Vaarties SR (Eds.). National Committee on Laser Safety, Laser safety in health care (second edition), The Netherlands Organization for Applied Scientific Research (TNO), Division Prevention and Health, Leiden, the Netherlands, p. $109-20$ (in press). 
Part Two - Effectiveness and Cost-Effectiveness of Selected Laser Applications 


\section{Introduction}

\subsection{Contents and structure}

In part 2 an update is presented of the results of a project that was supported during the period 1988-1991 by the COMAC-HSR of Directorate General XII of the European Commission (EC). A brief description of the EC-project can be found in Chapter 2. Briefly, part 2 aims at synthesizing six years of progress in clinical and economic evaluation in selected areas of the medical laser field and to guide future research. Chapter 4 provides a theoretical background for this exercise. Then major laser applications in six specialties are discussed: Dermatology (Chapter 5), Ophthalmology (Chapter 6), Otolaryngology (Chapter 7), Gastroenterology (Chapter 8), Urology (Chapter 9), and Gynecology (Chapter 10). Photodynamic Therapy (PDT) is not discussed as a separate issue but integrated in a discussion of the evidence for specific indications in each specialty, e.g. in treatment of dermatological tumours, esophageal cancer and bladder cancer. Chapter 11 summarizes and discusses the findings.

\subsection{Literature search strategy}

To include relevant articles, Medline and Embase searches were performed covering the period of 1976, when the first interim-analysis of a randomized controlled trial (RCT) on lasers was published, to a period ranging from mid 1994 (for Dermatology) to mid 1996 (for Gynecology). The closing date of the manuscript for each of the other specialties was in between the dates indicated above. Supplemental articles were found by reference tracking, by a survey of 'grey" literature including reports of the EC, government advisory bodies and national health lechnology assessment agencies, and by consulting experts.

As the gold standard for measuring effectiveness of therapeutic interventions is the (RCT) ${ }^{31 !}$, it is in particular RCTs that were selected. Likewise, as the quality of the evidence on cost-effectiveness is dependent on the quality of the underlying medical evidence ${ }^{|4.12|}$, evidence on the costeffectiveness of laser applications was preferred which was collected as an integrated part of an RCT. In case evidence based on RCTs was not available, evidence from other' studies was accepted, including for example controlled but non-randomized studies. Whenever case-series had to be relied upon, large, well-documented studies with an appropriate length of follow-up were selected. Likewise, if If no other evidence was available, cast descriptions based on case-series were inciuded. In general, it was tried to select the best available evidence.

The hierarchy in the quality of evidence was reflected in the computerized search strategy. Key words for identification of articles included combinations of the MeSH terms of the condition (e.g. "endometriosis') and the search terms "lasers" or 'laser surgery', or "laser coagulation' with the terms "randomized controlled trial', 'controlled clinical trial', 'review', 'meta-analysis', 'cost", 'cost-analysis', "economics", 'adverse events', and 'complications'. Additional search terms were used to retrieve background data. The publication language had to be either English or Dutch, although occasionally articles in French, German, Spanish or Italian were included. In addition, English language abstracts of RCTs published in other languages than those listed above were accepted for inclusion when they were clear and concise. 


\subsection{Evaluation of safety, effectiveness and cost-effectiveness}

\subsubsection{Safety}

As discussed in Chapter 3 , safety of medical laser applications can be a problem, and is obviously related to effectiveness and cost-effectiveness. Certain safety precautions are necessary in all cases $^{(16)}$. The mast important issues in laser safety are eye protection, dealing with the plume of vaporized tissue, and controlling potential fire hazards ${ }^{54}$. A common complication with lasers is burning deeper than planned, which can result in problems such as perforation of the bowel of other organs. In cancer treatment, the laser may make pathological testing of tissue or staging of the tumor impossible. These and other questions relating to the safety of laser procedures, and laser safety management, are discussed in conjunction with data on effectiveness and costeffectiveness.

\subsubsection{Effectiveness}

\section{Assessment}

When available in the form of RCTs, the quality of evidence on the safety and effectiveness of laser applications was assessed using guides formulated by Sackett, Haynes and Tugwell (1985), in particular those in Chapter 7 and Chapter $12^{[3 !]}$ and by Banta (1991) ${ }^{[4]}$. Examples of these guides, or questions are:

1 Was the assignment of patients to treatments really randomized?

2 Were all clinically important outcomes reported?

3 Was the outcome assessment 'blind'?

4 Were both clinical and statistical significance considered?

5 Were all the patients who entered the study accounted for at its conclusion?

When no information from RCTs was available, selected studies were assessed based on genera criteria for the reporting of clinical research, such as an adequate description of inclusion criteria and completeness of reporting[31]. By applying the literature search strategy outlined in paragraph 4.2 and the assessment strategy outlined above, it can be argued that the efficacy rather than the effectiveness of laser applications is documented in this assessment. Efficacy may be defined as 'the probability of benefit to individuals in a defined population from a medical technology applied for a given medical problem under ideall conditions of use'. Efficacy, then, is the best results possible, and is approximated by the results of good clinical trials done in the best medical centers Effectiveness refers to the results found in the community after widespread implementation of the intervention ${ }^{|4|}$.

\section{Clinical effect measures and quallity of life}

In the 1991 EC laser-project it was found that the advantages of lasers had usually been expressed using clinical measures, although accasionally improvements in quality of life for the patient had been claimed. In general, the advantages for laser therapy may include: no touching of the hands, reduced blood loss, reduced edema, reduced postoperative pain the possibility to apply less invasive techniques, easier access to difficult sites, a surgical field free of instruments, increased precision, selective absorption of light, a sterile procedure, less danger of metastasis during surgery, the possibility of outpatient treatment or a shorter length of stay ${ }^{\mid 5,46}$.

Since completion of the EC-laser project, the field of quality of life research has become more visible. Although the full potential of quality of life research for medical technology assessment has not yet been realized, the tools for measuring quality of life have increased in number and breadth $^{(7)}$. This development, pertaining both to specific and generic instruments, may have positively influenced the availability of data on the impact of laser treatment on quality of life. Although a summary of these developments is beyond the scope of this thesis, a few highlights wil be presented. In particular generic instruments are important here, because they offer the 
possibility of detecting treatment differences across a broad range of health-related quality-of-life dimensions, and they enable the comparison of outcomes across a range of different interventions, conditions and populations ${ }^{[28]}$. With this particular focus, it is perhaps appropriate to start with the World Bank Report "Investing in Health', which promotes application of the disability-adjusted lifeyear (DALY) as a generic outcome measure as well as a measure of the (global) burden of disease ${ }^{[2]}$. This report has received much attention within the international health community and among aid donors ${ }^{[7]}$. Of all generic health-related quality of life instruments, perhaps the Nottingham Health Profile (NHIP), the Sickness Impact Profile (SIP), the Medical Outcomes Study Short-Form 36 ltem Health Survey (MOS SF-36 or SF-36) "the EuroQol, the Dartmouth Primary Care Cooperative Information Project (COOPWONCA) chart system , the European Organization for Research and Treatment of Cancer (EORTC) Quality of Life Questionnaire (QLQ) and the World Health Organization's WHOQOL have diffused most widely ${ }^{\prime 1 !}$. Of these, contrasting with the other instruments, the COOPWONCA chart system is used to measure functional health status specifically in primary care ${ }^{\mid 32]}$. In the Netherlands, the Dutch Working Group on Health Status Assessment has recommended the use of the COOP/WONCA charts as a 'common core' generic instrument in studies aimed at assessing health status. The use of these charts has been recommended for a specific period (1994-1996) $)^{151}$. Of the instruments listed above, the World Health Organization's project to develop an international, multicultural quality of life instrument (WHOQOL), has been initiated too recent to have an impact in this assessment of laser applications ${ }^{10.37}$. This instrument in particular may hold a promise for intensified use of quality of life data on a global scalle ${ }^{[3 \% !}$.

\subsubsection{Cost-effectilveness}

\section{Assessment}

The medical literature abounds in statements that medical laser applications are cost-effective ${ }^{(5)}$. However, the underlying data and methods of data-analysis often do not allow for such a conclusion. Economic principles dictate that all costs and benefits of laser use should be analyzed. Analytical methods to determine economic effects comprise a spectrum ranging from sophisticated computer-based data-analyses to best-guess estimates of costs and benefits[25]. The term 'costbenefit analysis' refers to valuing all costs and benefits in monetary terms, and is difficult to apply in health care. A 'cost-effectiveness analysis", in contrast, produces a measure of the cost involved in terms of some desirable health-related effect, e.g. a life-year gained. Where the consequences of two or more alternatives are examined alongside costs, and are shown to be equivalent, the study is called a "cost-minimization analysis'. A "cost-utility analysis' uses an outcome measure that includes patient utilities of the outcome. Utility refers to the value or worth of a specific level of health status (or improvement in health status) and can be measured by the preferences of individuals or society for any particular set of health outcomes ${ }^{\{13\}}$. A common example is to use Quality-Adjusted Life-Years (QALY's), which would include pain and functioning as well as mortality. In this case, the cost-effectiveness ratio consists of the costs per quality-adjusted life-year gained.

In order to assess the quality of the evidence documenting the cost-effectiveness of laser applications, the checklist for assessing economic evaluations by Drummond, Stoddart and Torrance (1987) has been tentatively applied ${ }^{13 !}$. This checklist, which was slightly modified by Drummand (1991) for the case of lasers, basically includes ten questions: ${ }^{[14]}$

1 Was a well-defined question posed in an answerable form?

2 Was a comprehensive description of the competing alternatives given?

(i.e. can you tell who did what to whom, where, and how often)?

3 Was there evidence that the programs' effectiveness had been established?

Was this done through a randomized, controlled trial? If not, how strong was the evidence of effectiveness?

4 Were all the important and relevant costs and consequences for each alternative identified?

5 Were costs and consequences measured accurately in appropriate physical units? 
(e.g hours of nursing time, number of physician visits, or years of life gained)

6 . Were costs and consequences valued credibly?

7 Were costs and consequences adjusted for differential timing?

8. Was an incremental anallysis of costs and consequences of alternatives performed?

Were the additional (incremental) costs generated by the use of one alternative over another compared with the additional effects, benefits or utilities generated?

9 Was a sensitivity analysis performed?

10 Did the presentation and discussion of the study results include all issues of concern to users?

There is a close relation between these questions and the questions formulated by Sacket, Haynes and Tugwell for assessing the quality of evidence on effectiveness, in particular RCTs: Drummond (1991) notes that the availability of medical evidence is almost always a problem, and that the laser field is no exception ${ }^{1 / 4 !}$. Therefore, the following additional questions should be posed:

1 For which applications of lasers does reliable medical evidence exist, and does this provide an adequate basis for economic evaluation?

2 For which applications are clinical trials planned and is there any scope for undertaking economic analysis alongside these trials?

3 For which major applications is there yet no reliable medical evidence and what efforts should be made to assemble such evidence?

These questions, in particular the latter two, combined with the input of the EC-project and its update, have been used to formulate recommendations on future research. These recommendations have been included in Chapter 20. The combination of the checklist by Drummond (1991) and the guides by Sackett "Haynes and Tugwell (1985) constitute the analytical framework used throughout Chapter 5 to 10.

\section{Guidelines}

Since the time that the checklist for assessing economic evaluations was created by Drummond, Stoddard and Torrance (1987), many developments have taken place in the field of economic evaluation, which may have affected the data that have become available in the laser field. Although, again, a summary of these developments is beyond the scope of this thesis, some highlights will be briefly pointed out. Internationally, most progress has been made in the field of pharmacoeconomic evaluation. In August 1990, the Australian government was the first to release (draft) guidelines for the pharmaceutical industry on preparation of submissions to the Pharmaceutical Benefits Advisory Committee (PBAC). These guidelines were subsequently amended and the first revised guidelines were published in August 1992, for implementation from 1 January 1993. In November 1995 a revised, more demanding set of guidelines was released by the Australian government ${ }^{\mid 2,3}$. The Canadian Coordinating Office of Health Technology Assessment (CCOHTA) released the first edition of its 'guidelines for economic evaluation of pharmaceuticals in Canada' in 1994 other countries with similar initiatives could be added. Besides creating and updating these specific pharmacooeconomic guidelines, researchers have tried to extend the scope of these guidelines in order to allow them to be used with procedures and medical devices as well. An example here is the United Kingdom ${ }^{[24]}$. In the Netherlands, practical guidelines for economic evaluation and scenario-analysis have been produced in $1993^{\text {(30). }}$. Likewise, general (draft) guidelines for economic evaluation have been produced in Switzerland in $1995^{|b|}$ and in the United States in $1996^{\mid 18 t}$. Some of these latter developments are too recent to have an impact on the quality of data in this assessment, but they hold a promise for the future.

Progress has also been made in determination of utilities, which reflect the relative values individuals place on different states of health. Utilities, as outlined earlier, can be used as an input for cost-utility analysis, for which the Quality-Adjusted Life-Year (QALY) is the most frequently used output measure. The work of the EuroQol group may serve as an example of progress in this field. In the early nineties, a group of European researchers developed this instrument, which can be 
characterised as a standardised generic multidimensional instrument for both describing and valuing health-related quality of life. Based on a rating scale, the instrument can be used to express health-related quality of life as a single index value ${ }^{[2]}$. A major aim of the EuroQol Group in this collaborative exercise was to devise an instrument that would allow cross-national comparisons of health state valuations. Results obtained with the EuroQol are encouraging ${ }^{[9 !}$. The instrument has been translated to nine languages and more official translations are underway ${ }^{\mid t \eta}$. It should be recognized, however, that no single method of eliciting health state utilities (e.g. standard gamble, time trade-off) has yet been recognized as standard. Thus; the calculation and use of for example QALY's or any other such measure (e.g. Healthy-Years Equivalents (HYEs)) will continue to be controversial (see for example the 1993 and 1995 wolumes of the Journal of Health Economics): Nevertheless, some of the intensive activities in this area could be reflected in recent publications in the laser field.

Perhaps the most comprehensive actual source of information on recent developments in (pharmaco)economic evaluation is the second edition of a book by Spilker ${ }^{[35]}$, to which the readers are referred for additional information.

\section{Direct and indirect costs}

As a consequence of the checklist for assessing economic evaluations, what costs should be considered in the case of medical lasers? When adopting a societal point of view, both the costs of organizing and operating the laser facility, the costs which are borne by the patients and their families, and the cost of production losses should be included ${ }^{|13|}$. These categories of costs are usually referred to as direct health care costs, direct non health care costs, and indirect costs, respectively.

\section{Direct health care costs}

Lasers are expensive devices and can add considerably to the cost of therapy. As reported in an article in 1990, the costs of a $\mathrm{CO}_{2}$ laser amounted US\$40 000 in the case of a 25 Watt laser up to US\$100 000 for a 65 Watt laser ${ }^{|5|}$. The capital cost of an argon lasers ranged from US\$40 00060000 , compared with US\$65 000 for a 60 watt Nd:YAG laser and US $\$ 100000$ for a 100 watt Nd:YAG laser. Estimated costs of operating a laser are presented in Table 4.1.

Table 4.1 Estimated annual operating cost for $\mathrm{a} \mathrm{CO}_{2}$ and an Nd:YAG laser (1000 US\$), at 5 and 10 percent interest rate in 1990 ( 1 US $\$=2 \mathrm{DfI}$ ).

\begin{tabular}{lcccc}
\hline \hline Type of Costs & \multicolumn{2}{c}{$\mathrm{CO}_{2}$ laser } & \multicolumn{2}{c}{ Nd:YAG laser } \\
\hline Capital & $5 \%$ & $10 \%$ & $5 \%$ & $10 \%$ \\
Provisions & $20.8-27.7$ & $23.7-31.7$ & $20.0-26.0$ & $22.8-29.6$ \\
Running Costs & $1.8-2.7$ & $2.3-3.4$ & $1.9-3.0$ & $2.4-3.7$ \\
Maintenance & 1.3 & 2.0 & 1.3 & 2.0 \\
Personnel & .5 & .9 & .5 & .9 \\
Total & 25.0 & 25.0 & 25.0 & 25.0 \\
\hline
\end{tabular}

\footnotetext{
Capital = Capital costs of laser and accessory equipment;

Provisions $=$ Costs of provisions for special electrical power supply, cooling system, and safety measures;

Exploitation $=$ Costs of water, electricity, etc.;

Maintenance $=$ Costs of maintenance and repair;

Personnel $=$ Technical staff and administrative support; Physician costs are not included.
} 
Table 4.1 shows that the annual costs of use of a laser ranged from about US\$50 000 to US\$60 000 , depending on the interest rate Costs of personnel constituted about 50 percent of costs, even excluding physician time. Capital costs of the laser and accessory equipment accounted for approximately 45 percent of total costs. It should be noticed that since 1990 the costs of lasers have decreased. In addition, in many cases special facilities (for example provisions for a special electrical power supply and water cooling to carry away excess heat) are not necessary anymore, reducing the annual operating costs. On the other hand, the variety and costs of associated equipment (such as microscopes, fibers and tips) has markedly increased, which may partially or totally offset the reduction in annual operating costs outlined above.

Another reason to interpret these figures with caution is that they were best estimates and depended on important assumptions. The most important assumption was that laser treatment capacity is completely used. In practice, this situation is seldom found. Therefore, all variable costs could be lower than those presented here. Due to rapid developments, the life of a laser and asscciated equipment is assumed to be no more than 5 years, while depreciation of accessories is assumed to take 10 years. Costs of exploitation, maintenance, and personnel recur every year: The costs of one laser treatment of course depends on the number of times the instrument is used.

Direct non health care costs

These include for example the costs of travel and the costs of travel time and waiting time for the patient and his or her family. No evidence at all has been published on direct non health care costs in the laser field.

\section{Indirect costs}

These are the costs of forgone production of the patient related to treatment of the disease. These costs can be calculated using the Human Capital Method, but preferably by the Friction Cost Method ${ }^{|20|}$. This method, which has been refined and adapted for practical use ${ }^{|21.22|}$, has been applied once in the laser field ${ }^{[11]}$. Usually, indirect costs have been estimated indirectly by reporting the number of days absence from work.

Considering the requirements for economic evaluation and the actual availability of evidence, it can : be concluded that most evidence that will be discussed in Chapter 5 to 10 , pertains exclusively to: direct health care costs of laser treatment. 


\subsection{References}

1. Anderson RT, Aaronson NK Bubinger M, McBee WL A rewew of the progress towards developing health-Felated quality of life instruments for international clinical studies and outcomes reseanch. PhamacoEconomics $1996(10) 4: 36-55$

2. Appelman YEA, Piek UJ, Strikwerda S, Tissen JGP, de Feyter PJ, David GK, Serruys PW, Margolis JR, Koellemay MJ, Montauban van Swindregt EWJ, Koolen $\mathrm{JJ}$. Randomized trial of excimer laser angioplasty versus balloon angioplasty for treatment of obstructive coronary artery disease. Lancet $1996(347): 79-84$.

3. Banta HD, Schou 1 (Eds.). Lasers in Hoalth Care. Effectiveness Cost-Effectiveness and Policy Implications. Acrademic Publishing, Frederiksberg, Denmark, 1991.

4. Banta HD. Measuring the efficacy of laser applications in medicine: In: Eanta HD, Schou i (Eds). Lasers in Health Care. Effectiveness, Cost-Effectiveness and Policy Implications. Academic Publishing, Frederiksberg, Denmark, 1991, p. 54-62.

5. Banta HD, Vondeling H, de Wit $A_{n}$ Haan G. Economic appraisal of laser applications in medicine. Lasers in Medical Science 1990(5)3:253-69.

6. Banta $H D$, Schou 1 , Vondeling $H$, de Wit A. Economic appraisal of laser applications in health care. Report of a project. Lasers in Medical Science 1992(7)1:9-2\%.

7. Battista RR, Hodge MJ. Quality of life research and health technology assessment - a time for synergy: Quality of Life Research 1996(5):413-8.

8. Brandt A, Cranovsky R, Horisberger B (Eds.). Manual for the standardization of clinical and economic evaluation of medical technology. Swiss Federal office of Social Security, Berne, 1996.

9. Canadian Coordinating Office of Health Technology Assessmant (CCOHTA). Guidelines for economic evaluation of pharmaceuticals (first edition). Ottawa, Canadia, November 1994.

10. Commonwealth Government. Draft guidelines for the pharmaceutical indusitry on preparation of submissions to the PBAC including submissions including economic analyses. Camberra, Australia, June 1995.

11. Crijins HiHM. Diabetic retinopathy: a cost-effectiveness analys is of ophthalmoscopy and photocoagulation (thesis). Erasmus University Rotterdam, the Netherlands, 1993.

12. Drummond MF, Davies L. Economic analysis alongside dinical trials. Revisiting the methodological issues. International Journal of Technology Assessiment in Health Care 1991(7)4:561-73.

13. Drummond MF, Stoddart GL. Torrance GW. Melhods for the economic evaluation of heaith care programs. Oxford University Press, Oxford, New York, Toronto 1987.

14. Drummond MF. Economic evaluation of laser applications. In: Banta HD. Schou \#(Eds.). Lasers in Health Care. Effectiveness, Cost-Effectiveness and Policy implications. Acadlemic Publishing. Frederliksberg, Denmark "1991, p. $72-81$.

15. Essink-Bot ML. Bonsel G.J. Towards stamdardisation of instruments for health status assessment. Huisarts en Wetenschap 1995(38)3:117-24 (in Dutch).

16. Eglgink GJ, Kaptein $C$, van Kempen RJ, van der Meulen FW, Teirlinck CJPM, Vaartjes SR (Eds). National Committee on Laser Safety, Laser safety in health care (second edition). The Netherlands Organization for Applied

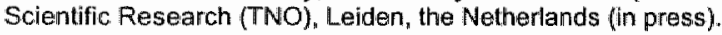

17. EQ-5D User Guide. A measure of health-related quality of life developed by the EuroCol group. EuroQol Business Management, Rotterdam, 1996.

18. Gold MR, Siegel JE, Russell LB, Weinstein MC (Eds.). Cost-effectiveness in thealth and medicine. Oxford Universily Presss, Oxford, New York, Toronto, 1996.

19. Kind $P$. The EuroQoL instrument: an index of healthrelated quality of life. In: Spilker B (Ed.). Quality of life and pharmacoeconomics in clinical trials (second edition). Lippincott-Raven Publishers, Philadelphia, New York, 1996 , p. 191-201.

20. Koopmanschap $M_{\mathrm{B}}$ Ineveld $\mathrm{BM}$ van. Towards a new approach for estimating indiract costs of diserse. Social Science and Medicine 1992(34):1005-10.

21. Koopmanschap MA. Rutien FFH, van Imeveld BM, van Roijen $L$. The friction cost method for measuring indirect costs of disease. Joumal of Health Economics 1995( 14):17/-89.

22. Koopmanschap MA, Rutten FFH. A practical guide for calculating indirect cost of disease. Pharmeco Economics $1996(10) 5: 460-6$.

23. Langley PG. The November 1995 revised Australian guidelines: for the economic evaluation of pharmaceuticals. PhamacoEconomics $1996(9) 4: 341-52$.

24. Mason J, Drummond M. Reporting guidelines for economic studies. Health Economics 1995(4):85-94

25. Office of Technology Assessment. Strategies for medical technology assessment. Washington. DC: US Gavernment Printing Office, 1982.

26. The EuroQol Group. EuroQol - a new facility for the measurement of health-related quality of Hife. Health Policy $1990(16): 199-208$ 
27. The Word Bant World Clevelopment Report 1993. Investing in Health. Wond dewelopment indicators. Oxtond Umiversity Press, Oxford, New York, Toronto; Delhi; Bombay, Calcutta, Madras, Karachi, Kuala Lumpur, Singapone, Hong Kong, Tokyo, Narobi, Dar es Salaam, Cape Town "Melboume, Aucktand, 1993.

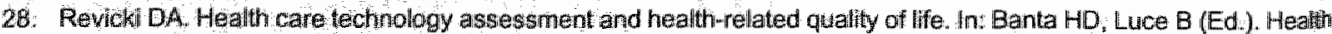
care technology and its assessment. An intemational perspective. Oxford University Press, Oxford, New York. Tokyo; $1993,114-31$.

29. Rovira I. Standardizing economic appiraisal of health technology in the European Community. Sacial Science ant Medicine 1994(38)12:4675-8.

30. Rutten $\mathrm{FFH}$, Ineveld BM wan, Ommen $\mathrm{R}$ van, Hout BA wan, Hulisman R. Scienario-development and costing in health care, practical guidelines; STG/Jan van Arkel, Utrecht the Nethertands (in Dutich).

31. Sackett DL, Haynes RB, Tugwell P. Clinical epidemiology. A basic science for clinical medicine. Little, Brown and Company, Boston, Toronto, 1985.

32. Scholten $\mathrm{HHG}$ yan Weel (Eds.). Functional status assessment in family practice. The Dartmouth COOP Functional Health Assessment Charts WONCA MEDITekst; Lelystad, 1992.

33. Stieldon TA. Problems of using modelling in the economic evaluation of health care. Health Economics 1996(5):111.

34. Sliney DH. Laser safety: Lasers in Surgery and Medicine 1995(16):215-25.

35. Spilker $B$ (Ed.), Quality of life and pharmacoeconomics in clinical trials (second edition). Lippincott-Raven Publishers, Philadelphia, New York, 1996.

36. Torrance GW, Blaker D, Detsky A, Kennedy W, Schubert F, Menon D, Tugwell P, Konchak R, Hubbard E, Firestone T. Canadian guidelines for economic evaluation of pharmaceuticals. PharmacoEconomics 1996(6):535-59.

37. The WHOQOL Group. The World Health Organization Quality of Life Assessment (WHOQOL): position paper from the World Health Organization. Social Science and Mledicine 1995(41)10"1403-9. 


\section{Lasers in dermatology}

\subsection{Introduction}

The first laser to be used in humans was a ruby laser studied by Leon Goldman, a dermatologist, in 1962. Lasers became widely used in dermatology with the development of the argon and $\mathrm{CO}_{2}$ lasers in the early $1970 \mathrm{~s}^{\mathrm{i} 11}$. At present, the argon laser is predominantly employed in treatment of a widle variety of cutaneous vascular lesions while the $\mathrm{CO}_{2}$ laser is widely used in skin surgery ${ }^{27,30 ?}$ The third "classical" laser, the Nd:YAG laser, has found limited application in dermatology ${ }^{1+1}$.

The understanding of laser-tissue interaction in dermatology was greatly enhanced with the theory of selective photothermolysis, which was published by Anderson and Parrish in $1983^{11 !}$.. The theory of selective photothermolysis stimulated fundamental and clinical research in this field, encouraging the introduction of new types of lasers e.g. the krypton-, the frequency doubled Nd:YAG-, coppervapour-, and alexandrite laser. The first laser to be based on this theory was the flashlamp pumped pulsed dye laser. This laser was developed especially to treat cutaneous vascular lesions and in particular portwine stains in children ${ }^{\mid 11,29 !}$. In the course of 30 years, laser therapy has become fully established in dermatological practice ${ }^{[29]}$. Evaluation of treatment alternatives never included RCTs. For some indications, e.g. portwine stains and tattoos, no effective treatment existed before the adwent of lasers. In many other indications it is often unclear whether laser treatment or conventional treatment should be the treatment of choice. Another common problem is, if lasers are used, which type of laser should be the system of choice. For portwine stains the flashlamp pumped pulsed dye laser has become the laser treatment of choice at the expense of the argon laser. But in the case of tattoo removal the choice of a specific (combination of) laser(s) is dependent on the colour(s) of the tattoo. For the majority of indications, however, it seems that many laser systems can be used with only marginal differences in effectiveness. As in other specialties, the development of patterns of use of existing lasers in dermatology is an ongoing process. At the same time still new lasers are introduced, e.g. titanium-sapphire lasers and diode lasers, which are sometimes judged to hold great clinicall potential|11.29!

A gross subdivision of lesions in dermatology is that in vascular and pigmented lesions. In case of vascular lesions (oxy)hemoglobin occurs in excess to the skin, usually producing a red discoloration. Other skin pigments can also cause a discoloration. The most important example is melanin, which, if it occurs in excess in the skin, produces a brown discolloration. Any such abnormal discoloration inevitably becomes the basis of notice, questions, and so on "from others. This often results in a desire for removaliz3? This does not apply to tattoos, however, which mostly are intentionally decorative. But also in this case the wish is often expressed to have them removed at some point. In contrast with e.g. hemoglobin and melanin, which are labelled as endogenous pigments, tattoo pigments are labelled as exogenous pigments, that is, originating from a source outside the body ${ }^{[23]}$.

A second gross subdivision is that between benign and malignant tumors. This division is superimposed on that between vascular and pigmented lesions. In accordance with the clinical subdivisions, in this assessment firstly the results of treatment of cutaneous vascular lesions are discussed, focusing particularly on two congenital lesions (hemangiomas and portwine stains) and one acquired lesion (telangiectasias). Secondly, treatment of pigmented lesions is discussed, including tattoos and a variety of endogenous lesions. Then surgilcal laser applications are assessed covering, among other indications, treatment of warts. 


\subsection{Cutaneous vascular lesions}

\subsubsection{Introduction}

A cutaneous vascular lesion is a skin lesion that arises either from the proliferation of cells lining the blood vessels (endothelial cells) or from vascular abnormalities and malformations of capillaries, veins, arteries, and/or lymph vessels ${ }^{(4)}$. The most important congenital lesions that are amenable to laser treatment are hemangiomas and portwine stains. With respect to treatment of acquired lesions, most evidence is available on telangiectaslas appearing on the face, which is discussed in some detall. For other indications, evidence is usually scattered. These indications are therefore discussed in general terms only.

\subsubsection{Hemanglomas}

Hemangiomas llook like small red marks, and are usually distinguished in three categories: superficial, deep and mixed. Superficial hemangiomas are also termed 'strawberry nevus' or 'capillary hemangioma'. Deep hemangiomas are also termed 'cavernous hemangioma', and mixed hemangioma is also known as "capillary-cavernous hemangioma". Many other terms have also been used ${ }^{[4,26]}$. Jacobs et al, quoted by ECRI, identified superficial hemangiomas in about $2.6 \%$ of neonates in a study comprising 1,058 life births in the US in $1976^{[a]}$. These hemangiomas occur three times as frequently in females than in males. In total, it is estimated that about $12 \%$ of children develop hemangiomas, and $70 \%$ to $90 \%$ of cases appear within one month after birth. Firstly they show a period of rapid growth lasting 6 to 8 months (proliferative phase), befare gradually fading or disappearing (involutive phase). About $50 \%$ to $65 \%$ of hemanglomas completely fade by 5 years of age, $75 \%$ disappear by age 7 , and $90 \%$ by age 9 . The most common complication of hemangiomas is ulceration caused by spontaneous necrosis. Other complications include for example severe bleeding, recurrent infection, and rapid uncontrolled growth with possible disfigurement. Most clinicians recommend observation and no specific treatment of uncomplicated superficial hemangiomas as long as they pose no threat to normal body functions. If complications develop, topical therapy (e.g., hydrogen peroxide, Burow's solution with topical antibiotic ointment), surgical excision, or laser therapy may be applied ${ }^{[4]}$.

\section{The role of lasers}

What is the quality of the evidence to support any statements on the effectiveness of laser therapy for this indication? No comparative data are available. In 1995, ECRI published an assessment o three laser applications in dermatology (hemangiomas, portwine stains and telangiectasias) basec on an extensive review of literature published up until February $1995^{[4]}$. The three laser systems most frequently used for hemangiomas were the argon-, Nd:YAG-, and pulsed dye laser, Al studies included a relatively small number of patients. Studies employing an argon laser includec a median number of 8 patients. These studies often combined results of superficial and deef lesions. A range of 8 to 37 patients was present in studies using an Nd:YAG laser, in which result: of superficial and deep lesions were usually separated. A range of 6 to 24 patients was includec in studies using a pulsed dye laser. These studies included superficial, deep and mixed lesions o both adults and children. In a quantitative analysis, mean response rates and the standare deviation were calculated. In this exercise, the results of each study were weighted by the numbe of patients enroled. A good or excellent response rate was achieved in $95 \%(5 \%)$ (mean ani standard deviation) of patients in studies using a pulsed dye laser, $82 \%$ (17\%) by Nd:YAG laser and $76 \%(20 \%)$ by argon laser therapy. Dye lasers produced $88 \%(12 \%)$ excellent response rates Argon laser treatment resulted in $16 \%(14 \%)$ excellent responses, and the corresponding figure: for Nd:YAG laser treatment was $53 \%(33 \%)$. Also, pulsed dye laser studies, unlike argon o $\mathrm{Nd}: Y A G$ laser studies, reported no postoperative complications. Postoperative scarring rates of । to $25 \%$ were reported in argon laser studies. Although statistical testing was deemed impossible the authors concluded that pulsed dye laser therapy appears to be the most effective laser therap 
for most hemangiomas ${ }^{(4)}$. Unfortunately, neither the number of studies nor the number of patients per study was mentioned. Also, due to low patient numbers no distinction was made between superficial, deep and mixed hemangiomas in the analysis.

Other authors, e.g. Wheeland in his 1995 review, also arrive at the conclusion that the pulsed-dye laser is the most effective laser treatment option in patients with superficial hemangiomas ${ }^{2}$. The use of the pulsed dye laser for other types of hemangiomas may be questionable. For example. Lask and Glassberg (1995) state that deep hemangiomas are not responsive to this laser ${ }^{[t ?}$. Neither of these authors provide information on other treatment options.

For superficial hemangiomas, Lask and Glasberg (1995) recommend laser treatment in the first few weeks of life, when the lesions are still small and macular ${ }^{[4 !}$. In this phase treatment is easier and provides better results than in mature lesions. They also claim that the results of laser treatment are superior to those obtained if the lesion were left to resolve by itself. Wheeland (1995) reports that in patients where hemangiomas are associated with complications laser treatment is most effective during the early proliferative phase of their development ${ }^{\{29}$. So, although laser treatment of (at least) superficial hemangiomas is established by clinical experience and the pulsed dye laser has bécome the laser of first choice, there is no consensus on the timing of treatment and, related to this, who is eligible to treatment. Either (almost) every patient should be treated at a very young age or only those (older) patients should be treated in whom hemangiomas did not fade and/or in whom complications develop or where the hemangioma interferes with normal functioning. As there are a variety of treatment methods available, both laser-and non-laser methods, this could possibly justify the organization of RCTs for specific indications, perhaps also including 'early" versus "late' treatment groups. No data on cost-effectiveness are available.

\subsubsection{Portwine stains}

Portwine stains (PWSs) are ectatic (dilated or distended) capillaries within the upper dermis. Untike hemangiomas they have normal endothelial cell development. PWSs usually appear on the head and the neck, at equal rates in males and females ${ }^{|4|}$. PWSs are fiat and sharply demarcated. Their colour varies from pale pink to deep red during infancy and deepens to red or purple during middle age. The skin overlying PWSs eventually becomes raised with nodular lesions. This hypertrophic skin is a significant long-term complication that increases the risk of spontaneous bleeding and hemorrhaging ${ }^{\mid 4 !}$. Maim and Lundeberg, in their 1992 review, refer to authors, who documented that PWSs occurred in $0.3 \%$ of the (white) population in the US in the mid seventies ${ }^{[20]}$

Several studies have tried to assess the burden of having PWSs. The two largest studies are discussed. In 1981, Kalick, Goldwyn and Noe assessed the psychological status of 82 patients with PWSs before laser treatment was applied ${ }^{[13\}}$. The questionnaires included the Eyseneck Personality Inventory (EPI), which measures neurotic tendencies as well as extroversion versus introversion, the Multiple Affect Adjective Checklist (MAAC), which measures anxiety, depression, and hostility, and the State-Trait Anxiety Inventory (STAI), which measures anxiety as a transient mood and as a long-term character disposition. The results were compared with results of 'normal' comparison groups. None of the differences between both groups approached statistical significance except the one based on the MAAC measure on anxiety (Student's t-test, $p<0.05$ ). It was concluded that the test instruments did not detect greater emotional disturbance in patients with PWSS compared to "normal" control populations. Additional, PWSs specific questionnaires however, revealed that in many patients, having a PWS had been a source of stress and psychological burder. Nevertheless these patients, by en large, had managed to build a self-concept sufficiently strong to keep them on a reasonably even keel through their lives ${ }^{\text {1": }}$ !

In 1989, Lanigan and Cotterill assessed psychological morbidity of PWSs before laser treatment in 110 patients, of whom $71(64.5 \%)$ responded to a self-devised questionnaire designed to elicit feelings of stigmatization, poor self regard and difficulties in interpersonal relationships ${ }^{(15)}$. In addition, two standard psychiatric screening tests, the General Health Questionnaire (GHQ) and the Hospital Anxjety and Depression Scale (HAD) were given to the first 30 and 25 patients in the 
study, respectively. Results of the PWS psychological morbidity questionnaire showed that $50 \%$ of the patients felt embarrassed, anxious or depressed about their birthmark. Forty-one percent felt envious of people with normal skin and the same number felt people avoided looking at them Twenty-three per cent said they avoided sexual activity because of their birthmark. Especially older respondents felt different from other people more offen, which is a central feature of stigmatisation. They were more often embarrassed because of their birthmark and avoided social situations because of it. It was concluded that these results indicate that patients with PWSs suffer 3 significant degree of psychological morbidity. This morbidity was not detected in the results of the GHQ, as compared to a general population Also the scores obtained with the HAD questionnaire did not indicate a higher level of emotional disorders than in other groups of patients, e.g. women attending a general practitioner. The lack of differences with other groups was interpreted as being. the result of attempts of patients to suppress their psychological difficulties ${ }^{15}$. Of course, it may: also be that general psychologiical screening tests are not sufficiently sensitive to detect differences between individuals with PWSs and those without. This may apply both to this study and the study by Kalick et al .in $1981^{143 !}$.

\section{Treatment alternatives}

A variety of treatment modalities in patients with PWSs have been tried which "according to Malm and Lundeberg in their 1992 review, for the most part had no effecti2u. When effects were achieved and the red lesion was bleached it was replaced by disabling scars in far too many cases. Sometimes even more serious side-effects occurred such as development of malignant neoplasms after application of lionizing radiation ${ }^{\{20 \mid}$. Combining data from Malm and Lundeberg (1993) and a 1995 ECRI publication, an impressive list of ineffective treatment methods can be assembled ${ }^{[4,22 \mid}$. The earliest treatment was vaccination in and around the stain itself. Other, more modern remedies included radicactive phosphorus, radiotherapy with thorium-X. Bucky (Grentz) rays, ultraviolet and infrared radiation, carbon dioxide snow, liquid nitrogen ${ }_{n}$ cryocautery, sclerosing agents, cortisone, protamide, heparin, compression wraps or garments, obliteration by ligature of vessels; dermabrasion, surgical excision, skin grafting and over-tattooing the stain with masking pigmentation. The most successful 'treatment' according to ECRI, was cosmetic cover-up. However, Malm and Lundeberg report that camouflage covering by thick cosmetics is expensive, takes a lot of time to apply and results in an unnatural appearance. In one survey, quoted by these authors, only $20 \%$ of patients were consistently using camouflage ${ }^{|f, 20| !}$. In this situation it may not be surprising that most of the laser types that are presently in use have been tried to treat patients with PWSs ${ }^{(1,20)}$.

\section{The role of lasers}

Initially, the argon laser became the laser of choice in treatment of PWS ${ }^{112^{29}}$. Relativelly good fading was reported in over $80 \%$ of adult patients, but a $4 \%$ to $15 \%$ incidence of scarring was seen $^{11,25 \mid}$. Even in the early nineties, in optimal treatment situations it was estimated that scarring would follow treatment in $4 \%$ to $6 \%$ of patients. Children with light-pink PWSs appeared to be at increased risk of scarring, which occurred in up to $40 \%$ of cases treated ${ }^{(1,25)}$. Therefore, several authors recommended that children younger than 18 not be treated ${ }^{225}$. This advice would later be repeated by the European Community Hemangioma Working Party ${ }^{228:}$ Also, textural changes and permanent hypopigmentation were reported after argon laser treatment. These side-effects limited the potential usefulness of this laser system ${ }^{[29]}$.

In the eighties, laser treatment went through a technological evolution. Treatment techniques changed and new types of lasers were introduced. Initial treatment techniques included the 'point by point' technique " the '(polka)dot" or 'pointillistic' technique and the "painting" or 'stripe" technique. These all required great expertise and its results showed great variability with poor reproducibility. Therefore, robotical optical scanning devices and automated handpieces were developed, which provide greater consistency in the treatment of PWSs while also reducing both the time required to treat large areas and the tedium associated with the older techniques ${ }^{2 a 9}$. Because of improved results compared to conventional techniques the European Community Hemangioma Working Party recommended that an argon laser should only be used with scanning devices ${ }^{286]}$ 
in 1983 , the understanding of laser-tissue interaction was greatly enhanced with the introduction of the theory of selective photothermalysis 11.299 . The first laser to be based on this theory was the flashlamp pumped pulsed dye laser. This laser was developed especially to treat cutaneous vaiscular lesions and in particular portwine stains in children ${ }^{\text {thal }}$. Good results have been reported and the flashlamp pumped pulsed dye laser ( 450 microsecond, $585 \mathrm{~nm}$ ) is nowadays accepted as treatment of choice in PWSs in children and, by increasing numbers of authors, in adults as well:1.28].

Pulsed dye laser treatment is applied in the outpatient department. Patients may require 5 to 15 therapy sessions over many months or a few years. A dye laser session usually averages between 1 and 2 hours, but may last from 5 minutes to five hours. Session length depends on patient tolerance and anesthesia ${ }^{[4]}$. Adults may not require local anesthesia at all ${ }_{i}$ but the treatment of large areas in younger children, where immobilization is required, may require sedation as well as the use of topical, regional or general anesthesia ${ }^{[14.12]}$. In a double-blind RCT including 73 children with PWSs between 5 to 16 years of age it was demonstrated that EMLA 5\% (an eutectic mixture of lidocaine base and prilocaine base formulated into a cream) resulted in lower pain scores during flashlamp pumped pulsed dye laser treatment than a placebo cream or no analgesia ${ }^{(25)}$ Interestingly, none of the children in this study needed general anesthesia. It should be noticed here that the level of randomization and how it was achieved are completely undocumented. This applies as well to blinding the patients and the physician administering the treatment, which seems impossible.

What is the quality of the evidence underlying the conviction that the pulsed dye laser should be the laser of choice for treatment of PWSs? As noticed above, portwine stains were one of the three indications included in a 1995 assessment published by ECR ${ }^{[4]}$. The authors noted that due to reporting variability, the assessment of overall results was difficult. In early studies treatment effectiveness was exclusively reported subjectively in terms of 'poor' to 'excellent' blanching. Later studies added other criteria to this, such as skin texture, colour and scarring. Also, in more recent studies, attempts have been made to assess treatment effectiveness both subjectively and objectively, e.g. by using colormeters and reflectance spectrophotometry. $\| \mathrm{n}$ order to use all available data, it was decided to base the analysis exclusively on subjective assessments. The number of patients included in each study and the number of studies per laser varied widely. For example, 24 studies were retrieved in which an argon laser was used, including 2 to 1,064 patients. Nearly all of these studies excluded children. It was suggested that a lower number of studies was available on all other types of lasers than argon lasers and that especially in case of copper vapour lasers few clinical data were available. In general, non-argon laser studies did not include more than 30 patients. Therefore, in a quantitative analysis of subjective criteria, the results of the studies were weighted by the number of patients enrolled.

Good or excellent results were found in $85 \%$ (11\%) (mean and standard deviation) of patients having (flashlamp pumped) pulsed dye laser therapy, in $74 \%(11 \%)$ by continuous wave (argon pumped) dye laser, in $54 \%$ (14\%) of patients having argon laser therapy, in $66 \%(2 \%)$ of patients undergoing $\mathrm{CO}_{2}$ laser therapy, in $80 \%(9 \%)$ of patients having Nd:YAG or frequency-doubled Nd:YAG laser therapy, and in $72 \%(11 \%)$ of patients undergoing copper-vapour laser therapy ${ }^{[(4 !}$. It is rather surprising that the Nd:YAG and frequency-doubled Nd:YAG are listed together here, because one would expect differences in laser-tissue interaction (and thus differences in results) depending on the wavelength used ${ }^{[29]}$. Pulsed dye laser therapy yielded a much higher excellent response rate $(43 \%(14 \%))$ than argon laser therapy $(7 \%(7 \%))^{1 / 4}$. This comparison is exclusively based on adult patients, because after disappointing results in early studies, hardly any children were treated with argon lasers anymore ${ }^{[25,29]}$. Pulsed dye laser therapy appeared to be equally effective for children and adults, with excellent response rates of $41 \%(22 \%)$ and $43 \%(14 \%)$ respectively. Patients receiving pulsed dye laser therapy also had a low postoperative scarring rate $(3 \%(2 \%))$. Based on these results it was concluded that pulsed dye laser treatment appears to be the treatment of choice for PWSs ${ }^{(4)}$.

Complications with the pullsed dye laser are rare. According to Lask and Glassberg (1995), scarring has been reported in less than $1 \%$ of cases, whether treatment was applied for PWSs or other indications. When it does occur, it is usually atrophic in nature ${ }^{[1]}$. The reported percentage of 
scarring by these authors is somewhat lower than reported by ECRI. Hyperpigmentation, which is the most common complication, results in approximately 10 to $15 \%$ of cases and resolves within: 2 or 3 months. Hypopigmentation occurs in less than $5 \%$ of patients, and usually resolves in a couple of monthis ${ }^{17)}$. Notwithstanding these results, some authors, e.g. Wheeland (1995) claim that in selected adult patients with small, thick, blue, or nodular PWSs at anatomic locations with a low potential for scarring can still be effectively treated with the argon lase ${ }^{[29]}$. In children and the vast majority of adults, the pulsed dye laser has clearly become the treatment of choice.

\section{Cost-effectiveness}

Although exact data on cost of treatment are unavailable it is clear that laser therapy for PWSs incurs considerable additional health care costs. Thus, good economic evaluation is essential prior to widespread diffusion, to ensure that the benefits to patients justify the resource costs involved. The additional resources associated with laser treatment of PWSs are best justified when they are spent at the time when the anticipated benefits are highest. Several studies, e.g. by Tan et al. in 1989, Reyes and Geronemus in 1990, and by Ashinoff and Geronemus in 1991, have provided evidence that treatment can be successfully administered to children of any age and that patients less than 7 years of age require fewer treatments to attain the same (complete) response $e^{[2.22 .25]}$. In a study by Tan et al, all 35 patients, aged between $0-14$, achieved excellent results. The 19 patients who were less than 7 years old required (mean and standard deviation) 5.8 (1.1) sessions whereas older children required 7.1 (1.1) sessions, which was a significant difference (Student's t-test, $p<0.05)^{[25]}$. It is thought that treatment at a very young age, especially before formal education begins and the child is forced to interact with many other children, could prevent considerable psychological impairment, prevent later complications, and provide for a more complete response $e^{[2]}$. Thus, at first sight, treatment before the age of 7 could be the most costeffective treatment timing. Of course, this picture would change dramatically whenever general anesthesia would be required in young children.

Neither in this nor in other studies by Tan et al. general anesthesia was applied. Children below 3 years of age or more than 12 years of age were not premedicated. Children between these ages were given a single dose of triazolam ${ }^{[25]}$. However, in a report evaluating a two-year experience with the pulsed dye laser in an Australian hospital, the authors felt that they were severely hampered in treating children with PWSs by the inability to provide general anesthetic services, recovery, admission and discharge series ${ }^{[12]}$. Patients were labelled as children when they were at most 14 years of age. In this Australian series, approximately 40 children were waiting for these facilities before treatment could proceed. Many more children had not been referred to the clinic by pediatric dermatologists because of their known inability to provide needed anesthetic facilities.

Overall, marked clinical variation in the (anticipated) need for anesthesia could result in marked differences in the results of economic evaluation of laser treatment of PWSs.

Although this is another highly interesting theme, no studies to date have systematically estimated the cost-effectiveness of early versus late treatment. Some model calculations, using different age limits than those used by Tan et al. have been published by ECRI ${ }^{[4]}$. The authors calculated that treating all children 15 years of age or younger by pulsed dye laser therapy is less costly than delaying treatment until they are 15 years old, when lesion-associated psychological disturbances are more likely to have developed, or until they are 35 years old, when they are more likely to devellop skin hypertrophy. Likewise, treating PWSs with pulsed dye laser therapy for prophylaxis against skin hypertrophy in all 15 year-old adolescents is less costly than delaying treatment until they are 35 years of age (and more likely to develop skin problems associated with PWSs) ${ }^{\text {it }}$. Unfortunately, no monetary values were specified here. In the Netherlands, an Investigational Medicine project has evaluated effects and costs of pulsed dye laser treatment of a case-series of patients with PWSs ${ }^{[2]}$. The main hypothesis in this project was that treatment at an early age is easier and results in higher benefits than treatment at a later age, e.g. in adulthood. The results of this study have not yet been published. In an interview, the Head of the project (Prof. van Gemert) explained that the result of this project show the collour of the lesion is a more important prognostic factor for treatment success than the age of the patient $t^{(5)}$. Best treatment results are 
achieved in patients with light PWSs. However " 10 avoid psychological problems, Prof. van Gemert recommends treatment at an early age.

Besides this issue, earlier versus later treatment, one could also think of evaluating one type of laser versus another in the design of an RCT, enrolling patients of the same age or age group. whenever appropriate.

In any economic evaluation alongside a trial evaluating PWSs in children it seems appropriate to include direct costs borne by the parents of the patients, and parental indirect costs due to absence from work besides the direct cost of treatment. Of course, a standard approach can be applied in adult patients. In the Australian study discussed above, using a variety of assumptions (see Intermezzo 5.1), the direct cost per half hour treatment session was calculated to amount US $\$ 184^{[12]}$. It was estimated that in half an hour an area of $8 \times 8 \mathrm{~cm}$ of a PWS can be treated. Simply implementing data from other studies on the number of treatment sessions per patient, 5 to 15 treatment sessions for adults with PWSs and on average 7 for children (disregarding the need for anesthesia), would result in a range of costs of at least US\$920 and at most $\$ 2760$ for adults. and average costs of $\$ 920$ for children.

Evaluation of the effects of treatment on quality of life have been restricted to measurements of changes in psychological morbidity "behaviour or personality. Few studies have been done. Kalick (1983) attempted to measure personality change before and after laser treatment and found no significant changes in the instruments used. The author concluded that the data suggest that appearance-altering procedures, particularly in adults, alter not personality but modes of coping within established personality ${ }^{1 / 4}$. There are reasons to believe, however, that improvement in overall quality of life can be realized. Nearly three quarters of patients in Lanigan and Cotterill's study, discussed earlier, felt the need to hide their birthmark and a large majority felt that laser treatment would significantly improve their quality of life $e^{[15]}$, indicating anticipated value on the part of patients for the therapy.

In summary, there was no satisfactory treatment of PWSs before the advent of lasers, although cosmetics can cover the lesion to some extent. Although RCTs have not been organized in evaluation of treatment of PWSs, the combination of evidence on the natural history of PWS and the data on the effectiveness of laser treatment results in accepting the clinical results of laser treatment of this condition without too much scepticism. However, no judgment on costeffectiveness can be made. Obviously, there is a need to develop standardized PWS specific quality of life questionnaires, in adults and particularly in children. New instruments, focusing on dermatological disease in general, could be used to supplementary assess quality of life in patients with PWSs and other skin disease. Examples are the Children's Dermatology Life Quality Index (DLQI) and the DLQI for use in adults ${ }^{[19}$. Again, these data could be supplemented with utility measurements from adolescent and adult patients and perhaps of parents of very young children using a variety of methods (e.g. the direct time trade-off technique) to allow a comparison across a variety of diseases in a variety of specialties.

\subsubsection{Other congenital vascular lesions}

Other, less frequent congenital lesions than hemangiomas or portwine slains in which laser therapy has been successfully applied include angioma serpiginosa "blue rubber bleb syndrome and angiokeratoma ${ }^{1291}$. Although laser treatment for these indications has been established by clinical experience, the available evidence on the safety and effectiveness of these procedures is often far from convincing. 


\subsubsection{Telangliectastas}

Telangiectasias are noncongenital vascular malformations of dilated capillaries, arterioles, or venules. They may appear as red lines on the face, cheeks and nose, as blue, branched lines on the lower extremities, as spiderlike configurations and as papular (small, round, elevated) lesions ${ }^{64}$. Laser treatment has been described as offering effective treatment in a wide variety of telangiectasias, including acne rosacea, 'essential' telangiectasia, collagen vascular disorders, solar-induced telangiectasia, poikiloderma of Civatte, hereditary hemorrhagic telangiectasia (see Chapter 7), postrhinoplasty red nose, and spider telangiectasia (nevus araneus) ${ }^{[2 a]}$. According to ECRI, in their 1995 assessment, studies of patients with these lesions used argon-, coppervapour-, or dye laser therapy. Using the methodology described in the previous paragraphs, good to excellent response rates for telangiectasia were found in $83 \%(14 \%)$ of the patients in whom an argon laser was used, compared to $74 \%(27 \%)$ for pulsed dye laser therapy and $67(7 \%)$ for copper-vapour laser therapy: No subdivision per type of telangiectasia was made although, apparently, most studies included patients with facial telangiectasia. It was stated that the analysis had to be based on a small number of studies. Based on these findings it was tentatively concluded that all three types of lasers appeared to be effective for treatment of this condition ${ }^{[4]}$.

Wheeland (1995) arrived at the same conclusion for a large variety of types of lasers (including the argon-, argon pumped tunable dye-, copper vapor-, copper bromide-, krypton- and frequencydoubled Nd:YAG laser) in treatment of facial telangiectasias ${ }^{[29]}$. In patients with a mixture of both large and small calibre blood vessels this author recommends to treat the large vessels first using either of the lasers listed above (green light lasers), followed by treatment of the finer vessels with a yellow light laser (e.g. a pulsed dye laser) ${ }^{|29|}$. Geronemus (1995), on the contrary, specifically recommends the argon laser for use in larger diameter telangiectatic blood vessels, while smaller vessels are judged as best handled with pulsed laser light. At the same time this author does nol exclude argon laser treatment of smaller vessels, permitted that this is done with the aid of a scanning device ${ }^{171}$. In another review, Lask and Glassberg (1995) state that facial telangiectasias have a good response rate to the pulsed dye laser ${ }^{[17]}$. According to ECRl, the pulsed dye laser is completely effective in removing facial telangiectasias in children ${ }^{14 *}$. This is in line with some of the findings of the previously cited authors, because children's vessels are relatively small. The limiting factor for use of the pulsed dye laser in these patients, according to both Lask and Glassberg (1995) and Geronemus (1995) is the development of postoperative purpura. Therefore, many patients are reported to prefer other modalities, including argon- and copper-vapour lasers, which can have good results and do not yield the postoperative purpura, and may be more tolerable to the patient ${ }^{[7.17]}$. Thus, it is as yet unclear which laser should be the laser of choice for each vessel size in facial telangiectasia.

In ECRl's view there are insufficient data to arrive at a judgment on the effectiveness of laser therapy on specific subindications, although some preliminary evidence may be available, e.g. on acne rosacea $^{\mid{ }^{|l|}}$. For this indication (severe acne rosacea) and another one, poikiloderma of Civatte, several authors recommend the flashlamp pumped pulsed dye laser ${ }^{7.17 .291}$. Poor results are reported in treatment of lower extremity telangiectasia with red vessels of $0.2 \mathrm{~mm}$ and larger using any of the lasers currently available. Several authors note that the small matted vessels that sometimes appear after sclerotherapy seem to be responsive to the pulsed dye laser ${ }^{\{7,29 !}$.

Overall, laser treatment of telanglectasia, in particular facial telangiectasia, is established by clinical experience. Which laser should be the laser of choice for specific vessel calibers and subtypes of telangiectasia, however, is still unclear. In general, for other indications than (simple) facial telangiectasia the evidence is still scattered.

\subsubsection{Other acquired vascular lesions tumors}

Of a variety of other acquired vascular lesions, laser treatment has been successfully applied in angiolymphoid hyperplasia, Kaposi's sarcoma, pyogenic granuloma, cherry angioma, and venous lakes $^{\mid 29}$. Geronemus $(1995)^{(7)}$ produces a similar list, including angiolymphoid hyperplasia with 
eosinophilia, Kaposi"s sarcoma, pyogenic granuloma, while adding angiofibroma. This author includes these indications under the heading of neoplasms. Specifically, these lesions can successfully be treated with the argon laser when they contain a predominant vascular component $^{\text {t }}$. Wheeland (1995) also lists angiofibroma (adenoma sebaceum) as an indication for laser treatment, but he labels this under the heading benign appendigeal tumours, and adds glomus tumour here ${ }^{[29]}$.

\section{Intermezzo 5. The operating costs of a pulsed dye laser facility in dematology}

As the pulsed dye laser can be used for many indications in dermatology, its annual runming costs are detemined by its frequency of use for all different pumposes. To provide an impression of the direct annual exploitation costis of a pulsed dye laser, and the way in which these costs are calculated, data from Australla ar used as an

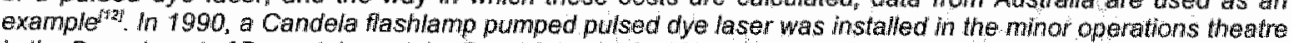
in the Department of Dermatology at the Royal Prince Alfed Hospital in Carnperdown. New South Wales, Australia. Data derived from a two-year experience were extrapolated to estimate the total annual costs when the service would be offered full-time. The costs have been recalculated in US\$, using mid-1995 exchange rates.

The capital costs of the laser were US\$180 000, which were linearly depreciated in a 5-year poriod resulting in annual equivalent casts of US\$36 000. The (fixed) costs of maintenance were US\$14250 per annum, or $7.9 \%$ of the capital costs. It was assumed that the clinic where the laser was installed wowd be operating 44 weeks/year. that there would be 9 clinjos per week, lasting 3.5 hours per session and that there would be 7 patients in each session, each on average requiring a half an hour treatment session. This would result in a maximum number of 2770 treatment sessions per year. Furthemore, it was assimed that 200 pulses per patient treatment session would be needed and that a flashlamp would last for 50 000 pulses. At this rate the flashlamp would frave to be replaced 10 times per annum, incurring US $\$ 4000$ (the first fiashlamp is included in the capital costs of the laser). The costs of changing the dye were calculated at US\$185 000, assuming a change of dyes too times per armum as a fumction of the presumed intensity of use, at a cost of US $\$ 1850$ per change. The costs of space rental were US $\$ 7500$, based on a tariff of US\$50 per $\mathrm{m}^{2}$, and use of a room of $50 \mathrm{~m}^{2}$. The annual costs of photography were calculated at US\$22 500. Personnel costs, including physicians, nurses and administrative stiaff amounted US\$206 500. No allowance was made for electricity, water, down time on machine, overheads related to employment, the need for analgesia, the costs of safety measures, the costs of education and training of physicians and supportive staff, etc. Nor was an allowance made for extra cosits associated with theating onildien which, as we have seen, may include the costs of general anesthesia, admission, recovery, and theatre fees. Blased on these assumptions and calculations, the total costs of running the clinic per year would be US\$509750, with a cost par half hour treatment session of US\$184.

Clearly, this is a consenative estimate of the actual costs per treatment session and, following fron this, the total costs of treatment. Besides that a variety of costs were not included in the calculation perthaps more important is that lasers are havdly ever employed at their full capacity (see Chapter 12). In the two-year period in which this laser was employed, 210 adults had been treated, 95 for PWSs (45\%) and 115 for other conditions, mainly telangiectasias. Twentynine children (defined as 14 years of age or youngerd had been treated, 22 for PWSs (76\%) and 7 for other conditions. In both groups only a minority of patients had completed treatment. Although the total number of treatment sessions was not specified, it is asy to see that a total anmual number of 2,700 sessions in these patients is highly unlikely, because this would have required an average number of almost 23 sessions por patient in the two-year period of the study. Of course, capilal costs and maintenance cost, usually $8 \%$ of the capital costs on an annual basis, stay the same independent of the irtensily of use. This will result in an iricreese in cosis per treatment session. Also, the relative contribution of each (sublindication is important because of the impact on the average length of a treatment sessions. In this example, the contribution of PWSs was $45 \%$ in adult patients, and $76 \%$ in children. If a larger proportion af patients with PiWSs would have been included, this would probably have had an increasing effect on the average length of a treatment session. In addition, patients with large lesions who have to travel far to obtain treatment, not unusual in Australid, may wish to heve a larger area treated than is possible in a standard treatment session "This may contrast with the effect of an increased frequency of other conditions than PWSs, as each treatment session in these cases may last for only 5 minutes. Therefore, in data documenting annual operating casts of a medical device, the types of costs included, tha relative contribution of each (sublindication, and the assumptions on the infensity of use should always be clearly specifled. A final consideration here is that the capital costs of lasers tend to coma down over time. In 1995, the capital costs for pulsed dye laser units were estimated to wary between US\$50 000 to 130000 in the US "which is remarkaby lower than the 1992 (Australian) price level of US\$180 000. In the example discussed here, fhe equivalent anivial costs of the capital costs were calculated by simply dividing the capital costs by the length af its techmical life (in? years). However, an estimate of the useful clinical life of the device is preferred as a basis ror calculation of the anmual equivalent costs over its actual mechanical life, since it may be superseded by more up-fo-date models ${ }^{\text {jal }}$. In addition, if the laser has a resale value this should be taken into accourt as well. 


\subsection{Cutaneous pigmented lesions}

Cutaneous pigmented lesions are subdivided into tattoos and other pigmented lesions.

\subsubsection{Tattoos}

The word tattoo is derived from the Polynesian word tau-tau and refers to the accidental or volitional acquisition of pigmented particles in the skin. In the United States, estimates have put the prevalence of tattoos at 5 to $10 \%$. Decorative tattoos subserve a variety of aesthetic social, tribal, cultural and occasionally criminal purposes ${ }^{19}$. In this case tattoos are either used a membership sign of a criminal organization or as a means to identify prisoners. Cosmetic tattoos are applied to enhance the eyebrows, eyelids or lips ${ }^{\text {lat }}$. Traumatic tattoos are secondary to abrasive or explosive trauma ${ }^{[18]}$. Decorative and cosmetic tattoos have been made either by amateurs or professionals, which is usually closely linked to the anatomical position of the tattoo. Most professional tattoos inks are uniformly deposited in the papillary dermis, whereas amateur tattoo inks are variably deposited in the papillary and reticular dermis and even in subcutaneous fat. Amateur and most professional tattoos consist of a single colour, blue or black. Sometimes professionals apply multiple colours, e.g. green, yellow and red ${ }^{[18,29 !}$.

According to Wheeland in his 1995 review, dermatologists use a number of surgical procedures and techniques to remove tattoos. Removal is perhaps most worthwhile in (ex)prisoners who have been tattooed. Techniques for removal have included a number of ablative procedures such as salabrasion, dermabrasion, split-thickness excision, chemical scarification "excision and infrared coagulation. However, none of these techniques could completely remove all tattoo pigment in one procedure without also producing scars, hypopigmentation, or textural changes ${ }^{[29]}$. Also ${ }_{1} \mathrm{CW}$ argonand $\mathrm{CO}_{2}$ lasers did not produce satisfactory results, as this was always associated with scar formation ${ }^{118,21,29 !}$. Wheeland (1995) states that most of the difficulties with treatment were largely solved with the introduction of the short-pulsed high-intensity, qs lasers for this indication ${ }^{\text {[29]. This }}$ is in line with an observation by Geronemus (1995) who agues that argon laser treatment of tattoos will be less satisfactory compared with the results obtained with qs and pulsed lasers ${ }^{[7]}$.

As early as 1965, Goldman et al. reported results of the first qs laser, a ruby laser, in treatment of tattoos ${ }^{(9)}$.Although this and subsequent work allegedly stimulated great interest in improving the laser-tissue interaction, this interest gradually faded. The 'rediscovery" of ruby lasers has been attributed to several factors including the success of pulsed dye lasers and the development of the theory of selective photothermolysis in $1983^{1181}$. Wheeland points to publications by a Scottish team, led by Reid, which rekindled interests in this system by establishing the benefits of using this device in treatment of tattoos, first in a 1983 publication and then in a subsequent follow-up study ${ }^{309}$. Other research teams then picked up the subject as well and demonstrated this device offered many advantages over existing forms of treatment of tattoos by minimizing the risk of scarring, preventing textural changes, and reducing postoperative wound care ${ }^{i 30 \mid}$. Also, according to this author, technical problems of the original system contributed to the circumstance that it would take until 1989, 24 years after the first publication on the subject, that the ruby laser received approval from the US FDA for the treatment of tattoos ${ }^{(30)}$. Since then, the qs ruby laser $(694 \mathrm{~nm})$ has been joined by the qs Nd:YAG laser ( $532 \mathrm{~nm}$ and $1064 \mathrm{~nm}$ ) in 1991 and, more recently, by the qs Alexandrite laser $(755 \text { or } 760 \mathrm{~nm})^{[2.3,30)}$. Other qs laser systems that have been used for this indication are the Excimer laser $(351 \mathrm{~nm})$, and the flashlamp pumped pulsed dye laser $(510 \mathrm{~nm})^{111}$. Most experience has been gained with the qs ruby laser. Treatment is usually applied without local anesthesia. Retreatment is usually not performed until 6-12 weeks later ${ }^{\text {29: }}$. Many patients request intralesional lidocaine or, increasingly, topical $40 \%$ lidocaine or EMLA ${ }^{[4]}$. The total number of treatments needed is highly variable. Two to 3 retreatments may be necessary with traumatic tattoos, while 4 retreatments may be required to obtain maximum fading in amateur tattoos, and $6-8$ retreatments may be necessary for professional multicolored tattoos. Best results, according to Wheeland 
(1995), have been achieved with amateur, traumatic and black professional tattoos ${ }^{\text {legil }}$. The most common side effect is transient pigmentary change, which occurs in up to $50 \%$ of patients: Hypopigmentation is more common than hyperpigmentation, which may last months: A transient textural change is also quite common in these patients, but a permanent textural change occurs in less than $5 \%$ of cases, while hypertrophic scarring accurs in only $0.05 \%$ of cases ${ }^{11 \%}$.

In summary, in the past decade laser treatment of tattoos has been established by clinical experience in a situation where there was no existing effective treatment. A lot of research is now dedicated to the question which laser system is most suitable for which type of tattoo. As in many other indications, there is no single laser which is the first choice for all tattoos. This is understandable. Tattoo removal requires, in general, complementarity between the tattoo colour and the laser light colour. Thus, the (red) ruby laser is excellent for removing black, blue, green and some brown tattoos. However, red ink is often difficult to remove. In contrast, green qs frequency-

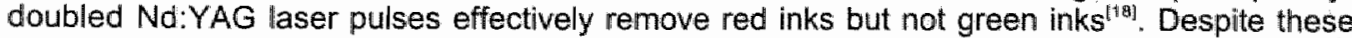
theoretical considerations, several authors agree upon the fact that the results of as laser treatment of tattoos are impossible to predict ${ }^{[23.29]}$. Stafford and Tan (1995), in a review, conclude that available evidence indicates that both the qs ruby-, qs Nd:YAG- and the qs Alexandrite laser appear to be effective in removing blue-black tattoos ${ }^{[23]}$. In removal of black tattoos, the qS Nd:YAG laser may be slightly more effective than the qs Alexandrite laser" ${ }^{\text {t1 }}$. This situation is more complicated with respect to coloured tattoos. Neither one of the three laser types discussed above is judged effective in removing yellow or orange tattoos by Stafford and Tan ${ }^{[23]}$. This contrasts with Wheeland (1995), who states that the qs Nd:YAG laser is suitable for this purpose ${ }^{[29 !}$. Stafford and Tan judge the qs Alexandrite laser as the only of these three lasers to effectively remove green and red tattoos ${ }^{[23\}}$. Wheeland, however, concludes that the qs frequency-doubled Nd:YAG laser can be used to remove red tattoos, while Hruza et al. in a 1993 review, quote evidence that the qs pulsed dye laser is effective in removal of green tattoos ${ }^{[1,29)}$. With respect to green tattoos Stafford and Tan quote evidence favouring the qs ruby lasers over the qs Alexandrite laser ${ }^{-23 !}$. Obviously, this issue is part of the mainstream of actual scientific debate in this field. What is clear is that at this time no one laser is able to remove all tattoo colours well ${ }^{[1]}$. In practice, one can see combinations of different lasers in treatment of multicolored tattoos. For example, in a black tattoo with focal areas of red pigment, successful fading was achieved after subsequent treatment sessions with a qs Nd:YAG laser (removal of black particles) and the qs frequency-doubled Nd:YAG laser (removal of red particles) ${ }^{[16.29 !}$

Another issue of major importance is treatment safety. Stafford and Tan (1995) suggest, based on theoretical considerations and a limited number of observations, that the qs Alexandrite laser may be safer than the qs ruby laser and the qs Nd:YAG laser with respect to the occurrence of traumatic rupture of the skin surface during treatment ${ }^{123 !}$. The concern here is, besides negative implications for the patient, the transmission of HIV and hepatitis viral particles in the plume as well as from the skin surface when frank bleeding occurs. No definite conclusions can yet be drawn on this matter.

\subsubsection{Other pigmented' lesions}

Conventional treatment modalities for benign cutaneous pigmented lesions include cryosurgery, dermabrasion, electrocautery, depigmentation creams (e.g. hydroquinone), and retin A. Several types of lasers are also used, including the argon-, qs dye-, qs ruby-, qs frequency-doubled Nd:YAG-, Nd:YAG- and $\mathrm{CO}_{2}$ laser. With any of these modalities, scar formation has occasionally been reported, just as transient or permanent hypo- and hyperpigmentation ${ }^{|z \||}$. For laser treatment, the location of the pigmentation in the skin is quite important. A common division is epidermal, dermal and mixed (epidermal and dermal) pigmentation. In conjunction with this often a distinction is made between visible light lasers, which penetrate exclusively the epidermis, and lasers that emit light in the invisible part of the spectrum, such as the Nd:YAG and the $\mathrm{CO}_{2}$ laser. These lasers can penetrate both the epidermis and the dermis and are predominantly used to treat either dermal or 
mixed lesions ${ }^{294}$. Other subdivisions are between congenitall and acquired lesions and premalignant and malignant lesions. The use of lasers is widespread for many types of pigmented lesions. Some examples are covered, following one or a combination of subdivisions outlined above.

\section{Congenital benign pigmented epidermal lesions}

Becker's nevus. Becker's nevus is usually located on the shoulder or chest of a man. These lesions usually respond well to the gs frequency-doubled Nd:YAG laser. A few treatments are usually required and recurrence is a possibility ${ }^{161}$. The pulsed dye laser ( $504 \mathrm{~nm}_{\%}, 300 \mathrm{~ns}$ ), has been reported in 2 cases to successfully treat patients with Becker's nevus. Two and six treatment sessions were required ${ }^{\text {respectively }}$.

Cafe au lait macules. Café au lait macules are tan or light-brown hyperpigmentated macules, which occur sporadically. The qs ruby laser has been reported to effectively clear café au lait macules in two sessions without any scarring. According to Levine and Anderson (1995), long-term follow-up is needed to assess the true recurrence rate, which may be as high as 50 to $80 \%{ }^{104}$. Also, the pulsed dye laser ( $504 \mathrm{~nm}, 300 \mathrm{~ns}$ and $510 \mathrm{~nm}, 300 \mathrm{~ns}$ ) and the qs frequency-doubled Nd:YAG laser have successfully been used for this indication ${ }^{[4,23,24]}$. In 1992, Tan, Morelli and Kurban treated 18 patients with the pulsed dye laser $(504 \mathrm{~nm}, 300 \mathrm{~ns})$ in whom a range of 1 to 7 treatment sessions was needed (average 3.33 ) to obtain good results. No complications occurred ${ }^{[24]}$. Also in case of the qs frequency-doubled Nd:YAG laser several treatment sessions may be required for maximum lightning or clearing of these lesions, according to Lask and Glassberg (1995) ${ }^{16}$. These authors report that about $50 \%$ of patients attain clearing. Just as Levine and Anderson (1995), Lask and Glassberg (1995) point at a significant rate of recurrence in patients in whom treatment is initially successful ${ }^{[16]}$.

\section{Acquired benign pigmented epidermal lesions}

Ephelides. Many treatment options exist for treatment of ephelides (freckles), including cryotherapy, low-dose $\mathrm{CO}_{2}$ laser irradiation, or qs ruby- or qs Nd:YAG lasers. According to Levine and Anderson, all appear to be equally effective ${ }^{[t]}$. Wheeland adds the copper bromide-, copper vapour- $(511 \mathrm{~nm})$, and the qs pulsed dye laser to this lis ${ }^{[29]}$.

Lentigines. Lentigines (e.g. lentigo benign and lentigo simplex) can be treated by multiple modalities including cryotherapy, low-dose $\mathrm{CO}_{2}$ laser irradiation, argon lasers, pulsed- or qs ruby lasers, or qs Nd:YAG laser therapy. Of these modalities the qs ruby laser and the qs Nd:YAG laser are thought to be associated with the lowest risk of depigmentation or scarring ${ }^{17,501}$. Both Wheeland (1995), Tan, Morelli and Kurban (1992) and Stafford and Tan (1995) add the 504 an the $510 \mathrm{~nm}$ pulsed dye laser to this short list while Wheeland also adds the copper-vapour-, copper-bromideand krypton laser (521 and $530 \mathrm{~nm})^{[23,24,29]}$. The series published by Tan, Marelli and Kurban (1992), using a flashlamp pumped pulsed dye laser ( $504 \mathrm{~nm}, 300 \mathrm{~ns}$ ), included 52 patients. Of these, 25 patients received a range of $1-3$ treatments to remove senile lentigines (average 2.18) and 4 patients received a range of $1-4$ treatments for eradication of lentigines (average 2.5). Treatment was successful and no complications were reported. This implies that the skin healed with retention of normal skin colour, texture, markings and mobility. Hyperpigmentation occurred sporadically and was transient, persisting anywhere between 3 and 6 month $\mathbf{s}^{[24]}$.

\section{Congenital benign pigmented dermal lesions}

Nevus of Ota. Nevus of Ota is the eponym for the blue, dark brown, or mixed-colour macules that occur in the distribution of the first and second branches of the trigeminal nerve. The incidence in Japanese populations has been estimated at 1 to $2 \%$. Multiple treatment modalities, such as cryosurgery, dermabrasion, microscopic surgical removal, argon-, (cw and qs) ruby-, and $\mathrm{CO}_{2}$ lasers have been used. Although often satisfactory results were achieved with these modalities, scarring was frequently encountered ${ }^{|19|}$. For this reason, Geronemus (1995) states that the argon laser should not be considered treatment of choice for dermal pigmentation ${ }^{[71}$. The only exception with respect to the occurrence of scarring is the qs ruby laser, according to Levins and Anderson (1995). In a series of 16 patients treated with this laser by Lowe et al. in 1993, discussed by Levins and Anderson, $85 \%$ of patients treated three times showed at least $50 \%$ lightening, and all patients treated at least four times showed from 50 to $100 \%$ lightening. Combined with the absence of 
scarring, the qs ruby laser is regarded (for the time being) as the treatment of choice for these lesions by these authors ${ }^{[16]}$. Lask and Glassberg (1995) report a minimal incidence of complications with this $\|$ aser, mainly hypopigmentation ${ }^{[16]}$.

\section{Acquired benign pigmented dermal lesions}

Postinflammatory hyperpigmentation. The qs Alexandrite laser has been reported to successfully remove dermal postinflammatory hyperpigmentation ${ }^{(23)}$. Wheeland (1995) specifically mentions the copper-vapour and copper-bromide laser here ${ }^{291}$. However, these lesions usually appear as mixed lesions.

Melasma. Melasma is another acquired benign pigmented dermal lesions which is listed by Wheeland (1995) as amenable to laser treatment ${ }^{[29]}$.

\section{Congenital epidermal, dermal or mixed premalignant lesions}

Levine and Anderson (1995) report that controversy surrounds the treatment of compound and congenital dermal or mixed nevi in the United States using destructive or ablative laser techniques because of the natural potential of transformation of these lesions to melanoma ${ }^{[18]}$. As congenital nevi of a certain size are known to have an increased risk for malignant change, and even small congenital nevi pose a risk, most authorities do not recommend laser treatment in these cases. $A$ contrary argument, put forward by these authors, is that the destruction of any possible premalignant cells may benefit the patient ${ }^{[18]}$. Of the authors who treat nevi with lasers, e.g. Wheeland (1995) lists for example nevocellular nevi as an indication for use of this device. He simply categorizes this indication under the heading of acquired lesions ${ }^{\mid 291}$. Of premalignant lesions, only lentigo maligna is considered eligible to laser treatment by this author, while the pigmented type of basal cell carcinoma is the only malignant lesion with this qualification ${ }^{1298}$.

\subsubsection{Discussion}

In general, there is a wide variation in clinical practice related to benign pigmented lesions. The position of laser treatment versus other modalities and the position of individual lasers is often unclear. For example, in the United States, ruby lasers are used only in the qs mode in dermatology, while in Japan the cw mode ruby laser has been used successfully for years to treat a variety of pigmented lesions ${ }^{1(18)}$. In general terms, clinical evidence seems to favour qs lasers over ow lasers for many of the indications covered here. It has repeatedly been stressed that the response to laser treatment of individual lesions has been difficult to predict ${ }^{23,291}$. Finally, it not yet clear whether laser treatment of compound and congenital nevi increases the risk of the subsequent development of malignant melanoma. Perhaps this is the only exception where laser treatment has not (yet) been established by clinical experience in the field of dermatology. Nevertheless, for the remaining indications, the quality of the evidence on the safety and effectiveness of laser treatment is often rather poor. No data on cost-effectiveness are available.

\section{$5.4 \mathrm{CO}_{2}$ laser surgery in dermatology}

\subsubsection{Introduction}

The $\mathrm{CO}_{2}$ laser can be used to cul (excise, incise) or vaporize epidermal and dermal lesions. The rapid vaporization of superficial cutaneous lesions is the main modality for which the $\mathrm{CO}_{2}$ laser has been used in dermatologic surgery ${ }^{(6,8,29)}$. The laser is used either in the continuous wave--, chopped pulse-, superpulse- or ultrapulsed mode ${ }^{\left[{ }^{[}\right]}$. In general, results are better when the laser is used in a pulsed mode than in the continuous wave mode, which is consistent with the theory of selective thermolysis. However, this development is rather recent and the bulk of the evidence on the safety and effectiveness of $\mathrm{CO}_{2}$ laser surgery in dermatology has been generated using the laser in a continuous wave mode ${ }^{16.115}$. 
In many cases, laser surgery has to compete with conventional surgery using a scalpel andior with other forms of thermal destruction, such as cryosurgery and electrocautery. No RCTs have been done, and for many indications it is still uncertain if there are real benefits associated with laser surgery. In this situation, interestingly, several authors have tried to assess the available evidence. The two most elaborate examples of such assessments are discussed.

Gloster and Roenigk (1995) subdivide 34 indications for $\mathrm{CO}_{2}$ laser surgeny in three categories. The first category was labelled as "common indications for the $\mathrm{CO}_{2} l_{\text {aser' }}$ ( 7 indications), the second category comprises indications where the $\mathrm{CO}_{2}$ laser is used as one of several alternative therapies ( 13 indlications) and the third category includes indications where the $\mathrm{CO}_{2}$ laser is occasionally useful (14 indications) ${ }^{|8|}$. Fitzpatrick and Goldman (1995) subdivide 54 indications for $\mathrm{CO}_{2}$ laser surgery in four categories: lesions where the $\mathrm{CO}_{2}$ laser is potentially the treatment of choice (5 indications), lesions where the use of the $\mathrm{CO}_{2}$ laser offers distinct advantages (19 indications), lesions. where the use of the $\mathrm{CO}_{2}$ laser simplifies the procedure (22 lesions) and lesions for which the $\mathrm{CO}_{2}$ laser offers no advantage ( 8 indications) $)^{|6|}$.

\subsubsection{Indications where the $\mathrm{CO}_{2}$ llaser is potentially the treatment of choice}

Gloster and Roenigk (1995) state that although the $\mathrm{CO}_{2}$ laser has been used for many cutaneous disorders, this device is probably the treatment of choice in only three indications: recalcitrant warts, actinic cheilitis, and rhinophyma ${ }^{\mid 3]}$. Fitzpatrick and Goldman (1995) list 5 indications here: actinic cheilitis, eyeliner tattoo, rhinophyma, epidermal nevi and cutaneous resurfacing procedures $^{[\text {[e] }}$. For clarity, cutaneous resurfacing procedures include for example treatment of pitted acne scarring and revision of full-thickness skin grafts. What is the quality of the evidence for the indications listed here?

\section{Actinic cheilitis}

Actinic cheilitis is squamous cell carcinoma in situ of the lip. The judgment of Gloster and Roenigk (1995) is based on a series of 43 patients treated earlier by Roenigk and a colleague of his at the Mayo Clinic, in which the $\mathrm{CO}_{2}$ laser was shown to be effective in a long-term follow-up study in patients with this disease ${ }^{[32]}$. The procedure, according to these authors, is equally efficacious but much less invasive, bloody and time consuming than surgical vermillionectomy with advancement of intraoral mucosia. Other modalities, such as 5-fluorouracil and cryotherapy have a higher recurrence rate and longer healing time and generally are more painful when compared with the $\mathrm{CO}_{2}$ laser $^{(\mathrm{b} \mid}$. Fitzpatrick and Goldman (1995) document improving results depending on the mode in which the laser is used for 4 consecutive small series of patients with actinic cheilitis ${ }^{i 61}$. In 17 patients $\mathrm{a} \mathrm{CO}_{2}$ laser was used in the $\mathrm{cw}$ mode, resulting in scars in $44 \%$ of patients and an average healing time of 37 days. Best results were achieved in 20 patients treated with an ultrapulse $\mathrm{CO}_{2}$ laser. No scarring occurred in any of these patients, and the average healing time was 13 days. The other two series, where the $\mathrm{CO}_{2}$ laser was used in the superpulsed-and gated superpulsed mode, respectively, produced intermediate results ${ }^{\mid 6 !}$. Although laser treatment of actinic cheilitis seems to be established in clinical practice, its comparative advantage over other modalities is not beyond doubts. In a 1993 review by Hruza et al., one comparative study was included that was not covered in either of the two reviews cited here ${ }^{[1]}$. This study, by Robinson in 1989, was judged to show no clear advantage of lasers over electrosurgery in these patients.

\section{Rhynophyma}

Rhynophyma is a manifestation of chronic acne rosacea. "t consists of large, nodular masses around the end of the nose. Several case-series have documented the effectiveness of $\mathrm{CO}_{2}$ laser surgery for this indication ${ }^{[6,9]}$. However, in a 1993 review by Hruza et al., the only study comparing $\mathrm{CO}_{2}$ laser surgery and electrosurgery in these patients was judged not to demonstrate an advantage of laser surgery ${ }^{111}$. This study, by Greenbaum et al. (1988) was included in the review by Fitzpatrick and Goldman (1995) but was absent in the review by Gloster and Roenigk (1995). When checking the original publication it turns out this was a nonrandomized study including only 3 patients. One side of the nose was treated with the $\mathrm{CO}_{2}$ laser, the other side with electrosurgery. 
While adding that usually the more severe side was treated with electrosurgery, the authors concluded that both treatment modalities were equally effective. The operating time for electrosurgery was about one-half that with the $\mathrm{CO}_{2}$ Uaser in each patient "Obviously, no conclusions can be drawn based on the results of this study.

\section{Warts}

The warts included by Gloster and Roenigk (1995), are condylomata acuminata, plantar warts, warts found around the nails (periungual warts), and warts on the hands and fingers: These indications are part of their 7 'common indications' for $\mathrm{CO}_{2}$ laser surgery. The authors stress that only recalcitrant warts should be treated with the $\mathrm{CO}_{2}$ laser. To support this statement they refer th some of their own earlier work ${ }^{\text {litl }}$, reporting 6 months cure rates of 80 to $95 \%$ for plantar warts and warts around the nails that were refractory to other standard treatments such as electrodesiccation and curettage, cryotherapy and salicylic acid. The $\mathrm{CO}_{\vec{z}}$ laser is not considered a first-line treatment by these authors for small, simple warts because of the need for anesthesia, the long recovery periods, and the $\cos t^{[B]}$.

Fitzpatrick and Goldman (1995) list treatment of common warts as one of the indications where laser surgery offers distinct advantages ${ }^{[0]}$. They present three series of patients with warts on the hands and feet showing improved results depending on the mode in which the laser is used (continuous wave, superpulse, or ultrapulse). In at least $80 \%$ of patients in each series previous therapy had failed. Warts had recurred in the remainder of patients. Twenty-two patients were treated with the $\mathrm{CO}_{2}$ laser in the continuous wave mode. Of the 121 warts $82(68 \%)$ cleared, in 65 $(54 \%)$ a scar occurred and a recurrence was reported in $39(32 \%)$ of warts. Best results were achieved in 24 patients with 150 warts who were treated with the ultrapulse $\mathrm{CO}_{2}$ laser. $135(90 \%)$ Warts cleared, a scar was noticed in $11(7 \%)$, while 15 warts recurred $(10 \%)^{\mid 6]^{2}}$.

Overall, treatment of recalcitrant warts seems to be established by clinical experience. The evidence with respect to a specific type of warts, condyloma acuminata, is discussed in more detail in Chapter 10.

\section{Other indications}

Of the remainder of indications where $\mathrm{CO}_{2}$ laser surgery is listed as the potential treatment of choice by Fitzpatrick and Goldman (eyeliner tattoos, epidermal nevi, and cutaneous resurfacing procedures), not enough evidence is presented to reach a judgment on its status ${ }^{[6]}$. The same problem arises in the other categories which were distinguished by both teams of authors quoted above. Surprisingly, the lists produced by Gloster and Roenigk (1995) and Fitzpatrick and Goldman (1995) are virtually mutually exclusive ${ }^{\mid 6,9 !}$. Perhaps the indications that where included in both assessments are most interesting to discuss. In case of adenoma sebacecum (of tuberous sclerosis) Gloster and Roenigk arrive at the conclusion that the $\mathrm{CO}_{2}$ laser can be used here as one of several alternative therapies, while this is the only indication that has (mistakenly?) received two labels by Fitzpatrick and Goldman, who judge that the $\mathrm{CO}_{2}$ laser both offers distinctive advantages and simplifies the procedure ${ }^{[6]}$. Angiokeratoma (Fordyce) is the only indication where Gloster and Roenigk judge the $\mathrm{CO}_{2}$ laser as occasionally useful, while Fitzpatrick and Goldman state that the $\mathrm{CO}_{2}$ laser offers distinctive advantages here ${ }^{[6]}$. Likewise, trichoepithelioma is the only indication where Gloster and Roenigk judge the $\mathrm{CO}_{2}$ laser as one of several alternative therapies, while the other team of authors states that the $\mathrm{CO}_{2}$ laser simplifies the procedure. In case of both (superficial) basal cell carcinoma and squamous cell carcinoma (in situ, but excluding squamous cell carcinoma in situ of the lip " which is actinic cheilitis), Gloster and Roenigk judge the $\mathrm{CO}_{2}$ laser as one of several therapies, while Fitzpatrick and Goldman judge that the $\mathrm{CO}_{2}$ laser offers significant advantages ${ }^{[6 !}$. A more consistent pattern of differences in judgement is found for four indications, which are all judged as 'the $\mathrm{CO}_{2}$ laser is occasionally useful' by Gloster and Roenigk and as 'the $\mathrm{CO}_{2}$ laser simplifies the procedure' by Fitzpatrick and Goldman. This applies to the indications: balanitis xerotica obliterans, erythroplasia of Queyrat, Hailey-Hailey disease and Zoon's balanitis ${ }^{[5.8 \mid}$. A final category of indications that may be of interest here is the one that includes indications where, according to Fitzpatrick and Goldman, the $\mathrm{CO}_{2}$ laser offers no advantage. These indications are: angiomas, portwine stains, telangiectasia, (labial) lentigines, ephelides and café-au- 
lait macules ${ }^{101}$. As we have seen, these lesions are usually treated with other types of lasers than the $\mathrm{CO}_{2}$ laser.

The lists produced by Gloster and Roenigk (1995) and by Fitzpatrick and Goldman (1995) are impressive, but not exhaustive. Wheeland, in his 1995 review, lists perhaps 5 to 10 other indications than those already covered by the two teams of authors quoted above ${ }^{[29]}$.

Undoubtedly, it is possible to perform a wide range of excisional and vaporization procedures with the $\mathrm{CO}_{2}$ laser. The exact number of indications may not be the most important issue: it is their evaluative status that matters. One of the fathers of laser surgery, Leon Goldman; used to say, "if you don't need a laser; don't use one'. According to Mordon et al, in a 1991 review of lasers in dermatology, lasers should only be in routine use when it can perform a specific task better than established, conventional techniques ${ }^{121]}$. It still is as difficult as it was then to identify these advantages in many of the procedures described in the literature

\section{Cost-effectiveness}

According to Gloster and Roenigk (1995), despite the precision cutting and hemostatic properties of the $\mathrm{CO}_{2}$ laser, it has not replaced standard scalpel surgery because of the laser's expense and the awkwardness of handling the articulated arm and handpiece ${ }^{|B|}$. Considering the evidence on effectiveness, its potential for cost-effective use seems at best limited.

\subsection{Summary}

Table 5.1 summarizes the findings presented in this Chapter.

Table 5.1 Judgments on the effectiveness and cost-effectiveness of sellected laser applications in dermatology

\begin{tabular}{|c|c|c|c|c|c|c|c|}
\hline \multirow[t]{2}{*}{ Application } & \multicolumn{7}{|c|}{ Judgments (see definitions below) } \\
\hline & 1 & 2 & 3 & 4 & 5 & 6 & 7 \\
\hline Hemangiomas & & & $x$ & & & & \\
\hline Portwine stains & & & $x$ & & & $x$ & \\
\hline $\begin{array}{l}\text { Other congenital vascular } \\
\text { lesions }\end{array}$ & & & $x$ & & & & \\
\hline Telangiectasis & & & $x$ & & & & \\
\hline $\begin{array}{l}\text { Other acquired vascular } \\
\text { lesions }\end{array}$ & & & $x$ & & & & \\
\hline Pigmented lesions & & & $x$ & & & & \\
\hline Tattoos & & & $\mathrm{x}$ & & & & \\
\hline Laser surgery, e.g. warts & & & $x$ & & $x$ & & \\
\hline
\end{tabular}

Definitions:

1 Experimental $=$ still in development, llimited clinical data , no proof of effectiveness.

2 Promising $=$ some clinical data, but procedure not accepted as a routine.

3 Established by clinical experience $=$ no randomized trials, but sufficient data to reach a judgment.

4 Established by RCT = established by severall well-designed randomized controlled trials . 
5 Probably not cost-effective = limited data on cost-effectiveness, butreason to believe that the procedure will not turn out to be cost-effective.

6 Probably cost-effective = limited data on cast-effectiveness, but reason to believe that the procedure will turn out to be cost-effective.

Proven cost-effective $=$ adequate analysis of cost-effectiveness.

In the absence of RCTs no firm statements can be made in almost all laser applications covered in dermatology. Perhaps the only indication that could be considered an exception is treatment of PWSs, which is potentially cost-effective compared to "doing-nothing" or cosmetic surgery. In contrast, in particular $\mathrm{CO}_{2}$ laser surgery is likely not to be cost-effective for many indications. compared to the use of a scalpel.

\subsection{References}

1. Anderson RR, Parrish RR. Selective photothermolysis: precise microsurgery by selective absorption of pulsed radiation. Sicience 1983(220):524-7.

2. Ashinoff R, Geronemus RG. Flashlamp-pumped pulsed dye laser for portwine stains in infancy: earlier versus later treatment. Joumal of the American Academy of Dermatology 1991(24)3:467-72.

3. Drummond MF. Economic ewaluations of laser applications. In: Banta HD, Schou (Eds.). Lasers in Health Care. Effectiveness, Cost-Effectiveness and Pollicy Implications. Academic Publishing. Frederiksberg, Denmank, 1991 " P.72-81.

4. ECRl. Laser Therapy for Hemangiomas, Port-wine Stains, and Telangiectasias. Health Technology Assessment Information Service, Execullive Briefing, ECRI 1995.

5. Ekkelboomu لl. Light in medicine (interview with Prof. Dr. van Gemert). AMC Magazine 1995(4)9:4-7 (in Dutch).

6. Filzpatrick RE, Goldman MP "Advances in Carbon Dioxidle Laser Surgery. Clinics in Dermatology 1995 (13) 1,35 m47.

7. Geronemus RG. Argon laser for the treatment of cutaneous lesions. Clinics in Denmatology 1995(13)1:55-8.

8. Gloster Jr. HM. Roenigk RK. Carbon dioxide laser for the treatment of cutaneous lesions. Clinics in Dermaltology $1995(13) 1: 25-33$

9. Goldmen L, Wilson RG. Homby $P_{\mathrm{H}}$ Meyer RG. Radiation from a Q-switched ruby laser. Effect of repeated impacts of power output of 10 megawatts on a tattoo of man. Journal of Investigative Dermatology 1965(44)1:69-71.

10. Greenbeum SS. Krull EA, Watnick K. Journal of the American Academy of Dearmatology 1991(18)2(part 1):363-B.

11. Hruza Gil, Geronemus RG, Dover JS, Arndt KA. Lasers in Dermatology - 1993 (editorial). Archiwes of Derrnatclogy $1993(129) \div 1026-35$

12. Jarrett $R$, Stewart $M$. Interim report on the use of the Candela laser at Royal Prince Alfred hospital, Camperdown, NSW, Australlia, 1992.

13. Kalick SM. Goldwyn $\mathrm{RM}_{\text {"No }}$ NM. Social issues and body image concerns of port wine stain patients undergoing laser therapy. Lasers in Surgery and Medicine 1981(1):205-13.

14. Kallick. SM. Laser treatment of port wine stains: observations concerning psychologlcal outcome. Im: Arndt KA, Noe IM, Rosen S (Eds..), Cutaneous Laser Therapy: Principles and Methods. John Wiley and Sons, Chichester, Now York, Brisbane, Toronto, Singapore, 1983.

15. Lamigan SW, Cotterill $J$ A. Psychological disabilities amongst patients with port wine stains. British Journal of Dermatology 1989(121):209-15.

16. Lask GP, Glassberg E. Neodymium:Ytrium-Aluminum-Gamet laser for the treatment of cutaneous lesions. Clinics. in Dermatalogy 1995(13)1:81-6.

17. Lask GP. Glassberg E. 585-nm pulsed dye laser for the treatment of cutaneous lesions. Clinics in Dermatology 1995(13)1:63-7.

18. Lewine PC. Anderson RR. Q-switched ruby laser for the treatment of pigmented lesions and tattoos. Clinics in Dermattology $1995(13) 1: 75-9$.

19. Lewis dones MS. Finlay AY. The Children's Dermatology Lise Quality Index (CDLQI) initiall walidation and practical use. British Jounall of Dermatology 1995(132):942-9.

20. Mam M, Lundeberg T. Laser technology in plastic surgery. Scandinavian Joumal of Plastic Reconstructive Hand Surgery 1992(26):3-11.

21. Mordon S, Carruth JAS, Nikolaou A. Dermatology. Ini Banta HD, Schou I (Eds.). Lasers in Health Care. Effectiveness, Cost-Effectiweness and Policy Implications. Academic Publishing, Frederiksberg, Denmark, 1991 , D. $84-8$.

22. Reyes BA, Geronemus RG. Treatment of port-wine stains during childhood with the flashlamp-pumped pulsed oye laser. Jounal of the American Academy of Dermatology 1990(23):1142-8. 
23 Staffond T, Tan OT. 510 mm pulsed dye laser and alexandrite crystal laser for the treatment of pigmented lesions and tatoos. Chics in Dermatology $1995(13) 1: 69-73$.

24. Tan OT, Morelli $\mathrm{JG}$, Kurtan AK, Pused dye laser treatment of benign cutaneous pigmented lesions Lasers in Surgery and Medicine 1992(1,2):538-42.

25. Tan OT, Sherwood K, Glchrest BA. Treatment of children with port-wine stains using the flastilamp-pulsed tunable dye laser. Now England Journal of Medicine 1989(320):416-21.

20. Tan OT, Stafford T. EMLA for laser treatment of portwine stains in children. Lasers in Surgery and Medicine $1992(12): 543-8$.

27. Yan Gemert MJC, Beek UF, Laser treatment of portwine stains The younger the better? Research proposal submitted to the Investigational Mledicine Fund of the Dutch Health Inisurance Executive Council, Academic Medical Center, Amsterdam, 1961 (in Dutch).

28. van Gemert MJC, Camuth JAS, Skakespeare PG. Laser treatment of port wine stain. Newer lasers bring better treatments. British Medical Joumal 1993(306):4-5.

29. Whetand RG. Chinlcal uses of lasers in dermatology (review series article. Lasers in Surgery and Medicine $1995(16) \times 2 \times 23$.

30. Whe eland RG. History of lasers in dermatology. Clinics in Dermatology $1995(13) 1: 3-10$.

31. Street ML Roenigk RK Recalcitrant periungual verruciae: the role of carbon dioxide laser vaporization. Journal of the Amentcan Academy of Dermatology 1990(23)1:115-20.

32. Zelickson BD. Roenigk RK Actinitic cheilitis: treatment with the carbon dioxide laser. Cancer 1990(65)6:1307-11. 


\section{Lasers in ophthalmology}

\subsection{Introduction}

Ophthalmology is the most important laser-using specialty and at the same time the specialty in which most RCTs have been organised to evaluate laser applications. Furthermore, the quality of evidence on economic evaluation of laser applications is relatively good. Laser treatment has been assessed for a variety of conditions, such as diabetic retinopathy, age-related (or senile) macular degeneration, glaucoma and cataract. With increasing incidence of the underlying chronic conditions, e.g. diabetes mellitus, and with an ageing population in the Western world, the public health importance of treatment of these conditions is growing. Many of the studies reviewed in this Chapter have been organized in the US, where the National Eye Institute (NEI) of the US National Institutes of Health (NIH) has played a major role in stimulating evaluation of lasers in ophthalmology by sponsoring RCTs. Although a large variety of types of lasers are in use in ophthalmology, the most important lasers in this specialty are the argon laser and the qs NId:YAG laser. One of the most important indications for argon laser treatment is diabetic retinopathy. For a detailed account of the evaluation of this particular case and its diffusion in the Netherlands, the reader is referred to Chapter 16. A summary and brief update of the results of this assiessment is provided in this Chapter. Although of the more recent types of lasers in particular the use of diode lasers is becoming more frequent, it is unlikely that this laser will supersede the argon laser for all of its major applications in the near future. Of the other remaining more recent types of lasers, Excimer- and Holmium lasers play an increasingly important role. These lasers are applied in photorefractive and phototherapeutic keratectomy, respectively. Photorefractive keratectomy is ailmed at correction of myopia, hyperopia, astigmatism and other refractive errors, whereas phototherapeutic keratectomy can be used in treatment of corneal scars, for example. No RCTs have (yet) been published here. Most information is available on photorefractive keratectomy (PRK) for correction of myopia, a procedure which has to compete with various existing methods for correction of this condition, e.g. wearing glasses or contact lenses. Besides a brief description of this particular case in this Chapter, evaluation of Excimer laser PRK is discussed in detail in Chapter 17.

\subsection{Diabetic retinopathy}

Diabetic retinopathy refers to degeneration of the retina secondary to microvascular disease, leading to gradual visual loss. In the US, diabetic retinopathy is the most important cause of blindness in the 20-6.5 age-group. In the age-group over 65, macular degeneration is the most important cause of blindness ${ }^{\{29\}}$. In general, the progression of retinopathy is orderly: mild 'background' ainormalities characterized by increased vascular permeability appear first, advancing to "preproliferative" retinopathy (moderately severe nonproliferative diabetic retinopathy) characterized by vascular closure, and then to proliferative retinopathy characterized by the growth of new blood vessels on to the retina and posterior surface of the vitreous ${ }^{|⿰|}$.

What is the role of lasers here? To address this issue, the National Eye Institute of the United States National institutes of Health organized two large scale multicenter randomized controlled trials with long-term follow-up of patients. These carefully organized trials demonstrated that argon laser photocoagulation is safe and highly effective in treatment of diabetic retinopathy and macular edema in patients with type I diabetes mellitus ${ }^{[29,66)}$. In the first of these studies, the Diabretic Retinopathy Study (DRS), panretinal photocoagulation was initially reported to reduce the likelihood of severe visual loss by $60 \%$ compared with no treatment. Based on the notion that many patients enroled in the DRS had advanced disease, and thus had worse outcomes than patients who are identified for care today, a reanalysis of the primary data was performed in the early nineties. The reanalysis suggested that the treatment effect may be over $90 \%{ }^{(29)}$. The second of these trials, the Early Treatment of Diabetic Retinopathy Study (ETDRS), established the benefit of argon laser 
treattment in eyes with macular edema, in particular those with "clinically significant macular edema?:?!?

Javitt (1995), in an ellaborate analysis, showed that detection and laser treatment of diabetic eye disease in both type 1 and type 2 diabetes mellitus patients in the United States and in Sweden is not only cost-effective, but is actually cost saving from the governmental perspective $\mathrm{e}^{2 \mathrm{z} \mid}$. This is a highly desirable, but also a very unusual outcome. Based on an analysis of a cohort of incident cases, potential savings were calculated to exceed US\$ 600 million annually in the United States with 112730 person-years of sight gained. In Sweden, potential savings of 36 million SEK might be realised, while gaining 3300 person-years of sight. These findings were judged comparable given the differences in population between the two countries.

The calculation was based on Monte Carlo simulation modelling, incorporating data from population-based epidemiological studies and multicenter trials, including the DRS and the ETDRS. All costs and savings , both monetary and sight years, were expressed in 1990 currency units, using a discount rate of $5 \%$. An elaborate sensitivity analysis demonstrated that the finding of cost savings was consistent over a broad range of assumptions regarding screening and treatment efficacy, screening strategies, and discount rate.

The author noted that in both countries the actual savings are much lower than the potential savings due to the fact that only about $60 \%$ of individuals with diabetes are receiving suggested ophthalmological care ${ }^{[29]}$. In a later article on this subject, it was demonstrated that in the United States in particular a large portion of elderly people with diabetes is not obtaining the necessary eye care, especially blacks, men, and those residing in poor areas with fewer ophthalmologists ${ }^{[29.11]}$

In the Netherlands, simulation modelling based on Markov processes was used to calculate potential lifetime costs and benefits of five scenarios for diagnosis and laser treatment of retinopathy in a cohort of type I diabetes patients (age of onset of diabetes 15 years) and three cohorts of type $1 /$ diabetes patients (age of onset of diabetes mellitus 35,55 , and 75 years, respectively) $)^{[7]}$. One scenario represented the option of "doing nothing'. Effectiveness was expressed in terms of "years of realised sight gain", and 'years of prevented vision loss', of which the former was preferred because it coincides with the definition of treatment benefit by ophthalmologists. Costs were expressed in 1992 Dutch guilders. For the purpose of this review, these costs were recalculated in US $\$$ (in 19961 US $\$$ equalled about Dfl 1.75). Direct costs included costs of diagnosis and treatment, minus savings in rehabilitation services. In addition, indirect costs outside the health care system (representing the value of production losses), were calculated using both the Human Capital Method (HCM) and the Friction Cost Method (FCM) (see Chapter 4). Both costs and effects were discounted using a discount rate of $5 \%$ and $10 \%$, respectively. These results were compared to a calculation without discounting.

The scenario that most closely resembles actual clinical practice guidelines in the Netherlands was the one in which diabetes patients without relinopathy are screened once every two years, with annual screening of diabetes patients with background retinopathy, and quarterly screening of diabetes patients with macular edema and/or proliferative diabetic retinopathy. Based on the results of the analysis and taking into account the effect of failure to comply with the recommended guidelines for ophthalmic examination, it is this scenario that received most support from the author. Using this scenario, in the cohort of patients with type I diabetes mellitus direct costs of screening and treatment per year sight gain were calculated to amount US $\$ 5021$. Including indirect costs in the calculation, screening and treatment turned out to be cost-saving from a macroeconomic perspective, using either the HCM or FCM. It should be noticed that production losses attributable to diabetic rellinopathy were calculated to be ten times as high based on the HCM compared to the FCM.

In the three cohorts of patients with type II diabetes mellitus, results were most promising in the cohort with the youngest age of onset. In these patients, the scenario for diagnosis and treatment of retinopathy described above, resulted in direct costs per year sight gain of about US\$12 570 . When a calculation of indirect costs was included based on the HCM, the strategy would be cost- 
saving. while the net costs per year of sight gain would be US\$8763 if the FCM would be applied for indirect cost calculation. In patients with type ll diabetes mellitus with age of onset of disease of 55 years, the scenario resulted in direct costs per year sight gain of US\$19 428. When a callculation of indirect costs was included based on the HCM; the strategy would be nearly cost neutral, while the net costs per year of sight gain were US\$16218 when applying the FCM. In the final cohort of patients, the corresponding figures were US\$101714, \$55 093 and $\$ 94159$, respectively. These latter figures can be seriously questioned as these refer to a period in life beyond retirement. However; these figures can be related to the origins of the model, assuming an average labor productivity of US\$17 143 in patients aged 75 at onset of disease.

A preliminary conclusion based on these data was that screening and treatment of patients with type II diabetes mellitus irrespective of the age of onset of disease, may not be cost-effective. It was added that, irrespective of the age of onset of disease, results were more favourable in patients with type II diabetes mellitus who are insulin dependent.

These results were complemented with a calculation of the costs and benefits for screening and treatment based on the scenario discussed above for the period 1993-2020. The direct costs of treatment per patient per year would be US\$84 in type I patients, and US\$47 in type II patients. Including indirect costs in the calculation, treatment would be cost-saving for both type I and type II patients using the HCM, while, although still cost-saving in type I patients, in type II patients the overall annual costs were calculated US\$41 when applying the FCM.

The combination of results suggest, according to the author, that one might consider to give precedence to patients with type I diabetes mellitus and to patients with relatively young onset type II diabetes mellitus in the detection of retinopathy. The older-onset type II patients, more specifically, patients who are 65 years of age or older, were thought to be likely to require the same ophthalmic care as the general population, because at older ages cataract, glaucoma and senile macullar degeneration threaten the vision of diabetic patients more than diabetic retinopathy ${ }^{[17]}$.

In summary, the case of diabetic retinopathy shows that good studies are needed to document effectiveness in a credible way. Also, modelling can be a powerful tool to demonstrate costeffectiveness. Overall, the conclusion is justified that screening for and treatment of retinopathy in diabetic populations is beneficial, both in terms of effects and costs. Perhaps more important than the discussion on age-limits is the issue of improving compliance with screening guidelines in the population with diabetes.

\subsection{Age-related (senile) macular degeneration}

The macula may be involved in several disorders, such as diabetes mellitus, but the commonest maculopathy is senile, or-age related macular degeneration. The main symptom of macular disease is a gradual decrease in centrall visual acuity, usually bilateral, but often unequal. Patients retain peripheral fields of vision. Age-related macular degeneration is subdivided clinically into 'nonexudative' or 'dry' and 'exudative" or 'wet". The 'wet' type occurs when the retinal pigment epithellum detaches from Bruch's membrane, which separates choroid from retinal pigment epithelium at the macula. This type is usually characterized by choroidal neovascularization (CNV) ${ }^{\mid 46 !}$.

Starting in 1982, the US Macular Photocoagulation Study (MPS) Group, sponsored by the NEI, has published a substantial number of reports on multicenter RCTs (12 centers) evaluating laser treatment for choroidal neovascularization (CNV) secondary to various conditions. In three RCTs with a follow-up of five years comparing blue-green argon laser to no treatment, laser treatment was demonstrated to be effective in preventing or delaying visual loss in patients with simple extrafoveal CNV secondary to age-related macular degeneration ( $n=236)$, ocular histoplasmosis $(n=262)$ and in idiopathic CNV $(n=67)^{[32,33,34,36,37]}$ 
In a related series of three RCTs comparing red krypton laser to no treatment of either extrafoveal or fuxtafoveal complex neovascular lesions secondary to age-related macular degeneration ( $n=$ 496 ; median follow-up 4 years), ocular histoplasmosis ( $n=288$, median follow-up 2.5 years) and idiopathic neovascularization $(n=50,65 \%$ of patients followed up for five years and $85 \%$ for three years) it was shown that laser treatment was beneficial in preventing or delaying visual loss ${ }^{135}$, 3e.4al.

In two final trials, patients with subfoveal CNV secondary to age-related macular degeneration were randomized into lase treatment versus no treatment In the first of these trials, patients were included with new subfoveal $\mathrm{CNV}^{[40}$. These patients never had laser treatment in the macula before. In the second trial, patients were included with recurrent subfoveall CNV, that is, after previous extrafoveal or juxtafoveal laser treatment ${ }^{\text {(4) }}$. This trial was organized because recurrent (and persistent) CNV was frequently reported in all trials discussed above, which was interpreted as a major factor in decreased visuall acuity after laser treatment ${ }^{\mid 391}$. In both trials " the patients randomized to laser treatment were again randomized in a green argon laser treatment group and a red krypton laser group. However, in the analysis comparing laser treatment to no treatment, both laser treated groups were analyzed as one group. In both the subfoveal new CNV Study ( $n=373$, follow-up 4 years) and the subfoveal recurrent CNV study ( $n=214$, follow-up 3 years) laser treatment was beneficial in curtailing long-term loss of visual acuity despite a decrease of visual acuity immedliately after treatment. However, in a few subgroups of patients no benefit of treatment could be demonstrated. Follow-up of patients in these trials was therefore continued untill the end of 1993 to determine whether eventual benefit is sufficient to offset the initial insult to visual acuity in different sub-groups of patients. Final reports are not yet available ${ }^{[40.41,42]}$.

In 1984, French authors published an RCT ( $n=60$, follow-up one year) comparing blue-green argon laser to no treatment in patients with subretinal parafoveal CNV secondary to age-related macular degeneration $^{[19]}$. The authors reported favourable visual acuity in the laser treated group. In 1991, these authors published the results of an RCT comparing laser treatment to no treatment ( $n=160$, follow-up one year) for the same indication in a new set of patients. In this trial either the green argon, the red krypton, or the yellow or orange dye laser was used for laser treatment, depending on the patient's fundus status. The krypton red laser was used if hemorrhage was present, while occult new vessels were treated with the green argon or yellow wavelength from the dye laser. Laser treatment was shown to reduce short-term visual acuity loss ${ }^{[15]}$.

In 1988, members of the MPSG organized an RCT to evaluate laser treatment versus no treatment in patients with subfoveal CNV secondary to ocular histoplasmosis. In two years time only 25 patients were enroled in the 6-center trial which therefore was terminated early. An analysis of results of patients who completed a one-year follow-up did not produce any evidence that laser treatment was or was not effective for this condition ${ }^{[24]}$.

In all the trials discussed in this section, the complication rate was about 30 to $40 \%$. In about $90 \%$ of these, new hemorrhage during treatment occurred, which is a relatively benign complication.

\section{The effectiveness of blue-green or green argon laser versus red krypton lasers}

In the clinical trials discussed in this paragraph, evaluating subfoveal new or recurrent CNV. extrafoveal or juxtafoveal CNV secondary to age-related macular degeneration, ocular histoplasmosis, and idiopathic neovascularization, it was suggested that blue-green or green argon and krypton red wavelengths were comparable with respect to visual outcomes and complications. However, it should be noticed that these trials were not designed to demonstrate clinical treatment differences between the two lasers. To address this issue, in 1986 the Canadian Ophthalmology Study Group (COSG) organized a multicenter (13 centers) RCT to evaluate green argon and red krypton laser treatment of extrafoveal CNV secondary to age-related macular degeneration ( $n=210$, follow-up one year). It was concluded that the krypton laser is no better than the argon laser in treatment of these patients ${ }^{[13 !}$. Follow-up of these patients was announced to continue to three years after enrolment. A final report is not yet available. 
In conclusion, the RCTs discussed here have demonstrated that laser treatment compared to no treatment is effective in delaying or preventing lass of visual acuity in patients with:

1 CNV secondary to age-related macular edema, regardless of the location of the neovascular lesion with respect to the fovea;

2. CNV secondary to ocular histoplasmosis or idiopathic CNV in extrafoveal or juxtafoveal locations.

No cost-effectiveness analyses have been reported in this field. In this case, laser treatment was introduced for a disorder that could not be treated previously. This creates a potential for costeffectiveness of the treatment-indication combination.

\subsection{Retinal vein occlusion}

Retinal vein occlusion may either happen in the central vein or in the branch vein, which is more common ${ }^{[46]}$.

\subsubsection{Retinal branch vein occlusion}

Occlusion of a temporal branch vein often affects the macular area with significant loss of central and peripheral vision. When a nasal vein is affected there is no central and peripheral field loss. One of the most frequent vision-limiting complications of retinal branch vein occlusion (RBVO) is macular ediema ${ }^{[25,46]}$. Neovascularization is uncommon following branch vein occlusion ${ }^{[46]}$.

The US Retinal Branch Vein Occlusion Study (RBVOS) Group, sponsored by the NEI published three multicenter RCTs on this issue, each involving 5 centers. In all trials blue-green argon laser treatment was compared to no treatment in patients with petinal branch vein occlusion. The first trial ( $n=139$, average follow-up 3.1 years) demonstrated that extrafoveal blue-green argon laser treatment resulted in an improvement of visual acuity in patients with macular edema secondary to retinal branch vein occlusion ${ }^{[64]}$. The second trial $(n=319$, average follow-up 3.7 years) showed that laser treatment resulted in less development of neovascularization and the third trial (n=82, average follow-up 2.8 years) allowed for the conclusion that in eyes with neovascularization present, laser treatment results in less vitreous hemorrhage ${ }^{\left|\mathrm{s}^{3}\right|}$.

In conclusion, the major result of the RCTs organized by the US Retinal Branch Vein Occlusion Study Group is that argon laser treatment compared to no treatment is effective in delaying or preventing loss of visual acuity in patients with macular edema secondary to retinal branch vein acclusion in extrafoveal locations.

The NEl-sponsored trials can be contrasted to RCTs organized in the UK on this subject. In 1984, wo RCTs were published by the same authors in this country, comparing extrafoveal blue-green argon laser versus no treatment of macular edema secondary to retinal branch vein occlusion ${ }^{|53|}$ The number of eyes included in the studies were 63 and 27, respectively, with a follow-up of 2 years. The trials did not demonstrate favourable treatment results in the laser treated patients ${ }^{1593}$. As more than half of the patients enroled in these small single-center trial were lost to follow-up, these results were not convincing.

No evidence on the cost-effectiveness of laser treatment is available. Because there was no treatment availlable for these conditions before the advent of the laser, treatment of this condition is potentially cost-effective. 


\subsubsection{Retinal central vein occlusion}

Occlusion of the central retinal vein cccurs at the optic disc. Vision is markedly reduced in the affected eye usually over a period of several hours ${ }^{\text {twbl }}$. Retinal central vein occlusion is often divided into an ischemic type and a hyperpermeability response type. In the ischemic type, neovascularization may develop in the optic disc, retina, iris and in the angle of the anterior chamber within three months. Also, neovascular glaucomas may develop in these patients. Patients of the hyperpermeability response type predominantly develop macular edema after central vein occlusion ${ }^{[28,46 !}$.

In 1990, an RCT was published comparing long-term results of argon laser photocoagulation and no treatment in patients with ischemic central retinal vein occlusion i $n=123$ eyes, follow-up ten years). The study was prepared in 1975, patient enrolment was started in 1977 and finished in 1986. Two years after starting enrolment only 21 patients had entered this study. The follow-up schedule had proven to be difficult to adhere to. Also, by then the National institutes of Health decided to stop funding the study on the ground that it was inappropriate to conduct a major study on vein occlusion that addresses a well-known fact that patients can be eliminated by panretinal photocoagulation (PRP). The NIH probably refers here to the interim-analysis of the Diabetic Retinopathy Study in 1976. However, photocoagulation of patients secondary to retinal vein occlusion does not necessarily needs to be as successful as photocoagulation of patients secondary to diabetic retinopathy. As a result of this criticism, and in the absence of external funding, the organizers of the trial decided to continue the trial but on the basis of a new randomization scheme.

In the new randomization scheme, implemented after 1977, the choice of treatment was left entirely to the patient. Because this may have introduced bias, only the results of the 'true' RCT will be discussed here. This part of the study comprised the first 21 patients enrolled, of which 10 had PRP and 11 had no treatment: The analysis included follow-up of patients of a maximum of 50 months. There were no lasting changes in any of the outcome parameters for the two patient groups, such as visual acuity and visual field function ${ }^{|26|}$.

While referring, among other studies, to the study discussed above in an editorial in the Archives of Ophthalmology in 1991, it was recognized that, because of the diversity of opinions resulting from conflicting research results, the optimum treatment of a patient with ischemic CRVO is uncertain $^{(14)}$. To reduce this uncertainty, in 1988 the US Central Vein Occlusion Study (CVOS) Group, sponsored by the NEI (perhaps a bit surprising in regard of the NIH remarks on the previous study), organized a multicenter RCT ( 9 centers, $n=728$ eyes of 725 patients, follow-up 3 years).

The trial was designed to answer four questions:

1 Does early panretinal photocoagulation (PRP) prevent iris neovascularization (INV) in eyes with ischemic CVO? To answer this question patients with a non-perfused retina (group N) were randomly assigned to either PRP or observation.

2 Is early PRP more effective than PRP at first identification of INV in preventing further ocular morbidity in eyes with ischemic CVO? This question will be answered by a sub-group analysis of group $N$.

3 Does macular-grid pattern laser photocoagulation improve visual acuity in eyes with reduced vision due to macular edema? To answer this question, eyes with macular edema (group M) were randomly assigned to photocoagulation or observation without treatment.

4 What is the natural history of eyes with CVO that have little or no evidence of ischemia? To answer this question, a group of patients with a perfused retina was included (Group $\mathbb{P}$ ). Besides being followed up for natural history, these eyes served as a pool for possible later entry into group $M$. After a change in protocol, a new randomized group of eyes with intermediate perfusion was enrolled (Group 1). Two of the patients in group $P$ transferred to the 
Group I. These eyes were followed up on a monthly basis until they became eligible for either group $N$ or group $P$.

So far, only a baseline and early natural history report has been published ${ }^{113}$. The results of the two randomized studies incorporated in the study, which should provide convincing evidence on the value of (early) treatment, are not yet available.

\subsection{Retinal detachment}

Detachment of the retina describes the situation where the neurosensory retina becomes separated from the underlying retinal pigment epithelium (RPE). Retinal detachments generally lead to severe visual loss if not successfully treated ${ }^{1 / 46}$.

In 1982 the Moorfields Macular Study Group published an RCT comparing argon laser treatment to no treatment in elderly patients with retinal pigment epithelial detachments (RPED) which in these patients was part of the spectrum of age-related macular degeneration $(n=49$, follow-up 18 months) ${ }^{693}$. The major purpose of the study was to determine whether argon laser treatment altered the central vision prognosis of pigment epithellial detachments in the elderly. At the time of analysis it was shown that treated patients were prone to a large deterioration in visual acuity shortly after treatment. Such a deterioration was not seen in patients in the observation group during the early period after entry in the trial. In the observation group rapid loss of vision could occur at any time. This contrasted with the treated patients, in whom additional visual loss was unusual after the initial period. However, at the 18-month visit the convergence between the 2 groups was insufficient to cancel out the early differences. Overall, it was shown that photocoagulation did not result in a (short-term) visual benefit for patients with RPED.

Of the three types of retinal detachments, rhegmatogenous detachment is the most common form, occurring in approximately 1 in 10000 persons per year. Rhegmatogenous detachments are caused by a tear or hole in the retina, through which vitreous cavity fluid passes, which results in the actual detachment ${ }^{\mid[j}$. Nowadays, according to an assessment of the American Academy of Ophthalmology (AAO), in patients with rhegmatogenous retinal detachment, retinal reattachment is performed with the use of cryopexy, diathermy or laser in conjunction with indentation of the sclera by the placement of a scleral buckle, or retinal reattachment surgery with the use of pneumatic retinopexy.

Sometimes, when retinal detachments are limited and associated with minimal subretinal fluid, laser photocoagulation or cryopexy alone can be used ${ }^{[6]}$. Furthermore, according to the AAO assessment, either laser photocoagulation or cryopexy can be applied to treat retinall breaks as a prophylactic treatment for retinal detachment. These procedures are usually performed in the ophthalmologist's office or in an outpatient surgical facility ${ }^{[6.4 b \mid}$

As far as we know, no RCTs have compared the safety and effectiveness of cryopexy, diathermy. laser photocoagulation and reattachment surgery for "prophylactic) treatment of retinal detachment. Obviously, no evidence on the cost-effectiveness of the procedure is available. Until 1992, before the reform of the US Medicare Fee Schedule, prophylactic laser photocoagulation and cryopexy were reimbursed with US\$ 718 and 705 , respectively ${ }^{870 !}$. 


\subsection{Glaucoma}

\subsubsection{Introduction}

Glaucoma is an eye disease characterized by a deterioration of the optic nerve. The disease, which becomes manifest in the optic disc first, is slowly progressive and asymptomatic in most cases. Visual dysfunction in the disease is first manifested in the mid-peripheral field of vision. Centrall vision functions such as acuity are involved relatively later in the disease. Glaucoma is the third most prevalent cause of visual impairment and blindness in the United States, after cataract anid age-related macular degeneration ${ }^{[4] \mid}$ "In at least half of the cases the disease is preceded by an abnormally high intraocular pressure, caused by an obstruction of the outflow of ocular fluid ("aequeous humourt) at the angle of the anterior chamber of the eye.

Laser treatment is applied in various stagés of the disease, which therefore needs some additional explanation. Glaucoma can be distinguished in primary and secondary disease. In secondary forms glaucoma is caused by another, underlying disease. Primary glaucoma is classified into open-angle (chronic simple), closed-angle (angle-closure) and congenital or infantile glaucoma ${ }^{[45)}$. In openangle glaucoma, the escape from the aequeous humour from the anterior chamber of the eye through the trabecular meshwork and Schlemm's canal into the episcleral space is impeded. In closed-angle glaucoma, the above described 'filtration-angle', the juncture of the iris and the cornea, is (increasingly) blocked, reducing the outflow of aequeous humour: In the course of the disease the aqueous humour increasingly meets resistance at the pupil. This stage is termed "relative pupil block". In older publications, sometimes CAG is therefore called pupillary-block glaucoma, although pupillary block actually precedes glaucoma. Pupillary block of aqueous humour causes ballooning of the iris, termed iris bombé. Ultimately, the base of the iris blocks the trabecular meshwork. If this happens suddenly, this is called acute CAG. If this happens gradually, the proces is called chronic CAG ${ }^{[4] !}$.

\subsubsection{Primary open-angle glaucoma}

In the United States, primary open-angle glaucoma is seven times more prevalent than primary closed-anglie glaucoma ${ }^{[48]}$. In the elderly population of the US, the prevalence of primary open-angle glaucoma has been estimated 2 to 3 per 1,000 in $1988^{[471}$. In a textbook by Philips, Clark and Tsukahara (1994) it is stated that a level of $16 \mathrm{mmHg}$ is regarded as the mean narmal intraocular pressure but that, like blood pressure the level varies quite widely between individuals even of the same age and sex, and at different times in 24 hours $^{[46]}$. As a result, according to these authors, a strict 'upper limit of normal' would be $21 \mathrm{mmHg}$, and a less stringent limit $24 \mathrm{mmHg}$. Values exceeding the latter limit would be agreed by most ophthalmologists as definitely pathological, i.e. as a level at which the field of vision will be affected.

Before the advent of laser treatment, reduction of intraocular pressure in primary open-angle glaucoma was managed by a variety of medications or, in more advanced disease, by surgery ('filtering' surgery; trabeculectomy). In the early eighties, laser trabeculoplasty became a popular treatment modality to reduce intraocular pressure in patients who had uncontrolled open-angle glaucoma on maximally tolerated medical therapy ${ }^{154}$. In these patients laser therapy was provided in the hope to either postpone or obviate the need for surgery. In addition, a few studies suggested that laser treatment could have a role as initial treatment.

Both these tentative conclusions were confirmed as the result of an assessment of argon laser trabeculoplasty in 1984 by the National Center for Health Services Research ${ }^{[231}$. In this report, an assessment of laser trabecular surgery for open-angle glaucoma by the American Academy of Ophthalmology (AAO) was cited ${ }^{151}$ that supported the use of lasers for these patients. More specifically, according to the AAO, laser therapy seemed most beneficial in patients with 
ineffectively medically managed intraocular pressure in primary open-angle glaucoma and (secondary), pigmentary and exfolliate glaucoma. This statement would again by made in an update of the assessment in $1989^{17}$. Although this conclusion was in part based on a few controlled studies, none of these had a randomized design. In this period, however, some RCTs had been published.

\section{Evaluation of laser treatment in patients with advanced primary open-angle glaucoma}

Smith (1984) compared the effectiveness of blue-green argon laser trabeculoplasty and green argon laser trabeculoplasty in two groups of patients ( $n=50$, average follow-up about 10 months) with chronic (advanced) open-angle glaucoma in whom intraocular pressure was judged to be uncontrolled on maximum tolerated medication ${ }^{[58]}$. Medication was unchanged and continued in every patient after laser treatment. A comparison of intraocular pressure in eyes before and after treatment showed that both blue-green and green argon laser treatment reduced intraocular pressure to a similar extent. Although the study was presented as an RCT, the method of randomization was not clarified. Information in the summary suggested that the two groups of patients had been sequentially treated. Although a baseline comparison of both patient groups did not reveal selection bias, the conclusion of the author that blue-green and green argon laser treatment were equally effective in reducing intraocular pressure, can only be interpreted as suggestive.

Also in 1984, a Swedish RCT compared the effectiveness of one- and two session argon laser trabeculoplasty in patients with advanced primary open-angle or pseudoexfoliation glaucoma in whom intraocular pressure was medically uncontrolled $(n=77$ eyes of 67 patients, mean follow-up 12 months $)^{[27]}$. The method of randomization in this study was questionable (randomization by (even or uneven) birth date) and inclusion criteria were not strict. The results suggested that a one session laser treatment is slightly more effective in reducing intraocular pressure, but that a two session laser treatment, scheduled one month apart, results in slightly less frequent complications, such as a temporary increase in intraocular pressure immediately after treatment.

In summary, the quality of these 'RCTs' was not very high and the scope of these studies was limited to different laser treatment alternatives- and procedures. In practice, argon laser trabeculolpasty has become the first line surgical treatment of patients with advanced, medically uncontrolled glaucoma, but several reports have appeared that indicate substantial loss of effectiveness of argon laser trabeculoplasty to control pressure within 2-5 years, despite continued use of medications ${ }^{|62|}$.

\section{Evaluation of laser treatment in newly diagnosed patients with primary open-angle glaucoma}

After presentation of the first results of another RCT in $1984^{|44|}$. Migdal and Hitchings published a second report in 1986 of an RCT comparing medical, surgical, and laser treatment for untreated patients with open-angle glaucoma ( $n=168$, one eye randomized per patient; 56 medical treatment, 57 surgical treatment, 55 laser treatment, follow-up ranging from 7 to 36 months) ${ }^{[45]}$. The mean pretreatment intraocular pressure for these three groups was similar, $33.8 \mathrm{mmHg}$ in the surgical treatment group, $34.9 \mathrm{mmHg}$ in the laser treatment group, and $33.0 \mathrm{mmHg}$ in the medical treatment group.

The non-study eye received conventional medical treatment. Laser treatment was provided in two sessions. Whenever laser treatment could not control intraocular pressure "pilacarpine was regarded as permissible adjuvant therapy. Medical treatment included stepped medication with four different compounds. In an analysis of an unspecified number of patients followed-up for two years, intraocular pressure had decreased $18.4,16.6$ and $13.8 \mathrm{mmHg}$ in the surgicaltreatment groups, respectively, resulting in a mean intraocular pressure of $15.4,18.3$ and 19.2 $\mathrm{mmHg}$ in these groups. An analysis of patients with one year follow-up showed that in $2.5 \%(1 / 40)$ of surgically treated patients $11.1 \%$ (4/36) of llaser treated patients and $16.6 \%(6 / 36)$ of medically treated patients, ocular pressure was not adequately controlled. The authors concluded that, in terms of intraocular pressure control, primary surgery proved the most successful method of 
treatment. There were no differences between the medical and the laser treated group in this respect. The authors did not present a systematic comparison of all complications and side-effecis of the treatment alternatives: Therefore, no conclusions were drawn from the study in terms of a treatment strategy. It was decided to continue follow-up in these patients ${ }^{[45]}$.

A third report by these authors was published in the proceedings of an ophthalmological congress in Singapore in 1990, which could not be traced. However, in 1993, the authors presented the main results of this study in a discussion paper ${ }^{5 i s}$. With a minimum follow-up of 4 years, the surgical group consistently showed the lowest intraocular pressure, with a mean pressure of $13.3 \mathrm{mmHg}$ compared with $16.8 \mathrm{~mm} \mathrm{Hg}$ and $17.3 \mathrm{mmHg}$ in the medical and laser groups, respectively. The succes rate of intraocular pressure control (defined as an intraocular pressure $<22 \mathrm{mmHg}$ ) at four years was $98 \%$ in the primary surgery group, $80 \%$ in the medical group, and only $60 \%$ in the laser group. No differences in the patient groups were found with respect to visual field loss and visual acuity.

These findings suggest that if only long-term effectiveness of treatment in terms of intraocular pressure control is considered, surgery could be regarded as treatment of choice. However. because the surgical procedure is invasive and often associated with complications, non-surgical treatment as initial treatment is preferred. Furthermore, the data suggested that medical treatment is more effective than laser treatment in controlling intraocular pressure in newly diagnosed patients with extremely elevated baseline-values of intraocular pressure.

\section{Evaluation of laser treatment of newly diagnosed and patients with advanced primary open- angle glaucoma by the National Eye Institute}

The studies discussed above were either of low quality, limited in scope or limited in size. All these drawbacks were addressed by the National Eye Institute in two RCTs focusing on glaucoma treatment. To assess the value of laser therapy in this fieid the NEI sponsored two investigatorinitiated multicenter RCTs comparing treatment strategies including blue-green argon laser treatment as initial treatment versus medical treatment as initial treatment in newly diagnosed patients (the Glaucoma Laser Trial (GLT)), and laser treatment as initial treatment versus surgery as initial treatment in patients in whom initial medical treatment had failed (the Advanced Glaucoma Laser Trial (AGLT) ${ }^{19 !}$.

\section{The Glaucoma Laser Trial}

Eight centers were involved in the Glaucoma Laser Trial (GLT), enrolling patients starting February 1984 and ending April 1987 ( $n=271$, follow-up in these patients was planned to be continued until 3 years after treatment). Patients with newly diagnosed, previously untreated primary open-angle glaucoma (POAG) of 35 years or older with intraocular pressure of at least $22 \mathrm{mmHg} \mathrm{Hg}$ were included in the study. In addition, a glaucomatous field defect in at least one eye or disc abnormalities in the presence of extremely elevated intraocular pressure had to be present for a patient to be eligible for enrolment. In both groups, mean intraocular pressure at baselline was 27 $\mathrm{mmHg}$. Each patient had one eye assigned by randomization to argon laser trabeculoplasty first (LF) and medical treatment first (MF). Medication was initiated or changed in either eye according to the siame stepped regimen if the intraocular pressure was not controlled.

A first publication of the Glaucoma Laser Trial Research Group (GLTRG) focused on the acute effects of argon laser trabeculoplasty on intraocular pressure ${ }^{[89]}$. Of the 269 patients who, in accordance with the protocol, underwent two laser treatment sessions, 91 (34\%) had a transient IOP rise of more than $5 \mathrm{~mm} H \mathrm{Hg}$ immediately after treatment. Forty-six percent of eyes developed greater than or equal to $1^{\circ}$ of peripheral anterior synechiae (PAS). The authors concluded that the abserved frequency and degree of pressure rises and PAS formation with in 3 months after the first treatment session would not outweigh the benefits of ALT if ALT were effective in lowering intraocular pressure and preventing visual field deterioration. 
The two-year follow-up analysis showed that, after a mean decrease of 9 and $7 \mathrm{mmHg}$ three months after treatment of the LF and MF eyes, respectively, LF eyes had lower mean 10 Ss than MF eyes $(1-2 \mathrm{mmHg})$ during the remainder of the follow-up period ${ }^{\text {th? }}$. Considering the mean baseline values of intraocular pressure, $27 \mathrm{mmHg}$ in both groups, the decrease can be considered as relevant A cross-over effect of medication, could only account for about $25 \%$ of this difference. At all times during follow-up, more than twice as many MF patients than LF eyes required two or more medications to control intraocular pressure. Overall, in the LF group, $89 \%$ of patients could be managed within the stepped medication regimen, compared to $66 \%$ of the MF patients. There were no differences in the treatment groups with respect to changes in the visual field. Therefore, the authors were cautious in their conclusions. They stated that it appeared that ALT is at least as good as if not better than medications as a starting treatment for patients with $\mathrm{PAOG}^{[07]}$.

This conclusion was heavily criticized by Lichter (1990) in an editorial in the journal 'Ophthaimology'. The author argued that the results could be interpreted differently, showing that the balance was in favor of medical treatment ${ }^{\mid 311}$. Other authors, however, supported the conclusions of the trial|is| ${ }^{\mid 30}$. Because there were no differences in the treatment groups with respect to changes in the visual field, it has been suggested that the two-year follow-up period was too short to reveal differences in clinical outcomes that are most relevant to patients. Long-term followup in the GLT is ongoing ${ }^{[67]}$. In other publications, controversy has primarily focused on surgery versus medical treatment as initial treatment ${ }^{[20]}$ and on medical treatment versus no treatment at all $^{\mid 52 !}$. Overall, it is still unclear which treatment, or treatment strategy is the best for patients with newly diagnosed open-angle glaucoma.

\section{The Advanced Glaucoma Intervention Study}

The Advanced Glaucoma Intervention Study was designed to study, in advanced glaucoma, the long term clinical course and, in a randomized trial, the comparative outcomes of two sequences of surgical treatments ${ }^{[62]}$. Towards these goals, 789 eyes in 591 patients were enrolled at 11 clinical centers in the US between 1988 and 1992, with a projected follow-up until the end of 1996 . Eligible eyes were randomly assigned to one or two intervention sequences. One sequence begins with argon laser trabeculoplasty (AL.T), is followed by trabeculectomy should ALT fail to control the disease, and by a second trabeculectomy should the first trabeculectomy fail. The other sequence begins with trabeculectomy; is followed by ALT should trabeculectomy fail, and by a second trabeculectomy should ALT fail. The main outcome of interest is visual function (visual field and visual acuity). So far, only the study design and methods and baseline characteristics of study patients have been published ${ }^{(62)}$, It is expected that this RCT will provide convincing evidence on the best treatment strategy in these patients.

\subsubsection{Primary closed-angle or angle-closure glaucoma}

About $75 \%$ of cases of closed-angle glaucoma (CAG) present as acute CAG, $25 \%$ as chronic $\mathrm{CAG}^{[4]}$.

\section{Acute closed-angle glaucoma}

Before the advent of the laser acute CAG was managed by one out of two surgical procedures, peripheral iridectomy or trabeculectomy. In the first case, medical treatment preceding further treatment had been successful in reducing intraocular pressure to normal levels and in reopening the angle far enough to allow iridectomy, which comes down to creating a hole in the iris to allow the aequeous humour to flow out of the posterior chamber to the anterior chamber ${ }^{[46]}$. Trabeculectomy used to be performed as a standard inpatient procedure "but is nowadays occasionally performed in an out-patient setting (Prof. Dr. F. Hendrikse, personal communication). Surgical iridectomy is either performed as an inpatient procedure or as a procedure in an ambulatory surgical center. 
With the advent of the laser in the seventies it became possible to perform iridectomy standard as an outpatient treatment. Case-series provided evidence for the safety and effectiveness of the procedure, which compared favourably to surgery. Thus, without any randomized clinical trials carried out, in the early eighties argon laser iridotomy became the treatment modality of choice in performing iridectomy. In patients with acute CAG, which is only occasionally bilateral, the felloweye is also at risk of acute CAG. Before the advent of the laser this eye was therefore prophylactically treated by surgical iridectomy. Later, laser iridotomy was preferred in these eyes as we |[3, In an ophthalmological textbook (1994), both laser treatment and surgical treatment of the fellow-eye are presented as optional ${ }^{[4]}$

\section{Chronic closed-angle glaucoma}

In chronic $C A G_{\text {, }}$ as a result of an analogous proces compared to acute $C A G$, argon laser treatment had become the treatment of choice compared to surgical iridectomy. This proces has been influenced by a favourable review of laser studies by the NCHSR in 1984 and by positive assessments of the procedure by the AAO in 1988 and $1989^{[3: 4 !}$. What is the quality of the evidence?

In the late seventies, the qs Nd:YAG laser was introduced in ophthalmology. In 1984, a $n$ RCT was published by Robin and Pollack, comparing qs Nd:YAG versus argon laser iridotomy in patients with bilateral chronic CAG ( $n=20$ patients, 40 eyes, follow-up 1 month $)^{[5.1]}$. All patients had bilateral iridotomies with one eye randomly assigned to argon laser treatment and the fellow-eye to qs Nd:YAG laser therapy. In all eyes a patent iridotomy was created in one treatment session. No (qs) Nd:YAG- and 30\% (6/20) argon laser iridotomies had marked iridotomy closure requiring retreatment. These could easily be reopened. The mean (standard deviation) preoperative intraocular pressure of the argon- and (qs) Nd:YAG laser treated eyes were 23 (8) $\mathrm{mmHg}$ and $23(7) \mathrm{mmHg}$, respectively. The one-month post-operative pressures for the argon- and the (qs) Nd:YAG treated eyes was 20 (8) and $18(4)$, respectively: The authors state that there were no significant intraocular pressure differences at any time interval (paired Student's t-test ${ }_{n} p>0.05$ ). Immediate postoperative intraocular pressure elevation is the most important complication, because it is a risk factor for irreversible visual loss. Intraocular pressure elevation greater than 10 $\mathrm{mmHg}$ was seen in $35 \%(7 / 20)$ in the argon laser treated eyes and in $30 \%(6 / 20)$ of the (qs) Nd:YAG laser treated eyes. There was no major progression of visual field loss or damage in any eye. The authors concluded that there were no differences in short-term treatment effectiveness (in terms of relief of pupillary block) and complications between the two treatment modalities.

In 1988, the authors published long-term follow-up results of patients in this trial, which had continued to enrol patients. At the time of analysis, 61 patients ( 122 eyes) had been included in the study ${ }^{[13]}$. Of these, 18 patients $(30 \%)$ were lost to follow-up before 20 months after treatments primarily because of the referral nature of the population. The analysis, which may be heavily biased, focused on 43 patients that had been followed-up for at least 20 months. Mean follow-up was 27 morths, maximum follow-up in these patients was 42 months. Iridotomy closure, shortly or weeks after treatment, was not observed in the (qs) Nd:YAG laser treated eyes, but nine (21\%) argon laser iridotomies required retreatment. The mean (standard deviation) preoperative intraocular pressure was $22.1(5.7) \mathrm{mmHg}$ in the argon laser treated eyes and $24.9(10.4) \mathrm{mmHg}$ in the (qs) Nd:YAG laser treated eyes. The final results at 20 months were $19.7(5.4) \mathrm{mmHg}$ (argon) and $19.4(5.2) \mathrm{mmHg}$ (Nd:YAG).

Although, just as in the previous publication, a paired Student's tt-test showed that preoperative differences were not significantly different, it is unclear whether or not the post-treatment values in both groups are significantly lower than base-line values. No swstematic information was provided on the occurrence of immediate postoperative intraocular pressure elevation, which may ocuur in eyes after (transient) bleeding, a minor complication predominantly associated with (os) $\mathrm{Nd:YAG} \mathrm{laser} \mathrm{treatment.} \mathrm{However,} \mathrm{in} \mathrm{contrast} \mathrm{with} \mathrm{the} \mathrm{previously} \mathrm{published} \mathrm{short-term} \mathrm{data-}$ analysis, a variety of other minor complications was less frequently observed in (qs) Nd:YAG laser 
treated eyes than in argon laser treated eyes. Also, the (qs) Nd: YAG laser was easier to use for physicians and its use was also more comfortable far patients.

For this combination of reasons, the authors concluded that (qs) Nd:YAG laser treatment suggested some advantages over argon laser treatment intraoperatively and short-term postoperatively. Laser treatment was shown to have no effect on visual acuity, which stayed at a constant level in the study periad. So there were no long-term differences between the treatment modalities. Newertheless, based on these data; it was concluded that the (qs) Nd:YAG laser should be the treatment of choice. This conclusion seems hardly justified: $30 \%$ of patients initially randomized was lost to follow-up, two different types of (qs) Nd:YAG lasers were used and no longterm differences in visual acuity were demonstrated ${ }^{[18]}$.

In 1988, the American Academy of Ophthalmology (AAO) reviewed the literature on laser peripheral iridotomy for $\mathrm{CAG}^{[3 !}$. While including the results of the RCT discussed above, it was concluded that a major advantage of laser iridotomy over surgical iridectomy is the greater patient safety. Furthermore, without formal evidence, it was concluded that laser iridotomy was costeffective compared to surgical iridectomy and that the procedure has dramatically improved the quality of care given to patients with all forms of angle-closure glaucoma. In addition, it was stated that, although argon laser was the most commonly used laser for creating an iridotomy, in wiew of the advantages listed above, the (qs) Nd:YAG laser could probably become the preferred modality in the future. For specific subgroups of patients (e.g. elderly patients) the (qs) Nd:YAG laser was recommended as first choice $e^{[3]}$.

An AAO assessment of Nd:YAG photodisruptors (predominantly qs Nd:YAG lasers in iridotomy and posterior capsulotomy) in 1989, was based on a lot more information, all case-series. For laser iridotomy the conclusion was that the $Q$-switched technique offered advantages over the modelacked technique, as initially used by European pioneers in the field ${ }^{14 !}$. Both in 1989 and 1993 , when the assessment was updated again, the basic conclusions with respect to laser iridectomy had not changed compared with the original 1988 AAO assessment of laser iridotomy for pupillary-block glaucoma ${ }^{[1,4]}$. In the 1993 assessment it was written that in the meantime qs Nd:YAG lasers had become the method of choice for many ophthalmologists when creating iridotomies ${ }^{[1]}$.

In summary, in the absence of RCTs, laser iridotomy has replaced surgical iridectomy. It is unclear which laser should be the treatment of choice, the argon laser or the qs Nd:YAG laser. Perhaps the choice of laser should be predominantly based on the pigmentation of the iris. Another possibility is to use the Q-switched Nd:YAG laser in patients in whom the argon laser fails ${ }^{150]}$.

\subsubsection{Uncontrollable glaucoma}

In a range of glaucomatous patients in whom intraocular pressure can not be controlled, despite (repeated) application of the treatment modalities discussed in this paragraph or when these therapies are not possible or are felt to have a low chance of success, usually cyclocryatherapy, a surgical procedure, is applied.

Since 1973, transscleral Nd:YAG laser cyclophotocoagulation (TSYLC) is documented as an alternative to cyclocryotherapy. More recently, the diode laser is used for this purpose as well (Prof. Dr. F. Hendrikse, personal communication). TSYLC is a cyciodestructive procedure by which the ciliary body is coagulated and consequent lowering of the production rate of aqueous flow and of intraocular pressure are expected to be achieved. No RCTs comparing TSYLC and cyclocryotherapy have been done ${ }^{[0 ! !}$.

In 1993, an RCT was published comparing the safety and effectiveness of two energy levels for non-contact transscleral Nd:YAG cyclophotocoagulation ${ }^{[57]}$. In the trial, patients were randomized to approximately 4 or $8 \mathrm{~J}$ of laser energy per application ( $n=89$, average follow-up 1 year). Although patients in the $4 \mathrm{~J}$ group showed less early anterior chamber inflammatory reactions, there were 
no differences in both groups with respect to control of intraocular pressure and visual acuity. In this case, a comparison of laser treatment to another treatment modality would have been more informative on its relative vallue. In the absence of comparative RCTS, TSYLC can be considered established by clinical experience.

\section{Summary: laser treatiment of glaucoma}

It has been documented by one RCT in patients with primary open-angle glaucoma indicated thai surgery first is most successful in controlling long-term intraocular pressure compared to laser: treatment first (including one additional drug) and stepped medical treatment in patients with newly diagnosed glaucoma. However, surgical treatment was considered undesirable in this stage of the disease due to the invasiveness of the procedure, the need for general anesthesia and the frequency of serious complications. It should be noticed, however, that filtering surgery nowadays can be done as an outpatient procedure, requiring local anesthesia ${ }^{[56]}$.

In the Glaucoma Laser Trial, excluding the option of initial surgery. 2 year follow-up results of laserfirst treatment (with stepped medication) of patients was at least as good as (stepped) medicationfirst treatment. However, no differences were found with respect to visual field loss and visual acuity. Therefore, and because not all aspects of the various treatment strategies have been systematically included, no definite choice of treatment strategy can be made until results of longterm follow-up become available.

In case of medically uncontrolled primary open-angle glaucoma, acute and chronic angle-closure glaucoma and uncontrollable glaucoma, laser treatment has become either the treatment of choice or an established modality without convincing (long-term) evidence of RCTs comparing laser therapy to conventional treatment. Whenever RCTs were designed to compare the effectiveness of two (combinations of) wavelengths of one laser or of two types of lasers, no clinically significant differences were found.

No formal evidence is available on the cost-effectiveness of laser treatment of glaucoma. An economic evaluation of laser treatment in primary open-angle glaucoma should take into account that laser treatment is performed rather as part of a treatment strategy than as a stand-alone procedure. Contrasting with surgical procedures, laser therapy can always be performed as an outpatient procedure requiring topical anesthesia. In particular where lasers replace surgical procedures, such as in uncontrollable glaucoma, it has a potential for being the most cost-effective treatment option.

\subsection{Cataract}

Cataract can be described as an opacity (a loss of transparency) of the crystalline lens of the eye, leading to visual impairment. When cataract surgery is performed, the lens is extracted from the eye. The lens is usually replaced by an artificial lens which is designed to restore the lost focusing power of the removed natural lens. This lens is usually implanted immediately behind the iris ${ }^{[12,22,45]}$. In the US cataract surgery is the most common surgery procedure in citizens aged 65 and over. Based on data from 1986 and 1987 , it was estimated that 1.35 million procedures were performed in 1991, at a Medicare expenditure of approximately US\$ 3.4 billion ${ }^{[6]]}$

During the postoperative period following lens extraction patients develop an opacification of the posterior capsule. As a result of this proces, which is considered a natural consequence of cataract surgery, there is a gradual deterioration in visual function that ultimately can become symptomatic to the patient. In these cases, a surgical incision was performed to open an opacified capsule (posterior capsulotomy). This procedure could be done either as an office procedure or as an inpatient procedure (requiring an operating room), depending on the technique. 
In the early eighties, Q-switched and mode-locked Nd YAG laser treatment started being offered as an alternative method for opening the opacified capsule, negating the need for conventional surgery ${ }^{i 2,22,45 !}$. At that time, the US National Eye Institute (NEl) recognized that (GS) Nd: VAG pasterior capsulotomy was becoming a widespread ophthalmic procedure. As a resul, the US Office of Health Technology Assessment (OHTA) was requested to assess the procedure, and, as part of this proces, obtain the opinion of the American Academy of Ophthaimology (AAO) on this procedure ${ }^{221}$. In 1983, the AAO assessed the procedure. Its conclusion, based on case-series, was that $q$ S Nd:YAG laser therapy appeared to be safe and effective for this indication. Because the technique had an extremely low complication rate it was a preferred alternative to surgical incision ${ }^{21}$. In 1984, the OHTA assessment of the procedure was published by the National Center for Health Services Research (NCHSR). While using the results of the assessment of the AAO, but in the absence of RCTs comparing laser therapy to surgery, it was concluded that laser posterior capsulotomy is safer and at least as effective in opening the posterior capsule as surgery ${ }^{222}$. The only complication of any note at that time was the development of a temporary rise in intraocular pressure in some patients.

Also for this indication (see paragraph 6.6.3 on closed-angle glaucoma) the $Q$-switched technique was favoured over the mode-locked technique. The conviction put forward in the NCHSR assessment was repeated in an FDA report on the safety and effectiveness of the procedure in $1985^{[59]}$ and by renewed assessments of qs Nd:YAG capsulotomy (and a variety of other Nd:YAG laser procedures) by the AAO in 1989 and $1993^{\{1,4\}}$.

In 1993, the US Agency for Health Care Policy and Research (AHCPR) issued a guideline for functional impairment due to cataract in adults ${ }^{\text {"2) }}$. Part of this guideline was directed at Nd:YAG laser capsulotomy after posterior capsual opacification. Based on an extensive literature review, which did not contain RCTs comparing laser therapy and surgery, it was concluded that Nd:YAG capsulatomy is a highly successful procedure. In eyes without comorbidity, a visual improvement can be expected in $65 \%$ of the cases. The major complications of Nd:YAG laser capsulotomy include elevated intraocular pressure, retinal detachment, cystoid macular edema, damage to the intraocular lens (IOL), hyphema, dislocation of the IOL, and corneal edema. Although these complications are not common (the majority of complications is found in less than $1 \%$ of patients). some of these, most notably retinal detachment, are potentially blinding.

Despite this information, it was also concluded in the AHCPR guideline that although Nd:YAG posterior capsullotomy is a commonly performed procedure (600 000 in 1991 in the US), there are few rigorously documented data available on indications, the rate of immediate and long-term complications, and outcome from the perspective of the patient's quality of life. Finally, there is also no information on the rate of occurrence of visually significant posterior capsular opacification and the appropriate timing and rates of performance of the procedure ${ }^{[12]}$. One year earlier though, Javitt and colleagues ${ }^{[28]}$ had convincingly argued that capsulotomy should be deferred untill the patient's impairment caused by capsular opacification warrants the increased risk of retinal complications associated with performance of capsulotomy ${ }^{\mid 28 !}$.

In conclusion, the safety and effectiveness of qs Nd:YAG laser capsulotomy has been established by clinical evidence outside RCTs. In the AAO assessment of Nd:YAG photodisruptors of 1993, an explanation was provided for the lack of RCTs. The physiclans' perception of increased safety of the laser as compared to conventional capsulotomy has made it impossible to achieve controlled prospective studies ${ }^{\text {th }}$.

There is no formal information available on the cost-effectiveness of the procedure, which is potentially cost-effective because it replaces a surgical treatment (performed as an inpatient or as an office procedure, depending on the technique) by an outpatient procedure which only requires topical anesthesia. In the US the average Medicare charge for Nd:YAG capsulotomy was US\$511 un 1991. This is about $20 \%$ of the average charge for a 'typical' cataract surgery procedure, 
estimated to be as high as US $\$ 2500$ in $1991^{1003}$. Of course, the real costs of these procedures may differ largely from the (negotiated) average charge.

\subsection{Photorefractive and phototherapeutic keratectomy}

Contrany to the previously discussed mature, well-evaluated applications for the use of lasers, laser treatment for correction of myopia, hyperopia, astigmatism (in short photorefractive keratectomy) and treatment of comeal scars (and other comeal lesions; in short phototherapeutic keratectomy) are relatively immature. Of these applications, Excimer laser treatment of myopia has received most attention. This particular case is discussed in detail in Chapter 17.

\subsection{The potential role of diode lasers}

It has sometimes been speculated that the dominant position of the argon laser may be challenged by the diode laser ${ }^{[0]}$. At least two small RCTs have been reported comparing argon- and diode laser treatment of proliferative diabetic retinopathy and trabeculoplasty for primary open-angle glaucoma, respectively ${ }^{10,11]}$. Although these small studies did not detect any clinically significant differences between both types of lasers, the diode laser has the advantages of a lower purchase price, liberal installation requirements (including the ability to run off ordinary household current and no need for water cooling), lower maintenance costs, and portability.

This combination of data stimulated Sculpher (1993) to publish a preliminary economic evaluation of the diode laser in ophthalmology from the perspective of the hospita| $\left.\right|^{|55| \mid}$. Five cost components were included in the study to calculate the cost per patient-treatment course of the diode laser relative to the argon laser: staff cost, consumable cost, overhead cost, and the capital- and maintenance cost of equipment. The calculation was adapted to five different contexts of purchase and use, including routine replacement of equipment, early replacement of equipment, creation of a new facility, condition-specific provision and creation of a new form of care.

Most detailed information was available for the first situation, routine replacement of equipment, showing that the total costs of a course of diode laser treatment would be BP 100 versus BP 120 with the argon laser (1991-1992 price-base). In the base-case analysis the capital costs of the argon laser system, and the costs of its maintenance, represent about $32 \%$ of the total cost of an average treatment course. The author concluded that, even if the diode laser thalves the capital and maintenance cost, this would only reduce the total cost of a treatment course by about $16 \%$ (aboult BP 20).

In a sensitivity analysis, the cost difference was shown to decrease with increasing utilization rates and the number of treatment sessions required. For example, if one more treatment session would be needed with the diode laser to achieve the same clinical result as with the argon laser, an annual utilization rate of 800 patients would result in equal total costs of a course of treatment with either laser. The calculations for the remaining situations suggested that a significant cost advantage of the diode laser is unlikely. Based on both preliminary data on effectiveness and costs, the author concluded that a rapid replacement of argon lasers by diode lasers is unlikely ${ }^{155 .}$. The diode laser may still have the potential to replace the argon laser, but in the absence of convincing clinicall data this process continues to be speculative. 


\subsection{Summary}

Table 6.1 summarizes the results of this Chapter. As can be seen, the effectiveness of the use of lasers in a variety of indications has been well-documented by RCTs. Only argonlaser treatment of diabetic retinopathy can be considered as "proven' cost-effective. This case demonstrates extremely well that good studies are needed to document effectiveness in a credible way. Also, modelling can be a powerful tool to demonstrate cost-effectiveness. Overall, the conclusion was justified that screening for and treatment of retinopathy in diabetic populations is beneficial, both in terms of effects and costs. For other indications, although evidence of effectiveness is often excellent, hardly any evidence on cost-effectiveness is avallable. Compared to no treatment, which would be the realistic alternative for many indications, laser treatment is judged as potentially casteffective. In general, of all different types of lasers in ophthalmology, argon-, krypton and qs Nd:YAG lasers have been best evaluated. Of these types, the differences in effectiveness between the krypton laser and the argon laser are marginal for many indications. Nevertheless; the argon laser is the most important in terms of adoption and use.

Table 6.1 Judgments on the effectiveness and cost-effectiveness of selected laser applications in ophthalmology

\begin{tabular}{|c|c|c|c|c|c|c|c|}
\hline \multirow[t]{2}{*}{ Application } & \multicolumn{7}{|c|}{$\begin{array}{l}\text { Judgments } \\
\text { (see definitions below) }\end{array}$} \\
\hline & 1 & 2 & 3 & 4 & 5 & 6 & 7 \\
\hline Diabettic retinopathy & & & & $x$ & & & $x$ \\
\hline Age-related macular degeneration & & & & $x$ & & $x$ & \\
\hline $\begin{array}{l}\text { Retinal vein occlusion } \\
\text { Branch vein occlusion } \\
\text { Centrall vein occlusion }\end{array}$ & & & $x$ & $x$ & & $x$ & \\
\hline Retinal detachment & & & $x$ & & & & \\
\hline $\begin{array}{l}\text { Primary glaucoma } \\
\text { Newly diagnosed open-angle glaucoma } \\
\text { Advanced open-angle glaucoma } \\
\text { Angle-closure glaucoma } \\
\text { Uncontrollable glaucoma }\end{array}$ & & & $\begin{array}{l}x \\
x \\
x\end{array}$ & $x$ & & $\begin{array}{l}x \\
X\end{array}$ & \\
\hline Cataract (posterior capsulotomy) & & & $x$ & & & $x$ & \\
\hline $\begin{array}{l}\text { Phatorefractive keratectomy } \\
\text { Phototherapeutic keratectomy }\end{array}$ & $x$ & & $x$ & & $x$ & & \\
\hline
\end{tabular}

judgment of myopia correction only

Definitions:

1 Experimental = still in development, limited clinical data, no proof of effectiveness.

2 Promising = some clinical dlata, but procedure not accepted as a routine.

3 Established by clinical experience $=$ no randomized trials ${ }_{\text {, }}$ but sufficient clinical data to reach a judgment.

4 Established by RCT = established by several well-designed randomized controlled trials.

5 Probably not cost-effective = limited data on cost-effectiveness, but reason to believe that the procedure will not turn out to be cost-effective.

6 Probably cost-effective = limited data on cost-effectiveness, but reason to believe that the procedure will turn out to be cost-effective.

7 Proven cost-effective $=$ adequate analyses of cost-effectiveness. 


\subsection{References}

1. American Academy of Ophthamology (AAO). Ophthalmic Procedures Assessment. Nid:YAG photodisruptor: Opluthaimology $1993(100) 11: 1736-42$

2. American Academy of Ophthalmology (AAO). Assessmemt of the safety and efficacy of the Neodymium:YAG laser American Academy of Ophthaimology, San Francisco, 1983.

3. American Academy of Ophthamology (AAO). Infomation About Eye Care. Laser peripheral iridotomy for pupillary. block glaucoma. Ameritan Acaidemy of Ophthalmology San Francisco, 1988.

4. American Academy of Ophthalmology (AAO), Ophthalmic Procedures Assessment. Nd YAG photodisruptors. If: Ophthialmology, Instrument and Boid lssue, 1989, p. 46.51.

5. American Academy of Ophthalmology (AAO). Ophthalmic Procedures Assessment. Recommendations. Laser trabecular surgery for open-angle glaucoma, American Academy of Ophthalmology, San Franicisco, 1983.

9. American Academy of Ophthamology (AAO). Information Statement. The repair of rhegmatogenous retinat detachmente Ophthaimology 1990(97) 11:1562-72.

7.: American Acadenty of Ophthalmology (AAO). Ophthaimic Procedures Assessment. Laser trabeculoplasty tor apenangle glaucoma. In: Ophthalmology, Instrument and Book issue 1989, p. $42-5$.

8. American College of Physicians, American Diabetes Association, and American Academy of Ophthalmology. Screening guidelines for diabetc retinopathy. Clinical guideline. Ophthalmology 1902(99)10:1626-8.

9. Aron-Rosia D. Ophthalmology - Effectiveness. In: Banta HD, Schou ll (Eds) Lasers in Health Care. Effectiveness Cost-Effectiveness and Policy Implications. Academic Publishng, Frederiksberg, Denmark, 1991, p. 90m6.

10. Bandello $F$, Brancato $\mathbb{R}$, Trabucchi $G$, Lattanzio $R$. Malegoni $A$. Diode versus argon-green laser panretinal photocoagulation in proliferative diabelic retinopathy: a randomized study in 44 eyes with a long follow-up time. Craefe's Archive for Clinical \& Experimental Ophthalmology 1993(231)9:491-4.

11. Brancato $R$, Carassa $R$, Trabucchi $G$. Diode laser compared with argon laser for trabeculoplasty. American Journal of Ophthalmology $1991(112): 50-5$.

12. Cataract Management Guideline Panell. Management of Functional Impaiment Due to Cataract in Adults. Guideline report, Number 4. US Department of Health and Human Services (DHHS), Public Heallth Service (PHS), Agency for Health Care Policy and Research (AHCPR). AHCPR Publication No. 93-0541, Rockwille MD, August 1983. Published in: Ophthalmology 1993(100)8(Supplement 1):1-343.

13. Central Vein Occlusion Study Group. Baseline and early natural history report. Archives of Ophthalmology 1993(111):1087-95.

14. Clarkson JG. Central Ven Occlusion Study. Photocoagulation for ischemic central retinal vein occlusion (editorial) Archives of Ophthalmology 1991(109):1218-9.

15. Coscas $C$, Soubrane $G$, Ramahefasolo $C$, Fardeau $C$. Perifoveal laser treatment for subfoveal choroidal mew vessels in age-related macular degeneration. Results of a randomized clinical trial. Archives of Ophthalmology $1991(109): 1258-65$.

16. Coscas C, Soubrane $\mathrm{G}$. Indications, techniques et résultats de la photocoagulation au laser à argon des néovaisseaux sous-rétiniens maculaires. Journal Français d"Ophthalimology 1984(7)2:99-105.

17. Crijns HHM. Diabetic retinopathy. A cost-effectiveness analysis of ophthalmoscopy and photocoagulation (thesis). Erasmus University Rotterdam, the Netherlands, 1993.

18. Dell Priore JV, Robin AL, Pollack PP. Long-term follow-up in a prospective randomized clinical trial. Ophthalmology $1988(95) 9: 1207 m 1$ 1

19. Department of Health and Human Services (OHHS), Public Health Service (PHS), National Institutes of Health (WIH). Clinical trials supported by the National Eye Institute. Evaluating new approaches to the treatment of eye and vision disorders. NHH Publication No. 87-2910, US DHHS, PHS, NHH, Bethesda, MD, Nowember 1987.

20. Dutton $\mathrm{d}$, Slamovits T (Eds.), Initial treatment of glaucoma: surgery or medications. Survey of Ophthalmology $1993(37) 4: 293 \times 305$.

21. Early Treatment. Diabetic Retinopathy Study Research Group. Early photocoagulation for diabetic retinopathy: ETDRS Report No. 9. Ophthalmology 1991(78):766-85.

22. Erlichman M. Assessment of Nd:YAG laser for posterior capsulotomies. In: US Department of Health and Human Services (DHHS), Public Health Service (PHS), National Center for Health Services Research (NCHSR). Health Tachnology Assessment Report No. 231, US DHHS, PHS, Rockville, MO, 1984, p. 317-35.

23. Feigenbaum E. Assessment of laser trabeculoplasty for open-angle glaucoma. lin: US Department of Health and Human Services (DHHS). Public Health Service (PHS), National Center for Health Services Research (NCHSR). Health Technology Assessment Report No. 23. US DHHS. PHS, Rockville, MO, 1984, p. $371-90$.

24. Fine SL, Wood WJ, Isemhagen RD, Singerman Lل, Bressler NM, Folk JC, Kimura AE, Fish GE, Maguire MG, Alexander J. Laser treatment for subfoweal neovascular membranes in ocular histoplasmosis syndrome: results of a pilot randomized trial (letter to the editor). Archives of Ophthalmology 1993(111):19-20.

25. Finkelstein D. Argon laser photocoagulation for macular edema in branch vein occlusion. Ophthalmology $1986(93) 7: 975-7$ 
26. Hayreh SS, Kugman MR, Podhajsky P. Seruais GE, Perkins ES. Argon laser panratinal photocoagulation in ischemic retinal vein occlusion. A 10-year prospective study. Graefe"s Archive of Clinical amd Experimental Ophthalmology $1990(228): 281,96$.

27. Heij A. Onew and two session laser trabeculoplasty. A randantized, prospective study. Acta Ophthalmologica $1984(62): 715-24$

28. Javitt $J C$, Tiessch $J M$, Canner JK, Kolb MM, Sommer $A$, Steinberg EP, on behalf of the Catanact Patlent Outcomes Research Team. National outcomes of cataract extraction, lncreased risk of retinal complications associated with Nd:YAG laser capsulotomy. Optithamology 1992/99y10:1487-97.

29. Jawitt JC. Cost savings associaled with detection and treatment of diabetic eye disease. PharmacoEconomics 1995 (8) Supplement 1 $33-9$.

30. Letters to the Editor. Ophthalmology 1991(98)Supplemen $1: 841-3$

31. Lichter PR. Practice implications of the Glaucoma Laser Trial (editoriai). Ophthalmology 1990(97)11:1401.

32. Macular Photocoagulation Study Group (MPSG). Argon laser photocoagulation for senile macular degeneration. Results of a randomized clinical trial. Archives of Opththalmology 1982(100):912-8.

33. Macular Photocoagulation Study Group (MPSG). Argon laser photocoagulation of neovascular maculopathy: three year results from randomized clinical trials. Archives of Ophthalmology 1986(104):694-701.

34. Macular Photocoagulation Study Group (MPSG). Argon laser photocoagulation of neovascular maculopathy. 情传e year results from randomized clinical trials. Archives of Ophthalmology 1991(109):1109-14.

35. Macular Photocoagulation Study Group (MPSG). Kirypton laser photocoagulation for meovascular llesions off ocular histoplasmosis: results of a randomized controlled trial. Archives of Ophthalmology 1987(105):1499-507.

36. Macular Photocoagulation Study Group (MPSG). Argon laser photocoagulation for ocular histoplasmosis: results of a randomized clinical trial. Archives of Ophthamology 1983(104):1347-57.

37. Macular Photocoagutation Study Group (MPSG). Argon taser photocoagulation for idiopathic patients. Results of a randomized clinicial trial. Archives of Ophthatmology 1983(101):1358-61.

38. Macular Photocoagulation Study Group (MPSG). Krypton laser photocoagulation for idicpathic lesions. Results of a randomized clinical trial. Archives of Ophthalmology 1990(108):832-7.

39. Macular Photocoagulation Study Group (MPSG). Persistent and recurrent neovascularization after krypton laser photocoagulation for neovascular lesions of age-related macular degeneration. Archives of Ophthalmology $1990(108): 825-3 \%$.

40. Macular Photocoagulation Study Group (MPSG). Laser photocoagulation of Subfoveal neovascular lesions in agerelated macular degeneration: results of a randomized clinical triall. Archives of Ophthalmology 1991(109)"1220-31.

41. Macular Photocoagulation Study Group (MPSG). Laser photocoagulation of subfoveal recurrent neovascular lesions in age-related macular degeneration: results of a randomized clinicall trial. Archives of Ophthalmology $1991(109): 1232-41$

42. Macular Photocoagulation Study Group (MPSG). Laser photocoagulation of subfoveal neovascular llesions of agerelated macular degeneration. Updated findings of two clinical trial: Archives of Ophthalmology 1993(111):1200-9.

43. Macular Photocoagulation Study Group (MPSG). Krypton laser photocoagulation for neo-wascularized lesions of age-related macular degeneration. Resulls of a randomized clinical triai. Archives of Ophthaimology 1990(108):81624.

44. Migdal C, Hitchings RA. Primary therapy for open-angle glaucoma. the role of argon laser trabeculoplasty. Transactions of the Ophthalmological Societies of the United Kingdom 1984(104):62-6.

45. Migdal $C$. Hitchings RA. Control of chronic simple glaucoma wilti primary medical, surgical and laser treatment. Transactions of the Ophthalmological Societies of the United Kingdom 1986(105):653-6.

46. Phalips Cl, Clark CV. Tsukahara S. Ophthalmology. A primer for medical students and practitioners. Bailliere Tindall. London, Philladelphia, Toronto, Sidney, Tokyo, 1994.

47. Power EJ, Wagner JL, Duffy BM. Screening for open-angle glaucoma in the elderly. A Staff Paper in OTA's Series on Preventive Health Services Under Medicare. Paper 1.number 20510-8025. Health Program, office of Technology Assessment, Congress of the United States, Washington D.C, 1988.

42. Quigley H. Open-angle glaucoma. New England Journal of Medicine 1993(328):1097-106.

49. Rivera AH, Brown RH, Anderson DR. Laser iridotomy vs surgical iridotomy. Have the indications changed? Archives of Opththalmology $1985(103): 1350-4$.

50. Robin AL. Pollack P. Q-switched Nd:YAG laser iridotomy in patients in whom the argan laser fails. Archives of Ophthalmology 1986(104):531-5.

51. Robim AL, Pollack UP. A comparison of Nd:YAG and argon laser indotomies. Ophthalmology 1984(91):1011-6

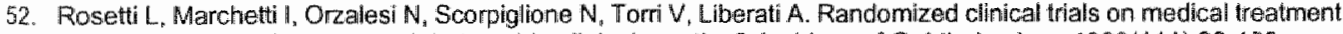
af glaucoma. Are they appropriate to guide clinical practice? Archives of Ophthalmology $1993(111) \cdot 96$ 103.

53. Schiling JS, Jones CA. Retinal branch vein occlusion: a study of argon laser photocoagulation in the treatment of macular edema. British Jourinal of Ophthalmology 1984(68); 196-8.

54. Schwartz AL, Kopelman J. Four-year experience with argon laser trabecular surgeny in uncontrolled open-angle glaucoma. Ophthalmology $1983(90) 7: 771.80$ 
55. Scupher M. A preliminary economic evaluation of the diode laser in ophthalmology. Lasers in Medical Science $1993(8): 163-9$

56. Shenwood MR, Migdal CS, Hitchings RA I. Fitration surgery. In: Dutton J Slamovits T (Eds, Inital trealment of gllaucoma: surgery or medications. Survey of Ophthalmology 1993(37)4:293-305.

57. Shields MB, Wikerson MH, Echelman DA. A comparison of two energy levels for noncontact transscleral Nd:YAG cyclophotocoegutation. Archives of Ophthaimology 1993(111):484-7

58. Smith J. Argon laser trabeculoplasty: comparison of bichromatic and monochiomatic wavelengths. Ophthalmology $1984(91) 4: 355-60$.

50. Stark WJ, Worthen D. Holladay JT, Murray G. NeodymiumiYA lasers: an FDA report Ophthalmolog $1985(92): 209-12$

60. Steinberg EP Jawit JC, Shankey PD. Zuckermann A Legroo MW, Anderson GP, Bass EB, ODay D. The content and cost of cataract surgery. Archives of Ophthalmology 1993(111):1041-9.

61. Suzuk Y, Araic M. Yumita A, Yamamoto T. Transscleral Nd,YAG laser cyclophotocoagulation verseds cyclocryotherapy. Graefe's Archive for Clinical and Experimental Ophthalmology 1991(229):33-6.

62. The AGIS Investigators. The Advanced Glaucoma Intervention Study (AGIS).\#. Study design and methods and basieline charactertistics of study patients. Controlted Clinical Trials 1994(15):299-325.

63. The Branch Vein Occtusion Study Group. Argon lasier scatter photocoagulation for prevention of neowascularization and vitreous themorhage in branch wein occlusion: a randomized clinical trial. Archives of Ophthalmology $1986(104): 34-41$.

64. The Branch Vein Occlusion Study Group. Argon laser photocoagulation for macular edema in branch vein occlusion. Artierican Journal of Ophthaimology 1984(98):271-82.

65. The Canadian Ophithalmology Study Group. Argon green versus krypton red laser photocoagulation of extrafovewl choroidal neovascular lesions. One-year results in age-related macular degeneration. Archives of Ophthalmology $1993(111): 181-5$.

66. The Diabetic Retinopathy Study Research Group. Photocoagulation treatment of proliferative retinopathy. Clinical application of Dilabetic Retinopathy Study (DRS) findings. DRS Report No. 8. Ophthalmology 1981(88):583-600.

67. The Glaucoma Laser Trial Research Group. The Glaucoma Laser Trial (GLT). 2. Results of argon laser trabeculoplasity versus topical medicines. Ophthalmology 1990(97)11:1403-13.

68. The Glaucoma Laser Trial Research Group. The glaucoma laser trial 1: acute effects of argon laser trabeculoplasty on intraocular pressure. Airchiwes of Ophthatmology $1989(107): 1135-42$.

69. The Moorfield Macular Study Group. Retinal pitgment epithelial detachments in the elderly: a controlled trial of argon laser photocoagulation. British Journal of Ophthalmology 1982(66):1-16.

70. Verrilli DK, Dunn DL, Rand $L$. The resource-based relative walue scale. Methods, results and impacts for ophthalmology. Archives of Ophthalmology 1993(111):41-9.

71. Wang $F$, Jawitt JC. Eye care for elderly Americans with diabetes mellitus. Ophthalmalogy 1996(103)11:1744-51. 


\section{Lasers in otolaryngology}

\subsection{Introduction}

Otolaryngologists have been involved in laser surgery since the first inception of this modality as a surgical tool. After three years of animal experimentation, Strong and Jako presented the first clinical report on the use of the $\mathrm{CO}_{2}$ laser in otolaryngology at the 1972 Annual Meeting of the American Broncho-Esophagological Society where its use was described in 15 procedures in 12 patients with laryngeal pathology ${ }^{|36|}$. Today, the most commonly used type of laser in otolaryngology still is the $\mathrm{CO}_{2}$ laser ${ }^{(13,35)}$. Usually, $\mathrm{CO}_{2}$ laser light is delivered to tissue either through a handpiece for macroscopic surgery or adapted to an operating microscope for microscopic surgery. This can be done either in the continuous wave- pulsed-or superpulsed mode ${ }^{[13.35 y}$. At present, if the use of a laser is deemed appropriate, the $\mathrm{CO}_{2}$ laser is the laser of first choice for mast microlaryngoscopic applications ${ }^{135 \mid}$. In the future its use is expected to expand in otolaryngology, for example in pediatric applications due to miniaturisation of bronchoscopes and more generally due to the commercial availability of hollow wave guides and increased transportability of recently developed types of $\mathrm{CO}_{2}$ lasers. This facilitates the use of these devices not only in a hospital environment but also in the physicians's office ${ }^{[35.45]}$. Of the other two 'classical' lasers the Nd:YAG laser plays a role especially in the nasal cavity, while the use of argon lasers seems limited to a single otological application, stapedotomy. Of the "new" types of lasers especially the KTP laser is becoming more important. For some indications this laser is in a proces of sulbstituting for the $\mathrm{CO}_{2}$ laser ${ }^{19,35 !}$. Other relatively new types of lasers are the diode-, Ho: YAG- and dye lasers, of which the latter are especially used in treatment of vascular lesions in head-and neck surgery ${ }^{13,391}$. No RCTs have been performed in this field. Combinations of clinical studies and the few cost studies that have been done suggest that laser treatment is potentially cost-effective in treatment of early laryngeal carcinoma. In general, however, the potential for cost-effectiveness of laser procedures is decreasing because an increasing number of surgical procedures that used to require hospitalization are presently carried out on a day-case basis ${ }^{[59}$.

The safety of otolaryngological llaser applications deserves special attention. If safely protocols are not strictly adhered to very serious complications, e.g. endotracheal tube fires, may occur. The complications that may accompany otolaryngological laser applications and related safely issues are therefore discussed first.

\subsection{Safety and complications of $\mathrm{CO}_{2}$ laser microsurgery}

The most serious complications of $\mathrm{CO}_{2}$ laser surgery arise from ignition by the laser beam of flammable materials in the operative field in the oral cavity, larynx, pharynx, and trachea: An airway fire will ensue, which is a potentially fatal situation ${ }^{\left[{ }^{3 !}\right]}$. In non-fatal cases, according to the Dutch National Committee on Laser Safety, airway fires can result in serious handicaps in patients ${ }^{|18| \mid}$. Most accidents in otolaryngological laser surgery have been reported as a result of endotracheal tube ignition, but also tooth guards, throat packs, nasal packs or airways may all burn ${ }^{\text {"3i }}$. In reviews such as those by Ossoff et al. in 1994, besides endotracheal tube ignition also complications such as hemorrhage, edema, perichondritis, vocal cords webs and glottic incompetence are listed as resulting from laser treatment ${ }^{355}$. What is the frequency of these and other complications? In the largest case-series published to date, including $4,416 \mathrm{CO}_{2}$ laser procedures, Healy et all. (1984) reported a complication rate of $0.2 \%$ (9 complications). Four of these complications, as reviewed by Ossoff in 1989, were associated with endotracheal tube fires, all of which occurred in their early experience, prior to protecting the tubes with reflective metallic tape $e^{[36]}$. A fifth fire occurred during rigid bronchoscopic laser surgery, when the latex cuff around the bronchoscope ignited. The sixth fire in their series occurred when a surgical sponge used to occlude a tracheostoma during bronchoscopic laser surgery suddenly ignited. None of these complications were life-threatening. The other complications in their series included a facial burn from an imperfectly aligned laser 
beam heating a bronchoscope that was in contact with the skin of the face, and two cases of bleeding while resecting obstructing tracheobronchial carcinoma. One year earlier, in 1983, Ossoff ef all. reviewed their experience with 204 cases at their institution over a 2 year period. ${ }^{[3 \hbar !}$ They noted 12 complications $(1,7 \%)$ including one extraluminal endotracheal tube fire in their early experience, which stimulated them to formulate and implement a laser safety protocol. The other complications included 2 finger burns suffered by the surgeon and 10 were minor patient-related accidents. No endotracheal tube fires occurred after implementation of the safety protocol. Also in 1983. Fried mailed a questionnaire on laser-related complications in laryngoscopy to 229 otolaryngologists, all members of a senior otolaryngological society in the United States. As reviewed by Ossoff in 1989 and 1994 , a response of 210 ololaryngologists was obtained $(92 \%)^{\mid 35,361}$. Of the respondents, $57(27 \%)$ did not use the laser. No complications had occurred in about two-third of the respondents who did use a laser, while about one-third of the laser-using otolaryngologists had experienced at least one complication. The total number of complications was 81 . The most frequent of these was endotracheal explosion, which was reported 28 times. Facial burns were reported 9 times, and laryngeal subglattic or tracheal stenosis were reported in 8 patients. There were 3 endotracheal cuff ignitions, and 2 cottonoid ignitions. Five cases of pneumothorax and two cases of subcutaneous emphysema were also reported. The 33 documented ignitions (tube, cuff and cottonoid) was both striking and alarming. Fried (as cited by Ossoff ( 1989,1994$)$ ) compared the results of the survey with the reporting of complications in the literature and concluded that complications are underreported in the literature. Furthermore, he stressed the importance of education and training programs because all complications occurred in patients who were treated by experienced otolaryngologists ${ }^{[36.36 \mid}$. It is interesting to note there that Hermens et al., (as reviewed by Ossoff in 1989) based on reported cases in 1983, estimated the incidence of endotracheal tube ignition in otolaryngological laser surgery to be between $0.4 \%$ and $1.5 \%$

Since the mid-eighties a lot of research has been done aiming at the prevention of complications in this field. Also anesthetic techniques have been developed that do not depend on the use of end otracheal tubes ${ }^{\{13,10\}}$. Laser safety standards have been developed and in many specialties education and training programs have been set up ${ }^{[15.18]}$. Although this may have contributed to a decrease of the frequency of laser-related complications, the actual complication rates are not welldocumented. In 1994, van Duyne et al., quoting many of the reports cited above, estimated that the complication rate of otolaryngological laser surgery varies from $0.2 \%$ to $1.7 \%{ }^{115 !}$. This implies that continuous attention to safety aspects of the use of lasers in otolaryngology is still required.

\subsection{Laryngeal disease}

\subsubsection{Introduction}

Laryngeal microsurgery is routinely performed in all otolaryngology departments. The patient is usually admitted to hospital the day before surgery and is usually discharged on the evening after surgery or on the following day. Although the larynx is a delicate organ, and as such very prone to iatrogenic damage during microlaryngeal surgery ${ }^{(13)}$, conventional microlaryngeal surgery has a very low morbidity. Open surgical procedures, however, are associated with substantial morbidity and prolonged hospitalization periods ${ }^{[7]} \cdot \mathrm{CO}_{2}$ laser microsurgery has become an alternative to conventionat microlaryngeal surgery in both benign and malignant lesions. 


\subsubsection{Benign laryngeal lesions}

\section{Stenosis}

Laryngeal stenasis is increasingly common, caused either by external trauma, commonly in roaid traffic accidents, or by internal trauma from prolonged endotracheal intubation. Any form of trauma to the larynx may cause fibrosis and scarring, narrowing the airway, which can necessitate a tracheostomy ${ }^{2}$. The management of laryngotracheal stenosis is a difficult problem for the otolaryngologist-head and neck surgeon. The first decision, according to Ossoff et al. in their 1994 review, whether open management is necessary or if endoscopic techniques alone are adequate. is probably the most demanding ${ }^{[35]}$. In the absence of RCTs, retrospective analysis of case-series has provided clues as to which stenotic lesions are suitable for endoscopic management. Firstly, all lesions to be treated with endoscopic techniques must have intact external cartilaginous support. Secondly, lesions appropriate for endoscopic management are usually $<1 \mathrm{~cm}$ in vertical length. Thirdly, total cervical tracheal or subglottic stenosis usually do not respond well to endoscopic treatment. In general, endoscopic management of laryngeal stenosis relies on mucosal preservation. The two techniques advocated for this task are radial incision with bronchoscopic dilation and the microtrapdoor flap technique. An indwelling laryngeal stent is sometimes needed after these procedures. In the first technique, the laser is used to make radial incisions in the stenotic area. Bronchoscopes are then sequentially passed through the stenosis to dilate it. The microtrapdoor flap technique utilizes the $\mathrm{CO}_{2}$ laser to make a horizontal incision in the mucosa overlying the stenosis. The laser is then used to vaporize the underlying scar tissue. Either microscissors or lasers can then be used to incise the lateral portions of the inferiorly based flap to permit redraping of the preserved mucosa.

The development of the microsubglottiscope has facilitated the use of the $\mathrm{CO}_{2}$ laser in the subglottis and cervical trachea. The $\mathrm{CO}_{2}$ laser can be attached to the operating microscope via a micramanipulator and lesions can be approached through a microlaryngoscopic technique as opposed to a bronchoscopic technique. Ossoff et al. (1994) conclude that of all types of lasers, the $\mathrm{CO}_{2}$ laser seems to be the most useful technique for the management of laryngeal stenosis, but add that favourable results have also been reported in lesions treated with the Nd:YAG laser. The Nd: YAG laser offers the advantage of a fiberoptic delivery system that facilitates its use in otherwise difficult to expose subglottic cervical tracheall lesions. However, due to its somewhat unpredictable penetration depth, there is a risk of producing thermal injury to the underlying cartilage. This can be avoided by using low power settings with a short pulse duration, according to these authors ${ }^{135 !}$.

In summary, based on clinical experience, in selected cases endoscopic procedures using a $\mathrm{CO}_{2}$ or an Nd:YAG laser may be used as a substitute for conventionall microlaryngeal procedures. No direct comparisons of laser versus non-laser microlaryngeal procedures and of (laser or non-laser) microlaryngoscopic procedures versus open surgery have been published. The evidence on the use of lasers is therefore, rather cautiously, judged as promising.

\section{Bilateral true vocal cord immobility}

Bilateral vocal cord immobility or paralysis is a potentially life-threatening condition that frequently requires some kind of surgical intervention to prevent acute asphyxiation or chronic centrall airway obstruction ${ }^{\mid 15 !}$. Surgical (total) arytenoidectomy as described by Woodman is the gold standard for this indication ${ }^{[16,35\}}$. According to Ossoff et al. (1994) endoscopic arytenoidectomy (with or without lasers) are accepted techniques as well ${ }^{[35]}$. The technique of endoscopic laser arytenoidectomy is described in detail by these authors, who were the first to apply this procedure clinically (in $1984^{116 i}$ ). Both the open and the endoscopic procedures, designed to statically open the glottis, will by necessity also decrease voicing. Thus, rehabilitation of the glottis for air passage is a compromise accepted to eliminate the need for tracheostomy in patients with serious symptoms who had to undergo a tracheostomy upon admittance ${ }^{\{39\}}$. In patients with less severe symptoms also a compromise must be made between retaining voice quality and restoring an adequate 
translaryngeal pathway, but without having to take into account the necessity of a preoperative tracheostomy:

Some evidence of the safefy and effectiveness of endoscopic laser arytenoidectomy can be inferred from a review by Crockett and Reynolds, published in 1990 101 . These authors reviewed an early series of 11 patients with bilateral vocal cord paralysis by Ossoff and colleagues, of whom 10 were successfully treated with "minimal voice loss". This study is probably the best of all early case-series (about 10 in total) which individually never ncluded more than 35 patients. As reviewed by Crumley (1993) (118i $^{1 / 2}$ patients included in these series all suffered from severe glottic airway obstruction secondary to bilateral laryngeal paralysis and/or $g$ lottic stenosis. As a consequence, the authors of these studies primarily focused on the possibility of tracheotomy decannulation following the procedure, at the expense of attention for phonatory function. As treatment in terms of the possibility of postoperative decannulation was usually successful, the more recent literature consists of patient-series with less severe symptoms. In these series, more attention is paid to postoperative phonatory function.

According to Eckel et al. (1994), endoscopic laser arytenoidectomy is a challenge to the laryngologist, while conventional microlaryngoscopic arytenoidectomy is even more demanding ${ }^{i \text {. }}$. Besides devising treatment procedures for patients with relatively mild symptoms, the technical difficulty of the procedure may also have been a stimulus to develop alternatives, including (posterior) cordotomy/cordectomy and medial arytenoidectomy ${ }^{11.16 .351}$ : In (posterior) cordotomy, a $\mathrm{CO}_{2}$ or a KTP laser is used to incise the vocal fold at the junction of the vocal process. In medial arytenoidectomy one of these lasers is used to shave the medial surface of the arytenoid body ${ }^{\text {[3] }}$ : Crockett and Reynolds (1990) reviewed a case-series of 6 patients by Prasad, in whom posterior cordotomy was successfully applied with good preservation of speech ${ }^{10 \mid}$. Eckel and colleagues (1994) compared results of 18 patients treated with laser cordectomy with 10 patients who were treated with laser arytenoidectomy ${ }^{\text {ing }}$. Treatment was applied under intravenous anesthesia. After extubation, patients in both groups were observed for 4 to 24 hours in an intensive care unit. Patients were usually kept in the hospital for 7 days after surgery. Six of the patients needed an indwelling tracheostomy on admission, while none of the patients required a tracheostomy for endolaryngeal intervention. Both treatment modalities were assessed for respiratory and phonatory function preoperatively and 6 and 12 months postoperatively. In both patient groups these functions improved substantially, but there were no differences in improvement between the groups. Within each group preoperative and postoperative phonatory results varied considerably. Subclinical aspiration was not encountered in any of the patients treated with laser cordectomy, whereas this happened in 5 of the 10 patients who underwent laser arytenoidectomy. Cordectomy was also considered easier and faster to perform than arytenoidectomy. Results in both groups were judged as encouraging ${ }^{|16|}$.

In summary, some preliminary evidence indicates that endoscopic procedures including a laser may be helpful in avoiding or delaying a total arytenoidectomy in patients with bilateral true vocal cord paralysis.

These procedures are still in development and it is as yet unclear which type of procedure offers best results for each of the two indications. A totall arytenoidectomy may follow any of the endoscopic (laser) techniques if this is considered to be required to further improve the glottic airway. This will, however, (further) decrease the quality of voice. According to Ossoff et al. (1994), when a microsurgical total arytenoidectomy is required, again lasers can be used as part of the procedure ${ }^{|35|}$. Overall, these results are regarded as promising.

\section{Recurrent respiratory papillomatosis}

Recurrent respiratory papillomatosis is a condition which often presents in childhood, with sometimes a continuation in adult-life. In this condition, the laryngeal inlet becomes filled with frondlike papillomas which cause severe voice problems and, more particularly in children, airway obstruction. No reliable method has been found to prevent recurrence. With conventional surgery, papillomas are removed as often as necessary to preserve the voice. Surgical removal causes 
significant bleeding, and prevents accurate and complete removal of the papillomas. If the diatherny loop is used to control the hemorthage, significant thermal damage to the tissues can result with increased scarring and contracture ${ }^{[7]}$. When using the $\mathrm{CO}_{2}$ laser all papillomas can be removed without significant bleeding and with minimal damage to the normal laryngeal structures $^{[7,35]}$. For this reason the $\mathrm{CO}_{2}$ laser has become the standard treatment modality for this condition $^{10,35]}$. In a series reported and reviewed by Ossoff et al. (1994), 22 patients successfully underwent $105 \mathrm{CO}_{2}$ laser excisions without intraoperative complications. Two patients suffered delayed complications $(9.1 \%)$. In one case a slight true vocal vold scarring occurred and in another case a small posterior web developed. These results compared favourably to other published complication rates of series of laser-treated patients of 28.7 and $45 \%$, respectively. These rates are historical and unlikely to be repeated because in the mean time a microsubglottiscope has been developed, facilitating $\mathrm{CO}_{2}$ laser treatment ${ }^{(35)}$. Ossoff and colleagues advocate extensive treatment of both vocal cords in one session with a standard repeat operation about six. weeks. later. Further treatment intervals can then be based on the rate of reexpression ${ }^{|35|}$ "In the past, there has been considerable debate on optimal laser treatment scheduling in these patients ${ }^{10 !}$. Conflicting results have been reported on the presence of human papilloma virus in the laser plume. To lessen the potential risk of exposure the use of adequate smoke evacuation and wearing of high filtration face masks is recommended ${ }^{(35 !)}$.

In conclusion, despite shortcomings in documentation of case-series and low numbers of patients the use of llasers for this indication seems to be established by clinical experience. Considering the morbidity associated with conventional surgery, and the potential difficulties arising when retreatment of scarred tissue is needed laser treatment may potentially be cost-effective in avoiding airway obstruction and maintaining voice quality.

\section{Vocal cord polypi, nodules, granulomas and generalized edema of the mucosa}

Vocal cord polypi (also called polypoid degeneration), nodules, gramulomas and generalized edema of the mucosa will cause varying degrees of hoarseness of the voice $e^{[7,35]}$. The treatment of patients with polypoid degeneration includes preoperative and postoperative voice therapy. Also the patient is advised to stop any laryngeal irritants such as smoking products and alcoholic beverages. Only when conservative management fails surgical excision is indicated. Both the $\mathrm{CO}_{2}$ laser and $a$ microsickel knife are used for this purpose. Normal voice can be expected after a period of 6-8 weeks. In this period, the patient will have a weak, breathy voice. There is no evidence to suggest that efficacy of laser surgery is greater than that of conventional techniques ${ }^{[i, 35 !}$.

Voice therapy is the treatment of choice for nodules ${ }^{i 35]}$. If conservative management fails, microsurgical cold knife techniques are used to remove the nodules. Although the $\mathrm{CO}_{2}$ laser can be used, Ossoff et al. (1994)point to the potential danger of thermal injury to surrounding tissues ${ }^{[26]}$. If dysphonia or airway compromise is present resulting from granuloma of the larynx, surgical excision is indicated, either by conventional microlaryngeal or laser techniques. No difference in recurrence rates of granulomas have been reported using either of these techniques ${ }^{[35]}$

Generalized edema of the vocal cords develops from chronic infection, smoking, and voice abuse. A wide range of conventional techniques have been described for this condition, which almost all have been modified to utilize the laser. The voice returns to normal in most patients, and the quality of the voice can be maintained if causative factors are removed. Case-series of laser-treated patients by Yates \& Dedio and Kolle, reviewed by Carruth in 1991, reported results somewhat better than those achieved by conventional techniques. Carruth concluded that short-term laser treatment may be marginally more effective than conventional treatment of these patients ${ }^{[i]}$.

Overall, for these indications there are no well-documented differences in effectiveness between laser treatment and conventional treatment. Perhaps with an exception for treatment of nodules, lasers are established for these indications based on clinical experience and used as a virtually bloodless scalpel. For these indications, the cost-effectiveness of lasers is questionable. Due to a lack of data, however, no explicit judgment can be made. 


\subsubsection{Carcinoma of the larynx}

Patients with $\Gamma 1$ glottic squamous cell carcinoma have been treated with external-beam radiation, laryngofissure and cordectomy (hemilaryngectomy), and endoscopic excision with or without lasers. External beam radiation is the most commonly prescribed treatment modality for patients with early glottic cancer. This position is challenged by the advent of lasers. According to soma authors ${ }^{[35]}$ hemilanyngectomy is most commonly reserved for patients with large or bulky cancers involving a mobile true vocal cord. However, other authors state that surgery is still commonty applied in all categories of patients ${ }^{[20)}$. In cases with deep cancer invasion, total laryngectomy may be necessary to achieve local control. Besides local control (cure) rates, in recent literature the quality of the voice is becoming an increasingly important outcome measure. In these articles: subjective measures are usually complemented with objective parameters ${ }^{191}$ : In generall, the quality of the voice is best preserved if surgery can be avoided or minimized. Patients who undergo total laryngectomy loose their voice. Some degree of voice rehabilitation can be achieved in these patients using one of a variety of techniques, e.g. by insertion of a prosthesis ${ }^{|30|}$. In the discussion a distinction is made between early (stage I and II) wersus late or advanced (stage III and IV) glottic and supraglottic carcinoma.

\section{Early glottic and supraglottic carcinoma}

In 1975 Strong, as reviewed both by Carruth in 1991 and Ossoff et al. in 1994, reported no recurrences in the first series of 11 patients with $\mathrm{T} 1 \mathrm{~g}$ glotic carcinoma treated with $\mathrm{CO}_{2}$ laser ${ }^{17,34 y_{1}}$, In the procedures, the lesions were excised with appropriate margins and biopsies were taken from the edge of the defect and studied by frozen section. The removal of tumour is continued, as possible, until the margins of the defect are tumour free. With this technique, called excisional biopsy, diagnostic examination, biopsy and treatment are carried out with one anesthetic ${ }^{[7]}$

In 1978, Ossoff et al began using the $\mathrm{CO}_{2}$ laser to manage patients with early mid-cordial T1 glotic tumours. Of 25 selected previously untreated patients, 17 were managed by $\mathrm{CO}_{2}$ laser excision alone; in 6 patients this was followed by radiation and in 2 patients this was followed by partial. laryngectomy. At three year follow-up 24 patients were alive and free of disease $(96 \%)$. One patient died of both local and regional recurrence. The authors concluded that endoscopic $\mathrm{CO}_{2}$ laser excision of midcordial micro- and minicancers can be curative, with success rates equal to radiation therapy and laryngofissure with cordectomy ${ }^{[35]}$. However, the cases were selected and the number of cases was small.

In a more recent study, Cragle et al.(1993) reviewed the results of 22 radiotherapy studies includim 3,357 patients with T1 glottic tumours published in the period 1975-1987 $7^{[9]}$. A comparison was made with the results of 7 studies including 142 patients with $T 1$ glottic cancers who received $\mathrm{CO}_{2}$ laser cordectomy. These studies, excluding the two early studies cited above but including an often cited study by Blakeslee el al. in 1984, were published in the period 1984 - 1988. A limited costanalysis was also included (see below). Average follow-up of these patients was 24 months, ranging from 5 to 58 months. Data on average length and range of follow-up of patients in the radiotherapy studies were not provided. Local control after initial treatment was achieved in $87.5 \%$ of patients receiving radiotherapy and in $87.3 \%$ of the patients receiving laser treatment. In patients where initial treatment falled, one of a variety of salvage procedures was performed, resulting in an ultimate control rate of $94.8 \%$ after radiotherapy and $98.6 \%$ after laser treatment. In only $2 \%$ of the patients where laser treatment failed a salvage total laryngectomy had to be performed, compared to $11 \%$ of patients in 5 of the radiatherapy studies. Based on these data, the main conclusion of the authors was that cure rates of both treatment modalities are equivalent ${ }^{9 !}$. Of course, selection bias may play a role here. Therefore, these results should be interpreted with caution.

Davis and Steiner (1994) reported abstracted results of $\mathrm{CO}_{2}$ laser excision in 84 patients with stage II glottic cancer ${ }^{42 \|}$. Patients were subdivided in three groups. The 34 patients in group 1 had T2 glottic cancer with normal cord mobility. These patients underwent $\mathrm{CO}_{2}$ laser excision only. With a five year follow-up, only 2 patients developed local recurrence in this group, giving a local control 
rate of $94 \%$. Five-year survival was $78 \%$ with all deaths coming from either second primary cancers or intercurrent disease. Patients in group 2 and 3 had carcinoma in situ with microinvasion of the vocal cords. The 25 patients in group 2 received $\mathrm{CO}_{2}$ laser therapy anlly whereas the 23 patients in group 3 received endoscopic $\mathrm{CO}_{2}$ laser microsurgery followed by postoperative radiation. At fiveyear follow-up, local recurrence was $32 \%$ in group $2(n=8)$. Two patients $(8 \%)$ underwent salvage laryngectomy whereas 6 patients ( $24 \%$ ) underwent secondary laser procedures in an attempt to gain local control. Ultimate local control at five years was achieved in 23 patients $(92 \%)$. Five year survival was $80 \%$ (20 patients) with three deaths due to second primary cancer and two deaths due to uncontrolled laryngeal cancer. At 3.5 years follow-up in group 3, local recurrence was noticed in 5 patients $(22 \%)$, while regional and distant metastases occurred in $2(9 \%)$ and $3(13 \%)$ patients; respectively. Ultimate local control was achieved in $96 \%$ of the patients, with three patients ( $13 \%$ ) undergoing salvage total laryngectomy: Survival at 3.5 years follow-up was $83 \%$. Twenty percent of the total group of 84 patients required postoperative feeding tubes for a period of 1 to 6 weeks. No data on woice quality were presented. Also, a comparison with other patient-series was lacking $^{[12]}$. These results, while not allowing a valid comparison between group 2 and 3 , are not as good as those achieved in patients with T1 glottic carcinoma.

Rudert and Werner (1995) presented results of transoral $\mathrm{CO}_{2}$ laser resection of 114 patients with early glottic cancer treated in the period 1979-1993 (group 1), and of 47 patients with variously sized supraglottic cancer (group 2) treated in the period 1981-1993 $3^{[4 \mid}$. Patients in group | were treated curatively for Tis to T2 tumours of the vocal cord. The average age of these patients was 66.4 years and average follow-up was 40 months, ranging from 13 months to 14.3 years. Nine recurrences $(7.9 \%$ ) developed which were treated with good results by irradiation ( 3 cases; $2.6 \%$ ) and repeat laser surgery ( 3 cases; $2.6 \%$ ). Salvage laryngectomies were performed in 3 patients $(2.6 \%)$. These patients were free of recurrences at the time of writing the article. None of the patients had died of a tumour-related cause.

of the 47 patients in group 11,17 were treated with laser surgery for tumour debulking to avoid tracheostomy before laryngectomy. Thirty patients were treated for cure by laser surgery, with 11 patients requiring neck dissections. In 21 cases, additional radiotherapy was applied. Average follow-up of these patients was 24 months. Eighteen patients $(60 \%)$ were alive without evidence for local or metastatic disease. Six patients $(20 \%)$ had died, but without evidence of local disease. Two patients $(6.7 \%)$ died of secondary malignancies without evidence of local recurrence and 3 patients $(10 \%)$ died because of their tumours. One patient was apparently lost to follow-up. The authors concluded that laser surgical resection for vocal cord carcinomas larger than T1a should be included in the repertoire of therapeutic measures for laryngeal carcinoma. Although a direct comparison is lacking, they feel that the advantages of laser surgery outweigh what they feel is the only advantage of irradiation, which is a slightly better voice. Although a discussion of the 17 patients in group II who underwent laser tumour debulking is lacking, the authors also arrive at a favourable conclusion for this procedure ${ }^{[4 * 1]}$.

In a review of endoscopic $\mathrm{CO}_{2}$ laser management of supraglottic cancer, Zeitels and Davis $(1995)^{|4 a|}$ state that laser treatment can be successfully applied in small-sized lesions especially in the suprahyoid epiglottis, the aryepiglottic-and the vestibular fold. Small lesions in the first two listed sites often show early invasion of the paraglottic space, placing the neck at risk for occult metastasis. If this is the case, postoperative radiotherapy and/or a neck dissection is applied. In an earlier study by these authors, which was referred to in the review, 19 patients underwent endoscopic resection without postoperative radiotherapy for $\mathrm{T} 1$ or $\mathrm{T} 2$ lesions of the three supraglottic sites referred to above. Six of the patients $(32 \%)$, in wham treatment of larynx lesions was successful, had a short follow-up because of incurable primary cancers at other sites, most frequently in the lung. The remaining 13 patients were followed-up for a median period of 53 months, with a minimum of 24 months. Treatment for larynx lesions was successful without impairing swallowing function. One patient in this subgroup developed a metachronous neck recurrence, which was successfully treated with a neck dissection and radiotherapy. These data suggest that, according to these authors, endoscopic excision of small supraglottic cancers can 
be performed during the staging endoscopy. The transoral resection is both diagnostic and therapeutic ${ }^{[4]}$

In summary, results with laser surgery in terms of cure rates for early glottic and supraglottic carcinoma are at least promising and for stage llesions laser therapy may be on the brink of being established by clinical experience. But how about voice quality? Carruth (1991) and Ossoff et al: (1994) state that local control rates with radiotherapy vary from $80-90 \%$ with few immediate complications, and preservation of a normal volce ${ }^{7 / 35}$. Carruth recognized that laser therapy on the one hand spares the patient the short-term morbidity and long-term risk of a course of radiotherap but on the other hand, if a portion of the vocall cords is excised (with lasers), the voice will not be normal ${ }^{71}$. This suggests that a complicated trade-off may exist between woice quality and treatment morbidity. Other authors are also of the opinion that the quality of the voice is better after radiotherapy than after laser surgery ${ }^{[11]}$. However, there is no universal agreement on this matter. For example, as part of their study Cragle et al. (1993) subjectively and objectively assessed voice quality of 11 patients who recelved laser treatment for $T 1$ glottic carcinoma and compared these results with voice quality after radiotherapy for the same indication in 20 patients as published in the literature ${ }^{[9]}$. It was concluded that voice quality after laser therapy was just as good as after radiotherapy.

McGuirt et al. (1994) compared subjective and objective quality of the voice in 13 men after radiotherapy with the quality of the voice of 11 men who received outpatient laser treatment of either T1 or Tis vocal cord carcinoma ${ }^{[31]}$. It was concluded that there were no significant differences between both groups as assessed 6 months after treatment. Quoting literature claiming that laser treatment offers at least equal cure rates to radiation therapy, thesie authors felt that radiation therapy should be reserved for possible future needs ${ }^{[34]}$. Mahieu et al. (1994) arrived at a similar conclusion after analyzing cure rates and voice quality of 31 patients who received $\mathrm{CO}_{2}$ laser treatment of early glottic carcinoma ${ }^{[29 !}$. These authors, recognizing that this claim is based on limited data, called for prospective comparative studies of these modalities ${ }^{[29]}$. One may then envisage a role for laser therapy as part of a strategy aimed at curing patients while preserving voice quality as long and as much as possible. Surgery would be avoided or (further) delayed.

The combination of data allows for the conclusion that laser therapy is established by clinical experience for this indication. Well-designed, multi-institutional studies seem justified to assess laser therapy versus radiotherapy both in terms of morbidity, mortality, quality of the voice (as part of quality of life) and costs at the same time in patients with early vocal cord carcinoma.

\section{Cost-effectiveness}

Based on comparisons of charges of only three patients, it was suggested that the initial dired costs of laser therapy (based on a one-night stay in hospital) would be much lower than the initial direct costs of radiotherapy ( $\$ 5945$ versus US $\$ 14150$, respectively) ) $^{[s]}$. In another study ${ }_{n}$ again based on charges and using many assumptions, it was suggested that the direct costs of outpatient laser therapy for patients with T1 glottic carcinoma (including salvage procedures) would be U $\$ 15000$, compared to $\$ 36000$ for radiotherapy (also including salvage procedures) and $\$ 39000$ for hemilaryngectomy, respectively ${ }^{[21}$. However, due to major deficiencies, these data can only be regarded as speculative. It can be concluded that, permitted laser therapy was demonstrated to be at least as effective as radiotherapy, it has the potential to be cost-effective compared with radiotherapy and, even more likely, also with hemilaryngectomy.

\section{Advanced lesions}

In either large and/or advanced glottic or supraglottic lesions $\mathrm{CO}_{2}$ laser surgery may be beneficial preceding additional treatment including radiotherapy, chemotherapy or (hemi)laryngectomy ${ }^{[25,41,48 \text {. }}$ In these cases $\mathrm{CO}_{2}$ laser surgery can be applied for tumor debulking in order to reestablish a safe airway. This avoids the need for a postoperative artificial airway or emergency laryngectomy, in particular in patients with large exophytic supraglottic cancer who present with stridor and airway compromise. Hospitalization for these procedures in these patients is usually 1 to 3 days. More 
extensive resections may predispose to aspiration from several days to 6 weeks and patients may require nasogastric feeding during this time, according to Zeitels and Davis (1995) in a review Occasionally, small case-series are reported in which laser debulking of obstructing laryngeal tumours is successfully applied in the majority of patients ${ }^{403}$. These studies do not allow a firm judgment on the safety and effectiveness of the procedure.

It has previously been demonstrated that some patients with advanced laryngeal cancer will choose the lower cure rates of radiotherapy in preference to surgery because of preservation of the voice and better quality of life. In this special category of patients, laser surgery has more or less speculatively been recognized as having the potential to be quite cost-effective, perhaps because it could improve results obtained with radiotherapy ${ }^{[2]}$. However, no special studies involving lasers have been oriented to these patients.

\subsection{Pharyngeal disease}

\subsubsection{Pharyngeal pouch}

A pharyngeal pouch (or hypopharyngeall diverticulum or Zenke's diverticulum) can be found immediately above the esophageal inlet on the posterior wall of the distal part of the hypopharynx. A pharyngeal pouch is twice as common in men than in women. The vast majority of patients is at least 40 years of age. Symptoms are dysphagia, foetor oris, gurgling noises in the neck on swallowing, coughing and repeated infections of the bronchi due to aspiration. The duration of symptoms may vary from a few weeks to many years ${ }^{[3]]}$. In 1960, Dohlman described a new endoscopic procedure for this condition and although reports were published presenting good results with Dohlman's technique, there was a tendency away from endoscopic techniques in favour of an external surgical procedure because it was thought that the end results would be superior, despite a significantly greater morbidity and prolonged stay in hospital ${ }^{[3,3]}$. In the external surgical procedure the pouch is either excised or invaginated into the pharynx. As part of the procedure, the cricopharyngeus muscle (the upper esophageal sphincter) is divided surgically in order to reduce the risk of recurrence ${ }^{[7,37]}$.

Van Overbeek et al. (1988), started endoscopic treatment of patients with pharyngeal pouch in 1964 , using and later modifying Dohlman's technique ${ }^{[37]}$. In 1981, using a specially designed scope and an operation microscope, the procedure could be performed using microsurgical techniques. Besides electrocoagulation by means of insulated microsurgical instruments, this development also allowed to use the $\mathrm{CO}_{2}$ laser to divide the tissue bridge between the esophagus and diverticulum. In the course of time, based on subjective impressions, these otolaryngologists started to use the $\mathrm{CO}_{2}$ laser as the preferred treatment modality. To investigate the value of endoscopic (laser) treatment, they retrospectively analysed 377 patients that had been treated endoscopically in the period 1964-1984 with a follow-up of at least 1 year. Electrocoagulation was applied in 308 patients and $\mathrm{CO}_{2}$ laser treatment was applied in 69 patients. Of the total number of patients, $339(90 \%)$ were highly satisfied and $36(9.5 \%)$ were fairly satisfied with the results obtained. One patient in the electrocoagulation group died two days postoperative by cardiac failure whereas no patients died in the laser group. In the electrocoagulation group complications included 5 cases of mediastinitis $((1.6 \%)$, cured by conservative therapy), 4 cases of hemorrhage $(1.3 \%)$, one esophageal fistula $(0.03 \%)$ and a tendency to stenosis in 8 cases $(2.6 \%)$. In the laser group there was one case of mediastinitis (treated by mediastinotomy) and hemorrhage in one case $(1.4 \%)$. Radiological follow-up revealed no essential difference in the final results between the patients treated with the electrocoagulation technique or with the $\mathrm{CO}_{2}$ laser. The authors concluded that $\mathrm{CO}_{2}$ laser microsurgery is an improvement on the original Dohlman's method ${ }^{[37]}$.

Although this is only a retrospective historical comparison based on experience in one center and data on treatment efficacy of the conventional procedure have not been considered, one can speculate that there are advantages of the microsurgical procedure compared to the external 
surgical procedure. Despite this potential only marginal attention is paid to this indication in reviews

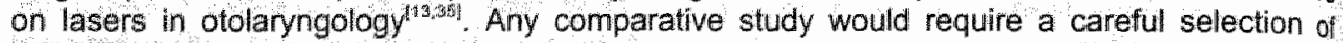
pafients, taking many patient characteristics into account. For example, laser treatment is applied under general anesthesia with intubation and general anesthesia is also preferred with the electrocoagulation technilque. But in patierits in whom there is a contraindication to general anesthesia, the electrocoagulation technique is performed using only local anesthesia ${ }^{\{3]}$. Finally, due to a complete lack of data, no informed judgment can be made on the potential cost. effectiveness of this laser procedure.

\subsubsection{Snoring and other sleep-related breathing disorders}

The $\mathrm{CO}_{2}$ laser was introduced for treatment of snoring and other sleep related breathing disorders in France in the late eighties. A few years later the $\mathrm{CO}_{2}$ laser procedure, called laser-assisted uvulopalatoplasty (LAUP), was introduced in the US. In this country LAUP has continued to grow in popularity, with more than 500 otolaryngologists offering the procedure in 1994 to increasing numbers of patients ${ }^{\mid 17,35,45 !}$.

Snoring is a loud recurrent breath sound during sleep that occurs on inspiration. It is produced by the vibration of soft tissue, including the tonsillar pillars, soft palate, uvula, pharyngeal folds and other structures of the oropharyngeal airway. Epidemiological studies indicate that approximately $19 \%$ of adults describe themselves as habitual snorers. Snoring is about twice as common in males than in females and its incidence increases with age in both sexes ${ }^{[47}$. Snoring may result in significant sleep disruption or fragmentation, which may lead to daytime fatigue, sleepiness or occupational and safety risks caused by performance impairment. In addition, recent data suggest that snoring is associated with a number of health risks. For example, it has been shown that habitual snorers are as much as 10.3 times more likely to suffer a stroke than those who never or occasionally snore ${ }^{[47]}$. Most snorers do not hear themselves, although they can generate sounds as loud as 85 decibels (dB). This may wreck relationships, disrupt families, and cause problems with neighbours, on holiday and in hotels ${ }^{\mid 199}$. Perhaps the most significant health risk of snoring is its association with upper airway resistance syndrome (UARS) and obstructive sleep apneu syndrome (OSAS) ${ }^{\mid 24 !}$. Patients with UARS show modest, short reductions in airflow during sleep. The OSAS patient experiences apneic episodes, defined as the cessation of breathing for at least 10 seconds, and hypopneic episodes, defined as a $50 \%$ reduction in respiratory flow with oxygen $\left(\mathrm{O}_{2}\right)$ desaturation. The patient may or may not awaken during these episodes. OSAS is thought to affect between $1 \%$ and $10 \%$ of the general population, predominantly in men, with a pasitive age dependent incidence in both sexes ${ }^{|47|}$. UARS and OSAS have a lot of symptoms in common such as memory difficulties, attention/concentration deficits, behavioural and affective changes, impotence and loss of alertness ${ }^{[23,24 !}$. The most important health risk of OSAS is increased mortality. In a 9-year longitudinal study of untreated patients with OSAS it was found that those with 20 or more apneic events per hour of sleep had a high mortality rate, with a cumulative probability of survival of only 0.63 , whereas those with fewer than 20 events had a cumulative probability of survival of $0.96^{[24,4 n}$.

Treatment of snoring, upper airway respiratory syndrome and obstructive sleep apneu syndrome begins by eliminating or reducing causative or exacerbating factors. The traditional plan involves weight loss, which is an important risk factor, alteration of sleeping position from supine (face-up) to prone (face-down), and avoidance of sedatives, smoking, and alcohol. If this does not result in sufficient improvements additional treatment is advised ${ }^{\mid 23\}}$. The US Patent and Trademark Office has registered approximately 350 antisnoring devices. Patient compliance is often a problem because many patients find these devices uncomfortable $e^{117.24 !}$. Medical management is often helpful if nasal allergies or endocrine problems underlie snoring problems ${ }^{[24]}$. If surgical management is deemed necessary in patients who snore, until recently the treatment of choice was uvulopalatopharyngoplasty (UPPP). This is a single-stage maximal removal of the soft palate, tonsills, and uvula, performed with conventional surgical equipment ${ }^{[23]}$. UPPP is usually an inpatient 
procedure, requiring approximately one hour of operating room time and general anesthesia. Average hospital stays vary from 2.8 to 5.5 days ${ }^{[17 ?}$ ?

In patients with OSAS, nasal continuous airway pressure (CPAP) has been the mainstream therapy in cases where conservative therapy has failed. This noninvasive treatment modality requires patients to wear a self-sealing, light weight mask atlached to a bedside positive pressuregenerating machine. In case of insufficient results UPPP is performed; sometimes extended with one or more additional surgical procedures depending on OSAS severity and anatomical conditions. Examples are mandibular osteotomy, tongue base surgery and nasal reconstruction ${ }^{\text {[3is! }}$ According to ECRI, UPPP is the most commonly performed surgical procedure for the treatment of habitual snoring and OSAS ${ }^{[17}$. What is the role of lasers here?

In patients with loud, habitual grade II or grade III snoring which is disruptive to others, and in patients with OSAS, the position of UPPP is challenged by the proponents of laser-assisted uvulopalatoplasty (LAUP) ${ }^{123,24 !}$. In LAUP, a $\mathrm{cW} \mathrm{CO}_{2}$ laser with special attachments is used to vaporize the uvula and the free margins of the palate in a series of procedures until the patient and/or his/her partner is satisfied or when the snoring is gone ${ }^{(35)}$. In patients with grade II and grade III snoring (with or without UARS or mild OSAS), LAUP can be performed in an office setting under local anesthesia. Treatment is usually completed in two to three sessions. In patients with severe OSAS LAUP should be applied in a hospital setting using general anesthesia. In these patients LAUP may be combined with laser-assisted tonsillectomy (LAT). This is then called extended LAUP, involving resection of the soft palate; Uvula, the palatine arches and the palatine tonsills: An extended LAUP may consist of four to five treatments, with at least five weeks between cansecutive sessions ${ }^{[35]}$.

In 1995, ECRI published an assessment of LALP versus UPPP in patients with severe snoring or OSAS ${ }^{13}$. The assessment included publications and abstracts published in Eriglish up to February 1995. Three uncontrolled studies and four uncontrolled studies published as abstracts were identified, discussing results in patients with snoring treated with LAUP. These studies reported $81 \%$ to $100 \%$ reductions in snoring as rated subjectively (i.e. by patients). Ten UPPP studies (controlled and uncontrolled) reported similar success rates, ranging from 62 to $100 \%$. In the only controlled study, published by Carenfelt in 1991,85\% of 60 LAUP-and $89 \%$ of 36 UPPP-treated patients reported improvement in snoring, and $71 \%$ of LAUP and $77 \%$ of UPPP patients reported improvement in daytime somnolence ${ }^{[17]}$. There were no significant postoperative complications among LAUP-treated patient in the studies discussed in the ECRI-report. Postoperative complications after UPPP reported in the literature include: temporary postoperative nasal regurgitation in 20 to $60 \%$ of patients, hemorrhage in $2 \%$ and permanent velopharyngeal insufficiency in $0.5 \%$. Long-term complications include voice/resonance changes in a minority of patients and a foreign body sensation of increased thick mucus in the back of the throat ${ }^{[23\}}$. Based on their findings, ECRI concluded that LAUP appears to be as effective as UPPP for the treatment of snoring. It was immediately addled that both the LAUP and UPPP studies which were included in the analysis had similar design and reporting flaws, which does not allow a definite conclusion ${ }^{[1 /]}$. With respect to LAUP in patients with OSAS, ECRI found one study and several abstracts and meeting presentations. It was concluded that there are insufficient data to assess whether LAUP can be effective in treatment of patients with OSAS ${ }^{[17}$.

To illustrate the difficulty of interpreting existing data, the largest study published to date will be presented in more detail. In late 1994. Krespi et al. published results of 450 patients who underwent a total of more than 1,000 LAUP procedures in the period January 1993 - May $1994^{\mid 23 !}$. Four hundred twenty-three patients underwent LAUP. Three hundred fifty-one (78\%) were deemed suitable for in-office LAUP, $72(16 \%)$ were admitted to the hospital for extended LAUP and $27(6 \%)$ were not surgical candidates. In total, in about $30 \%$ of patients LAUP was combined with other upper airway procedures including submucous resection of the septum, laser turbinectomy, LAT, or laser lingual tonsillectomy. In addition, all patients with severe sleep apneu were treated with CPAP as an adjunct to surgical intervention in the hospital. 
Of the total group, 118 were simple snorers, 42 had UARS, 155 had mild OSAS, 65 had moderate OSAS, and 43 had severe OSAS. The quality of sleep was improved in $28 \%$, slightly improved in $39 \%$ and $37 \%$ experienced no change. The overall energy level of the patients was improved in $42 \%$, unchanged in $49 \%$, and there was evidence of deterioration of the energy level in $9 \%$, which was attributed to weight gain. An improvement was found in a variety of reported symptoms including daytime alertness and sleeptime choking and gasping. A moderate to severe sore throat Was the major side-effect following LAUP. Very mild bleeding was encountered in approximately $2 \%$ of the patients. An overall success rate of $85 \%$ for reduction or elimination of snoring was reported ${ }^{\mid 23\}}$. Because of the aggregate level of data-analysis, the lack of objective measures and the combination of procedures, no firm conclusions can be drawn on the safety and effectiveness of LAUP for specific indications. At best, the evidence is promising on the short-term. Nevertheless, LAUP has been approved by the American Academy of Otolaryngoly-Head and Neck Surgery for the treatment of snoring. This contrasts with the American Sleep Disorders Association, who state that patients should understand that the risks, complications and efficacy of LAUP in treatment of snoring have not been objectively evaluated and that, to date, there is no printed documentation that LAUP is effective in the treatment of sleep apneu ${ }^{[17]}$

\section{Cost-effectiveness of LAUP versus UPPP}

There are no published studies on the cost-effectiveness of LAUP ${ }^{17}$. If the effectiveness of LAUP would be firmly established, it may have the potential to be cost-effective compared to UPPP for patients with snoring and/or mild to moderate OSAS because patients do not require hospitalization after LAUP and because the investment in the laser is rather modest, about US\$55 $000^{[17]}$. At present, according to ECRI, in the US the direct cost per case of successful snoring reduction with LAUP (based on charges) ranges from US\$3529 to $\$ 5882$, whereas these costs vary from US\$12 592 to $\$ 14960$ after UPPP. However; because LAUP can be applied as an office procedure, there may be a danger that the procedure also replaces conservative treatment modalities, which would dilute its overall potential for cost-effective use.

\subsection{Lesions of the mouth}

A wide range of benign, premalignant and malignant lesions can be excised or vaporized from the mouth or the tongue using the $\mathrm{CO}_{2}$ laser as a light scalpel. It appears certain that all operations that can be performed with the $\mathrm{CO}_{2}$ laser could also be performed using conventional instruments using the scalpel, scissors and cutting and coagulation diathermy. However, the use of the scalpel and scissors provokes significant bleeding in the highly vascularized tissues of this region and this necessitates hemostasis using diathermy which causes thermal damage to normal tissues. With the use of the cutting diathermy as a scalpel, there is a profound thermal damage to normal tissues $^{|7|}$. Although any systematic comparison with conventional procedures is lacking, stated advantages of laser surgery include extra precision with minimall blood loss during surgery and less post-operative pain and oedema. Especially reduced edema is thought to reduce the need for tracheotomy, which is often associated with scalpel excision of tissue in the oral cavity ${ }^{7,13,43 !}$.

Clinical experience is illustrated by means of a relatively recently published, large case-series. In 1994, Barak and colleagues reported their experience with the $\mathrm{CO}_{2}$ laser in ambulatory oral and maxillofacial surgery in the period 1986-1994, covering 1,150 procedures. A variety of benign, premalignant and malignant lesions were excised and/or vaporized with the laser. The vast majority of cases were treated under local anesthesia, in the remainder of patients no anesthesia was needed. Benign oral lesions comprised more than 40 percent of all cases, with fibroma covering about half of these cases. Other indications in this category included: papilloma $(9.3 \%)$, condyloma $(4.6 \%)$, giant cell granuloma $(2.6 \%)$, pyogenic granuloma $(2.3 \%)$, lipoma $(3.0 \%)$, and nevi $(0.9 \%)$. Minimal bleeding was encountered. The lesions to be removed were under traction. Sutures were required in only 17 patients because of continuous oozing immediately after surgery. Inflammatory phenomena developed in only 17 cases and were eliminated by antibiotic and/or steroid therapy. 
The ability to perform accurate waporization or excision of superficial lesions of the oral cavily with hemostasis and minimal scarring and postoperative pain and edema, gives the $\mathrm{CO}_{2}$ laser a decided advantage over conventional modalities, according to these authors. Other indications where they successfully applied the $\mathrm{CO}_{2}$ laser include for example ankyloglossia, buccal frena, haemangioma and/or wascular proliferation, lymphangioma, lichen planus, leukoplakia and other premalignant mucosal lesions, mucocele, ranula and salivary gland stones ${ }^{i 3:}$ :

Other authors, including Schuller (1990) and Ossoff et al. (1994), point at the suitability of the $\mathrm{CO}_{2}$ laser to treat verrucous and superficial T1 carcinoma of the oral cavity: No clinical data on these procedures were provided ${ }^{[35]}$. Various authors point to the fact that although the $\mathrm{CO}_{2}$ laser is very good at vaporization of tissue, many of the lesions accurring in the oral cavity are very vascular with blood vessels somewhat larger than that capable of being sealed by the $\mathrm{CO}_{2}$ laser. For this reason, also lasers that allow deeper thermal coagulation, e.g. the Nd:YAG laser, have found applications in the oral cavity ${ }^{7,33,35\}}$. Overall, lasers, and especially the $\mathrm{CO}_{2}$ laser, seems to be established in treatment of a variety of indications in the oral cavity based on clinical experience.

\subsection{Tonsillitis}

In literature, palatine tonsils are distinguished from lingual tonsils. According to Ossoff et all. (1994), the $\mathrm{CO}_{2}$ laser is the laser of first choice for vaporization of lingual tonsils in case of recurrent tonsillitis or hypertrophy ${ }^{[35]}$. Other authors also report successfull laser treatment of lingual tonsils with KTP- or Ho:YAG lasers ${ }^{|i 3.45|}$. Ossoff and colleagues also list two indications for laser resection of palatine tonsils: recurrent tonsillitis and obstructive sleep apneu syndrome ${ }^{\mid 35]}$. Of these indications, chromic tonsillitis is the most common one for tonsillectomy, a procedure that inumbered 74,000 cases, mostly pediatric patients, in 1990 in the US ${ }^{[22]}$. For the resection of palatine tonsils Ossoff et al. prefer the KTP laser over the $\mathrm{CO}_{2}$ laser ${ }^{[35\}}$. Other authors, e.g. Dilkes (1994), are positive about both the KTP and the Ho:YAG laser for this indication ${ }^{[13]}$. How does this relate to published evidence, especially in comparison with conventional techniques?

Oas and Barteis (1990) reviewed two case-series of $\mathrm{CO}_{2}$ laser tonsillectomy published by Matinez et al. in 1987 and by Baron in $1987^{[33]}$. These case-series included 1,110 and 500 patients, respectively. In both series a postoperative haemorrhage rate of less than $2 \%$ was reported. This compares favourably to case-series of patients treated with conventional tonsillectomy, where the postoperative hemorrhage rate varies from 2 to $5 \%$. Besides a reduction in postoperative bloodloss, the authors noted other benefits of $\mathrm{CO}_{2}$ llaser excision: decreased postoperative pain and accelerated healing ${ }^{|33| \mid}$

Other early series reported promising results with the KTP laser, which is much easier to handle than the $\mathrm{CO}_{2}$ laser. Oas and Bartels (1990) included 32 patients in a study where one tonsil was removed with a KTP laser and the other was excised with standard dissection and snare techniques ${ }^{|33|}$. Allocation of each of two tonsils in each patients to either treatment was random. Patients were not informed which tonsils was removed with each treatment. Alf patients in both groups stayed at least overnight in hospital. Follow-up was one week. In one case, brisk bleeding occurred during laser treatment, which resulted in a shift to conventional techniques in finishing the procedure. This patient was excluded from the analysis. The average blood loss after laser tonsillectomy was $40 \mathrm{cc}$ versus $95 \mathrm{cc}$ on the contralateral side. There were no differences in healing in both groups. Postoperative pain immediately after operation was lower in the laser group in $83 \%$ of patients, but after three days about half of the patients reported more pain in the laser resected site, and at the end of the weak $63 \%$ of the patients felt more pain on the laser dissection site. The early reduction of postoperative pain in the laser group was attributed to sealing of peripheral small nerve endings, while a later increase of pain was attributed to secondarilly infection of necrotic proteinaceous debris left in the tonsillar fossa following laser tonsillectomy. In each group there was one case of mild bleeding. No other complications occurred in these series. The authors concluded that KTP laser tonsillectomy leads to reduced blood loss and less postoperative 
pain immediately after operation compared with conventionall tonsillectomy. However one week postoperatively, laser treated patients experience more pain than conventionally treated patients

Krespy and Ling (1994) presented preliminary evidence that a new $\mathrm{CO}_{2}$ laser procedure, tonsi cryptolysis, may be an improvement over both conventional tonsillectomy and laser tonsillectomy of palatine tonsils ${ }^{22 x}$ in the new procedure, using a $\mathrm{CO}_{2}$ laser with a special attachment, only the surface crypts of the palatine tonsils are ablated (cryptolysis). The tissue of the tonsills is conserved in this procedure. In a series of 120 patients with a median follow-up of 20 months, the procedure was pierformed in an ambulatory or office surgery setting in 110 patients $(92 \%)$. The procedure required either topical anesthesia, local anesthesia complemented with oral anesthesia, or general anesthesia. 95 Patients $(80 \%)$ reported total elimination of symptoms of their recurrent tonsilla inflammation, and 20 patients $(17 \%)$ reported only milld reduction of their symptoms. These patients underwent near total laser tonsillectomy. Only 5 patients $(4 \%)$ reported insufficient improvemen and underwent completion tonsillectomy using traditional methods under general anesthesia. There were no immediate postoperative complications in any of these patients. Postoperative pain was well-tolerated with administration of oral analgesics ${ }^{|22|}$.

These results of laser tonsillectomy are promising, compared with conventional tonsillectomy. Oas and Bartels (1990) hypothesized that laser tonsillectomy, due to low postoperative pain immediate after treatment, has the potential to be performed as an outpatient procedure ${ }^{[331}$. Besides developments in laser tonsillectomy, as reported e.g. by Krespi and Ling (1994), there are also developments in conventional tonsillectomy. For example; de Carpentier and Timms (1994) reported excellent preliminary results in 70 patients applying a microscopic tonsillectomy technique with a bipolar diathermy forceps ${ }^{|i|}$. Besides these technological developments, it should be noticed that the indications for tonsillectomy are far from clear (Chapter $2^{[42]}$ ). As a consequence, better documentation of the indications for tonsil excision is necessary, for example by means of RCTs with a 'do-nothing' arm as part of the treatment strategy. In those cases where treatment is beneficial using commonly employed techniques, RCTs could be organized comparing these techniques with promising new modifications and/or laser techniques.

\subsection{Tongue lesions}

Carruth (1991) reported a series of 100 patients in whom substantial benign or premalignant tongue lesions were resected by using the $\mathrm{CO}_{2}$ laser. None of the defects needed to be sutured or grafted and the residual muscle hypertrophied, allowing the tongue to regain maximal residual function. The post-operative analgesia requirements were minimal and more than $80 \%$ of cases could be discharged on the first or second post-operative day ${ }^{(7)}$. According to Ossoff et al. (1994) tongue resections are especially amenable to the use of the lase ${ }^{35}$. Besides benign lesions, these authors list T1 and selected cases of T2 tongue carcinoma as suitable for $\mathrm{CO}_{2}$ laser treatment. referring to precise cutting, less pain and edema after treatment and lack of musculature artefact and motion, which they state is an explicit advantage of laser surgery over the electrocautery system in treatment of these patients. Unfortunately, these claimed advantages are nol substantiated. in 1991, as part of an EC project, the use of $\mathrm{CO}_{z}$ lasers for tongue resections was judged as established by clinical experience and potentially cost-effective compared to conventional surgery ${ }^{(2)}$. This may still hold true. Unfortunately, since then no new evidence on this subject has been published. 


\subsection{Otology}

\subsubsection{Stapedectomy}

Stapedectomy is a demanding middle ear procedure that is applied in treatment of otosclerosis. In this procedure the stapes is replaced by a prosthesis. In the past, usually a total stapedectomy was performed, involving a total removal of the stapes footplate. This technique is called large fenestra stapes surgery, or the large fenestra technique (LFT). Nowadays in most cases only a small hole is created in the stapes footplate, a technique which is referred to as the small fenestra technique (SFT) or stapedotomy. The aim of both procedures is a complete hearing recovery with a complication rate as low as possible. It has been suggested that the SFT provides better shortand long term results than the LFT. The 'gold-standard' for these procedures is a less than a 10 $\mathrm{dB}$ conductive deficit in $90 \%$ of cases. In practice this result, defining successful treatment, is achieved in 62 to $96 \%$ of patients. Total loss of hearing, the most serious complication, is rare after stapedotomy. Significant sensorineural hearing loss, defined as a greater than $10 \mathrm{~dB}$ drop in the speech range, occurs in $0.8-2.7 \%$ of patients ${ }^{[14,26,46]}$.

The first clinical application of lasers in otology was reported by Perkins, who used an argon laser coupled to a microscope in treatment of 11 patients with otosclerosis. In 1983, as reviewed by Carruth in 1991 and by Strunk and Quinn in 1993, Mc Ghee treated 100 patients with otosclerosis using an argon laser attached to a micromanipulator. He found that the postoperative course in the laser cases was rather smoother than after conventional surgery, but no significant benefit in terms of long term hearing preservation was found ${ }^{[7.46]}$. Nowadays, not only the argon laser but also the $\mathrm{CO}_{2}$ - and KTP laser are used in primary stapedotomy as an alternative to conventional surgery. Ossoff et al. (1994) reported that considerable controversy exists in the field as to which type of lasers is the most appropriate. Every type of laser has its own theoretical and practical advantages and disadvantages ${ }^{[35]}$. For example, Strunk and Quinn introduced the KTP laser in treatment of otosclerosis patients in $1986^{[46]}$. When evaluating their early experience with the KTP laser, the authors noticed an improvement of success rates from $82 \%$ using cold steel instruments to $86 \%$ when using the KTP laser. However, the technique in using the KTP laser was cumbersome. Therefore, in 1989, they started to use a handheld argon laser probe for stapedotomy, a so-called endo-otoprobe, which is more easy to use. They compared the results obtained with both lasers in primary stapedotomy in 50 consecutively treated patients, with twenty-five patients in each group. Either general or local anesthesia was used, in the latter case supplemented with intravenous sedation. The argon laser treated patients showed a similar success rate as the KTP laser treated group, $96 \%$. In the KTP laser treated group there was one patient who suffered total hearing loss (4\%). This was the only patient who was not routinely dlischarged the morning after operation. There were no complications in the argon laser treated group ${ }^{\text {abl }}$. A preliminary conclusion at this point is that argon laser stapedotomy may offer results that are somewhat better than obtained with the KTP laser. Using either of these lasers, results may be as good as those obtained with conventional stapedotomy.

\subsubsection{Revision stapes surgery in otosclerosis}

Patients who do not obtain optimal hearing improvement following an operation for otosclerosis and patients who later develop conductive hearing loss will be offered a revision procedure, in which the prosthesis is replaced. Revision surgical procedures are performed in a small percentage of patients. For example, in a Danish consecutive series of 4,000 patients, 186 patients were offered a revision procedure on average 8 years after the primary procedure. The most common clinical findings at revision in this series included adhesions in the middle ear, an acentric (displaced) prosthesis either with or without adhesions, and reobliteration of the oval window. Revision surgery was most successful in patients in whom the small fenestra technique was applied in the primary procedure ${ }^{\mid 3.8 !}$. The results of revision procedures are by far not as goad as those obtained after 
primary procedures. Success rates in revision surgery vary from 30 to $50 \%$. Eight to $33 \%$ of patients develop worse hearing, with profound deafness occurring in 2 to $15 \%$ of patients. Significant nerve deafness (sensorineural hearing loss), defined as a greater than $10 \mathrm{~dB}$ drop in the speech range, varies from 3 to $20 \%$. These data are derived from case-series of patients published in the seventies and eighties, as reviewed by Lesinski and Stein in 1992 and by Horn ei al, in $1994^{\mid 20.27 !}$. However, in more recent series, complication rates after revision surgery are only slightly higher than those after primary procedures ${ }^{\text {isa! }}$. What is the role of lasers here?

Lesinski and Stein (1992) reported results of $89 \mathrm{CO}_{2}$ laser revision surgery procedures (superpulsed mode) in 89 consecutively treated patients ${ }^{[27]}$. This is the largest case-series published to date. Nearly half of these patients had undergone one or more previous unsuccessful nonlaser attempts at stapedectomy revision before being referred to $\mathrm{CO}_{2}$ laser revision. Laser surgery was applied under local anesthesia combined with intravenous sedation. Mean follow-up was 40 months, with a range of 6 months to 7 years. Eighty-two patients (92\%) were explored because of significant conductive hearing loss (> 20 dB). In 63 patients $(71 \%)$ the stapedectomy prosthesis was displaced out of the oval window. Success, defined as a less than $10 \mathrm{~dB}$ conductive hearing deficit, was achieved in 63 patients $(71 \%)$. 18 Patients $(20 \%)$ had a conductive hearing deficit in the range of $11-20 \mathrm{~dB}$, whereas in 9 patients $(10 \%)$ revision surgery resulted in additional hearing loss. These fallures could almost always be related to abnormalities in the malleus or incus: There was one patient who exhibited significant sensorineural hearing loss in the speech range. The authors attributed these relatively good results to three advantages of laser surgery over conventional surgery: 1) improved diagnostic precision, 2) the ability to stabilize a new prosthesis in the center of the oval window, and 3 ) reduction of inner ear trauma ${ }^{[27]}$.

In 1994, Horn and colleagues published the first series of 30 patients who underwent revision stepedectomy in 32 ears using an argon laser with an endo-otoprobe ${ }^{|20|}$. Laser surgery was performed under local anesthesia with intravenous sedation. Follow-up of patients was at least 6 months. A conductive deficit less than $10 \mathrm{~dB}$ was achieved in $75 \%$ of cases. There was no loss of speech discrimination or sensorineural function in any of the patients nor did intraoperative or postoperative complications occur. The authors concluded that argon laser revision stapedectomy is a safe and effective alternative to mechanical revision stapes procedures ${ }^{201}$. According to Ossoff et al. (1994), the laser has shown itself to be possibly the best instrument to use in revision stapes procedures, significantly decreasing postoperative complications in this very difficult procedure ${ }^{[35 !}$.

In summary, considering both primary and revision laser stapedotomy, it can be concluded that the results achieved with this device are promising. Although laser stapedotomy may be advantageous compared to conventional surgery in primary procedures, it has not conclusively been shown that this results in better short- and long term outcomes. In addition, both laser techniques and conventional surgical techniques are continuously evolwing, hampering a direct comparison ${ }^{[4,38]}$. In revision surgery, lasers may have definite advantages over conventional surgery. However, due to the low numbers of patients treated the lack of long-term follow-up results and the absence of adequate comparative studies, a well-founded conclusion on the superiority of this laser procedure over conventional surgery can not yet be drawn.

\subsection{Tumours}

In particular $\mathrm{CO}_{2}$ lasers thave been used to remove a variety of tumours in otology and otoneurosurgery, including tumours of the external ear or external auditory meatus, and tumours in the cerebello-pontine angle in small numbers of patients with promising results ${ }^{17.33}$.

According to Carruth, in a 1991 review, no laser has proved to be of significant value over conventional surgery in the field of otology and oto-neurosurgery ${ }^{[7]}$. In more recent reviews of lasers in otolaryngology, relatively little attention is paid to the field of otology. In one of these reviews Dilkes (1994) states that laser surgery has failed to take off in this area ${ }^{\mid 12 \|}$. This may change with 
the development of endoscope-assisted surgeny in otology and oto-neurosurgery, which at present though is still in its infancy ${ }^{[(1)]}$.

\subsection{Nasal lesions}

\subsubsection{Introduction}

The use of lasers in treatment of nasal lesions has been stimulated with the introduction of sinus endoscopes in the mid 1980 s and by advances in computerized tomography scanning of the paranasal sinuses in the late 1980s. Lasers may have a role especially in treatment of epistaxis of the nasall cavity (nose bleeding). A distinction is made between epistaxis secondary to hereditary hemorrhagic telangiectasia (HHT) and intractable epistaxis of unknown origin. Other nasal lesions where lasers can be applied include polyps, concha bullosa and turbinate hypertrophy in treatment of chronic rhinitis ${ }^{[35]}$.

\subsubsection{Hereditary hemorrhagic telangiectasia of the nasal cavity}

Hereditary hemorrhagic telangiectasia $(\mathrm{HHT})$, or Osler-Weber-Rendu disease, is an autosamal dominant disorder. The homozygous form is uniformly fatal at an early age. The incidence is about 1 to 2 cases per 100,000 population. The first symptom in a majority of patients is recurrent epistaxis, the usual onset being in adolescence. Roughly $80 \%$ of symptomatic patients have epistaxis. Frequently, patients have other manifestations, reflecting the systemic nature of the disease. Cutaneous telangiectasias are found in over one half of the patients, usually on the cheeks, tongue, lip, hands, fingertips and feet (see Chapter 5 Lasers in Dermatology). Other structures that may contain these lesions include the gastric mucosa, vaginal mucosa, bladder, uterus, liver, brain, spinall cord and lungs. Besides telangiectasias, one also finds arteriovenous malformations (AVMs) and aneurysms on the mucosa of the entire gastrointestinal tract, that can be the source of massive gastrointestinal bleeds (see Chapter 8 Lasers in Gastroenterology). Recurrent epistaxis can be debilitating and life threatening. Treatment is aimed at palliation of symptoms. Nasal packing, with a variety of agents, is usually the first line of therapy to stop bleeding. Other treatment modalities for this disorder include local pressure, topical vasoconstrictors " chemical cautery, cryotherapy, arterial embolization and arterial ligation, which have all been applied with limited success. Patients with severe HHT may require repeated blood transfusions if treatment is unsuccessful. Moderalle long-term success has been achieved with estrogen derivatives and septodermatoplasty, which may be accompanied with serious side-effects and complications ${ }^{[5,39]}$.

In the past 15 years, a variety of types of lasers has been tried in treatment of HHT, including the $\mathrm{CO}_{2}$ - dye- argon- Nd:YAG-, Ho:YAG- and KTP laser. Commonly, patients were referred for laser treatment after other therapies had failed. Only two case-series, both using an Nd:YAG laser, included more than 20 patients ${ }^{[5.39]}$. The largest of these, published by Rebeiz et all. in 1991 . included 41 patients with HHT who underwent 85 treatments in the period 1982-1990 ${ }^{\mid 39 !}$. The nasal lesions of these patients were visualized with a microscope. Nd:YAG laser energy was delivered via a fiber, inserted in a handpiece with suction chaninel. Follow-up of these patients ranged from 1 to 8 years, with an average of 3.5 years. Treatment was applied on an ambulatory basis using local anesthesia with intravenous sedation in the majority of cases. General anesthesia with endotracheal tube intubation was reserved for elderly patients with severe disease. Response to treatment was defined as a $50 \%$ reduction in number of bleeds and number of blood transfusions. A response to treatment lasting at least one month was achieved in 35 patients $(85 \%)$. An excellent response, defined as a response to treatment lasting 8 months or longer, was achieved in 15 patients (37\%) with mild to moderate bleeding. Mild bleeding was defined as 0.3 bleeding episodes per week without the need for blood transfusion, whereas moderate bleeding 
Was defined as 1-2 bleeding episodes per day including the need for blood transfusion. Mo response occurred in $50 \%$ (6 out of 12) patients with severe bleeding, defined as $2-5$ bleeding episodes per day including the need for blood transfusion. One patient developed a sepla perforation. The authors concluded that laser therapy is beneficial in particular in young patienls with mild to moderate bleeding. In these patients treatment can easily be repeated if necessary In patients with severe disease, surgical techniques (e.g. dermatoplasty), were thought to be more appropriate than laser treatment ${ }^{\mid 30 !}$.

In 1994, Blitzer reported results in 21 patients with HHT treated with the KTP and/or the Nd:YAG laser in a ten-year period ${ }^{\text {Is. }}$. Except in severe cases, treatment was applied in the office on an ambulatory basis. The lesions were observed using a rigid nasal endoscope. Laser energy was: delivered using fibers inserted in a handpiece. Follow-up was unspecified. While applying the classification of Rebeiz et al. Blitzer found a response to treatment lasting at least one month in all but one patient $(95 \%)$. This patient suffered from severe bleeding. An excellent response was found in 5 patients $(24 \%$ ) with mild to moderate bleeding, and four of these had no recurrent: epistaxis for a period of at least 2 years. Three patients $(14 \%)$ developed a septal perforation after Nd:YAG laser treatment. The author concluded that KTP and Nd:YAG laser treatment may be: beneficial in patients with mild to moderate bleeding secondary to $\mathrm{HHT}^{\text {[j] }}$.

In summary, in selected patients with HHT laser treatment may have the potential to substitute fot surgical treatment of recurrent bleeding. The advantages and disadvantages of each type of lasers: have only partly been clarified. Ossoff et all, in their 1994 review; list the Nd:YAG laser as the lase of first choice for this indication, with the KTP laser as a second choice ${ }^{[35]}$. Dilkes $(1994)$, however: prefers the KTP and argon lasers for this procedure ${ }^{[13]}$. A systematic comparison of different types: of lasers with each other and with conventional techniques seems justified.

\subsubsection{Intractable epistaxis of unknown origin}

Epistaxis, unrelated to HHT, is a common condition that often resolves with home remedies or with the attention of a primary care physician. However, 5 to $10 \%$ of patients suffer intractable epistaxis and are referred to an otolaryngologist. These patients have not responded to conventional: methods of packing and external chemical or diathermic cauterization. In these cases vascular control can be achieved by angiographic arterial embolization or by surgical ligation of the major feeding vessels ${ }^{12 !}$. Again "perhaps laser treatment may have the potential to substitute for surgeny in selected patients. For example, Krespi and Ling (1994) reported preliminary evidence indicating that sinus endoscopic visualisation of the vascular architecture of the perichondrium combined wilts a nasal probe delivering argon- or Nd:YAG laser energy offers good hemostatic results with septal perforation as the most important complication ${ }^{[2]}$. Ossoff et al.(1994), without providing any evidence, listed the KTP and the Nd:YAG laser as the lasers of first choice in treatment of patients. with recurrent epistaxis ${ }^{|3|}$. Although the short-term clinical evidence as reported by Krespi and Ling (1994) is promising, more evidence is needed to allow a judgment.

\subsubsection{Chronic rhinitis}

Chronic nasal obstruction is one of the most common otolaryngological complaints. Patients complain about habitual mouth breathing, dryness of mouth and throat, snoring, sleep apneu, and geneiral malaise. Chronic hypertrophy of the inferior turbinates is a common cause of chronic nasal obstruction, either alone or in combination with other disease entities or abnormalities in the nose ${ }^{[3.4]}$. Medical management, e.g. with corticosteroids, is preferred as initial treatment to reduce the mucosal swelling of the inferior turbinates ${ }^{25.28 .341}$. In cases where medical therapy fails to promote nasal ainway patency a variety of interventions may be applied. Simple lateral outfracture of the inferior urbinates by pressure is the major mechanical modality employed. The most important surgical techniques are inferior turbinectomy and inferior turbinoplasty. Other techniques 
include cryosurgery and electrocoagulation ${ }^{[2,25,29]}$. It is unclear which technique should be the first choice of treatment after failure of medical management. Most experience seems to have been gained with inferior turbinectomy. In an analysis of 2,400 cases with a mean follow-up of 6.3 years, Ophir (1991) reported successful treatment in $92 \%$ of patients. Postoperative bleeding requiring repacking or tamponade occurred in 96 patients $(4 \%), 12$ of whom required blood transfusion $(0.5 \%)$. Postoperative infection occurred in 8 cases $(0.3 \%)$ and intranasal adhesions were observed in 142 patients $(5.9 \%)$. In 67 of these patients surgical lysis was required ${ }^{344}$.

Without providing more than preliminary evidence, many types of lasers have been suggested to replace or assist in the procedures listed above. The $\mathrm{CO}_{2}$ laser, using conventional delivery systems, has been used as an alternative for conventional turbinectomy in the mid-eighties. More recently, the Ho:YAG laser has been used for turbinectomy ${ }^{13.359}$. Several authors report the use of either Nd:YAG or diode lasers to coagulate the turbinates prior to their removal ${ }^{13,45 \mid}$. In a similar procedure both these lasers and the KTP laser have been used to shrink the turbinates, in some cases to replace submucosal diathermy ${ }^{[1,3,35]}$.

Krespi and colleagues (1994) developed two other laser procedures to replace surgical procedures, which can both be performed in a doctor's office or ambulatory setting using topical and/or local anesthesia. In 50 patients an Nd:YAG laser was used for interstitial pholocoagulation of the inferior lurbinate submucosa. In another 40 patients, a $\mathrm{CO}_{2}$ laser (superpulsed mode) delivered by a hollow wave guide was used to directly ablate the turbinate mucosa and glandular tissue. The authors judged treatment results as satisfactory, with minimal crusting postoperatively and a minimal risk of postoperative blleeding ${ }^{[2: 5\}}$.

Overall, the evidence specullatively suggests that lasers may play a role in treatment of patients with chronic rhinitis, eilher preceding or replacing surgical or other conventional procedures. Although many laser systems are available and evidence is scattered, Ossoff et al. (1994) list the $\mathrm{CO}_{2}$ laser (conventional delivery system) as the laser of first choice for this indication, and the Nd:YAG laser as second choice ${ }^{[35 !}$. At the moment it is difficult to devise a strategy of comparative studies for this indication that could include one or more laser applications. This is due to the preliminary character of the evidence, the large variety of procedures already in use for this indication and ongoing developments in both surgical and non-surgical techniques. For example, turbinoplasty is less invasive than turbinectomy, and offers promising results compared to the conventional technique ${ }^{[2]}$. 


\subsection{Summary}

Table 7.1 summarizes the findings of this Chapter.

Table 7.1 Judgments on the effectiveness and cost-effectiveness of selected lase applications in otolaryngology

\begin{tabular}{|c|c|c|c|c|c|c|}
\hline \multirow[t]{2}{*}{ Indication } & \multicolumn{6}{|c|}{$\begin{array}{c}\text { Judgments } \\
\text { (see definitions below) }\end{array}$} \\
\hline & 2 & 3 & 4 & 5 & 6 & 7 \\
\hline Laryngeal stenosis & $x$ & & & & & \\
\hline Vocal cord immobility & $x$ & & & & & \\
\hline Respiratory papillomatosis & & $x$ & & & $x$ & \\
\hline $\begin{array}{l}\text { Vocal cord polypi, nodules, } \\
\text { granuloma and edema }\end{array}$ & & $x$ & & & & \\
\hline Carcinoma of larynx & & $x$ & & & $x$ & \\
\hline Pharyngeal pouch & $x$ & & & & & \\
\hline Severe snoring and sleep apneu & $x$ & & & & & \\
\hline Lesions of the mouth & & $x$ & & & & \\
\hline Tonsillitis & $x$ & & & & & \\
\hline Tongue lesions & $x$ & & & & & \\
\hline Stapedotomy & $x$ & & & & & \\
\hline Ear tumours & $x$ & & & & & \\
\hline Nasal surgery & $x$ & & & & & \\
\hline
\end{tabular}

Definitions:

1 Experimental $=$ still in development, limited clinical data, no proof of effectiveness.

2 Promising = some clinical data, but procedure not accepted as a routine.

3 Established by clinical experience $=$ no randomized trials, but sufficient clinical data to reach a judgment.

4 Established by RCT = established by several well-designed randomized controlled trials.

5 Probably not cost-effective = limited data on cost-effectiveness, but reason to believe that the procedure will not turn out to be cost-effective.

6 Probably cost-effective = limited data on cost-effectiveness, , but reason to believe that the procedure will turn out to be cost-effective.

7 Proven cost-effective = adequate analysis of cost-effectiveness. 


\subsection{References}

1. Banta $\mathrm{HD}_{1}$ Schou $\mathrm{f}_{\mathrm{n}}$ Vondeling $\mathrm{H}_{\text {}}$ de Wit A. Economic appraisal of laser applications in health care. Repont of a project. Lasers in Medical Science $1992(7)$ 1:9-21.

2. Banta HD, Schou I. Otolaryngology - Costeffectiveness (editor's comment). Hin: Banta HD, Schou I (Eds.). Lasers in health care. Effectiveness, cost-effectiveness and pollicy implications. Academic Publishing, Frederiksbeng, Denmark, $1994,10.113$.

3. Barak $S$, Mintz $S$, Katz J. The role of lasers in ambulatory oral maxillofacial surgery. Operative Techniques in Otolaryngology - Head and Neck Surgery 1994(5)4:244-49.

4. Barbara M, Monini $S$, de Seta $E_{\text {s }}$ Fillpo R. Early hearing evaluation after microdrill stapedotomy. Clinical Otolamngology $1994(19) 1: 9-12$

5. Blitzer A. Laser photocoagulation in the case of patients with Osler-Weber-Rendu disease. Operative Techniques in Otolanyngology - Head and Neck Surgery 1994(5)4:274-7.

6. Bottrill 10, Poe DS. Endoscope-assisted ear surgefy. The American Journal of Otology 1995(16)2:158-63.

7. Carruth JA. Otolaryngology. In: Benta HD, Schou I (Eds.). Lasers in health care. Effectiveness, cost-effectiventsss and policy implications. Academic Publishing. Frederiksberg. Denmark, $1991, \mathrm{p} 105-13$.

8. Carpentier $\mathfrak{J}$ de, Timms M. Preliminary experience with microscopic tonsillectomy. Clinical Otolaryngology $1994(19) 4: 352-4$

9. Cragle SP. Brandenburg $\mathrm{JH}$. Laser cordectomy or radiotherapy: cure rates, communication, and cost. Otolaryngology, Head and Nexk Surgery 1993(108)6:648-54.

10. Crockett. DM, Reynolds BN. Laryngeall laser surgery. In: Parkin JL. (Ed.). Lasers in otolanyngology. The Otolaryngological Clinics of North America 1990(23)1:49-66.

11. Crumley RL. Endoscopic laser medial arytenoidectomy for ainway management in bilateral laryngeal paralysis. Annals of Otology, Rhinology and Laryngology 1993(102):81-4.

12. Davis RK, Steiner W. Transoral $\mathrm{CO}_{2}$ laser excision of stage II glottic cancer: current indlications and results in 84 patients (abstract). Lasers in Surgery and Medicine 1994(9)Supplement 6:34.

13. Dilkes MG. Invited review. Lasers in otolaryngology. Lasers in Medical Science 1994(9):71-9.

14. Dornhoffer JL, Bailey, Jr. T, Graham SS. Long-term hearing results following stapedotomy. The American dournal of Otology $1994(15) 5: 674-8$.

15. Van Duyne JA, Coleman JA, Ossoff $\mathrm{RH}$. Laser safeby in the atolaryngologist's office. Operative Techniques in Otolaryngollogy - Head and Neck Surgery 1994(5)4:298-302.

16. Eckel HE, Thumfart $M$, Wassermann $K$, Vössing $M$. Thumfart WF. Cordectomy versus arytenoidectomy in the management of bilateral wocal cord paralysis. Annals of Otology, Rhinology and Laryngology 1994(103):852-7.

17. ECRl. Laser-assisted uwulopalatoplasty for the treatment of simple (nonapneic) snoring and abstructive sleep apneu. Health Technology Assessment Information Service, Executive Briefings, ECRI, March 1995.

18. Eggink GJ, Kaptein $C$, van Kempen RJ, van der Meulen FW, Teirlinck C.JPM, Vaartjes SR (Eds.). Natianal Committee on Laser Safety. Laser safety in health care (second edition). The Netherlands Organization for Applied Scientific Research (TNO) "Leiden, the Netherlands (in press).

19. Hinton A, Moore-Gillon V. Otorhinolaryngology. British Medical لournal 1994(309):651-4.

20. Horn $\mathrm{KL}$. Gherini $\mathrm{SG}$, Franz DC. Argon laser rewision stapedectomy. The American Journal of Otology $1994(15) 3: 383-8$

21. Krespi $Y$, Ling EH. Laser management of anterior epistaxis. Operatiwe Techniques in Otolaryngology - Mead and Neck Surgery $1994(5) 4: 271-3$.

22. Krespi $Y$, Ling EH. Tonsil cryptolysis using $\mathrm{CO}_{2}$ Swiftlase. Operatiwe Techniques in Otolaryngology - Head and Neck Surgen 1994(5)4:294-7.

23. Krespi YP, Keidar A. Khosh MM, Pearlman SJ, Zammit G. The efficacy of laser-assisted uvulopalatoplasty in the management of obstructive steep apneu syndrome and upper airway resistance syndrome. Operative Techniques in Otolanyngology - Head and Neck Surgery 1994(5)4:235-43.

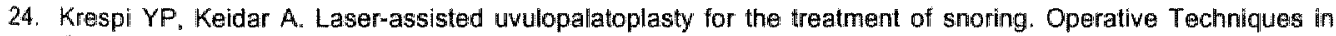
Otolaryngology - Head and Neck Surgery 1994(5)4:228-34.

25. Krespy $Y P$. Mayer $M$. Slatkine $M$. Laser photocoagulation of the inferior turbinates. Operative Teachniques in Otolaryngology - Head and Neck Surgery 1994(5)4:287-91.

26. Kürsten $R_{\text {}}$ Schneider $B$, Zrunek $M$. Long-term resul ts after stapedectomy wersus stapedolomy. The American Jounal of Otology $1994(15) 6: 804-6$.

27. Lesinski SG, Stein JA. Lasers in revision stapes surgen. Operatiwe Techniques in Otolaryngology - Head and Neck Surgery 1992(3)1:21-31.

28. Mabry RL. Inferior turbinoplasty. Operative Techniques in Otolaryngology - Head and Neck Surgery 1991(2)3:183-8.

29. Mahieu HF, Patel P, Annyas AA, van der Laan T. Carbon dioxide laser vaporization in early glottic carcinoma. Archives of Otolaryngology and Head and Neck Surgery 1994(120)4:383-7. 
30. Manmi JU, van der Hoogen FJA, Oudes M. Erahrungen milt der Groninger Stimprothese nach Laryngectomy HNo $1994(42) 3: 358 \sim 61$

31. MEGuin WF, Blalock D, Koufman JA, Feehs RS, Hillard Aw "Greven C, Randall M. Archives of Otolaryngology and Head and Neck Surgery 1994(120)9:951-5.

32. Myers EN. Wagner RL, Johnson IT. Microlanymgoscopic surgery for T1 glattic lesions: a cost-effective option Annalis of Otology, Rhinology and Laryngology 1994 (103):28-30.

33. Oas RE Jr, Bartels JP. KTP-532 laser tonsillectomy: a comparison with standard techniques. Lanngoscope $1990(100) 385-8$.

34. Ophir D. Inferior turbinectomy. Operative Techniques in Otolanyngology - Head and Neck Surgery 1991(2)3:189-93

35. Ogsoff $\mathrm{RH}$, Colleman JA, Courey MS, Duncavage JA, Werkhaven JA, Reinisch $R$. Clinical applications of lasers in otolaryngology-head and neck surgery. Lasers in Surgery and Medicine 1994(15):217 -48.

36. Ossoff RH Laser safety in otolaryngology-head and neck surgery. anesthetic considerations and educationat considerations for laryngeal surgery. Laryngoscope 1989(89)8 Part 2(Supplement no 48):1-26.

37. wan Overbeek. JJM. Micromendoscopic $\mathrm{CO}_{2}$ laser surgery of the laryngopharyngeal diverticulum. In: Carrutin JAS Simpson GT (Eds.), Lasers in otolaryngology: Lasers in Medicine and Surgery Series, Chapmann and Hall Medical Chapmann and Hall, London, 1988 , p. 133-45.

38. Pedersen CB. Revision surgery ü otosclerosis - operative findings in 186 patients. Clinical Otolaryngology $1994(19,5: 446-50$

39. Rebeiz EE, Parks S, Shapshay SM. Management of epistaxis in hereditary hemorrhagic telangiectasia with Neodymium:Yttrium-Alumimum-Gamet laser photocoagulation. Operative Techniques in Otolaryngology - Head and Neck Surgery $1994(5) 4: 17 \% \div 82$.

40. Robson AK, Herrema I, Stafford FW. Laser debulking of obstructing laryngeal tumours. Clinical Otolaryngology $1995(19) 5,430-2$.

41. Rudert $\mathrm{HH}_{4}$ Wener JA. Endoscopic resections of glottic and supraglottic carcinomas with the $\mathrm{CO}_{3}$ laser. European Archives of Otorhinolaryngology 1995(252)3:146-8.

42. Sackett DL, Haynes RB, Tugwell P. Clinical epidemiology. A basic science for clinical medicine. Little, Brown and Company, Boston, Toronto, 1985.

43. Schuller DE. Use of the laser in the oral cavity. In: Parkin JL (Ed.). Lasers in otalaryngology. The Otolanyngological Clinics of North America 1990(23)1:31-42.

44. Silverstein $H$, Bendet $E$, Rosenberg $S$, Nichols $M$. Revision stapes surgery with and without laser: a comparison. Laryngoscope 1994(104)12:1431-8.

45. Slatkine $M_{1}$ Krespi YP. Instrumentation for office laser surgery. Operative Techniques in Otolaryngology - Head and Neck Surgery 1994(5)4:21:1-7.

46. Strunk CL Jr. Quinn FB Jr. Stapedectomy surgery in residency: KTP-532 laser versus argon lasier. American Joumal of Otology $1993(14) 2: 113-7$.

47. Zamitt $G K$, Lund $S L$, Ghassibi JG. Clinical polysomnography in the evaluation of snoring and sleep-related breathing disorders. Operative Techniques in Otolaryngology - Head and Neck Surgery 1994(5)4:221-7.

48. Zeitels SM, Davis RK. Endoscopic management of supragloftic cancer. American Journal of Otolanyngolagy 1995(16)1:2 11. 


\section{Lasers in gastroenterology}

\subsection{Introduction}

Lasers were introduced in gastroenterology in 1976, when an argon laser was used for the first time as part of an endoscopic procedure to control gastrontestinal bleeding. One year later, as reviewed by the Office of Health Technology Assessment in the US, Kiefhaber and associates reported the use of an Nd:YAG laser to photocoagulate bleeding uicers ${ }^{|\hat{4}| !}$. The first RCT on laser treatment of upper gastrointestanal (ugi) bleeding was published as early as $1981^{12 !}$. At the same time, other endoscopic modalities were successfully introduced in gastroenterology, resulting in increased evaluation activities. In the eighties more than $30 \mathrm{RCTS}$ evaluated treatment of ugibleeding, including 13 on lasers and 17 on other endoscopic modalities ${ }^{i 2\}}$. For a related indication, bleeding resulting from esophageal varices, Nd:YAG lasers have found limited use. Both argon and Nd:YAG lasers have been successfully applied in treatment of bleeding resulting from vascular anomalies, for example angiodysplasia and Hereditary Hemorrhagic Telangiectasia (HHT). No RCTs have been published for these indications.

The applications of lasers aimed at hemostas is were followed by palliation for advanced cancers of the upper and lower gastrointestinal tract and treatment of benign tumours of the colon ${ }^{\text {(89) }}$. In addition, limited experience in curative laser treatment of small cancers has been gained ${ }^{[4 \mid}$. According to Nishioka, in his 1995 review, relief of malignant dysphagia is the most common gastrointestinal use of the Nd:YAG laser in most centers ${ }^{[169]}$. This application, palliation of esophageal cancer, has been evaluated in a number of prospective studies and RCTs. One of these compared Nd:YAG laser therapy with PDT ${ }^{|24|}$. In contrast, no RCTs have been published an laser palliation of colon cancer and of treatment of sessile colorectal adenomas. A final indication that is discussed is treatment of hemorrhoids. Various modalities are used in treatment of hemornoids, including Nd:YAG- and $\mathrm{CO}_{2}$ lasers. Many RCTs have beem published, but the quality of these studies is rather mixed $d^{[41]}$. For all indications listed here, laser therapy has to compete with a variety of endascopic methods. Together, these endoscopic methods have largely substituted for conventional surgical procedures.

\subsection{Upper gastrointestinal bleeding}

\subsubsection{Introduction}

Upper gastrointestinal bleeding may be caused by peptic ulcer disease, esophageal varices and a number of vascular anomalies such as telangiectasia and angiodysplasia ${ }^{\left[{ }^{(8)}\right]}$.

\subsubsection{Blleeding resulting from peptic ulcer disease}

Bleeding is the most common complication of ulcer disease and either fails to stop spantaneously or recurs in about 20 percent of patients, requiring some form of therapy ${ }^{|\beta 3|}$. These patients represent about half of all cases of ugi-bleeding ${ }^{[84,896]}$. In the Netherlands it has been estimated that each year 5,500 patients are admitted to hospital due to ugi-bleeding, of whom about $10 \%$ decease ${ }^{\left[{ }^{[4]}\right.}$. The international mortality rate from bleeding ulcers averaged between $6 \%$ and $10 \%$ in the period 1959-1989 despite advances in diagnosis and treatment. The $10 \%$ mortality rate refers to surgery performed in emergency conditions ${ }^{1544}$. The possibility of an intervention that could reduce not only bleeding but also emergency surgery and mortality rates, was (and still is) of great importance. This explains the introduction of a variety of endoscopic modalities for treatment of ugibleeding in the late sixties and early seventies. Besides laser treatment (argon-and Nd:YAG laser), also contact thermal methods (heater probe or monopolar or bipolar electrocoagulation), and injection therapy (e.g. epinephrine, alcohol, polidocanol) have been applied in these patients ${ }^{(2)}$. 
With the publication of increasing numbers of both RCTs and uncontrolled studies, various: attempts have been made to review the safety and efficacy of lasers and other modalities.

As early as 1985, the American Medical Association (AMA) produced a Diagnostic and Therapeutic Technology Assessment (DATTA) Report on laser and other endoscopic treatment modalities of ugi-bleeding ${ }^{[13]}$. The majority of consultants for this report indicated laser photocoagulation to be an established therapy for bleeding of the stomach and duodenum. Nd:YAG laser therapy was fudged as more effective than argon laser therapy, In 1987, the Office of Health Technology. Assessment (OHTA) of the United States reviewed the literature on laser treatment of ugi-bleeding. At that moment data were available on 11 mostly small RCTs comparing laser therapy to no active treatment $O$ f the three RCTs including argon lasers, one concluded that the laser was not influential in affecting outcome whereas the other two smaller trials demonstrated that the laser improved both initial hemostasis and outcome. Of the eight RCTs on Nd:YAG laser treatment four: did not demonstrate benefit and four did demonstrate benefit. Based on these trials, the OHTA concluded that reassessment should be done in about two years ${ }^{[61 !}$. In the meantime, RCTs evaluating the heater probe and electrocoagulation were published. Mathus-Vliegen (1988) " ${ }^{47}$ using her own clinical experience and these (laser and non-laser) trials, ranked the heater probe and bipolar electracoagulation as superior to laser treatment in bleeding peptic ulcers, but laser treatment as better than conventional therapy. In 1989, the National Institutes of Health (NIH) of the US convened a consensus conference on therapeutic endoscopy and bleeding ulcers ${ }^{154}$. Multipolar electrocoagulation and the heater probe were judged as the most promising techniques: $\mathrm{Nd}$ YAG laser therapy, although 'appearing to be effective for achieving immediate hemostasis and preventing rebleeding', was judged as difficult to master, difficult to apply, and costly in relation to other modalities. Argon laser treatment was judged to be superseded by other, more effective: treatment modalities. Finally, insufficient data prevented formulation of recommendations on injection therapy.

Sacks et al. (1990) reported a meta-analysis of RCTs on endoscopic hemostasis against standard

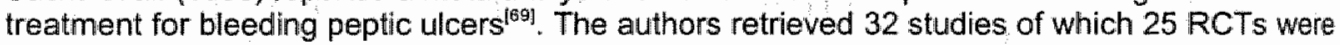
included based on methodological quality criteria. Eight RCTs focusing on laser treatment were included ( 3 on argon lasers and 5 on Nd:YAG lasers). The conclusions were clear. Endoscopic hemostasis was proven to be effective against active bleeding. It reduced recurrent bleeding and the need for emergency surgery. Overall, mortality was reduced 30 percent by treatment. At the same time, because the trials were small and because few of them tested treatment modalities against each other, direct comparisons between modalities were not possible. Therefore, more studies comparing the different treatment alternatives against each other were recommended. In these studies it would no longer be ethical to test treatment against no treatment.

Two years later, in 1992, Cook et al. published a meta-analysis including 30 RCTs focusing on endoscopic therapy for acute nonvariceal ugi-bleeding ${ }^{\mid 12 !}$. Compared to Sacks et al., a few additional studlies and unpublished data were included, primary authors actively participated in the overview and the reasons for heterogeneity in study results were explored. Of the selected trials, 13 compared contact thermal modalities (mono- and bipolar electrocoagulation, heater probe) with no endoscopic treatment 13 compared laser therapy with no active treatment ( 9 on Nd:YAG lasers, 4 on argon lasers) and 7 compared injection therapy with no active treatment. The RCTs on lasers included in the meta-analysis were published by thre et al. (1981), Vallon et all. (1981), Swain et al. (1981), Lebodic and Sudry (1981), Escourrou (1981), Rutgeerts et al. (1982), Jensen et al. (1982), MacLeod et al. (1983), Swain et al. (1986), Krejs et al. (1987). Buset et al. (1988).

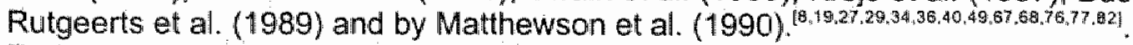

Endoscopic therapy significantly reduced rates of further bleeding (odds ratio, $0.38 ; 95 \%$ confidence interval, $0.32-0.45$ ), surgery (odds ratio, $0.36 ; 95 \%$ confidence interval, $0.28-0.45$ ), and mortality (odds ratio, $0.55 ; 95 \%$ confidence interval, $0.40-0.76$ ). The three treatment groups, when analysed separately, all significantly reduced further bleeding, and surgery rates. The reductions in mortality were comparable for all three forms of therapy, but, in the words of the authors, the decrease reached conventional levels of statistical significance only for laser therapy. No distinction 
Was made between results from RCTs using an argon laser or an Nd: YAG laser. A sensitwity analysis restricted to trials of the highest methodological quality showed greater uniformity of trial results than was found across all studies. Endoscopic therapy in these trials was found better than no active treatment with respect to the three main outcome variables discussed above. The risk of therapeutic endoscopy was low for all endoscopic modalities. In case of laser treatment, the incidence of bleeding induced during endoscopy was $0.39 \%(95 \%$ confidence interval $-0.140 .87)$. The reported incidence of perforation in the laser trials was $0.9 \%(95$ confidence interval $0.11-1.6)$. It was noticed that these low figures may be subject to reporting bias and thus may not reflect the risk of endoscopic hemostasis in all centers.

A subgroup-analysis indicated that the effectiveness of endoscopic treatment is limited to patients with endoscopic features predictive of a high risk of persistent and recurrent bleeding, which was supportive of the National Institutes of Health Consensus Conference discussed eartie ${ }^{[54 !}$. This document stated that endoscopic hemostasis is indicated for the treatment of bleeding ulcers in selected high-risk patients, defined as rapid bleeding with substantial blood loss, ongoing transfusion requirement, red hematemesis, red stool, age over 60 and major associated diseases $^{i 54}$. In these patients, the strategy of no active treatment, with surgery when needed, shoulld no longer be applied.

At present many endoscopic methods, including laser treatment, are judged as equally safe and effective ${ }^{[64]}$. In practice, despite the successful use of lasers to arrest hemorrhage from bleeding peptic ulcers in many studies, the use of lasers in this setting has fallen out of use because of the widely held perception that other modalities can produce similar results at much less expense ${ }^{\text {j5:98y? }}$ Data on the cost-effectiveness of laser treatment are discussed in the next paragraph. Future RCTs, when organized in the Netherlands, should always include injection sclerotherapy, because this has become the most frequently used treatment modality in this country ${ }^{(\theta a)}$. A number of new (mechanical) treatment modalities have been developed, including metal clips, clamps, rubber band ligation and sewing. Although none of these are yet established in clinical practice ${ }^{(6 a)}$ "they may become candidates for future RCTs. In addition, it has been suggested that endoscopic treatment followed by elective surgery could be beneficial in those patients in whom now emergency surgery is performed without an attempt at endoscopic control ${ }^{[6] \mid}$.

\section{Cost-effectiveness}

What is the evidence on the cost-effectiveness of laser treatment? Historically, one could distinguish two situations here: the cost-effectiveness of laser treatment versus conservative treatment, and the cost-effectiveness of laser treatment versus other endoscopic modalities. Firstly, a cost comparison with surgery is discussed. Overholt (1987) compared total physician and hospital charges for a patient receiving laser therapy for an antral ulcer with a visible bleeding vessel to those of a surgically treated patient who suffered from recurrent rebleeding of a pyloroduodenal ulcer ${ }^{63}$. Total charges for laser therapy were calculated as US $\$ 4500$. The surgery costs amounted to US\$7000. Overholt concluded that if laser therapy is successful, total costs and lost time from work are clearly less than if surgery is necessary. Nishioka and Richter (1986) calculated that for patients with acute active hemorrhage, in whom bleeding stops spontaneously in only 25 percent, laser therapy with an efficacy of 80 percent reduces hospital costs by US\$3000 per patient treated. For patients with peptic ulcers who are bleeding from clearly defined visible vessels, hospital cost reductions of more than US $\$ 2000$ per patient treated were said to be

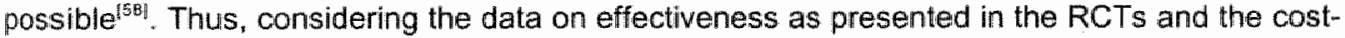
data presented above, laser therapy appears to be cost-effective compared to conventional therapy.

The more relevant comparison is that between laser treatment and other endoscopic treatment options. The highest quality evidence on the cost-effectiveness of laser treatment versus other endoscopic modalities would be gained from economic evaluations based on or carried out alongside RCTs evaluating two or more of these modalities. Evaluation of the safety and effectiveness of laser therapy and at least one other endoscopic modality and a control group was. 
carried out in only two studies included in the meta-analysis by cook et al. ${ }^{127}$. These studies were performed by Rutgeents et al. in 1989, and by Matthewson et al. in $1990^{149,889}$, In only one of these, by Matthewson et al., comparing Nd:YAG laser, heater probe and no endoscopic therapy, a retrospective economic evaluation was carried out ${ }^{[065)}$. The trial itself showed that laser treatment results in a reduction of the rate of rebleeding compared to the control group (chi-square test: $p<0.05$ ). There were no differences between the three treatment groups with respect to the rate of emergency surgery and the mortality rate ${ }^{\mid 49)}$. In the context of this trial Rees (1987) found that both the heater probe and Nd:YAG laser treatment for bleeding peptic ulcer costs considerably less than conventional therapy, considering only the costs at the time of initial hospitalization and not including non-National Health Service costs ${ }^{(6)}$. The cost of the conventional treatment, using drugs and other non-invasive methods, followed by surgery with rebleeding, was about 4500 British pounds per patient. The cost per patient of laser- and heater probe treatment were both about 2000 pounds ${ }^{(6)}$. In other words, there seemed to be no direct cost-differences between the Nd:YAG laser and the heater probe. This result is rather surprising, given the relatively thigh capital cast of laser equipment. Furthermore, with the introduction of injection sclerotherapy and other minimallyinvasive treatment modalities, which are equally effective, simple to administer and relatively cheap, laser therapy nor therapy with heater probe may be cost-effective compared to these modalities. Empirical evidence to support this hypothesis is lacking. Clearly, a systematic approach to the cost-effectiveness of various endoscopic modalities for this indication is required. In the case of lasers, this may be too late. Ironically, one of the best evaluated laser applications in medicine is one of the first which has been abandoned in clinical practice based on a presumed lack of cost: effectiveness and because it is relatively difficult to apply ${ }^{[59,64]}$.

\subsubsection{Treatment of esophageal variceal bleeding}

In the Netherlands, esophageal variceal bleeding accounts for 6 to $10 \%$ of all ugi-bleeding. In other countries, a percentage of at most 30 is reported ${ }^{\mid 84 !}$. Variceal hemorrhage may occur in up to $30 \%$ of patients with chronic liver disease. There is an estimated mortality in the region of $50 \%$ for the initial bleed and a hospital inpatient mortality for subsequent blleeds of $30 \%$. Endoscopic techniques are accepted as the first line treatment for bleeding of these lesions and for their long term management ${ }^{\{0 \mid}$. What is the rale of lasers here?

In 1987, Fleischer published a double-blind RCT including 20 patients with active bleeding esophageal varices who underwent either $N d$ :YAG laser treatment $(n=10)$ or sham endoscopy and standard medical therapy $(n=10)^{[23]}$. Patients in the laser group were on average 64.4 years of age while the average age in the control group was only 49.8 years. Initial hemostasis was achieved in 7 patients in the laser treated group and in nome of the patients in the control group ( $p<0.002$, Fisher's exact test). There were no differences between the treatment groups with respect to

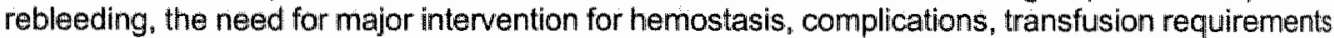
and death. Due to the small number of patients enrolled in the trial no definite conclusions could be drawn.

In 1983, as reviewed by Fleischer (1985), Kiefhaber and colleagues published the results of Nd:YAG laser treatment in 174 patients $^{[32]}$. Initial hemostasis was reported in $160(92 \%)$. The overall rebleeding rate was $30 \%$. Because of the frequency of rebleeding these authors begun early injection sclerotherapy to achieve more permanent hemostasis. Other authors too, e.g. Bown (1991), consider injection sclerotherapy a much better option than laser treatment in these patients ${ }^{[i s]}$. Williams and Westaby (1995) reported that $90 \%$ of all cases can be controlled with injection sclerotherapy. These authors judge the introduction of endoscopic banding ligation as the most important recent advance in this field, while not spending a single word on lasers ${ }^{\text {[8B: }}$. Assessment of laser treatment has been limited. As concluded earlier by Bown (1991), lasers do not seem to play a role for this indication ${ }^{[89]}$. 


\subsubsection{Vascular anomalies}

The most important vascular malformations responsible for bleeding in the gastrointestinal tract are angiodysplasia and Hereditary Hemorthagic Telangiectasis (HHT), also known as the Oster-Weber Rendu syndrome ${ }^{[59]}$. The natural history of these lesions is one of both acute and chronic blood loss, which in many cases becomes more severe, more frequent, and from more sites over a period of months or years ${ }^{[99 !}$. Surgery in these patients is associated with high morbidity and mortality. Surgical resection of all involved areas may be impossible in instances where the lesions are found in many different parts of the gastrointestinal tract, which may be the case especially in HHT patients ${ }^{59,89 !}$. Therefore, many endoscopic modalities have been applied in these patients including the argon- and NdYYAG laser, heater probe, multipolar electrocoagulation, and injection therapy. No RCTs have been published. Of these modalities, the argan- and the Nd:YAG laser are the most commonly used, according to Nishoka in his 1995 review! ${ }^{159}$. Bown (1991) states that treatment is usually quite successful whichever modality is used ${ }^{[39 !}$. Likewise, Nishioka (1995) concludes that promising results have been reponted with all modalities; and that it may be that comparable results will ultimately be demonstrated for each method ${ }^{159}$. Although treatment of vascular anomalies has not yet been the subject of cost-effectiveness analysis, it may well be that, assuming Nishioka's prediction is correct, the role of lasers may decrease for these indications in the future due to a perceived lack of cost-effectiveness compared to other endoscopic modalities.

\subsection{Esophageal cancer}

Esophageal cancer accounts for around $2 \%$ of cancer deaths in the United Kingdom annually ${ }^{151}$. The most common problem with esophageal cancer is blockage of the tract, making nutrition difficult or impossible $e^{[23]}$. The diagnosis is associated with a median survival of ten months, and fewer than $5 \%$ of patients are cured ${ }^{[45}$. If curation is judged to be possible, radical esophagectomy is performed. Some preliminary evidence has been published suggesting that both Nd:YAG laser therapy and Photodynamic Therapy may play a role in curing patients with early esophageal carcinoma. In other, low-risk patients laser therapy may be applied preceding surgery ${ }^{[155,59 !}$. The vast majority of patients with esophageal cancer will need palliative treatment in order to maintain quality of life as much and as long as possible. The morbidity of any procedure undertaken must be balanced against the likely benefits for each indwidual both in terms of relief of dysphagia and of prolongation of survival|igl. More specifically. Fleischer (1989) defined the aims of palliative management as intended to relieve dysphagia, to allow the patient oral intake that will meet his/her caloric needs and to allow him/her to feel comfortable eating in public ${ }^{[2.2]}$.

Of the available options, surgery provides good palliation but the complication rate can be as high as $35 \%$. Treatment related mortality varies from 10-30\% seen after age 70, many patients are poor risks for surgical palliation. Therefore, radiation therapy, chemotherapy, and a number of endoscopic methods are used for this purpose. Methods include dilatation, insertion of an endoprosthesis, percutaneous gastrostomy, alcohol injection, monopolar or bipolar electrocoagulation, endoluminal treatment with iridium-192, Nd:YAG laser therapy and Photodynamic Therapy|22.26!. According to Bown (1991), Carter et al. (1992) and Ellis and Cunningham (1994) of all endoscopic methods, intubation (insertion of an endoprosthesis) is the most commonly used ${ }^{10.15 .9}$. What is the role of lasers here?

The Nd:YAG laser was introduced for palliation of advanced esophageal cancer by Fleischer and colleagues in 1982. The Nd:YAG laser is used to create a hole through the obstructing tumour. The procedure can be done quickly on an outpatient basis or as a day-case and produces improvement of symptoms in most patients in a few days. General anesthesia is not necessary. To achieve recanalization ${ }_{n}$ usually a series of every-other-day treatments is required. In many patients symptomatic relief is shortlived (four to six weeks), requiring repeated treatments ${ }^{15,22,55,8941}$. Fleischer (1987) summarized the results in more than 2,000 reported patients treated by laser in 
the United States, Europe, and Japan. In more than 90 percent, the esophagus can be opened II allow swallowing. This is defined as technical success. Because of other problems, such as pain functional success was seen in about 70 percent ${ }^{23}$. The major complication, accurring in i-11 percent of patients, is perforation ${ }^{(22)}$. Other complications that can result from endoscopic lase therapy include the formation of tracheo-esophageal fistulas, bleeding, low-grade fever an odynophagia ${ }^{[59 !}$. The overall mortality of laser therapy has been estimated at around 1 percent ${ }^{22, y^{3}}$

The exact role of lasers compared to other palliative methods is difficult to define. According in Fleischer (1989) there is a common axiom that when a long list of therapies exist, it is likely tha none has emerged as a clearly superior method ${ }^{227}$. At that time, only one small RCT including lase therapy had been publisthed. This study, by Carter and Smith in 1986, included 20 patients who either received repeated laser therapy or repeated bougienage and intubation for palliation o esophageal carcinoma or carcinoma of the gastro-esophageal junction ${ }^{[9]}$. Although the results it the laser group were better than in the intubation group, no firm conclusions could be drawn dus to an inadequate randomization procedure (randomization was achieved by primary referral to a particular surgical team). The authors noted that, although out-patient therapy with laser could sometimes be done, over-night stay in hospital was usually required for "socio-economic' difficulties.

Since then, a small number RCTs have been published, not including more than 20 patients in each treatment group. Usually, swallowing ability was assessed on a 0 to 4 scale in these studies, with 0 being normal swallowing and 4 total dysphagia.

The first of these RCTs, by Alderson and Wright (1990), compared Nd:YAG laser recanalization $(n=20)$ with endoscopic intubation $(n=20)$ in palliation of patients with widely disseminated or locally advanced carcinoma in the middle or lower third of the esophagus ${ }^{[1]}$. In the laser group, patients were retreated every 4 weeks, receiving laser treatment either with or without dilatation. In the intubation group $p_{n}$ patients were intubated after preliminary dilatation. Technicall success (recanalization) was achieved in 17 of 20 endoscopic laser therapy patients ( $85 \%$ ) and in 18 of 20 endoscopic intubation patients (90\%). Both technical failures in the laser group were successfully intubated and one of the three technical failures in the intubated group was successfully treated by laser. Only patients in whom technical success was achieved were included in the analysis. All these patients experienced an improvement in the grading of their dysphagia. Seven patients $(41.2 \%)$ in the laser group scared grade 0 or 1 on the swallowing ability scale posttreatment, whereas none of the patients in the intubation group scored grade 0 and only 2 patients scored grade 1. Patients in the laser group received on average 4 treatments. Retreatment was necessary in 16 out of 17 patients $(94 \%)$. In the intubation group, retreatment was judged necessary in only two patients ( $11 \%)$. Complications, including perforations and hemorrhage, occurred in 4 patients in the laser group $(23.5 \%)$ and in 1 patient in the intubation group $(5.5 \%)$. One patient in the laser group died due to myocardial infarction, which was probably related to failed attempts to stop bleeding of the esophageal carcinoma by means of photocoagulation. Median survival in the laser group was 12 weeks (range $12-36$ weeks) and median survival in the intubation group was 16 weeks (no range provided). The authors concluded that both endoscopic laser therapy and endoscopic intubation provided effective palliation for nearly all patients with malignant obstruction of the esophagus.

The second RCT, by Barr et al. (1990), compared Nd:YAG laser therapy only to Nd:YAG laser therapy followed by endoscopic intubation for the palliation of imalignant dysphagia ${ }^{[4]}$. Forty-six patients were included in the trial, of whom 40 were included for analysis. There were 20 palients in each group. After initial recanalization, patients in the laser group received treatment at monthly intervals until death to prevent recurrent dysphagia. For each laser session the patients were admitted overnight and discharged the following day.

Besidles assessing the swallowing ability of the patient on a 0 to 4 scale, the patient's quality of life was assessed using a physician's assessment (by means of a quality of life index) and the patien's own assessment using a linear analogue self assessment (LASA). Actually, this scale comprised 
25 Visual Analogue Scales encompassing various health dimensions. The individual VAS-Scores were simply added for analysis. In the analysis it turned out to be impossible to strictly judge the two treatment arms as separate alternatives. In the laser only group, six patients $(30 \%)$ were intubated after initial laser treatment because of rapid tumor growth or extrinsic compression of the esophagus. In the laser/intubation group four patients $(20 \%)$ received laser treatment after initial laser treatment plus intubation because of recurrent dysphagia caused by tumour overgrowth of the tube. Statistical testing was done using the Mann-Whitney U-test, Fisher"s Exact test and Wilcoxon's signed-rank test for paired observations. There was no significant difference in the mean pretreatment, mean posttreatment and mean best posttreatment swallowing grade in each group. However, 8 patients $(40 \%)$ in the laser group were eating a normal diet for at least three weeks at some time after the start of treatment, while none of the patients in the laser/intubation group consistently reported a normal diet at any time after intubation. Dysphagia recurred in 5 patients in the laser group (25\%) and in 9 patients in the laser/intubation group ( $45 \%)$. There were less complications in the laser group (2 patients; $10 \%$ ) than in the laser/intubation group ( 9 patients; $45 \%$ ). All complications, which often were not major, could be successfully treated using conservative means. Both the quality-of-life scores measured by the physicians and the patients remained at a constant level pretreatment and posttreatment. Unfortunately, no exact figures were provided. The graphs in the article show that the mean pretreatment quality of life index score in both groups was a little higher than 5 on a scale ranging from $0-10$. The mean posttreatment quality of life index score in both groups was about 5.3 in both groups. The mean LASA scores (0-250) varied between about 105 and 115 in both groups both pretreatment and posttreatment. There was no procedure related mortality in either group of patients. The mean length of survival was 18.3 weeks in the laser group and 16.1 weeks in the laser/intubation group. The mean number of nights in hospital in the laser group was 13.7 , compared to 11.2 in the laser/intubation group. All these differences were not statistically significant. Although there was no improvement between pretreatment and posttreatment scores the authors concluded that both laser therapy and intubation are effective at relieving malignant dysphagia, noticing that the course of disease is one producing a general decline of the patient. The results of the trial suggested, according to the authors, that intubation should remain an effective first line treatment in most patients and that laser therapy should be restricted to specialist centers and selected patients. Both techniques should be available for the management of patients with malignant dysphagia ${ }^{|4|}$.

In 1992, Carter and colleagues published a second RCT comparing Nd:YAG laser recanalization $(n=20)$ versus endoscopic intubation $(n=20)$ in palliation of patients with esophageal carcinoma ${ }^{[0]}$ Pakients were admitted to hospital to undergo palliative treatment, and were followed-up until they died. Patients in the laser group underwent 96 endoscopies: 79 with laser and dilatation; 11 with dilatation alone; 1 with dilatation and intubation; 1 intubation alone; and on 4 occasions no trealment was required. In the group that was intubated the patients required 37 endoscopies (only $38.5 \%$ of endoscoples in the laser group): 19 with dilatation and intubation; 7 with dilatation and laser therapy" 6 for clearance of food bolus; 2 dilatations alone; 2 laser therapy alone; and on one occasion no treatment was required. Obviouslly, after initial random treatment, subsequent treatment decisions were not dictated by the randomization scheme, essentially changing the nature of the trial to a comparison of treatment strategies. Swallowing ability was graded on a numerical scale, ranging from 0 (absolute dysphagia) to 4 (able to eat anything). All patients in both groups swallowed better as a result of treatment but the improvement was higher in the laser group (Mann-Whitney U-test, $p<0.001)$, where 19 patients scored grade 4 at some time during their illness, compared to 1 patient in the intubation group. However, all remaining (eighteen) patients in the intubation group scored swallowing grade 3 at some time during their illness. Recurrent dysphagia occurred in one patient in the laser group and in four who underwent intubation. Median weight loss during follow-up was $2 \mathrm{~kg}$ in the laser group, which was less than in the intubated patients (median weight loss $3 \mathrm{~kg}$; (Mann-Whitney U-test, $p=0.04$ ). Median survival from the time of diagnosis in the laser group was 21.5 weeks (range 4-62 weeks) compared to 14.5 weeks (range $7-102$ weeks) in the intubated group (ns, Mann-Whitney U-test). The median proportion of survival spent as an inpatient was 14 (range 4-100) percent in the laser-treated group and 15 (range 3-35) percent in the intubated group. One patient in the laser group died after developing a perforation 
which was surgically treated. Two other patients in this group who developed a perforation survived after conservative treatment. Another patient developed a fistula and there was also a case of aspiration pneumonia requiring admission. In the intubated group three patients required in total 6 admissions for obstruction due to a food bolus. Four patients required admission due to aspiration preumonia. Secondary obstruction, requiring treatment, occurred in one patient. None of the patients in this group died as a complication of treatment. The authors concluded that both methods relieved symptoms, but that palliation in the laser group was better in terms of swallowing ability and weight loss, although there was no improvement in survival. Both treatments were judged to be associated with significant complications. The authors, in a final comment, stated that they had come to view both treatment alternatives as complementary and, while referring to some of their earlier work, that these methods may safely be combined in the same patient ${ }^{\text {iol }}$.

How to interpret these data? In a comment on the second RCT by Carter and colleagues by Kim and Goldschmid $(1992)^{1331}$ it was suggested that the decease of one patient in the laser group is not representative for the outcome of laser therapy versus intubation in general. Large, retrospective studies, according to these authors, suggest that mortality and morbidity are higher in patients receiving an endoprosthesis than in those receiving laser therapy. However, according to Nishioka (1995), in his review, the complication rates arising from laser therapy do not appear to be significantly different than those associated with other treatment methods ${ }^{[59]}$. Kim and Goldschmid ${ }^{[321}$ interpreted the data presented by Carter et al. as being suggestive of a trend towards longer survival in the laser group, but that the groups were too small to reach statistical significance. Nishioka (1995) discusses other small studies that show a similar trend, but none of these studies is regarded as convincing ${ }^{[59 !}$. Finally, Kim and Goldschmid (1992) state that the decision as to which endoscopic technique to employ should be dependent on the endoscopist's expertise, the ability of the patient to return frequently for procediures (in case of laser treatment) and the efficacy and risks of the procedure ${ }^{[33 !}$.

In summary, the combination of small RCTs presented here suggest that Nd:YAG laser therapy and intubation can be used in a complementary fashion to produce, at least for some time, an improvement of the swallowing ability of the patient. In general, quality of life was not well-assessed in these studies. Therefore, although improved swallowing will have a positive impact on overall health-related quality of life, it is unclear which strategy produces best palliative care. If one approach fails, either initially or subsequently, the other may succeed. This conclusion basically confirms conclusions drawn based on non-randomized studies ${ }^{[89]}$.

One more treatment modality, which has not been discussed yet, may have something to offer for patients with esophageal carcinoma. This is Photodynamic Therapy (PDT). The first RCT evaluating the role of PDT for this indication has been published in 1995 by Heier and colleagues ${ }^{\left[2^{2}{ }^{4}\right.}$. The RCT compared PDT $(n=22)$ and Nd:YAG laser treatment $(n=20)$ in patients with obstructive esophageal cancer. Patients were enrolled in the study if they were unsuitable for or had refused or failed surgery, radiotherapy or chemotherapy. Patients in the PDT group were photosensitized to laser light using dihematatoporphyrin ethers (DHE, also known as porfimer sodium or Photofrin $\left.{ }^{\top M}\right)$. An argon pumped dye laser $(630 \mathrm{~nm})$ was used for photoactivation. Patients received a standardized light dose $(300 \mathrm{~J} / \mathrm{cm})$ for both their first and (when necessary) second light applications. The dose was selected from an analysis of preliminary results in 10 patients, of which an account was included in the article. Patients in the Nd:YAG laser treatment group underwent one to four initial treatment sessions to obtain luminal patency with subsequent courses of therapy delivered when the patient developed recurrent symptoms.

Both PDT and Nd:YAG laser therapy ablated sufficient tumor to achieve luminal patency. One week after baseline data were collected complete relief of dysphagia was achieved in 12 patients in the PDT group versus 11 patients in the Nd:YAG laser group. Partial relief of dysphagia was achieved in 8 patients in both groups. After follow-up of one month a careful analysis showed that dietary performance, esophageal grade and Karnofsky performance status were better in the PDT group (PDT +1.8 standard deviation (sd) 1.2 vs +1.0 sd 1.0 ; PDT +22.4 sd 15.4 vs +7.0 sd 17.5 ; PDT +7.2 sd 14.5 vs -7.2 sd 3.1 , respectively). Irrespective of therapy, however, weight (kg) at 1 
month decreased (PDT -1.7 sd 2.4 vs -1.4 sd 3.1 ). Duration of response was longer in the PDT group: 84 days, vs 53 days for Nd: VAG laser therapy. There were no differences in mean duration of survival, which was 145 days in the PDT group and 128 days in the Nd. YAG laser group. A complete response was obtained in 1 patient in each treatment group. Complications, including fistula and stricture formation, fever, and luminal plugging by necrotic debris occurred in 11 patients in the PDT group (50\%), and in 10 patients of the Nd: YAG laser treatment group (50\%). The most important of these complications, fistula formation and stricture, occurred in 4 patients in the Nd:YAG laser group $(20 \%)$ and in one patient in the PDT group $(4.5 \%)$. Skin photosensitivity reaction to sun exposure occurred in 4 patients (of the PDT group) ( $18.2 \%$ ).

The authors concluded that PDT can relieve esophageal obstiruction and is an alternative to $\mathrm{Nd}$ YAG laser therapy with longer duration of response. At the same time the authors considered that there were only narrow differences in efficacy. Skin photosensitivity was judged the most significant of the differences between the two therapies because it requires patients precautions and possible changes in lifestyle for long periods of time beyond the actual delivery of therapy (at least 30 days). Therefore, it was recommended to restrict PDT to those patients in whom precautions would not require a change in lifestyle or to those whose tumours are not amenable to or difficult to treat with Nd:YAG laser therapy, e.g. patients with cervical or completely obstructing tumors. In the latter circumstance, PDT could be performed by embedding the fiber into the tumour to deliver laser light interstitially. Finally, it was recognized that the present generation of photosensitizers have many disadvantages which should at least partly be overcome by the coming second-generation photosensitizers, e.g. benzoporphyrin derivative.

Although these data are promising, it is still unclear how PDT will fit in the overall strategy of palliative management of patients with esophageal cancer. In a general comment, Nishioka (1995) considered the use of PDT in gastroenterolagy as clearly being in its infancy ${ }^{\mid 59 !}$. In the case of esophageal cancer too, PDT is not (yet) accepted and practised as a real therapeutic option $n^{[55 !}$.

\section{Cost-effectiveness}

There is little cost data on esophageal cancer. Fleischer (1987) compared the costs of palliative surgery versus palliation by laser, using data from a university medical center on the East Coast of the United States. The surgical treatment required 11 days in the hospital, compared with 6 days for the laser therapy. With laser therapy, three different treatments sessions were assumed. In addition, the operating room was not used with the laser treatment. The surgical treatment was calculated to cost US\$12 710 , compared to US\$4800 for the laser treatment. The problem with this study is that the two groups of patients are not comparable, because laser therapy (or other endoscopic modalities) was selected in patients who were unfit for palliative surgerylaz,80].

Kelly et al. (1986), in an abstract, compared a group of 7 male patients treated with the traditional methods (including dilatation, radiation and insertion of a prosthesis) before the laser was introduced to a group of 7 male patients after the laser was introduced, when the laser was added to other palliative methods of treatment ${ }^{[31 !}$. The median survival in the traditional treatment group was 126 days, compared to 109 days in the 'laser' group. Mean hospital stay was 76 days in the traditional treatment group versus 83 days in the 'laser' group. The mean cost of care for the traditional treatment group was US $\$ 18287$ per patient, and the cost of care for the laser group was US\$21 977 per patient. The authors did not mention extra benefits in the "laser' group, the average extra expense was US $\$ 1041$. Of course, because the second group of patients received laser therapy in addition to traditional therapy, this is not a true comparison. Also, assessment of effectiveness was limited to survival, although quality of life is the most important outcome parameter in these patients.

In 1995, Sculpher et al. pubiished a cost analysis of Nd:YAG laser ablation versus endoscopic intubation for the palliation of malignant dysphagia ${ }^{[72]}$. The analysis considered the costs of the major health service resources used by patients undergoing the two forms of palliation over their lifetimes. The analysis was based on a number of assumptions, one of which was that patients 
survive, on average, for the same length of time whatever their primary palliative therapy. The results, which were subjected to an extensive sensitivity analysis, showed that, over the short time that patients with malignant dysphagia are likely to live, palliation of their symptoms using Nd:YAC laser will cost between 153 and 710 British pounds more than endoscopic intubation. Factors that to some extent reduce or even eliminate the cost differential included e.g. increasing the number of laser procedures; undertaking more laser procedures as day-cases, and using (more expensive) metal stents for intubation. The authors caution that, based on these data, it would not be justified to conclude that intubation is the mone cost-effective form of palliation. This depends on the rallo of the costs and health outcomes of both forms of therapy. The authors notice that there have been no studies with sufficiently large sample size to identify statistically significant overall differences between the primary therapies in health-related quality of life.

Overall, in agreement with Sculpher et al. (1995), the combination of data presented here does no: allow a judgment on the cost-effectiveness of laser therapy versus conventional therapy in these patients. More studies are meeded in this area, especially focusing on alternative endoscopic: treatment strategies in patients who are unfit for surgery.

\section{Strategies for prolonging the dysphagia-free period after initiall treatment succes}

If a treatment strategy aimed at recanalizing the esophagus is successful, a dysphagia free period starts. In theory, this period can be prolonged by slowing down regrowth of exophytic tumour or by reducing the volume of extrinsic tumour. This can be achieved by combining the therapy or strategy that achieved initial relief of symptoms with for example radiotherapy or chemotherapy ${ }^{[89]}$. A few RCTs using the Nd:YAG laser for initial treatment, have addressed this subject.

The first of these studies, by Sander et al. (1991) compared Nd:YAG laser treatment to Nd:YAG laser treatment plus afterloading with iridium-192 in palliative treatment of mallignant stenosis of the esophagus ${ }^{70 !}$. Forty-three patients with carcinoma of the esophagus and/or cardia who were inoperable or who refused surgery were entered in the two-center trial. There were 22 patients in the laser group and 21 patients in the laserlafterloading group. Initial laser treatment was applied on an inpatient basis with subsequent treatment on an outpatient basis. Patients in the laserlafterloading group received the same initial treatment. When luminal patency was achieved, irradiation with iridium-192 was applied in three sessions with weekly intervals $(3 \times 7$ Gy) Irradiation was applied endoscopically on an outpatient basis. In case of restenosis, afterloading treatment was repeated to a maximum of $6 \times 7$ Gy. Patients were followed-up until death at least at monthly intervals. Swallowing was assessed on a 0 to 3 scale with 0 being no dysphagia and 3 absolute dysphagja. Four patients (two in each group) were excluded from the final analysis due to non-compliance in two patients, a change in the therapeutic regimen (1 patient) and additive chemotherapy (1 patient), resulting in 20 patients in the laser group and 19 in the laserlafterloading group.

In all 39 patients, complete patency of the esophageal stricture was obtained with the laser. All patients experienced a dysphagia free interval after treatment, which was an improvemenl compared to the mean pretreatment dysphagia grade, which was 1.5 in both groups. It was suggested that there were no differences between the groups in mean posttreatment dysphagia rate. Patients in the laser group needed 1.8 treatment sessions per month, compared to a mean of 3.0 sessions in patients in the lasertafterloading group. Although no exact data were provided it was concluded that there were no differences in overall survival times in both groups. Likewise, there were no differences between the groups in terms of the length of the overall dysphagia-free period, which was about $50 \%$ of the overall survival time in both groups. In the treatment period, six fistulas occurred, three in each group, which were closed off with an endoprosthesis. One covered perforation occurred in the laser group, while esophagitis occurred in 4 patients in the laser/afterloading group. There were no fatal complications in either of the two treatment groups. The authors concluded that endoluminal treatment with iridium-192 as the sole adjunctive therapy to palliative laser treatment of malignant esophageal stenoses cannot be generally recommended. 
In an abstract, Tan et al (1995) presented preliminary results of an RCT comparing NdYAG laser therapy versus Nd:YAG laser therapy followed by brachytherapy for palliation of esophageal carcinoma. In a similar design as compared to Sander et al. (1991), patients in the brachytherapy group were treated using a Selectron to deliver $1500 \mathrm{cG}$. Twentysix patients were randomized, 14 in the laser group and 12 in the laseribrachytherapy group and followed-up until death. One patient was lost to follow-up and 4 patients randomized to the combined treatment group were too unfit to receive brachytherapy. These patients were included in the analysis on an intention to treat basis. In the laser group, the average improvement in dysphagia score was 1.8 compared to 2.2 in the laserbrachytherapy group. The mean therapeutic interval, defined as the dysphagia-free period after initial treatment, was 36 days (range 6-90) in the laser group versus 83 days (range 14-277) in the laseribrachytherapy group. The mean survival time in the laser group was 110 days (range 8-369), compared to 114 (range 30-277) in the laserlbrachytherapy group. There were two fatal perforations in the laser group (14\%), one bleed and one patient with symptoms of esophageal reflux. One patient in the laserkbrachytherapy suffered form transient confusion after brachytherapy. It was concluded that adding brachytherapy to laser therapy seems to increase the therapeutic interval; from about 5 weeks with laser only to about 12 weeks when combined with brachytherapy ${ }^{\text {[ral }}$. However, the length of the initial dysphagia-free period may not be predictive for the total dysphagia free period as percentage of total survival time, as shown by Sander et al. ${ }^{[70]}$ Therefore, and considering the low number of patients, these data are considered preliminary.

Sargeant et al. (no date) performed an RCT comparing laser therapy and laser therapy added by external beam radiotherapy ${ }^{[71]}$. Ellis and Cunningham (1994) discussed the trial which was then referenced as being in press. The article seems not yet to have been published. Ellis and Cunningham reported that initial data of this trial suggested that the combined treatment method produced a longer symptomatic response $e^{\mid 15]}$.

In summary, the combination of Nd:YAG laser treatment with radiotherapy, either afterloading with inidium-192, brachytherapy or external beam radiotherapy, has not (yet) convincingly been shown to affer benefits over Nd:YAG laser therapy as a stand alone therapy. Likewise, other prospective, uncontrolled or controlled but non-randomized studies, suggest that laser therapy combined with chemotherapy or bipolar electrocoagulation (BICAP) produces excellent palliation of symptoms, perhaps better than laser therapy alone in selected patient $s^{i 5.591}$. Considering the combination of evidence presented in this paragraph it is clear that the management options for esophageal cancer are increasing, both initially and after successful first treatment. This process is ongoing. For example, a promising new therapy that has not been discussed is endoscopic injection of esophageal tumours with $100 \%$ ethanol ${ }^{[60]}$. For this reason, and due to the fact that so far onlly relatively small RCTs have been published, more and larger multicenter trials are needed to convincingly demonstrate any advantage of one treatment strategy over the other. As a result the position of laser therapy, which seems established at present, may rapidly change in the future. However, this may not be a revolutionary change for the patient. Of course, a diagnosis of esophageal carcinoma early in the course of disease would produce most dramatic improvements in patient outcome. Unfortunately, esophageal cancer is an example of an indication where high technology medicine still seems to have relatively little to offer, as its diagnosis carries a death sentence within a year for almost every patient $t^{(22)}$.

\subsection{Colorectal carcinoma}

\subsubsection{Introduction}

Cancer of the colon and rectum is among the leading causes of morbidity and mortality in the Western world. Among cancers, it is the second most frequent cancer in incidence ${ }^{144.894}$. Of all patients diagnosed with colorectal carcinoma $80 \%$ dies within 3 years ${ }^{i 5 !}$. This is, among other 
bactors, related to the fact that in only $3.3-17 \%$ of patients presenting with colorectal malignancies, colorectal carcinoma is diagnosed as being in an early stage ${ }^{|46|}$ (see intermezzo 8:1).

Intermezzo 8. 1 Progrosis of patients with colorectal carcinoma

The prognosis of patients who are diagnosed with colorectal carcinoma is dependent on several factors, including the stage and location of the carcinoma, whether the procedure is carried out on an elective or emergency basis the age of the patient and comorbidity. These factors are discussed, followed by a summary conclusilon.

\title{
Stage of disease
}

In $3.3 .17 \%$ of patients presenting wh colonectal malignancies, colorectat carcinoma is dragnosed as being in an early stage" Donald and Bumenne (1993) state that when detected early at a localised stage, the 5 -year surviva rate is $87 \%$ for colonic cancer and $79 \%$ for rectal cancer ${ }^{44}$. This compares with an appraximate $40 \%$ Hyear surwit rate among patfents in whom the disease has spread ${ }^{\text {M. }}$.

\begin{abstract}
Elective versus emergency surgery
hinathus-Wegens' analysis (1988) of 24 studves including 66,414 cases published in the period $1964-1986$, the age-conected 5 year survival rate of patients who undenwent resection with curative intent an an elective basis was about $65 \%$, whive the age-corrected 5 -year surwival rate of patients undergoing emergency surgeny with curative intent was abou 36\%. Operative montalty was 7 to $10 \%$ in patients undergoing elective surgery, but was as high as $22 \%$ in patjents undergoing emergency surgen. The age-corrected 5-year survival rate in obstructive versus non-obstructive emengency surgery was 37 and $52 \%$, respectively ${ }^{4 \% t}$.
\end{abstract}

\begin{abstract}
Age
The effect of age on major surgical procedures, such as abdominoperineal resection, is considerable: Operative mortality ranges from approximately $5 \%$ in patients under 70 to $20 \%$ in patients over $80^{[53 t}$. Recent data from ithe Netherlands presented by Bonneux and associates indicate that the age-adjusted 5-year sunwal rafe for relatively young patients (30-64 years of age) who had colorectal carcinoma as primary diagnosis at first hospital admission, increased from $42.6 \%$ to $54.6 \%$ in the period $1979-1989^{5}$.

\section{Comorbidity}

Fitzgerald et al. (1995) discuss two sudies that reported an increase in mortality from colon resection performed in patients over 70 years old of $2 \%$ in patients with zero or one concurrent diseases to a $16 \%$ percent montality when two or more diseases coexist, and an increase in mortalify for colectomy of $2 \%$ in patients over 70 years old with zero or one associated illnesses to a $3 \% \%$ mortality in those with two or more coexistent diseases: respectively wat
\end{abstract}

\section{Overall}

Irespective of speciffc prognostic factors, the 5-year sunvival rate of patients with cclorectal carcinoma who are

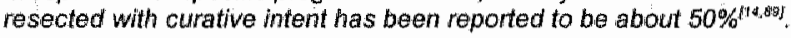

The median age of patients at diagnosis is 70 years with less than $4 \%$ of cases occurring in patients younger than 50 years ${ }^{[14 !}$. Surgical removal is the standard treatment for carcinoma of the bowe $\left.\right|^{\left|5 s_{1}(6)\right|}$. However, the effectiveness of different surgical approaches to the disease has not been tested in randomized trials ${ }^{\left[{ }^{3}\right]}$. Mathus-Vliegen (1988), in an analysis of 24 studies including 66414 cases published in the period 1964-1986, showed that only 5 to $10 \%$ of the patients were considered inoperable. In 8 to $28 \%$ of the cases the tumour was unresectable. In these cases only laparotomy, colostomy or by-pass was performed. Radical resection, that is resection with curative intent, was applied in 60 to $80 \%$ of patients. Age-adjusted 5-year survival rates in these patients waried between 37 and $66 \%$. These data imply that only a small number of patients will have good prospects of cure. Despite this situation the 5-year survival rates are modestly increasing in countries such as the US, the Netherlands and Sweden. This trend has been tentatively explained by increased rates of early detection and, simultaneously, a shift towards non-surgical treatment of early lesions ${ }^{|s|}$. Other developments include new surgical techniques, e.g. laparoscopic, microsurgical and sphincter-saving techniques, and new (adjuvant) therapeutic regimes, e.g. chemotherapy and radiotherapy ${ }^{[2,35: 52]}$. Notwithstanding these developments it is clear that a considerable number of patients will need adequate palliation already from the beginning. Also the rather poor 5-year survivall rate provides future patients for palliation, since they will suffer from bleeding, pain, mucous discharge, tenesmus or obstruction ${ }^{[4]}$.

No randomized trials have been done in a situation where many non-surgical treatment modalities are available, including radiotherapy (external or intracavity), chemotherapy, electrocoagulation, 
electrofulguration and cryotherapy. Most of these techniques, which may be used both in early and advanced lesions, can only be applied when the carcinoma is located th the part of the rectum below the peritoneal reflection. In addition, in case of early rectal carcinoma a variety of extraabdominal surgical procedures is avallable, e.g. posterior rectotomy and transsphincteric proctorectotomy ${ }^{47.59 .891}$. What is the role of lasers here?

Experience with lasers has been gained in patients in whom the risks associated with surgery were considered to outweigh the benefits and in patients who refused surgery. A small minority of these patients had only small lesions that were limited to the lumen of the bowel. Treatment of these patients sometimes resulted in cure. The results of case-series of laser treatment of both early and advanced lesions will be discussed.

\subsubsection{Early lesions}

Brunetaud and colleagues (1990), presented results of 200 patients with colorectal carcinoma who were treated in the period 1979-1989 with an Nd:YAG laser or an argon laser ${ }^{b_{1}}$. An argon laser was used when the carcinoma was close to the anall verge. This choice, quite exceptional in international literature, was made in order to avoid deep thermal injury and to reduce the uncomfortability of the procedure. Of all patients, 182 were treated for advanced lesions and there were 18 patients with early lesions. In all patients of the latter group complete local destruction with negative biopsy was obtained ${ }^{[6]}$.

In 1993, Mathus-Vliegen published results of 35 patients with early colon cancer who received Nd:YAG laser treatment in the period 1979-1992 in a Dutch referral center ${ }^{465}$. In the same period 209 patients underwent laser treatment for end-stage colorectal malignancies. There were either contraindications for surgery in the patients treated with early lesions or they had refused surgery, for example when abdominoperineal resection and colostomy needed to be considered. Laser treatment was given every 2 to 4 weeks on an outpatient basis until complete removal was achieved: Follow-up was scheduled once every six weeks for the first three months and every three months thereafter for a median period of three years (range $7.3-89.6$ months). The mean age of the patients was 74 years (range $50-96$ years). Treatment could be evaluated in 30 patients. The follow-up period was too short for two patients due to non-laser, non-cancer related death. One patient was still under treatment at the time of writing. Two patients withdrew from treatment, in one case after successful alleviation of symptoms. Complete eradication was achieved in 28 of 30 patients (93.3\%), after a median of two treatment sessions (range 1-9) given over a median period of 42 days (range 0-389 days) without amy complications or laser-related death. No recurrences were seen during the median follow-up period of three years (range 218-2,688 days). The cumulative probability of survival at one, two and five years was $94 \%, 84 \%$ and $62 \%$, respectively. Treatment failed in two patients $(6.7 \%)$. The author concluded that laser therapy should be applied in selected cases with early collon cancer ${ }^{[46]}$.

Mellow (1994) discusses evidence produced by Escourrou et al. (1988) ${ }^{[1]]}$ who reported that laser treatment resulted in local destruction in 18 of their 101 patients without recurrence of rectal carcinoma at a mean follow-up of 13 months. The lesions were no longer than $5 \mathrm{~cm}$ in length in this subgroup of patients ${ }^{\mid 53\}}$.

Although these data indicate that laser treatment is effective for early stage colorectal carcinoma, Mellow (1994) recommends to still limit endoscopic treatment to non-surgical patients because loco-regional spread of tumours can not be detected with certainty by current diagnositic technology ${ }^{1531}$. Theoretically, this risk could be balanced against the risk of not surviving a surgical procedure. This consideration implies that with improving diagnostic strategies, e.g. endoscopic ultrasonography (EUS) with injected contrast-agents or transrectal ultrasound-guided biopsy, the role of endoscopic treatment of patients with early colorectal carcinoma may become more important in the future ${ }^{[21,46,53]}$ 


\subsubsection{Advanced lesions}

Common symptoms of advanced cancer include obstruction and/or bleeding, and mucus discharge ${ }^{t+7 ?}$. Similarly to treatment of small lesions, laser treatment for advanced colorectal carcinoma is generally performed on an outpatient basis. Patients are treated every 2 to 4 days until the initial treatment goal is realized. In most cases, according to Mellow (1994) the goal is: simply to create a lumen sufficient for the effective passage of faeces ${ }^{|53|}$. Once the initial series of treatments has been completed, patients return at 6 to 8 weeks for repeat evaluation. Thereafter patient visits are scheduled depending on tumour regrowth characteristics and the ease of maintaining an adequate lumen. Usually, these periods do not exceed 10 weeks $^{[38,53]}$.

A large, well-documented case-series by Brunetaud et al. (1990) will be discussed as an example $e^{|(\mid)|}$ In the period 1979-1989 the authors treated 200 patients, of whom 182 were treated for an advanced tumour (in the previous section the results of laser treatment of 18 patients with early' lesions were discussed). The majority of the patients with advanced carcinomas $(n=139 ; 77 \%)$ were referred for laser treatment when they were considered inoperable. One hundred-nine of this subgroup of patients had metastases. Twenty patients $(11 \%)$ had a colostomy and abnormal discharge, 17 patients ( $9 \%$ ) had a recurrence after surgery and 6 patients $(3 \%)$ refused surgery. Of the 182 patients with an advanced lesion, in $159(88 \%)$ palliation was successful after an average of 2.5 laser treatment sessions. The average period of treatment was 15 days. In 128 patients in whom treatment was stopped the average duration of improvement of symptoms was 10.4 months. Of these patients, $77(60.2 \%)$ remained improved until their death, 60 patients dying from the cancer and 17 patients dying from other causes. At the time of writing, 31 patients were still followed. The average duration of improvement of symptoms in these patients was 13.4 months. There were five complications $(2.7 \%)$ after 2168 laser sessions. There were two perforations at the rectosigmoid junction $(1.1 \%)$, and one of these patients died $(0.5 \%)$. One patient developed a perirectal abscess $(0.5 \%)$ and there were two recto-vaginal fistulae $(1.1 \%)$.

As the number of studies using (Nd:YAG) lasers for this indication increased in the eighties, several authors tried to summarize treatment results. Mathus-Vliegen and Tytgat (1986) evaluated the experience of 7 laser centers with 181 patients with advanced colorectal carcinoma. Of the 181 patients, 60 presented with obstruction, 81 with severe bleeding, and 40 with both. Palliation was successful in 89 percent. The complication rate was 9.4 percent. Laser-related mortality occurred in 2 patients $(1.1 \%)^{[42]}$.

In his 1995 review. Nishioka ${ }^{1591}$ discusses a more recent review on this subject by Mathus-Vliegen (1991), in which she reported that the results of laser treatment were most satisfying in patients with bleeding as the predominant symptom ${ }^{\text {lims }}$. In over $90 \%$ of these patients laser treatment was successful compared to a $75 \%$ success rate in patients with predominantly obstructive symptoms. Complication rates were reported to vary between 0 and $13 \%$ with mortality rates between 1 and $3 \%$.

In another review, Banta (1993) Banta reported that the majority of patients remain symptomatically improved after laser therapy of colorectal carcinoma ${ }^{|3|}$. Overall success rates of laser treatment were in the range of 75-80 percent in terms of short-term relief of symptoms, and fell to 65-70 percent over the patient's lifetime. The success rate was higher for patients with bleeding or rectal discharge than for obstruction. These results basically confirm the findings of Mathus-V/iegen ${ }^{[4 !}$. In addition, many authors report that circumferential tumour extension is the only significant prognostic factor, with deteriorating success rates as the circumferential extent of the tumour
increases
$\mid 6,35,53 !$

Mellow (1994) compiled complication rates from surveys of "experienced laser endoscopists': including 500 patients ${ }^{\mid 53]}$. Anal stenosis was reported in 21 patients $(4.2 \%), 19$ patients needed surgery after failure of laser treatment $(3.8 \%)$, in 15 patients a local perforation occurred $(3.0 \%)$, 
while a free abdominal perforation occurred in 5 patients $(1.0 \%)$. Ten patients required bload transfusion (2.0\%) while 16 patients lost more than $100 \mathrm{ml}$, of blood without the need for transfusion ( $3.2 \%$ ). Fecal incontinence occurred in 5 patients $(1.0 \%)$, and 3 patients required narcotic analgesia for pain relief $(0.6 \%)$. Six patients died of laser-related causes $(1.2 \%)^{[53]}$.

McGowan et al. (1989) examined whether laser palliation for inoperable rectal cancer improves the quality of life and the dying process experienced by patients ${ }^{\text {i50! }}$. Quality of life was measured prospectivelly in 14 patients before, during, and after treatment using the Quality of Life index and a linear analogue self assessment (LASA). The authors concluded that in patients where local symptoms are predominant and well-controlled, the quality of life scores show consistent improvement. On the other hand, patients suffering from pain and the general effects of malignant disease do not benefit greatly, and the quality of life scores deteriorate. Bown made a similar statement in his 1991 review $^{[39]}$.

Overall, Nd:YAG- or argon- and Nd:YAG laser treatment is considered to be established by clinical experience in palliation of patients with advanced colorectal carcinoma. As a result of avallable clinicall data, there is a debate in literature both on the indications for surgery versus laser treatment and on the non-surgical treatment modality of choice if surgery is judged impossible. To start with the first theme, it has been estimated that approximately $10 \%$ of all patients with rectal adenocarcinoma would be better served by endoscopic laser therapy than by surgery. Patients most likely to benefit preferentially from laser treatment include those with unresectable metastases. and those whose medical condition would preclude surgery or make it an unduly high risk ${ }^{153,89 !}$. Another specific example is the group of elderly patients who are considered operable but whose tumour is unresectable. When surgery is applied, most of these patients receive a defunctioning colostomy. This operation, which is associated with a mortality around $6 \%$, rellieves obstructive symptoms but is of little value in palliating rectal bleeding, discharge, and tenesmus. Secondly, formation of a permanent colostomy is associated with a prolonged recovery period in patients with a limited survival. Thirdly, quality of life of stoma patients is impaired compared to non-stoma (surgical) patients ${ }^{[75]}$, suggesting even bigger differences in quality of life between stoma patients and non-surgically treated patients. This is relevant because laser therapy is often claimed to have prevented the need for a permanent colostomy in a considerable number of these patients ${ }^{\mid 3 \theta\}}$. Other authors, however, arrive at the conclusion that surgical treatment results in high-risk elderly patients are acceptable even in the presence of advanced disease ${ }^{|20|}$.

As a consequence of this debate, although clinical experience with the Nd:YAG laser is limited to patients who are considered to be unfit for surgical palliation, some authors have tried to compare treatment results after surgery and laser therapy. Meilow (1989) reported the results of 35 patients with surgical treatment and 21 with laser therapy for colon cancer ${ }^{514}$. Laser therapy was selected because of advanced metastatic disease ( 10 patients, $47.6 \%$ ), advanced clinical disease plus other medical problems ( 9 patients $\mathrm{s}_{i} 42.8 \%$ ), and refusal of surgery ( 2 patients, $9.6 \%$ ). Eight of the 35 patients treated with surgery had metastatic disease $(90.6 \%)$. The laser treated patients were 78 years old on average, and the surgically treated patients were 67 years old on average. Of the 35 surgically treated patients, 19 suffered 25 complications $(54.3 \%)$, and five required re-operation for complications (14.3\%). Three of the 21 laser patients had laser-associated complications $(14.3 \%)$; two had bleeding, and two developed a perirectal abscess. One patient died of pulmonary embolus after a laser treatment session $(4.8 \%)$, and two patients treated by laser became obstructed and required surgery $(9.5 \%)$. The author recognized that the two groups as a whole were not comparable, so compared mortality only for patients with advanced metastatic disease. Mean survival for the 10 patients treated by laser was 36 weeks, while that of the 8 patients treated by surgery was 28 weeks. Of course, these numbers are merely indicative. Obviously, more studies are needed in this area.

With respect to the discussion on the non-surgical treatment modality of choice if surgery is judged impossible, many authors can be cited who, in the absence of RCTs, have pointed cut that laser treatment offers the advantage over ather non-surgical modalities that it can be applied from the 
anus up to the coecum in ambulatory patients without the need for general anesthesia Furthermore, the use of lasers is neither limited by the volume of tissue nor by the accumulation of dose $\mathrm{e}^{[4,69]}$. For these reasons laser therapy has been claimed to be the most wersatile non surgical palliative method ${ }^{\left.15969^{6}\right)}$. Loizou and co-authors (1990) took a stronger position on this issus when they formulated the recommendation to use laser treatment as the treatment of choice in palliation of rectal carcinoma in patients not suitable for surgery ${ }^{323}$. Suggestions for RCTs comparing different (combinations of) non-surgical treatment modalities have been made, e.g. lase treatment with or without radiotherapy. Other authors have tried to retrospectively compar treatment results using laser versus electrofulguration, as discussed for example by Mellow $(1994)^{153 !}$. Of course, no definite statements can be made. Of the available options, cryotherapy contrany to laser therapy is limited to tumours below the peritoneal reflection of the recturn. It can just as laser therapy, be applied without general anesthesia, is well tolerated and can produce good tumour debulking. Electrofulguration and curettage requires general or regional anesthesila and also cannot be used above the peritoneal reflection. Radiotherapy (external or intracavity) can produce excellent results in small rectosigmoid tumours. In general, the results for palliation of advanced tumours with this modality are much less satisfactory ${ }^{\mid 8 \%}$. Clearly, more studies are needed in this area as well.

\section{Cost-effectiveness}

There are no data on the costs of laser treatment versus surgery in patients with either or advanced colorectal carcinoma. The latter comparison is the most complicated due to the controversy over indications. Similarly, there are no reliable data either comparing the costs of alternative non-surgical modalities in treatment of advanced lesions, although it has been suggested that electrocauterization is associated with higher morbidity and costs than laser therapy because the former procedure is performed in the operating room under general anesthesia with longer post-procedure recuperation than with laser therapy $y^{[52.53]}$.

Mellow (1987, 1989) made cost estimates based on his experience in a private University-affiliated hospital in the Southwestern United States. He calculated the cost for three groups: those admitted for surgery, those admitted for laser therapy, and those receiving laser therapy on an out-patient basis. Laser costs assumed three treatments the mean number in his institution. In his first report $\mathrm{t}^{152]}$, he found that surgical patients required 17 days hospitalization, and incurred expenses of $\$ 19767$, including $\$ 2267$ in surgical fees, $\$ 2590$ for the hospital room, $\$ 1650$ for the intensive care unit, and $\$ 1183$ for the operating room. Inpatient laser therapy was associated with a hospital stay of 5 days, and cost $\$ 4483$. Outpatient laser therapy cost $\$ 2460$. In a later report ${ }^{\$ 4}$ " he calculated that the mean total cost of hospitalization for the surgery group was $\$ 23156$, with range of $\$ 2331$. The laser patients treated with a hospitalization had a mean cost of $\$ 5333$, with a range of $\$ 391$. The mean cost in five patients with outpatient laser therapy was $\$ 2263$, with a range of $\$ 367$. Again, these groups are likely to be incomparable and merelly indicative of the costs that would actually result if surgically treated patients would have been scheduled for laser therapy instead. In addition, of course, dlata on costs would have to be related to data on effectiveness. Vondeling et al. (1991) compared the palliation of advanced colon cancer by laser and surgery in a retrospective study using matched historical controls from a laser and a non-laser university hospital in the Netherlands ${ }^{|(13)|}$. The main difference between the center that used an Nd:YAG laser and the one without this device was that in the latter case patients were operated upon in case of advanced metastases at the time of diagnosis. The two forms of palliation were found to be roulghly similar in their outcomes. The average cost of laser therapy, including the cost of follow-up, was US $\$ 9200$. The averagle cost of surgery, including follow-up, was US\$15 100 .

In summary, preliminary cost information indicates that laser palliation for obstructing colorectal cancer is an economic alternative to standard surgical therapy. Quality of life for pattents with advanced colon cancer could be improved with such therapy. RCTs are urgently needed to test both effectiveness and cost-effectiveness of lasers in palliation of advanced colorectal cancer. 


\subsection{Colorectal adenomas}

\subsubsection{Introduction}

Patients with colorectal adenomas may present with symptoms such as mucous discharge, diarthea, hematochezia, abdominal cramps, mild illeus and fecal incontinence ${ }^{[4]}$. Complete removal of colorectal adenomas is advised not only on the basis of symptoms, but also to prevent subsequent malignant transformation ${ }^{(8)}$. In the US, it has been estimated that one-third of the population have villous adenoma and that approximately $5 \%$ of these will become malignant if not removed ${ }^{[8]}$. Prognostic factors for malignant behaviour include villous types adenoma, large size; poor differentiation and severe dysplasia ${ }^{[4] \mid}$. More specifically, malignant transformation has been estimated to occur in $0.1 \%$ of diminutive polyps (less than $0.5 \mathrm{~cm}$ in diameter) ${ }^{[3]}$ and in $26-40 \%$ of llarge villous adenomas ${ }^{i 89 ?}$. The majority of adenomas will therefore be benign. Standard treatment for benign adenomas used to be surgery but this has largely been replaced by endoscopic techniques because this is less invasive. One of the most frequently used techniques is snare polypectomy, which is associated with a mortality rate of $0.05-0.1 \%$ and a complication ate of $0.2-1.8 \%$. Complications include predominantly hemorrhage and perforation ${ }^{[47}$. As a consequence of the advantages of endoscopic treatment of benign lesions, surgery remained standlard treatment only for melanomas with severe dysplasia and malignant polyps at diagnosis ${ }^{[09]}$. In the eighties and nineties, many authors have started questioning the role of surgery for this indication as well, favouring endoscopic treatment, including laser treatment. What is the role of lasers here?

In the late seventies, lasers have been introduced in treatment of benign adenomas as a logical next step to treatment with snare polypectomy, in particular because sessile lesions are often incompletely excised and likely to recur ${ }^{[47,69]}$. At the same time, many authors have pointed at a fundamental difficulty with laser treatment of melanoma which is that, contrary to surgery, no specimen is available for complete histologic study. Consequently, a tumour with an undetected invasive cancer could be inappropriately treated with laser. In other patients a melanoma that transforms to a malignancy during the period of laser treatment may be missed. Pre-treatment biopsies or regular biopsies during the treatment period can not conclusively rule out this possibility. Therefore in patients presenting with benign melanoma on biopsy, the risk of missing an invasive carcinoma or a malignant transformation should be balanced against the risks of surgery ${ }^{[6,7,21,47,89]}$. The magnitude of this problem and some proposed solutions will be discussed. To start with, the evidence on the safety and effectiveness of laser treatment of patients with benign melanoma will be addressed.

\subsubsection{Benign llesions}

Several treatment techniques are employed by different authors. Some investigators, e.g. MathusVliegen $(1988)^{[4]}$, use laser treatment as a combination treatment after snare polypectomy while others, e.g. Brunetaud and associates (1990) use laser treatment as a stand-alone technique for all types of lesions ${ }^{[6]}$. The largest case-series has been published by Brunetaud et al. in 1990, who reported results of 313 patients who received laser treatment for rectosigmoid villous adenoma in the period 1979-1989 in Lille, France ${ }^{i 5 !}$. It should be noticed that patients with these lesions have the highest chance of malignant transformation of all patients with adenomas. The indications for laser treatment were: patient inoperability $(n=127,41 \%)$, patients for whom surgical resection seemed too drastic for a tumour found benign on biopsy $(n=119,38 \%)$, recurrent tumour after a non-laser treatment $(n=64,20 \%)$ and patient refusal of surgery $(n=3,1 \%)$. Patients received treatment on an outpatient basis twice a week until complete destruction of the adenoma was achieved. Coagulation of the bulky parts of the adenomas was preformed using an Nd:YAG laser While the argon laser was used to vaporize smaller areas. The mean age of these patients was 72 years (range $32-96$ years). Results of 46 patients could not be analysed due to loss to follow-up, 
mortality due to another etiology during treatment, and unfinished laser treatment at the time of writing, resulting in an analysis of data of 267 patients. Initial treatment success was achieved 245 patients $(92 \%)$ after an average of 6 treatments over a period of several months. Treatmeri failed in 3 patients $(1 \%)$, and 19 patients $(7 \%)$ had positive biopsies for cancer during the treatment: This group of patients will be discussed separately. Of the 245 successfully treated patients, 2 suffered from fever for 2 days and 5 developed a stenosis requiring dilatation ( $3 \%$ ). Ih 35 of these 245 patients $(14 \%)$ the adenoma recurred after an average follow-up of 16.3 months. Two patients had two recurrences. By the life table analysis method the recurrence rate was $11 \%$ at 12 months and $22 \%$ at 24 months. In 32 patients $(91 \%)$ repeated laser treatment was successfit after an average of 2.8 sessions. At the time of writing one patient was still under treatment. In the last two patients, a cancer was biopsy detected. These patients were operated upon. In the 3 patients in whom treatment failed this was due to the impossibility to pass an endoscope through a stenosis in one patient, development of a stenosis as a result of laser treatment in one patient, and intolerance of the procedure in one patient.

The authors compared their results with four other series (by Escourrou et al. (1987), Mathus: Vliegen et al. (1986), Naveau et al. (1986), and Souquet et al. (1987)) including in total 269 patients $^{\{1+, 43,56,74||}$. The initial success rate in these series ranged from 72 to $89 \%$, with complication rates ranging from 6 to $15 \%$. Complications included hemorrhage, stenosis, perforation and the development of fistulae. None of these complications was fatal. Recurrence rates varied from $3.8 \%$ during an 8.4 months follow-up to $46 \%$ after a follow-up of 2 years. All recurrences weire successfully treated in all but one series, where retreatment failed in $66 \%$ of patients. The authors concluded that laser treatment for adenoma is safe and effective. The relatively good results in theil own case-series was attributed to the preferential location of the adenomas in the rectosigmoid and to the use of a combination of an argon and an Nd:YAG laser. In other series exclusively an Nd:YAG laser was used ${ }^{[7]}$.

Brunetaud et al. do not specifically address how they incorporated alleviation of symptoms in their definition of successfultreatment, which seems to be based only on removal of the adenoma. According to e.g. Mathus-Vliegen (1989) laser treatment alleviates symptoms in nearly all patients. after a few treatment sessions ${ }^{\mid 45 !}$.

\subsubsection{Adenomas that transform to mallignancies during the period of laser treatment}

In the series reported by Brunetaud (1990) 19 patients (7\%) had a positive biopsy during the period of laser treatment. In these patients, a pre-treatment biopsy had not revealed any malignancy. Nine of these patients could not tolerate surgery and continued to be treated by laser for palliation of symptoms. However, complete destruction of the tumour with negative biopsies was obtained in four patients without recurrence during an average period of follow-up of 22 months (range 2-60) months). One patient died from bronchogenic cancer, 2 died from rectal cancer, and two laser treated patients were still followed-up at the time of writing, at 4 and 15 months, respectively. Nine other patients had surgery, and the last patient had radiotherapy. Although the rate of undetected cancer in their series was $7 \%$, only half of these patients could be operated upon, while delaying surgery for a period of a few months in the remainder of patients. The authors concluded that the risk of missing an invasive carcinoma, $3.5 \%$, in their series, should not be overestimated. In othei patient-series the undetected cancer rate varied from 0 to $15 \%$. This should be balanced against the risk of surgery, which has a mortality rate of $2.5 \%$, a complication rate of $18.5 \%$ and recurrence rate ranging from 3.6 to $42 \%$. Balancing these risks, Brunetaud et al. suggested that laser treatment should be selected for frail elderly patients with rectosigmoid adenomas, provides that these patients are not eligible for transanal surgical techniques, and that the circumferential extension of the tumor is no more than $2 / 3$ of the entire lumen ${ }^{17}$. Other authors, e.g. Mathus. Vliegen (1989) basically confirm these criteria, but do not by definition exclude laser treatment if other parts of the colon than the rectosigmoid region ${ }^{[45 !}$. Notwithstanding these carefit considerations, treatment by snare polypectomy has never been compared with laser treatmen! 
as part of an RCT ${ }^{\text {ies! }}$ Such a comparison is needed. Depending on for example the size and location of the lesion one could think of a comparison of both techniques in a stand-alone design or snare polypectomy versus snare polypectomy followed by laser treatment.

\subsubsection{Malignant lesilons}

Williams and Saunders (1993), report that endoscopic management has become standard in patients with malignant polyps at the time of diagnosis. However, the endoscopist is advised not to attempt to treat larger malignant polyps in the rectum who fall into the sphere of the competence of the proctologist. Secondly, these authors claim that endoscopic treatment is becoming more widely accepted in elderly patients with sessile malignant lesions who are at high surgical risk ${ }^{[87 !}$. This discussion is of course closely related to the discussion on clinical decision making in case of malignant transformation of an adenoma that is benign at biopsy. In both cases, the definition of inoperability and the prognosis of patients in alternative management strategies play a central role. With the advent of new surgical techniques less patients will be deemed inoperable and with the advent of new diagnostic techniques a more precise distinction will be enabled between benign and malignant tissue. As progress is made in both surgical and endoscopic techniques, the current debate is expected to continue.

\section{Cost-effectiveness}

Waye (1987) compared the cost of removal of a polyp by endoscope to that of surgical removal in a Midwestern university hospital in the United States ${ }^{[86 !}$. Surgicall removal requires 7 days of hospitalization. Endoscopic removal is usually performed as an out-patient procedure. The endoscopic polypectomy was estimated to cost US\$1036, compared to $\$ 7590$ for surgical removal. Brunetaud et al. (1989), estimated direct costs of laser therapy versus conventional surgery, using hospital charges, physician fees, and charges for ambulance transportation in Lille, France, and Los Angeles ${ }^{[7]}$. The costs of both treatment modalities correlated positively with increasing circumferentiall extension of the adenoma base. However, the relative difference in costs of both treatments decreased as the circumferential extension of the adenoma base increased. The laser costs included a range of 3.2 to 13.5 treatments and 3 to 4 control endoscopies without treatment and the corresponding ambulance transportation. The surgical costs included the costs of surgery and a range of 6 to 14 days of hospitalization. This resulted in laser charges varying from 28 to 40 percent of the cost of conventional surgery in Lille, and from 31 to 69 percent of the cost of conventional surgery in Los Angeles. The main cost difference was because laser therapy was done on an outpatient basis.

In summary, preliminary evidence suggests that laser treatment may be cost-effective compared to surgery in patients with colorectal adenoma. More evidence is needed on this subject in carefully selected patient groups. Research on the most cost-effective endoscopic treatment strategy in those patients where the choice for conservative management is not controversial, e.g. in patients with benign lesions at biopsy, would benefit a relatively large number of patients.

\subsection{Hemorrhoids}

Hemorrhoids appear as a consequence of dilatations or varicosities of the upper and lower hemorrhoidal venous plexus, in the wall of the anal channel, and in the lower part of the rectum. Clinical hemorrhoids are characterized by bleeding after defecation with or without prolapse of the mucosa. Hemorrhoids are also associated with symptoms of pain, secretion and anal pruritis (itching) $^{1 / 99}$. In the United States, in the mid-eighties, 10 million people complained of hemorrhoids, corresponding to a prevalence rate of $4.4 \%$. In both sexes, a peak in prevalence was noted from age 45-65. Of these, about one third presented to physicians for evaluation. Hemorrhoids rarely lead to significant morbidity. Hospitalization accurs in only 12.9 per million population ${ }^{\text {[39! }}$. In the 
Netheriands, the incidence of hemorrhoids is estimated at $8 \%$ and the prevalence at $10 \%$. The male-female ratio is $1: 4$. On an annual basis, 50,000 patients are referred to hospital for treatment of hemorrhoids in this country ${ }^{28}$.

Hemorrhoids are classified according to four stages. in grade llesions, the hemorrhoidal tissue is: present and identifiable but rarely symptomatic, although these lesions may become irritated and cause bleeding. Grade II hemorrhoids actually prolapse with a bowel movement but return spontaneously, whle grade 111 hemorrhoids require manulal replacement. With grade $\mathrm{N}$ hemorrhoids, the tissue remains outside the anus despite all efforts to replace $i^{165 !}$. In recent literature the clinical value of this classification has been questioned due to the advent of a number of new therapeutic modalities ${ }^{1263}$. Conservative treatment, which is usually started with, includes promotion of a high-fiber diet, adequate fluid intake and a change in bowel habits. It has been: estimated that half of the patients presenting with hemorrioids can be successfully treated this way ${ }^{z e i}$. Treatment options that provide a temporary relief of symptoms include for example application of local anesthetics and local corticosteroids. Outpatient-and office treatment modalities that are intended to result in a permanent relief of symptoms include manual dilatation of the anus, rubber band ligation, bipolar electrocoagulation, low-voltage direct current coagulation, injection sclerotherapy, cryotherapy and infrared photocoagulation. Inpatient procedures include e.g. sphincterotomy and hemorrhoidectomy ${ }^{28,651}$. What is the role of lasers here?

According to Trelles et al. (1991), both the $\mathrm{CO}_{2}$ and the Nd:YAG laser are increasingly used in treatment of stage III and stage IV hemorrhoids, in principal as a substitute for the scalpel and the electroscalpe ${ }^{[79]}$ in these cases the laser is applied as part of the conventional surgical procedure which, according to these authors, is predominantly the Milligan-Morgan technique. This is an open hemorrhoidectorny based on resection of the hemorrhoids with a ligature below the pedicle. In contrast with the conventional surgical procedure, the laser-based surgical procedure does not require general anesthesia and can be performed on an outpatient basis or in the office. Based on an assessment of case-series, the authors concluded in a 1991 assessment that, compared to conventional surgical therapy, laser treatment offers the advantages of less post-operative pain. less intra- and postoperative hemorrhage, reduced or avoided post-operative edema, anal discharge and urine retention, more rapid wound healing and reduced recurrences. The authors noted that these observations need to be confirmed by RCTs, which had not (yet) been published at that time. For an appropriate assessment, it should be noticed as well that in many reports of case-series, lasers are used in treatment of both stage $11,11 !$ and stage IV hemorrhoids ${ }^{[25,75]}$.

In 1995, MacRae and McLeod published a meta-analysis of RCTs comparing two or more treatment alternatives for symptomatic hemorrhoids ${ }^{[41]}$. Based on a Medline search covering the period 1966 to February 1994, 32 RCTs were retrieved. The quality of the trialls was assessed, resulting in the inclusion of 18 studies for analysis. The excluded trials included the only two studies that focused on laser treatment. One study, by Senagore et al. (1993); compared Nd:YAG laser excision to scalpel excision when performing a standard Ferguson-closed hemorrhoidectomy in patients with stage III or stage $\mathrm{V}$ lesions ${ }^{\mid 73)}$. In the other study, by Wang et al. (1991), $75 \%$ of the patients enrolled suffered from stage III or stage IV hemorrhoids ${ }^{|15|}$. The study was similar in design and the same surgical procedure was applied as in the study by Senagore et al., but in the study by Wang et al. Nd:YAG laser coagulation was applied in internall hemorrhoids, while $\mathrm{CO}_{2}$ laser vaporization was applied in external hemorrhoids ${ }^{43 !}$. The reasons for exclusion of these studies in the meta-analysis were an extremely short follow-up period without specification of efficacy in the study by Senagore and colleagues, and inadequate randomization in the study by Wang and colleagues. In the latter study "randomization was done according to the day of the patient's initial visit to the clinic. In the trials which were included in the meta-analysis, virtually all patients suffered from grade $\mathrm{I}$ II or III hemorrhoids. The result of the meta-analysis were interpreted as showing that rubber band ligation is therapy of choice in patients with hemorrhoilds, irrespective of its grade. Hemorrhoidectomy was recommended to be reserved for patients whose symptoms are not rellieved ${ }^{\text {(41? }}$. 
At least one more RCT has been published comparing Nd:YAG laser treatment and surgical ireatment. This study, by Enriquez-Navasicues et al. (1993), was not considered in the meta-

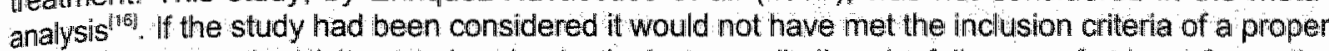
randomization method (alternate 'randomization" was applied) and a follow-up of at least 6 months was not completed in the majority of patients. It can be concluded that, based on available data from RCTs, there is insufficient high quality evidence to draw conclusions on the relative safely and efficacy of laser treatment versus conventional surgical hemorrhoidectomy.

How is the evidence on this issue judged in the Netherlands? In late 1994, a consensus statement on diagnosis and treatment of hemorrhoids was published by the Dutch National Organisation for Quality Assurance in Hospitals $(\mathrm{CBO})^{[28]}$. Conservative treatment was recommended as the initial treatment of choice. In patients in whom no relief of symptoms is achieved after conservative treatment, rubber band ligation was recommended. Depending on the characteristics of the hemorrhoids, rubber band ligation could be supplemented with either injection sclerotherapy or infrared coagulation. Hemorrhoidectomy should only be applied in those patients where other treatment options fail, which was considered highly unlikely. No considerations at all on laser treatment were included in the consensus statement. Other authors, e.g. Pfenninger and Surrell (1995), in line with the meta-analysis and the consensus statement discussed above, conclude that rubber band ligation is effective and remains the standard by which newer methods are compared ${ }^{\mid \theta 5}$.

In conclusion, in comparison with other modalities, laser treatment of hemorrhoids is not wellevaluated in RCTs. Although its use is established by clinical experience it is unclear which laser, the $\mathrm{CO}_{2}$ laser or the Nd:YAG laser, should be selected to achieve best results.

\section{Cost-effectiveness}

The cost of the equipment required for rubber band ligation ranges from US $\$ 250$ to $400^{[65]}$. Band ligation, according to Pfenninger and Surrell (1995), is easilly mastered ${ }^{165}$. Although two persons are required for the procedure - the operator and the assistant who holds the anoscope in place it will be difficult for any new treatment modality to be more cost-effective. This implies that whenever lasers are used in treatment of mild hemorrhoids, that is in cases where other modalities such as rubber band ligation could have been used as well, its use is unlikely to be cost-effective. For this reason Pfenninger and Surrell, while stating that neither the $\mathrm{CO}_{2}$ - nor the Nd:YAG laser provides distinct advantages over other outpatient methods, recommend the use of lasers exclusively in larger multispecialty groups ${ }^{[65]}$.

This does not address the potential cost-effectiveness of lasers as an alternative for conventional surgical hemorrhoidectomy. Trelles and colleagues (1991), assuming equal effectiveness of both procedures, calculated the costs of outpatient $\mathrm{CO}_{2}$ laser treatment to vary from 43300 to 47200 Spanish pesetas in a public outpatient clinic, compared to a minimum of 57900 and a maximum of 75000 pesetas in a public hospital ${ }^{[39,81]}$. The capital costs of the laser were depreciated in two years time, which is relatively short. The operating costs of the laser were calculated exclusively based on the annual number of treatments of hemorrhoids. For conventional surgery a hospitalization period of 3 days was assumed. Sick leave was estimated to vary from $7-12$ days after laser hemorrhoidectomy, compared to 20-25 days after conventional hemorrhoidectomy ${ }^{\text {rot }}$. Other authors have suggested that laser treatment results in higher costs than conventional treatment, without providing clear data ${ }^{[73]}$. It could be argued that laser treatment is more costly than conventional treatment when two separate lasers are used, such as in the study by Wang and colleagues $^{105 !}$. However, these authors failled to take the capital costs of the lasers into account in a calculation of the direct health care costs of laser treatment (US\$925) and conventional surgery (US\$1170). The difference was mainly based on reduced hospital stay after laser treatment ${ }^{(195)}$. Overall, combining all evidence, the use of lasers could potentially be cost-effective for this subindication. 
In summary, follents wh mith hemorhoids the application of tasers is unithely to be costeffective compared to rubber-band ligation and oher madalities. In patients whth severe disease: however, its use may be potentally be cost-effectve as a substitute for inpatient surgical hemorhoidectomy. Additional data would be desirable, In general, with the declining role of surgical hemormoidectomy, the role of laser treatment tor this indication seems to be decreasing as well. The potential for cost-effective use of lasers would therefore be increased when such a facilly would be made avallable on a regional basis.

\subsection{Summary}

Table 8.1 provides a summary of the findings presented in this chapter.

Table 8.1 Judgments on the effectiveness and cost-effectiveness of selected laser applications in gastroenterology

\begin{tabular}{|c|c|c|c|c|c|c|c|}
\hline \multirow[t]{2}{*}{ Appllcation } & \multicolumn{7}{|c|}{$\begin{array}{c}\text { Judgments } \\
\text { (see definitions below) }\end{array}$} \\
\hline & 1 & 2 & 3 & 4 & 5 & 6 & 7 \\
\hline Hemostasis of gastric ulcer & & & & $x$ & $x$ & & \\
\hline $\begin{array}{l}\text { Hemostasis of intestinal vascular } \\
\text { malformations }\end{array}$ & & $x$ & & & & & \\
\hline Cancer palliation of esophagus & & & $x$ & & & & \\
\hline Cancer palliation of colon & & & $x$ & & & $x$ & \\
\hline Sessile villous adenoma & & & $x$ & & & $x$ & \\
\hline $\begin{array}{ll}\text { Hemorrhoids } & \text { - mild cases } \\
& \text { - severe cases }\end{array}$ & & & $\begin{array}{l}x \\
x \\
\end{array}$ & & $x$ & $x$ & \\
\hline
\end{tabular}

Definitions:

1 Experimental $=$ still in development, limited clinical data, no proof of effectiveness.

2 Promising $=$ some clinical data, but procedure not accepted as a routine.

3 Established by clinical experience $=$ no randomized trials, but sufficient clinical data to reach a judigment.

4 Established by RCT = established by several well-designed randomized controlled trials.

5 Probably not cost-effective = limited data on cost-effectiveness, but reason to believe that the procedure will not turn out to be cost-effective.

6 Potentially cost-effective = limited data on cost-effectiveness, but reason to believe that the procedure will furn out to be cost-effective.

7 Proven cost-effective $=$ adequate analysis of cost-effectiveness. 


\subsection{References}

1. Alderson D, Wright PD. Laser recanalization versus endoscopic intubation in the palliation of malignant dysphag ia. British Journal of Surgery $1990(77): 1151-3$.

2. Baneilee $A_{1}$ Shorthouse $A$. Towards ewen fewer colostomies ... (commentary) Lancet 1995(346),859.

3. Banta HD. The cost-effectiveness of 10 selected applications of Minimally lnwasive Therapy. In: Banta $H \mathrm{C}$ (Ed.). Minimally invasiwe therapy in five European countries. Diffusion, Effectiveness and Cost.Effectiveness. Health Policy Monographs Vol. 3. Elsevier, Amsterdam, London, New York, Tokya, 1993, p. 135-51.

4. Barr H. Krasner N. Raouf A, Walker RJ. Prospective randomised trial of laser theirapy only and lasier therapy followed by endoscopic intubation for the palliation of malignant dysphagla. Gut 1990(31):252-8.

5. Eonneux J, Barendregit $J J$, Looman CWN, Van der Maas PJ. Divenging trends in colorectal cancer morbidity and mortality. Earlier diagnosis comes at a price European Journal of Cancer 1995(31|A)10:1665-71.

6. Brunetaud $\Omega M_{2}$, Manoury $V$, Cochelard D, Cortot $A$, Paris JC. Lasers in rectosigmoid tumors. In: Trelles MA (Ed.). Laser tumour therapy. European Commurity Medical Laser Concerted Action Program, Second Plenary Workshop. Cambrils/Tarragona, 28-29 November 1989. Ilustre Colegio Oficial de Médicos de Madrid "Spain, 1990, p. 5-17.

7. Brunetaud $\mathrm{JM}_{4}$, Manoury V, Cochelard D, Boniface $B$, Cortot A, Paris JC. Endoscopic laser treatment for rectosigmoid villous adenoma: factors affecting the results, Gastroenterology 1989(97):272-7.

8. Buset M, Des Marez B, Vandermeeren $A_{1}$ Baize $M_{4}$ Cremer $M$. Laser therapy for non bleeding vessels in peptic ulcer hemorrhage: a prospective randomized study (abstract). Gastrointestinal Endoscopy 1988(34):173-4.

9. Carter R, Smith JS. Oesophageal carcinoma: a comparative study of laser recanalization versus intubation in the palliation of gastro-esophageal carcinoma. Lasers in Medical Science 1986(1)4:245-51.

10. Carter $R, S m i$ th $J S$, Anderson JR. Laser recanalization versus endoscopic intubation in the palliation of malignant dysphagia: a randomized prospective study. British Joumal of Surgery 1992(79):1167-70.

11. Chia YW. Ngot $S S_{n}$ Goh PMY. Endoscopic Nd:YAG laser in the palliative treatment of advanced low rectal Garcinoma. Diseases of the Collon \& Rectum $1991(34) 12: 1093-6$.

12. Cook D., Guyatt GH. Salena B.J. Laine LA. Endoscopic therapy for acute monvaticeal upper gastrolintestinal hemorthage: a meta analysis. Gastroenterology 1992(102):139-48.

13. Diagnostic and Therapeutic Technology Assessment (DATTA Report). Journal of the American Medicall Association $1985(253): 2732-5$

14. Donald $J J_{1}$ Burhenne HJ. Colorectal cancer. Can we lower the death rate in the 1990 s? Canadian Family Physician 1993(39) 1:107-14.

15. Ellis P, Cunningham D. Management of carcinomas of the upper gastrointestinal tract. British Medica\# Jounal 1994( $(308): 834-8$.

16. Enriquez-Navascules JM. Devesa Mugica JM, Bucheli Proano P. Hemolroidectomia: convencional a con laser Nd:YAG de contacto? Estudio prospectivo y randomizado. Revista Espanola de Enfermedades Digestivas $1993(34) 4: 235-9$

17. Escourrou J, Delvaux $M$, deBellison $F$, Rumeau $J L$. Frexinos J, Ribet A. Laser for curative treatment of rectal cancer. Indications and follow-up (abstract). Gastirointestinal Endoscopy 198:8(34)2:195.

18. Escourrou J, Delvaux M, de Bellison F. Traitement par laser Nd:YAG des tumeurs villeuses rectales: experrience de 57 cas (abstract). Gestroenterologie Clinque et Biologique 1987(11):276.

19. Escourrou J. Endoscopic control of gastrointestinal hemorrinage. In: Papp JP (Ed.). European clinical experience in laser phatocoagulation in upper gastrointestinal tract. Boca Reton, FI: CRC, 1981, p. 103-10.

20. Fitzgerald SD, Longo WR Gayle R, Daniel RN, Vernava II, AM. Advanced colorectal neoplasia in the thigh-risk eiderly patient. Is surgical resection justfied? Diseases of the Colon \& Rectum 1993(36)2:161-6.

21. Fleischer DE. Concluding remarks: Endoscopic resection of gastrointestinal tumors. Endosicopy 1993(25):479-81.

22. Fleischer DE. Endoscopic laser therapy for esophageal cancer: present status with emphesis on past and future. Lasers in Surgery and Medicine 1989(9):6-16.

23. Fleischer DE. Esophageal ciancer: laser treatment In Ad Hoc Commitiee on Technology Assesisment. Therapeutic gastrointestinal endoscopy. American Society for Gastrointestinal Endoscopy Manchester, Masisachusetts, 1987. p. 29-31.

24. Heier SK, Rothman KA, Heier LM, Rosenthal WS. Photodynamic therapy for obstructing esophageal cancer: IIght dosimetry and randomized comparison with Nd: YAG laser therapy. Gastroenterology 1995(109):63-72.

25. Wollis JB. Office $\mathrm{CO}_{2}$ laser hemonhoidectomy on 200 patients by a gastroenterologist: a complete approach (abstract). Gastroenterology 1994(106)4:A11.

26. Horton R. Oesophageal cancer. Laser pallation of malignant dysphagia. Lancet 1993(341):348.

27. Ihre T, Johhans son $C_{i}$ Seligson U. Torngren S. Endoscopic YAG-laser treatment Hn massive upper gastrointestinal bleeding. Report of a controlled randomized study. Scandinavian Journal of Gastroenterology 198:1(16):633-40.

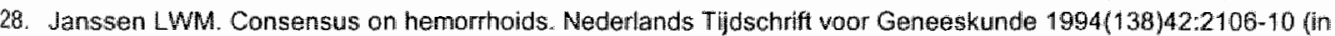
Dutchi) 
29. Jemsen DM, Machiciado GA, Tapia JI, Beilin DB, Mautner W. Endoscopic argon laser photocoagulation of patlents with severe upper gastrointestinal bleeding (abstract). Gastrointestinal Endoscopy 1982(28):151.

30. Johanson $\mathrm{JF}_{\mathrm{F}}$ Sonnemberg. A The prevalence of hemorrhoids and chronic constipation. Gastroenterolong $1980(98) 2: 380-6$.

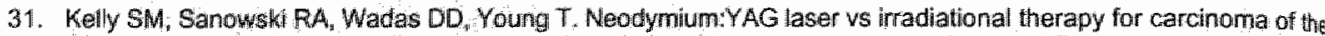
esophagus: a comparison of cost, in-patient days and survival (abstract). Gastrointestinal Endoscopy 1986 (32):145.

32. Kefhaber $P$, Kiehaber K, Huber $F$, Nath G. Usefulness of Nd:YAG laser applications in acute gastrointesthat hemorrhage. Lasers in Surgery and Mediche 1983(3),111.

33. Kim SL, Goldschmid S Paliation of malignant dysphagla carvers versus pumbers (comment). American Jlouna) of Gastroenterology 1995(90)3,512-3. Comment on: British Journal of Surgery 1992(79):1167-70.

34. Krejs GJ, Litti KH, Nestergaard H, Hamilon JK. Spady DK, Polter DE. Laser photocoagulation for the treatment of acute peptic ulcer bleeding. New England Jourral of Medicine $1987(316): 1618-21$.

35. Lambent R. Endoscopic detection and treatment of early esophageal cancer: a critical analysis. Endoscopy $1995(27) 1: 12-8$.

36. Lebodic L, Sudry P. Endoscopic control of gastrointestinal themorrhage. In: Papp JP (Ed.). European clinical experience in laser photocoagulation in upper gastrointestinal tracti. Boca Reton: Fl: CRC, 1981: p. $103-10$.

37. Lewis B. The only good polyp. (editorial). The American Joumal of Gastroenterology 1995(90) 1:1-2.

38. Loizou LA, Grigg D, Boulos PB, Bown SG. Endoscopic Nd:YAG laser treatment of rectosigmoid cancer. Gut $1990(31): 812-6$.

39. Lopez M. Adjuwant therapy of colorectal cancer, Diseases of the Colon \& Rectum 1994(37)2:S86-S91.

40. MacLeod IA, Mills PR Mackenzie JF, Joffe SN, Russell RL, Carter DC. Neodymium ytrium aluminum gamet laser photocoagulation for the major hemorrhage from peptic ulcers and single vessels: a single blind controlled study British Medical Journal $1983(286): 345 \cdot 8$.

41. MacRae HM, McLeod RS. Comparison of hemormoidal treatment modalities. A meta-analysis. Diseases of the Colon \&ectum 1995(38)7:687-94.

42. Mathus-V/legen EMH, Tytgat $\mathrm{GN}$. Laser ablation and palliation in colorectal malignancy. results of a multicenter enquiry. Gastrointestimal Endoscopy 1986(32):393 6.

43. Mathus-V/liegen EMH. Tytgat GN. Nd:YAG laser photocoagulation in colorectal adenoma. Evaluation of safiety, usefulliness and efficacy. Gastroenterology 1986(90):1965-73.

44. Mathus-Wtiegen EMH, Tytgat GNJ. Laser ablation and palliation in colorectal malignancy - results of a multicenter inquiry. Gastrointestinal Endoscopy 1986(32):393-9.

45. Mathus-Wliegen EMHH, Tytgat GNJ. Colorectal adenomas, in: Riemann JF. Ell C. (Eds.). Lasiers in Gastroenterology International Experiences and Trends. Georg Thieme Veriag, Stuttgart, New York, 1989; Thieme Medical Pubishers, Inc " New York, 1989, p. 72-80.

46. Mathus-Wliegen EMH. Laser ablation of early collorectal malignancy. Endoscopy 1993(25):462-8.

47. Mathus-Vliegen EMH. The role of lasers in gastroenterology. A descriptive anahsis of eight years experience (thesis). University of Amsterdam, the Netheriands 1988.

48. Mathus-Vliegen EMH. Treatment modaities in colorectal cancer. In: Krasner $N($ Ed. ). Lasers in Gastroenterology Willey Liss, New York, 1991.

49. Matthewson $K_{3}$ Swain CP, Bland M, Kirkham JS, Bown SG, Northfield TC. Randomized comparison of Nd:YAG, heater probe and no endoscopic therapy for bleading peptic ulcer. Gastroenterology 1990(98):1239-44.

50. McGowan I, Bari" H. Krasner N. Pallative laser therapy for inoperable rectall cancer - does ith work? Cancer $1989(63): 967-9$.

51. Mellow MH. Endoscoptc laser therapy as an alternative to palliative surgery for adenocarcinoma of the rectum comparison of costs and complications. Gastrointestimal Endoscopy 1989(35):283-7.

52. Mellow MH. Endoscopic laser therapy of colon cancer. In: Ad Hoc Committee on Techmology Assessment. Therapeutìic gastrointestinal endoscopy. American Society for Gastrointestinal Endoscopy, 1987, Manchester, Massechusetts, 1987, p. $32-35$.

53. Mellow MH. Endoscopic theory of colorectal neoplasms. Gastrointestinal Endoscopy Clinics of North America $1994(4) 2: 737-47$.

54. Miller-Catchpole R (Ed). Therapeutic endoscopy and bleeding ulcers. NHH Consensus Conference. Joumal of the American Medical Association 1989(262)10:1369-72.

55. Moghissi $K_{\text {. }}$ Dixon $K_{n}$ Hudson E. Stringer M. Photodynamic therapy of oesophageal cancer. Lasers in Medical Science 1996(10);77-81.

56. Naveau $S$, Perrier $C$, Zourabichwith $O_{1}$ Brumie F, Poirtine A, Chaput JC. Traitment par le laser Nd:YAG des tumeurs villeuses colo-rectales. Gastroenterologie Clinique et Biologique 1988(12)8-9:604-9.

57. Nicholls J. Large bowel cancer. In: Slewin ML., Staquet MJ (Eds.). Randomized trials in cancer: a critical review by sites, Raven Press, New Yorik, 1986, p. 241-71.

58. Nishioka N, Richter J. Endoscopic therapy of bleeding peptic ulcers: a cost-benefit analysis (abstract) Gastrointestinal Endoscopy 1986(32) 150 . 
59. Nishioka NS. Applications af lasers tn gastroenterology (review series article). Lasens in Surgery and Medicine 1995(16):205-14.

60. Nwokolo CU, Payne-James $d \downarrow$, Sik DBA, Misjewicz JJ, Loft DE. Palliation of malignant oysphagia by ethanol induced tumor necrasis. Gut 1994(35)299-303.

61. Affice of Health Technology Assessment (OHTA). Endoscopic laser photocoagulation in the treatmont of uppen gastrointestinal bleeding. Health Technology Assessment Reports 1987, Number 5. OHTA, Rockville, MD, US, 1987

62. Ortega AE, Beart Jr. RW, Steele GD, Winchester DP, Greene FL. Laparoscopic bowel surgery registry. prelininary results. Diseases of the Colon \& Rectum 1995(38)7:68 1-6.

6. Overholt BF. Ulcer hemorrhage: laser treatment. In: Ad Hoc Committee on Technollogy Assessment. Therapeutic gastrointestinal endoscopy. American Society for Gastrointestinal Endoscopy, Manchester, Masisachuselts, 1987. p. $4-5$.

64. Palmer KR. Endoscopic intervention in bleeding peptic ulcer. Gut $1995(37): 161-4$.

65. Pfenninger Jl, Surrell J. Nonsurgical treatment options for intemal hemomhoids. American Family Physician $1995(52) 3: 821-34$.

66. Rees M. The costing of a controlled trial of the use of laser and thermal probe for the treatment of bleeding peptic ulcer. Gastroenterological Unit, St. James Hospital, Balham, Londion, 1987.

67. Rutigeerts $P$, Vantrappen $G$, Broeckaent L, Janssens J, Coremans $G$, Geboes $K$, Schurmans $P$. Controlled trial of YAG laser treatment of upper digestive hemorrhage. Gastroenterology 1982(83):410-6.

68. Rutgeerts $P$, Vantrappen $G$, Broeckaert $L$, Coremans $G$, Janssens J. Hiele M. Comparison of endoscopic polidocanol injection and YAG laser therapy for bleeding peptic ulcers. Lancet 1989(1):1164-7.

69. Sacks HS, Chalmers TC, Blum AL, Berrier J, Paganu D. Endoscopic hemostasis: an effective therapy for blevding peptic ulcers. Joumal of the American Medical Assaciation 1990(264):494-9.

70. Sander $R$, Hagenmueller $F$, Sander $C$, Rless $G$, Classen M. Laser versus laser plus afterloading with iridiumy-192 in the palliative treatment of malignant stenosis of the esophagus: a prospective, randomized and controlled study. Gastrointestinal Endoscopy 1991(37)4:433-40.

71. Sargeant IR. Thorpe S, Tobias JS, Blackman G, Glover JR, Lees WC. Radiotherapy enhances laser palliation of malignant dysphagla: a randomized study. Gastroenterology (in press).

72. Sculpher MJ, Sargeant IR, Loizou LA, Thorpe SM, Spencer GM, Bown SG. A cost-analysis of Nd:YAG Iaser ablation versus endoscopic intubation for the palliation of malignant dysphagla. European Journal of Cancer $1995(31 \mathrm{~A}) 10: 1640-6$

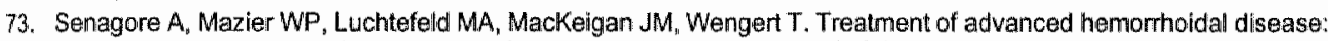
a prospective, randomized comparison of cold scalpel vs, contact Nd:YAG laser. Diseases of the Colon \& Rectum $1993(36): 1042-9$.

74. Souquet JC, Sabben G, Chavaillon A, Bomoissin S, Olve C, Boustiere B, Lambert R. Traintement laser des tumeurs villeuses rectales. Annales de Gastroenterologie et d'Hepatologie 1987(23)6:311 14 .

75. Sprangers MAG. Taal BG, Aaronson NK, te Velde A. Quality of life in colorectal cancer. Stoma ws. nonstoma piatients. Diseases of the Colon \& Reclum 1995(38)4:361-9.

76. Swain CP, Bown SG, Storey DW, Kirkham JS. Northfield $T C_{n}$ Salmon $\mathrm{PR}_{n}$ Controlled triall of argon llaser photocoagulation in bleeding peptic ulcers. Lancet 1981(2):1313-6.

77. Swain CP, Kirkham JS, Salmon PR, Bown SG, Northfleld TC. Controlled trial of Nd:YAG laser photocoagulation in bleeding peptic ulcers. The Lancel 1986(2):1113-16.

78. Tan CC, Freeman JG, Holmes KT, Benghiat A.. Prospective randomised trial of laser treatment versus laser followed by brachytherapy for palliation of esophageal carcinoma (preliminary report) (abstract). Gastroenterology 1995 (108)4:(Supplement):A54,4.

79. Trelles MA, Rovira J. Sanchez J, Vellez M. Trinxet C, Sala P. Treatment of Hemorrhoids - Cost-effectiveness. Im: Banta HD and Schou I (Eds.). Lasers in Health Care, Effectiveness, Cost-Effectiveness and Policy Implications, Academic Publishing. Frederiksberg, Denmark, 1991, p. 151-4.

80. Trelles MA, Rovira J, Sanchez J, Velez M, Trinxet C, Sala P. Trealment of Hemorhoids - Effectiveness. In: Banta HD and Schou I (Eds.). Lasers in Health Care, Effectiveness, Cost-Effectiveness and Policy Implications, Acadermic Publishing. Frederiksberg, Denmark, 1991, p. 145-50.

81. Trelles MA, Sanchez „, Sultan R, Sala P. Laser hemorrhoidectomy in an outpatient setting" a cost-analysis. Journal of Clinical Laser Medicine \& Surgery 1991:373-5.

82. Vallon AG, Cotton PB, Lawrence BH, Armengol Miro JR, Salord Oses JG. Randomized krial of endoscopic argon laser photocoagulation in bleeding peptic ulcers. Gut $1981(22)$ 228-33.

83. Vondeling H. Mathus-Vliegen EMH, Kievit J, Banta D. Palliation of advanced colon cancer. Hn: Banta HD, Schou I (Eds.). Lasers in health care. Effectiveness, Cost-Effectiveness and Policy Impliciations. Academic Publishing, Fredeniksberg, Denmark, 1991, p.140-7.

84. Vreeburg EM, Rauws EA. Diagnosis and treatment of upper gastrointestinal bleeding. Modern Medicine $1994(12): 861-8$ (in Dutch). 
85. Wang $\mathrm{Y} Y$, Chang-Chien $\mathrm{CR}$, Chen JS, Lai $\mathrm{CR}$, Tang R. The role of lasers in hemorhoidectomy. Diseases of the Colon \& Rectum $1991(34) ; 78: 92$.

86. Way JD. Cotonoscopic polypectomy. In: Ad Hoc Committee on Technology Assessment Therapeutic castroirtestinal endoscopy. American Sociey for Gastrointestinal Endoscopy, Manchester, Massachusets; 1987, 0. 26.7 .

87. Willams $\mathrm{B}$, Saunders $\mathrm{BP}$. The rationale for current practice in the management of malignant colonic polyps. Endoscopy $1993(25) 469-74$.

88. Willams $5 G J$. Westaby D. Recent adwantages in the endosicopic management of variceal bleeding. Gut $1995(36) \cdot 647 \times 8$.

89. Bown SG. Gastroenterology - Effectiveness In Banta HD, Schou ( Eds.). Lasers irt Health Care. Effectiveness, Cost-Effectiweness and Policy Implications. Academic Publishing, Frederiksberg, Denmark, 1991, p. $122-34$. 


\section{Lasers in urology}

\subsection{Introduction}

Lasers were used in urologically oriented animal experiments in $1966^{\text {is }}$. Since then laser enthusiasts have promoted their use in an ever increasing range of urological conditions. Clinical studies resulted in the approval of the use of the Nd:YAG laser (1984), argon laser (1987), KTP laser (1988) and Ho:YAG laser (1992) in urology by the US Food and Drug Administration ${ }^{\text {[36! }}$. At present, also $\mathrm{CO}_{2}$, pulsed dye- and diode lasers are used in urology. Of all these types of lasers, the Nd:YAG laser is the one that is most commonly used, while diode- and Ho:YAG lasers are in a stage of early clinical evaluation ${ }^{[3,36]}$. Nevertheless, the overall role of lasers in urology has stayed limited, although this may change as lasers have become more easy to use and particularly now that encouraging results of Nd:YAG laser treatment of benign prostatic hyperplasia (BPH) have been published ${ }^{[3,36]}$. Besides in $\mathrm{BPH}$, lasers are used in treatment of prostatic carcinoma, condyloma acuminata, a variety of bladder- penile, and urethral lesions, upper tract urothelial tumors, cystitis, hemangiomas, selected tissue welding applications, urolithiasis (stone disease) and other miscellaneous conditions ${ }^{i 36]}$. PDT plays a role in treatment of superficial bladder cancer. In selected applications, in particular treatment of $\mathrm{BPH}_{\text {, }}$ multiple small RCTs have been done. A few cost studies provide complementary information.

\subsection{Prostate disease}

\subsubsection{Introduction}

Benign prostatic hyperplasia (BPH) and prostate cancer (adenocarcinoma) affect middle-aged men and beyond with a markedly increasing incidence in the US due to improved detection methods and the ageing of the population, respectively ${ }^{[s \mid}$. In this country, having a population of 250 million people in 1992, in 1995600,000 men were expected with newly diagnosed prostatic disease of whom 400,000 were expectied to suffer from BPH and 200,000 from prostatic cancer. Prostate cancer has become the most commonlly diagnosed cancer in men and the second most common cause of death from cancer in men ${ }^{[31,49,59\}}$.

In the late eighties total health care expenditure for BPH and prostate cancer in the United States were estimated about US\$ 5 billion and US $\$ 1.5$ billion, respectively ${ }^{136,583}$. In 1992 , US $\$ 810$ billion was spent on health care ${ }^{30 !}$, thus costs for BPH and prostate cancer treatment represented at least $0.6 \%$ and $0.2 \%$ of total annual thealth care spending in the US, respectively ${ }^{30,69 !}$. Other countries show a similar pattern. For example in the United Kingdom It has been estimated that the annual maximum cost of treatment of BPH to the national health service amounted to $0.5 \%$ of total expenditure in $1990^{128]}$. In addition, the indirect costs of prostate cancer in the United States were estimated as high as US\$ 2.5 billion a year ${ }^{159 !}$. No indirect cost estimaltes for BPH have been published.

\subsubsection{Benign prostatic hyperplasia}

Benign prostatic hyperplasia (BPH) or prostatism causes urinary difficulties with symptoms classified as either obstructive or irritative. Obstructive symptoms are often associated with lower flow rates and include urinary hesitancy, weak stream force, straining, interruption of the stream, urinary retention and dribbling. Most common adaptations to BPH in daily routine of patients include limiting intake of fluid before going to bed or before travelling or, at least some of the time, avoiding places that may not have a toilet. Irritative symptoms include urgency, frequency, and nocturia and may be seen in the absence of urinary retention. ${ }^{\text {(2) }}$ 
Treatment of obstructive BPH is aimed at increasing the diameter of the prostatic urethra. The most frequently employed surgical technique for BPH is transurethral resection of the prostate (TURP) with an electrocautery loop, a procedure that almost completely replaced open surgery for this condition in the eighties ${ }^{2,26 !}$. TURP, which is associated with a hospital stay of a few days, has become one of the most frequently performed operations in the US ${ }^{30 \text {. }}$. After years of an increasing rate, since 1987 its rate is declining on average $4 \%$ annually, resulting in a total of 196,000 TURP procedures in men covered by Medicare in the United States in $1992^{\text {|28? }}$. This process is thought to reflect an increasingly critical assessment of outcomes after TURP and the advent of inew treatment modalities ncluding watchful waiting, medical management with alpha-sympatholytic agents or andragen suppression, insertion of coils and stents, balloon dilatation, microwave therapy, radiofrequency, focused ultrasound and a variety of laser techniques, almost always using the (cw) Nd:YAG laser $r^{10,2 ; 2,30,36,401}$. What is the role of lasers there?

Early attempts to use laser energy to ablate prostatic tissue endoscopically were mot very successful due to difficulties in adequately directing laser energy into the prostate. But when sidefiring laser catheters were developed in the late eighties, Nd:YAG laser prostatectomy became clinically feasible. In 1991 an article was published presenting the technique of a side-firing laser catheter with positioning monitored by transurethral ultrasound. An acronym for this technique is TULIP: Transurethral Uitrasound-guided Laser-Induced Prostatectomy ${ }^{[3]}$. Also in 1991, the first angled delivery system capable of being used in conjunction with standard operative endoscopes was presented. Thus, using this system, Nd:YAG laser energy can be delivered under direct vision and off-axis to lateral (prostatic) tissue $e^{\mid 3,36 ! !}$.

A second method to use lasers in treatment of BPH is contact laser prostatectomy. This can be applied either by using a synthetic sapphire tip with an Nd: YAG laser to vaporize prostatic tissue or by means of, for example, a KTP laser coupled to a ball-ended fibre ${ }^{(3,36)}$. An acronym for this techinique is VLAP: Visual Laser Assisted Prostatectomy.

A third technique consists of interstitial insertion of laser fibers into prostatic tissue followed by low power Nd:YAG laser energy delivered over prolonged periods of time (e.g. 5-10 watts for 5-10 min) resulting in tissue coagulation. These fibers are either placed transperineally or transurethrally into the lateral prostatic lobes. Promising results with these techniques have been achieved albeit in a limited number of patients, especially in contact laser prostatectomy and interstitial laser coagulation. According to some authors, the promising results are a result of case selection "which has favoured smaller prostates and patients with uncomplicated bladder outflow obstruction ${ }^{[3,30 \mid \eta}$. All techniques listed here are in its evolutionary stage. Nevertheless, a few RCTs have been organized comparing TURP and side-firing laser-catheter treatment techniques. The one RCT comparing direct contact Nd:YAG laser vaporization of the prostate versus TURP has so far enrolled 50 patients, of whom no data have as yet been analysed ${ }^{[67]}$.

\section{Transurethral ultrasound-guided laser-induced prostatectomy (TULIP)}

Schulze et al. (1994) organized an RCT comparing TULIP and TURP in treatment of symptomatic obstructive $\mathrm{BPH} \mathrm{f}^{(73)}$. At the first time of reporting, 40 patients had been enrolled in the ongoing study, of whom 13 in each group were in acute retention prior to treatment. In an interim analysis" presented in an abstract, they reported results with a 6 month follow-up. Mean symptom score was reduced from 18.9 at baseline to 3.0 after 6 months in the TULIP group, comparable with 17.7 and 1.8 in the TURP group. Peak flow increased from 3.2 to $18.5 \mathrm{ml} / \mathrm{s}$ in the TULIP group and from 2.4 to $24.4 \mathrm{ml} / \mathrm{s}$ in the TURP group. Post void residual urine volume decreased to $42.5 \mathrm{ml}$ in the TULIP group and to $12.1 \mathrm{ml}$ in the TURP group. One patient in each group developed complications related to treatment. Mean blood loss was 51 and $545 \mathrm{ml}$ in the TULIP and the TURP group, respectively, not requiring blood transfusion in any patient. After TULIP patients had a suprapubic catheter for 34 days on average. This is not required after TURP. Mean hospital stay after TULIP was 2.6 days, compared to 6.7 days after TURP. It was concluded that using the TULIP device results can be achieved comparable to that with TURP, reducing blood loss and hospital stay substantially. However, it was noticed that after TULIP it takes substantially longer time to obtain improvements than after TURP. 
Babayan et all. (1994) organized a multicenter national cooperative study of TULIP in the United States ${ }^{\text {i? }}$. In late 1994230 patients with bladder outlet obstruction secondary to BPH had entered the study, which is ongoing of whom 40 had completed a follow-up of two years. Their one-year follow-up results, presented in an abstract, demonstrated that after one year symptom scores and peak uroflow significantly improved over preoperative scores and that these improvements lasted at lleast for another year. This study is performed in the context of US Food and Drug Administration regulated human clinical studies of TULIP, organized to establish the safety and efficacy of the procedure. In an earlier publication of this group, 63 patients were analysed with 6 months follow-up with encouraging results. At that time 150 patients had been enrolled in the study. No intraoperative complications had occurred, no blood transfusions had been necessary and average hospitall stay after TUL\|P was 1.7 days, while $83 \%$ of the patients went home after a 1 night stay ${ }^{4.7 !}$.

It is concluded that TULIP may be as effective as TURP in treatment of obstructive BPH. Of course, patient numbers are still small and the length of follow-up is still short, so no definite slatements can (yet) be made.

\section{Visual Laser Assilsted Prostatectomy (VLAP)}

Kabalin et al. (1995) published results of an RCT comparing VLAP $(n=13)$ and TURP ( $n=12)$ in

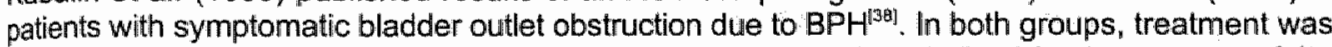
performed using regional (spinal) anesthesia. All patients were hospitalized for the purpose of the study. Normally, according to the authors, VLAP is performed as an outpatient procedure. Besides 18 month follow-up data, the article included in an addendum 24 month follow-up data in 9 patients. These data are discussed here. Average symptom scores, assessed by means of the check-list of the American Urological Association, decreased from 20.9 (se 1.9) to 4.7 (se 1.7) in the VLAP group and from 18.8 (se 1.8) to 6.8 (se 1.7) in the TURP group. Average peak urinary flow rates increased from 8.5 (se 1.1) $\mathrm{ml} / \mathrm{s}$ to 18.8 (se 1.7) in the VLAP group and from 9.0 (se 1.1) to 23.6 (se 4.9) in the TURP group. Average residual urine volume decreased from 236 (se 74) $\mathrm{ml}$ to 148 (se 28) $\mathrm{ml}$ in the VLAP group and from 291 (se 88) $\mathrm{ml}$ to 103 (se 22) $\mathrm{ml}$ in the TURP group. Two patients in each group failed to achieve clinically significant improvements in voiding after three months and were considered as failures. Follow-up in these patients was terminated. Subjective patient assessment of symptom improvement confirmed improvements in objective clinical scores in the remainder of patients. Absolute end points and durability of results were observed to be equivalent in the course of follow-up $(3,6,1218$ and 24 months). The mean length of postoperative catheterization was 2.7 days in the TURP group and 4.7 days in the VLAP group. VLAP was judged to show considerable advantages over TURP in terms of safety and morbidity. A preliminary conclusion is that VLAP compares fawourably in overall efficacy compared to TURP in treatment of $\mathrm{BPH}$.

Dixon et al. (1994) reported in an abstract the interim-results of a double-blind RCT comparing VLAP to TURP in the U.S. ${ }^{\mid 200]}$. 60 Patients were so far enrolled in the study, in 48 patients 6 month follow-up data were available of whom 20 underwent laser ablation, 24 underwent TURP and 2 elected not to participate after being randomized to laser ablation. Two laser patients falled and underwent TURP. The patients and the study nurse assessing efficacy were blinded to the treatment rendered. It is quite surprising to read that patient blinding has been achieved. The symptom score fell from 16.5 at baseline to 11.9 in the VLAP group and from 20.3 to 7.6 in the TURP group. Maximum flowrate increased from 9.2 to $14.1 \mathrm{~m} / \mathrm{s}$ in the VLAP group and from 8.5 to $14.9 \mathrm{ml} / \mathrm{s}$ in the TURP group. Residual urine fell from 131 to $84 \mathrm{ml}$ in the VLAP group and from 166 to 74 in the TURP group. Neither group suffered any adverse effects during the pracedure. In the absence of a description or an analysis of patient characteristics at baseline these data suggest that, according to the authors, while both modalities are effective, TURP results in greater symptomatic improvement, greater improvement in peak flow and greater decrease of residual urine volume. However, operating time, blood loss and hospital stay all favoured the laser group. but irritative symptoms tended to be more bothersome during the early postoperative period in the laser group. 
Buckley and colleagues (1994) presented in an abstract results of a completed multicenter RCT comparing VLAP (or ELAP: endoscopic laser ablation of the prostate) and TURP in the United Kingdom ${ }^{15}$. 151 Patients were enrolled in the study, $n=77$ (VLAP) and $n=74$ (TURP) , with a follow. up of one year. Data were presented for 139 patients. The symptom score fell from 19 to 4.5 in the TURP group and 19 to 6 in the VLAP group at 6 months. Maximum flowrate increased from 9.5 to $19.7 \mathrm{ml} / \mathrm{s}$ in the TURP group and from 9.9 to $17.5 \mathrm{~m} / \mathrm{s}$ in the ELAP group at 6 months. Residual urine fell from 147 to $54 \mathrm{ml}$ in the TURP group and from 140 to $79 \mathrm{ml}$ in the VLAP group at 9 months. It was concluded that VLAP offers results similar to TURP in the short term. Laser ablation had fewer problems with bleeding and was performed either as a day-case or with overnight stay: No data on complications after both procedures were provided.

Costello and Crowe (1994) reported results in an abstract of a completed RCT in Australia comparing VLAP $(n=35)$ to TURP $(n=37)$ in patients with obstructive BPH with a follow-up of 1 year ${ }^{\| 16]}$. No baseline data were provided. After 12 months symptom scores had decreased to 7.3 in the VLAP group and to 6.75 in the TURP group. Urinary flow increased to $20.8 \mathrm{~m} / \mathrm{s}$ in the VLAP group and to $16.8 \mathrm{ml} / \mathrm{s}$ in the TURP group and residual urine volume had decreased to $34.6 \mathrm{ml}$ in the VLAP group versus $26.4 \mathrm{ml}$. in the TURP group. Three Patients in the TURP group required a blood transfusion after treatment whereas this was not required in any patient in the VLAP group. A complicating factor in the analysis of this trial is that during enrollment indications for TURP and discharge policies were changed. Therefore, no conclusions can be drawn from this information ${ }^{[32]}$.

These data can be complemented with data collected in multicenter studies of lasers and TURP. Leach et al. (1994) presented results in an abstract of a multicenter study in the United States enrolling 117 patients with symptomatic BPH of whom 95 were followed up for 6 months and 50 for one year after VLAP ${ }^{\mid 42 !}$. Mean symptom score decreased from 21 to 8.3 at 12 months, mean peak uroflow rates increased from 7.4 to $16 \mathrm{ml} / \mathrm{s}$ and mean residual urine volumes decreased from 145 to $84 \mathrm{ml}$. Of all patients enrolled, 46 were treated under local anesthesia, the remainder of patients was treated using either spinal or general anesthesia. Despite the overall results, in 12 patients $(12.5 \%)$ obstructive symptoms returned, $48(50 \%)$ had transient irritative symptoms, infections or haematuria, $9(9.5 \%)$ reported retrograde ejaculation, there were $3(3.1 \%)$ bladder neck contractures and one patient $(1 \%)$ had venous thrombosis. Five men reported loss of potency on long term follow-up.

How do these data compare to TURP? Mebust et al. (1989) retrospectively analysed immediate and postoperative complications after TURP in 13 centers with 3,885 patients ${ }^{48 \mid}$. Nearly $80 \%$ of these patients had been treated in the period 1984-1987. A mortality rate of $0.2 \%$ was registered, and a morbidity rate of $18 \%$. Common operative complications included bleeding requiring transfusion (3.9\%), clot retention $(3.3 \%)$ and genitourinary infections $(2.3 \%)$. Seventy-eight percent of patients were discharged from the hospital by day 5 . A total of 93 patients $(2.4 \%)$ were dischrged from the hospital with indwelling urethral catheters. Of course, this is a historicall account and operation techniques have evolved in the meantime, reducing mortality and morbidity rates. Bishop (1994), in a brief review, quoted literature documenting that strictures of the urethra and bladder occur in about $10 \%$ of patients after TURP ${ }^{112]}$. Seven percent of patients will have retrograde ejaculation and impotence is likely to occur in about $10 \%$ of patients. Seventy-five percent of patients are satisfied with TURP as treatment of $\mathrm{BPH}$.

In summary, primarily based on summaries of interim-analyses of RCTs, it can be concluded that VLAP is likely to be as effective as TURP in treatment of patients with symplomatic BPH. It is encouraging to see laser treatment properly evaluated for this indication. However, definite results, including a detailed assessment of postoperative morbidity after both procedures, have to be awaited. A major difference between the efficacy data as reported in the trials and the effectiveness data based on registries and other sources is that TLRP is associated with a small mortality rate, contrasting with laser-treated patients. 
In addition, it should be taken into account that laser therapy of BPH is not the only modally that is or has been studied in RCTs. For example, transurethral microwave hyperthermia is presently being studied in a placebo-controlled RCT ${ }^{111}$ and has been studied before in this design compared to TURP(30il. Likewise a variety of medications have been studied with modest results in placebocontrolled RCTs ${ }^{\left[30_{1} 40\right]}$. In other words, besides lasers, depending among other factors on inclusion criteria $^{(4)}$, there may be other treatment modalities, pharmaceutical strategies or other strategies (including watchful waiting) that may be able to compete with TURP. To find out, further testing is required ${ }^{[12,40]}$. Furthermore, there are at present at least three laser treatment options available, including a variety of (predominantly) Nd:YAG lasers, laser catheters and fibers produced by perhaps as many as 10 manufacturers that can be applied in many different treatment protocols ${ }^{i 50.53]}$. Rapid technological developments, impressive clinical practice variation in diagnosis and treatment rates of $\mathrm{BPH}$ and a relatively conservative profession contribute to a complex environment for evaluation ${ }^{[28,31,36]}$.

\section{Cost-effectiveness}

There is as yet no convincing evidence to support the claim of cost-effectiveness of laser treatment of BPH compared to TURP. Even the most straightforward TURP requires a hospitalization of at least 3.5 days $^{33 \mid}$ while laser treatment can be applied as an outpatient procedure or with a one-day hospital stay in the majority of cases. Thus, assuming lasers to be as effective as TURP with probably less associated morbidity, laser therapy could potentially be cost-effective compared to TUIRP in case of high patient throughput. Without providing data, it has been reported that in two RCTs laser therapy resulted in lower and equal total costs of treatment, respectively, compared to TURP ${ }^{[18.20]}$. Even if the cost-effectiveness of lasers would be well-documented compared to TURP, the question which treatment modality is the most cost-effective would still not be answered. This would require a comparison to other minimally-invasive modalities or pharmaceuticals, if these have been shown to be equally effective to TURP in RCTs as well. Also, a meta-analysis could be helpful at this stage. Finally, one could think of laser therapy as part of a strategy, for example. combined with pharmaceuticals, that possiblly could compete with TURP.

These uncertainties should be taken into account when analysing the results of the most detailed study on this issue to date, by Vale et al. ${ }^{[641}$. In 1995 these authors analysed, based on a number of assumptions, the costs and the relative cost-effectiveness of four treatment modalities for BPH, including TURP, two transurethral microwave devices (the Leo Microthermer and the Prostatron), and VLAP ${ }^{[64]}$. The direct cost of treatment of TURP was estimated BP 1350 , compared to BP 322 for the Leo, BP 975 for the Prostatron, and BP 788 for VLAP. Cost-effectiveness was expressed in terms of both the cost per millilitre/second increase in peak urinary flow rate and the cost per percentage reduction in symptom score. Using the first expression, the cost per unit benefit amounted $153 \mathrm{BP}$ for TURP, $140 \mathrm{BP}$ for the Leo, $238 \mathrm{BP}$ for the Prostatron, and 178 BP for VLAP. Using the latter expression, the cost per unit benefit amounted $18 \mathrm{BP}$ for TURP, 5.1 BP for the Leo, 13.5 BP for the Prostatron, and 13.6 BP for VLAP. Based on these data, the Leo transurethral microwave device may offer the most cost-effective treatment option. Compared to TURP, VLAP would be either slightly more or slightly less cost-effective, depending on the choice of primary outcome measure. However, considering the uncertainties in the calculation, the lack of a sensitivity analysis, and the uncertainties regarding the data on effectiveness, these data have to judged as preliminary.

\subsubsection{Prostatic carcinoma}

Until recently, localized prostatic carcinoma, representing about $60 \%$ of all cases, was either treated by radical prostate resection and/or external radiotherapy or watchful waiting with treatment focused on symptom relief. There is no consensus on treatment strategy for prostatic carcinoma, nor is there consensus on the benefits of early detection and treatment of prostate cancer ${ }^{\left[{ }^{7 t}\right.}$.

Since 1984 the Nd:YAG laser was studied clinically to treat patients with early, localized prostatic carcinoma. In mast of these studies, as reviewed by Johrison and Costello $(1994)^{1361} \mathrm{Nd}$ :YAG laser 
heatment was appled some weeks after an extended TURP. The vast majority of patients stayed disease-free or showed ho tumour progression with an average follow-up of 2 years. Based on these encouraging results in patients with early stage disease Nd:YAG laser energy has been. attempted in treatment of patients with more advanced disease. For example, in a small caseseries of 10 patients, 5 out of 9 evaluable patients had negative bropsies at 4-6 month follow-up. In these patients laser treatment, either once or repeated, was started immediately following after extended TURPl3al. These results are considered as promising. Considering the increasing inportance of prostate cancer, this is an area that deserves more clinical research with lasers it should be noticed, however, that the use of lasers does not seem to play any role in the present debate on early detection and treatment of prostate carcinoma.

\subsection{Bladder disease}

\subsubsection{Introduction}

Bladder cancer is the tenth most prevalent cancer of the world's population. It makes up 4.7 percent of new cancers and its incidence is rising. 3.3 Percent of cancer deaths are caused by bladder cancer ${ }^{\text {sis } 1}$. The prevalence of the disease in men is higher than in women, probably related to smoking habits. Among men in the European Community, bladder cancer ranks number five of all types of cancer in terms of both incidence and mortality ${ }^{[4 / 4]}$. Among patients presenting with a transitional cell carcinoma of the bladder (representing $90 \%$ of all cases), nearly $70 \%$ have a non. invasive tumour ( $\mathrm{Ta}, \mathrm{T} 1$ ). Of these, 50 to $80 \%$ will recur after standard treatment, which is transurethral resection (TUR) and adjuvant intravesical chemotherapy or immunotherapy, and subsequent invasive disease will occur in about 10 to $30 \%{ }^{\{24,44\}}$. Invasive disease $(T 2, T 3, T 4)$ is usually treated with radical cystectomy. The 5 year survival rate of patients with $T 2$ and $T 3$ tumors is $80 \%$ and $40 \%$, respectively ${ }^{144]}$. A cystectomy is also indicated in the case of clear progression of the stage and/or grade of recurrent superficial tumours with beginning infiltration after conventional treatment ${ }^{11,44}$. Besides cystectomy, which is a mutilating procedure, also radiotherapy and general chemotherapy have a place in treatment of patients with invasive bladder cancer ${ }^{\text {Ad }}$

\subsubsection{Superficial bladder carcinoma}

Standard treatment, TUR, requires spinal or peridural anesthesia, and complications include bleeding, perforation and the necessity of the long use of catheters with related problems such as infection and reduced bladder capacity due to scar contracture. Hospitalization is usually 3-5 days after surgery ${ }^{15 * t}$. Johnson and Costello (1994) ist a number of advantages of lasier treatment over TUR including 1) delivery through standard endoscopes with local anesthesia, applied as an outpatient procedure, 2) lack of need for an indweiling catheter, 3) absence of obturator nerve reflex with the risk of bladder perforation, 4) absence of bleeding and 5) a very low complication rate $^{\text {inal }}$

One of the pioneers investigating the use lasers in urology was Hofstetter in Germany, who clinically applied the bare fiber Nd:YAG laser as early as 1976. After having satisfactorily treated more than 500 patients with bladder cancer an RCT was started in 1981. Sixty-five Patients with $\mathrm{Ta}_{\mathrm{i}} \mathrm{T} 1$ or T2 bladder tumors were enrolled in this trial by the end of 1983. In 1984 an interimanalysis was presented distinguishing treatment results in 40 patients with primary tumours at the time of enroliment, and 25 patients who had recurrent tumaurs at the time of enrollment ${ }^{[3]}$. In the first group 20 patients had received either laser therapy plus chemotherapy, scheduled for 2 years (mitomycin $(n=15)$, laser therapy ( $n=5)$, TUR plus chemotherapy scheduled for 2 years (mitomycin $n=12$ ) or TUR ( $n=8)$. Mean follow-up was 19 months in the laser plus chemotherapy group, 21 months in the laser group, 17 months in the TUR plus chemotherapy group and 7 months in the TUR group. Calculated and presented as rate of recurrence after one year in neither of the two 
laser groups recurrences were observed compared to recurrences in $16.7 \%$ of patients in the TuR plus chemotherapy group and $54.5 \%$ in the TUR group.

In the second group, the 25 patients with recurrent tumours, the laser plus chemotherapy group included 15 patients, the laser group 5 patients, the TUR plus chemotherapy group 1 patient and the TUR group 4 patients, with an average follow-up of $12,3,11,6,14.0$ and 9.8 months, respectively. Five of 15 patients $(33 \%)$ in the laser plus chemotherapy group showed recurrences, all 5 patients in the laser group, the only patient in the TUR plus mitomycin group and 3 of 4 patients $(75 \%)$ in the TUR group. The authors concluded that treatment results in the laser group (with or without mitomycin) were better than treatment results in the TUR group (with or without mitomycin), especially in patients with primary tumours. Recruitment in this trial was not completed, according to Muschter in 1991 reviewis $^{[51]}$. Furthermore, the impact of this trial is limited due to small numbers and short-term follow-up. Subsequently, Hofstetter would, as discussed by Müschter, publish case-series including 600 and 1,000 patients, respectively, with a local recurrence rate of $1.5 \%$ depending on the stage of the tumour ${ }^{[5 \%}$.

Beisland (1986) conducted a study in the mid-eighties including 100 patients who all received either cw bare fiber Nd:YAG laser treatment as a stand-alone procedure or as a combination procedure after resection of the bulk of the tumour. Results were encouraging and justified an RCT ${ }^{19}$. In the RCT 122 patients with urinary bladder cancer were randomized for $N d$ : $Y$ AG laser treatment $(n=63)$ or TUR $(n=60)$. The randomization procedure, however, was inadequate. The two groups were then subdivided according to the size of tumour being smaller or larger than the diameter of the diathermy loop $(6 \mathrm{~mm}$ ), The small tumours were all of stage T1 (laser $n=18$; TUR $n=16$ ). The smaller tumours were either laser irradiated or removed transurethrally in one piece. Large tumours (laser $n=29$; TUR $n=34$ ) were transurethrally resected with subsequent laser irradiation of the tumour base $1-2$ weeks later or transurethrally resected only. TUR was carried out under spinal anesthesia, laser treatment was carried out under local anesthesia. There were no complications in either group. Laser treated patients left the hospital the same day or the next morning. Follow-up was 2 years, there was no dropout. Statistical analyses were performed according to the two-sided Chi-square test of homogeneity. Recurrence of tumour in the treated area was seen in 3 out of 62 patients ( $4.8 \%$ ) in the aser group and in 19 out of 60 patients $(31.6 \%)$ in the TUR group $(p<0.001)$. New tumours in the non-treated area occurred in 12 patients in the laser group and in 13 patients in the TUR group. Occurrence of new tumours in non-treated areas was independent of traatment modality. Small tumours did not recur irrespective of the type of treatment. In large tumours of stage T1 and T2 which were transurethrally resected recurrences only occurred in 13 of 34 cases $(38 \%)$ whereas no recurrence occurred when combined with laser irradiation $(p<0.001$ and $p=0.042$, respectively). The authors stated that the results suggested that Nd:YAG laser treatment should be the treatment of choice in urinary bladder tumours of stage T1. The size of the tumour is decisive in the choice between laser treatment alone or combined with TUR. According to Hofstetter $(1984)^{|34|}$ and Beisland and Seeland $(1986)^{[B]}$, the laser could be applied technically successfully in tumours of maximally 15 to $20 \mathrm{~mm}$ in diameter. No definite recommendations could be provided in treatment of T2 tumours due to small numbers of patients. No data were provided on length of hospitalization of transurehthrally resected patients ${ }^{\mid \theta\}}$.

In reviews on lasers in urology it has repeatedly been stated that other authors, trying to apply the same technique, could not reproduce the results achieved by Hofstetter et al. and Beisland and Seeland ${ }^{[3.36 .58]}$. This could, among other factors, be due to a biased distribution of prognostic factors of patients included in the studies (e.g. the size and number of tumours present, number of prior lumour recurrences, failure to respond-to previous intravesical chemotherapy, and differences in skills of individual urologists or irreproducible dosimetry regimes ${ }^{[3.37]}$. Thus, although the safety and efficacy of laser treatment of superficial bladder cancer had been established both by a few RCTs and by numerous case-series sometimes including 1,000 patients, its superiority over TUR had not been convincingly demonstrated according to the urological community ${ }^{[24,37,58\}}$. Therefore, In 1990, the European Organization for Research and Treatment of Cancer (EORTC) organized a multinational, multicenter RCT to compare the efficacy of TUR and Nd:YAG laser treatment followed by intravesical chemoprophylaxis with epirubicin on the recurrence rate and progression 
of single recurrent, multiple primary or recurrent, Ta or T1 transitional cell carcinoma of the bladde: less than $2 \mathrm{~cm}$ in diameter ${ }^{264}$. As far as we know "this RCT failed because the urologists involved in the trial failed to adhere to the randomization scheme whenever a patient was allocated to laser treatment (Prof. Dr. F. de Bruyne, personal communication). Instead, the EORTC and other international research groups have focused on the role of warious schemes of adjuwant chemotherapy after TUR on recurrence rates and progression of superficial bladder cancer ${ }^{[144}$. This contrasts with Johnson and Castello (1994), who, in their review of lasers in urology, state that the Md YAG laser has become widely accepted around the world for treating superficial bladdef tumours $^{|3| 3 \mid}$ : Overall, I would say there is sufficient evidence to accept the Na:YAG laser as a treatment modality for superficlal bladder cancer (Ta, T1). Its superiority to TUR, however, has never been fully convincingly demonstrated. Its superiority too TUR is likely.

\section{Cost-effectiveness}

No formal cost-effectiveness analyses have been done in this field. In the United States, in 1987, estimates have been made on the cost of inpatient treatment of bladder-tumours compared with outpatient treatment with laser. In neither case is the physician fee included: Inpatient treatment was found to cost US $\$ 3818$ per case, whereas laser treatment costs US\$681, for a saving of $\$ 3137$ per case. The basis for the cost estimates is not clear, although the author states that it is based on "actual data"(5). Overall it seems that even if laser therapy would just be as effective as TUR it could potentially be cost-effective due to its low morbidity, outpatient treatment and lack of the need for general or spinal anaesthesia.

\section{Photodynamic therapy in superficial bladder cancer}

In a review on clinical and preclinical photodynamic therapy; Fisher et al. (1995) report that PDT phase III trials are underway for prophylaxis of recurrent papillary bladder cancer ${ }^{25 !}$. In these trials, Photofrin and low dose light is used after TUR, with irradiation of the whole bladder. Nonrandomized studies had demonstrated that widespread superficial lesions and tumours $<2 \mathrm{~cm}$ in diameter can be completely eradicated using PDT. Single treatment PDT in these patients was reported to result in cure rates of $70-95 \%$. In addition, according to Fisher et all. a phase II study is being performed in the United States and Europe for carcinoma in situ (CIS) of the bladder, in which PDT is an alternative to cystectomy ${ }^{(25)}$. These data suggest that PDT could potentially play a role in a treatment strategy of superficial bladder cancer aimed at preventing recurrences and. ultimately, in preventing or delaying the need for cystectomy.

\subsubsection{Invasive bladder carcinoma}

Both cystectomy, radiotherapy and chemotherapy play a role in treatment of invasive carcinoma, either alone or as a combination therapy ${ }^{[0]}$. Laser treatment has been applied in patients who were either unfit for radical surgery or refused the operation or other treatment options.

Hofstetter (1989) presented results of 49 patients of whom 45 received Nd:YAG laser therapy when tumours were smaller than $2 \mathrm{~cm}$ in diameter and combined transurethral resection and laser therapy in case of larger tumours ${ }^{[34]}$. Four patients underwent cystectomy after laser therapy. Additional therapies were administered as well including topical chemotherapy and pelvic lymphadenectomy in unspecified numbers of patients. Mean follow-up was 36 months. Of the 49 patients, 20 had grade T2 tumours and 3 patients had grade T3 tumours. Of the first group, 9 patients were free of recurrence, in another 9 patients the tumour recurred and in 2 patients the tumour progressed. In all three patients in the second group the tumour progressed. No data on the use of anesthesia, complications and length of hospitalization were provided.

Other authors, e.g. Beisland and Seeland (1986) have produced similar results, suggesting that combined TUR/Nd:YAG laser therapy could be beneficial for selected patients with stage T2 bladder tumours ${ }^{[\theta]}$. Overall, however, the role of lasers in treatment of invasive bladder cancer seems limited $\mathrm{d}^{[3,36,54]}$. 


\subsection{Benign urethral and penile lesions}

\section{Condyloma acuminata}

Condy oma acuminata is a sexually transmitted disease caused by infection with the humane papilomavirus, often occuming on the external genitals and in the urethra of (often) young patients. Treatment of these warts has included systemic or local drug administration, electroresection and fulguration, oryotherapy and laser treatment:

Typical results of Nd:YAG laser treatment of condyloma in the urethra, according to Muschter in a review ${ }^{254 !}$, were published by Schilling et al. in $1986^{\mid 160]}$. In 48 patients, including 7 severely affected, all patients were free of warts after 3 sessions on the average. After a mean follow-up of 1.9 years, only one patient needed retreatment due to recurrence at the region of the externat sphincter. Other authors have reported similar results ${ }^{[51]}$.

Treatment of external condyloma acuminata has been done using Nd:YAG lasers and to a lesser extent, by using $\mathrm{CO}_{2}-$ Ho:YAG-, argon- or KTP lasers, achieving greater than $90 \%$ success rates for grossly visible lesions ${ }^{[36]}$. According to Muschter, the recurrence rate after laser coagutation is much lower than that experienced with conventional methods, while providing excellent cosmetic results, even in severe cases. Severe complications after laser surgery are not known ${ }^{[5+1}$. Because viable virus. DNA particles have been shown to be present in the laser plume, several safety measures have to be applied, including the use of a laser smoke evacuator, wearing of a laser surgery mask, gowns and gloves by the surgeon and assisting personne:|[36!

Overall " laser treatment for condyloma acuminata in the urethra be judged as established by clinical experience. Compared to surgery, assuming at least a few days of hospitalization, higher morbidity and recurrence rates with relatively modest cosmetic results, laser therapy could potentially be cost-effective. More information on laser treatment of condylomata acuminata, but now in gynecology, can be found in Chapter 10.

\section{Uncommon urethral lesions}

There is limited experience in treatment of urethral lesions such as urethral strictures, neonatal posterior urethral valves and bladder neck contracture, using either contact or non-contact Nd:YAG-; -KTP or Ho:YAG lasers. It is as yet unclear if the laser offers advantages over conventional therapy for these lesions including for example cold knife urethrotomy for urethral strictures, and transurethral incision by means of either electrocautery or a urethrotome for bladderneck contracture ${ }^{[3,36]}$.

Only one of these indications, treatment of urethral strictures, has been subject to a small RCT. In this trial, Vicente et al. compared direct-vision endoscopic urethrotomy (cold-knife incision) and endoscopic urethrotomy (single cold-knife incision) followed by Nd:YAG laser coagulation of the borders of the incised area in treatment of 30 patients with single, iatrogenic, annular strictures of the bulbar urethra ${ }^{[05 !}$. Follow-up was 2 years. Succesful treatment was defined as satisfactory voiding, a maximum urine flow equal or greater than $15 \mathrm{ml} / \mathrm{s}$ and a normal eretrograde-voiding urethrography. After one year, treatment was judged succesful in 12 out of 15 patients in the traditional treatment group $(80 \%)$. After two years tratment was judged succesful in $60 \%$ of patients. In the "laser" group, in 11 out of 15 patients $(73.3 \%$ ) treatment was judged succesful both after one and two years followup. Without presenting data on the type of analysis, these differences were reported not to be statistically significant. However, the results were interpreted as indicating a lesser tendency for late recurrences when the Nd:YAG laser was used as part of the procedure. The authors noted that treatment results for this particular patient group were better than those reported in literature for all types of urethral strictures. 


\section{Uncommon penile lesions}

Of the more uncommon benign penile lesions treated with laser, including balanitis xerotica obliterans and Peyronie's disease, no more evidence than just case-reports are available? Peyronie's disease is caused by the formation of fibrotic or sclerotic plaque in the penis, resulting in painful erections and deviations, making sexual intercourse difficult or impossible. Conventional treatment includes a variety of medical treatments, surgical excision with or without grafting and radiation. None of these techniques are well-established ${ }^{127]}$. Treatment of 4 patients using an Nd:YAG laser resulted in straight erections and was performed with minimal blood loss. In another series of 5 patients a $\mathrm{CO}_{2}$ laser was used in patients with thin plaques with moderate success in subsecuent patients, venous grafts were preferred as single treatment in patients with thin plaques while the $\mathrm{CO}_{2}$ laser was used only for patients with large wide plaques in preparation of graff placement. Most promising results were achieved in 20 patients with a low power Ga-Al-As diode laser. Pain was relieved in $90 \%$ and curvature was reduced in $80 \%$ of these patients ${ }^{[30]}$.

\section{Bowenoid papillosis}

Bowenoid papillosis is a benign lesion appearing as rounded, reddish to violaceous papules arising on the glans or, more commonly, the shaft of the penis. The lesions are usually small (median 7 $\mathrm{mm}$ ) but they are often present in groups of 5 or more nodules that may coalesce to form larger plaques. A strong association with the presence of humane papilloma virus has been reported, The standard therapy is superficial excision strictly restricted to the lesion. $\mathrm{Nd:YAG}$ and $\mathrm{CO}_{2}$ lasers have been successfully applied to treat these lesions, just as a variety of other techniques including cryotherapy, electrocautery and topical administration of 5-fluorouracil. These techniques are recommended in case surgical excision would be disfiguring. In a series of 75 surgically treated patients, 15 patients had a recurrence after a median follow-up of five years. Recurrence rates when other techniques are applied are unknown. Laser therapy appears to be effective at obtaining local control of disease while achieving excellent cosmetic results ${ }^{[29]}$

\subsection{Penile precancerous and cancerous lesions}

The incidence of penile intraepithelial neoplasia $(P I N)$ is undocumented. The incidence of carcinoma of the penis (carcinoma in situ and invasive carcinoma) is estimated to occur in less than $1 \%$ of all malignancies in the American male population ${ }^{[29 !}$. Worldwide the tumour incidence varies markedly with standards of hygiene and with cultural and relligious practices ${ }^{[13 !}$. The incidence of penile carcinoma is expected to increase, as it is thought to be caused by humane papilloma virus infection, which prevalence is increasing rapidly ${ }^{(25)}$. Standard treatment for penila carcinoma is mainly surgical. Depending on the stage of the disease, either local surgical excision with a $5 \mathrm{~mm}$ margin of healthy tissue or partial or total penectomy is applied with or without groin dissection, with or without circumcision and sometimes followed by radiotherapy ${ }^{[13,29]}$. This leads to recurrence rates up to $50 \%$ after local excision, overall 5 -year survivall rates between $70-80 \%$, with loss of sexual function in the majority of $\operatorname{cases}^{[13,29,45 !}$.

In 1976. Hofstetter and Frank introduced the Nd:YAG in treatment of a variety of penile lesions. Since then a limited number of case-series including at most 49 patients have been published where lasers were used in treatment of these patients, either as a stand-alone procedure with the $\mathrm{Nd}$ :YAG-, $\mathrm{CO}_{2}$ - or KTP laser or after local surgical excision. Sometimes the $\mathrm{CO}_{2}$ laser was used in combination with the Nd:YAG laser. The latter laser was used in the majority of cases (either contact mode using sapphire tips or bare fibers in a non-contact fashion) under general anesthesia ${ }^{[13,29,36,45]}$. In a combined analysis of 5 case-series including 56 patients, Maiek concluded that the local recurrence rates for carcinoma in situ and T1 tumours after laser therapy (16\%) were as good as those obtained after traditional penectomy $(17.6 \%)$, but average rates of local recurrence were significantly worse (57\% vs $17.6 \%$ ) for stage T2 tumours and hopelessly high $(100 \%)$ for stage $\mathrm{T} 3$ tumours ${ }^{(45)}$. Maleks own report was based on 19 patients with penile intraepithelial neoplasia and 11 patients with either carcinoma in situ $(n=6)$ and invasive carcinoma $(n=5)$ of whom 23 were followed up for at least two years. His results compared favourably to those 
reported in the literature, with the exception of recurrence rates after treatment of T3 tumours which was $100 \%{ }^{1453}$. He concluded that these results were clinically equal and cosmetically and functionally far superior to partial penile amputation

Since then other authors. presenting cases-series including maximally 59 patients have reported similar results and arrived at the conclusion that laser therapy, especially thinking of the NdYAG laser, should be the first choice of treatment in both precancerous and cancerous lesions. It seems that only deep invasive disease (T3) can not be controlled with laser treatment ${ }^{\mid 36)}$. Overall, laser treatment is established by dinical experience for this indication. Although formal cost studies are lacking, laser treatment could potentially be cost-effective for this indication, with the highest probability in cases where a penis amputation is avoided.

\subsection{Upper urinary tract urothelial tumours}

Transitional cell carcinoma of the renal pelvis and ureter are estimated to arise in the upper urinary tract only once per 70 urothelial tumours. Standard treatment of these fumours has been a nephroureterectomy (removal of the ureter and the kidney) including removal of a cuff of bladder surrounding the ureteric orifice. The first successful endoscopic Nd:YAG laser treatment of distal ureteral tumours was reported by Hofssteter et al. in 1983, reporting 4 patients to be tumour free after one year of follow-up ${ }^{[36.51]}$. Subsequent case-series including up to 20 patients have confirmed the impression that patients with low-grade, low stage ureteric tumours can be satisfactorily treated with laser photoirradiation. However, local recurrence, strictures or both can develop, which can be treated by laser fulguration or transurethral resection ${ }^{[36]}$. Overall, I would regard these results as promising. Likewise, laser therapy has been used in treatmentt of small numbers of patients with fumours of the renal pelvis with encouraging results ${ }^{[36]}$. In the literature, laser therapy is recommended for highly selected patients only (including low grade tumours in solitary kidneys, renal failure and those unfit for extensive surgery $)^{3.51 !}$

\subsection{Hemangioma, Cystitis and Vasovasostomy}

\section{Hemangioma}

Hemangiomas are uncommon lesions that may occur anywhere in the urinary tract, but most often inwolve the kidney, bladder and urethra. They usually present as isolated lesions, but can be diffuse as well. Treatments for these lesions have included TUR and electrocoagulation; injection of sclerosing agents, ilradiation and open surgical resection. A few case-series have been published where Nd:YAG laser energy was applied, with encouraging results ${ }^{[3.36]}$. The largest of these caseseries consisted of 13 patients in whom Nd:YAG laser energy was applied under general anesthesia through a rigid cystoscope in 4 patients with local disease and in 9 patients with diffuse disease. In 3 of the four patients with local disease no residual hemangioma was found after 3-6 months, whereas a marked decrease in the size of the hemangiomas in the remainder of patients was found, who had extensive bladder involvement. In five patients treatment was performed on an outpatient basis, with overnight observation in 8 patients. No patients required catheterization, hospitalization or transfusion ${ }^{[36]}$

Based on such encouraging data laser treatment is adwocated as the preferred therapeutic method for most of these patients ${ }^{|3.3|}$. Of course, evidence is still limited with laser treatment and a systematic comparison with other treatment modalities is lacking.

\section{Cystitis}

Of the three types of cystitis where Nd:YAG laser energy has been applied, cystitis glandularis, themorrhagic cystitis and interstitial cystitis, only for the latter two indications there is evidence available other than case-reports ${ }^{[36,75]}$. Ravi (1994) treated 42 patients with radiation-induced hemorrhagic cystitis with the cW Nd:YAG laser under local anesthesia and achieved control of bleeding in 39 patients after one treatment and in two additional patients after two treatments ${ }^{[7)^{5 j}}$. 
There were no complications of the procedure. Cystitis did not recur in any of the 18 patients who were willing to undergo a follow-up cystoscopy after 6 months.

In the largest case-series of patients with interstitial cystitis, by Shanberg \& Malloy, reviewed by Johnson and Costello (1994), 86 patients of whom 27 had Hunner's ullcers were treated with the cW Nd:YAG laser ${ }^{\text {ralj }}$. Frequently the endoscopically applied Nd:YAG laser was combined with laparoscopy to be sure there was no risk of injury to the intra-abdominal organs. In $21(78 \%)$ af the patients with Hunner's ulcer, symptoms such as nocturia and pain showed immediate or marked improvement. Nine of these patients improved permanently, in the other 12 patients symptoms recurred within 18 months follow-up. Of the remaining 59 patients, 20 (34\%) showed marked symptomatic improvements during the initial post-operative period. Within 12 months, however, 10 of them $(50 \%)$ required further treatment. It was concluded that laser treatment is most beneficial in patients with Hunner's ulcer. Based on these results the authors recommended laser therapy in patients with interstitial cystitis that have failed more conservative therapy including hydrodistension, oral drug therapy and intravesical therapy with dimethylsulfoxide (DMSO).

\section{Vasovasostomy}

Since 1980 , when Jain reported the first successful sutureless microvascular anastomosis using an Nd:YAG laser, much research has been directed at using laser energy, either alone or as an adjunctive procedure in tissue-welding, e.g. to perform ureteric reimplantation, enterocystoplasty, ureteroureterostomy, patch-graft urethroplasty, sutureless bowel anastomosis and vasovasostomy. Of these laser-tissue welding applications only vasovasostomy has been applied clinically in caseseries of patients. In the largest case-series of 32 patients, reviewed by Johnson and Costello (1994) a $\mathrm{CO}_{2}$ laser was used to assist in vasectomy reversal, with encouraging results (expressed in terms of patency rates for sperm and pregnancy rates) compared to conventional microsurgical techniques $^{(36)}$. Overall, these applications are still experimental, to some extent due to an incomplete understanding of the mechanism(s) whereby laser photoirradiation "bonds" or "fuses' tissue together ${ }^{|36|}$.

\subsection{Urolithiasis}

Urinary tract stones occur with an incidence of about 1 in 1,000 population per year ${ }^{[6]}$. In the United States it has been estimated that approximately $5 \%$ of the women and $12 \%$ of the men will have at least one episode of urolithiasis during their lifetimes. Worldwide, the prevalence of urolithiasis in men by the age of 70 years varies from approximately $4 \% \mathrm{~m}$ England to $20 \%$ in Saudi Arabia. Stone disease is not fatal and as many as $70 \%$ of urolithiasis patients do not require hospitalization. Incapacitation is usually short-lived and most return to their normal life-styles. Recurrence rates increase with follow-up, with $1-5$ - and 10 -year recurrence rates being $13.7 \%$, $34.8 \%$ and $52 \%$, respectively ${ }^{156]}$.

Traditional open surgery for urinary tract stones has almost entirely been replaced by minimally invasive techniques in the seventies and eighties ${ }^{\mid 41,71 !}$. Extracorporeal shock wave lithotripsy (ESWL) has become the treatment of choice for most renal and ureteral stones and is by far the most frequently used treatment modality ${ }^{[19]}$. For example, In the United States in 1992, combining data on renal and ureteral stones, ESWL accounted for $66 \%$ of all procedures, followed by ureteroscopic modalities (27\%), percutaneous neprolithotomy $(6 \%)$ and open lithotomy (1\%) ${ }^{[56 !}$. No randomized trials have been done comparing ESWL or any other treatment modality, such as percutaneous nephrolithotomy ( $\mathrm{PCN}$ ), to open surgery ${ }^{[i]}$. As far as I know only one relatively small RCT has compared minimally invasive treatment modalities, focusing on ESWL versus PCN in medium sized renal stones ${ }^{[6]}$. Another study compared ureteroscopy and ESWL in treatment of lower ureteral stones, using matched historical controls ${ }^{[22]}$. Due to methodological and organizational problems, especially in the study comparing ESWL to PCN, and due to rapid technological progress, these studies have not been very influential. The present status of treatment modalities for specific indications has been determined by clinical case-series. For 
example, PCN is the first choice of treatment in patients with simple renal stones of at least $2 \mathrm{~cm}$ in diametter ${ }^{\text {(9) }}$. In smaller-sized stones ESWL, the least invasive treatment modality, is tried first; when ESWL fails, PCN is attempted. Information on the diffusion of these techniques can be found in Chapter 14 and 15. In renal stones, besides ESWL and PCN, in selected cases (e.g. in large stones) ureteropyeloscopic modalities are applied. In this technique, access is gained from below.

Technological developments are rapid in this field. New devices are developed and at the same time existing devices, including lasers, are refined. Especially miniaturisation and increased flexibility of endoscopes (nephroscopes and ureteroscopes) and associated working instruments (including baskets, graspers, forceps and snares) are important in this respect ${ }^{\text {t4.695 }}$. Of course, this situation complicates evaluation. Before discussing the clinical evidence on the use of lasers here, one may wonder about the actual position of the use of this device in lithotripsy. A distinction is made between renal stones, ureterall stones, and bladder stones.

Laser lithotripsy is one of the modalities that can be used as an adjunct to or as part of PCN and ureteropyeloscopic procedures ${ }^{[4.43]}$. In 1992, in the US, $1 \%$ of all procedures was performed using PCN for stones more than $2 \mathrm{~cm}$ in size ${ }^{[56]}$. Besides lasers, also techniques such as electrohydraulic and ultrasonic lithotripsy can be applied as an adjunct to $P C N^{[4]}$. In ureteral stones, besides ESWL, laser lithotripsy is one of a large variety of ureteroscopic modalities ${ }^{[3,4,57]}$. Referring again to the United States in 1992, urolithiasis procedures in which ureteroscopy was combined with lithotripsy: and thus potentially with a laser; represented $8 \%$ of all procedures ${ }^{|56|}$. In bladder stones, ESWL and a limited number of cystoscopic modalities are used, including, occasionally, laser lithotripsy ${ }^{14,19 !}$. In the Netherlands, the total percentage of urolithiasis patients eligible for laser lithotripsy was estimated to be no higher than $10 \%{ }^{1571}$. Thus, notwithstanding the notice that 'lasers are 'sexy' and often sought at after by patients ${ }^{154 !}$, their actual frequency of use in stone disease is rather limited. In how far is the (limited) use of lasers for these indications supported by clinical and economic evidence?

No RCTs have been published comparing laser lithotripsy with other ureteroscopic modalities or 10 ESWL. Evidence of the safety and effectiveness of laser lithotripsy is therefore restricted to case-series. Of all types of lasers used, most experience has been gained with the Candela pulsed dye laser (504 nm, coumarine green), which was introduced in treatment of ureteral stones in 1985 by Watson and associates ${ }^{[68,69)}$. When it was demonstrated that the pulsed dye laser could successfully be applied in treatment of ureteral stones its use was broadened to include renal slones and bladder stones.

Other types of lasers that have been introduced for lithotripsy include the qs Nd:YAG laser, the Alexandrite laser, the Excimer laser, the Holmium laser and the Lithognost, a dye laser which uses modamine as a laser medium. Clinical experience with these lasers is limited. There is evidence that different types of lasers can be most successfully applied in stones with a specific composition $(\text { colour })^{4,40,21 !}$.

A nice example of the impact of technological progress on the safety and effectiveness of laser lithotripsy and of the impact of the availability of more than one treatment modality is provided by Watson et al. (1993), where they analyse results of more than 1,000 patients treated with the pulsed dye laser in the period 1985-1990/69! . Best results were achieved in their latest series, comprising 74 patients in whom predominantly a small calibre ureteroscope was used ${ }_{*}$ the Candela Miniscope, made by the manufacturer that also produces the pulsed dye laser. Furthermore, in these patients laser lithotripsy was combined with stone basketing during the initial procedure. In $89 \%$ of patients complete stone clearance was achieved after at least three months follow-up. All remaining stones were cleared by additional ESWL in one case $(1.5 \%)$ and a second ureteroscopy in 7 cases $(9.5 \%)$. Complications in their latest series of in total 224 patients, where laser lithotripsy was used either as monotherapy (139 patients) or in combination with basketing ( 74 patients, the patient group just discussed) included perforation of the ureter ( 3 patients), postoperative fever ( 4 patients), a transient ischemic attack (1 patient), and a possible myocardial infarction (1 patient). in total complications occurred in 9 of 224 patients (4\%). All patients recovered. No ureteral 
strictures occurred; in contrast with older series. No data were provided on the use of anesthesia and the average length of hospital stay ${ }^{\text {(6). }}$ : In general, ureteroscopic procedures were considered as inpatient procedures requiring regional or general anesthesia with an average hospital stay of a few days ${ }^{2}$. But the development of smaller ureteroscopes and miniaturised equipment has enabled urologists to gradually perform ureteroscopy as an outpatient procedure with low complication rates under local anesthesia in the majority of cases ${ }^{\mid 72 !}$. This may increase the role of ureteroscopic procedures in the future.

In summary, the use and role of lasers in lithotripsy is complex ${ }^{\text {[3. }}$. Its effectiveness, especially in treatment of ureteral stones, seems well established and, according to Bagley (1994), pulsed dye laser lithotripsy has the largest margin of safety of all ureteroscopic modalities ${ }^{[4]}$. In well documented case-series using the latest instrumentation, laser lithotripsy, either applied as monotherapy or in conjunction with other ureteroscopic modalities, seems to approach or even exceed the effectiveness of ESWL. Clearly, there is a need for randomized trials in this field. In the Netherlands, a large $(n=1,200)$ multicenter RCT is being organized to compare ESWL and a number of ureteroscopic modalities for middle and lower ureteral stones, including pulsed dye laser lithotripsy. Other ureteroscopic modalities included in this trial are the Lithoclast (a mechanica technique) and the electrohydraullic technique (A. Hendrikx, MD. personal communication). Interestingly, only the initial treatment is determined by randomization. If a second procedure is needed, the urologist is completely free in his choice, which will be dependent on the availability of specific modalities at the institution. This will no doubt result in all sorts of combination therapies, presumably creating difficulties in formulating clear clinical practice guidelines. Despite this initiative, due to both the large variety of instruments, rapid technological progress and the relative scarcity of RCTs the field, the present lively debate on optimal treatment strategies for individual stones (in particular depending on its location, size, composition and/or local anatomical condition. such as obstruction, impaction and infection), is likely to continue ${ }^{[26.35 .54: 55]}$.

\section{Cost-effectiveness}

To start with it is important to notice that as yet there is no conclusive evidence of the relative effectiveness of laser lithotripsy. It is often doubted whether laser lithotripsy can be cost-effective. One reason is the high capital costs of the laser (US\$286 000 for a pulsed dye laser and US\$200 000 for a (solid state) Alexandrite laser (1994 prices, A. Oosterlee, M.Ba.. personal communication). Other reasons are the need, depending on the intensity of use, to replace the container with dye at a cost of US\$1500 ${ }^{\mid 23)}$, and the costs of (single use) laser fibers (about US $\$ 150$ per fiber) ${ }^{[52]}$. Although lasers are not cheap, the same holds true for ESWL machines, which could be purchased in the late eighties in the Netherlands for about US\$1 $250000^{[6 \% ?}$.

There are several options to assess the cost-effectiveness of laser lithotripsy, which perhaps can be subdivided in four categories. Firstly, one can think of the cost-effectiveness of laser lithotripsy monotherapy versus ESWL. A second option is laser lithotripsy monotherapy versus other individual ureteroscopic modalities. Thirdly, a combination therapy of endoscopic treatment modalities compared to ESWL and, finally, one could integrate ESWL and endoscopic treatment modalities in unified strategies and evaluate the most common and/or promising strategies. Reading the literature it is amazing to see how little is known on the issue of cost-effectiveness of treatment alternatives in urolithiasis ${ }^{[56]}$. At the same time, here and there some attempts related to laser lithotripsy have been traced, but these all suffer from serious problems including highly selected patients, extremely low patient numbers, limited data on costs etc. Some information is available on each of the options listed above, with the exception of the first one, laser lithotripsy wersus ESWL. The remaining options are briefly discussed.

\section{Laser lithotripsy versus other individual ureteroscopic modalities}

Esuvaranathan at al. (1992) tried to assess the cost-effectiveness of pulsed-dye laser lithotripsy $(n=64)$ versus ultrasonic lithotripsy $(n=98)$ in two selected consecutive series of patients with stones in all parts of the ureter ${ }^{23 !}$. The success rate for a first attempt at laser lithotripsy was $92 \%$ versus $71 \%$ for the ultrasound series. When the stone could be reached both treatment modalities had a 
fragmentation rate of $75 \%$. Overall morbidity was lower in the laser group, of which half were trealed as outpatients. In a hypothetical cohort of 100 patients in each treatment group, the average hospital costs per laser lithotripsy were calculated US\$1445, compared to US\$973 for Uittrasound lithotripsy. In the laser group the costs included a fee of US\$937 for each Use of the laser, levied in order to recapitulate capital- and maintenance costs of the laser in three years time; based on an annual case-load of 100 patients and a repeated use of the laser in a third of all patients" in total US\$324900.

The authors suggested that the increased costs of laser therapy is justified due to its increased success rate at the initial attempt, in other words that laser lithotripsy is cost-effective. The costs of laser treatment were shown to be sensitive to annual case-load and depreciation time. However; there were several methodological problems in this stuidy. For example, laser lithotripsy patients were followed prospectively for 6 to 12 months whereas ultrasound lithotripsy patients were assessed retrospectively for a period of one year. Furthermore, in laser lithotripsy, treatment was delivered using a small semirigid ureteroscope, whereas ultrasound lithotripsy was delivered using a relatively large, rigid ureteroscope. Therefore, one might suggest that the discrepancy in successful stone removal was due to the type of ureteroscope used and not to the mode of stone fragmentation $^{[54]}$. As smaller ultrasound probes have become avalable and noticing that the capital outlay for ultrasound probes is small compared to pulsed dye lasers, it could well be that laser lithotripsy is not cost-effective compared to this ureteroscopic modality for treatment of ureteric stones.

In another study Naqvi and colleagues (1994) compared the cost-effectiveness of Alexandrite laser lithotripsy (LL) and pneumatic lithotripsy (PL) using the Lithoclast ${ }^{[52 !}$. The analysis included treatment of 200 stones, predominantly in the lower ureter, in 194 patients. 48 Stones were fragmented using the laser and 152 with the Lithoclast. All patients were treated under regional or general anesthesia and allways the same ureteroscope was used. The overall stone-free status at 4 weeks was $95 \%$ for $\mathrm{PL}$ and $84 \%$ for $\mathrm{LL}$. The non-fragmentation rate was $10 \%$ for $\mathrm{LL}$ and $1 \%$ for PL. Post treatment complications were encountered in $8 \%$ of the patients treated by LL and $7 \%$ by $\mathrm{PL}$. In the cost-analysis the authors only included capital costs and costs of consumables (e.g. the laser fiber, arguing that all other cost components were identical. Capital costs of the laser was US\$200 000, each laser fiber (about one per patient) costs US\$150 and the capital outlay for the Lithoclast is about US $\$ 25000$. Based on capital cost depreciation of an unspecified number of years using an estimate of the annual case load of 200 patients, the cost difference per patient was calculated US $\$ 305$ in favour of the Lithoclast (differential average direct costs per laser treatment were US $\$ 350$, compared to US $\$ 45$ for the Lithoclast). The authors concludied that PL is costeffective compared to $\mathrm{LL}$. This conclusion is not convincing, among other factors due to limited data on direct cost components and because roughly the first half of the patients discussed here consisted of two consecutive case-series, whereas in the second half of the series both techniques were used concurrently, where PL tended to be used for larger and radiologically harder stones, possibly introducing selection bias.

\section{ESWL versus combination strategies of endoscopic modalities}

Laser lithotripsy is often combined with other endoscopic modalities, as demonstrated by Watson et al. (1993) (teg) $^{\text {. Of course }}$, this is not the only combination possible. Hofbauer et al. (1993) published a case-series where in some patients laser lithotripsy was used as first endoscopic treatment in the entire ureter, with the Lithoclast as second option in those patients where laser lithotripsy failed. In contrast with Watson et al., no basketing was used in the study by Hofbaver et al. where a stent was inserted instead ${ }^{\mid 32 !}$. Another example is provided by Bagley (1994) who reported a case-series where either laser lithotripsy or electrohydraulic lithotripsy was used ${ }^{[4]}$. Combination strategies may represent a realistic alternative for ESWL. This hypothesis is presently tested for a number of endoscopic modalities in the Netherlands (see previous section). No information on economic evaluation is available. 
Combination of ESWL and ureteroscopic modalities in one strategy

Some authors, e.g. Hofbauer et al. (1993) take matters even further and see both ESWL and endoscopic modalities as part of one strategy in urolithiasis ${ }^{[32]}$. Some of the authors advocating integrated strategies, e.g. Eisenberger (1993), see an increasing role of laser lithotripsy in the ureteroscopic modality mix $x^{[21]}$. As yet there no economic evidence available documenting a mixed ESWL/ureteroscopic modalities strategy. As an example of (ultimately) integrated strategies, but without laser lithotripsy, a study by Kapoor et al. (1992) is briefly discussed. These authors compared the cost-effectiveness of ESWL versus a combination of simple extraction electrohydraulic or ultrasonic lithotripsy in 43 patients with lower ureteral stones ${ }^{\text {is? }}$. Of ureteroscopically treated patients all but one $(97.5 \%)$ were stone-free after three months of follow up compared to $90 \%$ ( 18 out of 20) of ESWL treated patients. The overall average costs for ESWL was US\$7320 and for uneteroscopic treated patients it was US\$4569. Interestingly enough, the ureteroscopy failure was successfully managed by ESWL, and both ESWL failures had uneventfu ureteroscopic stone removal. Although these data can at best be considered as indicative, sometimes a lot of uncritical attention is paid to them in editorials $\mathrm{s}^{[621}$. This demonstrates that it is both necessary and difficult to organize potentially influential RCTs (including an economici evaluation) in this highly interesting field and that laser lithotripsy deserves to be included in such evaluations.

Overall, laser lithotripsy may not be cost-effective as a stand alone treatment option but in contrast, it could potentially be cost-effective as part of a strategy, including other endoscapic modalities and ESWL, aimed at avoiding open surgery.

\subsection{Summary}

Table 9.1 summarizes the findings presented in this Chapter.

Table 9.1 Judgments on the effectiveness and cost-effectiveness of selected laser applications in urology

\begin{tabular}{|c|c|c|c|c|c|c|c|}
\hline \multirow[t]{2}{*}{ Indication } & \multicolumn{7}{|c|}{$\begin{array}{c}\text { Judgments } \\
\text { (see definitions below) }\end{array}$} \\
\hline & 1 & 2 & 3 & 4 & 5 & 6 & 7 \\
\hline $\mathrm{BPH}$ & & & $X^{1}$ & & & $x$ & \\
\hline Prostate cancer & & $\mathrm{x}$ & & & & & \\
\hline Bladder cancer & & & $x$ & & & $x$ & \\
\hline Condyloma acuminata & & & $x$ & & & $x$ & \\
\hline Benign urethral and penile lesions & & $x$ & & & & & \\
\hline Upper tract carcinoma & & $x$ & & & & & \\
\hline Penile carcinoma & & & $x$ & & & $x$ & \\
\hline Hemangioma & & $x$ & & & & & \\
\hline Cystitis & & $x$ & & & & & \\
\hline Vasovasotomy & $x$ & & & & & & \\
\hline Laser lithotripsy & & & $x$ & & & $x$ & \\
\hline
\end{tabular}

"The short-term results of laser treatment of BPH in a number of small, ongoing RCTs is not (yet) considered as convincing evidence. 
Definitions:

1 Experimental = still in development, limited clinical data, no proof of effectiveness.

2 Promising $=$ some clinical data, but procedure not accepted as a routine,

3 Established by clinical experience $=$ no randomized trials, but sufficient clinical data to reach a judgment.

4 Established by RCT = established by several well-designed randomized controlled trials.

5 Probably not cost-effective = limited data on effectiveness, but reason to believe that the procedure will not turn out to be cost-effective.

6. Probably cost-effective = limited data on effectiveness, but reason to believe that the procedure will turn out to be cost-effective.

7 Proven cost-effective $=$ adequate analyses of cost-effectiveness

\subsection{References}

1. Abdel-Razzak OM, Bagley HG. Clinical experience with flexible ureteropyeloscopy. The Joumal of Urology $1992(148) 6:$ : 1788-92.

2. Anderson KR, Keetch DW, Albata DM, Chandhoke PS, McClennan BL, Clayman RV. Optimal therapy for the distal ureteral stone: extracorporeal shock wave lithotripsy versus ureteroscopy. The Joumal of Unology 1994(152)1:62-5.

3. Anson $K$, Seenivasiagam $K$, Miller $R$, Watson $G$. The nole of lasers in urology. British Journal of Urology $1994(73): 225-30$.

4. Bagley DH. Ureteroscopy. American Urological Association (AUA). Update Series 1994(13)26:206-11.

5. Banta HD, Schou I. Urology - Cost-Effectiveness (editor's comment). In: Banta HD, Schou I (Eds.). Lasers in Health Care. Effectiveness, Cost-Effectiveness and Policy Implications. Academic Publishing, Frederiksberg, Denmark. 1991, p. 188 .

6. Banta HD. The cost-effectiveness of 10 selected applications of Minimally Invasive Therapy. In: Banta HD (Ed.). Minimally invasive therapy in five European countries. Diffusion, effectiveness, cost-effectiveness. Health Policy Monographs, Val 3. Elsevier Science Publishers, Amsterdam, London, New York, Tokyo, 1993, p. 135-51.

7. Barry M.J. Proving early detection and treatment of prostate cancer does more good than harm: the need to support randomized clinical trials actively (editorial). The Journal of Urology 1994(1 52)5(part 2 of 2$): 1903-4$.

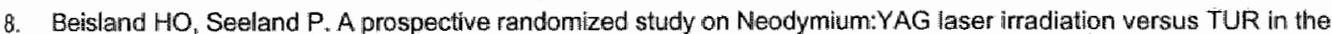
treatment of urinary bladder cancer. Scandinavian Journal of Urology and Nephrology 1986(20):209-12.

9. Beisland HO. Neodymium:YAG laser in the treatment of urinary bladder carcinoma and localized prostatic carcimoma. Journal of the Oslo City Hospitals 1986(36):63-84.

10. Benizri E, Wodey J, Amiel J, Toubol J. Comparison of 2 pulsed lasers for lithotripsy of ureterall calcull: report on 154 patients. The Journal of Urology 1993(150)16:1803-5.

11. Billebaud T, Mechali P, Astier L, Monneins F "Savatovsky I, Coloby P. Transurethral microwave thyperthermia for benign prostatic hyperplasia: is it effective? Results of a multicentric ramdomized prospective study versus placebo with 6 month follow-up (abstract). The Journal of Urology 1994(151)5:417A

12. Bishop MC. Are the days of Iransurethral resection of prostate for benigin prostatic hyperplasia numibered? Alternatives are still unproved. British Medical Joumal 1994(309):717-8.

13. Boon TA. Sapphire probe laser surgery for localized carcinoma of the penils. European Journal of Surgical Oncology $1988(14)-193-5$.

14. Bouffioux $\mathrm{Ch}_{1}$ Kurth $\mathrm{KH}_{\text {"Bon }}$, Oosterlinck W, Boeken Kruger $\mathrm{C}$, de Pauw M, Sylvester R and the Members a the European Organization for Research and Treatment Of Cancer Genitourinary Group (EORTC GU Group). Intrawesical adjuvant chemotherapy for superficial transitional cell bladder carcinoma: results of 2 Europeam Organization for Research and Treatment of Cancer randomized trials with mitomycin $C$ and doxorubicin comparing eariy versus delayed instillations and short-term versus long-term treatment. The Jounal of Urology $1995(1: 33) 3$ (part 1 of 2):934-41.

15. Buckley JF, Ligam $V$. Paterson $P$ on behalf of the British ELAP Group. Endoscopic llaser ablation of the prostatte gland (ELAP) (abstract). The Journal of Urology $1994(151) 5: 229 \mathrm{~A}$.

16. Carlsson $P_{*}$ Kinn $A C$. Tiselius $H G$, Ohlsén $H_{*}$ Rahmqvist $M$. Cost-effectiveness of extracorporeal shock wave Wthotripsy and percutaneous nepholitotomy for medium-sized kidney stones. Scandinavian dournal of Urology and Nephrology 1992(26):257-63.

17. Cohen JK, Berg G. Benz TB. Cost-effectiveness of the $504 \mathrm{~nm}$ pulse dye laser in the treatment of urinary stome disease (abstract). The Journal of Urology $1990(143)($ part 2 of 2$) 268 \mathrm{~A}$.

18. Costello AJ, Crowe HR. A single institution experience of reflecting laser fibre prostatectomy over four years (abstract). The Jourmal of Urology $1994(151) 5: 229$ A.

19. Dawson $\mathrm{C}$. Whitfieid $\mathrm{HN}$. The long-term results of treatment of urimary stones. British Joumal of Urology $1994(74) 4: 397-404$. 


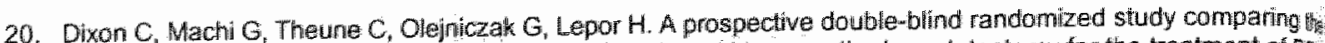
satety, efficacy and cost of laser ablation of the prostate and transurethral prostatectomy for the treatment of $B$ ? (abstract). The Joumal of Urology 1994(151)5:229A

21. Eisenterger F, Schmid A. ESWL and the future of stone management. World Joumal of Urology $1993(1 / 12,2$.

22. El.Faqh SR. Husain 1, Ekman PE, Shama ND, Chakrabanty A, Talie R. Primany cho ce of intervention for disa ureteric stione: ureteroscopy or ESWL? British Journal of Urology 1988(62)1:13-8.

23. Esuvaranathan $K$. Tan EC, Tan PK, Tung KH. Does transurethral laser ureterolithotomy Justify its cost? The Joumet of Urology $1992(148): 1091-4$.

24. European Organization for Resaarch and Treatment of Cancer Genito Uninary Group (EORTC GU Group). Proton 30901 . A phase Ifl multicenter prospective randomized trial to compare the efficacy of transurethral resection and Neodymum: YAG laser treatment followed by intravesical chemoprophylaxis with epirubicin on the recurrence rate and progression of single recurrent, imultiple primary or recurrent, Ta or T1 transitional cell carcinoma of the bladder EORTC GU Group. Brussels, Betgium, 1990.

25. Fisher AMR, Murphree AL, Gomer CJ. Clinical and precinical photodynamic therapy, Lasers in Surgery and Medicine $1995(17): 2-31$.

26. Fuchs Gu. interventional urinary stone management (editorial). The Journal of Urology 1994(151)3:668-9.

27. Ganabathi $K$, Dmochowski $R$. Zimmem PE, Leach GE. Peyronie"s disease: surgical treatment based on pende figidity. The Journal of Urology $1995(153)$ (part 1 of 2):662-6.

28. Garraway W. Kirby RS, Benign prostatc hyperplasia: effects on quality of life and impact on treatment decisions Urology $1994(44) 5: 629-36$

29. Gerber GS. Carcinoma in situ of the penis. The Joumal of Urology 1994(151)4:829-33.

30. Goluboff ET, Olsson CA. Urologists on a tightrope: coping with a changing economy. The Joumal of Urology 1994(151) $1: 4-4$.

31. Goluboff ET, Olsson CA. Urologists on a tightrope: coping with a changing economy (reply to a letter to the editor) The Joumal of Urology 1994(152)4:1215-6.

32. Hofbauer $J_{v}$ Tuerk $C$, Höbarth $K$, Hasun $R$ Marberger $M$. ESWL in situ or ureteroscopy for ureteric stones? World Jourriall of Urology $1993(11): 54-8$.

33. Hofstetter AG, Keiditsch E, Schmiedt E, Frank F. Der Neodym-YAG Laser in der Urologie. Derzeitiger Stand der klinisichen Erfahrungen. Fortschritte der Medizin 1984(102)36:885-90.

34. Hofstetter AG. Bladder cancer, In: Smith Jr JA, Stein BS, Benson RC (Eds. Lasers in urologic surgery (second Edition). Year Book Medical Publishers, Chicago, London, Boca Raton, 1989.

35. Jenkins AD. ESWL: distal ureteral stone management - 1993 (editorial). Urology 1993(42)4:353-5.

36. Johnson DE, Costello AJ. Lasers in Urology (invited review). Lasers in Medical Science 1994(9):145-60.

37. Johnson DE. Use of the Holmium:YAG (Ho:YAG) laser for treatment of superficial bladder carcinoma. Lasers in Surgery and Medicine 1994(14):213-8.

38. Kabalin JN, Gill HS, Bite G, Wolfe V. Comparative study of laser versus electrocautery prostatic resection: 18 month follow-up with complex urodynamic assessment. The Joumal of Urology 1995(153)4:94-8

39. Kapoor DA, Leech JE, Yap WT, Rose JF, Kabler R, Mowad JI. Cost and efficacy of extracorporeal shock wave lithotripsy versus ureteroscopy in the treatment of lower ureteral calculli. The Journal of Urology 1992(148): 1095-6.

40. Kirby RS. Are the day:s of transurethral resection of prostate for benigrn prostatic hyperplasia numbered? Urologists nust grasp the future. British Medical Journal 1994(309):716-7.

41. Kirchberger $\$$. The diffusion of two technologies for renal stone treatment across Europe. In: Stocking B (Ed). A shudy an the diffusion of medical technology in Europe. King's Fund Center for Health Services Development London, England, 1991.

42. Leach GE, Sirls LT, Dmochowski R, Ganabathi K, Zimmern P. Visual laser prostatectomy with local anesthesia (abstract). The Journal of Urology 1994(151)5:4177 A.

43. Loughlin $K R_{n}$ Sharpe $\Downarrow F$. Preliminary experience with the pulsed dye laser for treatment of urolithiasis. Lasiers in Surgery and Madicine 1991(11):1-4.

44. Lycklama à Nijeholt AAB. Bladder carcinoma. Nederlands Tijdschrift yoor Geneeskunde 1992(136)23:1097-1102 (in Dutch).

45. Malek RS. Laser treatment of premalignant and malignant squamous cell lesions of the penis. Lasers in Surgeny and Medicine $1992(12): 246-53$

46. Marks LM. Serial endoscopy following visuall laser ablation of the prostate (VLAP). Urology 1993(42) 1:66-71.

47. NcCullough DL, Roth RA, Babayan RB, Gordon JO, Reese JH, Crawford DE, Fuselier HA, Smith JA, Murchison Rul. Kaye KW. Tramsurethral ultrasound-guided laser-induced prostatectiomy: national human cooperative study restits. The Journal of Urology $1993(150)$ s(part 2 of 2):1607-11.

48. Mebust WK, Hollgreve HL, Cockett ATK, Peters PC. Transurethral prostatectamy: immediate and postoperative complications. A cooperative stludy of 13 participating institutions evaluating 3,885 patients. The Journal of Urology $1989(141) 2: 243-7$.

49. Montie JE. Observations on the epidemiology and natural history of prostate cancer. Urology $1994(44) 6 \mathrm{~A}: 2 \times 8$. 
50: Muschter R, Permutter AP. The optimization of laser prostatectomy part Il: other lasing lechniques. Unology 1994 (44)6:856-61.

51. Muschter R. Urology. In: Bamta HD and Schou l (Eds.). Lasers in Health Care. Effectiveness, Cost-Effectivensss and Policy Implications. Academic Publishing, Frederiksberg, Denmark, 1991, p.180-7\%.

52. Naqwi SAA, Khaliq M, Zafar MN, Rizvi SAH. Treatment of urinary stones. Comparison of laser and pneumatic lithotripsy. British Joumal of Urology 1994(74)6:694-8.

53. Fermutter A.P, Mischter R. The optimization of laser prostatectomy pant : free beam side fire coagulation. Urology $1994(44) 6: 847-55$.

54. Preminger DM. Management of ureteral calculi. The debate continues... The Joumal of Urology 1992(148):1102 4.

55. Preminger GM. Editorial: techniques versus technology: what is the most appropriate method for the remowal of wreteral calculi? The Joumal of Urology $1994(152): 66-7$.

56. Resnick M1. Persky L. Summary of the National Institutes of Arthritis, Diabetes, Digestive and Kidney Diseases Conference on Urolithiasis: state of the art and future research needs. The Journal of Urotogy 199:5(153)1:4-9.

57. de Reyke ThM, Zeijlemaker BYW. Sterenborg HCJM, Kurth K(H. Use of laser in treatment of urohithiasis. Nederlands Tijdschrift voor Geneeskunde 1991(135)50:2394-4 (in Dutch).

58. Ruston MA, Fowler CG. Lasers in treatment of bladder cancer. British Journal of Urology 1991/67):449-55.

59. Scardino PT. Problem of prostate cancer (editorial). The Journal of Urology 1994(152)5(part 2 of 2 ):1677-8.

80. Schilling $A$, Bowering $R$, Keiditsch $E$. Use of the Neodymum-YAG laser in the treatment of ureteral tumours and urethral condylomata actuminata. European Urology 1986(12)Supplement 1):30-3.

61. Seerden $R$, van Ineveld BM, Nijs HGT, Plaisier PW, van der Hul RL, Berger MY, Duckers 1, van Hout BA. Costeffectiveness analysis of ESWL versus cholecystectomy. IMTA Report Number 92.22. Unstitute for Medical Technology Assessment, Erasmus University Rotterdam, the Netherlands, 1993 (in Dutch).

62. Segura JW. Urateroscopy for lower ureteral stones (editarial). Urology 1993(42)4:356-7.

63. Shaffer B, Bolton D, Costello Au. Evolution, technique and preliminary results of Nd:YAG laser ablation of the prostate using a right angle delivery system wersus TURP: a randomized study (abstract). Lasers in Surgery and Medicine 1993 (13)Suppiement 5:64.

64. Vale JA, Bdesha AS, $N$ Witheraw RO. An analysis of the costs of altermative treatments for benign prostatic hypertrophy. Joumal of the Royal Society of Medicine 1995(88):644P-648P.

65. Vicente $\mathrm{J}$, Salvador $\mathrm{J}$, Caffaratti $\mathrm{J}$. Endoscopic urethrotomy versus urethrotomy plus Nd:YAG laser in the treatment of urethral stricture. European Uirology 1990(18):166-8.

66. Vogelzang NJ. The cure of bladder cancer: the meed for multidisciplinary efforts (editorial). The Journal of Urology $1994(151) 3: 605-6$.

67. Watson $G$, Anson $K$. An indepth evaluation of contact laser vaporization of the prostate (abstract). The Joumal of Urology $1994(151) 5: 231 \mathrm{~A}$.

68. Watson $\mathrm{G}$, Murray $\mathrm{S}$, Dretler $S F$, Parrish JA. The pulsed dye laser for fragmenting urinary calculi. The Joumal of Uralogy 1987(138):195-8.

69. Watson GM, Landers H, Nauth-Misir R, Wickham JEA. Developments in the ureteroscopes, techmiques and accessories associated with laser lithotripsy. Warld Journal of Urology $1993(11), 19-25$.

70. Whitmore WF. Management of clinically localized prostatic cancer. An unresolved problem. Journal of the American Medical Association 1993(269):2676-7.

71. Wickham JEA. An introduction to Minimally Invasiwe Therapy. In: Banta HD (Ed.). Minimally trvasive therapy in five European cotintries. Diffusion, effectiveness, costteffectiveness. Health Policy Monographs, Vol 3. Elsevier Science Publishers, Amsterdam, Londion, New York, Tokyo, 1993, p.7-18.

72. Wils TE, Bums JR. Lreteroscopy: an outpatient procedure? The Journat of Urology 1994(151)5:1 1485-7.

73. Schulze H. Martin W, Hoch P. Senge T. Tulip vs. Turp: a prospective randomized study (abstract). The Journal of Urology $1994(151) 5: 228 \mathrm{~A}$.

74. Babayan RK, Roth RA, Mc Cuillough DL, Salem W, Gordon JO, Reese JH, Fuselier HA, crawford ED. Smith JA, Murchison RJ Kaye KW. Tulip(0) - wo years results (abstract). The Journal of Liology 1994(151)5:228A.

75. Ravi R. Endoscopic neodymium-YAG laser treatment of radiation-induced hemorhagic cystitis. Lasers in Surgery and Medicine 1994(14)1:83-7. 


\section{Lasers in gynecology}

\subsection{Introduction}

The laser has been used in gynecology since 1973, when Kaplan first published the treatment of erosions of the uterine cervix by means of the $\mathrm{CO}_{2}$ laser ${ }^{(433)}$. In the early eighties, $\mathrm{CO}$ laser ireatment of cervical, vulvar and vaginal neoplasia and genital warts was assessed as safe and effective by the United States' Office of Health Technology Assessment ${ }^{\left({ }^{33 \mid}\right.}$ and the Diagnostic and Therapeutic Technology Assessment (DATTA) program of the American Medical Association ${ }^{[22]}$. In the course of the seventies and eighties, the $\mathrm{CO}_{2}$ laser has become a part of routine therapy in many gynecological departments, and the indications listed above are still common today. In particular treatment of CIN has been well-evaluated ${ }^{[62,84]}$. In 1981. Goldrath and colleagues introduced the Nd:YAG laser for treatment of patients with menorrhagia ${ }^{[34]}$. Ablation of the endometrium for menorrhagila is nowadays one of the most important gynecological applications of this laser. In the past decade, as part of the minimally invasive therapy "revolution", laparoscopic ireatment has become more important in gynecology, in particular in infertility treatment ${ }^{[2 ! 2}$. After introduction of the $\mathrm{CO}_{2}$ laser in reproductive surgery by Bellina in 1974 , as described by Bhatta et al|:21, Bruhat et al. adapted the $\mathrm{CO}_{2}$ laser to laparoscopic treatment in $1979^{\mid 14 i}$. Various lasers now play a role here, including the $\mathrm{CO}_{2}$, argon, and KTP laser ${ }^{68]}$. Common indications in infertility treatment are reopening of occluded fallopian tubes, removal of adhesions, and treatment of endometriosis ${ }^{(62.84)}$. The Nd:YAG- and KTP laser play a role in particular in hysteroscopic surgery ${ }^{(69)}$. Of all types of lasers, however, the $\mathrm{CO}_{2}$ laser is by far the most important in this field, and its use is still expanding ${ }^{[62]}$. The evidence for the use of lasers for the most important indications listed above has been assessed, starting with $\mathrm{CO}_{2}$ laser treatment of neoplasms and genital warts.

\subsection{Cervical intraepithelial neoplasia}

\subsubsection{Introduction}

Cervical cancer is generally divided into cervical intraepithelial neoplasia (CIN) and invasive carcinoma. In 1987, the World Health Organization (WHO) estimated that 460,000 cases of invasive cervical carcinoma occur annually worldwide, and 230,000 women die of cervical cancer each year ${ }^{[30]}$. Cervical cancer ranks as the sixth most frequent type of cancer for women in the United States, making up about 1.4 percent of all cancers. The major advance in dealing with cervical cancer has been large scale screening programs to allow early detection and treatment ${ }^{(7)}$. While in countries that have organized large scale screening programs deaths from cervical cancer have fallen during the last decades, the incidence of its premalignant stages is increasing ${ }^{|40|}$. In the early nineties, the peak incidence of $\mathrm{CIN}$ was between the ages of $20-30$ years $^{[8 \mathrm{~d} \mid}$. CIN can be divided into CIN I, CIN II, and CIN III reflecting mild, moderate, and severe dysplasia. CIN III also includes carcinoma in situ. CIN is thought to slowly develop into invasive carcinoma, and therefore must be carefully followed. If it does not spontaneously disappear, it must eventually be treated. When malignant conversion (change to a malignant cancer) is seen in cases of CIN, the rate of malignant conversion is more rapid in the transformation zone between the cervix and vagina, which is called the squamocolumnar junction ${ }^{\mid(62)}$.

Hysterectomy is the definitive treatment for early invasive cervical carcinoma ${ }^{(20)}$. Photodynamic Therapy (PDT) may also play a role in treatment of early invasive cervical carcinoma. In general, PDT in gynecology is considered experimenta! ${ }^{32 !}$.

The classical treatment of premalignant cervical disorders is surgical conization of the cervix (also known as cold knife conization) $)^{|40|}$. In rare cases, hysterectomy may be necessary for cervical intraepithelial neoplasia grade III that cannot be completely removed by conization ${ }^{[2,621}$. Conization leads to a cure rate of more than $95 \%$, and therefore may be considered an established and 


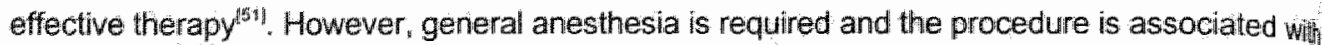
an overall complication rate of 15 to $30 \%$, of which postoperative hemorrhage is most frequent, thi 15 to $20 \%$ of women of childbearing age, surgical conization is associated with complications with regiard to fertility, the course of pregnancy, and delivery ${ }^{k 40.62 !}$. This caused a trend toward alternatives for cold-knife conization, and for the entire technique of conization.

For small localized lesions, local destruction technicues were introduced as early as the fifties and sixties, followed by $\mathrm{CO}_{2}$ laser treatment in the seventies. Originally, $\mathrm{CO}_{2}$ laser treatment was introduced as an alternative for cold-knife conization, but by varying treatment parameters of the laser, local destruction of tissue (vaporization) can be achieved as well ${ }^{[0,49,62]}$. Depending on the technique, operations with the $\mathrm{CO}_{2}$ laser can be performed using a colposcope, an operating microscope or al handpiece ${ }^{40.62]}$. When a conization procedure is selected, this can be done eithe creating a broad, deep cone, a long cylindrical cone, a combination cone (implying a combinalion of a conization and vaporization procedure), and a minicone. The choice of technique is dependen on the geometry of the lesion. For example, when a vaporization procedure is selected, a 'cowboy. hat' geometry of treatment is advocated. The peripheral disc of the 'cowboy hat' should have a vertical depth of $5-7 \mathrm{~mm}$ and a lateral diameter proportionate to the width of the lesion to te destroyed ${ }^{162 !}$.

The major advantage of laser vaporization and other modalities that are used for conservative treatment is, besides the use of local anesthesia in most cases, that the anatomic function and reproductive function are maintained in almost all patients ${ }^{[3,62\}}$. The major disadvantage dt modalities for conservative treatment is that no tissue is obtained for histology after the procedure. Authors that support conservative treatment claim that through a carefully carried out diagnostic protocol ("triage rules') combining cytology, colposcopy, and histology, the potential presence of invasive cancer can be ruled out ${ }^{10,621}$. As inappropriate conservative treatment of an unrecognized invasive cancer results in a poor prognosis, the choice between conization and conservative treatment has long been debated in literature. The distinction between 'satisfactory' and 'unsatisfactory" colposcopies has long been the basis of choice. According to Reid and Absten (1995), historically, British and American gynecologists restricted conization to patients with lesions extending into the endocervical canal (resulting in 'unsatisfactory' colposcopies), cases in which there is definite suspicion of occult invasion, unexplained high-gradle cytologic atypia, and smears showing abnormal glandular cells ${ }^{|62|}$.

When conservative treatment seems justified, laser vaporization is not the only treatment option, Another widely used treatment modality for conservative treatment of premalignant cervical lesions is cryotherapy. Laser vaporization, cryotherapy and conization techniques have been challenged by the advent of a tectinique called LLETZ (large loop excision of the transformation zone). This technique, introduced in 1989 by Prendiville et al. ${ }^{[610}$ can be regarded as a modification of the electrosurgical technique introduced by Cartier et al. in 198 $\|^{\mid 15 y}$. In the paper introducing LLETZ, the authors explained its use as an alternative for laser vaporization and cryotherapy, while adding that it is possible to use a modified procedure to perform a cone biopsy ${ }^{\text {ist? }}$. In the latter case the excised tissue can be used for histologic examination. Actually, the paper examined the treatment potential of LLETZ as a conization procedure, while applying the inclusion criteria for vaporization. This has resulted in much confusion on the appropriate combination of indications and treatment modalities ${ }^{[5,4}$.

The net result of introducing LLETZ, according to Absten and Reid (1995) has been a blurring of the distinction between satisfactory and unsatisfactory colposcopies ${ }^{\mid 62]}$. The results in the original paper by Prendiville et al. were promising, and in subsequent RCTs the technique has been compared to both alternative excisional and vaporization techniques. In these RCTS, LLETZ has always been used to excise tissue. Now, after all this background information, what is the role of lasers here? 
After preliminary positive assessments in the early eighties (see paragraph 10.1), a continuous flow of case-series demonstrated the safety and effectiveness of $\mathrm{CO}_{3}$ laser treatment of $\mathrm{CIN}$, either by vaporization or conization. Subsequently, a number of RCTs has been published. To determine the comparative success of treatment, Pap smears which are repieatedly negative for CIN have most frequently been used in these studies. Long-term follow-up to assure success is mecessary in all cases. When abnormalities were found on follow-up further treatment has often been applied, with a still high probability of treatment success. Firstly, the RCTs comparing different procedures for conization are addressed, followed by the RCTs focusing an vaporization procedures. As outlined above; LLETZ is a competing procedure for both conization and vaporization techniques.

\subsubsection{Conization and excision}

\section{Laser conization versus cold-knife conization}

Conization procedures have been applied predominantly in patients with severe $\mathrm{CIN}$ and in patients with CIS. In 1982, as a result of an increasing number of promising case-series using either modality, Larsson et al. organized the first randomized controlled trial comparing laser conization and cold knife conization ${ }^{\mid 48 !}$. The study focused mainly on the impact of both methods on postoperative hemorrhage. Patients were eligible when they had severe dysplasia or carcinoma in situ (CIS). Fifty-five patients were enrolled in each group. All patients were hospitalized and all conizations were performed using general anesthesia. Laser conization was performed using a 25 to $30 \mathrm{~W} \mathrm{cW} \mathrm{CO}_{2}$ laser. The range of blood loss within 24 hours after operation was 0.4 to $155.4 \mathrm{ml}$ in the laser conization group versus 5.6 to $1570.9 \mathrm{ml}$ in the cold knife conization group. The incidence rate for bleeding complications requiring surgical intervention was 1.8 per cent for the laser group and 14.6 percent for the surgical group ( $p<0.015$, Fisher's exact test). The authors, based on these results, expected that laser conization would replace cold knife conization in the future.

The second RCT involving laser treatment of CIN was published by Bostofte et al. in $1987^{131}$. The authors compared the safety and efficacy of ( $\mathrm{OW}, 30 \mathrm{~W}$ ) $\mathrm{CO}_{2}$ laser conization $(n=59)$ and cold knife conization ( $n=64)$ in patients with atypia, CIN $\|$, II or III or with microinvasive carcinoma. Nearly all patients actually enrolled in the study had CIN II or CIN III. General anesthesia was used with both methods and the patients were kept in hospital for 4 days. Follow-up was scheduled 3 and 12 weeks after treatment, then every 6 months for 2 years and thereafter once a year. Mean follow-up of patients in the analysis was 36 months (range 28 - 48 months). Of the laser conization cases three underwent hysterectomy due to the presence of (micro)invasive carcinoma, compared to four patients in the cold knife conization group. All recurrences in the remainder of patients in bolh groups were intra-epithelial neoplasias. There were 4 recurrences in the laser-treated group $(6.8 \%)_{\text {, }}$ of which two were treated with hysterectomy, one with re-conization, and one patient was followed-up without treatment. There were 6 recurrences in the cold-kinife treated group $(9.8 \%)$, of which one was treated with hysterectomy, three with reconization, one with laser vaporization. and one patient was followed-up without treatment. The mean duration of both operations was about half an hour. In contrast with the cold knife conization cases, no hemostatic procedures were used in the laser conizations. Despite this discrepancy in techniques the results showed a tendency of reduced bleeding in the laser group. Postoperative bleeding requiring hosplital treatment occurred in 11 patients $(17 \%)$ in the cold knife series, compared to 3 patients $(5 \%)$ in the laser series ( $p<0.05$, chi-square test). Exclusively in the laser series most patients experienced a brownish discharge for two weeks. Postoperative pain was negligible in both groups, reflected by the use of minor analgesics in $25 \%$ of patients in the laser group and in $33 \%$ of the patients in the cold knife group. After 12 weeks the cervical canal could be passed by a cotton-tipped swab in $93 \%$ of patients in the laser group, compared to $73 \%$ in the conization group. Four patients in the laser group $(6.8 \%)$ and $2(3.3 \%)$ developed dysmenorrhoea due to stenosis of the cervical canal. The authors concluded that laser conization is a safe procedure and suggested that laser conization is superior to cold knife conization when additional hemostatic procedures would be applied after 
laser therapy. This trial did not demanstrate any advantages of laser conization in terms 0 . preventing cervical stenosis.

In a retrospective study comprising 884 patients, laser conization has been compared favourably with cold-knife conization with respect to intraoperative and postoperative bleeding, infection distortion of the cervix, and stenosis of the endocervical canal ${ }^{\left.\mid{ }_{9}\right]}$. In this series, a subset of patients received laser treatment on an outpatient basis, using local anesthesia, showing results as goo as in hospitalized patients. Other authors, sometimes including hundreds or sometimes thousand of patients, have produced similar results ${ }^{[3,4]}$. As a result, outpatient laser conization using loca anesthesia has become increasingly common, while cold-knife conization nowadays is still applied using general anesthesia ${ }^{[62]}$.

Overall, the evidence suggests that laser conization is at least as effective as cold knife conization The differences in complication rates as assessed by RCTs and retrospective studies favour lase conization, although not all advantages based on results of retrospective studies have been demonstrated by RCTs as well. More recent studies indicate that pulsed-and super-pulsed CO laser treatment using high power outputs and higher power densities produce better results that when $\mathrm{cw}$ lasers with modest power outputs- and densities ${ }^{[2]}$. This implies that actual treatment techniques provide perhaps better results than those in the RCTs discussed here, which all used $\mathrm{cw} \mathrm{CO}_{2}$ lasers with modest power settings. As cold knife conization needs to be performed will general anesthesia, outpatient laser conization using local anesthesia can be regarded as the: preferred method ${ }^{[02]}$.

\section{Laser conization versus LLETZ}

In 1993, Oyesanya et al. published an RCT comparing loop diathermy excision and (25 W CW) CO, laser excisional conization in patients with CIN I, II or III, without evidence of invasion and with the upper limit of the transformation zone visible on colposcopy ${ }^{[54\}}$. Three-hundred patients were enrolled. Randomization was achieved by the patient drawing a sealed opaque envelope froma box. Both laser treatment $(n=150)$ and loop diathermy excision $(n=150)$ were applied in an outpatient setting under local anesthesia. Although no specific referral was made to Prendiville and colleagues (1989), the method and technique used for loop diathermy excision was simillar to the method used by this group ${ }^{[6]}$. Follow-up was scheduled 4 months, 8 months and one year after treatment.

The time required to complete treatment and hemostasis was 2.5 minutes (sd 3.6 minutes) in the diathermy excision group compared to 24.2 minutes (sd 11.8 minules) in the laser excision group $(p<0.001$. Student's t-test). Mean operative blood loss was $2.8 \mathrm{ml}$ ( $\mathrm{sd} 3.8 \mathrm{ml}$ ) in the diathermy excision group, less than in the laser excision group $(27.2 \mathrm{ml}$ (sd $17.8 \mathrm{ml}), \mathrm{p}<0.001$, Student's : (est). The impact of diathermy excision on patient discomfort was non or minimal in $93 \%$ ano moderate in $7 \%$ of patients, compared to non or minimal in $55 \%$, moderate in $39 \%$, and severe in $6 \%$ of laser excision patients. The difference in favour of diathermy excision was significant ( $p<0.001$, chi-square test). There were 11 complications in the diathermy excision group and 18 in the laser excision group. Two patients in each group were seen in the hospital casually department with secondary hemorrhage which was managed successfully with vaginal packs. One patient in the laser excision group had to be admitted to hospital due to secondary hemorrhage and received a blood transfusion. Three patients in each group were treated with antibiotics after diagnosis of infection. Persistent vaginal discharge occurred in one patient in the diathermy excision group versus 4 patients in the laser excision group. A silmilar difference was noted in the occurrence of dysmenorrhea, indicating a narrowing of the endocervical canal. Data of all but two patients in the diathermy excision group $(n=148)$ and in all but 3 patients in the laser excision group $(n=147)$ were available after finishing follow-up. There were 17 cases with residual or recurrent $\mathrm{CN}$ in the diathermy excision group (11.5\%) versus 22 in the laser excision group $(15.0 \%)$. In the diathermy excision group, 12 patients were retreated with diathermy $(8.2 \%), 3$ patients underwent total abdominal hysterectomy $(1.9 \%)$ and 2 patients were treated with colld-knife conization $(1.4 \%)$. In the laser excision group, 19 patients were retreated with laser-excision (12.9\%), 1 patient 
underwent total abdominal hysterectomy $(0.7 \%)$ and 2 patients were treated with cold-knife conization $(1.4 \%)$

The authors concluded that loop diathermy excision and laser excision are aually effective, but that loop diathermy excision is safer, more comfortable to the patient, quicker and more reliable than laser conization for the management of CIN.

According to several authors, e.g. Crompton and Johnson (1994) both laser conization and LLETZ excision are equally safe and effective in treatment of patients with severe $C \mathrm{~N}^{[17]}$. Therefore, the preferred treatment, according to these authors, should be the one that causes the least pain to the patient. LLETZ, in the description of these authors, is quicker than laser excision but accasionally causes sharp pain that some women describe as electrocuting. The pain of laser treatment is different and is often referred to as a burning pain.

To assess the painfuness of excisional surgery by LLETZ and $\mathrm{CO}_{2}$ laser, Crompton and Johnson (1994) organized a randomized trial enrolling 80 woman with CIN III as diagnosed colposcopically. Randomization was achieved by a computer-generated simple randomization code which was held in a sealled opaque envelope. Patients were matched for age, parity and size of the lesion. All patients were treated as outpatients. Before operation $4 \mathrm{ml}$ of $2 \%$ lignocaine with 0.3 international units/ml octopressin was injected into the cervical transformation zone with a dental syringe. Pain was objectively assessed independently by the attendant nurse and the laser surgeon, scoring different variables that were combined for analysis in a single score (0-8). At the end of the procedure the patient scored pain on a linear visual analogue meter $(0-10)$. The mean and median pain scores as assessed objectively were 1 and 0.5 , respectively, in the laser group and 1.2 and 0.5 , respectively, in the LLETZ group. Subjective mean and median scores were 4 and 4 in the laser group, respectively, and 4.1 and 4 in the LLETZ group, respectively (ns. Mann-Whitney Utest). The authors concluded that there is no difference between the pain experienced by women having the two techniques. In the discussion the authors noted that in this study the average time from sitting in the chair to the end of the procedure was 13 minutes (sd 3.6 minutes) for the LLETZ group compared to 17 minutes (sd 5.3 minutes) for laser excision. This difference (Student's $t$-test, $p<0.001$ ) was judged important for both patients and the health service.

The results of these trials suggest that laser conization and large loop excision of the transformation zone (LLETZ) are equally safe and effective in treatment of CIN. The differences in complication rates are small but slightly favouring LLETZ.

\subsubsection{Laser conization versus laser vaporization}

When to use conization and when vaporization? In the trial by Bostofte et al. (1986), it is stated that if the laser vaporization technique would have been used in the patients actually enrolled in the study, the cases with (micro)invasive carcinoma would have been missed ${ }^{[3]}$. It was therefore suggested to use the vaporization method only in those cases in whom the transformation zone is fully visible and in whom invasive disease has been ruled out.

The only RCT that addresses the problem of selection of the right treatment technique was published by Partington et al. in $1989^{189 !}$. A total of 100 patients were enrolled when they had histologically confirmed CIN I, II or III, when there was no colposcopic evidence of invasive disease and when the upper limit of the lesion could be clearly visualized with the squamocolumnar junction less than $5 \mathrm{~mm}$ in the endocervical canal. Half of these patients underwent $(15-20 \mathrm{~W} \mathrm{cW}) \mathrm{CO}_{2}$ laser excision, the other half of the patients received laser vaporization. Randomization was achieved by drawing sealed envelopes. All patients were treated on an outpatient basis using loca! anesthesia. 
Based on quantified results of the first 30 patients in each group, the mean intraoperative blood loss was greater in the excision group $(15.5 \mathrm{ml}$ versus $4.5 \mathrm{ml}, \mathrm{p}<0.01$. Student's t-test). but it was suggested that this difference was not clinically relevant. In one excision patient, treatment had to be interrupted because of bleeding. Treatment of this patient was finished later that same day under general anesthesia. The mean time required for both excision and vaporization treatment was about 20 minutes. The majority of both procedures, $90 \%$ of vaporizations and $76 \%$ of excisions ${ }_{1}$ produced no pain or only mild discomfort that could be easily tolerated. No patient complained of severe pain and no trealtment was abandoned because of pain.

For analysis of follow-up, data were available of 47 patients who underwent laser vaporization and of 45 patients who underwent laser excision. Mean follow-up time was 9.3 months (range 2-17 months). There was no difference in postoperative bleeding in both groups, which occurred in 26 patients in the vaporzation group $(55.3 \%)$ and in 24 patients in the excision group $(53.3 \%)$. Vaginal packing alone was sufficient to stop bleeding in those patients requiring treatment. None of the patients required a blood transfusion. Symptoms suggestive of pelvic infection accurred in $6 \%$ of patients in the excision group and in $9 \%$ of patients in the vaporization group. Neither of the patients experienced secondary amenorrhea or dysmenorrhea as a result of stenosis of the endocervical canal. In almost all patients in both groups, the squamocolumnar junction was seen easily at subsequent colposcopy. No microinvasive disease was encountered in the histologic specimens of the excision group. Disease recurrence was suspected or confirmed in $5(11 \%)$ af the vaporization patients and in $4(12.5 \%)$ of the excision patients. Two of the recurrences in the vaparization group were retreated, the other three were left untreated due to mild $\mathrm{CIN}$. Only one of the four recurrences in the excision group required retreatment, the other patients had mild CIN:

Although the authors concluded that the results of their study suggested that there are no important differences between both treatment options, they favoured laser excision because of the availability of tissue for histologic examination. At the same time, however, their results suggested that a carefully carried out diagnostic protocol can result in inclusion of patients without (micro)inwasive carcinoma, not necessitating a choice between both options on this ground. In addition, laser treatment did not result in cervical stenosis in any of the patients.

Other authors too have concluded that both methods of laser treatment are highly safe and effective $^{[62]}$. For example, Baggish and colleagues (1989), evaluating results of 3,000 patients in whom $\mathrm{CO}_{2}$ laser vaporization had been applied and of 1,000 patient who underwent $\mathrm{CO}_{2}$ laser excisional procedures in a period of 10 years time, concluded that after at least one-year follow-up treatment success was $94 \%$ with an overall complication rate of about $4 \%$. Their findings reflected the results of an earlier study ${ }^{[3]}$, to which the author commented that the major weakness of the vaporization technique is that this operation does not leave a specimen for the pathologist.

In summary, the results of these RCTs suggest that of three modalities, cold knife conization, laser conization and large loop excision of the transformation zone, the latter two can be preferred in trealment of CIN where conservative treatment is deemed inappropriate. In the absence of striking differences in safety and effectiveness, most authors of the RCTs discussed here tend to favour LLETZ instead of laser conization for practical reasons. The safely and efficacy of laser treatment is wellestablished for this indication. In practice, according to Reid and Absten (1995), LLETZ has completely replaced $\mathrm{CO}_{2}$ laser treatment when a minicone can be performed ${ }^{[62 !}$. A mini-conization represents the most easy type of conization. For other types of conization both modalities may be to some extent competitive ${ }^{\left[{ }^{[2]}\right]}$. The results of one RCT, by Partington et al. (1989), and a number of retrospective studies suggest that laser conization and laser vaporization can be used interchangeably ${ }^{[8]}$. This inevitably results in the loss of the possibility of histologic examination but this may not be problematic provided that appropriate diagnostic procedures are applied.

\section{Cost-effectiveness}

In contrast with cold-knife conization, both laser conization and LLETZ are applied on an outpatient basis. Berget et al. (1987), addressing this issue, noted that laser conization was not associated with hospitalization, whereas cold knife conization required an average of 4.5 days of 
hospitalization ${ }^{\mid 91}$. In 1986 , assuming that one outpatient visit was equivalent to one fourth of a hospital day, the average hospital stay for treatment of CIN in his hospital in Denmark had fallen from 4.5 days in 1979-1982 to 1.3 days in 1986 (In other words, almost all procedures were done on an out-patient basis by 1986). This obviously resulted in a considerable saving of resources. In a similar analysis, Bekassy (1987) found that conization without laser (which is likely to be cold knife conization) required at least 3 days hospitalization, while laser conization did not require hospitalization. He calculated on this basis that 85 outpatient conizations would pay for the lasep ${ }^{\left[{ }^{*}\right]}$. Although these data are suggestive of the potential cost-effectiveness of laser conization versus cold knife conization, the cost-effectiveness of laser conization is questionable compared to LLETZ Formal cost-effectiveness analyses are lacking. The capital costs of a $\mathrm{CO}_{2}$ laser were estimated to amount BP 80000 in 1990 , compared to BP 4000 for the LLETZ units (the loops are disposable $)^{[3]}$. Assuming equal safety and effectiveness of both procedures, LLETZ is likely to be the most cost-effective option.

\subsubsection{Conservative treatment and excision}

The most important conservative treatment options include laser vaporization and cryotherapy. LLETZ is a competing technique.

\section{Laser vaporization versus cryotherapy}

Perhaps the first RCT comparing $\mathrm{CO}_{2}$ laser vaporization and cryotherapy in patients with histologically confirmed CIN I, II or III was published by Jobson and Homesley in 1984 ${ }^{|\alpha 2|}$. Both treatment modalities were provided as a single treatment in an ambulatory outpatient setting. Onehundredtwenty-five patients were enrolled in the study, of whom 43 were lost during a period of follow-up of one year. Of the patients included in the analysis, 39 had been treated with cryotherapy, and 43 with laser.

The succes rate was $90 \%$ in both groups. Both modalities were well tolerated and severe complications did not occur. Discharge was relatively heavy in cryotherapy patients during the first week postoperatively. The authors concluded that both modalities are effective in treatment of CIN. Because the proximal edge of the transformation zone could be seen in many more patients in the laser group $(36 / 42 ; 86 \%)$ than in the cryotherapy group $(20 / 39 ; 53 \%)$, facilitating follow-up, the authors preferred $\mathrm{CO}_{2}$ laser treatment. As more than a third of the patients enrolled in this study were lost to follow-up selection bias may have occurred. The results of this trial therefore have to be considered indicative.

In the period January 1979 - June 1983 Kwikkel et al. enrolled 105 patients in a randomized trial comparing $\mathrm{CO}_{2}$ laser vaporization and cryotherapy ${ }^{[46]}$. Patients were eligible when there was no suspicion of invasive carcinoma and when the lesion could be fully visualized at colposcopy. Succes was defined as no evidence of recurrence of $\mathrm{CIN}$ at follow-up. Follow-up examinations were scheduled at 6 weeks, and then every 3 months until 18 months following treatment. Only single treatments were entered in the study. A few patients did not (fully) comply with the follow-up scheme and were excluded from analysis. As a result, the analysis included 101 patients, of whom 50 had been treated with cryotherapy and 51 with laser therapy. The overall rate of success of cryotherapy was $86 \%$ and of laser vaporization $71 \%$ (ns, chi-square test). Treatment succes in each group was highest in small lesions. In patients treated with laser vaporization the median duration of heavy discharge was 0 days (range 0-46 days), compared to 8 days (range 0-28 days) in patients managed with cryotherapy (Wilcoxon's test, $p<0.01$ ). Blood loss during treatment occurred only in patients treated with laser vaporization (20\%). Also pain during treatment was experienced only in laser-treated patients. During the trial, however, analgesics had been introduced. The authors concluded that both treatment modalities are safe and effective in treatment of patients with $\mathrm{CIN}$. Considering the high costs of the laser and the relatively small differences in side-effects between both treatment modalities, the authors recommended 
investment in lasers exclusively in large centers with sufficient numbers of referred cases of CII: (and vaginal and vulvar intraepithelial lesions).

In a subsequent report, the authors presented an analysis of data of patients enrolled in the period January 1979 - December 1984 , representing an additional period of enrollment of 1.5 years compared with the first study ${ }^{400}$. Additional follow-up was scheduled annually following the 18 : month visit after initial treatment. Mean follow-up in the laser group was 25 months compared to 28 months in the cryosurgery group. The analysis included 165 patients, of whom 84 received laser therapy and 81 cryotherapy. All patients were treated in an outpatient clinic, without anesthesia. Treatment time varied from 15 minutes for cryotherapy to 25 minutes for laser vaporization. Success rates were now $79 \%$ and $88 \%$, respectively. The results confirmed the findings of the authors in their previous study. With more and longer follow-up data available it was added that both methods appeared not to interfere with subsequent fertility. Laser therapy, according to the authors, may offer advantages in particular in the case of extensive affected areas or lesions not easy in reach.

In the period June 1981 - March 1983, Wijnen enrolled 100 patients in a randomized trial comparing (cW) $\mathrm{CO}_{2}$ laser treatment $(n=50)$ and cryotherapy $(n=50)^{[83]}$. Patients with $\mathrm{CIN} \mathrm{I}$, II or III were eligible when they were 55 years of age or younger, when there was no suspicion of invasive carcinoma and when the lesion could be fully visualized at colposcopy. All patients were treated in an outpatient clinic in one treatment session. In the last 15 patients in the laser group local anesthesia was applied. No anesthesia was applied in patients receiving cryotherapy. No local hemostatic agents were applied in patients in either group. Follow-up was scheduled 6 weeks, 4-6 months and 10-12 months after treatment and was subsequently extended. Succes was defined as no evidence of recurrence of $\mathrm{C} \| \mathrm{N}$ at follow-up based on cytological and histological examination: In patients with cytological evidence of CIN without histological evidence, treatment was still regarded as successful when at the next follow-up the cytology reverted to normal.

The median duration of laser treatment was 20 minutes (range 4 - 40 minutes) longer than the median duration of cryosurgery (6.5 minutes; range 3 -18 minutes) (Mann-Whittney U-test, $\mathrm{p}<0.001)$. Laser treatment was more frequently painful and also more painful than cryosurgery, as assessed subjectively (chi-square test, $p<0.001$ ). Likewise, laser treatment resulted more often and in more severe bleeding than cryosurgery (chi-square test, $p<0.001$ ). None of the patients in either group needed a blood transfusion. Postoperative discharge was heavier and lasted longer after cryotherapy. The median period was 50 days after cryotherapy (range 11-99 days) versus 43 days after laser therapy (range 14-92 days) (Mann-Whittney U-test, $p<0.001$ ). Analysis of follow-up data after one year was based on all patients, although in a strict sense only 48 patients in the cryotherapy group and 47 patients in the laser group fulfilled the criteria for analysis. Treatment was successful in $96 \%$ of the cryosurgery group and in $86 \%$ of the laser treatment group. After laser therapy a larger number of patients $(77 \%)$ had a visible squamocolumnar junction than following cryotherapy ( $45 \%$ ), which facilitates follow-up. The authors concluded that both modalities are safe and highly effective in treatment of $\mathrm{CIN}$

After finishing the trial, the authors started using local anesthesia and local hemostatic agents in all patients eligible for laser treatment, resulting in virtually painless, faster and, according to the authors, more effective treatment. The use of a $\mathrm{CO}_{2}$ laser was noticed to have the additional advantage that in large and deeply located lesions an excisional conization can be executed under local anesthesia or a combination of laser excision- and vaporization may be performed. Cryocoagulation, however, was judged to be less costly and easier to learn than laser surgery.

In 1987, Berget and colleagues published interim-results of a multicenter randomized trial comparing $\mathrm{CO}_{2}$ laser treatment $(n=103)$ and cryotherapy $(n=101)$ in patients with $C \mathbb{N}^{\| 9 !}$. Patients were eligible when CIN I, II, or III was histologically confirmed, when the squamocolumnar junction was fully visible at colposcopy and when endocervical curettage had shown normal results. Al patients were treated on an outpatient basis, all but a few exceptional cases without anesthesia. 
Follow-up was schedulied $3,9,15,21,33,45$ en 57 months after treatment. The interim-analysis reported results obtained after 15 months of follow-up and included 55 patients in the laser group and 58 patients in the cryo group. CIN found in this period of follow-up was defined as residual.

Residual CIN was found in 10 patients in the laser group (18\%) and in 9 patients in the cryo group $(16 \%)$. Retreatment was performed with laser therapy in 9 cases of the laser group, all without recurrence. One patient asked for a conization as retreatment. Retreatment was performed with cryotherapy in the cryotherapy group in 3 patients, without recurrence. The remaining 6 patients were trealed with conization because the residual CIN was found both in the biopsies as well as in the endocervix, which was an exclusion criterion for conservative treatment. All patients treated with conization were regarded as treatment failures. No recurrences were found at follow-up after conization. Based on the definition above, treatment succes rates after one or two treatments were $96 \%$ in the laser group and $93 \%$ in the cryo series. There was no difference in succes rates depending an $\mathrm{CIN}$ status in either group. Peroperative pain was reported by $53 \%$ of patients in the laser group, compared to $33 \%$ in the cryo therapy group. Three percent of patients in the laser group judged the pain as unacceptable, one of these had local analgesia applied. None of the patients in the cryo group judged the pain as unacceptable. Peroperative hemorrhage did not exceed $25 \mathrm{cc}$, except for one laser therapy patient. No additional hemostatic procedures were needed in these patients postoperatively. Vaginal discharge was more common and more severe in the cryotherapy group than in the laser group ( $p<0.01$, chi-square test).

The authors concluded that both methods are effective in treatment of $\mathrm{CIN}$ and that certain complications and some discomfort are more frequent in cryo therapy than in laser therapy and vice versa, but that the differences are small. The versatility of lasers and the possibilities for sharing the apparatus between different hospital departments was emphasized. Although additional. follow-up reports were announced, these seem never to have been published.

Other authors, at best in prospective, controlled but non-randomized studies comparing laser vaporization and cryotherapy, have concluded that both methods are equally safe and effective ${ }^{[76]}$, basically repeating the conclusions which were based on the RCTs discussed here. In summaryn both laser vaporization and cryosurgery are equally safe and effective in treatment of patients with colposicopically fully visible CIN.

\section{Cost-effectiveness}

Wetchler (1984) examined the costs of laser vaporization of CIN compared to costs of cryotherapy in the context of a hospital in Virginia in the United States ${ }^{\text {182] }}$. He found that the cost of laser therapy is US\$636, while the cost of cryotherapy is US $\$ 105$. However, the majority of the difference was made up of his estimate that the physician fee for laser therapy was US $\$ 400$, while the fee for cryotherapy was US\$100. This estimate was presumably related to the fact that laser therapy takes somewhat longer than cryotherapy. In addition, in his most optimistic assumptions, he estimated that the laser would be used only 5 times a week. This means a high equipment cost for each procedure. With more intensive use, the cost of the laser would of course be less. These cost estimates were very high, and while probably valid in the US context ${ }^{[7]}$, can only be considered exemplary in other settings. He also concluded that cold knife conization is more expensive than the other two alternatives, since it requires general anesthesia, an operating room, and hospitalization, but he gave no figures. Pozzi et al. (1985) carried out a similar analysis in Italy. comparing cryotherapy and laser vaporization ${ }^{|60|}$. The calculation of costs included estimates of the cost of the equipment, maintenance and related costs, with an assumption of 150 treatments per year. They found that the cost of one cryotherapy is 21499 lire, whereas the cost of a laser therapy is 87166 lire. Although these data are far from convincing, they indicate that, with equal effectiveness, laser vaporization may be less cost-effective than cryotherapy. 


\section{Laser vaporization versus excision}

In 1990, Gunasekara et al. published a randomized trial comparing large loop excision of the transformation zone $(n=98)$ to $\mathrm{CO}_{2}$ laser vaporization $(n=101)^{136 !}$. Patients were eligible when histological examination had confirmed presence of $\mathrm{CIN} \|$ or $\mathrm{C}\|\mathrm{N} I\| \mathrm{I}$ and when the upper margin of the transformation zone was colposcopically visible. The patients were allocated to the LLET2 or laser group by hospital number. Even numbers were placed in one group and the odd numbers were placed in the other group. Patients in both groups were treated as outpatients and received local anesthesia in the form of a paracervical block and infiltration using $6.6 \mathrm{ml}$. of $2 \%$ lignocaine with $1: 80.000$ adrenaline using a dental syringe.

No patients were lost during a follow-up period of 6 months, showing a recurrence rate of $8.2 \%$ (CIN II) aind $7.5 \%$ (CIN III) in the laser group versus $5 \%$ (CIN II) and $5.3 \%$ (CIN 11 ) in the LLETZ group ( $\mathrm{ns}$, chi-square test). During treatment, patients in both groups experienced the same level of pain. In the laser group, $69 \%$ of patients judged treatment as unpleasant compared to $18 \%$ in the LLETZ group. This difference was attributed to the length of the laser procedure. Operative time for the laser group was 4-15 minutes (mean 6.5 minutes), compared with 20-50 seconds (mean 16 seconds) for the LLETZ group. Although $77 \%$ of the patients in the laser group did not bleed at the time of surgery, 4 patients required immediate admission because of excessive bleeding, and 3 patients were later admitted with a diagnosis of secondary hemorrhage. None of the patients in the LLETZ group required admission for hemorrhage $\left(p=0.008_{\mathrm{r}}\right.$ Fisher"s exact test).

In the discussion of their findings, the authors listed a number of advantages of LLETZ over laser therapy, including the avallability of tissue specimens after treatment, less bleeding shortened $^{2}$ operative time, the possibility to assess and treat patients at the first visit and reduced costs of treatment, although no data were presented on costs. The authors concluded by stating that they now only use the laser in treatment of patients with widespread vaginal extension of dysplasia from the cervix, and to perform dilatation of the cervical os for stenosis after previous therapy. It should be noticed that the results of this study should be interpreted with caution due to the highly inadequate method of randomization.

In the period March 1990 to December 1992, Awarez et al. enrolled 375 premenopausal women with a recent Pap smear suggesting atypia or $\mathrm{CIN} \mathrm{I,} \mathrm{II} \mathrm{or} \mathrm{III} \mathrm{in} \mathrm{a} \mathrm{randomized} \mathrm{study} \mathrm{comparing}$ primary large-loop excision of the transformation zone ( $L L E T Z, n=195$ ) versus the management approach of colposcopically directed biopsies of the cervix and endocervical curettage (ECC) followed by $\mathrm{CO}_{2}$ laser ablation if dysplasia was detected $(n=180)^{[1]}$. All patients with an adequate colposcopic exam with a distinct cervical lesion were eligible. Patients were assigned a treatment strategy by computer-randomized forms contained in sealed opaque envelopes. A follow-up pelvic exam and Pap smear was scheduled 3 and 6 months after treatment. In both patient groups anesthesla consisted of a paracervical block and local infiltration using $1 \%$ Lidocaine with $1: 100$ 000 epinephrine

The mean treatment time of LLETZ was 7.4 minutes versus 10.8 minutes for laser therapy. In both groups there were no infectious complications and none of the patients required hospitalization. Immediate complications, bleeding and pain, occurred in 2 patients $(1.1 \%)$ in the LLETZ group and in none of the patients in the laser group. Nearly $50 \%$ of patients in the study were compliant with follow-up 3 months after treatment $(n=190)$. Of these, 8 patients in the laser arm $(4.4 \%)$ and 13 patients $(6.7 \%)$ in the LLETZ group had cytologic evidence of persistent cervical dysplasia. Complications in the first period of three months after treatment included bleeding $(11.1 \%$ of patients in the LLETZ group versus $10.8 \%$ in the laser group), pain ( $3.2 \%$ of patients in the LLETZ group versus $2.9 \%$ in the laser group) and discharge (10.6\% of patients in the LLETZ group versus $6.9 \%$ in the laser group). At 6 months, 107 patients $(28.5 \%)$ returned for a second evaluation. Four patients in the laser arm (2.2\%) and 5 patients (2.6\%) in the LLETZ group had cytologic evidence of cervical dysplasia. 
As a result of a subgroup analysis Alvarez and colleagues concluded that their data suggest that LLETZ therapy is as safe and effective as laser therapy in patients with CIN $\|$ or CIN III on referral Pap smear. However, due to low compliance at follow-up and to a relative high percentage of patients with histologically documented CIN II or III in the LLETZ group, this conclusion can at best be tentative.

In summary, the RCTs comparing LLETZ with laser vaporization are of rather poor quality: Combined analysis of a variety of non-randomized studies of LLETZ in patients with CIN III $(n=3,581)$, suggest success rates of at least $91 \%$ after a follow-up of minimally one year ${ }^{621}$. For the time being, it can only be concluded that laser vaporization and LLETZ are equally safe and effective.

No RCTs have been published comparing LLETZ and cryosurgery or LLETZ, cryosurgery and laser vaporization with sufficient numbers of patients that would allow a choice of treatment based on CIN stage I, II or III on an individual basis. Based on a combined analysis of six studies including 995 patients with CIN 111 who underwent cryosurgery, the short-term success rate was $84 \%{ }^{|| 2: \mid}$ Other studies, including all types of CIN patients, report success rates of cryotherapy of at least $90 \%{ }^{\text {i62? }}$. Based on the results of the RCTs comparing laser vaporization to cryotherapy, laser vaporization to LLETZ, and non-randomized studies, the provisional conclusion is that all these methods are equally safe and effective in treating patients with $\mathrm{CIN}$ in whom invasive cancer has been ruled out.

\section{Cost-effectiveness}

In the RCT by Alvarez et al: (1994), a brief analysis of selected direct costs based on fees was included ${ }^{[1]}$. Assuming that both a biopsy and endocervical curettage were obtained for each patient, the costs of diagnosis were US $\$ 568$ per patient in the laser group, compared to US $\$ 587$ in the LLETZ group. The fee for treatment was US\$659 in case of laser treatment and US\$767 in case of LLETZ. The total costs per treated patient were US\$1483 after laser treatment compared to US\$1354 after LLETZ. It can be concluded that treatment costs in terms of fees do not differ much on the level of the individual patient. However, real costs may well be lower after LLETZ, due to reduced capital outlay, maintenance costs, etcetera, leaving the potential for this procedure to represent the most cost-effective approach. Some authors have speculated on this outcome, without presenting data ${ }^{[19]}$.

When judging the cost-effectiveness of treatment alternatives of CIN, besides the modality also the method of patient selection is extremely important. Alvarez et al. (1994), despite the fact that based on the results of their trial it could only be tentatively concluded that laser treatment and LLETZ were equally safe and effective, favoured the so-called 'see and treat' approach represented by LLETZ. The authors defended this choice by a number of arguments, for example referring to the fact that 12 patients $(10.5 \%)$ were noncomplicant with instructions to return for laser ablation after the initial diagnostic procedure, compared to only 4 patients $(2.1 \%)$ who were asked to return for LLETZ treatment but did not show up. Non-compliance of patients is avoided by the 'see and treat'

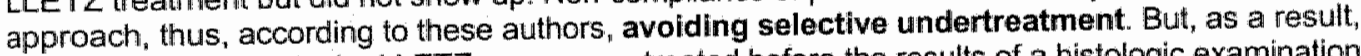
(almost) all patients in the LLETZ group were treated before the results of a histologic examination had become available. The weakness of such a "see and treat" approach, according to Reid and Absten (1995) is that it will lead to substantial overtreatment, additional morbidity and additional cost ${ }^{\mid 621}$. So there is a trade-off between the likelihood of missing pathology (and associated undertreatment) on the one hand, and the likelihood of overtreatment on the other hand, induced by the introduction of the "see and treat' approaches.

How to deal with this? Alwarez et al. (1994) noticed that the data in their study did not support the hypothesis of selective undertreatment and recognized that the "see and treat" approach resulted in overtreatment in the large category of patients that were (later) found to have normal histology or CIN I in both groups ${ }^{[1]}$. The presence of CIN I may not justify any treatment at all due to its high probability of spontaneous regression. In order to avoid overtreatment in some patients but at the 
same time avoiding to miss pathology, the authors suggest that LLETZ should be preferably used in patients with CIN II and CIN III on referral Pap smear while following up patients with CIN I on referral Pap smear. Of course, this only a partial solution to the problem of overtreatment. It can be added that in the trial by Alvarez and colleagues, not only patients in the LLETZ group, but also the majority of patients in the laser group (114 out of $190(60 \%))$ were actually treated after the results of biopsy had become available " of course, the cost-effectiveness of a treatment procedure is highly sensitive to the associated decision-making procedure. At present some authors, although recognizing the danger of overtreatment, accept or even recommend some 'see and treat' strategy, sometimes fawouring laser treatment but, more often, favouring LLETZ $Z^{11,62]}$. The fundamental problem with $\mathrm{CIN}$ is the inability to identify by cytological or histological examination those lesions that are destined to progress to invasive carcinoma from those lesions that are destined to remain stable or regress (see Intermezzo 10.1). This prablem is addressed in the nex? trial.

Writermezzo 10.1 Epidemiology and etiology of genital neoplasms and warts

Research has indicated that saxwally transmitted Human Papillomawrus (HPV) is the most common cause of the development of genital neoplasms, including cervicel intraepithelial neoplasta and genital wants, which ane aften called condylomata acuminata. More spectfically. HPV infections are usually muticentric and are found in women predominantly on the cerwix (70\%), wuve (25\%), vagina (10\%) and anus $(20 \%)$. In men, these infections most frequently occur on the inner surfice of the prepuce (70\%), the corona and frenulum (10\%, the shaft, glans and scrofum (10\%), anus (10\%) and urethra (10\%). Occasionally in both men and women, HPV infections are found on the wocal cords sol. The incidence of HPV infections is rapidly increasing, in particular in patients younger than 30 years of age $[$ [axt

More than 70 different types of human papillomawins have now been identified, but new ones are still being found. In a 1996 meeting organized by the Wortd Health Organization and the European Research Organization on Genilat Infection and Neoplasia, HPV types 16 and 18 were formally classified as oncogenic. The specialists in the meeling also agreed that HPV types 31 and 33 are probably' oncogenic" and that other types are "possibly oncogenic Furthermore, HPV types 6 and 11 are beliewed to be the mosit common cause of condylomata acuminata? in the past degade, the most general resukt of increased knowledge on the viral origin of neoplasms, and in particular of genital warts, has been that more attention has been paid to the treatment potential of antiviral agents in particutar interferons ${ }^{\text {(39:? }}$.

\subsubsection{An integrated treatment strategy for patients with CIN: the role of HPV, CIN status and interferons}

In the period October 1983 - July 1985, Woodman et al. (1993) organized a randomized trial to determine the efficacy of laser vaporization in the management of cervical Human Papilloma Virus infection occurring alone or in association with CIN I or CIN II, and to study the natural history of these lesions ${ }^{(a)}$. Patients were eligible when HPV with or without CIN I or CIN II was documented by histological examination. Threehundred-seventyone patients were stratified by the presence or absence of dysplasia and then randomized to treatment or non-treatment groups. Randomization was by telephone call to a central office. Follow-up was scheduled every 4 months and ended for each patient 1 February 1987, resulting in a median follow-up in both groups of 17 months (range 1-35 months). Disease progression was defined as histological evidence of a change from HPV infection alone to CIN, or an increase in the grade of CIN. Disease progression was coupled to the decision to treat patients in the untreated group and to repeat treatment in the treated group. Regression was considered to have occurred when two consecutive smears were negative. Disease status was considered unchanged when women with continuing cytological abnormality had no histological evidence of disease progression. When comparing outcome in treatment and non-treatment groups, the analysis was based on intention to treat. Of the 370 patients randomized, 365 were included in the analysis, 182 in the treatment group and 183 in the nontreatment group. All but one of the six excluded patients had failed to attend after initia! assessment.

Twelve months after randomization the proportion of women with progression to more severe disease was estimated to be $4 \%$ in the treatment group and $25 \%$ in the non-treatment group $(95 \%$ 
confidence interval for difference in proportions: 14-28\%). The risk of progression in the treatment group compared with the non-treatment group, expressed as a hazard ratio was $0.21(95 \% \mathrm{Cl}$ 0.08-0.34) for women with HPV infection alone, $0.23(95 \% \mathrm{Cl}$ 0.09-0.57) for those with CIN I and $0.18(95 \% \mathrm{Cl} 0.07-0.51)$ for those with CIN II. Sixty-two untreated women (34\%) with progressive disease were eventually treated by laser vaporization $(n=53)$ or by cone biopsy $(n=9)$. The remainder of women remained untreated, showing either unchanged disease state or disease regression. Thirteen women in the treatment group $(7 \%)$ required either further laser vaporization $(n=10)$ or cone biopsy $(n=3)$ to eradicate the disease. These data served to conclude that laser ireatment is efficacious in the short term to prevent progression of CIN associated with HPV infection.

Twelve months after randomization, in untreated women regression had occurred in $24 \%(95 \% \mathrm{Cl}$ $14-34 \%)$ of women with HPV infection alone, $33 \%(95 \% \mathrm{Cl} 19-47 \%)$ of those with CIN I and $18 \%$ $(95 \% \mathrm{C} \| 3-33 \%)$ in patients with $\mathrm{CIN} \|$. The total number of patients showing disease regression was $47(26 \%)$. When the log-rank test was used to compare time to regression between the three groups no significant differences were observed. The disease status of $63(31 \%)$ untreated women did not change. In total, in 110 patients $(57 \%)$ there was no indication for treatment.

Based on these data the authors concluded that the natural history of CIN associated with HPV is highly variable and that, as a consequence, some women had been treated unnecessary. A comparison of this study with other publications suggested that the presence of HPV presented no therapeutical disadvantage ${ }^{\left[6^{66}\right]}$. This may have been caused by the rather insensitive method of detecting HPV, by finding of koilocytosis in histological material, resulting in an underestimation of the prevalence of HPV.

Furthermore, no distinction could be made at that time between 'oncogenic" and "probablyoncogenic' and 'possibly oncogenic' HPV. Oncogenic HPV is currently believed to be an important prerequisite for disease progression ${ }^{831.621}$ (See Intermezzo 10.1). This classification, however, does not have immediate clinical implications because methods for cost-effective virall testing in routine clinical practice are not yet available ${ }^{[30]}$. It is expected that in the future cancer screening programs will be developed that try to screen for the oncogenic types of HPV or, even better, to develop vaccines against the virus ${ }^{[89]}$. The development of screening programs, as a first step, would solve the problem of selective undertreatment and overtreatment, while considerably reducing the number of diagnostic procedures. As a logical step in subsequent treatment decisions, antiviral agents could play a role.

The first randomized study comparing topically administered interferon gamma-1 beta gel and laser treatment in patients with cytologically and coloposcopically confirmed CIN II or CIN III was published in 1995 by Schneider et al..$^{64}$. The phase $1 \|$ trial included 33 patients of whom 9 were included in the laser treatment group. The remainder of patients were subdivided in three different dosage groups of IFN gamma-1 beta gel. However, at the end of the 6 month follow-up period laser treatment was shown to have superior results, with cures in 8 of 9 patients (89\%) compared to 10 cured patients $(42 \%)$ in the combination of all interferon groups (Fisher's two-tailed exact test, $p<0.05$ ). The three IFN dosage groups did not differ in their cure and response rates. Despite these results the authors suggest that IFN gamma-1 beta may have a future role when dosages, modes of application, and duration of treatment are better researched.

In summary, this is a highly dynamic area of fundamental and clinical research. The role of laser treatment, just as that of its most important competitors, is firmly established by RCTs. Although formal data on cost-effectiveness are lacking, the cost-effectiveness of laser treatment is questionable. In the future, treatment strategies may drastically change. More evidence and perhaps a meta-analysis of existing evidence would be desirable. 


\subsection{Vulvar, vaginal, anal and perianal neoplasia}

\subsubsection{Introduction}

Besides cervical neoplasia, neoplasia of the vulva (VIN), vagina (VAIN), anus (AIN) and perianal region (PAIN) have been distinguished. Just as with CIN, these lesions are considered to be preliminary stages of invasive carcinoma. Stages 1,11 and 111 are defined as mild, moderate, and severe dysplasia. The third stage also includes carcinoma in situ. These lesions are much less common than CIN but, unlike CIN, VIN, VAIN, AIN and PAIN is often multicentric and multifocal, complicating its management ${ }^{[6 ;: 84]}$. A prerequisite for non-surgical treatment of these lesions: including laser therapy, is that invasiveness has been excluded.

\subsubsection{Vulvar and vaginal intraepithellal neoplasia}

Both VIN and VAIN are discovered as a result of atypical smear and its diagnosis needs to be confirmed by colposcopic examination ${ }^{[34]}$. VIN is characterized by itching of the skin, and by changes in pigmentation of the skin, but in a third of all patients VIN is asymptomatic ${ }^{\text {gall }}$. Over the last decade, there has been a substantial increase in the prevalence of VIN in young women. The risk of progression to invasive cancer is estimated to be maximally $8 \%$, of which the vast majority is superficially invasive $(<1 \mathrm{~mm})^{[i] \mid}$. There is often a close relationship of the occurrence of VIN and CIN. Around $30 \%$ of the patients with VIN has been treated for CIN before ${ }^{[84 \mid}$.

In general, VAIN is an asymptomatic abnormality. The peak incidence of VAIN is in patients above 50 years of age and its incidence is increasing. VAIN has a well-defined potential for malignant progression. About one-third of the invasive cancers detected after therapy for CIN have occurred in the original squamous epithelium of the vaginal vault ${ }^{[62 !}$. Preliminary results indicate that Photodynamic Therapy may play a role in treatment of early invasive vaginal carcinoma ${ }^{[32]}$.

\section{Treatment of VIN and VAIN}

Conventional surgical approaches to VIN and VAIN were either total or partial vulvectomy or vaginectomy. Results of these procedures are far from perfect with high recurrence rates, frequent complaints of sexual dysfunction, and emotional problems following the mutilation of vagina or vulva $^{[84 !}$. In both cases, disease management is to some extent determined by the extent of the lesion.

\section{VIN}

Due to its disadvantages, surgery has become controversial particularly in patients where VIN is found in only one or two locations ${ }^{\mid 6,2]}$. In these patients, treatment alternatives for vulvectomy and related surgical techniques include wide local excision, cryotherapy, local chemotherapy (with 5fluorouracil creme), and $\mathrm{CO}_{2}$ laser vaporization ${ }^{[84 !}$. All the non-surgical modalities are reported to be associatted with recurrence rates of $30 \%{ }^{[8]}$. No RCTs have been reported, so it is unclear which treatment should be the treatment of choice. According to Reid and Absten (1995), wide excision of small foci produces excellent results ${ }^{162 !}$.

In patients with multifocal and/or extensive VIN, superficial 'skinning' vulvectomy with grafting has replaced conventional vulvectomy. However, the results of this technique, although better than corventional vulvectomy, are unpredictable in functional and cosmetic terms ${ }^{i 62 !}$. In a recent review, it is claimed that $\mathrm{CO}_{2}$ laser vaporization is an effective non-mutilating alternative to surgery in patients with bowenoid (warty) VIN $\|$ or VIN \|\|$^{\mid 6 i 2}$. No RCTs have been done in this field. In 5 case series, including a total of 201 patients with bowenoid VIN III, the average success rates for laser ablation was $86 \%$. No data on the length of follow-up were reported. Other case-series, however, have not distinguished between extensive lesions and small lesions. In a review of 9 case-series including minimally 6 and maximally 48 patients with VIN succes rates of laser treatment were reported to vary between 73 and $100 \%{ }^{\mid 841}$. In another series of 160 patients with unspecified VIN, 
the cumulative incidence of cellucitis, scarring, or secondary hemormage was $10.3 \%$ when a low

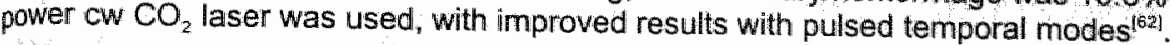

Overall, the evidence suggests that $\mathrm{CO}_{2}$ laser therapy is promising in treatment of VIN In particular when disease is extensive, $\mathrm{CO}_{2}$ laser therapy may have the potential to replace an invasive surgical procedure. In patients with small lesions, it is unclear which modality should be the treatment of choice. As the incidence of VIN is low multicentric and perhaps multinational studies are indicated to clarify these issues.

\section{VAIN}

In treatment of VAIN, various treatment techniques have been advocated to replace surgery. As no randomized trials have been done, discussions resemble those in VIN. According to Reid and Absten (1995) no single non-surgical method has been universally endorsed ${ }^{162}$. Despite conflicting results on the efficacy of $\mathrm{CO}_{2}$ laser treatment, which can be associated with serious complications such as vesicovaginal fistula formation, its use has been advocated as representing the only surgical method for destroying large ares of intricately contoured epithelium without causing vaginal scarring or shortening ${ }^{[62]}$. When $\mathrm{CO}_{2}$ laser treatment is selected, the lesions are ablated, excised or a combined vaporization/excision procedure is carried out.

In patients with extensive disease, excisional procedures can be carried out using a colposcope and either a laser or a cold knife, in both cases obviating the need for wide excision and concomitant skin grafting ${ }^{[62]}$. However, no specific case-series using the $\mathrm{CO}_{2}$ laser for this indication have been published. Excision is advocated when there is suspicion of invasion, if a high-grade lesion extends into a previous hysterectomy scar, and when there is a marked discrepancy between cytology and histology. The use of general anesthesia is recommended in these cases ${ }^{\text {iøy? }}$.

In patients with small lesions laser treatment is usually applied on an outpatient basis using local anesthesia ${ }^{[34]}$. In a series of 98 patients of VAIN treated by photovaporization, an initial failure rate of $11 \%$ was reported in women with unicentric disease, compared to $28 \%$ in patients with multicentric disease. Retreatment of initial failures resulted in an overall success rate of about $90 \%$ (652] Low-dose maintenance 5 fluorouracil therapy is optional after $\mathrm{CO}_{2}$ laser therapy ${ }^{[62\}}$. Other authors, however, have advocated local chemotherapy (such as 5 fluorouracil) as initial treatment of choice, suggesting a role for $\mathrm{CO}_{2}$ laser therapy only in patients with single keratinized lesions that are not due to HPV infection ${ }^{[84 !}$. In 11 case-series on laser treatment of VAIN including 6 to 41 patients, not distinguishing between small and extensive lesions, success rates after one or more treatment sessions ranged from 70 to $100 \% \%^{|64|}$.

Overall, in both VIN and VAIN, laser treatment may be the treatment of choice in selected cases. However, there is no evidence on its superiority. Advantages of laser treatment of vulvo-vaginal lesions are that good functional and cosmetic results can be achieved, and that in most patients treatment can be performed on an out-patient basis with local anesthesia ${ }^{[34]}$.

\subsubsection{Anal and perianal intraepithellial neoplasia}

Squarnous carcinoma of the anus is an uncommon tumour that accounts for about $2 \%$ of the cancers of the large bowel. Precursor lesions for invasive carcinoma of the anus have been considered to be very uncommon, generally being detected as a chance finding during histologic examination of tissue excised for benign ano-rectal disease. Extended $\mathrm{CO}_{2}$ laser ablation, carried out in an operating room, is advocated only in patients with PAIN 2 or $3^{\left[6^{2}\right]}$. 


\subsection{Condylomata acuminata}

Condylomata acuminata can be found on the cervix, vagina, vulva and penis, but also on othe sites such as the vocal cords. Possible complaints related to genital condylomata acuminata am: pain, pruritis, irritation and (malodorous) discharge ${ }^{[94]}$. When HPV infection occurs, it is classified as clinical, subclinical or latent. Clinical lesions can be diagnosed by gross observation. In 1987 , it was estimated that in the United States $2 \%$ of the sexually active population (about 2 million men and women) had cllinically visible HPV infections on the external anogenital skin ${ }^{1303}$. Subclinical lesilons may be detected by abnormal cytologic or histologic studies. They become clinically visib/e as white epithelial patches after application of $5 \%$ acetic acid and magnification by use of, for example, a colposcope. Latent infection is detected only by HPV DNA hybridization techniques ${ }^{\text {ton }}$. In some of these patients a form of neoplasia, e.g. VIN or VAIN can be present at the same time with condylomata acuminata. At present, condylomata acuminata are distinguished from neoplasms in most centers by a combination of cytological, colposcopical and histalogica!

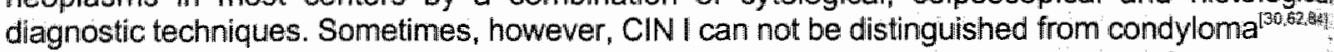
Virall testing of the biopsy sample is added to these techniques in expert centers only.

For early onset intravaginal or cervical condylomata acuminata, topical treatment using desiccant acids are described as highly effective ${ }^{[39 !}$. With these agents, e.g. bichloroacetic acid or trichloroacetic acid, success rates of 20 to $40 \%$ after 3 to 6 months of therapy have been reported ${ }^{\mid 62 !}$. The other popular topical agent is podophyllin, which biologic activity is due to an antimitotic effect ${ }^{390}$. This topical treatment can only be used in exophytic condylomata acuminatio and may have systemic side-effects. Podophyllin can therefore not be applied intravaginally, during pregnancy and in children. Podophyllin is currently being replaced by a $5 \%$ podophyllotoxin solution, podofilox (Condylox ${ }^{\mathrm{TM}}$ ). Complete clearance of external anogenital warts accurs in approximately $50 \%$ to $80 \%$ of patients ${ }^{130,39,52 \%}$. Besides topical treatment, scissor excision has been described as another technique for treating isolated external condylomas ${ }^{|62|}$.

When isollated condylomata acuminata are refractory to desiccant acids, podophyllin or podofilox; either localized, colposcopically guided $\mathrm{CO}_{2}$ laser ablation ('spot welding") hot cautery or cryosurgery (using either liquid nitrogen in drops or nitrous oxide cooled probes) can be applied.

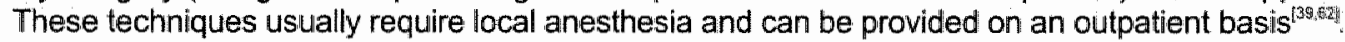
They have in common that scarring and local infection is more common than after topical treatment $t^{\mid 621}$. Systematic evaluation of each of these modalities is lacking. In general, however, locally destructive methods have been judged to often fail in controlling viral regrowth ${ }^{|39|}$. In the absence of data, none of these alternatives, including $\mathrm{CO}_{2}$ laser treatment, can be regarded as treatment of choice.

In patients with very extensive condyloma acuminata or condyloma acuminata that can not be controlled by at least 9 months of topical medical treatment, either conventional, extensive electrodiathermy ('Bovie destruction'), electrodiathermy loop excision (LEEP), surgical treatment (segmental excision and primary closure) or extended $\mathrm{CO}_{2}$ laser ablation have been used ${ }^{[39,62]}$. Reid and Absten (1995) reported that extended laser ablation in 117 patients with extensive or refractory condyloma was successful in 79 patients after one treatment $(67 \%)$, and that another $30(26 \%$ ) were contralled by either repeat laser surgery or the use of topical 5-fluorouracil as soon as recurrences appeared inevitable ${ }^{[(2)]}$. Ferenczy (1995) reported a complete or partial response rate of $76 \%$ and recurrence in $25 \%$ of patients after repeated $\mathrm{CO}_{2}$ laser treatment of an unspecified number of patients with external anogenital condylomata ${ }^{(30)}$. Exactly the same percentages were reported after LEEP, compared to a complete or partial response rate of $70 \%$ and recurrence rates of $25 \%$ after excisional biopsy ${ }^{\mid 30 !}$.

Of the inpatient procedures listed above, extensive diathermy has been outmoded in Westem society, while conventional surgical treatment has essentially been outmoded by $\mathrm{CO}_{2}$ laser surgery. according to Reid and Absten in their 1995 review of laser applications in gynecology/6a?. One RCT has addressed this latter question, published by Duus et al. in $1985^{24}$. In this study, $\mathrm{CO}_{2}$ laser 
surgery was compared to conventional surgical treatment in 50 male and female patients with condylomata acuminata resistant to theophyllin therapy. Patients in the laser group had been treated once weekly with theophyllin with a median treatment time of 33 weeks (range 8-115 weeks) versus 35 weeks (range 7-100 weeks) in the surgery group. Patients were excluded when they had condyloma acuminata of the Buschke-Lowenstein type. All patients were treated as inpatients. The patients who undenwent conventional surgery were treated by excision; electrocautery, or both. Follow-up was scheduled 2 weeks, one month and 6 months after treatment. When at the first follow-up visit recurrent disease was detected, treatment was repeated. Seven patients were lost in the follow-up period, resulting in data for analysis of 21 patients in the laser group and 22 patients in the surgery group.

After 6 months, cure rates were $43 \%$ after laser surgery and $36 \%$ after conventional surgery. Likewise, there were no differences between both groups regarding postoperative scar formation; healing time, or postoperative pain. The authors concluded that $\mathrm{CO}_{2}$ laser surgery did not show any advantage over conventional surgery in these patients. The result of this trial, while showing that treatment success is rather low conflicts with the judgement of Reid and Absten ${ }^{[62]}$.

This trial may be outdated in a sense that theophyllin has become obsolete and because monotherapy with whichever modality has become outdated as well. Why? In the eighties, as a result of disappointing treatment results and increasing knowledge on the role of HPV in the disease process, a number of predominantly small, double-blind placebo-controlled RCTs were organized studying the safety and efficacy of interferons in treatment of condyloma acuminata. Supported by the pharmaceutical industry, (type I) interferon-alfa, interferon-alfa $2 a$, interferon-alfa $2 \mathrm{~b}$ and interferon-beta were tested in small and large, single and multiple and early onset and refractory condyloma acuminata, using a variety of methods of administering the drug. Some of these RCTs showed moderately improved results compared to placebo, in particular when the drugs were intralesionally administered for a relatively long period using high dosages ${ }^{[39]}$. However, this was associated with serious systemic side-effects and disease often recurred after cessation of treatment. In addition, intralesional injections have been reported as very painful. As a result, interferons have not become established as primary therapy for condyloma acuminata. Instead, recommendations were published in international literature that stimulated researchers to evaluate combination therapy ${ }^{731}$. Subsequently, a number of RCTs have been published evalluating a variety of treatment combinations. Because of favourable results, besides interferon administration these combinations have included topical, non-cytolytic 5 fluorouracil cream in treatment of patients with papillary vaginal condyloma acuminata ${ }^{[39.62]}$. At least one study in the series evaluating combination treatlment, a single blind RCT published by Douglas et al. in 1990, has suggestied moderately improved short-term results when adding interferon-alpha $2 b$ to podophyllin compared to podophyllin alone in treatment of patients with small anogenital warts ${ }^{[23 \mid}$.

These results suggest that combination treatment strategies may result in a decrease of the number of patients that need additional therapy, e.g. laser therapy. Although no exact data are available, it can be inferred from published studies that no more than $10 \%$ of patients may have extensive lesions ${ }^{\mid 62 i}$. Both laser treatment and selected other treatment alternatives for mild disease may then may be most valuable in the category of patients with extensive lesions.

In one study, patients with extensive vulvar condylomata acuminata were randomized to either $\mathrm{CO}_{2}$ laser therapy alone or $\mathrm{CO}_{2}$ laser therapy plus either 5 fluorouracil or systemic interferon-aipha $2 \mathrm{a}$ injections ${ }^{163 i}$, another $\mathrm{RC}^{2}$ compared $\mathrm{CO}_{2}$ laser therapy plus placebo to $\mathrm{CO}_{2}$ laser therapy plus subcutaneous interferon-alpha $2 a$ injections in patients with multiple persistent lesions ${ }^{|56|}$, and a third RCT compared cryotherapy plus placebo versus cryotherapy combined with subcutaneous interferon-alpha $2 a$ in patients with primary anogenital warts ${ }^{|3|}$. In contrast with the results of the trial starting with cryotherapy, the results of both trials starting with $\mathrm{CO}_{2}$ llaser therapy suggested that the combination with IFN treatment resulted in greater cure rates. These trials were relatively small $_{\mathrm{a}}$ with about 20 patients in each treatment arm, follow-up was limited and in both cases the 
methodology and the analysis of results was heavily criticized by an international network of researchers in this field, the Condylomata International Collaborative Study Group (CICSG)

To overcome the methodological difficulties in these trials, the CICSG organized a multinational randomized placebo-controlled double-blind trial comparing $\mathrm{CO}_{2}$ laser therapy $(n=72)$ with $\mathrm{CO}_{2}$ laser therapy plus systemic interferon-alphal $2 a(n=74)^{[741}$. Patients $18-65$ years old with clinically visible genital or perianal warts (or both) that could be cleared by laser surgery within 4 weeks were eligible for the study. By applying these inclusion criteria, both patients with localized and extensive disease were included in the study. Patients with extensive disease were treated under general anesthesia, whereas those with localized disease were managed under local anesthesia with $2 \%$ xylocaine solution. $\mathrm{CO}_{2}$ laser energy was delivered in continuous or superpulsed mode. Interferon (IFN) therapy was initiated immediately after completion of $\mathrm{CO}_{2}$ laser ablation. IFN, 3IMU, was transcutaneously injected three times a week for 4 consecutive weeks. On the assumption that at 4 weeks one or more $\mathrm{CO}_{2}$ laser ablations had removed all clinically or colposicopically visible lesions, thie patients were assigned to one of the two study groups: complete responders (complete clearance of all external and intermal lesions) or non-responders (recurrence of lesions after complete response at the same or a new site). The complete responders were evaluated monthly during a follow-up period of 36 weeks or until recurrence, whichever came first. The number of laser treatments administered to patients in both groups was the same: 1 laser ablation in $75 \%$ of patients in the IFN group compared to $80 \%$ in the laser plus placebo group, two laser ablations in $14 \%$ of patients in the IFN group compared to $13 \%$ in the laser plus placebo group, and three laser ablations in $6 \%$ of patients in the IFN group compared to $3 \%$ in the laser plus placebo group. Of the 146 patients enrolled in the trial, data of 135 patients were available for analysis. Of the 11 patients whose data were not used, 7 had been lost to follow-up, while data of 4 patients were not used due to protocol violations, leaving 64 patients in the IFN group and 71 patients in the laser plus placebo group for analysis.

During the 36 week period of observation there were equivalent numbers of patients with recurrent disease; 48 in the IFN group (65\%) versus 40 in the laser plus placebo group (56\%). Similarly. there was no difference in the complete responders after 36 weeks of observation: 23 patients in the IFN group (32\%) versus 24 patients $(39 \%)$ in the laser plus placebo group. In the IFN group a variety of adverse effects were more common than in the laser plus placebo group. The authors concluded that $\mathrm{CO}_{2}$ laser ablation combined with systemic recombinant IFN-alpha 2 was ineffective as treatment of anogenital condylomata. Reconsidering these data focusing exclusively on lasers one would conclude that systemic recombinant IFN-alpha 2 did not increase the effectiveness of laser ablation, while resulting more often in systemic side-effects.

Overall, it is concluded that the safety and efficacy of $\mathrm{CO}_{2}$ laser treatment of genital and/or perianat condylomata acuminata is established by RCTs. There is no evidence on the superiority of one of the alternative treatment options for either small or extensive, refractory lesions. There is no evidence on the cost-effectiveness of laser treatment of condylomata acuminata. In patients with severe disease, laser treatment can be potentially cost-effective when it is the only alternative to conventional surgery. In the vast majority of patients, with mild disease, other treatment options may be more cost-effective than laser treatment. With respect to the position of interferon treatment it can be concluded that there is no evidence to support the replacement of either laser therapy or cryotherapy by interferon treatment, and interferon does not offer extra clinical benefits when added to laser therapy.

In literature, debate focuses mainly on the role of interferons. For example, other ways of administering interferons, e.g. intralesional, have been suggested to be more effective than subcutaneous injection as applied in the RCT by the CICSG ${ }^{\mid 30,39.74 !}$. Therefore "it is not impossible that antiviral agents such as interferon will become more important in treatment of condylomata acuminata in the future. Although the RCTs discussed here have not produced convincing results, in a recent review interferons have already been advocated as effective adjuvant therapy to conventional therapy, including as adjuvant therapy to topical treatment for early onset condyloma 
acuminatal ${ }^{39 !}$. Reid and Absten, in their 1995 review, conclude that low dose adjuvant interferon regimens are probably indicated in patients with severe disease ${ }^{|62|}$. If this is justified and, even more rigorous, if interferon treatment has the potential to actually replace other treatment options, including laser treatment, is highly speculative.

\subsection{Infertility due to occluded Fallopian tubes}

Infertility is thought to affect one in ten couples in the Western worl|d ${ }^{[27,04]}$. There are many causes for infertility, including occlusion of Fallopian tubes and endometriosis in the female, oligospermia and/or asthenospermia in the male. A separate category includes unexplained infertility. Up to $30 \%$ of the couples evaluated for infertility have occluded tubes as the primary cause of infertility ${ }^{271}$. The three most important treatments for tubal infertility are conventional microsurgery, laser laparoscopic microsurgery, and applications of assisted reproductive technology (e.g. in vitrofertilization) $)^{(27.84)}$. Conventional microsurgery is associated with hospital admission, while laparoscopic $\mathrm{CO}_{2}$ laser microsurgery is performed on a day-case basis ${ }^{(6) \mid}{ }_{\text {r }}$ and most assistive reproductive technologies are carried out as oulpatient procedures ${ }^{[27]}$.

Fertility-promoting procedures using the $\mathrm{CO}_{2}$ laser have been subjected to three $\mathrm{RCTs}$, comparing the procedure to microsurgery. These RCTs, including 45,67 and 63 patients, respectively, were carried out by Tulandi et al, in the mid-eighties ${ }^{\mid 77.78,800 !}$. Pregnancy rates in these subsequent studies were 22,30 and $57 \%$ in the laser group, respectively, and 27,23 and $57 \%$ after microsurgery, respectively. Ectopic pregnancy was reported as a frequent side-effect of both fertility promoting procedures. In 1987, Tulandi published a final article based on the RCTs discussed above, noting that there were no differences in adhesions reformation after either laparoscopic $\mathrm{CO}_{2}$ laser microsurgery or conventional microsurgery with the microdiathermy needle ${ }^{[79 ?}$.

A number of either non-randomized prospective studies and retrospective studies ${ }_{n}$ as assessed by de Wit and Trimbos (1991), suggested that laparoscopic $\mathrm{CO}_{2}$ laser treatment of occluded Fallopian tubes results in pregnancy rates of 21 to $42 \%$, which is in the same range as conventional microsurgery. The authors concluded that laser treatment is as effective as microsurgery for this indication. Improved results over time in the three RCTs by Tulandi and colleagues were interpreted as reflecting increased experience ${ }^{\left[{ }^{|d|} \mid\right.}$. In more recent assessments, a difference is made between laser laparoscopic salpingostomy for the division of peri-tubal or periovarian adhesions, as reported in the first two RCTs by Tulandi, and laser laparoscopic salpingoovariolysis in treatment of tubal occlusions, as reported in the third RCT by Tulandi and colleagues. Sutton (1993) reports case-series achieving pregnancy rates of 62 and $58 \%$ after $\mathrm{CO}_{2}$ laser treatment for this latter condition and states that similar treatment results can be achieved with conventional microsurgery using scissors (with or without electrodissection). Microsurgery, however, results in ectopic pregnancy rates between 5 and $8 \%$, higher than after laser treatmentiom: A similar, relatively high pregnancy rate was found in the third trial by Tulandi et all. in $1987^{\mid 99 !}$.

Both de Wit and Trimbos (1991) and Sutton (1993), in line with the findings in the first RCTs by Tulandi and colleagues, report pregnancy rates between 20 to $40 \%$ in patients after either laser microsurgery or conventional microsurgery for salpingostomy, the technique to reopen Fallopian tubes ${ }^{[6,8 ; 4]}$. Not only $\mathrm{CO}_{2}$ lasers are applied in these patients anymore. For example, a series discussed by Sutton (1993), reports 1 -year pregnancy rates of $31 \%$ with the KTP laser and $25 \%$ with the $\mathrm{CO}_{2}$ laser. The KTP laser, using flexible fibers, was reported to be easier to use, while generating less smoke than the $\mathrm{CO}_{2}$ laser. No new RCTs have been reported since the study by Tulandi et al. Both de Wit and Trimbos (1991) and Sutton (1993) emphasize that the main advantage lies in the minimally invasive approach afforded by laparoscapy, and the $\mathrm{CO}_{2}$ laser provides the most precise method of performing laparoscopic surgery ${ }^{[0,84]}$. Although treatment results after salpingostomy are much better than after salpingo-ovariolysis, for both indications there seem to be no differences in the safety and effectiveness of both pracedures. 
How do the results of (laser)microsurgery compare with alternative treatment modalities? Penzias and Dechemey, In an article published in 1996, without explicitly discussing laparoscopic techniques, seriously questioned the role of conventional tubal surgery compared with in witro fertilization and other reproductive techniques ${ }^{[55]}$. More specific publications on assisted reproductive technology confirm that in vitro fertilization-embryo transfer (IVF-ET) is now indicated especially for women with occluded (or absent) fallopian tubes ${ }^{\{27}$. Overall succes rate of NF-ET in 1991, based on the United States IVF-ET Registry and expressed in terms of the take-home baby rate "was $15.2 \%$. Al first sight these results are worse than those achieved after conventional or laser laparoscopic microsurgery, as demonstrated by Tuland and colleagues in their first two RCTs. Sutton (1993), without presenting data, suggests that for specific subindications, results of IVF may be comparable to those achieved with lasers ${ }^{[6]}$.

In summary, laparoscopic $\mathrm{CO}_{2}$ laser microsurgery seems well-established in infertility treatment of patients with peri-tubal and/or peri-ovarian adhesions and/or occluded Fallopian tubes. The role of lasers in this latter procedure, particularly in light of the advent and spread of a variety of assisted reproductive technologies, requires additional research.

\section{Cost-effectiveness}

No evidence has been published on the cost-effectiveness of laser treatment. Instead, studies have focused on other comparisons. For example, in retrospective studies published in the early nineties, as discussed by Penzilas et al. (1996), the costs per life birth have been calculated to amount US\$17 000 after conventional tubal surgery, compared to US\$12 000 after IVF $F^{[55}$. More comprehensive calculations indicate that the total cost of achieving a life-birth with IVF in the United States in 1992 were US $\$ 66667$ for the first cycle and US\$114286 by the sixth cycle $e^{27 !}$. These figures differ considerably, suggesting differences in methodology and perhaps differences in the viewpoint of the analyses.

\subsection{Endometriosis}

\subsubsection{Introduction}

Endometriosis is a non-malignant condition in which there is functioning endometrial tissue outside the uterus, e.g. On the Fallopian tubes or on the ovaries. The disease is usually limited to the pelvis ${ }^{[01.84\}}$. Diagnosis by clinical methods is unreliable and confirmation is required by laparoscopy or laparotomy. For these reasons, the true incidence and prevalence of endometriosis are unknown ${ }^{[75]}$. With these limitations, it has been estimated that endometriosis occurs in 1 to $7 \%$ of women 15 to 44 years of age in the US $S^{151}$. Data from the United Kingdom suggest that the incidence of endometriosis (in white women attending family planning clinics) increases with age, which peaks at about 6 per 1,000 woman years between 40 and 44 years of age ${ }^{[\theta 1]}$. Signs of the disease are found in 15 to 25 percent of women that have chronic abdominal pain, and in 25 to 35 percent of women with infertility problems. Other symptoms of endometriosis include painful menstruation (dysmenorrhea) and painful coitus (dyspareunia), but the disease can be asymptomatic as we $\left.\right|^{\left|{ }^{\mid 64}\right|}$ Some authors state that only symptomatic patients should be treated ${ }^{(35,75)}$. The reasons are that about half of the lesions progress, that progression not necessarily results in symptomatic disease and that the disease in the other half of cases either remains the same, improves or disappears The course of disease is unpredictable. Other authors, however, emphasize the probability of disease progression, and recommend treatment ${ }^{|44|}$.

Although both pain and infertility have been named as the most important indication for treatment, most studies have focused on the impact of treatment in women with fertility problems ${ }^{[35.75 .84 !}$. In these studies, treatment success has often been expressed in terms of pregnancy rates achieved ${ }^{[84]}$. A minarity of studies, including the only RCT involving lasers, have focused primarily on the impact of treatment on the relief of pain. 
Endometriosis is distinguished in stage I (minimal), II (mild), II (moderate) and stage V (advanced) disease ${ }^{72 ! 2}$. In stage / and stage 11 endometriosis, dysmenorrhea is reported in approximately $70 \%$ of women and intermenstrual pelvic pain and deep dyspareunia is reported in approximately $25 \%$ of women ${ }^{|69|}$. There is, however, considerable controversy over the role of minimal and mild endometriosis in the causation of these symptoms ${ }^{168,69, ; 3}$. For this reason, any treatment result should be interpreted very cautiously. Furthermore, as endometriosis may recur, prolonged periods of follow-up after treatment are required to reliably assess treatment success ${ }^{[8]}$.

Historically, infertility and pain followed by a diagnosis of endometriosis has been treated by pharmacotherapy as well as with surgery. Standard pharmacotherapy in the seventies and eighties, usually administered for a period of 6 to 9 months, was danazol, a synthetic androgenic ${ }^{144,84}$. When it was decided to apply surgical treatment, patients were treated by local excision or cautery during a llaparotomy procedure. These procedures, for which hospital admission and general anesthesia are mandatory, were adapted for laparoscopic application in the seventies ${ }^{[64]}$.

Laparoscopic $\mathrm{CO}_{2}$ laser treatment for endometriosis was introduced in $197^{14}$, followed by argon laser treatment in $1983^{1453}$, Nd:YAG laser treatment in $1985^{147 !}$, and KTP laser treatment in $1986^{\text {(181 }}$. Of course, laser treatment can be applied at laparotomy too, but this has hardly ever been done ${ }^{\text {fiel }}$. The advantages of treating significant abdominal disease through the laparoscope, instead of by laparotomy, have been called dramatic in terms of recovery time and patient discomforttial. Just as with infertility associated with occluded Fallopian tubes, assisted reproductive technology has become increasingly common for treatment of infertility associated with endometriosis. In the United States, for example, about $15 \%$ of all assisted reproductive procedures are performed after a diagnosis of endometriosis ${ }^{[27]}$.

Of the different types of lasers employed in laparoscopic treatment most experience has been gained with the $\mathrm{CO}_{2}$ laser and, to some extent, with the KTP laser ${ }^{[58]}$. When the $\mathrm{CO}_{2}$ laser is selected best results can probably be achieved using the superpulsed or ultrapulsed mode ${ }^{(62.68)}$. In practice, nearly all gynecologists have historically applied $\mathrm{cw}$ mode. To allow technically safe and most comfortable treatment, in modern $\mathrm{CO}_{2}$ lasers the articulated arm is fitted directly into the operating channel of the laparoscope ${ }^{[62]}$.

\subsubsection{Infertility associated with endometriosis}

In an assessment published in 1991, de Wit and Trimbos reported success rates of $\mathrm{CO}_{2}$ laser treatment of endometriosis-related infertility (any stage) ranging from 30 and $76 \%{ }^{184]}$. These figures were based on six case-series published between 1982 and 1989 including at least 10 and at most 243 patients. Success rates were expressed in terms of ongoing pregnancies. Abortion rates, only reported in the studies with the least and the most successful results, were about a fourth of the total number of pregnancies.

Chong et al. (1990) retrospectively compared results of treatment of 167 patients with minimal (stage 1) endometriosis with either danazol $(n=47), \mathrm{CO}_{2}$ laser treatment $(n=83)$ and $\mathrm{CO}_{2}$ laser treatment plus danazol $(n=37)$. In the majority of patients, besides endometriosis, other infertility factors were present as well. Pregnancy rates were $48.9 \%, 44.6 \%$ and $51.4 \%$, respectively ${ }^{[16]}$. No information was given on the course of the pregnancies in this non-randomized uncontrolled study. Nezhat et al. (1992), in a subgroup analysis of 61 patients who underwent $\mathrm{CO}_{2}$ laser treatiment of endometriosis related pain and infertility, reported that 14 out of 19 patients with minimal, mild and moderate (stage I,II and III) endometriosis achieved pregnancy $(73 \%)$, 10 out of 30 patients with severe endometriosis (stage IV) $(33 \%)$ and 1 out of 12 patients with extremely severe endometriosis (> stage IV) ( $8 \%$ ). The overall pregnancy rate was $65 \%$. No information was given on the course of the pregnancies ${ }^{\mid 52 !}$

Sutton (1993), in a subgroup analysis, evaluated the results of $\mathrm{CO}_{2}$ laser treatment in 88 patients with endometriosis as the only detected subfertility factor who had been followed-up for three years ${ }^{168 !}$. Of these patients, $70(79.5 \%)$ became pregnant, most of them $(75.8 \%)$ within a year. of those who did get pregnant, $57.6 \%$ had a successfull outcome, this low figure being accounted for 
by $14(20 \%)$ abortions, of which five $(7.1 \%)$ were spontaneous and nine $(12.9 \%)$ were induced, ore for a chromosome abnormality and the rest for social reasons: The success rate in patients wity: stage IV endometriosis was $63 \%$, rather high compared with pregnancy rates after $\mathrm{CO}_{2}$ laser treatment of patients with advanced endometriosis in other studies. Success rates in the studies quoted by the author ranged from 40 to $53 \%{ }^{\mid 106 \%}$. Although some authors, e.g. Sutton (1993) hawe generated hypotheses in order to explain a trend of increasing treatment succes rates with increasing severity of disease ${ }_{\text {actual results have shown opposite associations as welli5z.6el }}$.

How should these results be interpreted? A preliminary conclusion is that $\mathrm{CO}_{2}$ laser treatment: established by clinical experience for endometriosis related infertility, with treatment succes in most: studies ranging from at least 30 to at most $76 \%$. However, is laser therapy more efficacious than: for example, expectant management? A randomized study comparing treatment success of surgical intervention versus expectant management has never been carried out. According to Sutton (1993) 'expectant' management (consisting of remowal of fluid from the Pouch of Douglas accompanied by hydrotubation) has resulted in pregnancy rates of $50.3 \%$ (range 30.6-72.4) of patients with mild disease ${ }^{\mid t i b}$. Therefore, according to this author, any treatment for endometriosis associated infertility should be judged against this background. In that case, although the comparison is not entirely valid due to differences in the severity of disease, laser laparoscopic treatment may not have much to offer to patients with endometriosis.

Another source of information are the results of RCTs that evaluated medical treatment versus

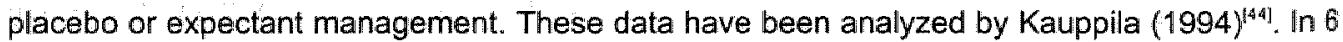
RCTs, danazol and/or MPA, gestrinone or buserilin were compared to either placebo (2 studies) or expectant management (4 studies) in treatment of endometriosis associated infertility. The number of patients in these studies varied from 42 to 144 . With a follow-up of 1 year, pregnancy rates in the treatment groups varied from at least 25 to at most $71 \%$, compared to at least $24 \%$ and at most $46 \%$ in the placebo-groups and at least $37 \%$ and at most $57 \%$ in the expectant management groups. In three other RCTs, directly comparing alternative hormonal agents, fertility rates ranged from 19 to $48 \%$. These latter studies included at least 62 and at most 182 patients. Although not based on a formal meta-analysis, the conclusion of the author that suppressive hormonal therapy has no place in the treatment of infertility associated with endometriosis, seems justified. Even stronger, the author concluded that it is a waste of time to expose patients desiring pregnancy to long-term suppressive hormonal therapy ${ }^{\left.[4]_{1}\right]}$. Furthermore, as these studies confirmed that expectant management can result in considerable pregnancy rates, as discussed by Sutton et al. (1994), the value of laser laparoscopic treatment can be seriously questioned as we ${ }^{[69]}$.

No RCTs have been done comparing the impact of surgical ablation of endometriosis either at laparoscopy or at laparotomy on future fertility. As a consequence, according to Thomas (1993) surgery can not be recommended ${ }^{(75)}$. This would pertain to surgical treatment irrespective of being performed during laparotomy or at laparoscopy. In practice, laparoscopic management of stage I and stage II endometriosis has become widely accepted in the United States in the nineties, while laparoscopic treatment of stage III and stage IV endometriosis is controversia|[5]. Small retrospective comparative studies using historical controls have, however, indicated that fertility rates in patients with stage IV disease are about $45 \%$ using either laparotomy or laparoscopy (using laser treatment) and other techniques, while reducing recovery time from a month to less than one week ${ }^{[6]}$.

Some preliminary data on costs have been published on this subject. In 1992, Luciano et al. reported the direct costs of treatment of 120 patients with stage III or stage IV endometriosis who underwent either laser laparoscopic treatment $(n=60)$ or conventional microsurgery at laparotomy $(n=60)^{i 50 l}$. Based on these data, the total average direct costs per patient in the laser group. including a one-year follow-up, amounted US\$3721, compared with US\$7075 in the conventional microsurgery group. The average number of days of incapacitation per patient was 3.6 in the laser group, versus 21.4 in the laparotomy group. These data suggest a clear advantage of laser laparoscopic treatment over conventional microsurgery at laparotomy. 
With respect to the role of assisted reproductive technology, the take-home baby rate after gamete intrafallopian transfer (GIFT) was reported to be $27 \%$ in the United States in 199127 . This particular procedure is applied not only in patients with endometriosis, but also for at least three other indications. Therefore, a direct comparison with other treatment alternatives is impossible.

In summary, laparoscopic laser treatment in treatment of patients with endometriosis associated infertility is established by clinical experience, in the absence of convincing evidence on the causal relation of minimal or mild endometriosis and infertility. Many new treatment options have been suggested in recent literature, including medical treatment alternatives (antiprostaglandins, immunomodulation agents), combined (selective) hormonal therapy and laparoscopic therapy, and new assisted fertilisation programs ${ }^{[4]}$. In the worst case, none of these treatment options is justified at all. lin the absence of clarity on these issues, if a choice has to be made between laparoscopic procedures and procedures at laparotomy, the former deserve the benefit of the doubt. Although some preliminary evidence has been produced suggesting that laser laparoscopic treatment is adwantageous both in terms of effects and costs compared to conventional microsurgery in patients with moderate or severe endometriosis, it is clear that both more fundamental research and more RCTs need to be organized in this rapidly evolving field.

\subsubsection{Pain associated with endometriosis}

In 1994, Sutton and colleagues published a double-blind RCT comparing laser lapraroscopy with no treatment in patients between 18 and 45 years of age with pain suggestive of endometriosis who at laparoscopy were found to have stage I, II or lll endometriosis ${ }^{(69)}$. Seventy-four patients were enrolled in the trial. Randomization was achieved by a compulter-generated randomization sequence. Laser treatment included vaporization of all visible endometriotic implants, adhesiolysis and uterine nerve transection with the $\mathrm{CO}_{2}$ laser or the KTP laser. Patients in the sham arm of the trial underwent diaginostic laparoscopy alone, although, according to the authors, it was necessary to remove the sanguineous fluid from the Pouch of Douglas to perform a thorough inspection of the pelvic peritoneum. To assure blinding all women were given the same number of incisions (three) as laser-treated patients. Follow-up was scheduled at 3 and 6 months after surgery by a blinded research nurse. Efficacy was based on changes in the symptoms of dysmenorrhoea. dyspareunia, and pelvic pain as assessed subjectively and by a visual analogue scale. Of the 74 patients enrolled, 63 completed the 6 month follow-up period. Data of 32 patients in the laser group and of 31 patients in the no-treatment group were analysed. Of 11 patients for whom all data were excluded, 2 became pregnant, 5 were started on hormonal contraception, 1 suffered from a depression and 3 were lost to follow-up. Five of the $32(15 \%)$ of women in the laser group and 4 of $31(13 \%)$ of patients managed expectantly had received previous medical treatment for endometriosis.

At three months after surgery, 18 of $32(56 \%)$ of patients in the laser group reported that their pain was better or improved compared with 15 of 31 patients $(48 \%)$ in the expectant group (ns, Fisher Exact test). At 6 months follow-up 20 of 32 patients $(62.5 \%)$ in the laser group reported less pain compared to 7 of 31 patients $(22.6 \%)$ in the expectant group $(p<0.01$ Fisher Exact test). At three months follow-up the median decrease of VAS-pain scores was 2.6 for the laser group and 1.2 in the expectant group ( $n s$, Mann-Whitney U-test). Compared to baseline scares, the median decrease in VAS pain scores after 6 months follow-up was 2.85 for the laser group and 0.05 in the expectant group ( $p<0.01$, Mann-Whitney U-test). There were no laparoscopic or laser complications in any of the women enrolled in the study.

The authors concluded that laser laparoscopy is safe and effective in treatment of stage I, II, and III endometriosis. Following this conclusion, laser laparoscopy was offered to patients who had been randomized to expectant management. The lack of differences between both groups after 3 months follow-up was attributed to the placebo-effect. It was also suggested that laparoscopy, performed in the way described above, results in a temporarily improvement of pain symptoms. A 
physiblogical explanation was provided for this result; which has been reported in other studies as: well. At the time of writing the article, 16 of the 31 women $(52 \%)$ who underwent expectart: management had undergone a second-look laparoscopy at 6 to 12 months after the original operation. Staging had not improved in any of these women; three cases (19\%) showed disease progression. Five women (15.6\%) continued to complain of pain despite laser treatment. In thee of these patients no endometriosis was found but in two cases pain was attributed to endometriasisi in new sites not lasered previously. Overall, this small RCT suggests some beneficial effects of laser treatment additional to physiological and placebo-effects of diagnostic laparoscopy.

A previous study by Sutton and colleagues (1993), focusing on patients with stage $\mathbb{V}$ endometriosis, had shown that either $\mathrm{CO}_{2}$ or KTP laser laparoscopy results in pain relief in $80 \%$ of patients ${ }^{\text {ros }}$. In contrast with the patients enrolled in the RCT, most of these patients had failed to respond to medical therapy.

How should these data be interpreted? As the findings in the RCT discussed above suggested that laser therapy was most effective in patients with stage III endometriosis, the combination of these findings suggests that either $\mathrm{CO}_{2}$ or KTP laser therapy is effective in pain relief in more severe stages of endometriosis ${ }^{[69]}$. As noticed at the outset of this section, the perceived lack of effectiveness of treatment in mild disease may reflect the probability that minimal and mild endometriosis may not be the cause of pelvic pain at all ${ }^{[69]}$ There are no RCTs comparing laser treatment to treatment alternatives. With respect to medical treatment, several placebo-controlled RCTs have demonstrated that hormonal agents such as danozol, MPA and various GnRH agonists analogues are effective in relief of pain associated with endometriosis ${ }^{[44]}$. Although hormonal agents are frequently associated with unpleasant side-effects, this type of treatment may be optional in women with pelvic pain who do not desire a pregnancy. In summary, as only one small $\mathbb{R C T}$ included laser treatment and as RCTs directly comparing medical treatment and laser laparoscopic treatment are lacking, no definite conclusions on the relative safety and effectiveness of these treatment alternatives can be drawn. Likewise, in the absence of data and reflecting the uncertainty on the cause of pain in these patients, no judgment can be made on the cost-effectiveness of laser treatment. Just as in case of endometriosis associated infertility, many new treatment options have been suggested in recent literature ${ }^{|44|}$.

\subsection{Menstrual disorders}

Menorrhagia, or excessive uterine bleeding, is a common complaint, estimated to occur in $9 \%$ to $14 \%$ of healthy women during their reproductive years ${ }^{[26]}$. Menorrhagia, although not lifethreatening, is a debilitating condition that gives rise to many consultations to general practitioners and gynecologists. In the UK, for example, in 1981-1982 the annual frequency of GP consultation rates for this condition was 31 per $1,000^{167 !}$. When a patient presents with menorrhagia to a primary care physician and the bleeding is associated with ovulation, it has been suggested that the patient should be referred to a gynecologist for further evaluation. When the bleeding is anovulatory in nature initial treatment depends on the severity of bleeding. If the bleeding is significant, or the patient is hemodynamically compromised, it is recommended to stabilize the patient, who should then be taken to the operating room for a dilatation and curettage. If the acute bleeding episode is less severe, a medical approach to treatment is warranted. The foundation of medical therapy has been hormonal treatment with estrogenic or progestational agents ${ }^{71}$. When hormonal therapy or repeated curettage fail to control the excessive bleeding, hysterectomy has been used as a last resort ${ }^{(26)}$. In the United States, in 1990 , about $20 \%$ of all 590,000 hysterectomies were carried out as definitive treatment of menorrhagia ${ }^{1201}$. In England and Wales, in $1985,28 \%$ of all 66,000 hysterectomies were performed to alleviate menstrual disorders ${ }^{[67]}$.

In the eighties, the development of a group of new techniques, collectively called endometrial ablation, has caused a reevaluation of the approach to the management of menorrhagia. There are three techniques for endometrial ablation: $\mathrm{Nd}$ :YAG laser ablation, electrosurgical ablation, and 
radio frequency electromagnetic wave ablation ${ }^{\mid 26 !}$. A final technique is by the use of microwaves ${ }^{\mid 66 !}$ Of these, only the oldest two, laser ablation and electrosurgical ablation, have been well-evaluated. These ablation techniques are carried out using a hysteroscope and are performed either on an outpatient basis or on a day surgery basis. With all techniques, outcome is improved by decreasing the thickness of the endometrium and the vascular blood supply. This is achieved by pretreatment administration of one of a variety of gonadotropin-releasing hormone (GnRH) agonists or danazol ${ }^{1261}$. The ablation techniques, when successful, avoid hysterectomy both in otherwise healthy patients and in patients with conditions that preclude the surgical approach of hysterectomy. While hysterectomy removes the uterus resulting in amenorrhea, ablation techniques result in a minority of patients just in a diminished menstrual blood flow. The reduction may be judged as either satisfactory or unsatisfactory by the patient. In cases of unsatisfactory treatment results, ablation can be repeated or a hysterectomy can be carried out ${ }^{[26]}$.

Nd:YAG laser ablation of the endometrium was introduced by Goldrath et al. in $1981^{[34]}$. A 1991 Diagnostic and Therapeutic Technology Assessment Report from the American Medical Association held that a consensus of panelists board-certified in obstetrics and gynecology considered laser ablation of the endometrium to be appropriate both in terms of safety and effectiveness for the treatment of menorrhagia unresponsive to conventional therapy ${ }^{[2] !}$. Two basic laser techniques have been described: touch (contact dragging) and nontouch (noncontact, blanching ${ }^{\mid 26)}$. In practice, most authors use a combination of these techniques depending on the specific area under treatment of the endometrium ${ }^{[6:]}$.

To assess the role of Nd:YAG laser ablation in this treatment strategy it should be noticed that, besides competitive ablation techniques, there are developments in the surgical technique of hysterectomy as well. Abdominal hysterectomy is the standard procedure, although indications for this operation as a treatment for benign uterine conditions have not been well established ${ }^{(20)}$. In the period 1988-1990 in the United States, abdominal hysterectomies accounted for over $75 \%$ of all hysterectomies ${ }^{[2]}$. Likewise ${ }_{n}$ in the early nineties, $80 \%$ of hysterectomies in Britain were performed abdominally ${ }^{\mid 59 !}$. An alternative is vaginal hysterectomy, which is repoited to result in lower postoperative rate of complications and morbidity ${ }^{[2,20]}$. In the United States, this technique is often performed by gynecological surgeons, while most general and oncological surgeons prefer abdominal hysterectomy. The abdominal route offers better exposure to complicated pathology and an easier operation. It has been suggested that in benign cases vaginal hysterectomy could be performed just as well ${ }^{[2]}$. In the United Kingdom, a move towards vaginal hysterectomy has been reported in the nineties (but again in the absence of RCTs) ${ }^{|59|}$. In some patients, therefore, the appropriate comparison to ablation techniques could be vaginal hysterectomy rather than abdominal hysterectomy.

In the eighties, laparoscopically assisted vaginal hysterectomy and hysterectomy performed through a laparoscopic incision have been developed ${ }^{[20]}$. This too has been recognized to thave implications for the appropriate selection of control groups for comparison of clinical results of ablation techniques ${ }^{\mid 26)}$. Two small Swedish RCTs comparing the safety and efficacy of (sub)total abdominal and laparoscopic hysterectomy in women with bleeding disturbances, of which one was accompanied by an economic evaluation, have suggested that the latter technique is cost-effective in patients with specific (sub)indications, in particular due to reduced indirect costs ${ }^{28.37 !}$. In particular the development of laparoscopically assisted vaginal hysterectomy may thus reduce the potential for cost-effective use of ablation techniques.

Three RCTs have been published comparing endometrial ablation (resection) with hysterectomy ${ }^{\mid 25,33,53]}$. Of these RCTs, the first two compared endometrial ablation to abdominal hysterectomy. These two RCTs, while not containing more than 25 patients per treatment arm, demonstrated favourable results for electrosurgical ablation ${ }^{25.331}$. A third $\mathrm{RCT}$, published by Pinion and colleagues in 1994, compared the results of abdominal or vaginal hysterectomy (12\% of cases randomized to hysterectomy), endometrial Nd:YAG laser ablation and transcervical endometrial ablation (resection) in patients with dysfunctional uterine bleeding that otherwise would have 
resulted in hysterectomy ${ }^{\text {set. }}$. Women were eligible to enter the study if they were under 50 years: Two hundred of the 204 women enrolled in the trial had medical treatment for menorhagia. Although randomization was primarily between hysterectomy (n=99) and consewative surgery $(n=104)$, those randomly allocated 10 conservative surgery were also randomly assigned to lasch ablation $(n=53)$ or endometrial resection $(n=52)$, anticipating the analysis of a subsequent tria: comparing the wo hysteroscopic treatments alone. Randomization was achieved by drawing of a series of numbered opaque enveloppes containing the treatment option. Patients undergoing contact Nd YAC laser ablation were treated at a power of $80 \mathrm{~W}$. Patients allocated to endometriat resection were treated by rollerball coagulation of the comula with resection af the rest of the cavily with a loop. Hysterectomy was performed according to standard procedures. Patients were followed-up after 1, 6 and 12 months. More than $90 \%$ of patients complied with follow-up after 12 months. Analysis was by intention to treat.

The analysis firstly showed that there were no clinicalty significant differences between both conservalive treatments. Operating time of patients who underwent laser ablation was on average 50 minutes (sd 16 minutes). longer than patients undergoing resection (on average 40 minutes: so 11 minutes). In the remainder of the analysis both groups that underwent consenvative treatment were combined and compared to the hysterectomy patients. At 12 month follow-up, 17 patients $(16.3 \%)$ assigned to conservative treatment had undergone hysterectomy for continuing menstrual symptoms. Eleven patients had undergone repeat hysteroscoplc surgery for continuing symptoms $(10.6 \%)$. At the end of the follow-up period, all patients in the hysterectomy group were by definition free of the symptoms that prompted surgery. Ninety-six patients in the conservative treatment group filled out an inquiry on symptoms. Twenty-one patients $(22 \%)$ treated by hysteroscopic surgery were amenornoeic at that time; a further 59 patients $(62 \%)$ were hypomenorthoeic. Three patients $(3 \%)$ reported an identical menstrual status. The remainder of 33 patients had undergone: hysterectomy. Was concluded that the success rate of treatment in terms of relief of symptoms. was higher in the hysterectomy group. However, it was judged far more important that the average hospital stay in the conservative treatment group was 2.5 days compared to 7.3 days in the hysterectomy group. Conservative treatment was less painful than hysterectomy, as measured by: visual analogue scale (mean 2.6 , sd 2.9 versus mean 5.2 , sd 3.0). Likewise, there was less morbidity in the conservative treatment group. The postoperative period until full recovery was $2-4$ weeks in the conservative treatment group compared to 2-3 months in the hysterectomy group: which coincided with differences in the time required to return to work. Elighty-nine percent af patients in the hysterectomy group (79/89) and 78\% (75/96) in the hysteroscopy group were very satisfied with the effect of surgery; $95 \%(85 / 89)$ and $90 \%(86 / 96)$ thought there had been an acceptable improvement in symptoms, and $72 \%(64 / 89)$ and $71 \%(68 / 96)$ would recommend the same operation in others.

The authors concluded that endometrial ablation compares favourably to hysterectomy in the treatment of dysfunctional uterine bleeding. Hysteroscopic surgery was recommended as an alternative for the majority of women when more conservative treatment has failed.

This trial evoked many reactions of readers of the British Medical Journal, both complimentary and highly critical| ${ }^{[59]}$. It can be concluded that the results of this trial are highly controversial although the authors, in their clear reply, stood by their recommendations ${ }^{[59]}$.

The combination of the three RCTs discussed here suggest that ablation techniques should be preferred compared to abdominal hysterectomy. Abdominal hysterectomy should then only be performed after ablation techniques have failed. However, these trials can collectively be considered more or less outdated for a considerable percentage of patients. As yet, no RCTs have compared different hysterectomy techniques or, in particular, (laparoscopically assisted) vaginal hysterectomy to ablation techniques, implying that the ultimate role of ablation techniques is still unsettled. 
If ablation techniques should be chosen, which technique is most effective? The trial by Pinion et al. (1994) suggests that there are no differences between laser ablation and surgical ablation in terms of safety and effectiveness ${ }^{[58]}$. ECRI, in its assessment of endometrial laser ablation therapy for menorrhagia, states that two non-randomized controlled studies comparing Nd: YAG laser with electrosurgical ablation demonstrated comparable efficacy of both techniques, with $87 \%$ to $94 \%$ of patients achieving a satisfactory responseitat.

Erian et al. (1994) published perhaps the largest case-series to date ${ }^{\mid 29 !}$. In three centers and in a period of five years, 2,342 women with disabling menorthagia that was unresponsive to medical therapy were treated with the Nd:YAG laser (contact technique) at power settings varying from 60 $1080 \mathrm{~W}$. All women were medically pretreated, either with danazol $(94 \%)$ or progestational agents plus one or two GnRH analogues $(6 \%)$. There were no major complications in this series, and no complications at all in 2,285 patients $(97.6 \%)$. The main complication in the study was fluid overload, which was suspected in 22 patients $(0.9 \%)$ and resulted in the development of hyponaetremia and pulmonary edema in 9 patients $(0.4 \%)$. Hospital discharge in these patients was delayed no more than 24 hours in these cases. There were 13 cases of infections $(0.6 \%)$. Hematometra (an accumulation of blood in the uterus) requiring balloon tampomade occurred in 8 patients $(0.3 \%)$. In five patients $(0.2 \%)$ the uterus was perforated with the hysteroscope. The use of the laser never caused perforation. One-year follow-up data were available of 1,866 women at the time of the analysis. Of these women, $1,043(56 \%)$ developed complete amenorrhea, 701 $(37.5 \%)$ reported continuing but satisfactorily reduced menstruation, and $122(6.5 \%)$ patients failed to improve with the first treatment. Hysterectomy was performed in 33 patients (27\%). Data of treatment of five patients in the initial failure group were lacking. Eighty-four patients were retreated with the laser, resulting in a satisfactory improvement in $57(68 \%)$. Data on treatment of patients after an unsuccessful second laser ablation was not presented. The overall, success rate of laser ablation was $96.5 \%$. The authors concluded that the procedure is acceptable for patients, moderately effective, and that it may be an effective alternative for hysterectomy in these patients.

An assessment by ECRI of results of 13 non-randomized uncontrolled studies on the use of laser ablation of the endometrium in the period 1981-1993, including 1,479 patients, showed that a satisfactory result was achieved in $92.2 \%$ of the cases ${ }^{|26|}$. Among the failures, usually incomplete procedures, a successful repeat ablation was performed in $3.4 \%$ of the cases. In some instances $(3.2 \%)$, the therapeutic failure led to hysterectomy. There were no differences between the touchand nontouch technique. In a similar assessment a series of 14 uncontrolled studies of 1,208 patients who have undergone the electrosurgical abllation procedure, the outcome was judged to be satisfactory in $92.5 \%$ of the cases. Repeat procedures due to incomplete ablation were required in $3.8 \%$ of the cases, and hysterectomy was elected in $2.2 \%$ of the cases ${ }^{[26]}$.

Overall, it can be concluded that there is no evidence to conclude that there are differences in the safety and effectiveness of both procedures. Other authors too "e.g. Reid and Absten (1995) state that experts will secure comparable results with either method ${ }^{[62]}$. Therefore, if ablation techniques should play a role, based on criteria of safety and effectiveness, both laser ablation and electrosurgical ablation would be good candidates.

In light of these data, it is interesting to note that both ECRI (1994) and Reid and Absten in their 1995 review of the use of lasers in gynecology, notice a shift away from laser ablation to electrosurgical ablation ${ }^{[2,62]}$. Costs are reported to be a factor, but of considerable importance is the idea that electrosurgery is a much easier procedure to learn and become comfortable with than is laser surgery ${ }^{126,62]}$. Reid and Absten, however, defend the use of the laser for this indication. particularly emphasizing the advantage of producing a well-delineated, visually recognizable zone of damage surrounding the sites of treatment ${ }^{[62]}$. In practice, a first RCT comparing two different techniques of endometrial resection has been published, apparently after having decided to abandon the Nd:YAG laser ${ }^{[57]}$. In the future, these techniques should probably be testled in comparison with (laparoscopically assisted) vaginal hysterectomy. The role of lasers, although safe and effective, seems to become marginal for this indication. 


\section{Cost-effectiveness}

Formal data comparing the cost-effectiveness of Nd:YAG laser ablation with other methods of ablation or laparoscopic hysterectomy are lacking. Data from the United States suggest that the direct costs of Nd:YAG laser treatment average US\$275 per patient, compared to US $\$ 1500$ for (abdominal') hysterectomy. However, the capital costs of the laser were not taken into accountilit this calculation ${ }^{226}$. Alongside one RCT comparing endometrial electrosurgical resection with abdominal hysterectomy, the one by Dwyer and colleagues ${ }^{[25]}$, an economic evaluation was carried out. Total direct costs up to 4 months after treatment averaged US $\$ 840$ per patient in the electrosurgical group compared to US\$1600 in the hysterectomy group. As the clinical results it the electrosurgical ablation group were better, this option can be considered cost-effective compared to abdominal hysterectomy ${ }^{[65]}$. As a result, many authors assume that endometrial ablation therapy, using any of the current methods, is cost-effective compared to abdominal hysterectomy. However, assuming equal safety and effectiveness, laser ablation is likely to be less cost-effective than electrosurgery, mainly due to a large difference in capital costs, maintenance costs, etcetera. In conclusion, if ablation techniques do have a role in treatment of menorrhagia, Nd:YAG laser treatment is unlikely to be the most cost-effective option.

\subsection{Summary}

Table 10.1 summarizes the main findings of this Chapter.

Table 10.1 Judgments on the effectiveness and cost-effectiveness of sellected laser applications in gynecology

\begin{tabular}{|c|c|c|c|c|c|c|c|}
\hline \multirow[t]{2}{*}{ Application } & \multicolumn{7}{|c|}{$\begin{array}{c}\text { Judgments } \\
\text { (see definitions bellow) }\end{array}$} \\
\hline & 1 & 2 & 3 & 4 & 5 & 6 & 7 \\
\hline $\mathrm{CIN}$ & & & & $x$ & $x$ & & \\
\hline VIN + VAIN & & $x$ & & & & & \\
\hline Condylomata acuminata & & & & $x$ & & $x$ & \\
\hline Fallopian tube reconstruction & & & $x$ & & & & \\
\hline Endometriosis & & & $x$ & & & & \\
\hline Menorrhagia & & & & $x$ & $x$ & & \\
\hline
\end{tabular}

Definitions:

1 Experimental $=$ still in development, limited clinical data, no proof of effectiveness.

2 Promising = some clinical data, but procedure not accepted as a routine.

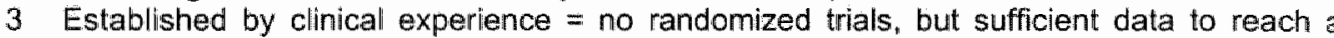
judgment.

4 Established by RCT = established by several well-designed clinical trials.

5 Potentially not cost-effective = limited data on cost-effectiveness, but reason to bellieve that the procedure will not turn out to be cost-effective.

6 Potentially cost-effective = limited data on cost-effectiveness, but reason to believe that the procedure will turn out to be cost-effective.

7 Proven cost-effective $=$ adequate analysis of cost-effectiveness. 


\subsection{References}

1. Alvanez RD, Helm W, Edwards RP, Wendel Naumann R, Partridge EE, Shingleton HIM, MeGee JA, Hall JB, Higgins RW. Malone Jr. JW. Prospective randomized trial of LLETZ versus laser ablation in pattents with cervical intraepithelial neoplasia. Gynecologic Oncology $1994(52)$ ) $175-9$.

2. Amini SB, Waight SA, Yuan Z, Rimm AA. Abdominal and vaginal radical hystenectony among U S. women aged 65 years and older. International Jounal of Technology Assessment in thealth Core 1996(12)2:377-401.

3. Baggishi MS, Dorsey JH, Adelson M. A ten-year experience treating cervical intraepithellal neoplasila with the Co. laser. American Journal of Obstetrics and Gynecollogy 1989(161)1:60-8.

4. Baggish MS. A comparisom between laser excisional conization and laser waporization for the treatment of corwical intraepithelial neoplasia. American Journal of Obstetrics and Gynecology 1986(155)1:39-44.

5. Barbieri RL. Etiology and epidemiology of endometriosis. American Journal of obstetrics and Gynecology $1990(162) 2 ; 565-7$

6. Batema BG, Kolp LA, Mills S. Endoscopic versus laparotomy management of endometriosils. Fertitity and Sterility 1994(62)4:690-5.

7. Bayer SR, DeChemey AH. Clinical manifestations and treatment of dysfunctional utterine bleading. Joumal of the American Medicall Association 1993(1269):1823-8.

8. Bekassy Z. Economical gains by laser conisations in gynecology (abstract). Abstract Baok of the First Meeting of the Scandinavian Society for Laser Therapy, Copenhagen, Dentrark, Ootober 1-4, 1987, p. 114.

9. Berget $A$, Andreasson B, Bock $ل$ E, Bostofte E, Hebjom $S$, Isager-Sally L, Philipsen T, Schantz A, Weber T. Outpatient treatment of cervical intra-epithelial neaplasia. The $\mathrm{CO}_{2}$ laser versus cryatherapy, a randomized trial. Acta Obstetrica Gynecologica Scandinavica $1987(66): 531-6$.

10. Benget A, Lenstrup C. Cervical intraepithelial neoplasia . Examinutions, treatment and followup. Obstetricial and G) necological Survey 1985(40)9:545-52.

11. Berget A. Economy in laser treatment in a gynecologic department (abstract). Abstract Book of the First Meeting of the Scandinavian Society for Laser Therapy, Copenhagen, Denmerk, October 1-4, 1987, p. 118.

12. Bhatta $N$, Isaacson $K$, Bhatta $K M$, Anderson $R R$, Schiff I. Comparatiwe study of different laser systems. Fertility and Sterility $1994(61) 4: 581-91$.

13. Bostofte E, Berget A, Falck Larsen J, Hjortkjaer Pedersen P. Rank. F. Conization by carbon dioxide laser or cold knife in the treatment of cenvical intra-epithelial neoplasia. Acta Obstetrica Gynecologica Scandinavica $1986(65): 199-202$.

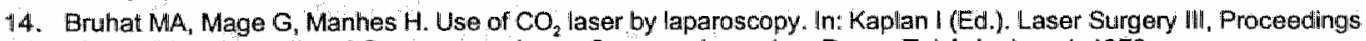
of the Third International Congress on Laser Surgery. Jeruzalem Press, Tel Aviv, Usrael, 1979.

15. Cartier $R$, Sopena B, Cartier I. Use of the diathermy loop in the diagnosis and treatnent of lasions of the uterine cervix. In: Proceedings of the Fourth World Congress of the International Federation for Cervical Pathology and Colposcopy. International Federation for Cerwical Pathology and Colposcopy, London, England, 1981.

16. Chong AP, Keene ME. Thornton NL. Comparisom of three modes of treatment for infertility patients with minimal pelvic endometriosis. Fertility and Sterility 1990(53):407-10.

17. Crompton AC, Johnson N. Which is more painful? A randomized trial comparing loop with laser excision of the cervical transformation zone. Gynecologic Oncology 1994(52) 392-4.

12 . Daniell JF. Miller W, Tosh R. Initial evaluation of the use of the patassium-titanyl-phosphate (KTP/532) laser in gynecologic laparoscopy. Fertility and Sterility 1986(46):373-7.

19. DelNolt RK. Laser ws cryotherapy for CIN and a LEEP of faith (letter to the editor). Joumal of the American Medical Associlation $1993(269) 1: 46$.

20. Desforges JF. Indications for hyskerectomy. New England Joumal of Medicine 1993(328):856\%60.

21. Diagnostic and Therapeutic Technology Assessment (DATTA). Laser ablation of the endometrium. Journal of the American Medical Association, 1991.

22. Diagnostic and Therapeutic Technology Assessment (DATTA). CO, kaser treatment of gynecologlic mallignancies. Journal of the American Medical Association 1983(250):672.

23. Douglas JM Jr. Eron Ld, Judson FN, Rogers M. Alder MB, Taylor E, Tanner D, Peets E. A randomized trial of combination therapy with intralesional interferon alphe $2 \mathrm{~b}$ and podophyllin versus podophyllin alone for the therapy of anogenitai warts. The Journal of infectious Diseases 1990(162):52-9.

24. Duus BR, Philipsen T. Christensen JD, Lundvall F, Sondergaard J. Refractory condylomata acuminata: a cantrolled clinical trial of carbon dioxide laser versus conventional surgical treatment. Gentlourinary Medicine 1985(61):59-61.

25. Dwyer $\mathbb{N}$. Hutton J, Stirrat GM. Randomised controlled trial comparing endometriall resection with abdominal hysterectomy for the surgical treatment of menorhagla. British Journal of Obstetrics and Gynecology $1993(100): 237-43$

26. ECRII. Endometrial ablation therapy for menornagia. Health Technology Assessment lnformation Service, Executive Briefings. ECRI. Plymouth Meeting, PA, US, May 1994

27. ECRI. Female Infertility Technologies, Part ll: Assisted Reproductive Technology. Health Technology Assessment Information Service, Executive Briefings. ECRI, Plymouth Meeting, PA, US, September 1994. 
28. Elstrom $M$. Hahlin M, Olsson $\mathrm{H} H$. Subtotal hysterectomy. a prospective randomised trial between laparoscopy and laparotomy (abstriaci). Acla Obstetrica Et Cynecollogica Scandinawica 1996(75ySupplement 162:54-5.

29. Erian 1 . Endometrial ablation in the treatment of menorihagia. British Joumal of Obstetrics and Gynecology $1994(101)$ Supplement 11:88-102.

30. Ferenczy $A$. Epidemiology and chinical pathophysiology of condylomata acuminata. American Journal of Obstetics and Gynecology $1995(172) 4$ part 2 ):1331-9.

31. Ferenczy A. Management of the patient whth an abnormal papanicolaou test. Recent developments. In: Cecil Wright $V$ (quest editor), Contemporary colposcopy. Obstetrics and Gynecology Clinics of North America 1993(20)1.169. 202.

32. Fisher AMR, Murphree AL, Gomer CJ. Clinical and preclinical photodynamic therapy. Lasers in Sungery and Medicine $1995(17): 231$.

33. Gannon MJ, Holl EM, Fairbank J, Fitzgerald M, Milne MA, Crystal AM, Greenhalf JO. Randamised trial comparing endometrial resection and abdominal hysterectomy for the treatment of menorhagia. British Medical Joumal $1991(303): 13624$

34. Goldrath $\mathrm{MH}_{3}$ Fuller T, Segal S. Laser photovaporization of endometrium for the treatment of menorhagia, American Journal of Obstetrics and Gynecology 1981(140):14-9.

35. Guillebaud J. Epidemiology of endometriosis (letter to the editor). British Medical Joumal 1993(306):9301.

36. Gunasekera PC. Phipps $\mathrm{JH}_{1}$ Lewis BV: Large loop excision of the transformation zone (LLETZ) compared to carbon dioxide laser in the treatment of CIN: a superior mode of treatiment. British Journal of Obstetrics and Gynecology $1990(97): 995-8$.

37. Hahlin M, Ellstrom M, Olsson JJ, Hellberg P. Heger M. Cost-effectivity and post-operative health status: a randomised trial comparing laparoscopic and abdominal hysterectomy (abstract). Acta Obstetrica Et Gynecologica Scandinavica 1996(75)Supplement 162:56-7.

38. Hamdey JM, Horner T, Maw RD, Lawther H. Dinsmore WW. Subcutaneous interferon alpha 2a combined with cryotherapy versus cryotherapy alone in treatment of primary anogenital warts: a randomized blind placebo controlled study, Genitourinary Medicine 1991(67)4:297-302.

39. Hatch KO. Clinica appearance and treatment strategies for human papillomavirus: a gynecologic perspective. American Journal of Obstetrics and Gymecology 1995(172):1340-4.

40. Helmemorst Th.JM, Kwikkel HJ, Siregar-Emck MTh.W, Stolk JG. Conservative treatment of premalignant cervical lesions. A prospective study. In: Helmerhorst Th. IM, Trends in management of female lower genital intraepithellal neoplasta (thesis). Vrije Universiteit Amsterdam, the Netherlands, 1988, p. 143-50.

41. Helmerhorst Th.JM. Laser vaporization in treatment of lower genital intraepithelial neoplasia. In: Helmerhorst Thull. Trends in management of female lower genital intraepithelial neoplasia (thesis). Vrije Universiteit Amsterdam, the Netherlands, 1988 , p. 125-42.

42. Jobson $\mathrm{VW}$, Homesley HD. Comparison of cryosurgeny and carbon dioxide laser ablation for treatment of cerwical intraepithelial neoplasia. Colposcopy \& Gynecological Laser Surgeny 1984(1)3:173-80.

43. Kaplan I, Goldman J, Ger R. The treatment of erosion of the uterine cenvix by use of the $\mathrm{CO}_{2}$ laser. Obstetrics \& Gynecology 1973(41):795.

44. Kauppila A. Changing concepts of medical treatment of endometriosis. Acta Obstetrica ef Gynecologica Scandinavica 1993(72)5:324-36.

45. Keye Jr WR, Dixon J.Photocoagulation of endometriosis by the argon laser through the laparoscope. Obstetrics and Gynecology 1983(62)3:383-6.

46. Kwikkell HJ, Helmerhorst Th.JM, Bezemer PD, Quaak MJ, Stol JG. Laser or cryotherapy for cervical intramepithelial neoplasial: a randomized study lo compare efficacy and side-effects. Gymecolog ic Oncology 1985(22):23-31.

47. Lamano JM. Photocoagulation of early pelvic endometriosis with the Nd:YAG laser through the laparoscope. Journal of Reproductive Medicine 1985(30)2:77-8

48. Larsson $\mathrm{G}, \mathrm{Alm} \mathrm{P}, \mathrm{Grundsell} H \mathrm{H}$. Laser conization versus cold-knife conization. Surgery, Gynecology and Obstetrics $1982(154): 59-62$.

49. Larsson $\mathrm{G}$, Gullberg B, Grundsell H. A comparison of complications of laser and cold knife conization. Obstetrics \& Gynecology $1983(62) 2: 213 m$.

50. Luciano $A A$, Lowney J, Jacobs SL. Endoscopic treatment of endometriosis-associated infertility. Therapeutic. economic and social benefits. Journal of Reproductive Medicine 1992(35)7:573-6.

51. McCartney AJ. Surgery of intraepithelial neoplasia, CIN, VAIN and VIN. Baillières Clinical and Obstetrical Gynecology $1987(1): 447-84$.

52. Nezhat $C_{n}$ Nezhat $F$ "Pennington $E$. Laparoscopic treatment of infiltrative rectosigmoid colon and rectovaginal septum endometriosis by the technique of videolaparoscopy and the $\mathrm{CO}_{2}$ laser. British Joumal of Obstetrics and Gynecology 1992(99):664-7.

53. Office of Health Technology Assessment. Carbon dioxide laser surgery. Assessment Report Series, Volume 2, Number 23. Washington, DG: Office of the Assistant Secretary for Health, Department of Health and Human Services, 1982. 
54. Oyesanya OA. Amerasimghe CN, Manning EAD. Outpatient excisional management of cervical intraepithelia neopiasia. A prospective, randomized comparison between loop diatherny excísion and laser excisional conization. American Journall of Obstetrics and Gynecology $1993(168) 2485-8$

55. Penzias AS, DeChemey AH. Is there ever a role for tubal surgery? Amierican Joumal of Obstetrics and Gynocology $1996(174) 4: 1248-21$

56. Peterson CS, Bjerring $P$, Larsen $J$, Blaakaer $J$, Hagdrup H. Systemic interferon alpha-2b increases the cune rate in laser treated patients with multiple persistent genital warts: a placebo-controlled study. Gonitourinary Modicine $1991(67) 2: 99-102$

57. Phillpsen $T$, Jorgensen $J C$ Pelle $J$. Endometrial resection versus rolterball ablation of the encometrim in the treatment of menorrhagia. A prospective randomized study (abstract). Acta Obstetrica Et Gyinecologica Scandinavica 1996(75)Supplement 162:57-8.

58. Pinion SB, Parkin DE, Abramowich DR, Naji A, Alexander DA, Russell VT, Kitchener HC. Randomised trial of nysterectomy, endometriat laser ablation, and transcervical endometrial resection for dysfunctional uterine bileeding. British Medical Joumal 1994(309):979-83.

59. Finion $\mathrm{SB}$, Parkin DE, Abramovich DR, Naji A, Alexander DA, Russell IT, Kitchenel HC. No title (author's reply). British Medical Joumal 1995(310):804

60. Pozzi V, Cappa F. Facchini D, Mascaretti G, Marino R. Crioterapia e lasenterapia nel trattamento della lesione intraepiteliali del collo dell' utero. Minerwa Ginecologica 1985(37):601-5.

61. Prendiville W, Cullimore J, Norman S. Large loop excision of the transformation zone (LLETZ). A new method of management for women with cervical intraepithellal neoplasia. British Journal of Obistetrics and Gynecology 1989(96): $1054-60$.

62. Reid $R$, Absten GT. Lasers in gynecology: why pragmatic surgeons have not abandoned this technique. Lasers in Surgery and Medicine 1995(17) $2011-301$

63. Reld R, Greenberg MD, Pizzuti DJ, Omoto H, Ruthledge LH, Soo W. Superficial laser vulwectomy V. Surgica debulking is enhanced by adjuvant systemic interferon. American Journal of Obstetrics and Gynecology $1992(166): 815-20$

64. Schneider A, Grubert T, Kirchimayr R, Wagner D, Papendick U, Schlunck G. Efficacy trial of topically administered interferon gamma-1 beta gell in comparison to laser treatment in cervical intraepithelial neoplasia. Archivess of Gynecology and Obstetrics 1995(256)2:75-83

55. Sculpher MJ, Bryan S, Dwyer N. Hutton J, Stirrat GM. An economic evaluation of transcervical endometrial resection versus abdominal hysterectomy for the treatment of menorhagia. British Journal of Obstetrics and Gynecology $1993(100): 244-52$

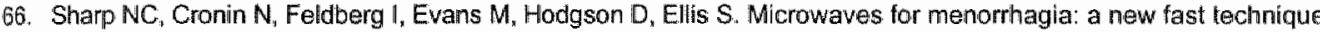
for endometrial ablation. Lancet 1995(346):1003-4.

67. Shaw RW. Introduction. British Joumal of Obstetrics and Gynecology 1994(101)Supplement 11:1.2.

68. Sutton C. Lasers in infertility. Human Reproduction Update 1993 (8) 1:133-46:

69. Sutton CJG, Ewen SP. Whittelaw N, Haines P. Prospective, randomized, double-blind, controlled trial of laser laparoscopy in the treatment of pelvic pain associated with minimal, milt, and moderate endometriosis. Fertility and Sterility 1994(62)4:696-700.

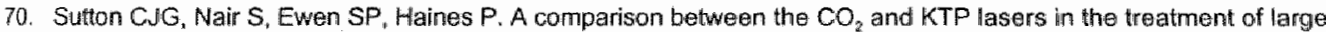
ovarian endometriomas. Gynecological Endoscopy 1993(2):113

71. Tchabo AG, Thomure MF. Tomail TP. A comparison of laser and colld knife conization. International Surgery $1993(78): 131-3$

72. The American Fertility Society. Revised American Fertility Society Classilication of Endometriosis: 1985. Fertility and Sterility $1985(43): 35$ - -2 .

73. The Condylomata International Collaborative Study Group. Recurrent condylomata acuminate treated with recombinant interferon-alfa 2a: multicenter double-blind placebo controlled clinical trial. Journal of the American Medical Association 1991 (265):2684-7

74. The Condylomata International Collaborative Study Group. Randomized placebo-controlled double-blind combined therapy with lasier surgery and systemic interferon-alfa $2 a$ in the treatment of anogenital condyloma acumimata. The Journal of Infectious Diseases 1993(167):824-30.

75. Thomes EJ. Endometriosis. Should not be treated just because it's there. Erittish Medical Journal 1993(306):158-9.

76. Townsend DE, Richart RM. Cyotherapy and carbon dioxide laser management of cervical intraepithelial neoplasia: a controlled comparison. Obstetrics \& Gynecology 1983(61):75-8.

77. Tulandi T, Sirag R, Mclnnes RA, Gelfand MM, Wright CV, Vilos GA. Reconstructive surgery wif hydrosalpinx with and without the carbon dioxide laser. Fertility and Sterility 1984(42):839-42

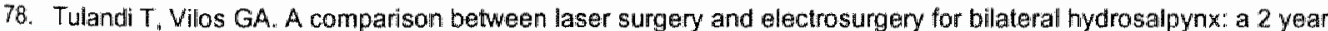
follow-up. Fertitity and Sterility 1985(44)6:846-8.

79. Tulandi T. Adhesion reformation after reproductive surgery with and without the carbon dioxide laser. Fertillty and Sterility 1987(47):704-6. 
61. Vessey MP. Wilard-Macintosch L, Painter R. Epidemiology of endometrosis in women attending family planning clnics. Britush Medical Jounal $1993(306)$ : $182-4$

82. Wetcher S. Treatment of cervical intraepithelial neoplasia with the $\mathrm{CO}_{2}$ laser laser versus cryotherapy. A review of effectiveness and costs. Obstetnical and Gynecological Survey $1984(39) 8: 469-73$

83. Wijnen JA. Cryocoaglation versusi laser vaporization in the treatment of CIN. In: Huisman WM, Winen HA Colposcopy in diagnosis and treatment of cenvical intraepithelial neoplasia (CIN) (thesis). Erasmus University Rotterdam, the Netherlands, $1987, p .177,212$.

84. de Wit GA, Trimbos $\mathbb{B}$. Gymecology - effectiveness. In: Banta HD and Schou I (Eds.). Lasers in Health Care. Effectiveness, Cost Effedtueness and Policy Implications. Academic Publishing. Frederiksberg. Denmark, 1991, p.189-200

85. de Wit GA, Vondeling H, Banta HD, Lasers in gynecology. A literature survey. Health Councit "Report No A90/07. The Hague, the Netherlands, 1990 (in Dutch)

86. Voodman $\mathrm{CH}$, , Byrie $P$, Kelly $\mathrm{KA}$, Hiton $\mathrm{O}$. A randomized trial of laser vaporization in the management of cervical intraepithelial neoplasia associated with Human Papilloma Virus infection. Journal of Public Health Medicine $1993(15) 4: 327-31$

87. Yliskosk M, Syrjanen K, Syrjanen S, Saarikoski S, Nethersell A. Systemic alpha-interferon (Welfieron) treatment of human papillomavinus (HPV) type $6,11,16$ and 18 infections: double-blind placebo-controlled trial. Gynecologic Oncology 1991(43):55-60.

88. Partingtion CK. Tumer MJ, Soutter WP, Griffiths M, Krausz T. Laser vaporization versus laser excision comization in the treatment of cervical intraepithelial neoplasia. Obstetrics \& Gynecology 1989(73)5([Part 1):775-9.

89. Mayor S. Human papillomavirus classified as carcinogenic. British Medical Joumal 1996(313):70. 


\section{Summary and Discussion}

The findings on the safety, effectiveness and cost-effectiveness of the use of lasers in various specialties are briefly summarized, followed by a general discussion.

\subsection{Individual specialties}

\section{Dermatology}

In dermatology much progress has been made in the understanding of laser-tissue interaction with the publication of the theory of selective photothermolysis by Anderson and Parrish in Science in $1983^{[1]}$. Although this has intensified evaluation efforts in this speciaity, this has never been done by means of RCTs. In some cases, where no accepted treatment existed before the advent of lasers, this can be judged as appropriate, e.g. in case of port wine stains and treatment of tattoos. However, in other cases good opportunities for evaluation by means of RCTs may be underused, for example when various types of lasers are hypothesized to differ slightly in effectiveness for specific categories of pigmented lesions. At present, as a result of this situation, all major laser applications in dermatology are judged as 'established by clinical experience', implicating that any judgment on cost-effectiveness is speculative.

\section{Ophthalmology}

Ophthalmology is the only specialty where the safety and effectiveness of a variety of major indications for the use of lasers has been convincingly demonstrated by RCTs. This can to a considerable extent be attributed to the strategy towards evaluation of the National Eye Institute of the National Institutes of Health in the United States ${ }^{(16)}$. The high quality of these large-scale multicenter trials was, among ather factors, associated with the institution of Data and Safety Monitoring Committees ${ }^{[i t]}$. The evaluation of ophthalmological laser applications still could serve as a model for evaluation of laser applications in other specialties.

Likewise, the quality of the evidence on economic evaluation of ophthalmological laser applications is unsurpassed in the laser field. As discussed in Chapter 16, the Diabetic Retinopathy Study has been subjected to a retrospective cost-effectiveness analysis followed by a translation of the findings to the United States' health care system by means of Monte-Carlo simulation techniques. The Diabetic Retinopathy Study and the subsequent retrospective economic evaluation stimulated worldwide research on (cost)-effective screening strategies for retinopathy in diabetes patients (see Chapter 16 for details). Likewise, most sophisticated evidence on indirect costs has been produced in this field (see Chapter 6).

In ophthalmology, argon laser treatment of diabetic retinopathy has developed with competition of the Xenon-arc. Otherwise, laser treatment has occupied niches without much competition of either conventional treatment or other minimally invasive procediures. The most important question seems to be which type of laser should be used for each indication or combination of indications. Although the diode laser may be in a position to compete with the argon laser "rapid replacement of argon lasers by the diode laser is not expected until (and if) it has been conclusively shown that the efficacy of the diode laser is at least as high as that of the argon laser.

\section{Otolaryngology}

Of all specialties, otolaryngology is perhaps the one where lasers have been least systematically evaluated. No RCTs on lasers have been published in this specialty. The majority of laser applications is judged as promising, with only a few indications judged as established by clinical experience. However, these are clinically important, including for example treatment of laryngeal carcinoma. In addition, laser treatment of laryngeal carcinoma is potentially cost-effective compared to traditional treatment. Additional research could be very beneficial here. Perhaps the lack of systematic evaluation of laser applications in otolaryngology is due to the fact that for many indications the laser is used as a very precise scalpel. The cost-effectiveness of these applications, although difficult to judge, is therefore subject to considerable debate. 


\section{Gastroenterology}

One laser application in gastroenterology, treatment of upper gastrointestinal bleeding, has been exceptionally well evaluated by RCTs. Meta-analysis has proven to be very helpful in interpretation of the combined results of a variety of trials in this case $e^{[s]}$. Assessment was complex because on of a variety of minimally invasive treatment modalities was compared to either conventiona: treatment or another minimally invasive technique. In the present assessment it was concluded that laser treatment, although effective, may not be the most cost-effective treatment option for this particular indication, which probably is the most important example of laser treatment becoming obsolete. This situation could have some predictive value for potential results of more systematic evaluation efforts of laser applications in other specialties.

For another indication, laser treatment of hemorrhoids, the methodological criteria for inclusion of RCTs in a metamanalysis resulted in exclusion of the trials focusing on laser treatment. In case of mild disease, laser treatment is likely to be obsolete, but laser treatment of severe hemorrhoids: warrants additional evaluation.

In other selected applications, e.g. laser palliation of esophageal cancer, judgments are difficul despite several but small RCTs. Probably application of the techniques of cumulative meta-analysis may speed up the process of drawing conclusions $s^{\mid 24\}}$.

\section{Urology}

In urology, treatment of benign prostate hyperplasia (BPH) is likely to become the best evaluated indication for laser use in this specialty. As multiple trials are ongoing, the outcome of this: assessment is still uncertain. In general, relatively many laser applications in urology are judged as 'promising'. Without exception, the indications that have been judged as 'established by clinical experience' are potentially cost-effective, warranting additional efforts in (economic) evaluation. Technological development is rapid in urology, in particular in treatment of BPH and in urolithiasis. complicating evaluation.

\section{Gynecology}

Laser treatment of CIN is the best-evaluated application of lasers in gynecoloy, followed by treatment of condylomata acuminata and menorrhagia. Formall costueffectiveness studies are lacking, resulting in at best preliminary conclusions on the cost-effectiveness of the use of lasers: in this specialty. Another factor that complicates formulation of conclusions on the (cost): effectiveness of laser treatment is the anticipated change of treatment concept for CIN (and perhaps other forms of neoplasia) "endometriosis and, more generally, infertility. Increased knowledge on the role of HPV and the advent of many new types of assisted reproductive technology, respectively, complicate the assessment.

In the 1991 assessment of the cost-effectiveness of gynecological laser applications by de Wit, laser treatment was compared to surgical treatment ${ }^{[30]}$. For one indication, treatment of $\mathrm{CIN}_{\text {, the }}$ comparison resulted in the judgment that laser treatment could be potentially cost-effective. In the present assessment, taking into account non-surgical interventions as well, there is now reason to believe that laser treatment may not be the most cost-effective treatment option.

\section{Photodynamic Therapy}

Although applications of PDT have been discussed as part of each specialty and for each indication where this therapy plays a role, for the combination of these applications it can be concluded that notwithstanding that PDT has always been judged as having a high potential to improve treatment results in cancer patients, actual developments have been extremely slow. On the basis of results of RCTs in the specialties covered in this thesis PDT may carve a niche in palliation of advanced esophageal cancer, and in curation of superficial bladder cancer. No information is available on the cost-effectiveness of these applications. 


\subsection{Combination of specialties}

Table 11.1 summarizes the findings from Chapter 5 to 10 . The Table shows that of the 58 (sub)indications included in the updated study, $2(3.4 \%)$ are judged experimental $17(29.3 \%)$ are judged as 'promising', $31(53.4 \%)$ are judged as "established by clinulcal experience, and $8(13.8 \%)$ are judged as 'established by RCTs'.

Table 11,1 Judgments on the effectiveness and cost-effectiveness of selected laser applications in six specialties

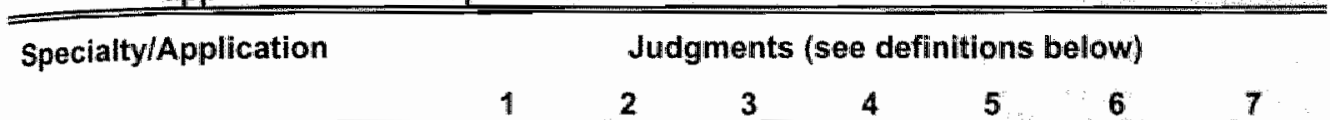

Dermatology

Hemangiomas

Portwine stains

Other congenital vascular

3

$5 \quad 6$

7

lesions

Telangiectasis

Other acquired vascular

$x$

lesions

Pigmented lesions

$x$

$x$

$x$

Tattoos

Laser surgery, e.g. warts

$x$

$x$

Ophthalmology

$x$

$x$

$x \quad x$

Diabetic retinopathy

Age-related macular

degeneration

Retinal Vein Occlusion

Branch Vein Occlusion

Central Vein Occlusion

Retinal detachment

Primary glaucoma

Newly diagnosed open-angle

glaucoma

Advanced open-angle

glaucoma

Angle-closure glaucoma

$x$

$\times$

$x$

$x$

Uncontrollable glaucoma

Cataract (posterior

capsulotomy)

Photorefractive keratectomy ${ }^{4}$

Phototherapeutic keratectomy

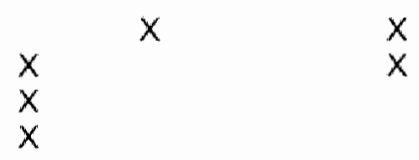

$x$

$x$

$x$ 


\begin{tabular}{|c|c|c|c|c|c|c|}
\hline Specialty/Application & \multicolumn{5}{|c|}{ Judgments (see definitions below) } & \multirow[b]{2}{*}{7} \\
\hline B & 2 & 3 & 4. & 5 & 6 & \\
\hline \multicolumn{7}{|l|}{ Otolaryngology } \\
\hline Laryngeal stenosis & $x$ & & & & & \\
\hline Vocal cord immobility & $x$ & $\therefore$ & & & & \\
\hline Respiratory papillomatosis & & $x$ & & & $x$ & \\
\hline $\begin{array}{l}\text { Vocal cord polypl, nodules, } \\
\text { granuloma and edema }\end{array}$ & & $x$ & & & & \\
\hline Carcinoma of larynx & & $x$ & & & $x$ & \\
\hline Pharyngeal pouch & $x$ & & & & & \\
\hline $\begin{array}{l}\text { Severe snoring and sleep } \\
\text { apneu }\end{array}$ & $x$ & & & & & \\
\hline Lesions of the mouth & & $x$ & & & & \\
\hline Tonsillitis & $\mathrm{x}$ & & & & & \\
\hline Tongue lesions & $x$ & & & & & \\
\hline Stapedotomy & $x$ & & & & & \\
\hline Ear tumours & $x$ & & & & & \\
\hline Nasal surgery & $x$ & & & & & \\
\hline \multicolumn{7}{|l|}{ Gastroenterology } \\
\hline Hemostasis of gastric ulcer & & & $x$ & $x$ & & \\
\hline $\begin{array}{l}\text { Hemostasis of intestinal } \\
\text { vascular malformations }\end{array}$ & $x$ & & & & & \\
\hline $\begin{array}{l}\text { Cancer palliation of } \\
\text { esophagus }\end{array}$ & & $x$ & & & & \\
\hline Cancer palliation of colon & & $x$ & & & $x$ & \\
\hline Sessile villous adenoma & & $x$ & & & $x$ & \\
\hline $\begin{aligned} \text { Hemorrhoids - mild cases } \\
\text { - severe cases }\end{aligned}$ & & $\begin{array}{l}x \\
x\end{array}$ & & $x$ & $x$ & \\
\hline \multicolumn{7}{|l|}{ Urology } \\
\hline $\mathrm{BPH}$ & & $x$ & & & $x$ & \\
\hline Prostate cancer & $x$ & & & & & \\
\hline Bladder cancer & & $x$ & & & $x$ & \\
\hline Condyloma acuminata & & $x$ & & & $x$ & \\
\hline $\begin{array}{l}\text { Benign urethral and penile } \\
\text { lesions }\end{array}$ & $x$ & & & & & \\
\hline Upper tract carcinoma & $x$ & & & & & \\
\hline Penile carcinoma & & $x$ & & & $x$ & \\
\hline
\end{tabular}




\begin{tabular}{|c|c|c|c|c|c|c|c|}
\hline \multirow[t]{2}{*}{ Specilalty/Application } & \multicolumn{7}{|c|}{ Judgiments (see definitions below) } \\
\hline & 1 & 2 & 3 & 4 & 5 & 6 & 7 \\
\hline Haemangioma & & $x$ & & & & & \\
\hline Cystitis & & $x$ & & & & & \\
\hline Vasovasotomy & $x$ & & & & & & \\
\hline Laser lithotripsy & & & $x$ & & & $x$ & \\
\hline Gynecology & & & & & & & \\
\hline $\mathrm{CIN}$ & & & & $x$ & $x$ & & \\
\hline$V: N+V A I N$ & & $x$ & & & & & \\
\hline Condyloma acuminata & & & & $x$ & & $\mathrm{x}$ & \\
\hline Fallopian tube reconstruction & & & $x$ & & & & \\
\hline Endometriosis & & & $x$ & & & & \\
\hline Menorrhaqia & & & & $\underline{x}$ & $x$ & & \\
\hline
\end{tabular}

\section{Definitions:}

1 Experimentall = still in development, limited clinical data, no proof of effectiveness.

2 Promising = some clinical data, but procedure not accepted as a routine.

3 Established by clinical experience $=$ no randomized trials, but sufficient clinical data to reach a judgment.

4 Established by RCT = established by several well-designed randomized controlled trials.

5 Probably not cost-effective = limited data on cost-effectiveness, but reason to believe that the procedure will turn out not to be cost-effective.

6 Probably cost-effective = limited data on cost-effectiveness, but reason to believe that the procedure will turn out to be cost-effective.

7 Proven cost-effective $=$ adequate analysis of cost-effectiveness.

Of the 58 indications included in the updated study, in 23 cases $(39.6 \%)$ there was sufficient evidence to reach a judgement on cost-effectiveness. In 6 of these cases $(26.1 \%)$ the laser application was judged as 'potentially not cost-effective', in 16 cases $(69.6 \%)$ the laser application was judged as 'potentially cost-effective', and one case (4.4\%), argon laser treatment of diabetic retinopathy, was judged as proven cost-effective.

The major difference between the original and the updated table is that in the latter case a comparison was made not only to the standard or traditional therapy, but to other minimally invasive treatment modalities as well. The main impact of this comparison was that some laser applications had to be judged as potentially not cost-effective. This judgment category was not included in the original table.

\subsection{Discussion}

\subsubsection{Selection of specialties and indications}

By selecting major laser applications in six specialties we intend to cover 'main-stream' laser use in medicine. The original selection of laser applications in the EC.Project was based on the criterion of therapeutical relevance in health care ${ }^{[31}$. As a consequence, both medical applications, 
paramedical applications (in general low power laser applications) and applications in dentistry were included.

For practical reasons, it was decided to limit the update to therapeutically relevant applications in medicine. Consequently, low power lasers were not included. These lasers are used in particular: in paramedics, for example in physiotherapy. In the EC-Project, low power laser applications were judged 'experimental". Likewise, dentistry was not covered. In the EC-Project, three laser applications in dentistry were distinguished, of which tooth restoration was judged "experimental: while applications in endodontics and periodontics were judged 'promising ${ }^{\text {i3! }}$.

Of the medical applications, lasers were not used for major therapeutical goals in neurosurgery. orthopedics, and head and neck surgery at the time when the selection of applications for the EC: project was made. Furthermore, the use of lasers as a scalpel was not extensively discussed in the EC-project, with the exception of lasers in otolaryngology due to the major importance of noninvasive treatment of vocal cord lesions.

Manitoring the field resulted in the conclusion that updating the use of lasers in pulmonology would not result in significant changes. The main application of lasers in this specialty is palliation of advanced lung cancer. For this indication. Nd:YAG laser treatment was considered 'established by clinical experience' in the EC-Project ${ }^{\text {II }}$. In the meantime, the use of PDT for this indication has become established by clinical experience as well. Presently, phase III randomized clinical trials are underway comparing both modalities in terms of relieving symptoms. Furthermore, PDT has been shown to have the potential to cure early lung cancer which is, however, difficult to detectif!

Due to the combination of results of new studies and rapid technological developments in the field of interventional cardiology, the use of lasers seems to have lost momentum here ${ }^{[2,5,19]}$. For this reason, the update does not include cardiovascular applications, although both in the context of Part 3 of the thesis and in Chapter 19 explicit attention is paid to an RCT evaluating Excimer laser treatment of coronary artery disease. In the EC-Project, coronary artery recanalization was judged as 'promising' indicating rapid technological change in this field. Likewise, peripheral artery recanalization was judged 'promising' in the EC-Project ${ }^{[3]}$.

Although the number of specialties in the updated study was limited to six, it was decided to include relevant developments in these specialties in the update, resulting in a net increase of the number of indications covered ( 58 versus 43 ). Examples are laser treatment of BPH in urology and laser treatment of snoring in otolaryngology.

Nevertheless, it can be argued that in a future update all relevant medical and non-medical health care applications of lasers should be included. In each specialty examples can be provided of indications that may become more important in the future. Three gynecological examples may serve as an illustration. The first example is endometriosis in other sites of the pelvis than on the ovaries or Fallopian tubes, as discussed in paragraph 10.6. In this case a specific study, published by Nezhat et al. in 1992, reported that $\mathrm{CO}_{2}$ laser treatment can prevent major surgery in patients with infiltrative rectosigmoid colon and rectovaginal septum endometriosis ${ }^{211}$. The second example is Nd:YAG- or KTP laser treatment of intrauterine abnormalities such as septae or adhesions that interfere with reproductive function ${ }^{[26]}$. The final example is the potential role of $\mathrm{Nd}$ : YAG lasers in reducing the mortality and morbidity associated with Twin-Twin Transfusion Syndrome ${ }^{2821}$. This latter application is still experimental.

Another field that promises to yield many new studies for assessment in the near future is PDT. Fisher (1995), besides confirming that stage III clinical trials are ongoing for indications covered in the thesis, states that phase III clinical trials have been organised in Japan to evaluate PDT in treatment of early stage lung-, esophagus-, gastric-, bladder and cervical tumours ${ }^{19 !}$. 


\subsubsection{Literature search strategy}

Originally, the updating exercise was intended to be limited to those laser applications which efficacy had been demonstrated by RCTs. This choice was made because randomized controlled irials represent the highest standard of evidence of effectiveness (efficacy), because the judgments of the international experts in the EC-Project, who often were pioneers in the field of medical lasers, may have been overly optimistic, and because the field had developed too fast to allow a complete review of all grades of evidence for the most frequent applications without substantial additional funding of the research team. However, when this work was carried out, it seemed in many specific applications impossible to add anything to existing knowledge without taking into account other evidence than just evidence provided by RCTs. The same consideration was felt even stronger when assessing cost-effectiveness. Only a limited number of cost-effectiveness studies had been done, despite frequent claims of cost-effectiveness in the medicall literature. Because there were hardly any data available of economic evaluations carried out alongside RCT's, which represents the highest quality of evidence in economic evaluation, a judgment on the quality of economic evaluation of these laser applications had to be based on predominantly partial evaluations, necessarily inducing (extra) subjectivity.

Notwithstanding these considerations, it could be argued that several RCTs have been missed due to limitations in the literature search strategy, in which Medline and Embase played an important role. The limitations of, for example, Medline in retrieving RCTs has been convincingly demonstrated by Dickersin et al. (1994) who, using ophthalmological RCTs published in 1988 as a case-study, showed that even the best search strategy would resuit in at most $87 \%$ of actually available RCTs ${ }^{[r]}$. Furthermore, these authors showed that the RCTs that can potentially be retrieved in Medline represented only half of the total number of ophthalmological RCTs published in 1988, including both indexed and non-indexed journals. Therefore, although in this study an extensive search strategy was applied combined with an effort to retrieve 'grey' literature (see Chapter 4), it may be expected that any new update of this study based on "state-of-the-art' search strategies would come up with a substantial additional number of RCTs.

A second improvement compared with the current search strategy would be to systematically include RCTs in other languages than English. The quality of these trials has been shown not to substantially differ from the RCTs published in the English language ${ }^{[20]}$. In this thesis, besides English language RCTs, occasionally Dutch, French, Italian and Spanish language data have been included. In a future update of this thesis, extending the number of languages included in the search strategy could be beneficial in for example assessing PDT, as a number of phase III studies focusing on PDT are presently ongoing in Japan (see previous paragraph).

A third improvement would be to include other databases than Medline and Embase, for example the Science Citation Index, the Cochrane Database of Systematic Reviews, and the NHS Economic Evaluation Database (see also Chapter 20).

\subsubsection{The quality of clinical evidence}

The most important factors in selecting a treatment are, or should be, safety and effectiveness of treatment alternatives. One would expect the treatment decision to be based on evidence of effectiveness from randomized controlled clinical trials (RCTs). However, as outlined above, few RCTs have been done in the field of laser therapy. In general, although available studies are suggestive of benefit, effectiveness has been demonstrated for relatively few laser applications. The argon laser in ophthalmology is most established. Few other areas of laser use have been as well-evaluated. Available studies may not be an adequate guide for clinical practice. Study design is often faulty ${ }^{|10|}$. For example, patient selection is often not carefully done ${ }^{[27]}$. Such important factors as age of patients and length of follow-up are sometimes not reported. Inadequate numbers of patients for a statistically significant result is a pervasive problem ${ }^{[2,27}$. Larger clinical trials are 
needed, but this requires multicenter studies that are difflcult to organize and support. In Chapter 19 the question why so few RCTs have been done is discussed in depth.

Almost all studies have used either only clinical parameters (e.g. intraocular pressure) or a "natural unit' of effectiveness. For example, the studies of $\mathrm{Javitt}$ et al. $(1989,1990,1991)$ included a "natural unit" in the form of "person-year of vision saved" "person-year of reading-vision saved", or "person

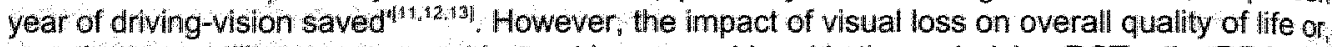
even better, a utility measurement has not been considered in the underlying RCTs, the DRS and the ETORS: This situation has changed for the better in the more recent RCTs in ophthalmology organized by the NEl, where quality of life has become a standard outcome measure.

In other fields, however, progress is slow. This is a particular problem in assessing many palliative procedures, where extended life expectancy cannot be anticipated. In these cases, the onty reasonable outcome measure is the quality of life of the patient. Despite the availability of validated measures of quality of life ${ }^{291}$, only a few studies of results from laser treatment have included prospective quality of life measurement as an outcome, e.g. the application of laser palliation of rectal cancer by McGowan et al. (1988) ${ }^{417}$. In still other cases, there may be a trade-off between the length and quality of life, having an impact on the choice of palliative treatment method, as demonstrated for example by McNell et al. (1981) in patients with laryngeal cancer ${ }^{[18\}}$ "In such cases utility measurement can be useful for both treatment decision-making and economic evaluation. However, utility measurement techniques have not yet been reported when evaluating laser application's, as far as they are overed in this thesis.

Overall, the laser is still a relatively new therapy in most areas. Evidence of effectiveness is often skimpy. This has repercussions on statements about cost-effectiveness.

\subsubsection{The quality of economic evidence}

In the scientific literature so far only a few examples can be found of economic evaluations carried out alongside clinical trials evaluating laser applications. These particular RCTs were carried out in the United Kingdom and the Netherlands, evaluating open surgery, Nd"YAG laser photocoagulation and the use of the thermal probe in treatment of upper gastrointestinal bleeding, and Excimer laser coronary angioplasty (ELCA) versus Percutaneous Transluminal Coronary Angioplasty (PTCA) in patients with coronary artery disease, respectively ${ }^{[2,23]}$. The British economic evaluation was never officially published, while the results of the economic evaluation were marginally referred to in a publication of the Dutch trial, focusing on the safety and efficacy of both interventions.

In none of the economic evaluations, whether performed alongside an RCT or not, a societal perspective has been applied. Not a single study has included direct costs outside the health care system, including for example the costs of travel, travel time and waiting time of patients and their relatives. Indirect costs have only been calculated by Crijns (1993) in a study on diabetic retinopathy ${ }^{|0|}$. It is interesting to note that the author applied the Friction Cost Method for this purpose. The Friction Cost Method has been shown to result in a more adequate calculation of indirect costs than the Human Capital Method ${ }^{[15}$. All other studies, whenever including indirect costs, have expressed these costs in terms of the number of days absent from work. The vast majority of studies that included direct costs of treatment have not adequately dealt with the capital cost of the laser. In none of these studies, real costs have been calculated. The highest quality evidence is available on economic evaluation of screening, diagnosis and treatment of argon laser treatment of diabetic retinopathy, produced by Javitt et al. $(1989,1990,1991)$ and Drummond et al. (1993) in the United States ${ }^{[8,31,12,13]}$ and Sculpher et al. in the United Kingdom ${ }^{[25]}$ and by Crijns (1993) in the Netherlands ${ }^{16 \|}$. In these studies future costs and effects have been discounted and an extensive sensitivity analysis has been applied. The remainder of data generally is of poor quality. Thus, the question of most interest still is whether the laser treatment is more effective or not, and 
at what cost. If the effectiveness is roughly the same, it is necessary to find out what the overall cost difference is. The little literature available to the present tends lo assume that treatment outcomes are equal, and estimates the cost of the laser treatment. This approach obviously has limitations. In most of these cases, in fact, equivallent outcomes have not been demonstrated.

In summary, since no complete cost-effectiveness analysis has been reported, most of the figures quoted are at best suggestive. More research is especially needed in this area, given the number of claims in the medical literature that lasers are more cost-effective than other therapies. A more extensive review of the limitations of the evidence on cost-effectiveness of laser applications can be found in Chapter 20.

\subsection{References}

1. Anderson RR, Parrish RR. Selective photothermolysis: precise microsurgery by selective absorption of pulsed radiation. Science 1983(220):524-7.

2. Appelman YEA, Plek JJ, Strikwerda S, Tijssen JGP, de Feyter PJ, David GK, Serriys PW, Margolis JR, Koelemay MJ, Montauban van Swijndregt EWJ, Koolen JJ. Randomized trial of excimer laser angioplasty versus balloon angioplasty for treatment of obstructive coronary artery disease. Lancet 1996(347):79-84.

3. Banta HD, Schou I, Vondeling H, de Wit A. Economic appraisal of laser applications in health care. Report of a project. Lasers in Medical Science 1992(7):9-21.

4. Cook DJ, Guyatt GH, Salena BJ, Laine LA. Endoscopic therapy for acute nonvariceal upper gastrointestinal hemorrhage: a meta analysis. Gastroenterology 1992("102):139-48.

5. Corr L. New methods of making blocked coronary arteries patent again. British Medical Journal 1994(309):579-83.

6. Crijns HHM. Diabetic retinopathy. A cost-effectiveness analysis of ophthalmoscopy and photocoagulation (thesis). Erasmus University Rotterdam, the Netherlands, 1993.

7. Dickersin $K$, Scherer R, Lefebvre $C$. Identifying relevant studies for systematic reviews. British Medical Journal 1994(309):1286-91.

8. Drummond $M$, Davies L, Ferris F. Assessing the costs and benefits of medical research: the diabetic retinopathy study. Social Science and Medicine 1993(34)9:973-81.

9. Fisher AMR, Murphree AL, Gomer CJ. Clinical and preclinical photodynamic therapy. Lasers in Surgery and Medicine 1995(17):2-31.

10. Garnier $H_{n}$ Flamant $R$, Fohanno $C$. Assessment of the role of randomized clinical trials in establishing treatment policies. Controlled Clinical Trials 1982(3):227-34.

11. Javitt JC, Aiello LP, Bassi LJ, Chiang YP. Canner JK. Detecting and treating retinopathy in patients with type: 1 diabetes mellitus. Savings associated with improved implementation of current guidelines. Ophthalmology 1991(98)10:156:5-73.

12. Javitt $J C$, Canner $J K$, Frank $R G$, Steinwsachs $D M$, Sommer A. Detecting and treating retinopathy in patients with type I diabetes mellitus: a health policy model, Ophthalmology 1990(97):483-95.

13. Javitt JC, Canner JK. Summer A. Cost-effectiveness of current approaches to the control of retinopethy in type I diabetics. Ophthalmology 1989(96):255-64.

14. Kaiser-Kupfer MI. Ethics in clinical research. In: Banta HD and Schou I (Eds.). Lasers in Health Care. Effectlyeness, Cost-Effectiveness and Policy-Implications. Academic Publishing, Frederiksberg, 1991, p. 68.71.

15. Koopmanschap MA, Rutten FFH, van Ineveld $B M$, van Roijen $L$. The friction cost method for measuring indirect costs of disease. Journal of Health Economics 1995(14):171-89.

18. Kupfer C. Clinical trials concerning the effectiveness of lasers. The case of ophthalmology. In: Banta HD and Schou ( Eds.). Lasers in Health Care. Effectiveness, Cost-Effectiveness and Policy-Implications. Academic Publishing. Frederiksberg, 1991, p. 63-8.

17. MCGowan 1. Barr H, Krasner N. Palliative laser therapy for inoperable rectal cancer-does it work? Cancer $1989(63): 967-9$.

18. McNeil B., Weichselbaum R, Pauker SG. Speech and survival: tradeoffs belween quality and quantity of life in laryngeal cancer. New England Journal of Medicine 1981(305):982-7.

19. Meiller AF, Rigter H. Developments in cardiovascular technology. The madern tools of cardiologist and surgeon. Health Councii ${ }_{1}$ Publication Number A 1993/3. The Hague, the Netherlands, 1993 (in Dutch).

20. Moher D, Fortin $P$, Jadad $A R$, Jüni $P$, Klassen $T$, Le Loirier J, Liberati $A_{s}$ Linde $K$, Penna $A_{u}$ Completeness of reporting of trials published in languages other than English: implications for conduct and reporting of systematic reviews. Lancet 1996(347):363-6. 
21. Wezhat $\mathrm{C}_{\mathrm{n}}$ Nezhat $\mathrm{F}$ Pennington $\mathrm{E}$. Laparoscopic treatment of infiltrative rectosigmoid colon and rectovaghn septum endometrosis by the technique of videolaparoscopy and the $\mathrm{CO}_{2}$ laser. British Journall of Obstetrics an Gynecology 1992(99),664-7.

22. Offce of Technology Assessment The impact of randomized clinical trials on health policy and medical practice Washington, DC, US Government Printing Office, 1983.

23. Rees M. The costing of a controled trial of the use pi laser and themal probe for the treatment of bleeding pent ulcer Gastroenterological Unit, St James Hospitai, Balham, London 1987.

24. Sackett DL, Cook DJ Can we leamanything from small trals? In: Warren KS, Mosteller F (Eds.). Doing more goo than harm the evaluation of health care interventions. Annals of the New York Academy of Sciences $1993(703) 25$ 32.

25. Sculpher Mu, Buxton MJ, Ferguson BA, Spiegelnalter DJ, Kirby AJ. Screening for diabretic retinopathy: a relath costeffectiveness analysis of altemative modalities and strategies. Health Economics 1992(1):39-51.

26. Sutton CJG. Lasers in infertifity. Human Reproduction Update 1993(8) 1:133-46.

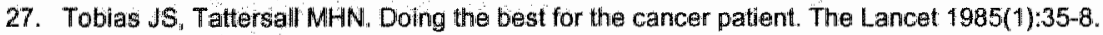

27. Wan Gement MUC, Scherjon SA, Borst C. Sterenborg HJCM, Wille Y, Hyett J, Nicolaides K. Can intra-uterine mortalit be redwced in Md:YAG laser treatment of win-twin transfusion syndiome? An analysis. Lasers in Medical Scieno $1995(10): 283-6 \%$.

29. Waker SR, Rosser RM (Eds.). Quality of life assessment. Key issues in the 1990s, Kluwer Academic Publisheis Dordrecht, Baston, London, 1993.

30. de Wit A. Gynecology - Costeffectweness. In: Banta HD and Schou I (Eds.). Lasers in Health Care. Effectiveness Cost-Effectiveness and Policy Implications. Academic Publishing. Frederiksberg, Denmark. 1991, p $201-3$. 
Part Three - Diffusion 


\title{
12 Diffusion of medical lasers in the Netherlands
}

\author{
Hindrik Vondeling", Henk Rosendaf', David Banta ${ }^{2}$
}

Vrije Universiteit Amsterdam

Department of Epidemiology and Biostatistics

Van der Boechorststraat 7

1081 BT Amsterdam, The Netherlands

2 The Netherlands Organization for Applied Scientific Research (TNO)

Division of Prevention and Health

P.O. Box 2215

2301 CE Leiden, The Netherlands

Originally published as: Diffusion of medical lasers in the Netherlands. In: Inzelt A, Coenen $R$ (Eds.). Knowledge, Technology Transfer and Foresight. NATO ASI Series, 4, Science and Technology Policy - Vol. 8, Kluwer Academic Publishers, Dordrecht, Boston, London, 1996, p. 7398. Reprinted with permission of the publisher.

\section{Summary}

Lasers represent a technological advance that has diffused in the Dutch health care system since 1972. Diffusion of medical technology is a complex phenomenon with many actors, such as physicians, manufacturers, governments, patients and so on. In general, diffusion processes are not well understood and there is no single explanatory model to elucidate these processes. The purpose of this study was to document and analyze the diffusion of medical lasers in the Netherlands. In 1989 all Dutch university hospitals $(n=8)$ and general hospitals $(n=139)$ were surveyed by telephone concerning their use of laser-technology. Because we anticipated rapid changes the survey was updated in late 1991. In both cases the response rate was $100 \%$. In late 1995, selected literature and expert opinion were used to update the main trends of the diffusion process. By the end of 1991 a total of 255 medical lasers had been installed, 64 in university hospitals and 191 in general hospitals. At least 5 lasers were operational in every university hospital, whereas in 106 out of 139 general hospitals $(76 \%)$ at least one laser had been installed. As in 1989, the Argon laser (105) was the most commonly used laser, followed by the Nd:YAG(56), the qs Nd:YAG- (32) and the $\mathrm{CO}_{2}$ laser (25). 157 Lasers were exclusively used in Ophthalmology. Other specialties that frequently used lasers are Gynaecology (22), ENT (17), Dermatology (16), Urology (15), Gastroenterology (14), Pulmonology (14) and Neurosurgery (13). A total of 16 medical specialties used lasers in clinical practice in 1991, compared to 15 in 1989. Sharing lasers among specialties was not very common in $1991(16 \%)$, and somewhat declined compared to $1989(20 \%)$. Of all lasers installed between 1989 and 1991 , only $10 \%$ was intended to be shared. The average use of lasers was not very high, about 7.5 hours a week. Only 16 lasers $(6 \%)$ were used at least 16 hours per week, 13 of these were applied in ophthalmology. Surprisingly, the average intensity of use of shared lasers, 6.4 hours a week, was somewhat lower than the average intensity of use. According to respondents in Dutch hospitals 20 hospitals planned to purchase a total of 28 lasers in 1992. The Nd:YAG laser was the most popular laser to invest in $(n=11)$ corresponding with $39 \%$ of the total investment plans.

We conclude that the rate of diffusion of medical lasers was rather low in the period 1972-1984. The diffusion rate increased in the period 1984 - 1991. Information collected in 1995 indicates that the diffusion rate has decreased, despite the growing importance of private clinics as a medical laser market. However, this needs to be differentiated between every individual laser system. For 
example, most new Argonlasers are purchased to replace old-ones, thus not increasing the tota: number of lasers used in clinical practice. This contrasts with investments in Nd:YAG- and other mostly new types of lasers which in most cases do not (yet) replace an old device. The Nd: YAG laser in particular has maintained its status as a popular device, particularly related to new applications, e.g. treatment of benign prositate hyperplasia (a urological condition), and its versatility. Associated with this, and contrasting with the laser field as a whole, sharing of this type of laser seems to have become more common.

Key-words: diffusion, medical lasers, the Netherlands

\subsection{Introduction}

Diffusion of technology refers to the process by which a technollogy enters and becomes part of the health care system. Most diffusion studies show that this process normally follows an S-shaped curve, representing the number of adopters over time (see Figure 12.1.1). The curve indicates that technology is subject to a "life cycle". The first stage is basic and applied research, which has uncertain outcomes. After the moment of innovation, that is, application to patients, the successful technology grows mature and finds its way into the health care system. At the end of the life cycle the market will be saturated or the technology becomes obsolete and will be replaced ${ }^{[3]}$.

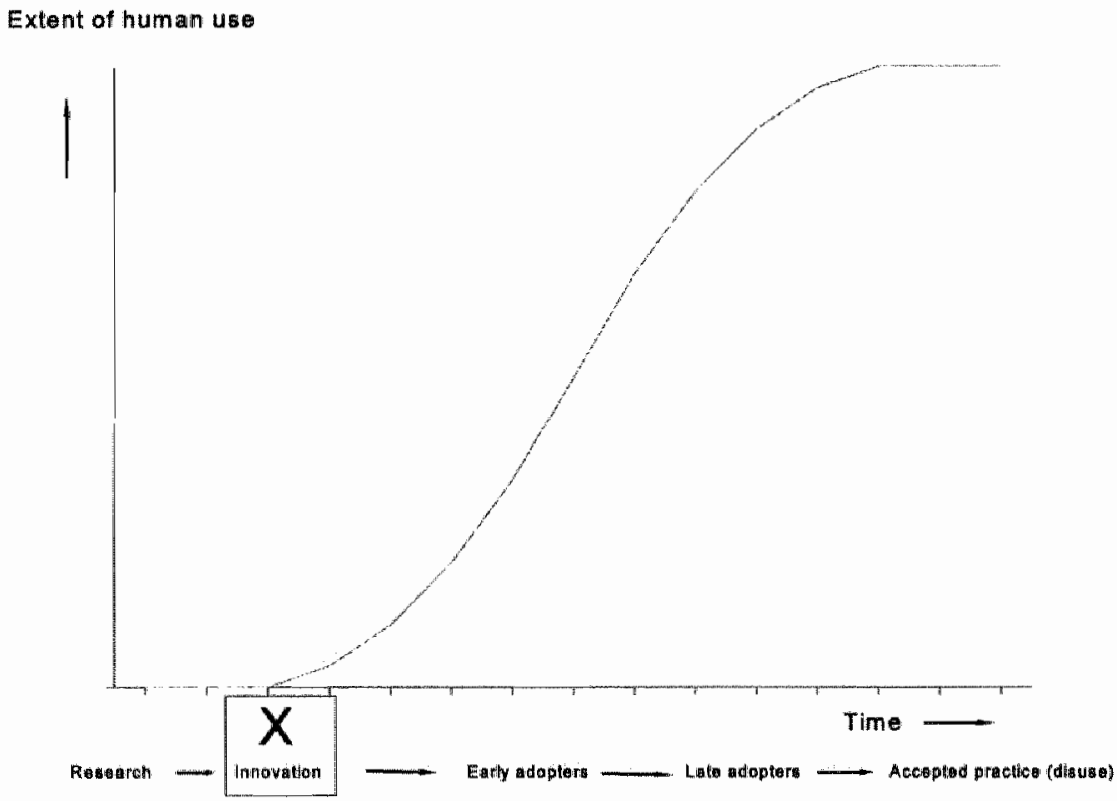

Figure 12.1.1 Development and diffusion of medical technologies (Source: ${ }^{[t]}$ )

The first laser was constructed in 1960. Two years later, the device was introduced in medicine in the US, followed by the Netherlands in the early seventies ${ }^{i 2 l}$. In 1989 the diffusion of lasers in Dutch hospitals was studied for the first time ${ }^{[15]}$. Because rapid changes were expected we updated this study in 1992. The methodology of the study is documented in paragraph 12.2. Results are presented in paragraph 12.3 , followed by conclusions and discussion in paragraph 12.4 . 


\subsection{Methodology}

The study was done by telephone surveys. This method was used because of the quick response and the relatively high percentage of answers, based on experience in the US, where diffusion research of CT-Scanners was carried out ${ }^{[1 !}$.

Informants for this study were, in contrast with the work on CT-scanners, hospital technicians only. The reason for this choice was that several sources were expected to give several kinds of information, and choosing one source would control information bias as much as possible. A second reason was that technicians are likely to be well informed about the presence of medical lasers, since laser installations require adaptations in rooms and, sometimes, buildings. These adaptations are carried out by hospital technicians most of the time. The third and last argument was a practical one: hospital technicians are relatively easy to contact.

All hospitals in the Netherlands were contacted. Just as in 1989 , there was a $100 \%$ response rate. In total there were 147 hospitals, including 8 university hospitals and 3 specialized hospitals. Two of these are cancer institutes and one center is specialized in treatment of patients with burns.

Questions concerned the number of lasers in the hospital, the types of lasers, the manufacturers, the year of installation, and utilization and investment intentions for the near future. For an example of the questionnaire used see Appendix 1.

Most participating hospital technicians were very cooperative. To check the correctness of their answers, $7 \%$ of the hospitals $(n=10)$ were chosen at random and the same questions were asked to laser-using physicians and representatives of the board. There appeared to be no difference in the information which was provided. We therefore assume that the information given by the technicians was correct. As in 1989 , a $100 \%$ response rate was achieved. Besides the choice of informant this may be attributed to the fact that the questionnaire contains a limited number of questions that are easy to answer. Obviously, the questions do not go into matters that are of strategic importance to hospitals.

Besides these hospitals 44 private clinics had been established in the Netherlands, of which 5 are so called "eye-clinics". These 5 clinics had in total 6 medical lasers in operation. These lasers were not included in this research. Low power lasers, although wide-spread in the Netherlands, e.g. in physiotherapy, were excluded from this study.

\subsection{Results}

Some general results will be presented first, followed by more detailed results.

\subsubsection{General results}

\section{Number of lasers}

The total number of medical lasers in Dutch hospitals was 255 in January 1992 (see Filgure 12.3.1). After a slow initial phase, diffusion has been rapid since the early 1980 s. 


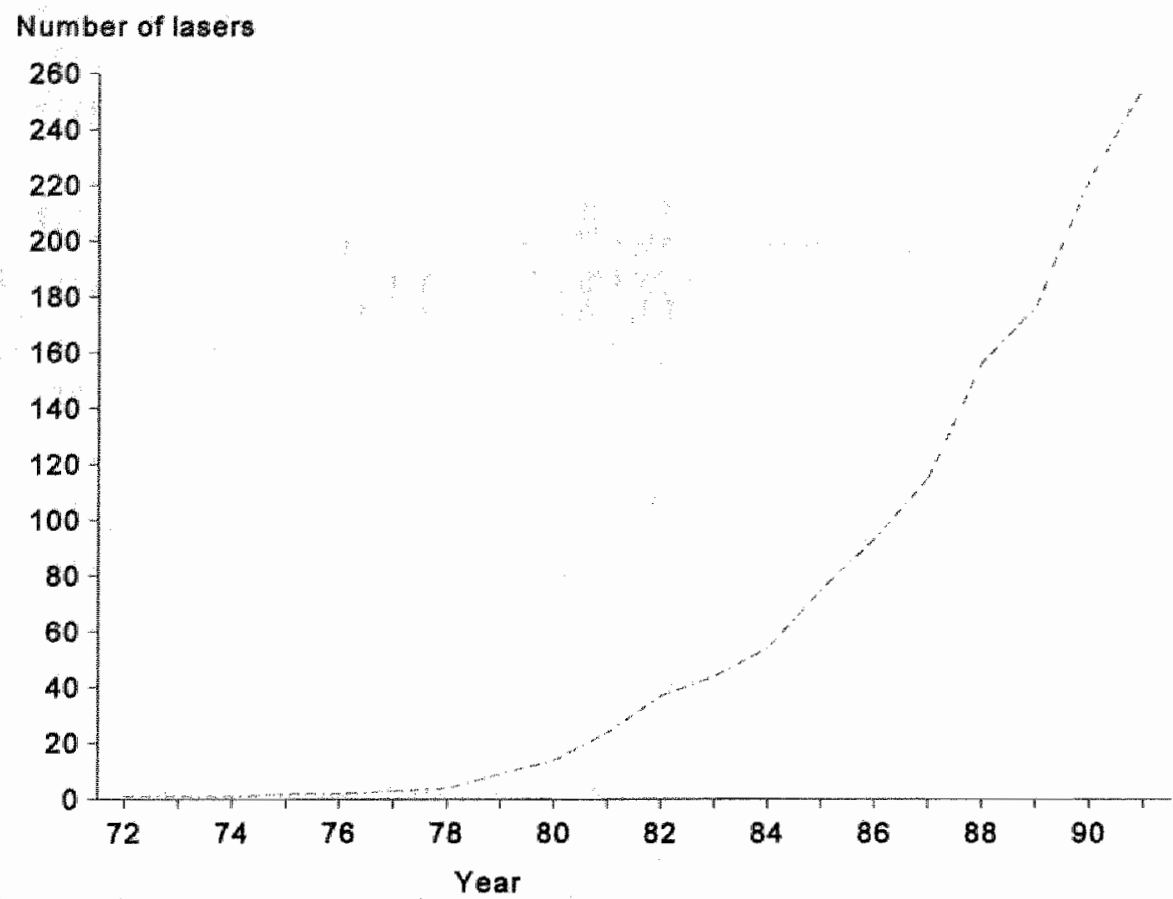

Figure 12.3.1 Diffusion of medical lasers in the Netherlands

Number of hospitals

Laser equipment was used in $78 \%(n=114)$ of Dutch hospitals. The increase in the number of hospitals that have adopted medical lasers in the period 1972-1992 is reflected in Figure 12.3.2. 


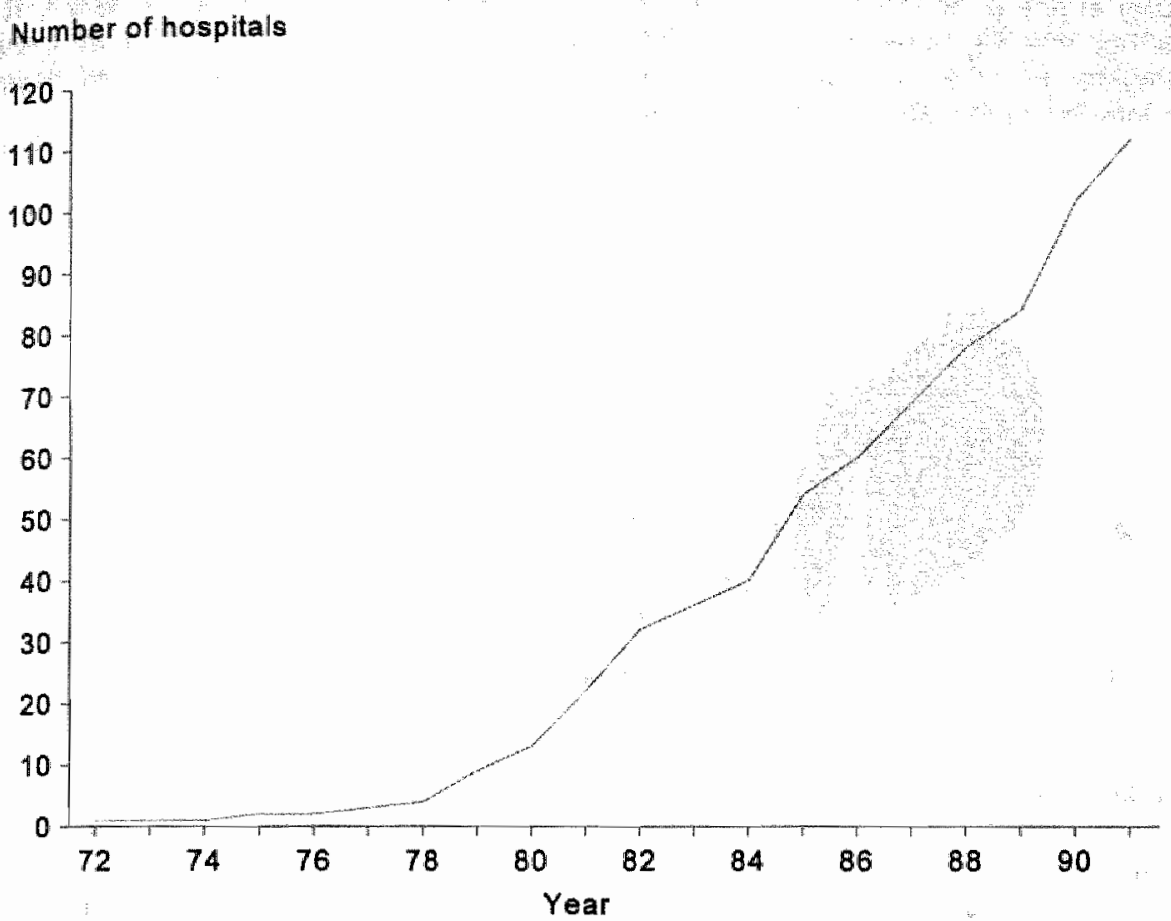

Figure 12.3.2 Number of hospitals with laser equipment

\section{Medical laser applications}

Medical lasers were used by 16 different specialties in the Netherlands. Table 12.3.1 lisis the specialties that employed at least ten lasers. About $64 \%(n=164)$ of all lasers were used in ophthalmology.

Table 12.3.1 Number of lasers per specialty

\begin{tabular}{lc|lc}
\hline \hline Specialty & No. of lasers & Specialty & No. of lasers \\
\hline Ophthalmology & 164 & Urology & 15 \\
Gynaecology & 22 & Pulmonology & 14 \\
ENT & 17 & Gastroenterology & 14 \\
Dermatology & 16 & Neurosurgery & 13 \\
\hline \hline
\end{tabular}

If a laser was shared by more specialties it was counted twice, or triple etc. Therefore the total number is more than 255 in this table. 


\section{Intensity of use}

The intensity of use of all medical lasers, in hours a week, is presented in Figure 12.3.3. The overall average intensity of use was about 7.5 hours per week. The figure shows that $80 \%$ of all lasers were used less than one day a week. Only 16 lasers $(6 \%)$ were used at least two days. week. It is interesting to note that 13 of these were ophthalmological lasers.

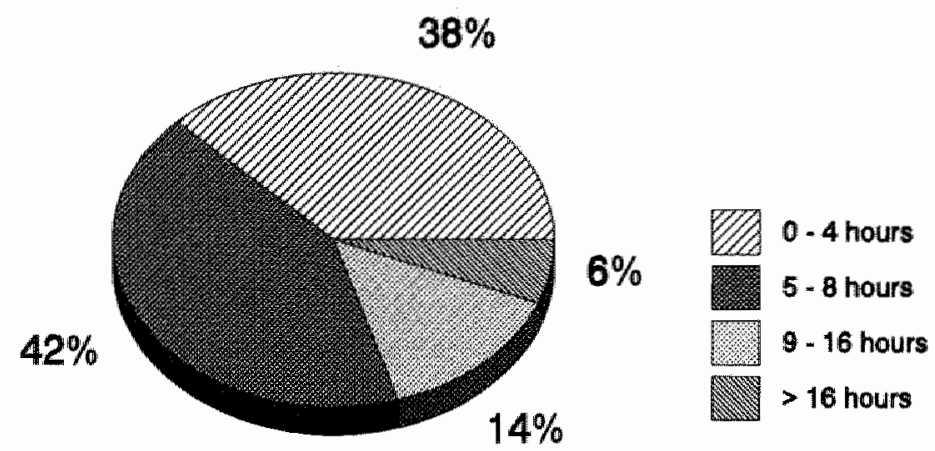

Figure 12.3.3 Intensity of use of medical lasers (hours a week)

\subsubsection{Specific results}

\section{Types of lasers}

The 255 medical lasers that were used in Dutch hospitals, embody 14 different types of lasers (see Table 12.3.2).

Table 12.3.2 Number of lasers by type of laser

\begin{tabular}{lcr}
\hline Type of laser & No. of lasers & Percentage \\
\hline Argon & 105 & 41.2 \\
Nd:YAG & 57 & 22.4 \\
qs Nd:YAG & 32 & 12.5 \\
CO. & 25 & 9.8 \\
Dye & 8 & 3.1 \\
Excimer & 3 & 1.2 \\
Copper-Vapour & 3 & 1.2 \\
Krypton & 2 & 0.8 \\
Erbium:YAG & 1 & 0.4 \\
Holmium:YAG & 1 & 0.4 \\
Diode & 1 & 0.4 \\
Argon/YAG & 8 & 3.1 \\
Argon/Dye & 7 & 2.7 \\
Argon/Krypton & 2 & 0.8 \\
\hline Total & 255 & 100.0 \\
\hline
\end{tabular}

Combined lasers, such as the Argon/VAG, Argon/Dye and Argon/Krypton laser, can be functionally used in two ways. For example, an Argon/YAG laser can be used as an Argon- and as a Nd:YAG laser. 
The four most commonly used types of lasers: the Argon, the $\mathrm{Nd:YAG}$, the qs Nd YAG and the $\mathrm{CO}_{2}$ laser, comprised more than $85 \%$ of all medical lasers in use in the Netherlands. Therefore, further results mainly focus on these four types of lasers. All other types of lasers are referred to as 'other types:.

Figure 12.3.4 presents the distribution of the four most important types of lasers.

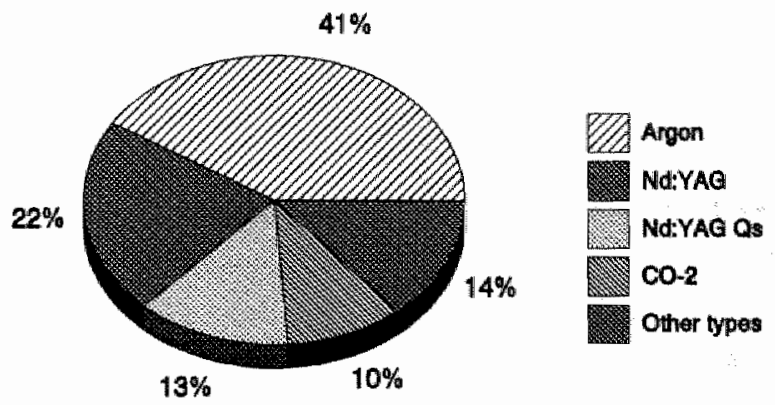

Figure 12.3.4 Distribution of four types of lasers

The diffusion of the four most commonly used types of lasers is shown in Figure 12.3.5. The Argon laser was not only the first to diffuse, but was also the most frequently found medical laser in 1992. The numbers of the other types had increased as well.

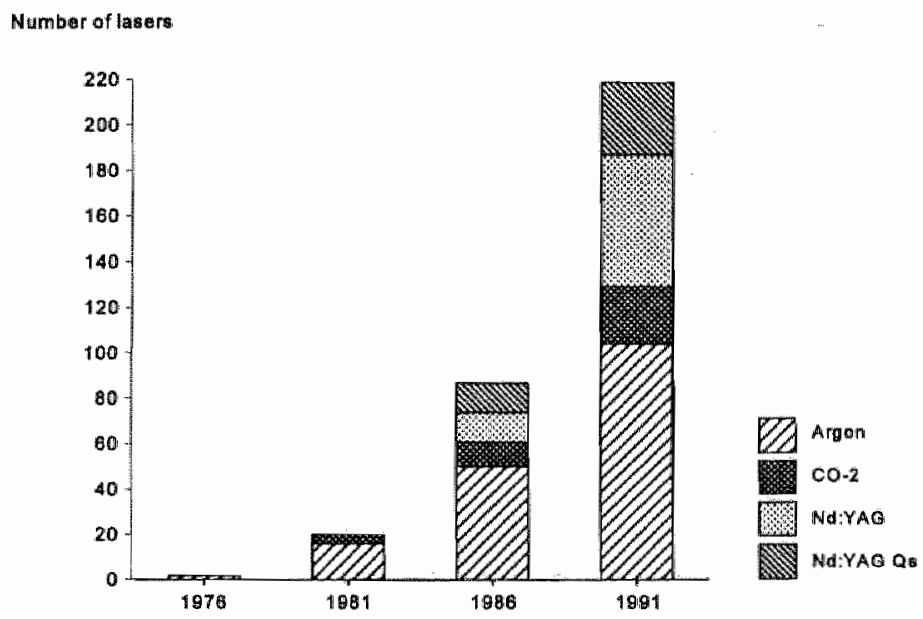

Figure 12.3.5 Diffusion of four types of lasers

\section{General hospitals and University hospitals}

In the Netherlands, the most important distinction between hospitals is whether or not they are university related. The diffusion pattern of lasers in both categories of hospitals is visualized in Figure 12.3.6. 


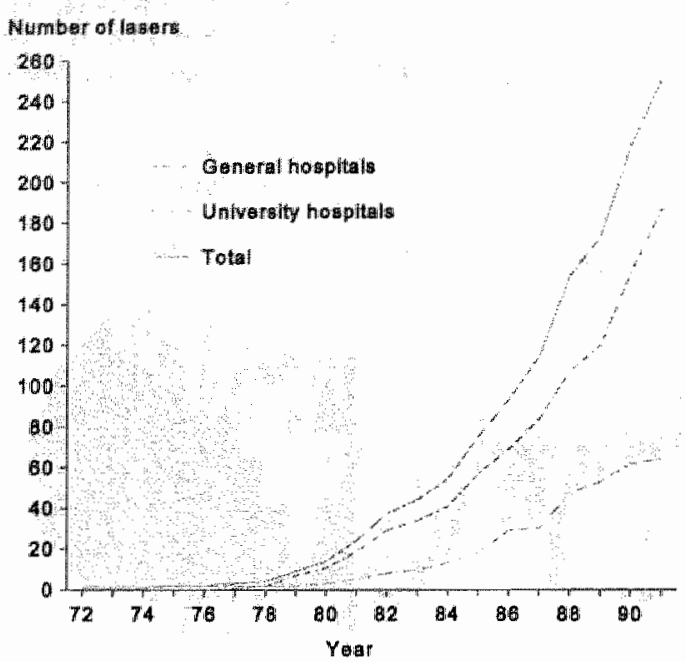

Figure 12.3.6 Diffusion of lasers by type of hospital

Although only $5 \%(n=8)$ of Dutch hospitals are university hospitals, they had installed about $25 \%$ $(n=64)$ of the total number of lasers. Figure 12.3.7 represents the beginning and speed of diffusion of lasers in each category of hospitals.

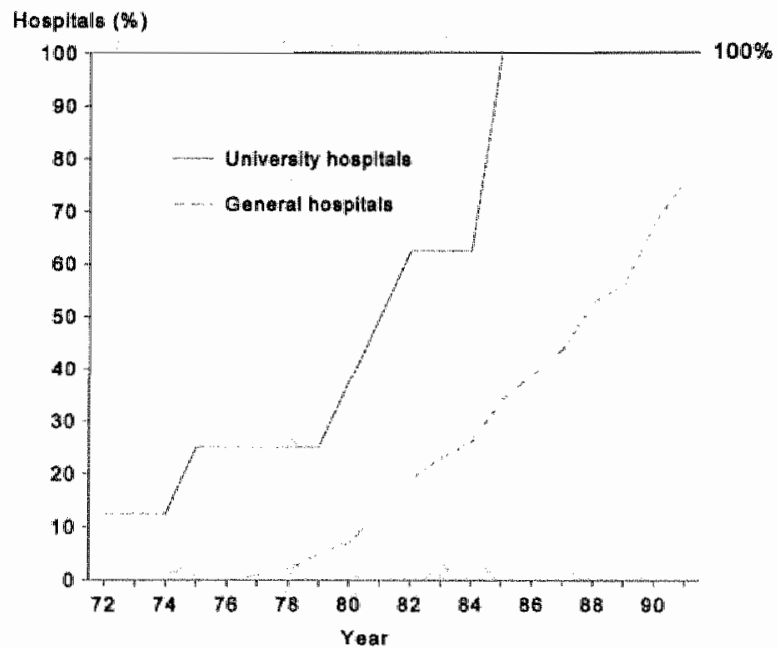

Figure 12.3.7 Diffusion of lasers by type of hospital (percent of hospitals)

Although the absolute number of lasers in general hospitals was higher than in university hospitals diffusion of medical lasers usually started in academic hospitals. Also, diffusion in academi hospitals was more rapid than in general hospitalls.

In Figure 12.3.8 - 12.3.15 the distinction between university hospitals and general hospitals $i$ combined with data documenting the absolute number and relative rate of diffusion of the four mos important types of lasers, the Argon, the Nd:YAG, the qs $\mathrm{Nd}: Y A G$ and the $\mathrm{CO}_{2}$ laser. On the lel 
the absolute number of each type of laser within the two categories of hospitals is visualized on the right side diffusion, by percentage of hospitals in each category is shown.

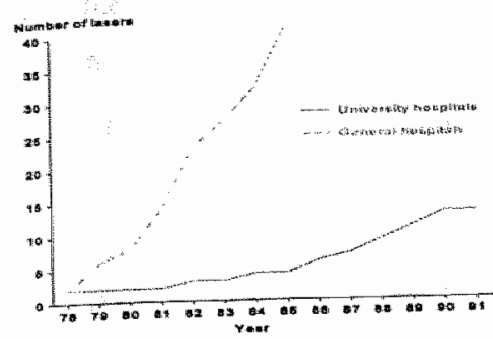

Figure 12:3.8 Diffusion of the argon laser

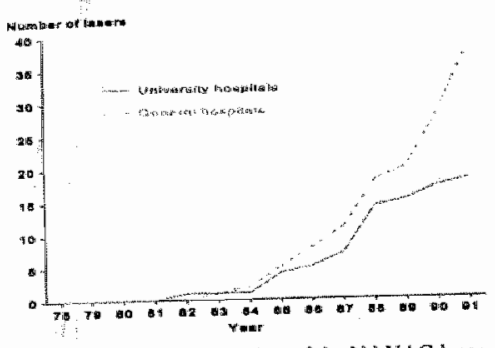

Figure 12.3.9 Diffusion of the Nd:YAG laser

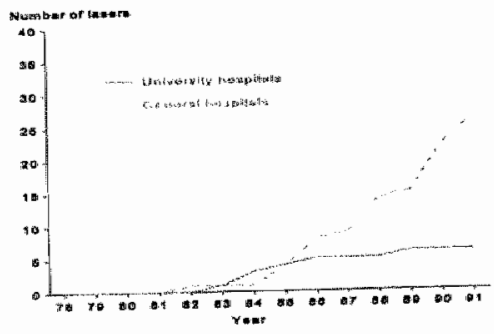

Figure 12.3.10 Diffusion of the qs Na:YAG laser

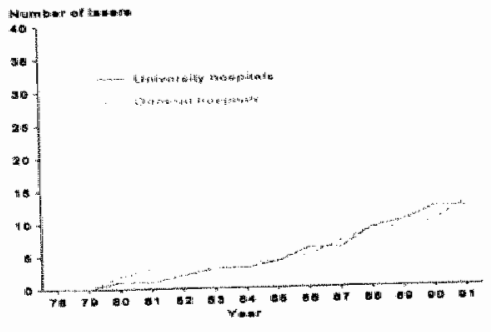

Figure 12.3.11 Diffusion of the 60, laser

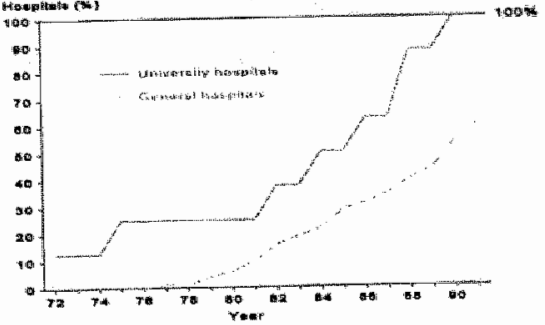

Figure 12.3.12. Diffusion of the argon laser

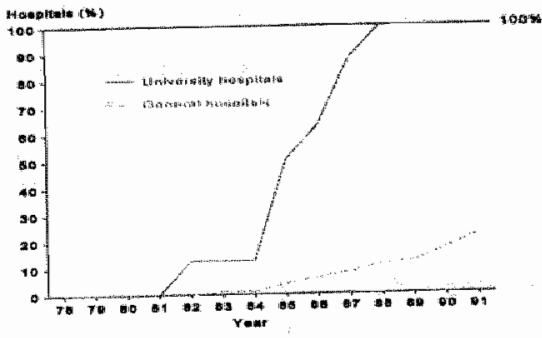

Figure 12.3.13 Diffusion of the Nd: YAG laser

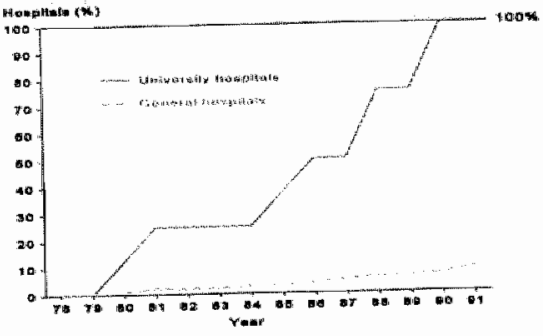

Figure 12.3.14 Diffusion of the $\mathrm{CO}_{2}$ laser

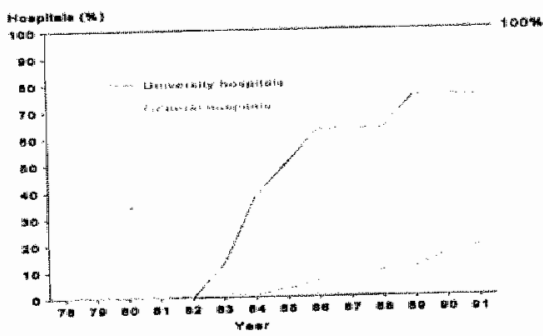

Figure J2.3.15 Difrusion of the qIS Fd: YAG laseer

case of the Argon and the Nd:YAG laser diffusion began in university hospitals. Diffusion of the $\mathrm{O}_{2}$ laser started synchronically in both types of hospitals whereas the qs Nd:YAG laser was dopted firstly by a general hospital. Initially, the number of lasers increased rapidly in university ospitals. However, in the course of time the total number of lasers in general hospitals outweighed 
Table 12.3.3 Differences in the diffusion patterns of four types of lasers

\begin{tabular}{|c|c|c|c|c|}
\hline Type of laser & $\begin{array}{l}\text { Type of } \\
\text { hospitall }\end{array}$ & $\begin{array}{l}\text { First year } \\
\text { of diffusion. }\end{array}$ & $\begin{array}{c}\% \text { of saturation } \\
(1992) \\
\end{array}$ & $\begin{array}{l}\text { Speed of } \\
\text { saturation (\%/year) }\end{array}$ \\
\hline \multirow{2}{*}{ Argon } & University & 1972 & 100 & 5,8 \\
\hline & General & 1976 & 60 & 3,8 \\
\hline \multirow{2}{*}{ Nd:YAG } & University & 1981 & 100 & 14,3 \\
\hline & General & 1982 & 22 & 2,2 \\
\hline \multirow{2}{*}{ qs Nd:YAG } & University & 1982 & 75 & 7,5 \\
\hline & General & 1981 & 19 & 1,9 \\
\hline \multirow[b]{2}{*}{$\mathrm{CO}_{z}$} & University & 1979 & 100 & 10 \\
\hline & General & 1979 & 9 & 0,8 \\
\hline
\end{tabular}

The table shows that every university hospital had at least one Argon, $\mathrm{Nd}: \mathrm{YAG}$ and $\mathrm{CO}_{2}$ laser. This contrasted with the situation in general hospitals, where only Argon lasers could be found in the majority of cases.

\section{Geographic diffusion}

The diffusion pattern of each type of laser can be visualized geographically (see Figure 12.3.16. 19). All top-left images show the location of the first two hospitals that installed a particular type of laser. Combined lasers, such as Argon-YAG and Argon-Dye lasers, are not included in these figures. 

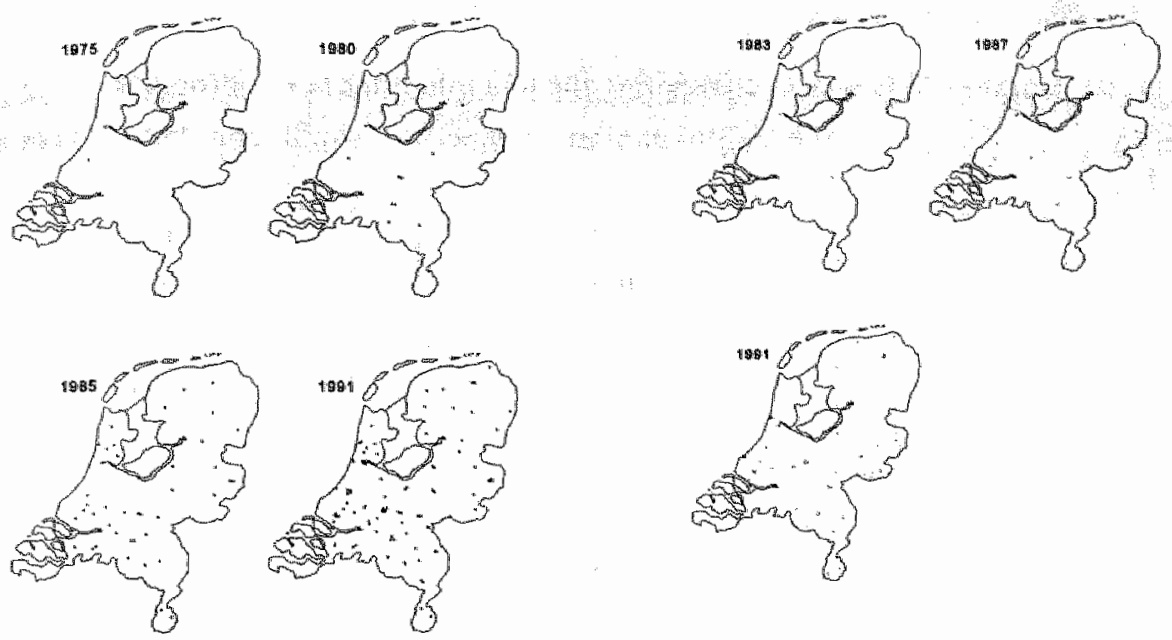

Figure 12.316. Geagraphical pattern of diffusion of the argon laser
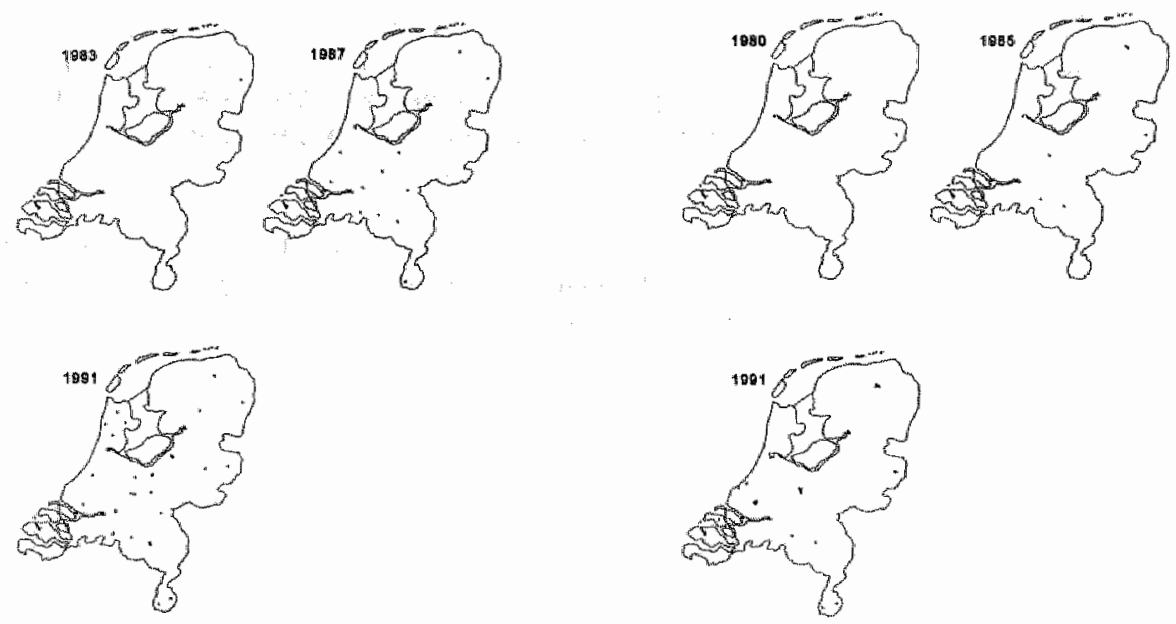

Figure 12.3.18 Geographical patten of diffusion of the Q⿱宀 Nd: YAG lasen"

Figne 12.3.19 Geographical pattern of diflusibon of the $\mathrm{CO}_{\mathfrak{p}}$ laser

The figures show that one of the first two installed lasers of every type was always located in the western part of the country, in particular in Amsterdam. Subsequently the device appeared in more peripheral areas. In addition "the figures show that the pattern of diffusion of these types of lasers has been rather irreguliar.

\section{Linking the diffusion of lasers to provinces and health regions in the Netherlands}

The Netherlands comprise twelve provinces with about 15.5 million inhabitants in 1995 . The western and southern part of the country are most densely populated. The number of ophthalmological and non-ophthalmological lasers that were available per 100,000 inhabitants per 
prowince in 1992 is shown in Table 12.3.4. At that time the number of Dutch citizens amounte almost 15 million people.

Table 12.3.4 Number of lasers avallable per 100,000 inhabitants per province

\begin{tabular}{lcc}
\hline \hline Province & non-ophthalmological lasers & ophthalmological lasers \\
\hline Groningen & 1,6 & 1,3 \\
Friesland & 0,0 & 1,3 \\
Drenthe & 0,0 & 0,9 \\
Overijssel & 0,5 & 1,1 \\
Gelderland & 0,2 & 1,1 \\
Utrecht & 1,7 & 1,6 \\
Noord-Holland & 0,7 & 1,1 \\
Zuid-Holland & 0,6 & 1,0 \\
Zeeland & 0,3 & 0,8 \\
Noord-Brabant & 0,4 & 1,2 \\
Limburg & 0,5 & 1,1 \\
Flevoland & 0,0 & 0,5 \\
\hline
\end{tabular}

One can see that with the exception of Flevoland, the availability of ophthalmological lasers was fairly constant. However, there were marked differences per province in the availability of nom. ophthalmological lasers. Inhabitants of the northern provinces Friesland and Drenthe and inhabitants of Flevoland, a province in the central part of the country, had to travel to othel provinces to receive any non-ophthalmological laser treatment.

In the context of the Hospital Provisions Act, the Netherlands have been divided in 27 'health regions". These regions serve as basic units for planning hospital facilities. In order to create a link with health policy-making it seems pertinent to provide a picture of the diffusion of medical lasers at this level of aggregation. These data are visualized in Figure 12.3.20. 

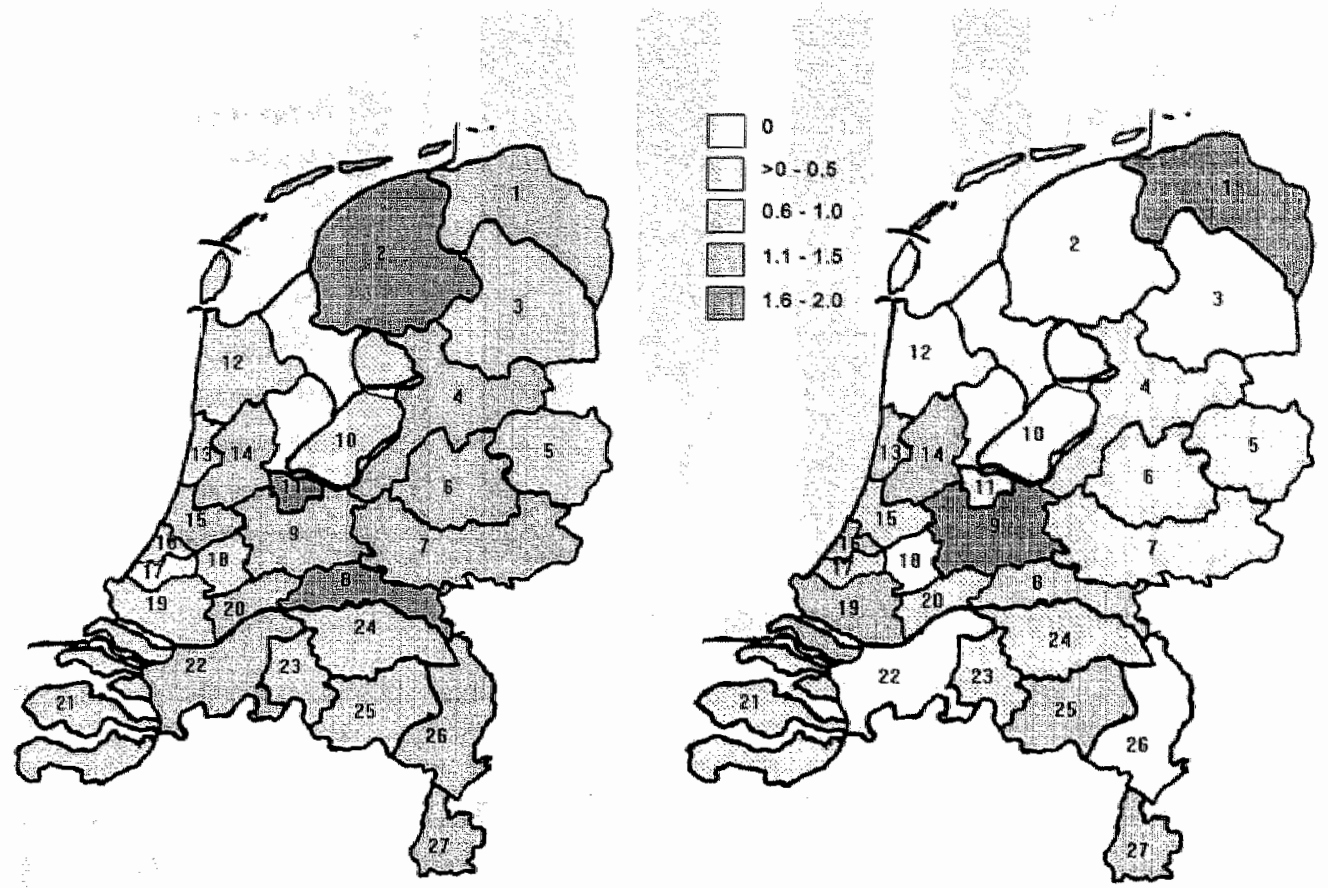

Figure 12.3.20 Diffusion of ophthalmological lasers (left) and non-ophthalmological lasers (right) in health regions in the Netherlands (number of lasers per 100,000 population per health region)

The availability of ophthalmological lasers varied between 0,3 and 1,7 per 100,000 population per region. These differences were minor compared to those in non-ophthalmologicall lasers. In eight health regions $(29.6 \%)$ patients had to travell to another region to receive non-ophthalmological laser treatment. Other health regions had almost two non-ophthalmologicall lasers per 100,000 population. As non-ophthalmological lasers entered the health care system more recently and because diffusion often started in university hospitals, these findings can be related to the distribution of university hospitals over health regions. University hospitals can be found in region 1 (Groningen), 8 (Nijmegen), 9 (Utrecht), 14 (Amsterdam, $n=2$ ), 15 (Leiden), 19 (Rijnmond) and 27 (Zuid-Limburg). With the exception of Leiden and Zuid-Limburg, the avallability of nonophthalmological lasers in these regions was higher than in any of the regions without university hospitals.

\section{The users of the 4 types of lasers}

The 4 most frequently found types af lasers were used in several specialties. The distribution of these types of lasers across the eight specialties that used lasers most frequently, is represented in Figure 12.3.21. Argon lasers and, espechally, qs Nd:YAG lasers were used mainly in ophthalmology. whereas the $\mathrm{CO}_{2}$ laser and the $\mathrm{Nd}$ Y YAG laser were employed in a variety of specialties. 


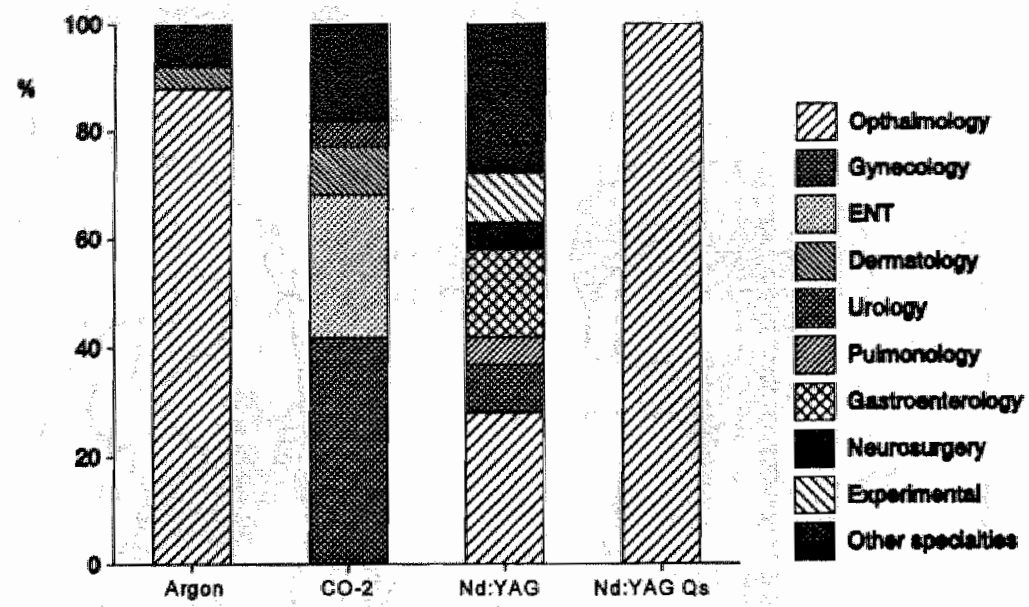

Figure 12.3.21 Number of specialties (\%) per type of laser

A mirror image of Figure 12.3.21 can be constructed if not the laser but the specialty is used as the starting point of the analysis.

Table 12.3.5 Most used types of lasers by specialty $(n, \%)$

\begin{tabular}{lllllll}
\hline \hline Specialty & Most used type & & $\%$ & Second most used type & $\%$ \\
\hline Ophthalmology & Argon & 94 & 58 & qs:YAG & 32 & 20 \\
Gynaecology & $\mathrm{CO}_{2}$ & 15 & 68 & $\mathrm{Nd}: Y \mathrm{AG}$ & 5 & 23 \\
ENT & $\mathrm{CO}_{2}$ & 12 & 71 & $\mathrm{Nd}: Y A G$ & 3 & 18 \\
Dermatology & $\mathrm{Argon}$ & 6 & 38 & $\mathrm{Dye} / \mathrm{CO}_{2}$ & 3 & 19 \\
Urology & $\mathrm{Nd}: Y A G$ & 11 & 73 & $\mathrm{CO}_{2}$ & 2 & 13 \\
Pulmonology & $\mathrm{Nd}: Y A G$ & 9 & 64 & $\mathrm{CO}_{2}$ & 3 & 21 \\
Gastroenterology & $\mathrm{Nd}: Y A G$ & 14 & 100 & - & & \\
Neurosurgery & $\mathrm{Nd:YAG}$ & 10 & 77 & $\mathrm{CO}_{2}$ & 4 & 23 \\
\hline \hline
\end{tabular}

Table 12.3 .5 shows that almost every specialty employed more than one type of laser. The $\mathrm{Nd}: Y A G$ laser was the most used type of laser in four specialties, whereas both the Argon- and $\mathrm{CO}_{2}$ laser were the most used in two specialties.

\section{Sharing of laser equipment}

Some types of lasers were shared by more specialties, whereas other types were used by only one specialty. The extent to which types of lasers were shared is presented in Figure 12.3.22. 


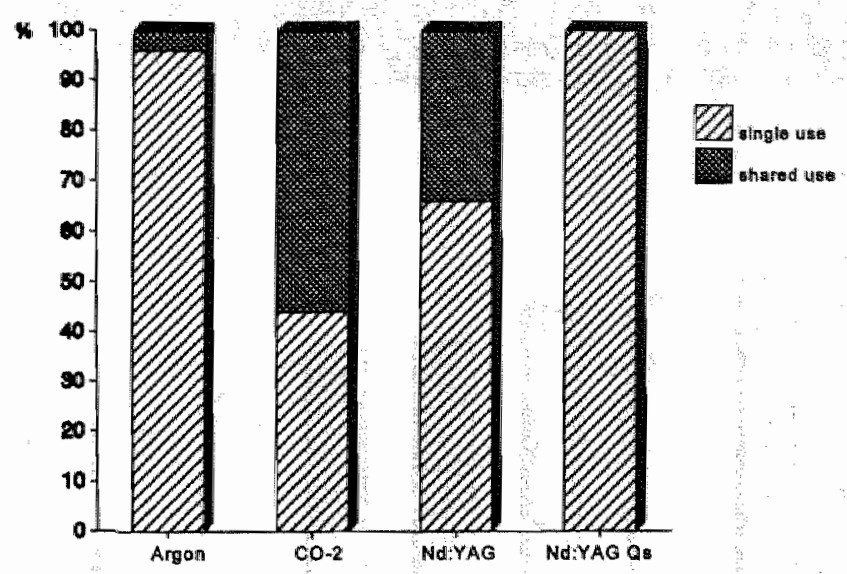

Figure 12.3.22 Percentage of shared lasers by type of laser

It appears that the Argon laser, and especially, the qs Nd:YAG laser were hardly ever shared by other specialties. This contrasts with the Nd:YAG and especially the $\mathrm{CO}_{2}$ laser, which were shared more often than they were purchased for single use only.

\section{Laser sharing specialties}

The percentage of 'sharing-specialists' in each specialty is presented in Figure 12.3.23. The Figure shows that sharing of lasers was uncommon in ophthalmology and, to a lesser extent, in dermatology. It should be noticed that shared devices are counted twice or three times, depending on the number of specialties sharing the laser. Therefore, the actual percentage of laser sharing specialties was lower than presented here.

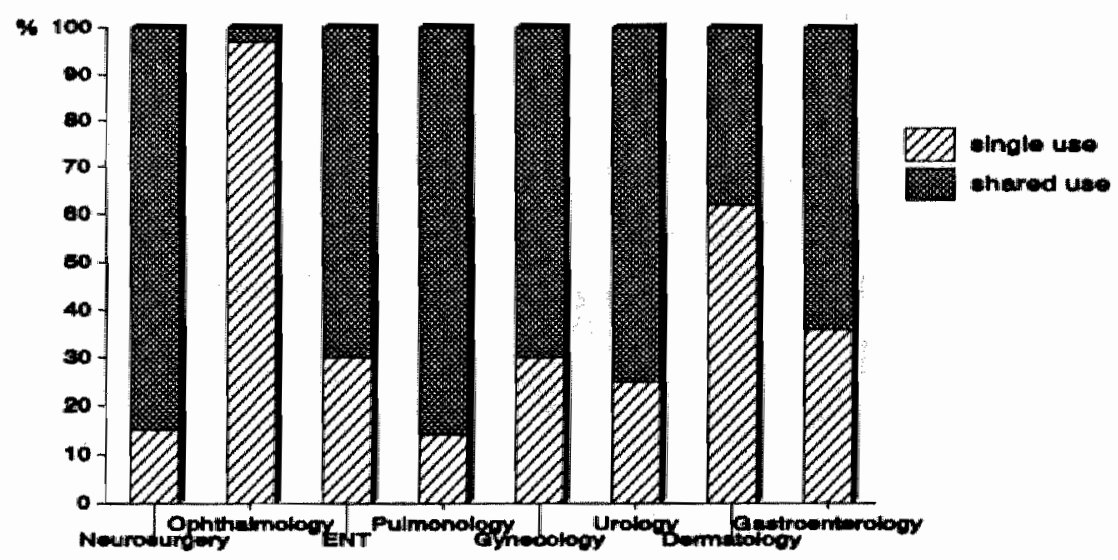

Figure 12.3.23 Percentage of individual departments per specialty that share lasers 


\section{Intensity of use}

The untensity of use of medical lasers is described in Figure 12.3.24. This intensity, however, was not simillar among all specialties, as presented in this Figure.

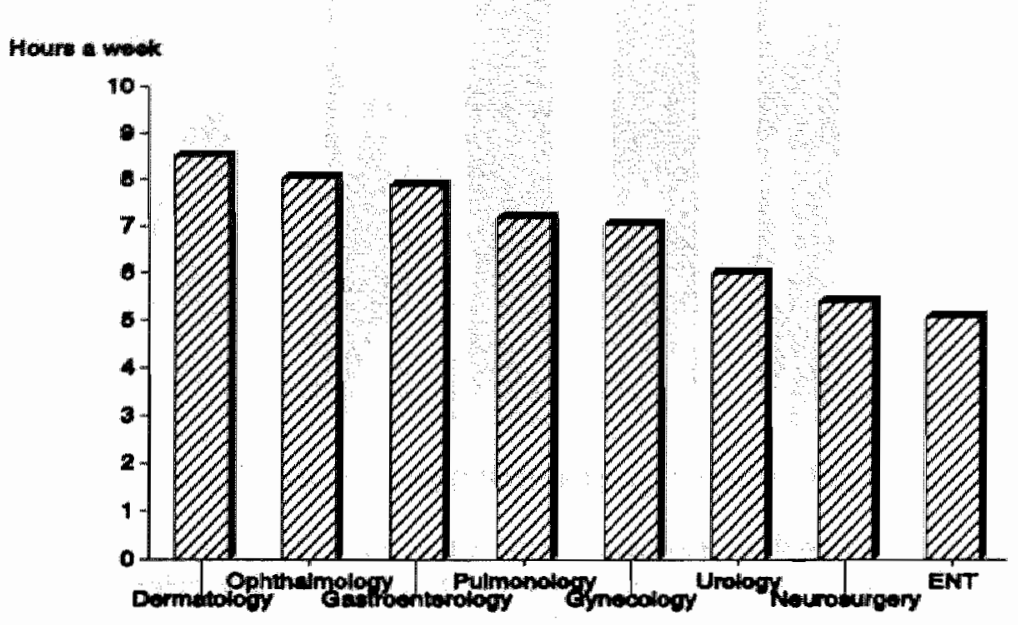

Figure 12.3.24 Intensity of use of lasers by specialty

The average intensity of use of lasers varied from a little more than 5 hours per week in ENT to almost 9 hours per week in dermatology.

\section{Investment plans}

In Figure 12.3.25 the percentage of investment-intentions for each type of laser are visualized. 20 Hospitals planned to invest in laser equipment in 1992, comprising a total of 28 new lasers. The: Nd:YAG laser was the first choice for investment in a laser $(n=12)$, whereas intentions to purchase Argon lasers declined $(n=3)$.
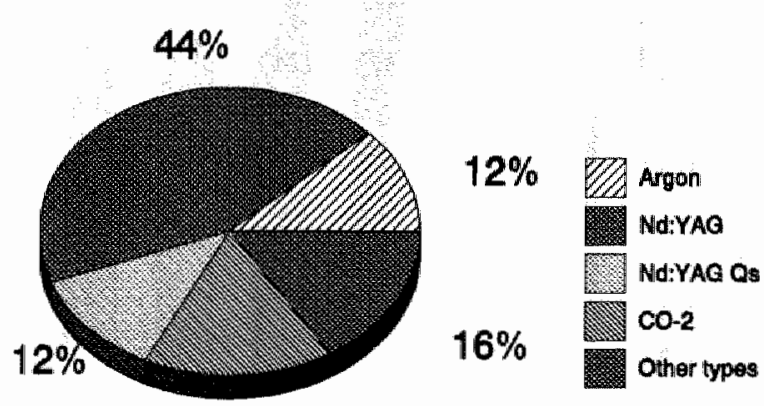

$16 \%$

Figure 12.3.25 Investment plans per type of laser (\%) in 1992

\section{Diffusion trend}

The number of medical lasers in The Netherlands was still rising in the early nineties. However, the relative importance of some types of lasers was subject to change. This is presented in Table 12.3 .6 
Table 12.3 .6 Investments in new medical lasers in 1992 compared to $19910 \%$

\begin{tabular}{lcc}
\hline Type of laser & $1991(\%)$ & $1992(\%)$ \\
\hline Argon & 41 & 12 \\
Nd:YAG & 22 & 44 \\
qs Nd:YAG & 13 & 12 \\
CO & 10 & 16 \\
Other types & 14 & 16 \\
Total & 100 & 100 \\
\hline
\end{tabular}

Compared to the distribution of types of lasers in 1991, the relative importance of the Nd:YAG laser .

In the previous sections a distinction was made between general and university hospitals. Purchases in academic and general hospitals are shown in Figure 12.3.26.

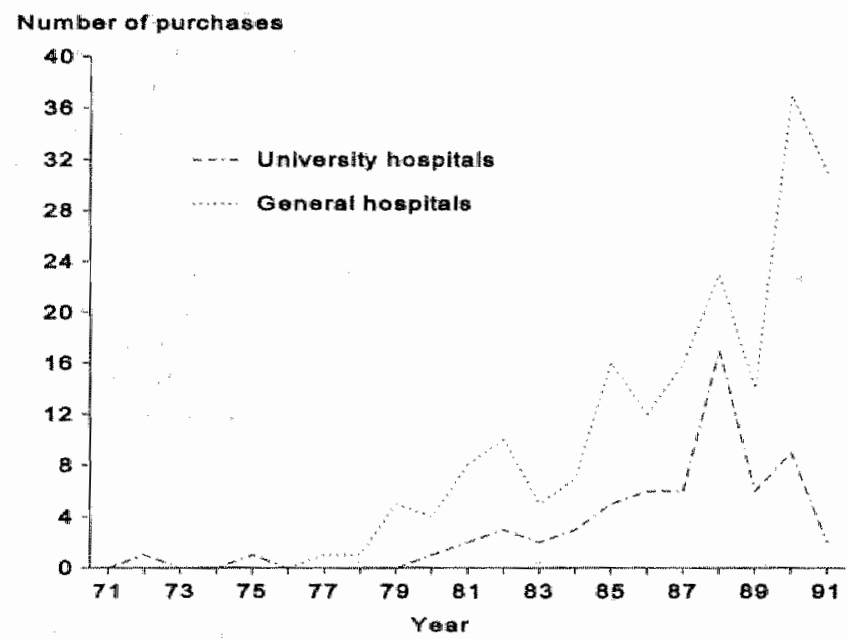

Figure 12.3.26. Number of purchases in academic and general hospitals

The figure shows that the number of purchases in genera! hospitals reached a peak of almost 40 in 1989 . In late 1991 the number of purchases was still high. The number of purchases in academic hospitals reached a peak in 1989, when 17 lasers were bought. In the two subsequent years the number of lasers that were bought in this category of hospitals decreased rapidiy to only wo in 1991.

\section{An international comparison}

In 1990, the number of lasers in Germany "excluding soft-lasers, was estimated to be $1352^{\text {i8l }}$. The Nd:YAG laser had diffused most widely in the German health care system, with 500 systems installed $(37 \%)$. Four hundred-seventy five argon lasers had been installed (35\%), of which 450 were used in ophthalmology. $\mathrm{CO}_{2}$ lasers ranked third in this list, with 200 systems installed (15\%). One hundred-twenty qs Nd:YAG lasers were used in ophthalmology (9\%). Of all other types of lasers, no more than 15 had been installed. The potential laser market was estimated at 100,000 lasers, indicating that the total number of installed lasers represented only $1.3 \%$ of the uitimate 
market The estimation of the potentiall size of the Geman laser market may have been targely overestimated Compared to the Netherlands, the Nd:YAG laser holds a relatively strong position: in Germany, which can be tentatively explained by the presence of both laser manufacturers and medical innovators who promoted the use of Nd:YAG lasers. Perhaps an important opinion leader has been Prof Hofstetter, a pioneer in the field of Nd:YAG laser applications in urology. In the Netherlands, opinion leaders have not have played such a prominent role in the diffusion process

As part of the European Community Concerted Action program on the development of medical laser applications, the Working Group on Laser Safety produced its final report in late $1994^{[137]}$. As part of its activities the Working Group had collected information on the diffusion of lasers in Europe, ncluding the frequency of use. To abtain information on this subject (and other, safety. related subjects), a questionnaire was sent to about 6000 hospitals in Germany, Spain. France, the United Kingdom, Ireland and the Netherlands in the period March 1990-May 1991. A second questionnaire was aimed at laser-using physicians in each hospital. Response was obtained from 845 hospitals $(14.1 \%)$, including 586 hospitals that actually used lasers $(9.8 \%$ of total). In these hospitals a response was obtained from 1042 physicians. The results show that about half of the lasers had been installed in hospitals of less than 500 beds. In the vast majority of hospitals one to three lasers were avallable. Ten lasers or more were available exclusively in a small percentage of those hospitals with 500 beds or more. Most frequently found were the argon laser, the Nd:YAG laser and the $\mathrm{CO}_{2}$ laser. The argon laser was used in more than $90 \%$ of the ophthalmological departments. In all other specialties argon lasers were available in less than half of all departments. The Nd:YAG laser was used in at least $75 \%$ of cases in gastroenterology, pulmonology and (cardio)vascular surgery. The $\mathrm{CO}_{2}$ laser was used in at least $75 \%$ of departments of gynecology. ENT and plastic surgery. Of the "new' types of lasers the dye laser was most frequently used. This laser had diffused to a wide variety of specialties as well.

The ophthalmologists in the survey reported a total number of more than 50,000 laser treatments in 4989. In both gynecology, gastroenterology, ENT, and dermatology between 10,000 and 20,000 treatments were reported. In other specialties less than 10000 treatments were reported. In all specialties except pulmonology the number of treatments increased in the period 1988-1989. Likewise, in all countries except Spain the total number of treatments had increased in this period.

More than half of the hospitals over 500 beds had investment plans for new lasers in the near future, compared with a percentage that increased from about $20 \%$ in hospitals of less than 100 beds to $30 \%$ in hospitals with 200 to 500 beds. The frequency of investment plans differed per country as well. In Spain. Italy and the Netherlands, more than $70 \%$ of the responding hospitals answered positively to the question on investment plans "compared with less than half of the responders in the remaining countries. In interpreting these results it should be noticed that nonresponse was extremely high and that the sample was biased to one country. France, as more than half of the hospitals responding and about $40 \%$ of all physicians responding were French. As nearly $40 \%$ of all physicians responding were ophthalmologists it is not surprising that the results indicate that ophthalmology is the most important laser-using specialty. A finding of more interest is that the presence of lasers is positively correlated with the size of a hospital. The level of investment in new lasers in the Netherlands may have been high in 1991 compared to other Western-European countries. The remainder of the reported trends are generally similar to those in the Netherlands.

It has been concluded that lasers have diffused sllowly in the Dutch health care system in the seventies, and more rapidly in the eighties. The Netherlands is not the only country where the diffusion of lasers exhibits this pattern. In Australia the number of lasers (excluding low power lasers) increased from 64 in 1983 to 289 in $1991^{[4]}$. Especially the early eighties have shown a large increase in the number of lasers in this country. In 1984, 84 lasers had been installed compared with 63 in 1983, representing an increase of $33 \%$. More than half of these lasers were used in ophthalmology, including about 40 argon lasers. Dermatology and gynecology were the only specialties where at least 10 lasers were used in 1984. Of all lasers installed, more than half could 
be found in one province, New South Wales. In this province, most lasers were installed in Sidney, the capital of the country ${ }^{[19]}$

In 1991,111 of the total of 289 lasers were used in ophthalmology (about $38 \%$ ), indicating that the role of ophthalmology in the overall diffusion process of lasers had declined somewhat compared 101984 A similar trend was described for the Netherlands. Although this was not specifled, the wast majority of ophthalmological lasers may still have been argon lasers. In non-ophthaimologica specialties, the $\mathrm{CO}_{2}$ laser had diffused most widely, with 79 systems in use. The majority of these fasers were used in gynecology. The Nd:YAG ranked third in this list with 32 systems, used in specialties including gastroenterology, urology, pulmonology and neurosurgery. All other types of lasers numbered less than five.

To correctly interpret these data it should be noticed that in the 1991 report by Dankiw et all. ${ }^{[4]}$ no data were provided on the response rate nor on the total number of hospitals and private clinics in Australia. This is important because the priwate sector has played an important role in the diffusion of lasers in Australia. For example, in early 1987 four gynecological lasers were in use in public hospitals, four were located in private clinics, and four were believed to be in use in private offices $^{[4]}$. In 1991, 60 ophthalmological lasers were used in specialists' rooms and other nonhospital settings, compared to 51 lasers in hospltals. Also in 1991, 25 lasers were reported to be in use in private clinics specializing in dermatological treatment, compared to 8 systems in hospitals ${ }^{[9]}$. In the Netherlands too, private clinics have become moie important in the diffusion process of lasers compared to regular hospitals. In the Netherlands, the role of private clinics became more important in the nineties, a decade later than in Australia.

The Australians considered the use of medical lasers in their country to be at a low level relative to other countries, as reported in $1991^{11}$. This was attributed in part to the isolation of the country and an, according to the authors, understandable lack of focus from manufacturers due to the relatively small, dispersed population. Although Australia is a continent and the Netherlands a very small country, it is striking that so many similarities in the diffusion of medical lasers can be pointed at.

Diffusion of lasers in Australia was achieved via enthusiastic cliniclans who encouraged institutions to purchase these devices. This often resulted in the purchase of inappropriate systems or the lack of consideration of other potentialusers. Unpublished research in the Netherlands has shown that physicians in this country too have been (and still may be) the most important actors in decisionmaking on the adoption of medical lasers ${ }^{|t 2|}$. Anecdotes tell that in exceptional cases physicians did not know how and why to use lasers, resulting in a situation where in a university hospital a new laser was not even unwrapped and left to dust in a storage.

So far, the comparisons have only inciuded total numbers of lasers and total numbers of lasers per specialty per country. Another comparison that can be made is that of intensity of use of these devices. In the United States, ECRI found in a 1991 survey of hospitals with general-purpose surgical lasers that, on average, these devices were used in less than $5 \%$ of total surgical procedures $^{[3]}$. This may correspond well to the findings in the Netheriands.

\subsection{Conclusions and discussion}

\subsubsection{Conclusions}

\section{General conclusions}

The first conclusion that can be drawn is that the number of medical lasers has increased in the Netherlands, predominantly in the eighties. Secondly, ophthalmology holds a unique position in the adoption and use of medical lasers. Over $2 / 3$ of all lasers are used in this specialty. Thirdly. of all different types of lasers, the Argon laser is by far the most frequently found. It is not surprising that this laser is mainly used in ophthalmology. A final general conclusion relates to the overall intensity of use of lasers, which is less than one day a week. In our opinion this is rather low. 


\section{Detalled conclusions}

Four types of laser appear to be most important in the Netherlands. Besides the argon laser these are the $\mathrm{Nd}$ Y YAG., the qs $\mathrm{Nd}$ Y $\mathrm{AG}$ - and the $\mathrm{CO}_{2}$ laser. These four types comprise more than $85 \%$ of all medical lasers avallable in the Netherlands. When adding combined lasers this percentage even rises to $92.5 \%$. Of these types, the Argon laser was the first to diffuse in the Netherlands, and It still is the most frequently found type of laser. At the same time the numbers of other types, especially the NdYAG laser, have also increased markedly.

Although only $5 \%$ of the Dutch hospitals are university hospitals, they have about $25 \%$ of all lasers at their disposal. Diffusion of medical lasers often starts in university thospitals. Therefore, these hospitals are important actors in the process of diffusion of medical lasers.

Considering the avallability of medical lasers to Dutch citizens, some inequality calls for attention. Especially differences in the avalability of non-ophthalmological lasers are striking in about $30 \%$ of health regions people have to travel outside their own region to receive any non. ophthalmological laser treatment.

$16 \%$ of all lasers $(n=41)$ are shared between specialties. If sharing occurs, most often Nd:YAG or $\mathrm{CO}_{2}$ lasers are involved. Argon lasers are hardly ever shared, and sharing never occurs in case of qs Nd:YAG lasers. These lasers are used predominantly and exclusively in ophthalmology. respectively. We therefore conclude that sharing of lasers is rather uncommon, primarily due to the absence of sharing ophthalmological lasers. Laser applications in ophthalmology requires specific ancillary equipment in a fixed formation, reducing the possibilities for sharing.

\subsubsection{Discussion}

\section{General pattern of diffusion}

A general pattern of diffusion of laser technology thas been constructed based on data of all types of lasers that have been introduced in the Netherlands in the 70 s and 80 s. Diffusion usually started in university hospitals, after some years followed by gieneral hospitals. In time the absolute number of each type of laser in general hospitals exceeded the number of all university hospitals. Considering this course of events, diffusion of lasers in university hospitals seems to have some predictive value for the diffusion process in general hospitals. Of all university hospitalls, those in Amsterdam have often been the first to adopt a new type of laser. Therefore, these hospitals are especially important actors in the process of diffusion of laser technology. It should be noted that Amsterdam is the only city that hosts two university hospitals. In addition, the National Cancer Hospital is located there as well. The concentration of innovative potential may serve as an explanation for the historical prominent position of this health region in the diffusion of medical lasers. This may continue in the future, as the first Dutch Laser Center was founded in Amsterdam in 1987. However, a second Laser Center was founded in 1992 in Utrecht, in the center of the country. As these Laser Centers are closely linked to the local university hospitals ane could predict that future diffusion of new clinical laser applications will most likely start in university hospitals in these regions. In the $90 \mathrm{~s}$, however, the diffusion pattern of new types of lasers has become more complex due to the increasing role of private clinics as a liaser market, especially with respect to ophthalmological laser applications.

\section{Speed of diffusion, absolute and relative numbers of llasers}

Diffusion of medical lasers in the Netherlands has been rapid, especially in ophthalmology. Im 1989 Dutch hospitals planned to purchase 66 new lasers in 1990 , of which $70 \%(n=46)$ was realized by the end of 1990. In 1992, Dutch hospitals planned to invest in 28 new medical lasers. Based on this experience it seems realistic to expect that $70 \%$ of these intentions were indeed realized by the end of 1992. Therefore, we speculated that the total number of lasers would amount 275 by the end of 1992. The Dutch National Laser Safety Commiltee estimated that in mid-1993 about 310 medical lasers were in use in the Netherlands ${ }^{\left|{ }^{\prime}\right|}$. Data prowided by industry in 1995 indicate that the rate of diffusion of medical lasers has decreased in the first half of the nineties compared with the second half of the eighties (P. Sterrenburg, personal communication). The only type of laser that has not shown a sharp decrease in sales is the Nd:YAG laser. The interest in Nd:YAG lasers is 
mainly attributed to new applications, e.g. treatment of benign prostate hyperplasia (a urological condition) and its versatility ${ }^{16 .}$. Basically, this confirms the documented change in the pattern of investment in new medicallasers in 1992, showing an increase in investments in No: YAG lasers and a marked decrease in investments in other types of lasers, especially in Argon lasers. Presently, the interest in $\mathrm{CO}_{2}$ lasers is increasing due to the development of flexible wave guides for this laser. which may stimulate new sales in the future.

\section{Ophthalmology, a story apart}

The decrease in investments in ophthalmological Argon lasers is interpreted as reflecting market saturation for this type of laser. As a result, investments in Argon-lasers have become predominantly replacement-investments. One could disagree with this statement pointing to the fact that one-third of all general hospitals did not have such a laser at the end of 1991. However, adetalled analysis showed that these were predominantly small hospitals which may not have been capable to raise the funds required to invest in such a laser. In addition, small hospitals are urged by the Dutch Ministry of Health to cooperate with larger hospitals, which may reduce investments of small hospitals in expensive high technology equipment.

In ophthalmology and perhaps other specialties as well the advent of the Diode laser was anticipated to have important consequences. This laser is small, it is relatively cheap and it can (theoretically) be used for a considerable number of indications that are now covered by the Argon laser. The Diode laser was the only new type of medical laser that was introduced in health care in the Netherlands in the period 1989-1991 $(n=1)$. However, recently other lasers have caught altention in this specialty. This may be related to the increased rate af establishment of private clinics. In 1991, there were 44 private clinics in the Netheriands of which five, specialized in ophthalmological care, had purchased 6 conventional lasers. In late 1993 the total number of private clinics had increased to 53 . In this period the private clinics that specialize in ophthalmological care introduced both the Excimer laser and the Ho:YAG laser in the Dutch health care system. These lasers are used in treatment of myopia and hyperopia, respectively. Subsequently, these lasers diffused primarily to other private clinics ${ }^{[14]}$. This example illustrates that private cinics have become more important as a laser market in ophthalmological care. A tentative explanation for this changing pattern of diffusion is that private clinics are not subject to the budgeting system. In the Netherlands, private clinics are allowed to offer treatment exclusively on an outpatient basis. Therefore, the impact of the increasing number of private clinics most likely affects the diffusion pattern of lasers in a limited number of specialties. Besides ophthalmology, one can think of dermatology, e.g. dye-laser treatment of portwine stains.

\section{Intensity of use and sharing of lasers}

Both the 1989 and 1991 data showed that the overall intensity of use of lasers is rather low. This is surprising because usually a relatively mew technology becomes more intensively used as it finds its way in the health care system. A common mechanism is broadening of indicalions. Nevertheless, the average use of lasers is (only) 7.5 hours a week varying from a little more than 5 hours in ENT to about 8.5 hours per week in ophthaimology. Of course, these estimates are rather crude. Therefore, we restricted ourselves to a general conclusion on the average intensity of use. However, because we also know that 13 out of 164 ophthalmological lasers $(7.9 \%)$ are used more than 16 hours per week, there are obviously marked differences in the intensity of use of lasers within this specialty.

The low overall intensity af use of lasers could explain the declining number af investments in new equipment, because intensifying the use of existing lasers seems a more cost-effective strategy then buying new ones. Sharing of lasers between different specialties is also a means to intensify the use of existing lasers.

Compared to 1989, the 1991 data showed an averall decrease of the intensily of use of lasers $(20 \%$ in 1989 versus $16 \%$ in 1992$)$. Only $10 \%$ of the lasers that were bought in the period 1989 1991 were intended to be shared. An indiciation that this trend may have changed is provided by the $\mathrm{Nd}$ :YAG laser. Investments in these lasers have been increasingly motivated in the first half of the nineties based on its versatility and, consequently il's possibilities to be used by more than one specialty. 


\section{Differences in the tife cycle between different types of lasers}

The different types of lasers represent different life phases of a technology. The life phase of 2 technology can be estabished by the type of adopters and by the extent of acceptance of its: application(s). Based on the results presented here and on existing evidence documenting the (cost)effectiveness and technological developments in the field of lasers ${ }^{[2]}$ it can be argued that the Argon laser has become a mature technology. The Nd:YAG laser has become a mature technology for certain indications. Other types of lasers, such as the Excimer and Dye-laser, are (still) dynamic. The lasers that are relatively early in their life cycle may have the biggest potential for additionall diffusion.

\section{Policy implications}

Data on the diffusion of medical technology can be used as an input for policy-making aimed at maintaining a high quality, efficient health care system for all citizens. Considering the irregular pattern of diffusion of medical lasers in the Netherlands and its low intensity of use, we conclude that there is a clear need for guidance of these processes. This need for guidance increases when the diffusion of lasers is combined with the evidence on the safety, effectiveness and costeffectiveness of laser applications (see Chapter 4 to 11). As a result, of all specialties, diffusion of lasers seems most appropriate in ophthalmology. These lasers are probably most widespread in industrialized countries.

Data on diffusion in the Netherlands showed that there are marked differences in the awailability of non-ophthalmological lasers between different health regions. This does not by definition justify policies aimed at stimulating new region- and type specific investments. In addition to the effectiveness and cost-effectiveness of treatmerit, recommendations on this subject depend on the availability of additional information on issues as demographic differences between regions and the relative number of patients receiving treatment abroad. To formulate recommendations on investments it would also be required to set a limit to the maximum travel time that can be asked of patients in the Netherlands to receive (laser) treatment. Determination of such limits should be considered in the light of the level of experience that laser-using physicians should be enabled to maintain, in order to assure safety and quality of care.

In addition to the types and numbers of lasers that are presently available, there is a continuous stream of new laser applications that for the most part diffuse in international health care systems without systematic evaluation. An example, as discussed above, is the introduction of Excimer laser treatment of myopia in private clinics. At present, private clinics are tolerated by the Ministry of Health. Although this is not expected to change in the future, additional regulation would be beneficial to promote rational adoption and use of new medicall technology in the Netheriands (see chapter 19).

When new laser applications are promising they could be evaluated early in their life cycle ina restricted number of centers. This policy has become more common in the Netherlands since the late eighties, when Investigational Medicine Fund was founded. The Investigational Medicine Fund has become the most important source of government funding in the field of medical technology assessment in the Netherlands. For example, Excimer laser coronary angioplasty (ELCA) thas been evaluated in the context of this Fund. The results of the study showed that ELCA offers no elinical advantages as an adjunct to PTCA in patients with long coronary artery stemosis, and that the procedure is associated with considerable additional costs. It was decided not to include the procedure in the health insurance benefit package ${ }^{[7]}$. Because other centers had refrained from adopting the new technology during the period of evaluation, the results of the evaluation coult guide the diffusion of the technology. In this case additional diffusion was discouraged. In other cases, diffusion could be stimulated. In each case, evaluation could support the rational adoplion and use of technology. 


\subsection{References}

1. Appendix 1. Establishment of a laser center. The example of Australla In; Banta HD, Schou I (Eds) Lasers in Health Care, Effectiveness, Cost-Etfectiveness and Policy Implications. Academic Publishing, Frederiksbery. Dienmark, 1991, p. 259-6i1.

2. Banta HD, Schou \& (Eds.). Lasers in Health Care, Effectiveness, Cost-Effectiveness and Policy Implications. Academic Publishing, Frederiksberg, Denmark, $199 \%$.

3. Banta HD. Behney CJ. Willems JS. Toward rational technology in medicine considerations for health policy. Springer-Verlag, New York, $199 \%$.

4. Dankiw W. Hailey D. Angel A. Lasers in medicine. Australlen Institute of Health and Welfare: Health Care Technology Series No, 8, AGPS, Canberra, Australla 1991.

5. ECRI. Lasers. In: Health Technology Checklist 1993/1994, Health Technology Trends 1993/Supplement):20 1.

6. Eggink G., Kaptein C, van Kempen RJ, van der Meulen FW, Tailinck CJPM. Vaartjes SR (Eds), Dutch National Committee on Laser Safety. Laser Safety in Health Care (second edition). Report of the Dutch Nationall Committee on Laser Safety. The Netherlands Organization for Applied Scientific Research (TNO), Leiden, the Netherlands, 1993 (in Dutch).

7. Health insurance Executive Council. Annual Report of Investigational Medicine. Investigational Medicine Committee, Health Insurance Executive Council, Amstelveen, the Netherlands, 1996 (in Dutch).

8. Muller GJ, Berlien HP. The medical laser market in Germany. In: Banta HD, Schou L (Eds). Lasers in Health Care, Effectiveness, Cost-Effectiveness and Policy Implications, Academic Publishing, Frederiksberg, Denmark, 1991 " p. $50-3$.

9. National Health Technology Advisory Panel. Lasers in gynecology. A report by the National Health Technology Advisory Panel, Canberra A.C.T. 2601, Australla, 1987.

10. National Health Technology Advisory Panel. Lasers in medicine. A report by the Nalional Health Technology Advisony Panel, Woden A.C.T. 2606, Australia, 1985.

11. Office of Technology Assessment. Strategies for Medical Technology Assessment. Washington DC: US Government Printing Office, 1982.

12. Rosendal $H$. Diffusion of medical technology. A case study on laser technolagy (doctoral thesis), University of Limburg, Maastricht, the Nethertiands, 1992.

13. Sterenborg HJCM, Müller GJ. European Community Concerted Action Program into the development of medical laser applications. Final report (unpublished), Working Group Laser Safety, 1994.

14. Vondeling $H$. Evaluation and diffusion of excimer laser treatment of myopia in the United States and in the Netherlands. Heaith Policy 1995 (34):21-33.

15. de Wit A, Banta HD. Diffusion of laser technology in hospitals. In: Banta HD, Schou I (Eds). Lasers in Heatth Care. Effectiveness, Cost-Effectiveness and Policy Implications, Academic Publishing. Frederiksberg, Denmark, 1991, p. $36-42$. 


\section{Appendix 12.1 Questionnaire}

The questionnaire is addressed to professionals working in hospitals, such as (hospital) technicians, laser using physicians or members of the Board.

- Are there any lasers in use in this hospital?

- If yes, which types?

- In what year did you buy them?

Which manuffacturer?

- In which specialty are they used?

- What is the average intensity of use?

- Are any lasers shared in your hospital?

- If yes, which type?

- By which specialties?

- By whiat percentage do each of these specialties use the device?

- Have any lasers been replaced?

- If yes, what type(s) has/have been replaced?

- What type(s) did you buy instead?

- Do you plan to buy lasers next year?

- If yes, which types?

- Which manufacturer?

- For which specialty?

- Are these lasers intended to be shared?

- If yes, by which specialties? 


\title{
13 Diffusion of lasers in medicine
}

\author{
H. David Banta", Hindrik Vondeling ${ }^{2}$, Ardine de Wit, Henk Rosendaf
}

* The Netherlands Organization for Applied Scientific Research (TNO)

P.O. BOX 2215

$2301 \mathrm{CE}$ Leiden, The Netherlands

2 Institute for Research in Extramural Medicine

(EMGO-Institute)

Free University Amsterdam

wan der Boechorststraat 7

1081 BT Amsterdam. The Netherlands

* Center for Health Pollicy and Law

Erasmus University

P.O. Box 1738

3000 DR Rotterdam, The Netherlands

4. Ministry of Heaith

P.O. Box 3008

2280 MK Rijswijk, The Netherlands

Originally published as: Diffusion of lasers in medicine. In: Szczepura A, Kankaanpäa J (Eds.). Assessment of Health Care Technologies. Case Studies, Key Concepts and Strategic Issues. John Wiley and Sons LId, Chichester, United Kingdom, 1996, p. 69-85. Reprinted with permission of the publisher.

\section{Summary}

The laser is a highlly technological tool that is diffusing into many areas of medical and surgical practice. In some cases, procedures can be done in a more effective manner, and perhaps in a more cost-effective manner, with lasers. In other cases, the conventional procedure seems at least as good. Diffusion of medical lasers is relatively rapid, aithough evidence for safety, efficacy, and cost-effectiveness is lacking in most cases. The most mature area "where a number of applications have been shown to be effective, is ophthalmology. Otherwise, most laser applications have not beem shown to be superior. There are a number of impediments to laser diffuslon. including a fragmented and weak industry, lack of evidence of value, rapid technological change, and lack of reimbursement for laser applications. These barriers to diffusion set up a "vicious circle" in which evaluation is difficult. Industry has limited profits to invest in evaluation. At the same time, policymakers have shown limited interest in lasers, despite the possibility that they can be quite costeffective. Few programs of health care technology assessment seek innovations such as lasers. for their evaluation. It would be beneficial if policy-makers paid mare explicit attention to lasers.

Key Words: Lasers, Diffusion, Efficacy, Effectiveness, Cost-effectiveness, Health policy 


\subsection{Introduction}

This chapter uses medical lasers as a case to illustrate the principles and techniques of health care technology assessment, focusing on the relationship between diffusion and information on safely, effectiveness, and cost weffectiveness.

The laser should be seen within the context of minimally invasive therapy (or minimally invasive surgery). It is one of a number of therapies that can reduce invasive surgery, especially when combined with endoscopes and vascular catheters. Therefore, lasers have the potential to increase the costeffectiveness of health care. Main considerations with lasers include which applications are beneficial and the financial implications of their use. One of the challenges raised by the case is to devise health policies that could be more effective in dealing with such technologies.

In the following section medical applications of lasers will be described, followed by information on diffusion in the health care system. Special attention is paid to the role of industry in this process. The evidence on the safety, efficacy and cost-effectiveness of laser applications is briefly discussed. The case is difficult because of the numerous types of lasers and the fact that lasers are being applied in many different specialties of medicine and surgery. The complexities are Illustrated by three cases, which are discussed in some detail: photodynamic therapy of cancer, laser treatment of diabetic retinopathy and laser treatment of coronary artery disease. The final section focuses on policy-aspects of lasers and discusses expected developments in this field.

\subsection{Medical applications of lasers}

Albert Einstein described the idea of the laser in 1912 as part of this quantum theary of radiation. The first functional laser was built by Theodore Maiman in 1960. Maiman also coined the name

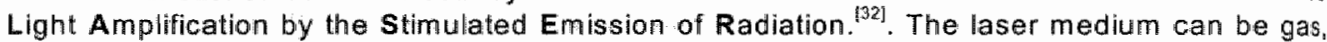
solid, or liquid. The name given to the laser $\left(\mathrm{CO}_{2}\right.$, argon-ion, $\mathrm{Nd}$ : $\mathrm{AG}$, ruby) is almost always equal to the lasing component of the laser medium. Exceptions are dye-lasers (a liquid laser medium), excimer lasers (a gas medium), and free ellectron lasers.

Dr. Leon Goldman of Cincinnati, Ohio began to use the first laser (a ruby laser) in medical treatment in the early 1960s. Medical applications of lasers were increasingly described in the medical literature through-out the late 1960 s and the early 1970 s. Today, there are more than 20 types of lasers in use in medicine. Their use is based on the ability to either cut, coagulate, vaporize, ablate, or atherwise interact with tissue. Some of the most common lasers include the argon laser, often used in ophthalmology, especially to treat diabetic retinopathy (see detailed description later); the $\mathrm{Nd}$ :YAG laser, often used in the palliation of cancer; and the $\mathrm{CO}_{2}$ laser, often used in surgery, including the treatment of pre-cancerous lesions. These lasers are "high-powered" lasers, as compared to "soft" or "low-power" lasers, which are mainly used in physiotherapy and dentistry. The soft laser is usually a helium-neon ( $\mathrm{He}-\mathrm{Ne}$ ) laser or a diode laser.

\subsection{Diffusion of lasers}

Relatively little is known concerning laser diffusion in Europe and North America or other parts of the world. A special study has demonstrated patterns of diffusion in the Netherlands ${ }^{[60]}$. Data were collected by contacting all 151 Dutch hospitals by telephone in mid-1989 to determine the number of lasers, their types, their dates of acquisition, and their patterns of use. The study was updated in $1992^{[43.57]}$.

In the Netherlands, the 1989 rate was 14.0 lasers per million population (excluding low power lasers). That could be compared to an estimated US rate of 45.5 lasers per million, a German rate 
of 22.1 lasers per milhon, and a rate in the UK of 8.8 lasers per million. The four most commonly used types of lasers - argon, $N d: Y A G, Q$-switched $N d, Y A G$, and $\mathrm{CO}_{2}$ laser, comprise more than $85 \%$ of all medical lasers in the Netherlands.

Diffusion of these 4 types of laser has thus far followed the classic s-shaped curve. The argon laser began to diffuse in the Netherlands about 1972. The $\mathrm{CO}_{2}$ laser began to appear about 4979 . The Nd:YAG began to be adopted about 1981, and it is now the most rapidy diffusing laser. By the end of 1991, 251 medical lasers had been installed; 64 in academic hospitals and 187 in general hospitals, for a rate of 16.8 lasers per million population. At least 5 lasers are operational in every academic hospital, whereas in 106 out of 139 general hospitats ( 76 percent) at least one laser has been installed (due to hospital closures and mergers, the number of hospitals in the Netherlands had fallen from 143 in 1989 to 139 in late 1991). The most commonly used laser was the argon laser (105), followed by the Nd:YAG (56), the Q-switched YAG (32), and the $\mathrm{CO}_{2}$ laser (25). 157 lasers were used exclusively in ophthalmology. Other frequent uses were in gynaecology (22), ENT (17), dermatology (16), urology (15), gastroenterology (14), pulmonology (14), and neurology (13).

Athough technically possible, sharing lasers among specialties is not very common. Only 10 percent of lasers being purchased are intended to be shared. The average laser is used about 7.5 hours a week. Only 16 lasers are used at least 20 hours a week, and 13 of these are in ophthalmology.

Low power lasers are much more frequent than high power lasers, but no figures on their absolute numbers are available. More detailed information on the diffusion of lasers in the Netherlands can be found in Chapter 12 .

\subsection{Industry and diffusion of lasers}

The total world market for lasers for medical applications was estimated at US\$ 400 million in $1987^{1551}$. In 1991, estimated worldwide medical laser sales amounted to US $\$ 1072$ million, including lower power lasers ${ }^{[30\}}$, about 3 percent of the world market for medicall equipment $t^{[11\}}$. About half of those sales were in the United States in 1987, but as lasers diffused through the rest of the world,

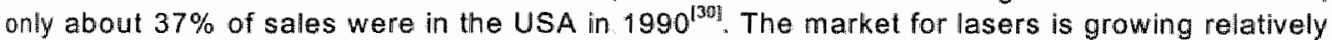
rapidy, perhaps 10 percent per year, and the market for laser accessories is growing more rapidly, perhaps 25-40 percent per year ${ }^{[37]}$. Technical developments come rapidly, but clinical application and proof of effectiveness is slower. In addition "the market is fragmented, because of the many different types of lasers, the many applications, and the lack of sharing lasers. Because of the fragmentation of the market, firms tend to specialize in one specialty or one medical condition, hampering multi-specialty use of lasers and creating inefficiencies. Companies are small and only 10 or 12 firms have a significant role in the world market ${ }^{[55]}$.

It. is clear that up to about 1985, most lasers sold were argion lasers. Diffusion in the late 1980s, however, was predominantly Nd:YAG lasers. The result is that the ophthalmology market is quite saturated, with growth of about 5 percent a year ${ }^{[55]}$. The manufacturers in this segment can make a modest profit, although the number of sales is small. The market is mature and sales are not active ${ }^{[10]}$, probably mainly to replace older lasers in many countries. A lower priced diode laser is beginning to replace the argon laser, and this has made new market opportunities.

The cardiovascular market is probably the most unstable part of the market. More than 20 different types of thermal, optical, and mechanical angioplasty devices are on the market, and none has been showm to be superior ${ }^{[15,34}$. It has been cllaimed that laser angioplasty is rapidly diffusing in

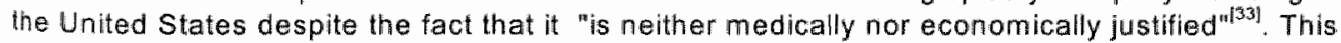
wouid suggest that laser manufacturers are succeeding in expanding their market for such technology ${ }^{[53]}$. 
A completely different market is that for low power or "soft" lasers. Such lasers are relatively inexpensive, ranging from about US $\$ 3000$ to a high of perhaps US\$50 000 ${ }^{[5, \text { al }}$. In $1989_{n}$ it was estimated that 500 low power lasers were in use in physiotherapy in the Netherlands ${ }^{[58]}$. In Europe there are at least 15 manufacturers marketing such lasers, with 10 manufacturers active in the Netherlands ${ }^{1 * 6,50,5 e \mid l}$. The Medical Laser Buyers' Guide lists 5 companies making "biostimulation. systems." In Germany, it was predicted that 500-700 soft lasers a year would be purchased for the next 10 years ${ }^{[3 ? !}$.

The large number of firms and the small and fragmented market mean that the laser market is no generally profitable. Bankruptcies, takeovers, changes in management, and poor stoo: performance are common. One important aspect of this insecure market is a limited amounto funds for research and development, including research on effectiveness and cost-effectiveness. in general, applied research on devices is funded by industry from its profits. Traditionally, lasei development has consisted of applying available lasers to coagulate or vaporize tissue, but now new laser-tissue interactions are being actively investigated. The lack of investment in effectiveness and cost-effectiveness research slows diffusion and leads to lower profits; this vicious circle prevents demonstrations of the value of medical lasers and may prevent diffusion of beneficial technologies. The rapid innovation cycle in the technology and the high maintenance costs of laser systems have also been identified as barriers to diffusion ${ }^{\mid 3 \% !}$ "

\subsection{Safety of lasers}

The hazards of lasers fall into two major categories:

1. Direct hazards to tissues such as eye or skin from the laser light.

2. Indirect hazards, including fire, $x$-rays and electrical high voltage, plus potential risks from exposure to mutagenic components and carcinogenic cells in laser smoke ${ }^{[26]}$.

These risks can affect both patients and health care personnel and extensive safety programs are necessary to minimize them.

The need for such safety programs may have reduced the diffusion of medical lasers. At the same time, control over diffusion is desirable to minimize safety problems, for example if lasers are used in small practices where safety programs may be difficult to implement.

\subsection{Efficacy and cost-effectiveness of laser applications}

Only a small number of well-designed studies of the efficacy of lasers have been published ${ }^{[7]}$. Same factors hampering evaluation have been mentioned above. Nevertheless, comparative studies are extremely important, since the laser in some cases will probably prove to be a superior alternative and should then become the standard of care. In other cases, the laser will not be superior ano should not become routine.

The most successful and best-demonstrated use of lasiers is in the treatment of diabetic change: in the retina of the eye. In general, ophthalmological applications of lasers are more mature ank better evaluated than other medical applications, in part because they have been in use longer. If other areas of medicine, few controlled studies have been carried out.

Statements often appear in the laser literature that the laser is a cost-effective medica modality $y^{19,29,411}$. These statements are based on observations that older procedures are generall more invasive, while lasers are often used in a less invasive way. Cost-effectiveness, then, refer to a comparison of older and newer treatment modalities. With laser use, the patient may not bi hospitalized or might have a shorter length of stay than when the conventional therapy is used 
Recovery time might also be shorter, meaning that the patient returns to work or normal activities more rapidly, reducing the number of working days $10 \mathrm{st}^{1 / 8,8]}$ ?

In the laser field, few formal cost-effectiveness analyses have been done. Generally "effectiveness has not been formally established, Costs may be lower with a laser treatment, but the cepital cost of the laser can be high. Claims of cost reduction following use of lasers are based mainly on the assumption that the equipment is used in a most optimal, that is, continuous way. However this. is not the everyday practice, where lasers are used as little as a few hours each week (see the section on diffusion). Furthermore, the laser procedure may take longer than a conventional procedure and sometimes must be applied several times, as in the case of cancer palliation. Thus, it is not clear that any particular laser procedure is more cost-effective than the conventional alternative unless a formal study has demonstrated it ${ }^{[6]}$.

$\mathrm{ACO}_{2}$ laser or an Nd:YAG laser might cost US\$100 000 or more. Amortized over several years: and depending on the discount rate used, the yearly cost of the laser can be estimated to be between $\$ 20000$ and $\$ 32000^{[6]}$. However, the laser is associated with other costs, including special provisions such as the cooling system, rebuilding and structural alterations to make suitable space for laser use, the costs of maintenance and repair, and the cost of technical staff and administration support. When all costs are added in, the annual cost of operating the laser is estimated to be between $\$ 45000$ and $\$ 60000$, excluding physician costs ${ }^{[8]}$.

in the Netherlandis, the average laser is used 7.5 hours per week. This means that the cost of one procedure is rather high. This high expense is a threat to cost-effectiveness and is probably a deterrent to the diffusion of lasers.

Table 13.1 shows some of the most important laser applications, the level of evaluation that has been carried out, and their estimated efficacy and cost-effectiveness. 
Table 13.1 Judgments on the effectiveness and cost-effectiveness for some important medical laser applications

\begin{tabular}{|c|c|c|c|c|c|c|c|c|}
\hline \multirow{2}{*}{ Specialty } & \multirow[t]{2}{*}{ Application } & \multicolumn{7}{|c|}{ Judgments (see definitions below) } \\
\hline & & 1 & 2 & 3 & 4 & 5 & 6 & 7 \\
\hline Dermatology & Portwine stains & & & $x$ & & & $\mathrm{x}$ & \\
\hline Ophthalmology & $\begin{array}{l}\text { y Diabetic } \\
\text { Retínopathy }\end{array}$ & & & & $x$ & & & $x$ \\
\hline$\therefore:$ & $\begin{array}{l}\text { Posterior } \\
\text { Capsulotomy }\end{array}$ & & & $x$ & & & $x$ & \\
\hline & Myopia & & & $x$ & & $x$ & & \\
\hline $\begin{array}{l}\text { Pulmonologyl } \\
\text { Urology }\end{array}$ & $\begin{array}{l}\text { PDT of lung- } \\
\text { and bladder } \\
\text { cancer }\end{array}$ & & $x$ & & & & $x$ & \\
\hline Cardiology & $\begin{array}{l}\text { Recanalization of } \\
\text { coronary arteries }\end{array}$ & & & $x$ & & $x$ & & \\
\hline Gastroenterolog & gy & & & & & & & \\
\hline & $\begin{array}{l}\text { Upper gastroin- } \\
\text { testinal } \\
\text { bleeding }\end{array}$ & & & & $x$ & $x$ & & \\
\hline Gynecology & $\begin{array}{l}\text { Cervical } \\
\text { intraepithelial } \\
\text { neoplasila }\end{array}$ & & & & $x$ & $x$ & & \\
\hline Physiotherapy & Biostimulation & $x$ & & & & & & \\
\hline
\end{tabular}

Definitions:

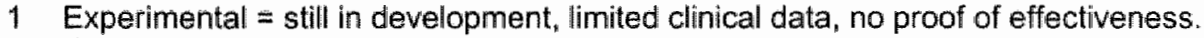

2 Promising = some clinical data, but procedure not accepted as a routine.

3 Established by clinical experience $=$ no randomized trials, but sufficient data to reach a judigment.

4 Established by RCT = established by several well-designed randomized controlled trials.

5 Probably not cost-effective = limited data on cost-effectiveness, but reasion to believe that the procedure will not turn out to be cost-effective.

6 Probably cost-effective = limited data on cost-effectiveness, but reason to believe that the procedure will turn out to be cost-effective.

7 Proven cost-effective $=$ adequate analyses of cost-effectiveness.

Note 1: Comparisons are to the standard or traditional therapy and alternative minimally invasive therapies, where appropriate. Although the laser applications are often potentially cost-effective compared with standard therapies, the judgment often becomes less favourable when compared to other minimally invasive modalities. See Part II of this thesis for more information on the cost-effectiveness of selected laser applications.

Note 2: In dermatology, portwine stains are treated using a pulsed dye laser. In ophthalmology, diabetic retinopathy is treated using an argon laser, posterior capsulotomy is treated using a Q-switched Nd:YAG laser, and myopia is corrected using an Excimer laser. In pulmonology and urology, photodynamic therapy of cancer is applied using a dye laser. In cardiology. 
recanalization of coronary arteries is carred out using an Excimer laser. In gastroenterology. an Nd:YAG laser is used in treatment of upper gastrointestinal bleeding. lin gynecology, a $\mathrm{CO}_{z}$ laser is used in treatment of cervical intraepithelial neoplasia: In physiotherapy, biostimulation is applied using a He-Ne laser.

As with efficacy, the best demonstration of cost-effectiveness is with diabetic retinopathy. Based on a formal weil-designed study, Javitt et al. $(1991)^{|28|}$ developed a model that predicted a cost of US\$966 per person-year of vision-saved from proliferative retinopathy and US $\$ 1118$ per personyear of centrall acuity saved from macular edema. The average eligible blind person receives US $\$ 6900$ annually from social security, including aid to dependents. Thus the US\$966 cost per person-year of sight saved is only $14 \%$ of the direct cost to the Federal government of a year of blindness-related disability. Expected savings on indirect costs of blindness, such as not being able to work, may be even higher. This indicates a high degree of cost-effectiveness. In a subsequent study, Javitt et al. demonstrated that screening for diabetic retinopathy is also highly cost-effective because of the ability of laser treatment to prevent bindnes ${ }^{[2,7]}$. This finding has been replicated in the UK $K^{[51]}$ and in Sweden ${ }^{[54]}$.

\subsection{Case studies}

\subsubsection{Intraduction}

All three case-studies in this chapter focus on relatively new types of lasers, the medical applications for which are still early in their life cycle. Photodynamic therapy (PDT) has been included because it provides an example of a siowly developing, potentially highly important category of new medical laser applications. Excimer laser treatment of coronary artery disease demonstrates the feasibility of policy-making towards medical technology early in its life cycle. Excimer laser treatment of myopia is discussed because the huge potential caseload and the high cost of the technology could have a substantial impact on health care costs and eye care practices. This case also demonstrates marked variations in policy making towards lasers, depending on the type of application, curative versus cosmetic.

\subsubsection{PDT}

Photodynamic therapy (PDT) is best characterized as an "eternal promise". In PDT a photosensitizer is injected, taken up by the cancer, and then activated by (laser) light, with the aim of destroying the tumour. Ever since the first clinical experience in the 1970 s with this technique, it has been stated that a breakthrough in cancer treatment was close. However most of the published clinical reports are anecdotall. No randomized controlled trials have been published, although RCTs are going on in the fields of pulmonology ${ }^{[6+1}$ and urology ${ }^{[18\}}$. Due to the experimental nature of PDT, the technique has not diffused into very many clinical settings. In the Netherlands, five hospitals (two cancer hospitals, two academic hospitals, one large general hospital) have PDT facilities.

In the past decade. PDT has been used to treat almost every type of malignancy. Most of the PDT applications that were initially examined, such as those in gynaecology and gastroenterology, have almost been abandoned. Currently, it is felt that these three clinical applications are still worth extensive research work ${ }^{[46,59]}$.

1. Pulmonology: PDT has been used to treat obstructing pulmonary cancers when the disease was considered inoperable, but still in an early stage. All initial reports to date have been encouraging. 
2. Urology, Transitional-cell carcinoma in situ of the bladder (Tis) appears to be the best indication for PDT. The number of recurrences, a common problem after treatment for Tis, seems much lower than with conventional therapy.

3. Dermatology: From the beginning of PDT development, dermatology was one of the main fields of interest: In general, small superficial tumours can do well with PDT.

In general, it is felt that most promising treatment sites may be those where there is limited thickness of the tumour, such as in superficial skin lesions or early-stage carcinomas involving the digestive tract, respiratory passages, or genitourinary tract ${ }^{\mid 451}$. However, PDT remains a treatment for tumours that are not accessible for or do not respond to conventional therapy. So far, PDT has been an investigational modality whose promise has yet to be realized. Since several phase III clinical trials are underway, more information on the walue of PDT will be available within the next few years.

\subsubsection{Excimer laser coronary angioplasty}

Cardiovascular disease is the greatest cause of death in industrialized countries. Coronary artery disease accounts for the greatest part of the deaths, primarily resulting from narrowing of the coronary arteries. Among the available methods of opening the coronaries are coronary artery bypass surgery (CABG) and percutaneous transluminal coronary angioplasty (PTCA) or balloon angioplasty ${ }^{1481}$. Because balloon angioplasty is less invasive than CABG, it is preferred, but $30-40$ percent of patient have arteries that obstruct again within 6 months or so. This has led to a search for alternatives, including laser coronary angioplasty. Among the laser applications in this area, (xenon-chloride) excimer laser coronary angioplasty (ELCA) has received the greatest attention.

The first ELCA procedure was carried out in 1988, and then two non-randomized multicenter trials were organized in the US by two competitive manufacturers. Initial results of the trials were promising, especially in treatment of long lesions, which are difficult to treatment with PTCA. As a result, a US Food and Drug Administration advisory panel recommended that the FDA should approve an ELCA system made by Advanced Interventionall Systems (AIS) for long lesions ${ }^{[21]}$. The FDA concluded that the technology should be limited to teaching and research facilities until there was more clinical consensus on its value ${ }^{[20]}$. In 1991, AIS sold 44 systems to such facilities ${ }^{[2 ! !}$. In 1992, FDA approved marketing of the system for treatment of long lesions, and a similar laser made by Spectranetics was approved for release in early $1993^{\mid 221}$. Before this approval was obtained, an estimated 100-200 systems were sold in the US by the two companies. The ELCA system costs about US $\$ 245$ 000. Single catheters sell for US $\$ 600$ to $\$ 1000$. Approximately 1.3 catheters are required per patient procedure. Profitability is therefore related to the number of catheters sold. The procedure is paid for by insurance companies, although its superiority over PTCA has not been demonstrated in RCTs ${ }^{1 / 51}$

This situation has been somewhat different in the Netherlands. Two university hospitals purchased a cardiological AIS excimer laser in 1990 and 1991, respectively. In 1991, an RCT comparing PTCA and ELCA for long lesions was organized by these centers, supported by the Health Insurance Executive Council's Investigational Medicine Program. Later, another Dutch center and a center in the United States would join the trial. In practice, ELCA was followed by PTCA in 98\% of all procedures and therefore the trial in fact comprised a comparison between combined ELCA and PTCA versus PTCA only. The results of this trial show that ELCA followed by PTCA provides no benefit additional to PTCA alone, both initially and after a follow-up period of six months. AS ELCA is associated with considerable additional costs the procedure is not cost-effective compared to PTCA as a stand-alone procedure ${ }^{[3]}$. Regardless the results of this trial, both PTCA and ELCA had already been regulated under Article 18 of the Hospital Provisions Act, which prevents the performance of these procedures without government approval. Until now, only 13 centers are 
licensed for PTCA/ELCA in the Netherlands. ELCA is actually performed in onlly three of these centers, which all were involved in the trial discussed above.

In summary, relatively rapid diffusion of ELCA has occurred in the US despite of limited evidence of increased benefits. Industry has profited from this development, but patient benefit is uncertain. In the Netherlands, regulation has prevented widespread diffusion of these devices in the period of evaluation. The results of the evaluation suggest that further diffusion should be discouraged.

\subsubsection{Excimer laser treatment of myopia}

Since the early $1980 \mathrm{~s}$, a laser techniques has been developed to treat patients with myopia, hyperopia and astigmatism. In some people, the laser procedure, photorefractive keratectomy. (PRK), could replace existing treatment methods, such as wearing glasses or contact lenses. At the end of 1993 it was estimated that 12,000 to 20,000 people had been treated with PRK woridwide ${ }^{\mid 2,31]}$. Perhaps 250-350 treatment systems have been sold worldwide, So far, in more than $90 \%$ of cases treatment was applied to correct myopia. As approximately $25 \%$ of the worid's population is myopic, there is a large potential market for ophthalmological Excimer lasers

PRK has been evaluated in two non-randomized clinical triais that included at least 500 patients each. The trials were mandated by the US Food and Drug Administration and sponsored by the two US manufacturers. Both of these companies submitted data to the FDA in 1993 claiming that over $90 \%$ of people treated have significantly improved vision. The technology was commercially released in the US in October $1995^{[56]}$.

In France the procedure has been regulated since 1991, although regulation is limited to public

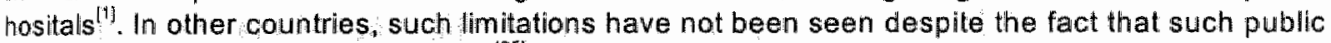
bodies as the Dutch Health Council ${ }^{25 i}$ and the Australian National Health Technology Advisory Panel $\left.\right|^{[16]}$ have concluded that the procedure is cosmetic, irreversible, possibly risky, and with insufficient evidence of long-term (5-year) effectiveness. Short-term results in people with low degrees of myopia are fair, but treatment results with more severe myopla are disappointing. No RCTs have been published ${ }^{(25)}$.

PRK is not reimbursed in any country, but it also is not regulated in any country except the US and France. People who have had the procedure pay for themselves. The procedure costs about $\$ 1900$ per eye in the US, the UK and the Netherlands and about US\$1250 in Australia. Although the laser is expensive, casting about $\$ 500000$ with annual operating costs as high as US\$200 000, the procedure appears to be highly profitable for providers ${ }^{[16,19]}$. Providers have aggressively marketed it in some countries, such as the UK and the Netherlands ${ }^{[14.25]}$. Despite the uncontrolled diffusion and the lack of evidence on the effectiveness of the procedure that has been recogrized by lechnology assessment organizations in France ${ }^{|1|}$, the Netherland $\mathrm{s}^{[25 \mid}$. Australia ${ }^{[46]}$ "and Canada ${ }^{|24|}$, policy makers in most countries are apparently not very interested in the technology because it is considered cosmetic and is not reimbursed by public funds, In the Netherlands, policymakers have recently expressed their concern about this uncontrolled diffusion, but there are no adequate tools for regulation ${ }^{\text {\{as? }}$.

In summary, the introduction of PRK shows how financial incentives for both industry and ophthaimologists have resulted in the uncritical introduction of a medical innovation. 


\subsection{Other issues concerning lasers}

As a consequence of the complexity of laser technology, safe and effective use of lasers requires: considerable education and skill on the part of physicians, nurses, laser safety officers and technicians. The different needs of these professionals should be reflected in specific programs: However, the situation in the European Union is very unsatisfactory in this respect because most education in laser technology is iragmented ${ }^{[23]}$ and because any practitioner may legally purchase a laser and begin to use it in clinical practice without the need for conclusively showing that he of she has acquired appropriate skills ${ }^{112\}}$. This situation has resulted in a number of cases of malpractice and, of course, to the awareness that lasers should only be used by those physicians who are fully trained and who can rely on a well-trained supporting staff.

\subsection{Policy implications of lasers in health care}

Policies in Europe have barely considered the new area of lasers in health care. In general, health policies have not been developed for influencing the development or diffusion of health care technology ${ }^{\gamma \mid}$, as illustrated in Table 13.2 which shows available policy instruments.

The problem of acquiring appropriate skills can be solved by a formal system of accreditation fior medical practitioners wishing to work with medical lasers ${ }^{[12]}$. In general, having surgeons with the appropriate credentials is important for a facility's legal protection, as well as its overall safeiy program. In the US, it is common to certify specialists as prepared to provide certain services, such as laser procedures. Ideally, hospital privileges to do the procedure would only be granted to those meeting such standards. This is not the case in Europe.

\begin{tabular}{ll}
\begin{tabular}{l} 
Table $\begin{array}{l}13.2 \text { Policies toward medical technology and the organizations that implement } \\
\text { them }\end{array}$ \\
\hline Policy
\end{tabular} & Organization \\
\hline Experiments & Research Institutes \\
Publication & Editors of journals \\
Education and training & Medical faculties, etc. \\
Planning & Planning organizations \\
Budgets, investment controls & Management, payers \\
Professional limits (certification) & Medical societies, etc. \\
Payment & Payers, tariff organizations \\
Evaluation (technology assessment) & TA organizations \\
Consumer information & Radio and TV \\
\hline \hline
\end{tabular}

The authors are grateful to Professor Evert Reerink, who suggested the formulation of palicies shown in Table 13.2

The most important general health policy in Europe for the last decades has been to control rising costs of health care by limited budgets in the hospital sector. This policy seems to have beer relatively effective in meeting the goal of controlling the share of societal resources going to heallt care $^{|45|}$. However, a policy of constrained budgets also has side effects, one of which is difficultie: in starting new services and purchasing new equipment. Without good information on positivt developments, a policy of restrained budgets can be expected to cause a general slowing it technological developments. This seems to be the case with minimally invasive therapy ${ }^{[4]}$. 
Several European countries, including France, Sweden, and the Netherlands have formal policies to use technology assessment in policy decisions. Increasingly, governments and insurers ask for evidence of effectiveness and costeffectiveness before they allow a new technology thito the benefit package or into health care. Technology assessment is necessary in such a setting to assist in choices. Several such assessments have dealt with laser applications ${ }^{16,25,38,39: 40,42,434}$

The increasing technological possibilities and the limited budget for health care have brought policy makers to the realization that the diffusion of new and promising procedures must be guided. Nonetheless, health policies are generally aimed primarily al slowing diffusion. There is essentially no mechanism for identifying particularly beneficial developments and encouraging their rapid adoption. Public and private health insurance bodies are generally wary of being overtaken by developments. They are often surprised by new technology. They prefer to be slow in decisionmaking rather than to risk serious mistakes.

An important policy used in several countries, including the Netherlands and France, is to centralize certain services for efficiency and quality reasons ${ }^{\{17,34,52\}}$. Such services are mostly highly sophisticated and the need for these services can be quantitatively assessed. Endoscopes and lasers, however, are considered to require a small enough investment that they are not directly regulated.

The fact is that European governments are taking a less active role in heaith policy making than before. In situations where governments withdraw from health care or where there is no strong government regulation of medical technology , physician societies and insurance companies need ta play a leading role to assure that innovations are not introduced in the health care system before convincing evidence on their (cost)-effectiveness has been generated. However, the case of excimer laser PRK demonstrates that in the European countries ophthalmological societies and insurance companies have not played an active role in evaluation. This issue will become more important in the future, since such developments as portable lasers, lower capital costs, and new applications may increase the diffusion of lasers, including diffusion to out-of-hospital settings.

As illustrated by the cases in this report, evaluation has generally not played a significant role in the acceptance of laser applications ${ }^{[4]}$. Perhaps this situation is beginning to change. The United Kingdom has inaugurated a new program to assess medical developments for effectiveness and cast-effectiveness ${ }^{[47]}$. One of the stated goais of this program is to assure that beneficial new developments are brought into health care. Other countries, such as Sweden and the Netherlands, are also trying to use assessments to guide such decisions. 


\subsection{References}

1. Agence wationale pour te Déweloppement de PEvaluation Médicale (AMDEM). Laser Excimer en ophthalmologit. ANDEM, Paris, 1992.

2. Anonymous, A year of growth for refractive surgery, Ocular Surgery News 1983(12):15-7.

3. Appelman YEA, Plek JJ, Strikwerda S, Tijssen JGP, de Feyter PJ, David GK, Serruys PW, Margolis JR, Koelenay M. Montauban van Swindregt EW, Koolen L. Randomised trial of ecimer laser angioplasty versus ballou angloplasiy for treatment of obstructive coronary artery disease Lancet $1996(347): 79-84$.

4. Banta HD (Ed.). Minmally lnvasive Therapy (MIT) in Five European Countries. Diffusion, Effectiveness and Cost. Effectiveness, Health Pollcy Monographs Vol 3. Elsevier, Amsterdam, London, New York, Tokio 1993.

5. Banta $\mathrm{HD}_{\text {, }} \mathrm{Kemp} \mathrm{KB}$ (Eds). The Management of Health Caire Technology in Nine Countries. Springer Verlag) Nev York, 1982.

6. Banta HD, Schod V Vondeling $H$, de Wit A. Economic appraisal of laser applications in heatth care: report of project. Lasers in Medical Science 1992(7):9-21.

7. Banta HD, Schou I (Eds). Lasers in Health Care, Effectiveness, Cost-Effectiveness and Policy Implications Academic Publishing, Fredoriksberg, Denmark, 1991.

8. Banta HD, Vondeling $H$, de Wit GA, Haan $G$. Economic appraisal of laser applications in medicine. Lasers in Medical Science 1990(5):253-69.

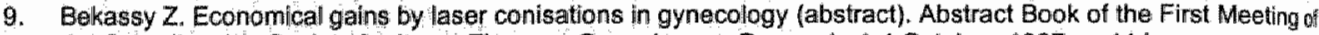
the Scandinavian Society for Laser Therapy, Copenhagen, Demmark, 1-4 October, 1987, p. 114.

10. Bertolero AA. The Laser lindustry: Present and Future. In: Joffe SN and Oguro $Y$ (Eds). Advances in Nd:YAG Laser Surgery, Springer Werlag, New York, 1988, p. 353-60.

11. Bloom $G$, Temple-Bird C. Medical equipment in Sub-Saharan Africa: a framework for policy formulation. uDS Research Report, The University of Sussex, Brighton, United Kingidom, 1990. Report Number WHO/SHSINHPi90.6 World Health Organization, Geneva, Switzerland, 1990.

12. Carruth JAS. Traiming in Great Britain. In: European Community Medical Laser Concerted Action Program Proceedings of the first plenary workshop on safety and laser-tissue interaction. Bailliëre Tindall "London, 1989. p. $373-4$.

13. CCOHTA. The excimer laser. Canadian Copdinating Office for Health Techriology Assessment (CCOHTA) Technology Brief 1992(2:0):1-4.

14. College of Ophthalmologists. Excimer Laser Photorefractive Keratectomy, College of Ophthalmologists, London, United Kingdom, 1993.

15. Corr L. Mew methods of making blocked coronary arteries patent again. British Medical Journal 1994(309):579-83.

16. Cowley DE. Laser corneal sculpting. A discussion paper. Australian Institute of Health, Australlan Government Publishing Service, Canberra, 1991.

17. Cromwell J, Mitchell JB, Stason WB. Learning by doing in CABG surgery. Medicall Care 1990(28):6-18.

18. Doughery TT, Marcus SL. Photodynamic therapy. European Jlournal of Cancer 1992(28A):1734-42.

19. van Duivemboden $Y$. Laser treatment of the comea. Graadmeter 1993(9):9-10 (in Dutch).

20. ECRI. Excimer laser for heart nears FDA OK. Health Technology Trends 1992(4):2.

21. ECRI. FDA panel okays excimer laser. Technology for Cardiology 1991(7):3.

22. ECRI. Health technology checklist. Health Technology Trends $1993 / 1994$ (Supplement).

23. ECRI. Lasers: facilitating their acquisition and wider use. In: Moretti $M$ (Ed.). Medical Lasers Buyers' Guide 1988 PennWell Publishing Co. Littleton, MA, 1987: p. 118-23.

24. Food and Drug Administration (FDA). Office of Device Evaluations, Division of Ophthalmic Dievicesi. Draft clinita guidance for the preparation and contents of an investigational device exemption (IDE) application for excimer laser devices used in ophthalmic surgery for myopic photorefractive keratectomy (PRK). Refractive Corneal Surgery 1990(4):265-9.

25. Health Council of the Netherlands: Standing Committee on Medicine. Lasers in Sight. Laser Correction of Refractive Errors. Health Council of the Netherlands, Publication Number 1993/19E. The Hague, the Netherlands, 1993.

26. Holmes JA. A summary of safetty considerations for the medical and siurgical practitioner. In: Apfelberg DB (Ed). Evaluation and Installation of Surgical Laser Systems. Springer Verlag, New York, 1987, p. 89-95.

27. Javitt $\downarrow$, Canner $M$, Frank $R$., Steinwachs $D M$, Sommer Al. Detecting and treating retinopathy in patients with fype 1 diabetes mellitus, a health policy model. Ophthalmology 1991(97):483-95.

28. Javitt J. Canner M, Sommer A. Cost-effectiveness of current approaches to the control of retinopathy in Type 1 diabetics. Ophthalmology 1989(96):255-64,

29. Kelly $S M_{1}$ Samowski $R A$, Wadas DD, Young T. Neodymium: YAG laser ws traditional therapy for carcinoma of the esophagus: a comparison of cost, in-patient days and survival (abstract). Gastrointestinal Endoscopy 1986(32): 145. 
30. Levit MR. The laser market in the 1990s. In. Forrest GT, Levith MR (Eds). The lasei market place in 1901, A seminar examining recent trends and ditections in the worldwide maket for lasers. The Society of Photo-Optical Engineers (SPIE) SPIE Publication 1520, SPIE, Belingham Washington, US, $1991,0.4-36$.

31. LUfH HS, Hunt SS, Maerki SC. The volume outcome relationship practice makes perfect or selective reternal patterns? Health Services Research $1987(22): 157-82$.

32. Maiman Th. Stimulated optical radiation in ruby masers. Nature $1960(187) ; 493-4$.

33. McLean $\mathrm{G}$, Burke $D$, Marinelli $D$. Comment on the clinical appropriateness of an emerging technology: Radiology $1989(172): 941-2$.

34. Meifler AF, Rigter H. Developments in cardlowascular technology. The modern too ls of cardiologist and surgeon. Health Council "Publication Number A 1993/3. The Hague, the Netherlands, 1993 (in Dutch).

35. Ministry of Welfare, Heaith and Culural Affairs. Letters to the Partiament's Chairman, 25 November 1993 and 8 March 1994. Ministry of Health, Welfare and Culfural Affairs, Rijswijk, the Netherlands, 1994 (in Dutch).

36. MTDITNO. Marktet survey, lasers, fysiotherapy. The Netherlands Organization for Applied Scientific Research (TNO). Leiden, the Netherlands, 1989 (in Dutch).

37. Muller GJ, Berlien HP. The Medical Laser Market in Germany. In: Banta HO, Schou ( (Eds). Lasers in Heaith Care " Effectiveness, Cost-Effectiveness and Policy Implications. Academic Publishing, Frederiksberg. Denmark, 1991. p. $50-3$.

38. National Center for Health Services Research. Laser trabeculoplasty (LTP) for open angle glaucoma. Report No. 23. In: Health Technology Assessment Reports 1988. Rockville, MD, DHHS Publication No. PHS 85-3373, p. 37190.

39. National Center for Health Services Research. Assessment of Nd:YAG laser for posteriar capsulotomies. Report No. 21. In: Health Technology Assessment Reports 1988. Rockville, MD, DHHS Publication No. PHS 85-3373, p. 317-35.

40. National Center for Health Services Research. Carbon dioxide lasers in head and neck surgery. Report No. 10. In: Health Technology Assessment Reports 1988. Rockville, MD, DHHS Publication NO. PHS 35-3373, p. 135-42.

41. Nishioka N, Richter J. Endoscopic therapy of bleeding peptic ulcers: a cost-benefit analysis (abstract). Gastrointestinal Endoscopy 1986(32):150.

42. Office of Health Techmology Assessment. Carboin Dioxide Laser Surgery. Assessment Report Series, Volume 2, Number 23. Washington, DC: Office of the Assistant Secretary for Health, Department of Heaith and Human Services, 1982.

43. Office of Health Technology Assessment. Endoscopic laser photocoagulation in the treatment of upper gastrointestinal bleeding. Health Technology Assessment Reports, number 5. Washington, DC: Office of the Assistant Secretary for Health, Department of Health and Human Services, 1987.

44. Office of Technology Assessment. The Impact of Randomized Clinical Trials on Health Pollicy and Medical Practice. Washington, DC: US Government Printing Office, 1983.

45. Organisation for Economic Cooperation and Development (OECD). OECD Health Systems, Facts and Trends 19601991. OECD, Paris, 1993.

46. Pass HKI. Photodynamic therapy in oncology: mechanisms and clinical use. Journal of the National Cancer Institute $1993(85): 443-56$.

47. Peckham M. Research and development for the National Health Service Lancet 1991(33):367-71.

48. Rienks. R, Borst C. Treatment of Atherosclerotic Cardiovascular Disease - Effectiveness. In: Benta MD, Schou: (Eds.). Lasers in Health Care, Effectiveness, Cost-Effectiveness and Policy Implications. Acadiemic Publishing, Frederiksburg, Denmark 1991 , p. 155-74.

49. Rosendal H. Diffusion of medical Hasers in the Netherlands (doctoral thesis), University of Limburg, Maastricht Netherlands, 1992.

50. Schut HA. Teirlinck C.JPM. Low-power lasers, an investigation of the Dutch market. Journal of Rehabilitation Sclence $1990(2) 4: 121-3$

51. Sculpher $M_{1}$ Buxton M, Ferguson B, Splegelhalter $D$, Kirby A. Screening for diabetic rotinopathy: a relative costeffectiveness analysis of alternative modalities and strategies. Health Economics 1992(1):39-51.

52. Showstack LJA, Rosenfeld KE, Garnick DW, Luft HS, Schaffarick RW, Fowles J. Association of volume with outcome of coronany artery bypass surgery. Journal of the American Medical Association 1987(257):785-9.

53. Strategic Business Development Irc. Angioplasty technologies, markets, business opportunities. Dell Mar, Cal. "US, 1987.

54. Swedish Council on Technology Assessment in Health Care (SBU). Diabelic retincpathy * importance of early detection. SBU, Stockhoim, 1993.

55. Thierry JP. Personal communication. 1989.

56. Voelker $R$. New techniques to resculpt the comea. Joumal of the American Medical Association 1995(247):1493-4.

57. Vondeling $H$, Rosendal H, Banta HD. Diffusion of medical lasers in the Netheriands. In: thzelt A and Coenen $R$ (Eds.), Knowledge, Technology Transfer and Foresight. NATO ASl Series, 4, Science and Technology Policy Volume 8. Kluwer Academic Publishers, Dordrecht, the Netherlands, 1996, p. 73-98. 
58. Wralik H. How soft is the laser in physiotherapy? The Wethentands Organization for Applied Scientific Reseand (TNO), Study Center for Technology and Policy (STB). Apeldoom, the Netherlands, 1989 (in Dutch),

59. Wieman TJ, Fingar VH. Photodlynamic therapy, Surgical Clinics of North America 1992(72):60922.

60. de Wh $A$, Banta, $H D$. Diffuston of laser technology in hospitals. The case of the Nethertands. In: Banta HD, Scho I (Eds). Lasers in Health Care, Effectiveness, Cost-Effectiveness and Policy Implicationis. Aciademic Publishing Frederiksberg, Denmark, 1991 , p. 36-42.

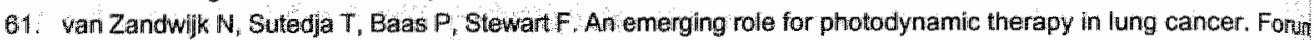
Trends in Experimental ard CHinical Medicine, 1993. 


\section{Appendix 13.1 Questions}

1. A weak medical industry has both advaniagles and disadvantages for a country. How is it an advantage? How is it a disadvantage?

2. Is it an advantage for industry to invest in evaluation of laser applications? Why or why not?

3. Can you suggest successful strategies for technology assessors in the laser case?

4. Why should policy makers be interested in encouraging the medical device industry?

5. What is industry's interest in encouraging policy-making?

6. Is it desirable to control low power lasers? Why or why not?

7. Why have so few RCTs been carried out in the laser field?

8. Why has the case of treatment of diabetic retinopathy been subject both to excellent RCTs and cost-effectiveness analyses?

9. Who should finance RCTs?

10. Should an RCT be done of all applications of any new technology?

11. In general, do RCTs affect diffusion of a new technology?

12. What role should studies of cost and cost-effectiveness play in diffusion? How can the effect of RCTs on diffusion be increased?

13. What social issues can be identified that result from laser diffusion? What could be the role of consumers/patients? 


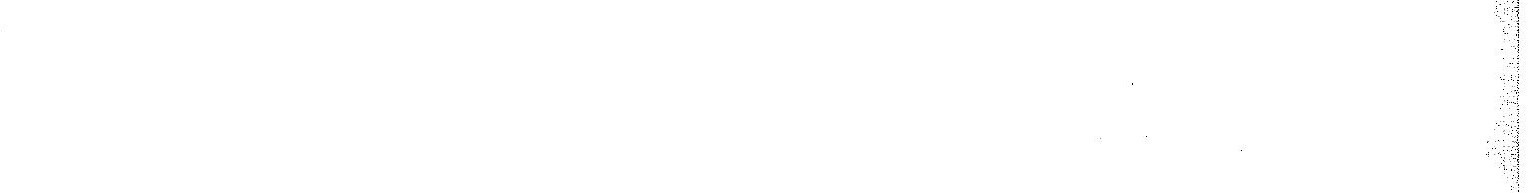




\section{Diffusion of minimally invasive therapy in the Netherlands}

Hindrik Vondeling, " Enria Haerkens, "Ardine de Wit, Michael Bos ${ }^{2} \mathrm{H}$. David Banta

1. Department of Health Economics, University of Limburg, Maastricht, the Netherlands

2 Center for Medical Technology, TNO Institute of Ageing and Vascular Research, Leiden, the Netherlands

3 Center for Health Policy and Law, Erasmus University Rotterdam, the Netherlands

4 Health Council, the Hague, the Netherlands

Originally published as: Diffusion of minimally invasive therapy in the Netheriands, Health Policy 1993(23):67-81. Reprinted with permisssion of the publisher.

\section{Summary}

In the context of a European project the diffusion of 10 selected cases of minimally invasive therapy (MIT) in the Dutch health care system was documented and analyzed. Literature on the cases was reviewed. Additional information was obtained by interviewing Dutch specialists, using a semi-structured questionnaire, focusing on different aspects of the diffusion process. In each case the diffusion pattern resulting from the balance of stimulating and impeding factors is described and summarized in a matrix framework. Budgetary pressures, lack of evidence on effectiveness and physician conservatism are the most important factors impeding diffusion of MIT. Patient demand, media reporting and presence of medicial innovators are important diffusionstimulating factors. Finally, a judgement is made on the policy implications of these findings, based on a comparison of the extent and speed of the diffusion of each case, the quality af evidence on effectiveness and cost-effectiveness and ${ }^{\text {in }}$ a few cases, existing policies. In most cases, more active policy making, either stimulating or otherwise guiding diffusion, would be desirable.

Key-words: Minimally invasive therapy, Diffusion, the Netherlands, Innovatlon, Evaluation, Health policy 


\subsection{Introduction}

In the context of a European project, the diffusion of ten cases of minimally invasive therapy (MIT) was studied in the Netherlands. Cases included 1) laser treatment of bladder tumours, 2) extra corporeal shock wave lithotripsy (ESWL) and percutaneous nephrolithotomy (PCN), 3) laparoscopic treatment of endometriosis, 4) laparoscopic removal of ovarian cysts 5) laparoscopic cholecystectomy, 6) laparoscopic appendectomy, 7) catheter treatment of coronary artery disease 8) palliation of colon cancer by endoscopic intervention 9) treatment of upper gastrointestinal (UG)] bleeding by endoscopic intervention and 10) arthroscopic knee surgery. An introduction to the project can be found in Chapter 2 . The role of the Dutch government in controlling health care technology is discussed in paragraph 2. Methods of data collection are described in paragraph 3 , followed by the results for each case in paragraph 4 . In the final paragraph, a judgment is made on the policy implications of these findings, based on a comparison of the extent and speed of the diffusion of each case, the quality of evidence on effectiveness and cost-effectiveness and, in a few cases, existing policies.

\subsection{Control of health care technology in the Netherlands}

The health care system of the Netherlands is pluralistic, with diagnosis and treatment primarily provided privalely. The government has the primary responsibility for providing preventive care services and for giving general guidance, mainly through the Ministry of Health, Welfare and Sport. In clinical health care, the government sees its role in the creation of appropriate conditions for the exercize of responsible private actions.

The government's role in health care was established in the Hospital Tariffs Law (Wet Ziekenhuistarieven) of 1965 , which regulated price setting for intramural institutions, and the Hospital Provisions Act (Wet Ziekenhuisvoorzieningen) of 1971, which regulates the building and renovation of institutions. In 1983, the Health Care Provisions Law (Wet Voorzieningen Gezondheidszorg) gave the government additional powers to control health care facilities, including non-hospitals. Under these laws, the Netherlands health care system is relatively heavily regulated. The insurance system can be divided into three parts. The first is sick fund insurance, which is a compulsory insurance scheme covering about 60 percent of the population. Members of the scheme include employees whose total wages fall below a certain level and those over the age of 65 with incomes below a certain level. The second part is private insurance, which covers almost everyone else in the population. The third part is the General Insurance Against Special Sickness Costs (AWBZ), which is a government fund covering every resident of the Netherlands.

Hospitals are paid a tariff that is regulated, and function within a global budgeting scheme, described below.

Specialists are paid fee-for-service for all patients under a defined benefit package. Fees are determined by negotiation between the physicians" organizations and the insurers. The relatively high fees for speciallists have been criticized in the Netherlands, and the government has attempted to reduce fees for some specialities, but has been faced with strikes and other actions and has been largely unsuccessful. As described below, technology assessment has begun to play an important role in defining the benefit package for payment.

Health care costs rose 11 to 18 percent per year between 1973 and 1977 , and by 1980 health care expenditures accounted for 8.4 percent of gross national product (GNP). The general impression in The Netherlands is that health care technology is a significant contributor to health care costs. These increases in costs were a stimulus to the development of health care technology assessment activities. In 1984, a government White Paper stated that technology assessmenl should be a part of policy making in all areas of science and technology. The Dutch Health Council (Gezondheidsraad) has an important role in advising the government on issues concerning science and technology and the Council has been active in doing assessments. Several academic programs have also developed. Perhaps the most important institution is the Health Insurance Executive Council (Ziekenfondsraad), which began to support technology assessment in the mid- 
1980s. The most important program is the Investigational Medicine fund fontwikkelings. geneeskunde). Jointly supported by the Health Insurance Executive Council, the Ministry of Education and Science, and the Ministry of Health (WWS). The fund began to support technology assessments in 1990 and has 27 million guilders available for such studies (including development and care costs) in 1996. As resuits of these studies have become available, they influence decisions as to whether or not a new technology is covered in the benefit package of the sick funds and other policy decisions.

Rises in health care costs also led to the development of the Financial Oversight on Care (Financieel Overzicht Zorg - FOZ), which is published every year by the Ministry of VWS. The amount for care is determined by the Ministry of VWS and approved by the Parliament every December. By 1989 , the care sector accounted for 9.6 percent of the GNP. The FOZ sets a maximum for the care sector that is then implemented in the health care system, as described Delow.

An important explicit control that the government itself has over technology is authorized by Article 18 of the Hospital Provisions Act, which gives the government the authority to license high technology services. About 10-15 such services are licensed and thereby centralized. Of the cases studied, only PTCA and CABG are regulated under Article 18.

The major control is now over budgets and tariffs, under the Law of Health Care Tariffs (Wet Tarieven Gezondheidszorg) of 1982. Under this law, all charges and fees in the health care sector are regulated, as well as the overall budget of each institution. The Central Health Care Charge Agency (Centraal Orgaan Tarieven Gezondheidszorg - COTG), made up of representatives of employers, employees, institutions, doctors" organizations, funding bodies, and independent experts, has the responsibility of implementing the $\mathrm{FOZ}$ and of drawing up guidelines for the setting of charges and fees. Each general hospital is now under a global budget. Under Article 17 of the law, medical equipment is included in that budget, which must in principle be approved in advance by the COTG. The actual amount of the budget is determined by historical experience and by negotiation. The COTG seldom interferes with the internal priorities of a given hospital. The hospital budget is determined before the year begins. The hospital cannot increase its budget, but it can change priorities with in that budiget to increase its avallable resources for certain purposes. For example, the hospital has an incentive for short-stay admissions under this plan, since admissions for specific conditions tend to receive a set amount. On the other hand, the hospital gains nothing fram moving services out of the in-patient sector.

\subsection{Methods}

Literature was reviewed on the 10 selected cases. In addition "Dutch specialists were interviewed personaliy using a semi-structured questionnaire, focusing on different aspects of the diffusion process. The questions had been formulated in a meeting of the research team that carried out the study in each of the five Europeam countries included in the project. The questionnaire can be found in Appendix 14.1. In most cases, both physicians who were actively involved in a specific procedure and physicians who were not (yet) involved in the new technology were interviewed, mostly by telephone. An initial list of key-informants was based on the literature review and, partly, an existing contacts of the members of the research team with innovators in various specialties. The methodology used was a simple 'snow-ball' approach. The innovators suggested additional physicians to be interviewed, including physicians who were not (yet) actively involved in a specific procedure. The total number of specialists interviewed was 25 . in the majority of cases, physicians were interviewed on two cases in the same specialty. There were 2 refusels. A report was drafted and used as part of the input for a meeting on the subject of Dutch experts and policy makers. Based on discussions in the meeting and on written comments, the report was revised and included in the final report that was presented to the EC. This article summarizes the main findings from these different sources. 


\subsection{Results}

\section{Laser treatment of bladder tumours}

Laser treatment of bladder tumours began in the Netherlands in the late 1970s. The early users were influenced by the techrical and clinical work of Hofstetter ${ }^{\left[t^{6}\right.}$, who started the procedure in Germany in 1976 . In the 1980s, the treatment spread to six centers in the Netherlands, treating low numbers of patients. Urologists in the Netherlands agree that there is no medical consensus concerning the effectiveness of laser treatment. Evidence of safety is considered adequate, bui evidence of efficacy is still limited.

Aside from the lack of evidence, other factors seem to impede diffusion of the technique. The urological profession in The Netherlands is rather strong, and is traditionally conservative about taking up new therapies. A particular problem in the view of same is that laser treatment prevents good histological examination of tumour tissue. The laser is also a problem in that it requires special experience and training. The urologist must first be a skilled endoscopist and then must have specific training in laser use. In the past few years training in laser use has gradually become avallable.

The cost of purchasing and operating the laser has been a problem in most settings. The financial incentives in the Netherlands generally favor TUR. There is no specific reimbursement for laser use. A hospital with a laser may favor its use because the laser procedure is faster, sparing physician time. Hospitals without a laser, however, have the incentive to do the TUR and not refer patients.

In summary, the factors impeding the diffusion of this procedure outweigh the stimulating factors. As a result, diffusion has been slow and is limited to only a few centers.

Extra-corporeal shock wave lithotripsy (ESWL) and percutaneous nephrolithotomy (PCN) Before 1980, the standard therapy for larger stones was open surgery. In the Netherlands, PCN began to be introduced in departments of urology around 1980 or so. By 1985 , all university departments and the majority of peripheral centers had adopted PCN ${ }^{\prime \prime}$. In 1984 ESWL was introduced. After a slow initial diffusion, the speed of diffusion increased in 1987-1988, resulting in the availability of 11 ESWL machines in the beginning of 1990.

The installation of the first ESWL in The Netherlands was delayed because the Sick Funds and the private insurance companies could not come to an agreement with the hospital concerning the capital costs of the project. The Kidney Foundation finally donated the ESWL to the hospital to solve the impasse. The first ESWL was a success, leading to attempts to purchase further machines. University hospitals, the Kidney Foundation, the kidney patients association, the medlia, and politicians all brought pressure on the Ministries of Health and Education and Science to allow more machines. The Health Council was asked by the Minister of Health to advise on the number of ESWL needed in The Netherlands. The Council recommended the establishment af $2-3$ machines in 1986. Such limitation could have been effected under Article 18 of the Health Care Facilities Act, but under increasing political pressure to allow more ESWL, the Minister decided not to regulate it. Nonetheless, diffusion of ESWL was constrained by continued reluctance of the insurance companies and Sick Funds to pay for the capital cost of the equipment. It took hospitals some time to find solutions to the resulting financial problem, but in 1987-1988 the number of ESWL machines increased rapidly. By the end of 1988, mine ESWL facilities were in operation and, as mentioned above, by the beginning of 1990, there were 11 machines including several mobile systems ${ }^{11}$. A report from the Dutch Health Council in 1990 recommended that 6 ESWL machines was sufficient for the Netherlands, based an a predicted number of 6500 treatments.

ESWL requires no particular skill, being a standardized method. PCN, on the other hand, requires considerable skill from the operator. An ESWL is a rewolutionary device, and was the subject of much publicity. Also the involvement of industry has been an important stimulating factor in the diffusion of ESWL. Both techniques are established in treatment of urinary tract stones.

In summary, the factors stimulating the diffusion of ESWL have outweighed the impeding factors, in this case resulting in rapid diffusion of $\mathrm{PCN}$ and, after a slow start, a rapid diffusian of ESWL. 
Laparoscopic treatment of endometriosis.

Or. A. ljzermans of the Diaconessen Hospital Eindhoven was the first to do laparoscople laser treatment in gynecology in The Netherlands, beginning in 1979. Dr. ljzermans has been almost alone in this field for ten years. One colleague began an active treatment program in 1988. In 1987, Dr. lizermans gave a lecture at the Dutch Society for Obstetrics and Gynecology on this subject and found no interest. However, in late 1989 he organized a symposium on the subject, and more than 100 Dutch gynecologists attended, showing great interest. He believes that developments in laparoscopic cholecystectomy have stimulated the interest of Dutch gymecologists in this treatment. still, there are probably no more than 5 hospitals doing the treatment now.

Despite the lack of RCTs, some gymecologists are convinced that lasertreatment of endometriosis can be considered established. However, other physicians state that the lack of RCTs is a hindrance. There is no consensus among Dutch gynecologists concerning either laparoscopic treatment or laser treatment. All informants believe that more trials of effectiveness and safety are needed. Some informants point to the Dutch gynecological specialty as particularly conservative with regard to innovation. However, other informants dispute this view, and state that Dutch gynecologists are appropriately prudent in accepting new treatments.

With some exceptions, hospital management is not supportive of the desires of gynecologists to acquire lasers. Physicians report that the budgeting system is the main reason. The hospital does not easily find room for such investments, which include not only the laser, but the laparoscope and the video equipment. Laser laparoscopic treatment in gynecology is not reimbursed by the Sick Funds, although diagnostic laparoscopy is reimbursed. Therefore, procedures are reimbursed as the standard operation or as diagnostic laparoscopy. Laparoscopic treatment of endometriosis recuires training and skill. A number of safety problems have been reported in the literature, but these are largely related to lack of skill. Lasers are not part of the routine training in gynecology in The Netherlands, and this helps explain their limited use. Physicians involved in laparoscopic treatments consistently showed concern about rapid diffusion of such treatments because patients will be harmed by unskilled operators. Some favor formal certification to address this problem. Another factor that slows diffusion in The Netherlands is a feeling against close cooperation with the industry among physicians.

in conclusion, the impeding factors actors are more important than the factors stimulating the diffusion of this procedure in The Netherlands.

\section{Laparoscopic removal of ovarian cysts}

Dr. A. ljzermans has treated ovarian cysts by laparoscopy since he began to use this technology in 1979. However, he apparently is the only gynecologist in The Netherlands to use the laparoscope in this way. The reason that, despite the evidence, the technique does not diffuse is because treatment of cysts is quite difficult in a technical sense, compared with treatment of endometriosis. Dutch gynecologists also point to concerns about malignant changes in such cysts and the fact that complete pathological examination cannot be done after treatment by laparoscope. There seems little doubt that advanced imaging, such as with ultrasound, makes it possible to diagnosis early malignancy reliably. However, Dutch gynecologists state that this makes removal of most ovarian cysts unnecessary. Otherwise, this case is quite similar to the case of endometriosis.

\section{Laparoscopic cholecystectomy}

The first laparoscopic cholecystectomy in the Netherlands was done by W.F.M. van Erp in February 1990. Van Erp was trained to do the procedure in France ${ }^{[3 !}$. In May 1990, the procedure was introduced to a second center ${ }^{|4|}$. In both centers a number of surgeons have been taught to do cholecystectomy by laparoscope.

The early diffusion process was stimulated by the lay media. Initially, surgeons were not very interested in the new method, because it takes longer than the traditional procedure. However " patients have demanded the procedure, and it has therefore spread rapidly in both hospitais and surgical practice. Hospitals have no financial incentive to provide the procedure, but they use it as a marketing point. In 1986 there were 14,731 cholecystectomies done in the Netherlands. Surgeons estimate that 80 percent of these cauld be done by laparoscope. 
By May 1991, there were about 60 hospitals doing this procedure as a routine, but mostly in low numbers because of difficulties acquiring equipment. These difficulties are partly due to a waitin period of some months before an operating laparoscope can be supplied by a manufacturertion Another reason is budgetary problems. From the standpoint of the hospital, the equipment is a new investment, and budgets are limited. It is therefore often difficult to acquire the equipment. In addition; Sick Funds and insurance companies provide only the fee to the surgeon for a standard procedure, while the extra investment in equipment; the longer time of the procedure, and the extra manpower necessary in the operating room are not taken into account. The payers are not veny interested in some cases because they say that the empty beds produced will just be filled by other patients, Insurance companies still must give specific permission before the procedure, and in one case such permission is still withtheld. One private insurance company will not provide payment because it considers the procedure experimental. Within the hospital, there are organizational problems. The anesthesiologists tend to give general anesthesia, which makes recovery time slower. Regional anesthesia is generally adequate. Nurses have not been trained to allow Immediate mobilization and nutrition of the patient. Training courses and seminars are necessary. The technology for the procedure is also not optimal. The laparoscope is adequate, but needs further improvement, and cannot presently be heat-sterilized. Also, associated equipment needs further development.

In conclusion, despite a number of difficulties, diffusion of this procedure proceeds rapidly in the Netherlands.

\section{Laparoscopic appendectomy}

Appendectomy by laparoscope was developed in Germany and has now been done for more than 10 years with good success ${ }^{10 !}$. Laparoscopic appendectomy began in the Netherlands in 1971 , carried out by H.J.M. de Kok, a surgeon ${ }^{[7 . a 1}$. De Kok developed the technique of bringing the appendix to the outside by laparoscope and he has performed more than 1500 laparoscopic appendectomies using this technique. With the growing popularity of the laparoscopic cholecystectomy in 1990, the technique was adopted by two additional centers.

Surgeons are less interested in this procedure than they are in laparoscopic cholecystectomy. One reason is that the standard procedure is done rapidly through a rather small incision. The laparoscopic procedure takes considerably longer. In addition, hospitals are not providing the equipment, as noted in the previous section. In conclusion, contrary to laparoscopis cholecystectomy, this procedure does not diffuse very rapidly, in part because the time for carrying out the procedure is longer than for the established appendectomy.

\section{Catheter treatment of coronary artery disease}

Dr. M.G.G. Ernst introduced PTCA to the Netherlands in 1980, after being trained by Dr. A.R. Grüntzig in Switzerland ${ }^{(2)}$. Other important opinion leaders have included Dr. H.W.M. Plokker and Dr. P.W. Serruys.

The number of PTCA procedures in the Netherlands has risen steadily, reaching 4400 in 1987 , 5600 in 1988, and 6400 in 1989. There are 12 PTCA centers in the Netherlands and 19 PTCA labs. PTCA is now the standard minimally invasive procedure for use in cardiovascular disease.

Evaluation of PTCA has played little part in its diffusion. Instead, local innovation and success has been the determining factor in its diffusion. Also, the fact that it could be done by cardiologists has been an important factor. Crardiac surgeons were already quite busy in the Netherlands, so they did not object too strongly to cardiologists taking some of the work load. However, competition between specialties has played a role, as cardiac surgeons have refused to cooperate with cardiologists in evaluative studies of PTCA versus CABG. Present strategies for treatment depend on treatment of isolated and shorter obstructions by PTCA whenever possible, expecting the likelihood that CABG will be needed in the future. The technology has been continually improved since its introduction. The entire system has become thinner and smaller and easier to use, which means improved treatment results and greater numbers of procedures. The result is that the technology is mature. The industry has been quite important in these improvements.

PTCA requires speciall skills, especially in catheterization techniques. A general training in cardiology is essential as a basis, in the opinion of Dutch informants " because of the possibility of 
mythm disturbances during the procedure. PTCA is not part of the training of cardiologists. however, and there is no certification. Therefore, each interested cardiologist must acquire special training supervised by an acknowledged expert.

Policy initiatives in The Netherlands have been aimed at limiting the annual number of PTCA procedures (and CABG procedures). Both procedures have been regulated under Article 18 of the Health Care Facilities Act. In 1987, the Supervising Committee on Heart Surgery was formed ${ }^{16}$. The committee was formed to plan facilities for interventional cardiology, including PTCA. The number of facilities has been limited following recommendations of this Committe, but attempts to limit the number of procedures have failed due to centers that exceed the limits. The increasing numbers are related to expanding indications for the procedure. Restriction of the number of facilities has been beneficial in promoting expertise. Each center does an adequate number of procedures, which means that the physicians are quite skilled. In addition, the institutions themselves oversee quality and assure appropriate training and supervision.

Financial constraints do not seem to play any significant role in this case. The procedure is fully reimbursed and hospitals do not slow down purchases. According to informants, the fee of US\$465 is higher than the costs of the procedure, so the hospital has an incentive to expand services.

The media has played some role in this case. Patients support the widespread availability of these treatments, including PTCA, and are organized in the Dutch Heart Foundation and the Friends of the Dutch Heart Foundation for the purpose of financially supporting scientific research and mobilizing media support. Both organizations are amongst the most powerful patient organizations in the Netherlands. A side effect of the media attention, however, is that patients are quite oriented to PTCA. In some cases, the procedure is applied inappropriately because of patient demand. in conclusion, PTCA has spread relatively rapidly into use and is fully accepted in the Netherlands. Evaluation has played little role in the diffusion. However, as in other cases, patient demands and media reporting seem to have worked to overcome barriers to diffusion.

\section{Palliation of colon cancer by endoscopic intervention}

Endoscopic palliation of colorectal cancer by Nd:YAG laser was begun in September 1980 in The Netherlands by Dr. E.M.H. Mathus-Vliegen ${ }^{[9]}$ in Amsterdam at the Academic Medical Center (AMC). Experience has gradually accumulated and the Amsterdam hospital has become a national referral center for this indication.

Before the laser was used in colorectal carcinoma, patients with colorectal adenomas and rectal stump polyps were treated by endoscope. However, this was not easy to achieve in the AMC because surgeons were reluctant to refer patients to the department of gastroenterology. The surgeons have obviously felt that internists were invading their realm, and this is true.

There is no medical consensus about the place of laser palliation. First, there is disagreement on patient characteristics that justify palliation, including laser palliation. Second, if palliation is required, most physicians refer to the local center; most physicians do not seem well-informed about alternatives. Each center offers a range of alternatives, and these available alternatives determine the palliation method. The diffusion of the laser therapy was also impeded by the perception that it was experimental and by its high expense.

There are no factors that specifically encourage laser use. The media has shown no interest in palliation of advanced cancer, probably because of the palliative nature of treatment and because there is a strong taboo concerning open discussion of cancer. The lack of information impedes patients from acquiring the most appropriate therapy. Patients readily accept laser therapy when it is offered.

There is no specific fee for laser therapy. Instead a fee for sigmoidoscopy (US\$196) or colonoscopy (US $\$ 260$ ) is received. The fee for both electrocoagulation and cryotherapy is US $\$ 206$. The AMC acquired its first laser without cost. Since then, it has made the lasers available through its budgets, but the latest accessories are not available.

The technology is not mature. The laser itseif is adequate, but the fibers and accessories need more development. Finally, improvements have been made in the structure and choice of materials for the heater probe.

In conclusion, laser therapy for this indication has not diffused in the Netherlands. However, there is considerable evidence to support more widespread use of the case for palliation. 


\section{Treatment of upper gastrointestinal (UGI) bleeding by endoscopilc intervention}

By the late $1970 s_{\text {, }}$ the endoscope was established as a diagnostic device, but therapy has been slow to follow. The first treatments of UGI bleeding were done in The Netherlands about 10 years ago. Monopolar electrocoagulation was the first modality to be used. Since then, a number of other modalities have been developed, and these have spread into use. Informants point to a number of international figures and to the Amsterdam Medical Center (AMC) group under Professor Tytgat as influential in influencing diffusion in The Netherlands. Diffusion has been slow but nowadays endoscopic methods are entirely established in treatment of UGI-blleeding in The Netherlands. In this case, evaluation is seen as a critical element in the development of the case ${ }^{[12]}$. There is also an awareness of cost-effectiveness of the treatment. However, there is no medical consensus on which specific modality should be used, and each center tends to use one therapy. There has been no national consensus meeting on the subject of this treatment, and such a meeting could be quite useful.

There are financial problems in mosit hospitals in acquiring the necessary equipment for this treatment. Ideally, a hospital active in the practice of giastroenterology should have both diagnostic and therapeutic endoscopes, but often the numbers are limited and hospitals hesitate to approve replacement technology because of the capital investment.

Therapeutic endoscopy requires considerable skill and there is a learning curve before one obtains the best results. At least one year of clinical training is required to achieve the best results. Bist internists do not necessary acquire this much training in gastroenterology. It might be desirable to certify physicians as having had sufficient training in this modality. Nurses also need special training with endoscopy. Training programs for physicians and nurses have been organized in at least one hospital in The Netherlands.

Reimbursement for the treatment is for a gastroscopy despite the choice of treatment modality and is about US $\$ 200$. The reimbursement is too low for a therapeutic endoscopy, which may take 2 hours or more, but is quite high for a diagnostic endoscopy. On the other hand olher gastroenterological procedures are reimbursed at low levels, so the high fee for the diagnostic gastroscopy compensates.

Patients may have little choice in this therapy. They are often in bad shape from severe bleeding. and may be in shock. Also, the media has played no role in the diffusion of gastroendoscopy. In conclusion, endoscopic treatment of gastrointestinal bleeding is entirely established in the Netherlands. Nonetheless, diffusion thas been relatively slow in The Netherlands, most likely because of lack of skilled physicians. The case also illustrates, with a number of others, the importance of constraints on acquiring equipment and of appropriate education and training in applying these technologies.

\section{Arthroscopic knee surgery}

The first meniscectomy by arthroscope was done in The Netherlands about 1978. Since then it has spread slowly. Evaluation has played no important role in the diffusion of arthroscopic surgery. However, orthopedic surgeons, influenced in part by literature concerning long-term problems after total meniscectomy, and in part by a better view of the menisci, have become more conservalive about removing them. Clinical experience indicates that there is no clear difference in the effectiveness of arthroscopic and open surgery. The advantages for the patient of the minimallyinvasive therapy are obvious. The procedure is potentially cost-effective. But no firm statements can be made on this subject because of lack of evidence. Barriers to the diffusion, except limited evaluation, include lack of skill among surgeons, less than optimal equipment, and hospital reluctance. The lack of skill was a serious problem, because in the beginning the arthroscopic procedure takes considerably longer. This was a problem because of waiting lists and pressing surgical schedules. Furthermore, the incentives for the hospital favored open surgery.

Instruments are expensive, with a cost of around US\$17 500 and hospitals are slow to make the capital investment. In addition, newer developments such as electrocautery and lasers are not used because of financial restrictions.

An important stimulating factor in the diffusion was the wishes of the patients, who gradually learned about the new alternative. In addition, arthroscopic surgery is now part of the training of the orthopedic surgeon, and open surgery will naturally continue to decline. 
Inconclusion, arthroscopic therapy appears to be effective for a variety of conditions based on dinical experience. Meniscectomy is the most common procedure, and has been the most evaluated.

Nonetheless, open surgery continues to be fairly widespread for various reasons.

\subsection{Discussion and summary}

The study discerned several factors that influence the diffusion of MIT. The factors can be subdivided in two categories: those that impede and those that stimulate diffusion, respectively.

Factors that may slow the diffusion include:

1) budgetary pressures on hospitals, which make them reluctant to undertake new, capitalintensive procedures or procedures that require extra time or personnel

2) financial incentives on hospitals, which make shorter stays disadvantageous

3) the absence of reimbursement of a new procedure, or a tediaus procedure of reimbursement

4) the fact that MIT procedures are often more time-consuming than the traditional procedure, at least until the 'learning curve' time is over

5) payment to physicians no higher than for the standard procedure, giving a disincentive if the new procedure is more time-consuming

6) Jack of convincing medical evidence (or any good information) on effectiveness

7) difficulties in organizing studies of effectiveness, including lagistical problems, lack of funds, lack of interest in the profession, resistance of patients to entering clinical trials, and regulatory requirements (especially) to assure that the study is ethical

lack of MIT procedures in present medical education, and a lack of training courses to bring skill to acceptable levels

9) physician, especially surgeon conservatism (that is, comfort with traditional procedures used in open surgery)

10) competition between different specialties in several areas

11) resistance to commercial pressures

12) rapidly evolving new procedures

13) policy measures, aiming to restrict the annuall number of procedures or the total number of devices

Factors that may facilitate diffusion of MIT are:

1) media reporting, fostering patient demand and physician interest

2) the availability of respected medical innovators in the Netherlands

3) the ability of physicians to get reimbursement for new procedures in most cases, even if they do not (yet) appear in the benefit package

4) commercial pressure and information

5) the availlability of appropriate training

6) maturity of the procedure or technology

7) policy measures encouraging the diffusion of a procedure, such as the decision not to regulate a procedure under Article 18 of the Health Care Provisions Act

8) convincing evidence on effectiveness

Table 14.1 summarizes the results in the 10 cases. Not every factor listed in the table is of importance for any particular case. Where appropriate, the individual factors have been weighed on a 1 to 3 impact-scale, from some influence to very strong influence.

The table shows that budgetary pressures and financiall incentives on hospitals, lack of convincing evidence on effectiveness and physician conservatism are the most often mentioned factors in the category of factors impeding diffusion. In the category of factors stimulating diffusion, patient 
dernand and media reporting, presence of medical innovators and evidence on effectiveness are most often mentioned and most influential.

Perhaps the most striking finding is the lack of interest and action by policy makers in a field that seems to have extraordinary potential to make health care more cost-effective.

Table 14.1 Factors affecting the diffusion of 10 cases of MIT in the Netherlands

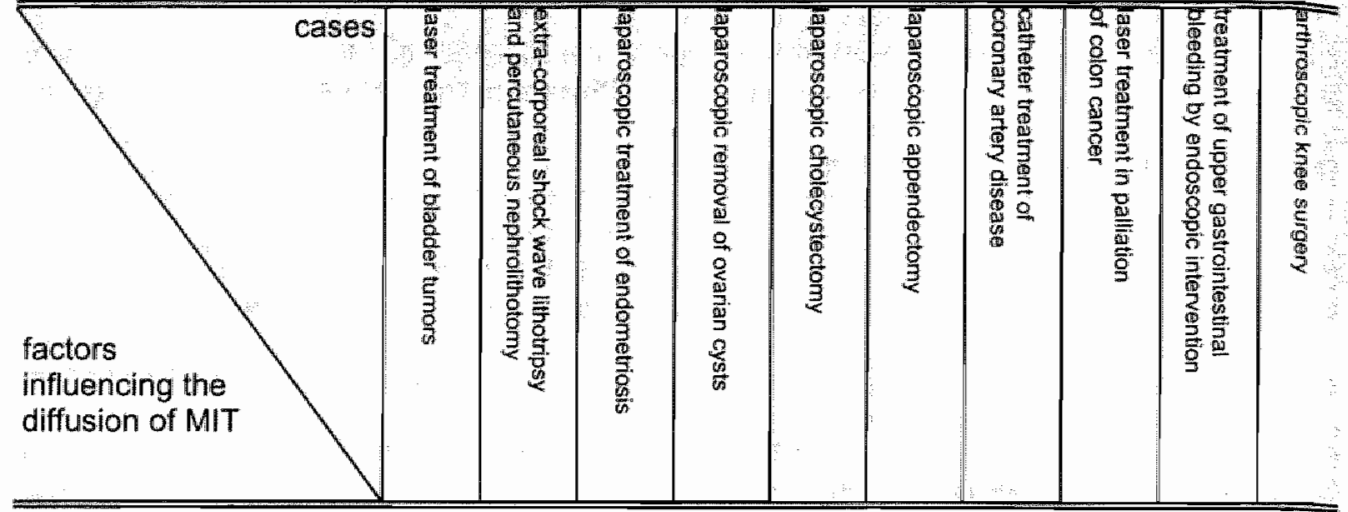

\begin{tabular}{|c|c|c|c|c|c|c|c|c|c|c|}
\hline \multicolumn{11}{|l|}{ impeding factors: } \\
\hline budgetary pressures & $x$ & $x x$ & $x x$ & $x x$ & $x x$ & $x \times x$ & & & $x$ & $x x$ \\
\hline $\begin{array}{l}\text { financial incentives on } \\
\text { hospitals }\end{array}$ & $x$ & & $x$ & $x$ & $\mathrm{x}$ & $x$ & & & & $x$ \\
\hline $\begin{array}{l}\text { no reimbursement or } \\
\text { difficult procedure of } \\
\text { reimbursement }\end{array}$ & & & $x$ & & $x$ & & & $x$ & & \\
\hline $\begin{array}{l}\text { procedure more time } \\
\text { consuming or more } \\
\text { difficult }\end{array}$ & & & $x$ & $x x x$ & $\mathrm{x}$ & $x$ & & & & $x x$ \\
\hline $\begin{array}{l}\text { no convincing evidence } \\
\text { on (cost) effectiveness }\end{array}$ & $x x$ & & $x x$ & $x x$ & & $x$ & & & & \\
\hline $\begin{array}{l}\text { difficulties in organizing } \\
\text { studies }\end{array}$ & $x$ & & $x$ & $x$ & & & $x$ & $\mathrm{x}$ & & \\
\hline $\begin{array}{l}\text { shortage of training } \\
\text { or education }\end{array}$ & & & $x$ & $x$ & $\dot{x}$ & $x$ & & & $x$ & \\
\hline physician conservatism & $x$ & & $x$ & $x$ & $x$ & $\bar{x}$ & $\bar{x}$ & & & \\
\hline $\begin{array}{l}\text { competition between } \\
\text { specialties }\end{array}$ & & & & & & & & $x x$ & & \\
\hline $\begin{array}{l}\text { resistance to commercial } \\
\text { pressures }\end{array}$ & & & $x$ & $x$ & & & & & & \\
\hline $\begin{array}{l}\text { immature procedure or } \\
\text { technology }\end{array}$ & & & & & $x$ & & & & & \\
\hline policy measures & & & & & & & $x$ & & $x$ & \\
\hline
\end{tabular}




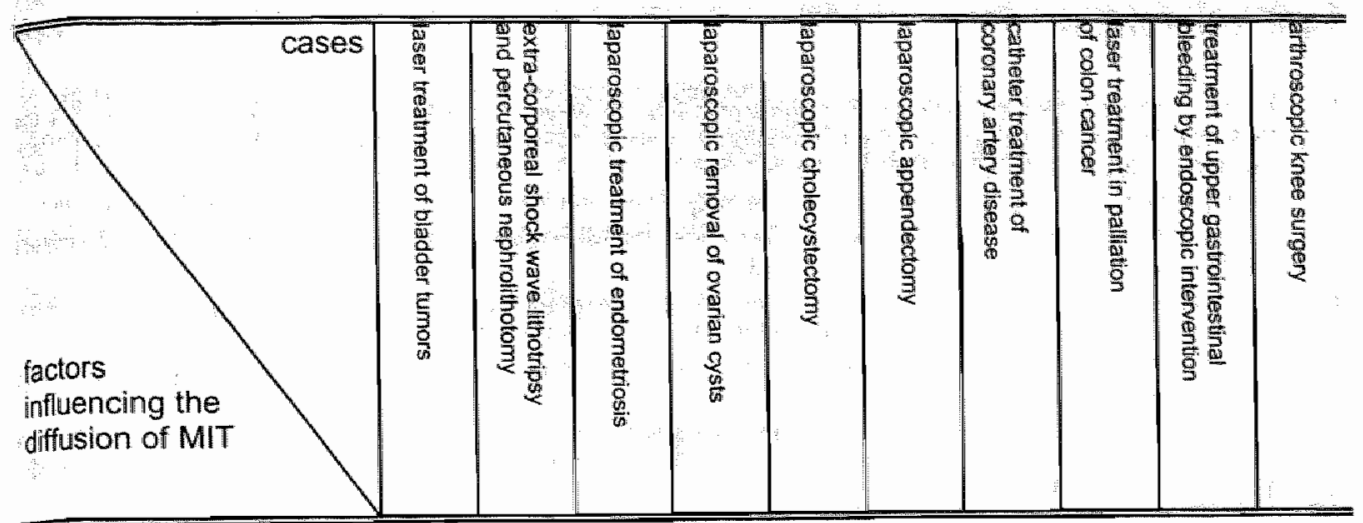

\section{stimulating factors:}

\begin{tabular}{l|c|c|c|c|c|c|c|c|c|c}
\hline $\begin{array}{l}\text { patient demand \& } \\
\text { media reporting }\end{array}$ & & $\mathrm{xx}$ & & & $\mathrm{xxx}$ & & $\mathrm{xx}$ & & & $\mathrm{xxx}$ \\
\hline medical innovators & $\mathrm{x}$ & $\mathrm{x}$ & & & $\mathrm{xxx}$ & $\mathrm{x}$ & $\mathrm{x}$ & $\mathrm{x}$ & $\mathrm{x}$ & \\
\hline reimbursement & & & & & & & $\mathrm{x}$ & & & $\mathrm{x}$ \\
\hline $\begin{array}{l}\text { commercial pressures } \\
\text { and information }\end{array}$ & & $\mathrm{x}$ & & & $\mathrm{x}$ & & $\mathrm{x}$ & & & \\
\hline $\begin{array}{l}\text { appropriate training } \\
\begin{array}{l}\text { mature procedure or } \\
\text { technology }\end{array}\end{array}$ & $\mathrm{x}$ & $\mathrm{x}$ & & & & & $\mathrm{x}$ & & $\mathrm{x}$ & $\mathrm{x}$ \\
\hline $\begin{array}{l}\text { evidence on (cost) } \\
\text { effectiveness }\end{array}$ & & $\mathrm{x}$ & & & $\mathrm{x}$ & & & $\mathrm{x}$ & $\mathrm{xxx}$ & \\
\hline
\end{tabular}




\subsection{References}

1. Bos M. The introduction and diffiston of extracorporeal shock wawe Whotripsy in the Netheriands. Reported to the Working Groupton the Diffusion of the Lithotripter. A study commisioned by the European Economic Community. Health Senvices Research Committee, March 1989.

2. Ernst MMPO. Percutaneous transluminal angioplasty. Short and Hong term angiographic results (thesis). 1987.

3. Van Erp WFN, Bruyninckx C. De eerste envaringen met laparoscopische cholecystectomy. Nedenands Tijdschif woor Genereshunde $1991(135): 2726$ (in Dutch).

4. Go P.Dauma D. De laparoscapisiche cholecystectomle, en Kijkoperatie'als alternatief woor galsteenvergnuzer. Nederlands Tijoschnift voor Geneeskunde 1990 (134):1681-2 (in Dutch).

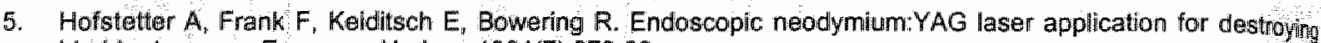
bladder tumours. European Urology 1981(7):278-83.

6. Jambroes $\mathrm{C}$ (Ed.). Begeleidingscommissie Hartchirurgie Nederland. Zorg om het hart: Hartchirurgie en PTCA de tachtiger jaren. Raming van de behoefte voor 1990 en 1991 . Hart Long Instituut. Academisch Zlekenhuis Utrecht: 7989 (1n Dutchy.

7. de Kok H. A new techuque for resecting the non-inflamed not-adhesive appendix through a mini-laparotomy with the aid of the laparoscope. Archivum Chirurgicum Neerlandicum (1977)29:195-8 (in Dutch).

8. de Kok $H$. The laparoscopic mini-appendicectomy. Acta Endoscopica (1983)1:3:347-52.

9. Mathus Vliegen EMH. Tytgat G. Laser photocoagulation in the palliation of colorectal malignancies. Cancer $(1986) 57.2212-6$

10. Semm K. Endoscopic appendectomy. Endoscopy 1983(15):59-64.

11. Wastell C. Laparoscopic cholecystectomy, better for patients and the health service. British Medicall Jouma: $(1991) 302: 30=1$. 


\section{Appendix 14.1 Semi-structured questionnaire for country case-studies}

1 Is (case) established as routine therapy in (country)?

2a (If yes) Please give a brief history of how (case) came to be established.

Was evaluation an important part of this process?

Which medical leaders were influential?

Were there any national conferences that encouraged diffusion?

Were there any policy initiatives that encouraged diffusion?

2b (If no) Please give a brief history of how (case) has come to be used in (country). Which factors were most important in delaying its diffusion and acceptance?

3 Do you think the technology underlying (case) is adequate for its routine use? if not, what changes or improvements are needed?

4 Do you think the evidence of safety and effectiveness is adequate to support more widespread use?

5 Is there evidence of cost-effectiveness of (case)?

6 Is there a medical consensus concerning (case)? Is there resistance to its adoption? If so, why do you think there is resistance?

7 Are the decision-making structures in your institution adequate to support (case)? What changes could be made?

8 Are resources available to support (case)? What is available, and what is not available?

9 Does (case) require special skill? Is education and training available to address this problem?

10 What types of community care are there to support (case)?

11 What policy factors need to be changed in relation to (case)?

12 Is reimbursement available for (case)? Is it a problem?

13 Are there any legal barriers or problems that prevent (case) from spreading?

14 is malpractice a potential problem?

15 Has the media played any role - positive or negative - in the diffusion of (case)?

16 Do patients accept (case)? What objections do they have? 


\section{Diffusion of minimally invasive therapy in Europe}

H.David Banta' and Hindrik Vondeling ${ }^{2}$

' The Netherlands Organization for Applied Scientific Research (TNO),

Division Prevention and Health

Leiden, the Netherlands

2 Department of Health Economics

University of Limburg

Maastricht, the Netherlands

Originally published as: Diffusion of mintmally invasive therapy in Europe, Health Policy 1993(23):125-33. Reprinted with permission of the publisher.

\section{Summary}

This paper considers the diffusion in Europe of the 10 cases presented in Chapter 14. Most of the cases have diffused relatively slowly. This slow diffusion can be explained by a number of factors, including budgetary restraints, financial incentives, physician conservatism, and lack of appropriate \#raining. A consistent thread in the cases is the argument that evidence of effectiveness is not convincing. The article considers whether further diffusion of each innovation can be justified from existing evidence. This information is important for policy purposes. Politics might be developed to encourage any potentially important innovation.

Key-words: Diffusion, Minimally invasive therapy, Incentives "Health Policy, Europe 


\subsection{Diffusion of the specific cases}

\section{Laser treatment of bladder tumours}

Laser treatment was developed in the late 1970s. Despite an accumulating experience of more than 10 years, none of the countries described in this volume have a consensus that the laser should replace traditionall (TUR) surgery'.

In this case diffusion is to a large extent hampered by a perceived lack of clinical evidence and budgetary pressures. Budgetary pressures make it difficult to acquire lasers and do not reward: those who use lasers. Therefore, it would be beneficial if governments would support clinical situdies of laser therapy versus TUR in this area. If effectiveness is equivalent, the advantages of the less invasive technique are obvious. Budgetary pressures could then be overcome by specific or general changes in reimbursement.

\section{Extra-corporeal shock wave lithotripsy (ESWL)}

ESWL has been used since 1982 and has diffused relatively rapidly in most countries. ESWL has been in competition with another less invasive procedure, percutaneous nephrolitotomy (PCN). since its beginning.

In this case, a number of countries, including Denmark, the UK, France, Germany and the Netherlands, attempted to constrain diffusion of ESWL for financial reasons ${ }^{18 i}$. All essentially failed, largely because of political and patient pressures. The rates of ESWL machine by population in a number of European countries can be seen in Table 15.1. However, in Denmark and France the constraint was applied to allow time for the national industry to produce its own machine, and these policies were relatively successful for this purpose.

Governments also failed in attempts to regulate ESWL because of private sector involvement. in all European countries studies, private funds were used to purchase lithotripters.

The availability of two procedures for treating stones and the availability of a relatively high number of ESWL machines has resulted in a relaxation of indications for treatment. More and more therapy is applied for small, asymptomatic stones and the total number of procedures has rapidly increased. This case, and others, has caused policy-makers to be cautious in approaching technologies associated with less invasiveness.

\section{Laparoscopic treatment of endometriosis and ovarian cysts}

Laparoscopic diagnosis has been used in gynecology since the 1960s, ad treatments have been done through the laparoscope almost since the beginning. Still, little evaluation of these procedures has been done, and they have diffused quite slowly.

Laparoscopic treatment is now beginning to be accepted as an alternative to conventional gynecological therapy in all European countries studies. However, diffusion is hampered especially by financial incentives. Acquiring the equipment, including lasers, is difficult in a situation of fixed budgets. Furthermore, payment for the procedure can be difficult to obtain. Finally, most gynecologists have not been trained in laparoscopy, so acquiring the skill with the procedure can be difficult. Usually, the surgeon must pay for special training him or herself.

There is little patient demand in most countries for laparoscopic procedures. This probably explains why general surgery has come to the laparoscope much later, but now accepts it much faster than gynecologists do. The case of laparoscopic cholecystectomy, discussed next, has stimulated gynecologists to become more involved in laparoscopic therapy. 


\begin{tabular}{lcccc}
\hline Country & \multicolumn{3}{c}{$\begin{array}{c}\text { Machines } \\
\text { (number) }\end{array}$} & $\begin{array}{c}\text { Machines } \\
\text { (per million population) }\end{array}$ \\
\hline Belgium & 1985 & 1988 & 1990 & $(1990)$ \\
F.R. Germany & 0 & 11 & 12 & 1.2 \\
Spain & 7 & 57 & 72 & 1.2 \\
Italy & 0 & 34 & 50 & 1.3 \\
Denmark & 6 & 48 & 69 & 1.2 \\
Greece & 0 & 2 & 3 & 0.6 \\
The Netherlands & 0 & 5 & 10 & 1.0 \\
Sweden & 1 & 8 & 11 & 0.8 \\
France & 1 & 5 & 6 & 0.7 \\
Ireland & 2 & 29 & 36 & 0.7 \\
Portugal & 0 & 2 & 2 & 0.6 \\
England & 0 & 3 & 4 & 0.4 \\
\hline \hline
\end{tabular}

Source: Reference ${ }^{[8]}$.

\section{Laparoscopic general surgery}

As mentioned above, general surgeons have been significantly slower than gynecologists to accept laparoscopic treatment. However, the field is now beginning to grow rapidly. The main force seems to be consumer demand, fueled by information from a few surgical innovators.

The first successful cholecystectomy via laparoscope was done by $P$. Mouret in Lyon, France in the Spring of 1987. Initially, the procedure spread in France, and in 1988, particularly after publications of the experience of the group of Dubois ${ }^{(4)}$ it began to spread internationally. The procedure seems to be spreading rapidly into use in a number of countries, but particullarly in the America ${ }^{[2]}$. The first procedure was done in the United States in $1988^{110]}$, but the most rapidi spread has occurred in 1990. A survey of teaching hospitals in Canada in late 1990 showed that more than two thirds of the 29 hospitals that responded were already in the laparoscopic cholecystectomy business $^{[3]}$. Surgeons in the United States and elsewhere have been skeptical initially; the development has been almost entirely consumer driven ${ }^{[2]}$. In other words, patients are demanding the less invasive procedure.

Now other procedures, such as appendectomy, hernia repair, and bowel resection, are beginning to be done by laparoscope.

Diffusion of laparoscopic cholecystectomy is beneficial and proceeds rapidly. However, this may result in treatment of patients by physicians who are insufficiently trained and skilled. It would therefore be preferable if the government would stimulate and at the same time guide the diffusion of the technique, for example by selecting centers offering a minimum number of patients, which enables physicians to maintain skills at a sufficient level. In a second phase of the diffusion of the procedure, it could be stimulated in smaller hospitals. In this way, the potential of the procedure, which is estimated to become first choice of treatment in $80 \%$ of all cholecystectomies, could be realized while assuring quality of care. 
Another problem in this area is similar to that with ESWL organs may be removed unnecessarily. This is especially likely when diagnostic laparoscopy is being done. For example, Nezhat and Nezhat ${ }^{[9]}$ describe incidental' appendectomies, removal of a normal appendix, during diagnostic gynecological laparoscopy.

\section{Catheter treatment of coronary artery disease}

Coronary artery bypass grafting (CABG) was introduced in 1967, followed by percutaneous transiuminal coronary angioplasty (PTCA) in 1979. These two procedures are now standard in all countries studied. The figures in Tables 15.2 and 15.3 canfirm that Denmark is behind other similar countries in implementing cardiac surgery, and that all European countries do these procedures at lower rates than is true in the United States ${ }^{[113}$.

PTCA and CABG have been subject to unsuccessful attempts to limit the annual number of procedures. Informants criticize government for attempting to restrict numbers of procedures without attention to quality issues. The prevailling impression among policy makers in Europe, at least in high rate countries such as Germany and the Netherlands, is that the procedures are overused. However, in the absence of more definitive evaluation, it is difficult to define appropriate use. Regulation has also failled in this case because of patient demand and resulting political pressures.

Table 15.2 Numbers and rates (per million population) of coronary artery bypass grafting (CABG) in different EC-countries and the United States, 1985 and 1989

\begin{tabular}{lcccc}
\hline Country & $\begin{array}{c}\text { Number } \\
(1985)\end{array}$ & Rate & $\begin{array}{c}\text { Number } \\
(1988)\end{array}$ & Rate \\
\hline Belgium & 3340 & 340 & - & - \\
Denmark & 414 & 83 & - & - \\
France & 5900 & 110 & 8280 & 583 \\
Italy & 8415 & 150 & - & \\
The Netherlands & 6800 & 480 & - & 480 \\
Spain & 5230 & 140 & 29000 & \\
England & 10840 & 195 & 206 & 1265 \\
F.R. Germany & 12460 & 20600 & \\
United States & & & 780 & 291000 \\
\hline \hline
\end{tabular}

Source: Reference $e^{[11]}$. 
Table 15.3 Numbers and rates (per million population) of percutaneous transluminal coronary angioplasty (PTCA) in different EC-countries and the United States, 1985 and 1989

\begin{tabular}{lcccc}
\hline Country & $\begin{array}{c}\text { Number } \\
(1985)\end{array}$ & Rate & $\begin{array}{c}\text { Number } \\
(1988)\end{array}$ & Rate \\
\hline Belgium & 1175 & 120 & 3400 & 344 \\
Denmark & - & & 350 & 68 \\
France & 3480 & 60 & 18000 & 324 \\
Italy & - & & 2500 & 44 \\
The Netherlands & 2556 & 190 & 6400 & 428 \\
Spain & - & & 1850 & 48 \\
England & - & & 6450 & 113 \\
F.R. Germany & 4490 & 90 & 18800 & 307 \\
& & & & 250000 \\
United States & 98100 & 470 & & 1100 \\
\hline
\end{tabular}

Source: Reference ${ }^{[11]}$.

\section{Laser palliation of colon cancer}

Laser palliation of colon cancer began slowly in the late 1970s. It has slowly spread in the countries studied. Part of the slow spread is explained by surgeon resistance to a procedure done by a medical specialist (gastroenterologist) The result is that generally only patients considered unsuitable for open surgery are referred for laser surgery.

The relatively small number of patients treated may justify onlly one national center in countries such as the Netherlands. On the other hand, the need to travel long distances, especially when the patient's condition is poor, sometimes prevents patients from receiving laser therapy. This is an argument in favor of regionalization of treatment. The efficacy of treatment depends greatly on the skill of the operator, and must begin with skill in endoscopy. Then expertise with the laser must be acquired. Also, kills can only be maintained if the experience is continuous. Therefore, and again considering the relatively small numbers of patients, a limited number of centers should be enabled to provide laser palliation.

The government could encourage developments in this area by establishing both technical and clinical standards for laser use. A system of quality assurance that was accepted and supported by all laser-using gastroenterologists could save considerable health care resources.

\section{Endoscopic treatment of upper gastroüntestinal (UGI) bleeding}

Although this case has been in use for more than 10 years, and has been well-evaluated, it still does not seem to be in routine use. The literature contains reports from community hospitals that they have only recently accepted the innovation ${ }^{(s)}$. In some countries, such as France, it is not fully accepted as routine therapy. The international literature indicated that death rates from UGI bleeding have not fallen in recent years ${ }^{128}$.

Financial constraints prevent some hospitals from buying lasers, and training for endoscopic therapy is also a problem in this case.

\section{Arthroscopic knee surgery}

The arthroscope was introduced in the 1970 s and was established as a diagnostic technology by 1975 or so $^{[6]}$. It has gradually become more and more of a therapeutic tool, in association with video-imaging, fiber-optics, and more precise instrumentation. Currently, about $80 \%$ of arthroscopic 
procedures are performed on the knee (removal or repair of the meniscus, the knee cartilage is the most common procedure), although arthroscopic treatment of a number of other joints is becoming increasingly common.

Despite an international consensus that arthroscopic knee surgery should replace open knee surgery, it has not. The main reason is probably that those doing knee surgery were not trained in arthroscopic techniques. It is difficult for practising surgeons to get the training necessary to change their routines

\subsection{Diffusion in relation to effectiveness and cost-effectiveness}

From a policy point of view, it is interesting to combine the data on the diffusion of the selected cases to the information on the effectiveness and cost-effectiveness of each case, as reported by Banta $^{11}$. This information can be used to judge the desirability of the diffusion of each case. This judgement can be compared to the diffusion of each case in the real world'. Any discrepancies can either result in policy recommendations to encourage, to discourage or otherwise guide the diffusion of a specific case, using the single two by two matrix in Table $15.4^{[13]}$.

Table 15.4 The decision whether or not to encourage diffusion

\begin{tabular}{rr|cc}
\hline \hline \multirow{2}{*}{ Encourage diffusion } & \multicolumn{3}{|c}{ Effective and cost-effective } \\
\hline & Yes & Yes & No \\
& No & A & B \\
\hline
\end{tabular}

In an ideal world a new technology would not be widely diffused until there was firm evidence of its effectiveness and cost-effectiveness. In that ideal world with its perfect information, no technologies would fall into boxes B or C. In reality information is imperfect; it is not certain whether technologies are effective and cost-effective, and it is usually a matter not of precluding diffusion or enforcing it, but of encouraging or discouraging of the technology given current imperfect information. The process involves balancing the risk of types I and II errors, as a result of imperfect information. A type I error leads to the encouragement of the diffusion of applications which are, in fact, inferior to existing modalities (Box B), and a type II error, leads to the discouragement of diffusion when the application, in fact, is effective and cost-effective relative to existing treatment $(\text { Box C) })^{[13 !}$.

We applied this simple model to the data avallable in this study. First the current diffusion pattern of each case was assessed, distinguishing three situations: a case has either (1) hardly diffused in the health care system or (2) diffused slow or (3) diffused rapid. Secondly, a judgement was made on the desirability of additional diffusion in each case. Table 15.5 shows that (curative) laser treatment of bladder tumours, laparoscopic cholecystectomy, laser treatment in palliation of colon cancer, treatment of upper glastrointestinal bleeding by endoscopic intervention and arthroscopic knee surgery have nol yet diffused as widely as can be desired. Considering the evidence, only a low probability of a type lerror would be associated with stimulating the diffusion of these cases

However, cancer therapy and palliation raise particular problems. In the case of cancer palliation, which is not expected to result in an extension of the life expectancy of the patient, the quality of life over the entire remaining life span is important. In the case of cancer treatment aimed at curing the patient, long-term

follow-up is necessary to assure that cancer did not recur more frequently when applying a new technique. In the particular cases of curative laser treatment of superficial cancer and, to some extent, laparoscopic removal of ovarian cysts, many specialists feel that complete pathological examination is very important. To clarify such issues will require relatively large studies carried out 
over long periods of time, which means that applications in cases of camcer will probably be slower to be accepted. An individual approach to each case seems to be indicated

Three cases, laparoscopic treatment of endometriosis, laparoscopic removal of ovarian cysts, and laparoscopic appendectomy, have not been evaluated to an extent that wartants encouragement of diffusion, without at the same time accepting a realistic chance of a type ll error.

The final two cases have diffused widely in the health care system: ESWL (and PCN) and catheter treatment of coronary artery disease. Any further diffusion of these cases is undesirable.

Table 15.5 comparison of the actual and desirable diffusion of ten cases of minimally invasive therapy

\begin{tabular}{|c|c|c|c|c|c|c|}
\hline \multirow{2}{*}{ 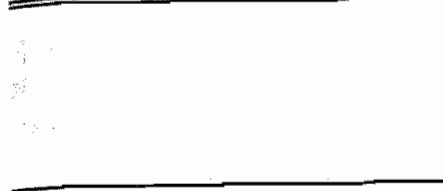 } & \multicolumn{3}{|c|}{ diffusion "in the real worlld" } & \multicolumn{3}{|c|}{$\begin{array}{l}\text { judgment on } \\
\text { desirability of } \\
\text { additional diffusion }\end{array}$} \\
\hline & hardly & slow & rapild & positive & neutral & negative \\
\hline $\begin{array}{l}\text { laser treatment of } \\
\text { bladder tumours }\end{array}$ & & $\mathrm{x}$ & & $\mathrm{x}$ & & \\
\hline $\begin{array}{l}\text { extra-corporeal shock wave } \\
\text { lithotripsy and percutaneous } \\
\text { nephrolithotomy }\end{array}$ & & & $x$ & & & $x$ \\
\hline $\begin{array}{l}\text { laparoscopic treatment of } \\
\text { endometriosis }\end{array}$ & & $x$ & & & $x$ & \\
\hline $\begin{array}{l}\text { laparoscopic removal of } \\
\text { owarian cysts }\end{array}$ & $x$ & & & & $x$ & \\
\hline laparoscopic cholecystectomy & & & $x$ & $x$ & & \\
\hline $\begin{array}{l}\text { laparoscopic } \\
\text { appendectomy }\end{array}$ & & $x$ & & & $x$ & \\
\hline $\begin{array}{l}\text { catheter treatment of } \\
\text { coronary artery disease }\end{array}$ & & & $x$ & & & $x$ \\
\hline $\begin{array}{l}\text { laser treatment in } \\
\text { palliation of } \\
\text { colon cancer }\end{array}$ & $x$ & & & $\mathrm{x}$ & & \\
\hline $\begin{array}{l}\text { treatment of upper } \\
\text { gastrointestinal bleeding by } \\
\text { endoscopic intervention }\end{array}$ & & $x$ & & $\mathrm{x}$ & & \\
\hline $\begin{array}{l}\text { arthrosicopic knee } \\
\text { surgery }\end{array}$ & & $x$ & & $x$ & & \\
\hline
\end{tabular}

\subsection{Other important factors in diffusion}

Perhaps the most important factor in diffusion of innovations such as MIT in European health care systems is the payment system. All countries examined in this study now have some sort of budget caps or prospective budgeting system for hospitals intended to limit hospital expenditures. Most of these have been implemented during the 1980 s.

The budget limitations are intended to force choice. The hope of policy-makers seems to have been that physicians and administrators would choose wisely in a situation of limited resources. The result does not seem to be so optimal. Without some sort of pressure, people continue to do 
what they were already doing In many cases, this is justified by the lack of evaluative studies. But governments that have limited budgets have been slow to increase budgets for evaluation. Where is the basis for choice?

As for physician specialists, they are quite free in most European countries to provide whatever treatment they find appropriate. There are limited requirements for training or experience before Using new tools such as endoscopes. On the other hand, this is a disadvantage, because training is not well-developed, which it might be if such requirements existed. Some physicians do not take up new procedures because of the difficulty in learning how to do them. Others take them up without appropriate preparation, and undoubtedly harm patients. In the countries studied, academic specialists seem generally conservative and skeptical concerning this new area. Many of this innovators practice in private hospitals or clinics.

The most important force facilitating the diffusion of MIT is patient demand, as has been seen in the cases of PTCA, ESWL, arthroscopic surgery, and laparoscopic cholecystectomy. Press reporting has fostered patient demand and physician interest, often to good effect, but not always.

\subsection{Conclusion}

Examination of the 10 cases in the European study illustrates the importance of financing of European health services as a force influencing innovation. Now some countries are beginning to understand the effects of their financing system and are discussing change. For example, in Germany it has been recognized that the system gives no incentive for shorter hospitalization, and lump sum payments are being discussed ${ }^{[?]}$.

Overall, the study shows that evaluation plays little role in choices in health care. In a time when the term 'cost-effectiveness' has become a faddish idea, this is a bit surprising. Certainlly, the lack. of policies toward evaluation in most countries is a partial explanation.

More evidence on the effectiveness of these procedures is needed. Governments could support additional efforts in evaluation. They could also link reimbursement to evaluation results, which could lead to decreased or increased use, as was appropriate. Developments in this direction in the Netherlands, France, and the UK seem constructive. Other countries need to become more involved in such activities. 


\subsection{References}

1. Banta HD. The cost-effectiveness of 10 selected applicalions in Minimally Invasive Therapy. Health Policy (1993), 23:135-51.

2. Cameron J, Gadacz T. Laparoscopic cholecystectomy. Annals of Surgen 1991/213):1-2.

3. Canadian Coordinating Office for Health Technology Assessment (CCOHTA). CCOHTA Update. Ottawa, Canada January 1991.

4. Dubois $F$, lcard $P$, Berthelot $G$ Levard $H$. Coelioscopic cholecystectomy, preliminary report of 36 cases. Annals of Surgery(1990)211:60-2.

5. Fullarton $G_{*}$, Bimie $G_{\text {, }}$ MacDonald $A$, Murray $W$. The effect of introducing endoscopic therapy on surgery and mortality rates for peptic ulcer hemorrhage, a single center analysis of 1,125 cases. Endoscopy 1990(22):110-3.

6. Jackson R, DeHaven K. Arthroscopy of the knee. Clinical Orthopedics (1995) 107:87-92.

7. Keuchel L, Beske F. Minimally invasive surgery in the Federal Republic of Germany. Health Policy 1993(23):49-65.

8. Kirchberger $S$. The diffusion of health care technologies in practice: stone treatment in urology, the diffusion of two technologies (draft). Report for the EEC Study on the Diffusion of Medical Technology in Europe. Munster. Germany, Institut für Medizinische Soziologie, 1990.

9. Nezhat C, Nezhat F. Incidental appendectomy during videolaseroscopy. American Journal of Obstetrics and Gynecology (1991)165:559-64.

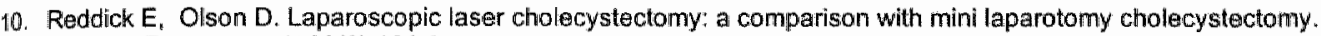
Surgical Endoscopy 1989(3):101-3

11. Rigter H, Bos M. Cardiovascular services in the Netherlands and Europe. In. Proceedings of the 12th Congress of the European Society of Cardiology, 1990.

12. Sacks H.S, Chalmers TC, Blum AL., Berrier J, Pagano D. Endoscopic hemostasis: an effective therapy for bleeding peptic ullcers. Joumal of the American Medical Association (1990)264:494-9

13. Sculpher M, Buxton M. Report of Phase 1 of the Medical Laser Technology Assessment. HERG Research Report No. 9. Health Economics Research Group, Brunel Universily, Uxbridge, United Kingdom, 1991. 


\section{Part Four - Case Studies}




\section{Evaluation of argon laser treatment of diabetic retinopathy and its diffusion in the Netherlands}

Hindrik Vondeling

Department of Health Economics, University of Limburg, PO Box 616, Maastricht, the Netherlands

Originally published as: Evaluation of argon laser treatment of diabetic retinopathy and its diffusion in the Netherlands. Health Policy 1993(23):97-112. Reprinted with permission of the publisher.

\section{Summary}

Argon laser treatment of diabetic retinopathy (DR) is the best evaluated case in the field of minimally invasive therapy. A well-organized randomized controlled trial was followed by formal cost analyses and cost-effectiveness analyses. Laser treatment of DR proved to be cost-effective in a situation where there was no satisfactory treatment previously. Subsequently, screening strategies for retinopathy were developed. Systematic screening for DR in diabetic popullations would be cost-saving from a societal perspective. The avallability of effective and cost-effective therapy and cost-saving screening strategies for DR warrants active policy making to stimulate the implementation of strategies to control retinopathy in diabetic populations. Such strategies would ideally include both guided diffusion of argon lasers and the organization of screening programs. Data from the Netherlands are used to illustrate the diffusion of argon lasers in health care. After a slow start, argon lasers have diffused widely in the Dutch health care system. This development is complemented by recommendations for screening of the European diabetic population, which have been issued last year. More active cooperation of all parties involved would benefit in preventing blindness from DR.

Key words: Diabetic retinopathy, Argon laser treatment, Effectiveness, Cost-effectiveness, Screening, Diffusion, Health policy, the Netherlands

\subsection{Introduction}

Epidemiological studies have shown a prevalence of diabetes mellitus in European populations of between 2 and $3 \%$. In 1991 in the United States a number of 12 million patients were estimated to suffer from diabetes mellitus ${ }^{[2 t}$. Diabetic retinopathy (DR) has been documented to be one of the most common long-term complications of diabetes mellitus ${ }^{471}$. Diabetic retinopathy refers to degeneration of the retina secondary to microvascular disease. Community screening programs in Australia have shown a prevalence of diabetic retinopathy in approximately $30 \%$ of the diabetic population ${ }^{[6]}$.

The prevalence of DR diepends very much on the length of time the patient has had diabetes. In a study of 996 insulin dependent younger onset diabetics in the US, Klein and colleagues ${ }^{19}$ found $17 \%$ of cases to have DR in those who have had diabetes for less than 5 years duration and $97.5 \%$ in those who have had the disease for over 15 years duration. The same authors ${ }^{1 / 91}$ also produced prevalence figures of 28.8 per cent ( 5 years) and 77.8 per cent ( 15 years) in those aged 30 years or more at the time of diagnosis. In contrast, proliferative DR is less common, occurring in 15.5 per cent of patients with diabetes of more than 15 years of duration ${ }^{[19]}$, but its implications are much greater. Briefly, it is characterised by new vessels appearing on the optic disc and/or retina - a 
process known as 'neovascularization'. If untreated about 50 per cent of patients with significal proliferative diabetic retinopathy will become blind within 5 years ${ }^{[7.4 \mathrm{t}}$.

It is estimated that 700000 Americans have proliferative retinopathy with a projected incidence 65000 new cases annually. Approximately 8000 new cases of blindness are caused annually b complications of diabetes ${ }^{[2 !}$. Overall, diabetic retinopathy accounts for at least $12 \%$ of the ne blindmess in the United States each year and it is the most common cause of blindness in the 45.7 year age group ${ }^{[5,21 !}$. In Europe, in the UK, diabetic retinopathy is the commonest cause of blin registration for individuals between the ages of 20 and 65 years ${ }^{[7]}$. And as many as $13-23 \%$ of th registered blind in Denmark have diabetes ${ }^{[1]}$.

Since the incidence of retinopathy increases with the duration of diabetes, and since improvement in diabetic care have resulted in prolonged survival of patients, DR may be expected to increas in the years to come. There is as yet no way of preventing the development of retinopath Therefore, emphasis has been placed on the treatment of its blinding complications. Obviously th blinding consequences of DR have a major effect on the quality of life of otherwise health individuals and their families and on health care expenditure $s^{[1.5]}$.

This article describes the development of treatment alternatives for $\mathrm{DR}$, and reviews evidence 0 effectiveness and cost-efectiveness of argon laser treatment of the disease. In addition, th development and cost-effectiveness of alternative screening strategies for DR in diabet populations is discussed. Subsequently " using data from the Netherlands, we focus on the diffusic of argon lasers in the health care system. This development is complemented by recommendation for screening of the European diabetic population, which have been issued last year. An importa goal of the screening program is to reduce new blindness due to diabetes by one third or more the next five years. Active support of all parties involved in the program seems to be required I reach this ambitious goal.

\subsection{Evaluation of effectiveness of alternative photocoagulation procedures in treatment of DR}

In 1855, just five years after the ophthalmoscope was develloped, von Jaeger described the fir case of retinopathy in a diabetic patient ${ }^{\prime \prime}$. More than 100 years later, xenon arc photocoagulatio developed and first described by Meyer-Schwickerath in 1959, was the first type photocoagulation used to treat patients with $\mathrm{DR}^{[2]}{ }_{\text {w }}$ The initial reports on the effectiveness of xenc arc photocoagulation were promising. The deve lopment of the ruby laser, in 1962, aroused intere in investigating its use for this purpose. The ruby laser proved to be poor in attempts to treat $D$ and other vascular conditions. The lack of effectiveness was attributed to an inappropria wavelength resulting in lack of absorbtion of the laser light by the retinal blood vessels. More rece work showed that the inadequacies of the ruby laser were not due to wavelength, but because th ruby laser was a slow laser. The instrument caused a high risk of choroidal hemorrhages. In 196 LEsperance documented the clinical potential of another laser, the argon blue-green laser, for th first time. Although subsequently numerous reports claimed a beneficiall effect in individuals trealt with photocoagulation "more than a decade later there was still considerable doubt within th ophthalmological community as to the safety and efficacy of this procedure ${ }^{[5,20]}$. The five be clinical studies, mone of which was an RCT, could not dispel this uncertainty ${ }^{20}$. In view of th magnitude of the public health problem of blindness from DR, the National Eye Institute of th National Institutes of Health organized the Diabetic Retinopathy Study (DRS) ${ }^{20 !}$. By that time xen arc photocoagulation was the mainstay of treatment in the US.

The DRS was the first randomized, controlled clinical trial to answer important questiol concerning DR. The DRS focused on the potential of two treatment alternatives in reducing the ra of occurrence of severe visual loss due to DR: argon laser photocoagulation and xenon a photocoagulation. Prospective design, careful adherence to protocol, masked measurements visual function, and ongoing statistical monitoring of the results led to the success of the study The study was prepared for in 1971. Between 1972 and 1975, 1758 patients were recruited 
fifteen centers. Eligible patients had proliferative diabetic retinopathy in at least one eye, or severe nonproliferative retinopathy in both eyes. As severe visual loss was defined as visual acuity less than $5 / 200$ at a visual acuity chart at two or more consecutively completed follow-up visits, visual acuity of $20 / 100$ or better in both eyes was required to be eligible to the study. One eye of each patient was randomly assigned to immediate photocoagulation and the other to follow-up without treatment regardless of the course followed by either eye. The eye chosen for photocoagulation was randomly assigned to either of two treatment technicues, one using the argon laser and the other using the xenon arc photocoagulator. Patients were followed for four years at four-month intervals. Patient follow-up was completed in 1979.

In 1976 an interim-analysis of results was published ${ }^{[0]}$. The demonstrated benefit of photocoagulation was enormous. Photocoagulation was found to reduce the risk of severe visual lass by $50 \%$ or more ${ }^{[20]}$. The protocol was then changed to allow photocoagulation of eyes initially assigned to the untreated control group, in which the risk of severe visual loss clearly outweighed the risk of harmful effects of treatment. In these eyes, at least three out of four identified risk factors had to be present: 1) presence of vitreous or preretinal hemorrhage 2) presence of new vessels, 3) location of new vessels on or near optic disc, and 4) severity of new vessels ${ }^{10 \text { ] }}$.

In 1981 a final analysis was published ${ }^{(s)}$, confirming the earlier findings. The difference in rates of severe visual loss between treated and control eyes appeared to be somewhat greater in the xenon than in the argon group, but the difference in treatment effect between argan and xenon groups was small, its statistical significance borderline, and its clinical importance was considered to be outweighed by the greater harmful effects observed following the DRS xenon technique. Twentyfiwe per cent of xenon-treated eyes suffered a modest loss of visual field and an additional $25 \%$ a more severe loss. In the argon group, $5 \%$ of eyes showed some constriction of visual field to the large test-object used ${ }^{[\theta]}$. The authors estimated that a persistent visual acuity decrease of one line on a visuall acuity chart could be attributed to treatment in $19 \%$ of xenon-treated eyes and a persistent decrease of two or more lines in an additional $11 \%$. Comparable estimates for the argon group were $11 \%$ and $3 \%$, respectively.

The clinical application of DRS results to eyes with high-risk characteristics was straightforward. The risk of severe visual loss without treatment substantially autweighed the risks of photocoagulation, particularly those observed following the DRS argon technique, and prompt treatment was considered to be usually advisable ${ }^{|8|}$

For eyes with severe (advanced) nonproliferative retinopathy or proliferative retinopathy without high-risk characteristics (mild proliferative retinopathy), a number of important questions remained unanswered ${ }^{[8]}$. One question that naturally arose was whether early treatment, that is, before the development of high-risk characteristics of DR, would be more effective than delayed treatment. Another question was whether or not photocoagulation would be effective in treatment of macular edema. These and other questions were addressed by another randomized controlled clinical trial, the Early Treatment Diabetic Retinopathy Study (ETDRS). In the period 1980 to 1985 the ETDRS research group enroled 3928 diabetic patients with early proliferative retinopathy, moderate to severe non-proliferative retinopathy, and/or diabetic macular edema in each eye. In 1985, first results of the ETDRS were published, focusing on treatment of macular edema ${ }^{\mid 13 !}$. In contrast to the complications of proliferative retinopathy, which occur relatively sudden and dramatic macular $_{s}$ edema accounts for milder visual disability, which is slowly progressive. in this part of the ETDRS, 754 eyes that had macular edema and mild to moderate DR were randomly assigned to focal argon laser treatment, while 1490 such eyes were randomly assigned to deferral of photocoagulation. The results indicated that eyes with clinically significant macular edema, defined as retinal thickening that involves or threatens the center of the macula, should receive prompt focal laser treatment. The results also indicated that eyes with macular edema that is not clinically significant can be watched and treated if the edema progresses to the clinically significant category. A later report of the ETDRS group, published in 1991, confirmed these findings ${ }^{|4|}$. This report also showed that early treatment, compared with deferral of photocoagulation, was associated with a small reduction in the incidence of severe visual loss, but 5-year rates were low in both groups (2.6 and $3.7 \%$, respectively). 
In summary, the DRS and ETDRS demonstrated that argon laser photocoagulation is high effective in treatment of diabetic retinopathy and macular edema. More specifically, th accumulated evidence indicates that laser photocoagulation is effective in preventing blindnes from proliferative retinopathy in at least $70 \%$ of cases, and there is follow-up evidence tha stabilization is achieved over at least ten years. Even in maculopathy, where results are les striking, maintenance of vision is possible in $60 \%$ of patients ${ }^{4 !}$.

\subsection{Cost-studies and cost-effectiveness analyses of argon laser treatment of DR}

Drummond ${ }^{(12)}$ reviewed the cost-effectiveness of laser applications in ophthalmalogy. He describe that The National Eye Institute estimated savings resulting from the Diabetic Retinopathy Stud (DRS). At the time of publication of the first results of the trial in 1976 , there were an estimated 11 500 people with advanced diabetic retinopathy in the US, of whom 85000 could be treated. It wa estimated that in the absence of treatment, 30000 of these would become bind by 1982 . Th costs of those 30000 blind people, assuming no treatment, were estimated as US $\$ 757.5$ million made up of $\$ 547.5$ million in lost wages, $\$ 150$ million in social welfare benefits, $\$ 30$ million medical assistance costs, and $\$ 30$ million in the cost of rehabilitative services (quoted Drummond ${ }^{(19 y)}$. The main problem in this 'cost-of-illness' approach is that it implicitly assumes tha no patients would be treated in the absence of the trial and that all patients would be treale following the publication of a positive trial result.

In his review Drummond ${ }^{(22)}$ refers to research by Drummond et al." ${ }^{1111}$, analyzing the costs an benefits of the DRS, using a decision-analysis framework. A decision tree was constructed showin the sequence of possible events that could have occurred for a person having proliferative diabeti retinopathy (PDR). Since laser photocoagulation was already in use, the main impact of the DR: would be to change the probabilities of key events. The key assumption in the analysis is that th DRS changed the probability of losing vision of those at high risk from diabetic retinopathy fron 0.75 to 0.50 . The cost of the DRS was US $\$ 10.5$ million. Using best guess assumptions, the ne savings of the trial for US society were found to total $\$ 2816$ million over 22 years use of lase photocoagulation, including the savings from averted lost production, or $\$ 2.31$ million if thes benefits were excluded. The benefits in relation to costs were not calculated for the best gues assumptions, since there were net financial savings from the trial, an unusual and highly desirabl outcome. However, it was calculated that even under pessimistic assumptions the net cost woul be small in relation to the benefits in vision years gained.

Based on the availability of effective treatment, Javitt et al. ${ }^{[17]}$. published an analysis of the cost effectiveness of argon laser treatment of diabetic retinopathy in patients with type I diabete mellitus in the US. The authors used a computer model that was constructed using a prospectiv population health event tabulation system. This program, based on Monte Carlo techniques analyzed events and costs incurred during the lifetime course of an irreversible chronic diseasi while considering each patient as a separate individual. A cohort of patients in a specific age grou in whom type I diabetes mellitus develops in a single calendar year, was entered into the model These individuals are subject to three ongoing risks: 1) the risk of proliferative retinopath developing with subsequent severe vision loss (visual acuity $<5 / 200$ ), 2) the risk of macular edemi developing with subsequent loss of reading and driving vision (visual acuity $<20 / 80$ ), and 3 ) the ris:

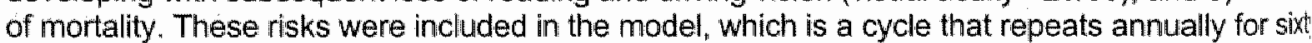
years. By then the model predicts a $95 \%$ mortality in the diabetic cohort.

The cohort first enters the 'mortality module' of the cycle, in which an annual mortality risk i assigned to each individual based on age and level of retinopathy, as documented by population based studies. All survivors pass on to the 'disease progression module' in which the likelihood o progressing to higher levels of retinopathy is assessed based on duration of diabetes, as observe in the DRS and the ETDRS. All members of the cohort then pass through the "screening an treatment' module in which screening visits are allocated according to existing recommendation 
of the Public Health Committee of the American Academy of Ophthalmology. According to these recommendations screening is assigned based on duration of disease and state of rethopathy. Treatment recommendations and treatment efficacy were drawn from the reports of the DRS and the ETDRS. Treatment is performed the year in which the conditions first develop. Once applied, treatment is protective against vision loss based on the treatment efficacy, as documented by the DRS and the ETDRS. The cohort then enters the next model year and the cycle is repeated as long as there are surviving members of the cohort.

The model was run for a cohort of an estimated 31,000 insulin-dependent diabetics, newly diagnosed at 12.5 years of age in one calendar year. In the 1988 and subsequent articles ${ }^{13.461}$ "the results were analyzed in the form of a cost-effectiveness analysis. In case of argon laser photocoagulation the cost-effectiveness ratio was expressed in terms of costs per person-year of vision saved. 'Severe visual loss' was defined to coincide with 'legal blindness', that is wisuall loss to the extent that makes patients eligible for disability payments. A similar ratio, noted as costs per person-years of reading and driving vision saved, was calculated for those individuals who could be prevented from doubling the visual angle by focal laser treatment for macular edema. This is equivalent to loosing three lines of wision on a visual acuity chart as measured in the ETRDS study. in this way the endpoints in the DRS and the ETDRS were used as a guide for endpoints in the model.

Charges for screening and photocoagulation of diabetics, which were taken from published Medicare data, were compared with federal budgetary expenses for blindness-induced disability under social security disability programs. Thus, in all articles, the authors analyzed treatment of diabetic eye disease from the viewpoint of the government agency or legislative body that is evaluating the relative expense of paying for blindness prevention versus paying disability benefits should blindness occur. All figures were represented in undiscounted 1986 dollars.

The model predicted that over a 60-year period, proliferative diabetic retinopathy, requiring panretinal photocoagulation, would eventually develop in $72 \%$ of type I diabetics and that macular edema, requiring focal photocoagulation, would develop in $42 \%$. If these treatments would be delivered as recommended in the clinical trials, the model predicted a cost of US\$966 per persionyear of vision-saved from proliferative retinopathy and US $\$ 1118$ per person-year of central acuity saved from macular edema. The average eligible blind person receives US $\$ 6900$ annually from sacial security, including aid to dependents. Thus the US\$ 966 cost per person-year of sight saved is only $14 \%$ of the direct cost to the Federal government of a year of blindness-rellated disability. The authors therefore concluded that the procedure is highly cost-effective.

Despite the use of interesting methodology the study of Javitt was judged to have a number of limitations $s^{[7]}$ : 1) it focused only on type I diabetics, 2) the screening element of the model was heavily simplified assuming $100 \%$ sensitivity and take-up 3 ) costs and outcomes were not discounted and 4) cost data was considered to be very rough and specific to the US. However, Drummond ${ }^{(11)}$ concluded that, while the analysis of Javitt et al. is not complete, in that it only examined one benefit and does not analyze all costs, the study is highly convincing in its conclusions.

\subsection{Rationale for and cost-effectiveness of alternative screening strategies for DR in the diabetic population}

In their first article, Javitt et al. ${ }^{[7]}$ employed recommendations for screening for DR from the American Academy of Ophthalmology. The rationale for screening is that clinical trials had shown that if high-risk DR and clinically significant macular edema are detected early, (cost)-effective lreatment can be provided. Although effectiveness of photocoagulation in preventing blindness from proliferative retinopathy is about $60-70 \%$ nowadays, it is generally accepted that an even befter success rate, up to $90 \%$ in cases with new vessels, could be obtained if patients reached ophthalmic care with less advanced lesions and ophthalmologists treated them early and adequately $y^{[1]}$. This conviction leads to the preference for the identification of patients at a 
presymptomatic stage, rather than, as is typically the case, diabetics being referred ophthalmologists" with advanced retinal changes and visual acuities so severely depressed th photocoagulation is ineffective ${ }^{\text {si }}$. The need for early detection and the evidence that a h proportion of patients are being referred too late for effective treatment resulted in calls systematic screening programs in the US and, among other countries, the UK. In this context American Academy of Ophthalmology issued screening recommendations which, as we have se were incorporated in the model by Javitt and colleagues. However, these screen recommendations had not been subject to a cost-effectiveness analysis. Given limited he: resources, however, choices have to be made about the most cost-effective means of screeni Therefore, in a second article Javitt et al. ${ }^{[15]}$, refined and extended their model to assess medical and economic implications of alternative screening programs for detecting and treal retinopathy in a type I diabetic population. As outlined earlier, the original model was criticized not discounting costs of screening and treatment and for assuming that ophthalmologi examination is $100 \%$ successfull in detecting disease. In the revised model the expenditures a savings were expressed in terms of their present net value, using a discount rate of $5 \%$. Based assumptions used in other studies and based on existing data, sensitivity of screening h estimated to be $80 \%$ and $100 \%$ for dilated ophthalmoscopy and fundus photography, respective Specificity, overall, was assumed to be as high as $97 \%$ and $100 \%$ for these methods, respective More specifically, based on existing evidence, in patients with no retinopathy, ophthaimoscopy assumed to result in a $0.3 \%$ false positive rate in detecting proliferative retinopathy. For patie with background or more severe retinopathy, the authors assumed a $5 \%$ false-positive rate. The model included five strategies of screening and treatment, all performed by ophthalmologist. Under all strategies, screening begins five years after the onset of type I diabet because there is little risk of proliferative retinopathy before that time. The model assumed that individuals with treatable retinopathy who are detected by screening will receive timely $a$ appropriate treatment. Although the authors confirmed that this is an optimistic assumption, is justified by stating that the purpose of the model is to determine potential benefit from alternal screening strategies. The frequency, sensitivity, specificity and cost of screening were specif at the start of each simulation. At the end of the simulation, a final calculation was made of pers years of reading vision saved and person years of severe vision loss prevented. Direct costs in analysis included the costs of screening and treatment. As standards of ophthalmologic care in US include a fluorescein angiogram for confirmation of proliferative retinopathy or macular ede before laser treatment, the costs of angiograms were included in the study. Since angiography by definition $100 \%$ specific in detecting proliferative retinopathy, the authors considered that th is little chance that a false-positive finding on initial screening would result in laser treatme Therefore, in the simulation, the consequence of a false-positive on screening was limited to cost of the fluorescein angiogram. Total costs of screening were divided equally over screening DR and macular edema. The price of treatment, based on national average charges to Medice was US\$2150. Government expenditures per year of blindness reflect only dollars that would paid from the federal treasury under social security programs, which was recalculated to amo US $\$ 6300$ annually for the average blind American. There were few good data on the likelihoodt a patient with vision loss from macular edema will be disabled. It was therefore assumed that 5 of individuals with untreated or unsuccessfully treated macular edema will be disabled, and that annual expenditure associated with vision loss from macular edema would be half of the aver: annual cost of total disability $(\$ 3150)$.

As noted above, benefits were expressed similarly to the first study. The benefit of treatment $v$ calculated as relative benefit compared with no treatment. Thus, although the effect of treatm is assumed to be permanent, treated patients were subject to continued, though reduced, ris vision loss. The results showed that all screening strategies were cost saving; the annual savil to the government varied from 62.1 to 108.6 million US $\$$, representing the discounted value savings in 1986 US $\$$ attributable to each annual cohort of patients with type I diabetes w! screening and treatment are provided. Between 71474 and 85315 years of sight and 76886 94705 years of reading vision could be saved in each cohort of such patients.

The results were subjected to an extensive sensitivity analysis. The value of a year of sight sai has the strongest effect on the predictions of the modiel. Screening and treatment of DR is $c$ 
sawing if the value of a year of sight saved is US\$2500 or more. At a value of $\$ 7500$ the strategy based on dilated ophthalmoscopy annually for all patients with no retinopathy and every 6 months for those with retinopathy becomes the most cost saving strategy as well as the most clinically effective. This strategy was roughly equivalent to the preferred practice pattern of the American Academy of Ophthalmology, which additionally calls for examination every 4 months in patients with macular edema and other signs of preproliferative retinopathy. This recommendation was not covered in the model but was expected to have little effect on the cost of care. The authors concluded that the simulation clearly demonstrates the enormous medical and economical benefits that may be realized by detecting and treating eye disease. It is cheaper to provide adequate eye care for patients with type I diabetes than to pay even the most minimal consequences of avoidable blindness.

Even though the availability of effective and cost-effective screening and treatment programs make the medical and economical argument for screening that leads to treatment a compelling one, Javitt et al. noted that there are substantial barriers to its universal implementation, resulting in a situation in the US where $25 \%$ to $50 \%$ of patients with 'high-risk' diabetic retinopathy are not receiving potentially sight-saving care. This situation motivated the authors to reanalyze the net federal budgetary savings achieved under current screening and treatment conditions and those that might be attained with increased enroiment of patients into appropriate ophthalmologic care ${ }^{[15]}$. For this purpose the model was refined again. The most important addition to the model was that under the prevaling screening and treatment strategies an estimate of $60 \%$ of the cohort meets the suggested guidelines for ophthalmic care, as issued by the American Academy of Ophthalmology. In addition, results from recently published clinical trials, especially the ETDRS, were incorporated in the model. Calculations, applied to a combination of data on age-related incidence rates and recent data of the American population, suggested that 17425 Americans develop Type I diabetes annually. Thus, the previous reports had overestimated the total annual incident cases by almost $80 \%$. Therefore, although the authors state that all conclusion remain valid, the absolute figures presented earlier are not realistic. All other assumptions of the model applied. Costs of screening and treatment were derived from the average Medicare charges of 1990 . The annual federal budgetary costs of blindness for patients younger than 65 years of age, based on social security programs, was US\$6615 on an annual basis. However, in this analysis also $\$ 150$ in tax expenditures, $\$ 3204$ in tax losses and $\$ 5236$ in Medicare and Medicaid payments made by the lederal treasury in 1990 to the average blind person with diabetes was included, predicting a total lederal expenditure of US\$15 205 annually for individuals younger than 65 years of age. Federal expenditures for persons 65 years of age and older were estimated at $\$ 484$ per year. All costs and savings were expressed in 1990 US\$, using a discount rate of $5 \%$.

The results of the simulation showed that at a $60 \%$ level of screening implementation 47374 person-years-sight and $\$ 101.0$ million dollar would be saved annually. Prevention of severe visual las from proliferative retinopathy accounted for $68.4 \%$ of sight savings, $79.4 \%$ of financial savings, and $61.6 \%$ of treatment costs. Persons with onset of Type I diabetes mellitus before age 30 accounted for $94.7 \%$ and $97.6 \%$ of total visual and financial savings, respectively.

Compared to the present situation, for any additional $10 \%$ of the cohort that enters into the screening, more than 7966 person-years-sight and $\$ 16.5$ million in further aninual savings would be acquired. The theoretical limits , with $100 \%$ screening and the previously discussed model assumptions, would be 79236 person-years sight and $\$ 167.0$ million in annual benefits. This represents a potential improvement over the current implementation of more than $65 \%$.

The authors concluded that the model demonstrates that it is of primary importance to recruit all possible patients into appropriate screening strategies. The expenditures represent both fruitful financial investments and appropriate medical care.

Adequate eye care includes both the availability of argon laser systems and the organization of screening strategies. Data from the Netherlands are used to illustrate the diffusion process. Then the implementation of screening strategies is discussed. 


\subsection{Diffusion of argon lasers in the Netherlands}

The diffusion of argon lasers in the Dutch health care system started in the early seventies. In diffusion was slow, with less than 10 lasers in use for a number of years. After 1978, argon las diffused more rapidly (Figure 16.1). In March 1992111 lasers (94 argon-lasers, 7 argon-dye lase 8 argon $Y$ YG lasers and 2 argon-Krypton lasers) were available in ophthalmological departmen corresponding with 1 laser per 136000 population ${ }^{123: 261}$. University hospitals were well-represen among the early adaptors of the technology. Nowadays all university hospitals $(n=8)$ have at le one ophthalmological argon laser at their disposal (Figure 16.2). In total 15 argon lasers have be installed in university hospitals.

In 1976, general hospitals started using argon lasers as well. Diffusion has been rather rapid general hospitals, resulting in a total number of 96 lasers in use in early 1992. The number hospitals using lasers is 93 , that is $68.4 \%$ of all general hospitals.

The number of argon lasers is expected to increase slowly in the near future, based on a sur of short-term investment plans ${ }^{\mid 23\}}$.

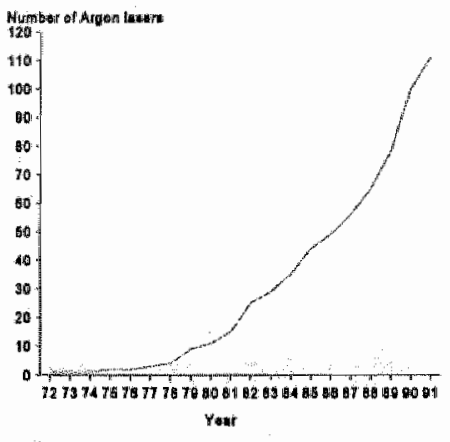

Figure 16.1

Diffusion of ophthalmological argonlasers

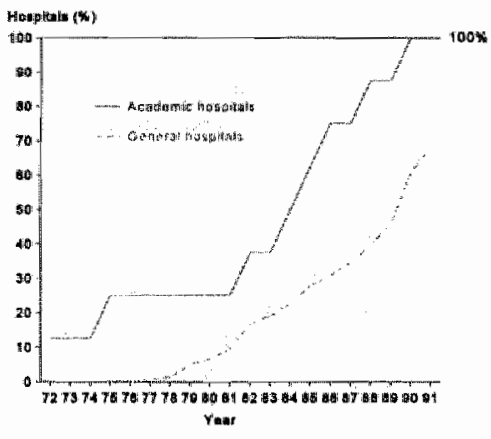

Figure 16.2

Diffusion of ophthalmological argonlasers in university hospitals and general hospitals 


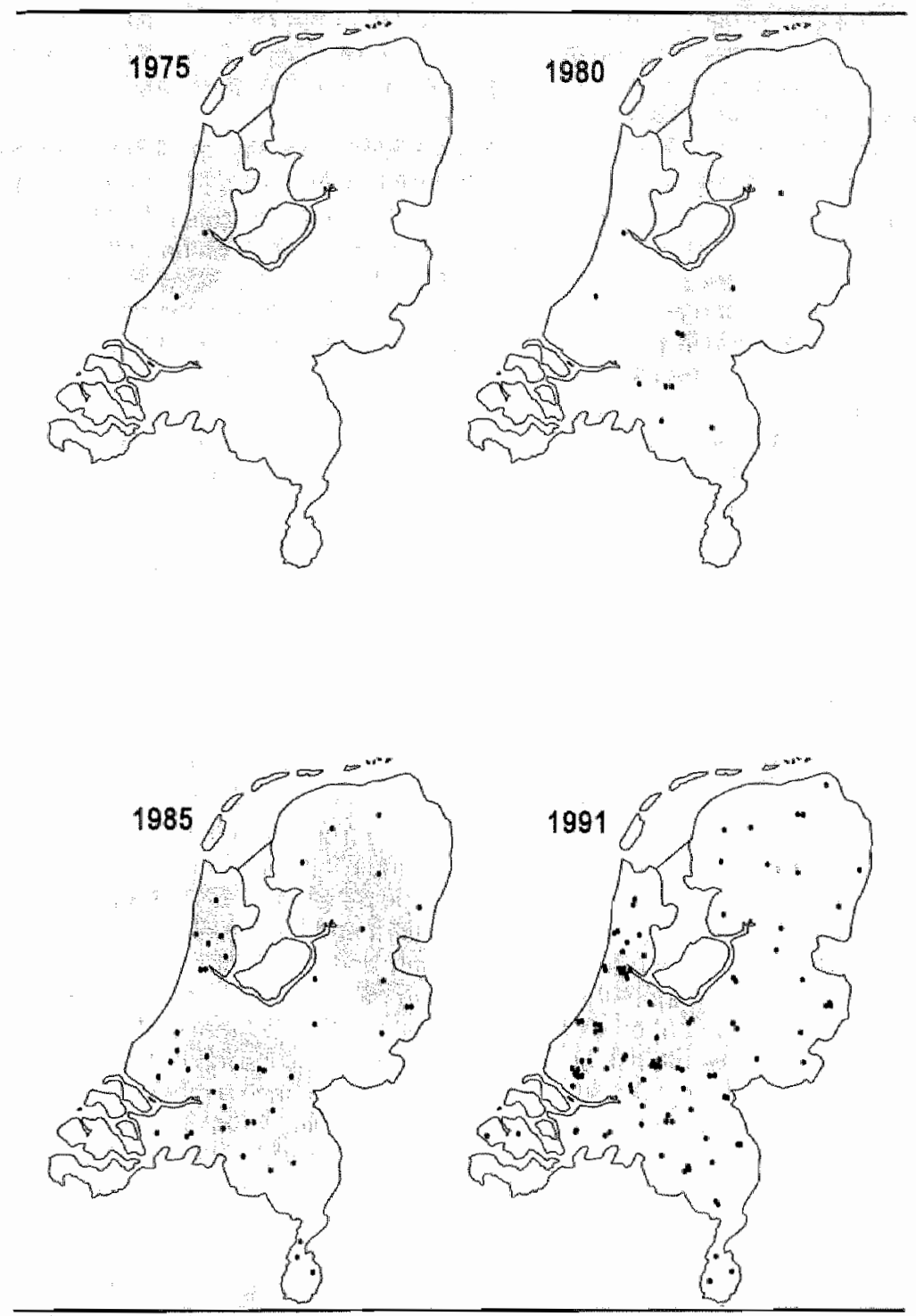

Figure 16.3 The geographic pattern of diffusion of ophthalmological argon-lasers in the Dutch health care system

Figure 16.3 shows that two hospitals in the western part of the country were the first to adopt an argon laser. These were university hospitals. Subsequently, the laser was adopted in centers in other regions of the country, showing an irregular pattern. Shaded areas in Figure 3 mark regions of the country that showed a relatively high speed of diffusion of argon lasers in a specific period of time. The innovative centers outside the western part of the country comprised both university and general hospitals. Nowadays the regional awailability of lasers to a large extent reflects the distribution of the population. 
Perhaps the irregular pattern of diffusion of the device in the Dutch health care system can be explained, at least partly, by the total absence of any policy regulations, most likely because this laser represents a "medium-ticket" technology. The capital costs of an argon laser are estimated to vary between US\$50 000 and $\$ 75000$.

No specific fees have been set for argon laser treatment. Actually, of all laser applications none is specifically reimbursed. Instead, most laser applications are billed as conventional procedures Argon laser treatment of DR is reimbursed under the heading 'coagulation of intraocular lesions: a label that has been in use at least since 1967. The fee was based on non-laser procedures using an operating room. Nowadays laser treatment is applied on an out-patient basis. Therefore, the fee is likely to be higher than the real costs of the procedure. This may have stimulated the diffusion of the device. The increase in the rate of diffusion of the argon laser after 1978 may have been fostered by publications on the DRS, in 1976 and 1981. In addition, laser treatment has become part of the training program of ophthalmologists since approximately ten years. Additional data on investment decisions of argon lasers support the impression that professionals have had a leading role in the introduction and diffusion of the technology ${ }^{\mid 231}$. It is unknown to what extent the xenon-arc photocoagulation technique and other techniques has spread in the health care system before the advent of the laser. It is likely that these treatment modalities have been gradually replaced by the argon laser. However, the latest Dutch textbook on diabetes mellitus makes mention of the use of the xenon-arc in treatment of diabetic retinopathy in a few centers ${ }^{[25]}$.

\subsection{Screening programs}

With respect to implementation of screening programs several initiatives have been launched in both the US and Europe. In the US, the American Academy of Ophthalmology started a long-term education project "Elimination of preventable blindness from diabetes by the year $2000^{\prime 21]}$. This program is intended to serve as a vehicle for translating clinical research findings more rapidly to the physicians responsible for the care of the diabetic patients. One of the project's key goals is to encourage effective partnerships between the patient and the patient's health care team. Also, the partnership between the primary care physician and the ophthalmologist takes on a high priority. Because diabetic retinopathy is often asymptomatic at its most treatable stages, the Diabetes 2000 program is intent on alerting physicians to the fact that early diagnosis and timely treatment can greatiy reduce the risk of visual loss. Proper application of the results of previously published studies and the recent results of the ETDRS should have a major impact on reducing the blinding consequences of diabetes. 'Diabetes $2000^{\prime}$ is paralleling a major diabetic retinopathy public information campaign by the National Eye Institute (NEI). The NEl's National Eye Health Education Program is fashioned along the lines of earlier federal initiatives against smoking and high blood pressure. The American Academy of Ophthalmology is a partner in this federal initiative.

In Europe, in 1990 the local regions of the World Health Organization and the International Diabetes Federation cooperated in producing a statement of intents with which governments of more than 30 countries acknowledged for the first time formally that diabetes is an important health and social concern and pledged themselves to implement policies to reduce its morbidity and mortality ${ }^{11}$. One of the targets of this statement, which has become known as the St. Vincent declaration ${ }_{n}$ is spelled out as reducing new blindness due to diabetes by one third or more in the next five years. As part of a strategy to reach the target, in 1991 an ad-hoc workshop was organised in London, intending to develop guidelines that could be acceptable and practical enough to work in all European countries, in spite of their differences ${ }^{|22|}$. The outcome was a protocol to screen for retinopathy and cataract and a set of guidelines for the treatment of retinopathy. The WHO has distributed these documents at the government level, in an attempt to encourage structured policies to screen for retinopathy. The protocol for screening for diabetic retinopathy in Europe recommends to abstain from eye examination before the age of ten years. The eyes should be examined at onset of puberty. At and after puberty, eyes should be examined at diagnosis, al least 2-yearly thereafter, at least annually if retinopathy appears, and more frequently, if deemed appropriate, in case of intercurrent illnesses and renal impairment. The number of individuals 10 
be screened is high, 30,000 per million total population per year. Unfortunately, the Retinopathy Working Party did not take considerations of cost-effectiveness into account in setting the protocol, and no reports of comparisons with other studies or recommendations have been published.

As we have seen, relevant studies on this subject have been published by Javitt et all: in the US. Also in Europe, in the UK, results on this subject have recently become availlable ${ }^{[24]}$. In their study, Sculpher et al. demonstrated that a clear trade-off exists with the screening of community-based diabetics for retinopathy: to increase the proportion of positive cases detected (ie. to increase sensitivity of screening programs) and hence to have the greatest impact on health outcomes, will necessitate the adoption of screening strategies that have lower specificities and higher expected costs per true positive case detected than those strategies that are presently available. A lot of research remains to be done in determining the optimal screening strategy in individual European countries.

Implementing the protocol may increase the need for skilled ophthalmologists, supportive personnel, and the need for argon lasers and accessory devices such as slit-lamps and operation microscopes. In the Netherlands, the average intensity of use of ophthalmological argon lasers is somewhat less than one day a week ${ }^{j 3.261}$. If these results are representative for the global situation in Europe, intensifying the use of existing lasers would be a cost-effective strategy compared with short-term investments in new laser devices.

in summary, the case shows that good studies are needed to document effectiveness in a credible way. Aiso, modelling can be a powerful tool to demonstrate cost-effectiveness. Overall, the conclusion is justified that screening for and treatment of retinopathy in diabetic populations is beneficial, both in terms of effects and costs. The results of the studies have been translated in appropriate changes in clinical practice. In the case of the Netherlands, these changes could have been implemented at a higher speed with more active governmental support. Another factor is the timing of the availability of data on cost-effectiveness. The DRS and the ETDRS were influential clinical trials, relatively early in the life cycle of ophthalmological applications of laser technology. If the National Eye Institute had performed a full economic analysis alongside these trials, the information on cost-effectiveness had become available many years before Javitt and colleagues created their model. This could have triggered policy makers to actively support the diffusion of the technology. As we have seen, despite the absence of any governmental support, conditions in the Netherlands were rather fawourable for the diffusion of the procedure. In other countries conditions may not have been as good, and this may have resulted in a relatively slow diffusion. This could have been counterbalanced by policy measures supporting the case. Finally, patients should play a role in the process. Patients should be made aware of the advent of a new, effective and costeffective therapy, inducing demand of the new procedure and thus stimulating change. 


\subsection{References}

1. Anonymous. Screening for dabetic rethopathy in Europe (edilorial). Diabetic Medicine 1991(8) 197-8.

2. Aron-Rosa D, Ophthalmology - Effectveness, In: Banta HD, Schoul (Eds.). Lasers in Health Came, Eftectivenss Cost-Efficutiveness and Policy Implications, Academic Publishing. Frederiksberg, Denmark, 1991, p. 90-6.

3. Bums-Cox $C_{y}$ Dean Hart $J C$. Screening for diabetics for relinopathy by ophthaimic opticians. British Medical Joum $290(1955)=1052-4$

4. Caird FI, Bundit AF, Draper GJ. Diabetics and the Eve, Blackwell, Oxford, 1969.

5. Canirill HL. The dabetic rethopatty study and he eary treatment diabetic retinopathy study. Internation Ophthaimology Clinics (1984)24:13-29.

6. Constabie W, Lm ASM. Laser. Its clinical use in eye diseasess (second edition). Churchill Livingstone, Edinburgr London, Melboume, New York, 1980.

7. Dlabetic retinopathy screening project: special medical development al Exeter, Oxford and Sheffield. Final repor Special medical development project team. HERG Reseanch Report No 8. Health Economics Research Group Brunel University, Uxbidge, London, United Kingdom, 1990.

8. Diabetic Retinopathy (DRS) Research Group. Preliminary report on the effects of photocoagulation therap: American Joumal of Ophthalmology $1976(81): 383-6$.

9. Diabetic Retinopathy Study (ORS) Research Giroup. Photocoagulation treatment of prollferative diabetic retinopatiny Clinical applications of diabetic retinopathy study (DRS) findings. DRS report number 8 . Ophthalmology $1981(88) 7: 5834600$

10. Diabetic Retinopathy Study (DRS) Research Group. Four risk factors for severe visual loss in diabetic retinopathy DRS report number 3. Archives of Ophthalmology 1979(97):654-5.

11. Drummond M. Davies L, Ferris F. Assessing the costs of medical research: the Diabetic Retinopathy Study. Repor to the National Eye Institute. Health Services Management Center, Birmingham. England, 1990.

12. Drummond M. Ophthalmology - Cost-effectlveness. In. Banta HD, Schou I (Eds.). Lasers in Health Care, Effectiveness, Cost-Effectiveness and Policy Implications. Academic Publishing, Frederiksberg, Denmark ${ }_{n} 1991$, p. 97-8.

13. Early Treatment Dlabetic Retinopathy Study (ETDRS) Research Group. Photocoagulation for diabetic maculal edema. ETDRS report number 1. Archives of Ophthalmology 1985(103):1786-1806.

44. Early Treatment Diabetic Retinopathy Study (ETDRS) Research Group. Early photocoagulation for diabelic rethopathy. ETDRS report number 9. Ophthalmology 1991(98)Supplement:766-85.

15. Jawitt JC, Allielo LP. Bassi LJ, Chiang YP, Canner JK. Detecting and treating diabetic retinopathy in patients with type I diabetic mellitus. Savings associated with improved implementation of current guidelines. Ophthalmolagy $1991(98): 1565-74$.

16. Javitt JC, Canner JK, Frank RG, Steinwachs DM, Sommer A. Detecting and treating retinopathy in patients with type 1 diabetes mellitus. A health policy madel. Ophthalmology 1990(97):483-95.

17. Javitt JC, Canner MK, Sommer A. Cost-effectiveness of current approaches to the control of retinopathy in Tyge 1 dilabetics. Ophthaimology 1989(96):255-64.

18. Keiln R, Klein BEK "Moss SE.et al. Provalence and risk of diabetic retinopathy when age at diagnosis is 30 years and older. Archives of Ophthalmology $1984 \mathrm{~b}(102): 527-32$.

19. Khin $R$, Klein BEK, Moss SE et al. Prevalence and risk of diabetic retinopathy when age at diagnosis is less than 30 years. Archives of Ophthalmology $1984 \mathrm{a}(102) \cdot 520-6$.

20. Kupter $\mathrm{C}$. Clinical trials concerning the effectiveness of lasers. The case of ophthalmology. Im: Banta HD, Schou I (Eds.). Lasers in Heaith Care. Effectiveness, Cost-Effectiweness and Policy Implications. Academic Publishing. Frederiksbarg. Donmark, 4991, p.63-8.

21. Patz A, Smith RE. The ETDRS and Diabetes 2000 (guest edilorial). Ophthatmology (1991)98(Supplement):739-40.

22. Retinopatty Working Party. A protocol for screening for diabetic retinopathy in Europe. Dlabetic Medicine (1994)8:263-7.

23. Rosendal $H_{3}$ Vondeling $H_{3}$ Banta HD. Diffusion of medical lasers in the Netherlands, an updated analysis (unpublished). Univarsity of Limburg. Maastricht, 1992.

24. Sculpher MJ, Buxion, MB, Ferguson BA, Spiegethalter D., Kirby Ad. Screening for diabetic retinopatry: a relative cost-effectiveness analysis of alternative modalities and strategies (draft). Health Economics Research Group, Burvel University, Uxbridge, London, 1992.

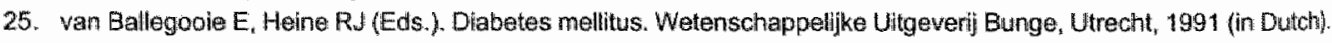

26. de Wit A Banta HD. Diffusion of laser technology in hospitals. The case of the Nietherlands. In: Banta HD. Schou I (Eds.). Lasers in Health Care. Effectiveness, Cost-Effectiveness and Policy Implications, Academic Publishing, Frederiksberg. Denmark, 1991, p. 36-42. 


\section{Evaluation and diffusion of Excimer laser treatment of myopia in the United States and in the Netherlands}

Hindrik Vondeling ${ }^{1,2}$, Henk Rosendal ${ }^{3}$ and David Banta

Vrije Universiteit Amsterdam, Department of Epidemiology and Biostatistics, Amsterdam, the Netherlands

2 University of Limburg, Department of Health Economics, Maastricht, the Netherlands

3 Ministry of Health (VWS), Department of Hospital Care and Top Clinical Care, Rijswijk, the Netherlands

4 The Netherlands Institute for Applied Scientific Research (TNO), Medical Technology Unit, Leiden, the Netherlands

Originally published as: Evaluation and diffusion of Excimer laser treatment of myopia in the United States and in the Netherlands. Health Policy 1995 (34):21-33. Reprinted with permission of the publisher.

Acknowledgements

The authors kindly acknowledge Dr. Yvonne A. van Duivenboden (Health Council of the Netherlands) and Prof. Dr. R. Leidl (University of Limburg, Department of Health Economics) for their comments and suggestions on a draft of this article.

\section{Summary}

Excimer laser photorefractive keratectomy (PRK) is an experimental treatment to correct myopia (short-sightedness) that is diffusing into use without convincing evidence of safety and efficacy. It has been claimed that PRK may render conventional methods of correcting myopia, such as wearing glasses or contact lenses, obsolete. Since about $25 \%$ of the world's population is myopic, the consequences of this technology in terms of benefits, risks, and costs could be truly enormous. The fee for the procedure in various countries such as the United States, the Netherlands, and Australia varies from about US $\$ 1500$ to US $\$ 2250$ per eye. In the United States, the Food and Drug Administration, acting as authorized in the Medical Devices Act of 1976, has limited the use of PRK to patients participating in clinical trials. In the Netherlands, in contrast, where medical equipment is not regulated, the method has diffused without controls, although it is not reimbursed by health insurance agencies. The procedure has become controversial in the Netherlands because it is provided privately and has been aggressively and inappropriately promoted. The case illustrates the limitations of heaith policy concerning technologies provided privately and raises a number of social and political questions.

Key-words: Excimer laser photorefractive keratectomy, Myopia, Policy-making, Evaluation, Diffusion, The United States, The Netherlands 


\subsection{Introduction}

Approximately one-fourth of the world's population is myopic, or short-sighted ${ }^{43}$. The causi myopia is generally a greater than normal axial length of the eyeball, not fully compensater flattening of the cornea and surfaces of the lens of the eye $e^{[42]}$. Spectacles and contact len provide good vision in most instances, but they have disadvantages, including functional: cosmetic objections from some people.

In the past 10 years, a technique has been developed that some claim has the potential to ren spectacles and contact lenses obsolete: excimer laser photorefractive keratectomy or PRK" Under topical anesthesia, a very thin layer of central cornea is "shaved-off" by a compu controlled excimer laser to make the anterior surface of the laser flatter ${ }^{\text {[42! }}$. The procedure is a called "corneal shaping" or "corneal shaving "mit:2? PRK seeks to alter the refractive state of the : permanently.

\subsection{Development, evaluation and diffusion of PRK in the United States}

The excimer laser was developed in the United States in $1970^{[7]}$. PRK was performed in the Uni States for the first time in 1983, although innovative research related to the procedure was carr out in the United Kingdom, France and Germany simultaneously. Industry began to fund sm clinical trials on sighted eyes in 1988 and $1989^{\mid i 33}$. PRK was introduced to ophthalmologists at i annual meeting of the American Academy of Ophthalmology in New Orleans, Louisiana by 1 three excimer laser companies exhibiting at the meeting (Summit Technology Inc., Taunt Technologies, Inc., and VisX Inc.), generating enormous excitement. Ophthalmologists wh reportedly frenzied in their attempts to make orders when sales representatives told them that o a few systems would be sold for participation in clinical studies ${ }^{\mid\langle 4|}$. This meeting attra ophthalmologists from all over the world, so it is likely that international pioneers also becar involved after this meeting.

In June 1990, an official statement from the American Academy of Ophthalmology express concerns about the long-term effectiveness of PRK ${ }^{[18]}$. Questions included the frequency infection and scarring, the ultimate effect of removing the corneal tissue, and the cancer causi potential of the laser's lightwaves.

In 1990 the U.S. Food and Drug Administration (FDA) published a draft clinical guidance for II preparation and contents of an investigational device exemption (IDE) application for excimer las devices used in ophthalmologic surgery for myopic PRK ${ }^{1221}$. The guidance was issued under 1976 Medical Devices Amendments, which give the FDA the authority to regulate the marketir of all medical devices in the United States. The two companies (Taunton Technologies had mergi with VisX) sponsored clinical trials for myopia under these guidelines ${ }^{44_{4}}$. The FDA defined a phast strategy of phase I, phase IIa, phase IIb, and phase III studies. The phase III guidelines requirt each of the two companies to present data on 500-700 patients from no more than 10 researr centers with a follow-up of two years ${ }^{10.4}$. After completing phase $I$ and $\mid I$ trials, both companit submitted data on phase III trials in 1993, although follow-up for the phase lli studies w: apparently not complete. Both companies reported that more than $90 \%$ of those treated achieve vision of $20 / 40$ or better. Although this vision is far from perfect, people can drive in mast state of the United States with this vision without corrective eyewear. FDA is considering the da (presumably including follow-up data as time goes on), and is expected to arrive at a decision 1995. If the FDA decision is positive, commercial marketing will begin in the US in 1995

It was estimated that about 2000 patients had been treated in the U.S. by August $1992^{[9 !}$. Clinic studies on these patients in the US have been funded largely by patient fees, which are US $\$ 150$ to US\$2000 for each eye treated ${ }^{\|6\|}$. 


\subsection{Early diffusion and evaluation of PRK in the Netherlands}

Adoption of an ophthalmological excimer laser was first considered by ophthalmologists affilated with the Nijmegen University Hospital in 1989. When the hospital board could not agree on terms for purchasing a device, the ophthalmologists decided to purchase the laser as a private investment. The laser was installed in 1991 $1^{[20,45]}$. Subsequently, the "Nijmegen Excimer Laser center" was opened, located in a building close to the University Hospital.

The Dutch Health Council, which has the statutory responsibility of giving scientific advice to the Dutch government, mentioned excimer laser PRK in its annual advice reports of 1990 and $1991^{127.291}$. In 1990, the Council concluded that it was too early to draw conclusions on the effectiveness of the procedure. In 1991, the Council judged that the procedure was experimental. This 1991 report also reported that the procedure had been done in some private clinics in the Netherlands (which was actually not yet true), and that this development was undesirable. This "early warning" was not heeded by the Ministry of Health, which showed no reaction to these reports.

In 1992, the procedure was adopted in other centers, so that it was available in six centers by early 1993, one in a general hospital, one in a specialized eye hospital, and four in private clinics. Three centers used a laser produced by Meditec-Aesculap (a German laser manufacturer), two used a Summit laser, and one used a Vis $X$ laser. The 14 ophthalmologists performing the procedure in 1993 were reportedly very enthusiastic about it ${ }^{[111}$. (These ophthalmologists represent only about $3 \%$ of Dutch ophthalmologists.) One of the centers closed in 1994 after completion of a lease contract on the laser, and the ophthalmologist involved became associated with two of the other centers, while a new private center opened. Of the 4 private centers, three are private companies or foundations of ophthalmologists, and one clinic is owned by a private insurance company.

Although no third-party payer in the Netherlands has decided to reimburse for the procedure, it is profitable on a self-pay basis. By late 1994, we estimate that the total number of people treated by excimer laser PRK was about 2000 . More than half were treated in two of the private clinics ${ }^{111}$. In the future, the largest private clinic, in Nijmegen, expects to treat about 300 patients a year ${ }^{200 !}$. Presently, the laser in this center is used about two hours a week ${ }^{15}$.

The potential population for treatment is Dutch citizens who are myopic and at least 18 years of age. The Dutch Ophthalmological Society estimated that refractive errors are present in 6 million people ${ }^{[15]}$, of whom $25 \%$ are younger than 18 years of age ${ }^{[10]}$. The potential case-load is therefore about 4.5 million people.

Capital costs of the laser are about US $\$ 500000^{|16,20|}$, and annual operating costs can be estimated to be as high as US $\$ 200000$. The treatment fee is about US $\$ 1900$ per eye ${ }^{[1.14,20]}$ Thus, about 50 patients, using conservative figures, can pay off the laser. If 300 patients are treated in a year, the procedure is very profitable ${ }^{[12,28]}$

By 1992, the centers using the Summit lasers and the Vis $X$ laser had been instructed by the laser companies to treat patients according to the FDA protocol, which meant only treating patients with relatively mild myopia (no more than -6D). The other centers treated much more severe myopia, despite evidence of disappointing results with severe myopia published in $1992^{[20]}$.

The innovators with PRK started a working group to coordinate development of treatment and follow-up protocols, patient information and informed consent forms in 1992. This working group was officially recognized by the Dutch Ophthalmological Society in $1993^{(5)}$. (It may be interesting to note that the Chairman and Secretary of the Dutch Ophthalmological Society in 1992 were among the providers of the procedure..) Also in 1992, the principal Dutch innovator in Nijmegen submitted an evaluation proposal to the most important national medical technology assessment 
program in the Netherlands, the Investigational Medicine Program (Ontwikkelingsgeneeskunde), funded by the government and the Health Insurance Council. The proposal was tumed down, according to the principal investigator, because PRK was deemed too experimental to evaluate. Actually, the rejection was based on a combination of factors, including the poor quality of the

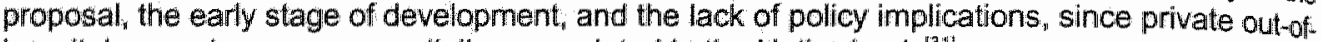
hospital procedures are essentially unregulated in the Netherlands ${ }^{[3]}$.

In 1994, the Nijmegen group presented results of PRK in their center to the Dutch Ophthalmological Society ${ }^{[191}$. The presentation covered 138 eyes with a follow-up of six months. The authors concluded that PRK was highly reliable for low to moderate myopia (below $-6 \mathrm{D}$ ). lin high myopia, results were not so good, and side effects were more common.

\subsection{Political visibility of PRK and further evaluation}

In January 1993, the Health Council published an article on PRK in its magazine Graadmete: ${ }^{144}$. Outcome results, quoted from a report published by the Australian Institute of Health ${ }^{[12]}$, showed that in $2-3 \%$ of patients visual acuity decreased after the procedure. Complications of the procedure were described, and profitability of the procedure was estimated. Finally, the article commented critically on the ease with which PRK had entered the Dutch health care system, given lack of evidence of efficacy and safety. This article was selected for reprinting by the General Dutch Press Agency and widely distributed. Subsequently, a prominent Dutch Professor of Ophthalmology from Rotterdam was interviewed by a local newspaper, quoting the Graadmeter article and asking the providers and the government to take steps to end such practice ${ }^{\mid 39]}$.

Also in 1993, an advertisement for PRK by the Nijmegen center in a secondary school magazine was noted by a Member of Parliament, who asked for information on the procedure from the Regional Medical Inspectorate for Public Health. This ad was considered highly inappropriate because of inflated claims and because myopia changes in the immature eye, making teenagers poor subjects for the procedure. The Inspectorate presented basic facts to the Director-General of Health in March 1993. Two members of Parliament important in the health area then formally asked for a response from the State Secretary of Health ${ }^{i 40}$. Perhaps the most interesting question was, "Do you share the view of (the Rotterdam ophthalmologist), who says that excimer laser treatment should only take place in one clinic and the results carefully recorded so that risks, cosis and results can be evaluated?" In a preliminary answer in April 1993, the State Secretary expressed his concern and announced that he would ask the Health Council, the Health Insurance Council, and the Chief Medical Inspectorate for Public Health to collect information related to the questions ${ }^{3: 33 !}$.

In the meantime, the controversy grew. The Rotterdam Professor again expressed his concern in an interview in a national newspaper in late Aprif ${ }^{201}$. He stated that recruitment and selection of patients might be done partly on commerciall grounds and partly as a result of overly optimistic; incomplete, or inappropriate patient outcome information. The innovators also used the media, with at least one interview in a national newspaper in March $1993^{[32]}$. Promotion was also carried oul by advertisements, stories in women's magazines, stories in periodicals of private insurance companies, by distributing leaflets on the procedure, and by organizing information meetings ${ }^{|20,28|}$.

Based on preliminary information, and withoult the information being collected by the different advisory bodies, the State Secretary attempted to answer the Parliamentary questions in a letter dated June $14,1993^{[28)}$. He provided data on the number of centers providing the procedure and preliminary information on effectiveness, based on the earlier Health Council reports. He stated that the procedure was still experimental and not without risks. He also stated that the recruitment of patients in these circumstances was too aggressive. 
On November 16, 1993, the Health Council presented its advice to the State Secretary "This report included a literature review covering the period to mid-1993. The Council concluded that PRK was experimental and should therefore not be offered routinely to patients. The procedure is irreversible, possibly risky, and medicaliy unnecessary because existing provisions are very effective in correcting myopia. The Council acknowledged some evidence of fair short-term effectiveness in case of low myopia, moderate effectiveness in moderate myopia, but pointed to poor effectiveness in severe myopia. The Council strongly objected to publications developed to inform patients, quoting texts such as, "PRK eliminates the need for spectacies and contact lenses." Overall, the Council considered PRK a medically unnecessary" cosmetic treatment that should not be eligible for reimbursement. It was also argued that PRK is not a high priority for evaluation because it was considered medically unnecessary. The Councill objected to the uncontrolled diffusion of PRK , but acknowledged the lack of policy instruments to guide diffusion.

The Health Insurance Council published its advice on November 26, 1993, focusing on the reimbursement question ${ }^{[31]}$. The Council stated that because PRK is almost always performed for cosmetic reasons, it is not eligible for reimbursement nor for evaluation in the Investigational Medicine program. The Council also stated that wearing glasses or contact lenses is cost-effective to correct myopia compared to PRK (this statement cannot be confirmed by existing studies, although it is probably true). The Council felt that coverage for high myopia could be considered if PRK were shown to be effective for this indication. Otherwise, the report agreed with the Health Council report.

Based on this advice (and a preliminary report by the Chief Medical Inspectorate on Public Health). the State Secretary issued a press release on November 24,1993 , stating that PRK is an experimental, cosmetic, medically unnecessary procedure that entails a number of risks ${ }^{|36|}$. Based on concerns with respect to the quality of patient information and the conditions for applying PRK. he stated that he would organize a meeting of the ophthalmological community to address these issues. He waited until after the Chief Medical Inspectorate completed its final report before organizing the meeting.

Parliament also asked further questions about possibilities of regulating the procedure. Members of Parliament doubted that a meeting could be useful where prominent ophthalmologists have great financial interests in performing the procedure ${ }^{[40]}$. In her answer, the Minister of Health stated that there are no legal possibilities of regulating cosmetic procedures in private clinics ${ }^{[35 !}$.

In April 1994, the Chief Medical Inspectorate published its final report, covering quality of care for patients treated with PRK ${ }^{[1] !}$. The overall judgment was that standards for good clinical practice had been met to a certain extent. Information given to patients was considered adequate, and misleading advertising was characterized by the ophthalmologists as a mistake. Major and minor differences were found in selection of indications and contra-indications, pretreatment examinations, treatment and post-treatment procedures and follow-up, despite the working group that had been established to standardize procedures. All clinics had by then agreed to treat only patients over the age of 18 . One striking difference between centers was that one center offered patients to treat the second eye during the same session as the first eye at half the costs of treatment of the first eye, leading to a total fee of US $\$ 2750$ instead of US $\$ 3600$. This practice was (and is) considered inappropriate. The report makes a number of recommendations. Data should be collected on efficacy and safety. Protocols for each phase of treatment should be developed. Advertising should be stopped. Medical ethical committees are needed by the centers that do not have one. Additional diffusion of the procedure should be avoided.

The meeting with the ophthalmologists was held in August 1994. On behalf of the State Secretary, the working group was asked to respond to a series of questions concerning rational diffusion and appropriate use. A definite answer to these questions if expected by the end of 1994 .

In late 1994 , the six centers continue to do the procedure with increasing patient numbers. 


\subsection{Discussion}

Despite the avallability of an identical technology and the same evaluative information, and despity the great enthusiasm of certain ophthalmological innovators in the United States and th Netherlands, the course of diffusion of PRK has been markedly different in the two countries largely because of the FDA program in the United States. Table 17.1 summarizes similarities an differences between the Netherlands and the United States.

\section{Effects of payment}

In the United States, the eagerness of ophthalmologists to adopt excimer laser PRK and other nen procedures has been explained by some by referring to anticipated reductions in Medicart payments for surgical procedures by $30-40 \%$ in the four years after $1992^{17,43)}$. The issue $a$ decreasing fees leading to induced demand for new medical technologies has been extensivelly analyzed in the United States with conflicting results $\left.\right|^{[21,25,34\}}$.

Table 17.1 Summary of findings with respect to the evaluation and diffusion of Excimer laser PRK in the United States and the Netherlands

\begin{tabular}{lll}
\hline & United States & the Netherlands \\
\hline Diffusion & controlled & uncontrolled \\
Evaluation & yes & no \\
Reimbursement & no & no \\
Financial incentives & present & present \\
Investment & predominantly private & predominantly private \\
\hline \hline
\end{tabular}

In other words, ophthalmologists are thought to adopt new procedures and expand indications for existing procedures as a way of maintaining or increasing their incomes. Tightened hospital medical equipment budgets and reduced numbers of hospitals are also hypothesized to stimulate the adoption of new technology directly by physicians. This hypothesis requires further research.

In the Netherlands, too, a policy aimed at controlling total physician care costs was implemented in the period 1989-1992 $2^{(3 r)}$. This policy was unsuccessfull. In 1993, it was calculated that overall physician care costs increased $20.7 \%$ in the period $1989-1992^{[37]}$. A new policy was implemented in 1993 to 1994 aimed at a generic physician reimbursement cut-back of $13.9 \%$ for sick funds and 12.1 for private health insurance ${ }^{|9|}$. Ophthalmologists were faced with a fee reduction of $20.2 \%$ from both the private and public health insurance sectors ${ }^{[\theta]}$. It has been suggested that ophthalmologists, like other physicians, have partly compensated for these measures by more precise billing and by changing treatment (and billing) strategies in favor of more profitable services. In Europe in general, physician incomes have gradually fallen in recent years due to controlled fees and physicians have responded by inducing demand and increasing some procedures ${ }^{[13,464]}$. This area also deserves further research in the Netherlands and Europe in general.

Still, one can wonder why in the Netherlands only one specialized eye hospital and one general hospital have begun providing PRK. One explanation is that a hospital buldgeting system was introduced in 1983 in the Netherlands. Un recent years, the annual budget for investments in medical equipment in hospitals has been set by a directive of the Central Health Care Charges Agency (COTG). An example of the results is that one academic hospital in Amsterdam, that of the Free University, had a 1993 total equipment budget of US\$ 7.7 million $^{[23]}$. An excimer laser costing US\$500 000 would thus represent a large investment for such a hospital. 
In summary, physician care cost containment measures and hospital budgeting policies have been implemented in both the United States and the Netherlands with largely unknown effects although observers in both countries state that ophthalmologists have increased volumes of certain procedures, such as cataract operations, as a method of maintaining income. The implemented policies in the Netherlands have not contained costs, but have resulted in a spiral of fee reductions, volume increases, and new fee reductions ${ }^{\text {(33! }}$.

\section{Private clinics}

It is especially interesting that diffusion of PRK has been predominantly in private clinics in the Netherlands. Perhaps the most important reason is that such climics are not subject to the buigeting system. Furthermore, private clinics can only carry out procedures that can be done as an out-patient procedure, as is the case with PRK and many other procedures in ophthalmology ${ }^{[33\}}$.

Private clinics are a relatively recent phenomenon in the Netherlands. In the period of the mid1980 s until 1993,53 have become active $e^{|35|}$. Entrepreneurial physicians who wanted to by-pass long waiting lists and/or wanted to pay more attention to the individual patient have established private clinics ${ }^{[2]}$. They may also have been motivated by the chance for higher incomes. Privale insurance companies have financed some of this development.

The government is ambiguous concerning private clinics. On the one hand, the importance of private clinics is considered marginal, as the costs were no more than US\$24 million in 1992, less. than $0.1 \%$ of total health expenditures ${ }^{[35]}$. Also, private clinics ease long waiting times. On the other hand, little is known about the quality of care in private clinics ${ }^{226 !}$. Private clinics are neither regulated nor encouraged, but merely tolerated ${ }^{[26.35 \mid}$. In ophthalmology, with lang waiting lists, especially for cataract surgery, there is a clear market for private clinics.

\section{The role of industry}

The laser industry is not a large or strong segment of the medical equipment industry. Total world sales of medical lasers were estimated to be US\$ 500 million in 1990 , with a projected rise to US\$ 1.2 billion by $1995^{[6]}$. The laser industry in general is characterized by the presence of a number of small firms.

The ophthalmology laser market is similar to the general market, but is perhaps less dynamic, because the argon laser in general use is already available in most departments of ophthalmology, meaning that most sales are for replacement. Industry therefore has an incentive to try to produce new and different laser systems in this area.

Al least three companies are known to make excimer lasers for PRK, two in the United States and one in Germany. These companies seem to have a world-wide marketing strategy. At the end of 1993, it was estimated that 12000 to 20000 patients have been treated worldwide with PRK ${ }^{|14,44|}$. industry considered that 1993 was a year showing a strong worldwide increase in interest in laser PRK $[1]$

\section{The role of ophthallmological societies}

As noted, the American Academy of Ophthalmology expressed its concerns about the long-term effectiveness of PRK in $1990^{14 !}$. This statement may have stimulated FDA to regulate the procedure rigorously.

In the UK, the Royal College of Ophthalmologists noted in a 1990 press release that the degree of myopia correctable by the procedure is probably limited. According to the College, the procedure was probably only effective for mild myopia. The College also stated that a follow-up of at least 5 years is necessary before firm statements could be made with respect to the long-term effectiveness of PRK ${ }^{[28]}$. 
In Germany, although the Deutsche Ophthalmologische Gesellschaft has stated that PRK clinically acceptable, it is felt that given its irreversible nature and high cost, spectacles and conta lenses are preferable. Patients should be given this information ${ }^{[28]}$.

In the Netherlands, although the Dutch Ophthalmological Society acknowledged the working gro on excimer laser surgery in 1993, common working protocols have not been developed. $\pi$ climate towards excimer laser PRK has probably been positively influenced by the former Chaim: and Secretary of the Society , who provide the procedure. The Society has made no critic statement on PRK, which fits with the usual practice of the Dutch medical societies. On the oth hand, the Dutch innovators were interested in formal evaluation of the procedure and developi a research proposal for this purpose that was not funded.

\section{The role of technology assessment agencles}

This case has been the subject of formal assessments by public technology assessment agenci in several countries, including the Netherlands (as noted). Australia ${ }^{[12]}$, Canada $a^{[7.44 !}$, and France $e^{\left[z_{1}\right.}$ The conclusions of these reports have been similar. All reports have also noted the difficulties controlling diffusion of an out-patient procedure that is not reimbursed by third-party payers.

\subsection{Conclusions}

Table 17.2 summarizes the impact of different factors on diffusion in the United States and th Netherlands. Table 17.2 shows that financial factors have probably played a large role in bol countries since the procedure is quite profitable, based on existing fees. The role of the Dutc Ophthalmological Society thas not been constructive in the Netherlands, which is unfortunal considering present policies that are beginning to ask physicians to take on a larger role in assurin the quality and appropriateness of their care ${ }^{[30 !}$.

Table 17.2 Summary of impact of several factors on the diffusion of Excimer laser PRK the United States and the Netherlands

\begin{tabular}{lll}
\hline \hline Factor & United States & the Netherlands \\
\hline $\begin{array}{l}\text { Effects of payment } \\
\text { Fee reductions }\end{array}$ & present & present \\
Volume increases & conflicting results & present \\
Hospital equipment budgets & decreasing & decreasing \\
Private clinics (NL) & increasing role & increasing role \\
$\begin{array}{l}\text { Physician offices (US) } \\
\text { Industry }\end{array}$ & incentives for marketing & incentives for marketing \\
Ophthalmological societies & critical & uncritical \\
Technology assessment & no active role & critical \\
agencies & & FDA \\
Regulation of market & & absent \\
\hline
\end{tabular}

While evaluation is still inadequate, the FDA regulations assure that at least a minimum 0 evaluation will be carried out.

In the United States, it has been estimated that of the 5000 medical devices that were introduce in 1993, only $10 \%$ were tested for safety and efficacy in FDA-guided protocols that can be 


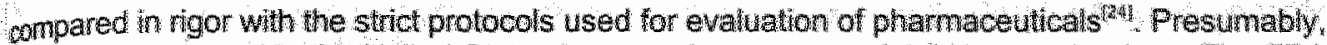
PR was evaluated in the Unted States because it was a completey new technology. The FDA protocols have also had a positve mpact in we Netherlands ${ }^{\text {t: }}$. This helps to confrm the FDA's joportant role as a reference center for the entire word ${ }^{2}$. If the FDA decides lo allow conmercial rease of excimer lasers for PRK in 1995 , is projected that 3.4 mullon procedures whin be pertomed in the Unted States in he following 5 years ${ }^{\text {t8y }}$. Assuming a number of patients treated y laser per year of 500 to 1000 , his would tead to sales of 680 to 360 excimer lasers in the United States, many of them directly to ophthatmologists.

It that should happen ofher countries why probably lollow the United States. Excimer laser PRK is aready more or less routinely available in France and Australia: ${ }^{\{t 2\}}$, and it is becoming mote frequent in the Netherlands. In the Nethertands, the story of PRK is considered to be an example of the problems that arise when govemment withdraws from heath care in the absence of suffictent guarantees for self-regutation by physicians ${ }^{349}$. There are also other developments in this field. including use of the erbium: $\mathrm{YAG}$ laser, which has lower size, cost, maintenance, and does not produce toxic gas, so may diffuse rapidy for this indication in the wure ${ }^{\text {i38? }}$

PRK was discussed in a 1994 public hearing on quality and efficiency in health care, organized by the Standing Committee for Public Health of the Dutch Parlament. The Chatrperson. Mrs. Netelenbos (one of the authors of the earler questions to the State Secretary), concluded that the Netherlands needs legislation to regulate this procedure ${ }^{[77}$. Although the poltical chmate is not lavorable to new regulation, the Ministry of Health is now seriously considering regulation of private dinics in arder to prevent "top clinical care" from being offered in prvate clinics. Since PRK is not considered top clinical care this will not change the present situation. Another factor is that the Vice-Chaiman of the Dutch Health Council during the period of the Health Council reports on PRK is now Minister of Heath. She may have a special interest in PRK and its implications.

The regulation of PRK can be justified rom a patient's protection point of view atone. However, the case has mplications that go well beyond patient protection.

The main interest of PRK is to show what can happen following policies aimed at controling heath care costs. In the Netherlands case, new perverse fnancial incentives seem to have acted on ophthatmologists, encouraging them to adopt this new technology. At the same time, industry has developed the strategy of seling medical devices directy to ophthaimologists. White PRK is not rembursed by public funds, other procedures provided publicly are. "Privatization" of the health care system withoul appropriate regulation can be expected to have a number of potentially destructive effects, including provision of inappropriate and unproven procedures. It therefore seems axiomatic that policies aimed at macro health care cost containment need to be complemented with policies regulating the introduction of new technology and its evaluation. irrespective of its "cosmetic" or "therapeutic" usages.

\footnotetext{
The myopic vision of Dutch policy makers and the lack of adequate instruments to regulate the diffusion of excimer lasers help to explain the differences between the United States and the Netherlands. For example, in the Netherlands, there has been no policy encouraging the evaluation of PRK. On the ather hand, the Netherlands medical profession has a tradition of professional selfregulation. Perhaps this will lead to a more controlled diffusion process later in development compared to that in the United States.

The United States, too, has not seen an ideal situation. The evaluation of PRK has been restricted to large numbers of case series. Such a design cannot answer questions such as what is the best treatment modality for myopia in individual patients. To answer such questions as relative effectiveness and cost-effectiveness, randomized trials are needed. These could include an economic evaluation aimed at improving decision-making concerning reimbursement of PRK.
} 
The official technolagy assessment agencies seem nol to have affected diffusion of the case ver much, primarily because PRK is provided privately and out of hospitals. As the case illustrates PRK is an international issue, but no internationall response to its diffusion has been seen. The fiet of health care technology assessment has become increasingly international in recent years, with the formation of such bodies as the International Society for Technology Assessment in Health Care (ISTAHC), the International Network of Agencies for Health Technology Assessmen (INAHTA), and the EUR-ASSESS program (a program developing coordinated strategies among European health care TA agencies funded by the European Commission). The internationa ophthalmological community could also play a larger role in such developments. The internationa industry needs to be encouraged or induced to assume a stronger role in evaluation. Attempts to thoroughly evaluate such new technologies as PRK also need to become more international.

\subsection{References}

1. 1993: a year of growth for refractlive surglery. Ocular Surgery News 1993(4)1:15-7.

2. Agence natlonale pour le développement de l'evaluation médicale (ANDEMM). Excimer lasers in ophthalmology. ANDEM, Paris;, 1992 .

3. Agence nationale pour le développement de l'evaluation médicale (ANDEM). Excimer laser in ophthalmology. Intemational Journal of Technology Assessment in Health Cari 1993(9):595-6.

4. American Academy of Ophthalmology (AAO). Ophthalmic procedures assessment. Radial keratotomy for myopia Ophthalmology 1993(100):1103-15

5. Beakhuis WH and Treskes DJ. Excimer laser surgery, structure and gaals of the Dutch Working Group. Medisch Contact 1994(49):265-6 (in Dutch).

6. Braver FA. CALAB. Presentation to the American Society of Lasers in Medicine and Surgery (ASLMS), 1990.

7. Canadian Coordinating Office for Health Technology Assessment (CCOHTA). The excimer laser. CCOHTA Technology Brief 1992(issue 2.0):1-4.

8. Central Health Care Charge Agency (COTGj. Annual Report, 1993. Central Health Care Charge Agency, Utrecht, 1993 (in Dutch).

9. Central Health Care Charge Agency (COTG). COTG Vademecum. Directives of the Central Health Care Charge Agency, Vol. 2. Bohn, Stafleu, van Loghum, Houten, 1993 (in Dutch).

10. Central Statistics Agency (CBS) Statistical Year Book 1994. Sdu Publishers, the Hague, the Netherlands, 1994 (lin Dutch).

11. Chief Medical Inspectorate for Public Health (CMIPH) and Inspectorate for Drugs (ID). Treatment of reffractive ertors using excimer laser surgery. CMIIPH, ID, Rilswijk, the Netherlands, 1994 (in Dutch).

12. Cowley DE. Laser comeal sculpting. A discussion paper. Australian Institute of Health, Canberra, 1991.

13. Culyer A. Cost containment in Europe. Health Care Financing Review (1989):Annual Supplement, p. 21-32.

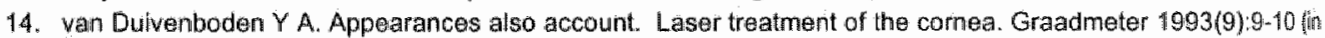
Dutchi).

15. Dutch Ophthalmological Society . Policy plan for eye care in the Netherlands. The future in sight, a strategic view on the future possibilities for eye care in the Netherlands. Dutch Ophthalmologilcal Society, 1992 (in Dutch).

16. ECVI. Excimer lasers. Health Technology Trends 99g:4(6):6.

17. ECRI. Hospitals tighten equipment spending. Health Technology Trends 1994(6):1.

18. ECRI. Ophthalmic community eyes excimer laser for vision correction. Health Technology Trends 1992(4):3,6.

19. Eggink CA, Deutman AF, Tilanus MAD, Meurs PJ, Schilt PD. Excimer lasers, one year in the Netherlands (abstract). Nederlends Tijdschift voor Geneeskunde 1994(138):273 (in Dutch).

20. van Eijk D. Laser operation for myopia "long-term effects are unclear due to limited research. NRC (daily newspaper) 28 April 1993 (in Dutch).

21. Escarce JJ. Effects of lower surgical fees on the use of physician services under medicare. Journal of the American Medical Association 1993(269):2513-8.

22. Food and Drug Administration (FDA). Office of Device Evaluations, Division of Ophthalmic Devices. Draft clinical guidance for the preparation and contents of an inwestigational device exemption (IDE) application for excimer laser devices used in ophthalmic surgery for myopic photorefractive keratectorny (PRK). Refractive Comeal Surgery 1990(0):265-9

23. Free University Hospital Amsterdam. Annual Plan 1993. Free University Hospital Amsterdam, Amsterdam, 1993 (in Dutchy.

24. Gelijns $\mathrm{AC}$. Letters from Washington, Food and Drug Administration and medical technollogy: an impasse? Nederlands Tijdschrift voor Geneeskunde $993(137): 146-50$ (in Dutch). 
25. Ginsburg $P B$, Hogan $C$. Physicians respond to fee charges, a contrary view Journal of the American Medical Association 1993(269):2550-2.

26. den Hartog M. Strategies of hospitals, priwate clinics are no serious treat to hospitals. Gezondheidszorg en Management (1994):24-7 (in Dutch).

27. Health Council of the Netherlands "Standing Committee on Medicine. Annual Actvice on Health Care 1991. Health Council, Publication no, 1991/24. The Hague, the Nethertands, 1991 (in Dutch).

28. Health Council of the Netherlands, Standing Committee on Medicine. Lasers in Sight. Laser Correction of Refractive Errors. Health Council, Publication no. 1993/19E. The Hague, the Netherlands, 1993.

29. Health Council of the Netherlands, Board of Advice and Deliberation. Annual Advise on Health Care 1990. Health Council, Publication no. 1990/25. The Hague, the Netherlands, 1990 (in Dutch).

30. Health Council of the Netherlands. Medicial practice at the crossroads. Health Council, Publication no. $1991 / 23 \mathrm{C}$. The Hague, the Netherlands, 1991 (in Dutch).

31. Health Insurance Executivie Council. Advice on the use of Excimer lasers in ophthalmology. Health Insurance Executive Council, Amstelween, 1993 (in Dutch)

32. de Leeuw K. Laser light stops hyperopia. Algemeen Dagblad (daily newspaper), March 20, 1993 (in Dutch).

33. Leusink AB. "Lasers in sight", Questions of Members of Parliament and the answers of the State Secretary, Graadmeter 1994(10) 10-11 (in Dutch).

34. MG Guire TG. Pauly MV. Physician response to fee changes with multiple payers. Journal of Health Economics $1991(10): 385-410$.

35. Ministry of Health, Weffare and Cultural Affairs. Document on independent non-clinicial treatment centers. Diutch Partiament, Second Chamber, Meeting Year 1991-1992, 22 311, nr. 6. Sdul Publishers, the Hague, 1994 (in Dutch).

36. Ministry of Welfare "Health and Cultural Affairs. Secretary of State Simons wants agreement on laser treatment of myopia. Press release of the Ministry of Health, Welfare and Cultural Affairs, Nr. 198, 24 November 1993 (in Dutch).

37. Ministry of Welfare, Health and Cultural Affairs, Financial Oversight on Care 1994. Dutch Parllament, Second Chamber, Meeting Year 1993-1994, 23407, nrs. 1-2. Sdu Publishers, the Hague, 1993 (in Dutch).

38. Moretti M. Solid state lasers compete with excimers for refractive surgery. Biomedical Optics $1992(1): 4$.

39. Neefjes $C$. "Use of laser in eye lesion irresponsible", Ophthalmologist criticizes expensive operation without guarantee of good results. Utrechts Nieuwsblad (dally newpaper), 25 February 1993 (in Dutch).

40. Netelenbos and van Otterloo (Members of Parliament). Questions to the State Secretary of Health on excimer laser treatment of myopia. Questions number 2929303860, $15 \mathrm{March} 1994$. In: Health Councill of the Netherlands, Standing Committee on Medicine. Lasers in sight. Laser correction of refractive errors. Publication no. 1993/19E. Health Council, the Hague, the Netherlands, 1993 .

41. Ophthatmologists choose excimers for corneal sculpting. Laser Focus World 1990(January).

42. Philips $\mathrm{Cl}$, Clark CV, Tsukahara S (Eds.). Ophthalmology. A Primer for Medical Students and Practitioners. Baillière Tirndall. London, Philladelphia, Toronto, Sidney, Tokyo, 1994.

43. PRK: a growth market for ophthalmology? Scope 1992 (January) (in Dutch).

44. Reports from the Canadian Coordinating Office for Health Technology Assessment (CCOHTA). The excimer laser. International Journal of Technology Assessment in Health Care 1993(9):145-9.

45. Rosendal H. Diffusion of medical lasers in the Netherlands (doctoral thesis). University of Limburg "Maastricht, the Netherlands, 1992

46. Sandier $S$. Health services ufilization and physician income trends. Health Care Financing Review 1999 (Annual Supplement): $33-48$.

47. Second Chamber of the Dutch Parliament. Minutes of a hearing on efflciency and quality in health care. Meeting Year 1993-1994, 23 400 XVI, nr. 80. Sidu Publishers, the Hague, 1994 (in Dutch).

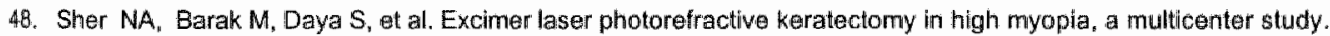
Archives of Ophthalmology 1992(110),935-43.

49. Spreeuwenberg $C_{x}$ Lasers in sight (editorial). Medisch Contach 1994(49):251 (in Dutch). 


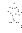


Part Five - Policy Implications 
$\therefore \quad \therefore \quad \therefore$

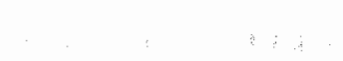




\section{Part Five - Policy Implications}


 Netherlands and in the European Union}

HVondeling", RJ van Kempen ${ }^{2}, S R$ Vaartjes $^{3}$, LCE Vlaskamp $p^{4}$, and the Dutch National Committee Laser Safety

1 Vrije Universiteit Amsterdam, Department of Epidemiology and Biostatistics, Amsterdam, the Netherlands

2 Ministry of Health. Welfare and Sport, Chief Medical Inspectorate for Public Health, Rijswijk, the Netherlands

3 Medisch Spectrum Twente, Department of General Clinical Physics, Enschedé, the Netherlands

4 Ministry of Social Affairs and Employment, Directorate General of Labor, Department of Radiation Hygiene, Rijswijk, the Netherlands

The members of the Dutch National Laser Safety Committee are: GJ Eggink, National Institute for Public Health and Environmental Protection (RIVM); Ph. J. Ham, TNO-Building; C Kaptein, Chief Medical Inspectorate for Public Health; FW van der Meulen, Academic Medical Center; RJ van Kempen, Chief Medical Inspectorate for Public Health; VLB Oei-Lim, Academic Medical Center; P Silliakus, Chief Medical Inspectorate for Public Health; HJCM Sterenborg, Academic Medical Center; PGJ Sterrenburg, Carl Zeiss Ltd.; Ir. CJPM Teirlinck, CMT-TNO (Secretary); SR Vaartjes, Medisch Spectrum Twente (Chairman); LCE Vlaskamp, Ministry of Social Affairs and Employment; HVondeling, Vrije Universiteit Amsterdam; RA Zandbergen, Academic Hospital Rotterdam-Dijkzigt.

Originally published as: Vondeling $H$, van Kempen $R J$, Vaartjes $S R$, Vlaskamp LCE. Regelgeving in verband met het veilig gebruik van medische lasers. In: Eggink $G J$, Kaptein $C_{\text {, }}$ van Kempen $R J$, van der Meulen FW, Teirlinck C.JPM, Vaartjes SR (Eds.), Laser safety in health care (second edition). National Committee Laser Safety, The Netherlands Organization for Applied Scientific Research (TNO), Center for Medical Technology, Leiden, the Netherlands, 1993 (in Dutch). A grant for a translation in English of this report was provided by the European Community as part of the European Year of Safety and Occupational Health. The translation was produced by Dr. A. Gaunt. As a result, the English version of the report of the Dutch Committee on Laser Safety in Health Gare is forthcoming. This chapter is reprinted with permission of the National Committee on Laser Safety. For the purpose of the thesis, the summary and discussion sections are written on the sole responsibility of the first author.

Acknowledgements: The authors kindly acknowledge the helpful comments of the co-members of the National Committee, Prof. Mr. FCB van Wijmen, Mr. NPYM de Bijl (Department of Health Law: University of Maastricht), Dr. Mr. LMC Faro (Orthomed B.V.) and Dr. P Ruys (The Dutch Quality Contral Institute for Electrical Materials and Appliances (KEMA). Dr. A. Gaunt (European Community) is acknowledged for translation of this Chapter in English.

\section{Summary}

This Chapter considers legislation in both the European Union (EU) and Dutch national legislation that is relevant to the safe use of lasers in health care. A broad range of laws and other, for the most part technical standards, are applicable to the use of medical lasers. Legislation on liability is covered as well. 
At the level of the EU, in particular Directives on products and goods and Directives occupational health and safety are relevant. The EU Directive concerning medical devices, whi comes into the first category, is officially applicable to the use of medical lasers and other medi devices in all member states since 1 January 1995. Of the Directives in the second category significant one is the 1989 framework Directive designed as an umbrella Directive for furth measures to improve the safety and health of workers. These Directives provide the legal basis a large number of measures that improve the safe use of medicall lasers.

There is no legislation in the Netherlands specifically on lasers. The National Committee beliey that there is a need for national legislation on medical lasers and that this legislation should dovel as far as possible with existing legislation.

Of the existing legislation, the Medical Devices Act, the Order on Criteria for the Recognition Hospitals and the Occupational Health and Safety Act are applicable to lasers. In particular th Safety Order for Factories and Workplaces and the Safety Order for Other Groups are importa in the laser field.

If necessary, legislation on liability is used to enforce good clinical practice. There are three typ of liability in the Netherlands: criminal liability, civil liability and liability under the Medical Disciplii Act. The rules on criminal liability are set out in the Criminal Code. Both a hospital and $i$ employees may be held criminally liable for measures carried out as part of hospital services. $\mathrm{Ci}$ (private-law) liability is defined in the Civil Code. The Civil Code has been specifically enlarged wi respect to health care by the inclusion in it of the Contract for Medical Treatment Act. Article 165 : of the Contract for Medical Treatment Act sets out rules for the liability of hospitals and equivale health care establishments in respect of all activities carried out by care personnell in sur establishments.

A hospital is also liable for objects it uses such as defective equipment employed in the treatme of patients unless such liability would be unreasonable. This is the case if the product is inherent defective. The risk is then borne by the manufacturer. Articles 185-193 of the Civil Code als incorporate an EU directive on liability for defective products.

Legislation on criminal liability and civil liability is complemented with legislation on liabili specifically for health care professionals covered by the Medicall Discipline Act (1928). The Act the

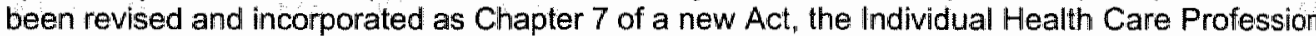
Act.

Hospitals, specialists and other employees can insure themselves against liability claims. Currer legal liability insurance for hospitals mostly provides "intramural cover", that is to say damage a patient sustained within the precincts of the hospital establishment is covered, irrespective of wh causes it.

Key-words: Lasers, Safety, Legislation, European Union, the Netherlands

\subsection{Introduction}

This chapter provides an overview of the laws and other, for the most part technical, standard: applicable to the use of lasers in the European Union, with a specification for the Netherlands Paragraph 18.2 looks at present and future EU policy which already governs or may govern th: use of lasers in the European Union. A distinction is made here between pollicy on products an goods and policy on occupational health and safety. We also look at how EU policy stands il relation to rules and standards devised by private-law bodies for the development and use 0 lasers. Paragraph 18.3 examines the Dutch national legislation relevant to laser use, namely th: Medical Devices Act, the Order on Criteria for the Recognition of Hospitals and the Occupationa Health and Safety Act. This paragraph also looks at the question of periodic eye tests. Paragrapt 18.4 considers the rules on liability in the event of injury resulting from laser treatment. Criminal an civil liability are considered together with liability under the Medical Discipline Act. The rules or liabillity in respect of the provision of medical lasers on approval and maintenance agreements fo them are also examined. The chapter ends with a brief discussion. Appendix 1 provides a list a translations of English terms for Dutch laws. 


\subsection{EU policy and standards bodies governed by private law}

\subsection{EU Directives}

Direches are an Importan part of European legistation on medical lasers. They are adopted by he Council on proposas by the Commissiont. An EU Directive is substantiaty binding on the nember slates. This meins hat the content of a Directwe has to be hooporated mo the member states ${ }^{2}$ own legislation. Usuath that boils down to hamonization measures. In general these are measures for the reciprocal mplementation of legal and acministrative provisions by menber states concerning the estabishment and operation of the single marke tit: Under the single market. or example, member states withave to open their maxkets to each ohers" products. Excessive differences in qualty due to differing ulues in the individual member states whil have to be roned out by he harmonization of quality criteria.

Medical devices, including lasers, are classifed as industrial products ${ }^{25}$. Eu policy an medicat Kasers thus derves from Directives on policy for goods and products and Directives on occupational hygiene and protection against occupational accidents.

\subsubsection{EU policy on products and goods}

There are different wcensing criteria in the individual EU member states for medical devices and other industrial products. These include different labeling requirements and registration methods, or different criteria for manufacture. Within the meaning of Community taw these tifferences constiute "barriers to rade" and are principally subjed to Article 30 of the EC Treaty ${ }^{235}$. As of 1985 . the so-called 'new-approach" has been followed in an effort to elminate barriers to trade. Under the new approach, instead of giving detated technical regulations, Directives contine themselves to a broader framework of objectives and criteria for safety and health. Whthin his framework essentia safety requirements (ESR) are formulated, the basic criteria for which are (usualy) set out in an Anex to the Directive concerned. The essential requitements in the form of technical specificautions are drafted by private standards bodies such as CEN (European Commitee for Standardization) and CENELEC (European Committee for Electroechnical Standardization). Industry needs these specifications in order to satisy the basic requirements laid down in the Ditectives. These standards are not in principle mandatory. But governments do have a duty to assume that products satisfy the essential requirements laid down in the Directive when they are manufactured in accordance with the harmonized standards ${ }^{\text {ins }}$. Such products can be considered for a CE marking (Conformite Europeenne). Responsibility for the affixing of a CE marking lies with the manufacturer. Because the harmonized standards are not mandatory manufacturing requirements the manufacturer must prove, if a product is not manufactured to these standards, that it meets the essential criteria of the Directive. The manufacturer must seek this proof either from independent notified bodies (testing houses) named by the member states or apply self-certification. The choice between both methods depends on the risk associated with the device and its complexity. Selfcertification, which comes down to drawing up an EU declaration of conformity, is restricted to class I medicall devices. Medical lasers are placed in a higher risk clas $s^{[1 \% 1,311}$. In the Netherlands the Ministry of Health, Welfare and Sport (VWS), as the competent authority for decisions of this nature ${ }^{\{32\}}$, has named KEEMA (the Dutch Quality Control Institute for Electrical Materials and Applicances) and CMT-TNO (the Center for Medical Technology of the Netherlands Organization for Applied Scientific Research) as notified bodies. A product which has a CE marking cannot be refused by the member states of the EU. The new approach does, however, in the case of an item that is regulated "generally" at Union level, allow member states the option of going further and conducting their own policy within their awn territory. But this policy must be consistent with Union policy, that is to say it must not form a (hidden) barrier to trade, and within the limits of Union policy it must produce the desired effect ${ }^{[?, 51\}}$ 
As part of the new approach the EU has commisioned a number of European manufacturers' associations to prepare draft Directives for policy on the licensing and quality control of medica devices ${ }^{77 !}$. A Directive on active implantable medical devices was adopted in 1990 (Directive $90 / 385 /$ EU). A Directive on in vitro diagnostic aids is scheduled for 1997 , and a proposall for this was published in mid-1993 $3^{13 \%}$. Following two proposals ${ }^{[0,121}$ the text of a Directive conceming medical devices was agreed in mid-1993 ${ }^{\text {in! }}$. Medical lasers are subject to this Directive, which has come into effect by 1 danuary 1995 (Article 22). The Directive on medical devices sets out to harmonize national rules and to safeguard the health of patients, users and, where the occasion arises, of third parties, when such devices are used ${ }^{[11]}$. The essential safety requirements are sel out in Annex 1 of the Directive. Under the Directive medical devices must from 14 June 1998 camy a CE marking (Articles 17 and 22). Until then national legislation remains in force. During this transitional period manufacturers have a choice. Until the middle of 1998 they can either continue to use the procedure for marketing medical devices which currently applies in each member state; or they can apply directly for a CE marking for a medical device provided the member states have incorporated the relevant Directive into their national legislation. As of 1 January 1995 this should have been done. In the Netherlands, the Directive was actually enforced 12 May 1995. If the procedure to obtain a CE marking is successful the device can thereafter be marketed freely throughout the whole of the EU.

The medical devices concerned are divided into three classes on the basis of the risk they represent: low (class I), moderate to high (class lla and IIb) and very high (class III). The higher the risk, the stricter the approval procedures ${ }^{1+1 !}$. Which category do medical lasers come into? Annex IX of the Directive lists classification criteria. In paragraph 1 of this Annex 'active medical devices" are defined as devices which can only operate when connected to a source of electrical energy: Active therapeutic devices are defined as devices intended to support, modify, replace of restore biological structures with a view to treatment or alleviation of an illness, injury or handicap. On the basis of this medical lasers may be classified as active therapeutic devices. Annex $I X$, paragraph 3 of this Directive places active therapeutic devices in risk class lla, unless their characteristics are such that they may administer or exchange energy to or from the human body in a potentially hazardous way, taking account of the nature, the density and site of application of the energy, in which case they are in class Ilb. Lasers should thus be placed in risk class Ila or Ilb. In the former case a notified body must be involved in the manufacture and final vetting of such devices if a CE marking is to be obtained. In the latter case the manufacturer must also involvea notified body at the design stage of the device ${ }^{[11]}$.

If there is the likelihood of a danger to the health and/or safety of patients, users and possibly third parties, a member state may under Article 8 of this Directive recall a medical devilce or prohibit or restrict ils use (the safeguard clause). The European Commission must be notified of this at ance. Article 10 stipulates that incidents occurring after medical devices have been placed on the markel will be recorded centrally and the details passed on if appropriate to other member states and to the Commission; the same goes for the measures taken. This can be the starting point for new measures at EU level. Under Annexes $\| I, I V, V, V I$ and $V I I$ of the Directive manufacturers are pirimarily responsible for a system of post-marketing surveillance (PMS). They are responsible. where relevant, for ensuring that a device can be traced. Instructions for use, checking and maintenance of devices must also help to ensure that they are sensibly and safely used ${ }^{\|, 13 n}$.

\subsubsection{Pollicies towards clinical research using medical lasers}

Under Article 15 of the Directive an medical devices ${ }^{3}$ such devices may be made available for clinical research only in certain circumstances. On the basis of this Article, Annex 10 of the Directive stipulates that confirmation of conformity with the essential requirements concerning the characteristics and performances under the normal conditions of use of the device and the evaluation of the undesirable side-effects must be based on cinical data in particular (bold printed by the authors) in the case of implantable devices and devices in class $\left[\|^{111}\right.$. The clinical data must. 
be based on either a compilation of relevant articles, a literature review, or the results of a clinical investigation. This suggests that manufacturers of medical lasers, which will be classified in class lla or I $_{\mathrm{b}}$ can freely choose whether or not to document the efficacy of the intended applications of their devices. When the choice is made to document efficacy, a clinical investigation seems again optional: However, if this option is selected, a number of preconditions have to be met. intended research must be submitted to a competent authority and authorized by a medical ethics committee. Clinical evaluation of medical devices musit be done on the basis of an appropriate state-of-the-art test protocol which is such that it enables the manufacturer's claims for the device to be vindicated or refuted. The research must, for example, contain enough evidence that its findings are sound. It must also be carried out in observance of the Helsinki Decilaration". A research project may be rejected for reasons of public health or concern for public order. The provisions on clinical research also cover research which is carried out using a device bearing the CE marking and when the device will be used for a purpose other than that referred to in the CE. conformity assessment procedure (Article 15(8)) ${ }^{[1.311}$. In theory this may mean that medical lasers bearing a CE marking have to be reassessed if they are used for a purpose other than described by the manufacturer in the context of the essential requirements:

\subsubsection{Coordination of EU measures on products and goods with the activities of standards bodies governed by private law}

Mention has already been made of the coordination of EU policy on products and goods with the work done by standards bodies. EU policy-making also takes account as far as possible of existing international standards such as those of the ISO (International Organization for Standardization) and the IEC (International Electrotechnical Commission).

What implications does this have for medical lasers? The manufacture of lasers may comply with the internationally recognized quality standards of the ISO (ISO 9000-1;2 and 3). The IEC also published a specific standard for the safe use of laser equipment (IEC 825) ${ }^{\mid 241}$. This standard gives definitions, requirements for classification of the laser beam, imarkings, maximum permissible exposures, a classification, and examples of how to calculate a hazard distance for stray laser light which may escape from the device. IEC-825 has since been adopted in amended form by CENELEC, of which the Netherlands is a member, as CENELEC EN 60825 ${ }^{[22]}$. This standard, ratified in 1991, was adopted by the Netherlands and published in 1992 by the Netherlands Standards Institute (NNI) as NEN-EN $60825^{128 !}$. When buying a laser both seller and purchaser should make sure that the equipment complies with this standard.

\subsubsection{EU policy on occupational hygiene and protection against occupational accidents}

In 1992 the EU published a proposal for a Directive designed to protect workers against actual or potential risks to their safety and health arising as a result of exposure to physical agents ${ }^{[4]}$. The proposal was an individual Directive pursuant to the Council's framework Directive 89/391/EEC. The Directive in question was incorporated into the Dutch Occupational Health and Safety Act in 1992 (see section 18.3.3). The proposal for a Directive on physical agensts applies only to nonionizing radiation. The proposal comprises a number of general articles which are elaborated per individual physical agent in Annexes. Annex 3 of the draft-Directive is relevant to medical lasers. This Annex is concerned with risks to eyes and skin in case of exposure to oplical radiation with a wavelength between $100 \mathrm{~nm}$ and $1 \mathrm{~mm}$. The most important articles are briefly discussed below. Under Article 3(4) certain activities are considered to present an increased risk. Annex 3 of the proposal defines such activities, with reference to amendment 1 of IEC 825 (1990), as activities involving lasers of class IIIb and above (it should be noticed that the IEC-classification differs from the classification in the Directive concerning medical devices). Such activities must be declared to the authority responsible. That authority must satisfy itself that appropriate measures are taken to control the risks associated with these activities. Under Article 9 persons working with sources of 
physical agents presenting an increased risk must receive special training and their competen must be checked. Article $5(1)$ says that efforts must be made to reduce the risks of exposure to physical agent to the lowest achievable level. Under paragraph 2 the employer must establish an implement a program to reduce the risk referred to in paragraph 1 by means of technical andli organizational measures. To reduce the risk of exposure the employer must, under Article provide workers with personal protection equipment. The employer must check the effectivenes of the personal protection equipment used. Under Article 8 workplaces to which specific protectiv measures apply must be marked accordingly. These workplaces must also be delimited and, when the exposure risk warrants it, access to them must be restricted. This Article is not elaborated o further in Annex 3 . Article 12 contains provisions on equipment aids and working methods. On th basis of this Article Annex 3 stipulates that lasers used at work must be labelled with the classification number according to amendment 1 of IEC 825 (1990). Article 13 (2) lays down rula for measures to be taken in the event of indirect effects of exposure to physical agents. On th basis of Annex 3 the provisions of Article 13(2) apply if optical radiation is likely to cause fire produce hazardous substances by decomposing or interfering with materials present. Thi explanatory memorandum justifies this Article by the example that a laser can cause material t decompose into toxic products or cause them to ignite.

This draft-Directive forms a potential legal basis for a large number of measures of which the mos important have been discussed in Chapter 3. However, until late 1996, a definite version of thi proposal has not been published.

\subsubsection{Coordination of EU policy on occupational hygiene and protection against occupational accidents with the work of standards bodies governed by private law}

The coordination of EU policy with the work of standards bodies governed by private law has beer expressed in the setting of various exposure levels for optical radiation. Article 2 of the proposa for a special Directive on physical agents distinguishes three exposure levels: a ceiling level, : threshold level and an action level ${ }^{5}$. The threshold level and the various action levels are all derive: from the ceiling level. In Annex 3 of the Directive reference is made, for the setting of a ceiling leve of exposure to optical radiation, to a publication by the ACGIH (American Conference o Government Industrial Hygienists $)^{12 !}$. The explanatory memorandum also makes reference to the exposure limits for optical radiation published by the Non-lonizing Radiation Committee of the International radiation protection Agency (INIRC-IRPA) ${ }^{[13]}$. This explanatory memorandum concludes that the proposed exposure limits of the ACGIH and INIRC-IRPA are based on a virtua consensus concerning the level of scientific knowledge. Thus the proposals largely agree with each other. As noticed, on the matter of lasers the Directive makes reference to IEC-825 and to the 1990 amended version of this standard (IEC 825-1). A further revision of this standard has been published in $1993^{\text {t23! }}$

\subsection{Dutch national legislation}

\subsubsection{Introduction}

In The Netherlands the Medical Devices Act, the Order on Criteria for the Recognition of Hospitals and the Occupational Health and Safety Act are relevant to laser safety 


\subsubsection{The Medical Devices Act}

In the Netherlands a Medical Device Act was introduced in 1970. This framework-Act included aspects of the quality (including safety) and use of medical devices. Thus the scope of this Act is wider than the scope of the present EU Medical Device Directives. Partly because of its relatively wide scope, the Medical Devices Act could potentially have had a major impact on the introduction of new medical devices in the Netherlands. However, since its inception the Act has only been implemented with respect to the sterility of certain devices and to rubber condoms. In the seventies only one executive at the Dutch Ministry of Health worked in the field of medical device regulation. The most important explanation for the low priority of medical device regulation was that accidents involving medical devices hardly ever happened, according to Dr. Faro. An affair with heart-valves in 1985 was the first time in the Netherlands that serious problems with medical devices were reported. However, this did not result in a policy change. Secondly, because the capacity for development of regulations for individual medical dlevices was limited and labour-intensive at the same time, the total number of 'regulations' was low. Subsequently, the philosophy of deregulation became dominant, conflicting with the aims and scope of the Act. Finally, when the EU announced its legal activities in this field (in the mid-eighties), the Dutch government decided to stop its own regulatory activities in this field.

Early 1993 the EU Directive on active implantable medical devices was incorporated in the Medical Devices Act. The second EU Medical Device Directive has been incorporated in the Medical Devices Act as well.

\subsubsection{The Order on Criteria for the Recognition of Hospitals}

All thealth care establishments providing services under the General Insurance Against Special Sickness Costs or the Sick Funds Act must be officially recognized. Recognition means that that the services taken up by insured parties will be of the requisite quality and quantity. These recognition standards thus require the hospital among other things to take measures to ensure that 'the safety (of patients and staff) is safeguarded as far as possible' (Article 11.2(b) $)^{[5,21]}$.

A 1985 study found, however, that the recognilion standards were very loosely enforced and that there was no active, coherent and coordinated policy in many hospitals ${ }^{[2]}$. Given that the recognition system was not working satisfactorily, the Dekker Committee advocated scrapping it in a 1987 report entitled 'The Will to Change ${ }^{|\theta|}$. Although that recommendation was based on research which is now out of date, it does seem that the recognition criteria are of little help in ensuring the safe use of medical laser equipment. Even so, the recognition criteria did prove important in 1988 in a criminal liability sult where the use of medical equipment was a major factor (see section 18.4.2).

\subsubsection{The Occupational Health and Safety Act}

The Occupationall Health and Safety Act primarily addresses employers and employees ${ }^{11}$. Laser safety falls under the purview of the Act in three different ways. Firstly, the Act itself contains general provisions which also apply to work with lasers. Secondly, more specific rules on noninizing radiation are contained in a number of safety orders, namely the Safety Order for Factories and Workplaces (last amended in 1991) ${ }^{|38|}$ and the Safety Order for Other Groups, which came into force on 1 October $1990^{16 !}$.

Article 4 of the Occupational Health and Safety Act requires the employer to desigin his policy with an eye to maximum possible safety. Article 6 requires the employer to provide the worker whom he recruits with information and training as often as is necessary for safety. Where workers are issued with personal protection equipment the employer must ensure that workers are familiar with its purpose and know how to use it. Article 9 sets out rules for the reporting of accidents and occupational illnesses. Article 11 requires employers to provide the right kind of personal protection 
equipment and ensure that it is used properly. Article 12 requires workers to exercise due care prevent dangers to their own safety or health and that of others. They are required to make prop use of the personal protection equipment issued to them.

\subsubsection{The Safety Order for Factories and Workplaces and the Safety Order for Other Groups}

The Safety Order for Factories and Workplaces ${ }^{(3)]}$ is addressed to workers in factories an workplaces in e.g: a technical department of a health care facility. The Safety Order for Oth: Groups $^{\text {id }}$ is applicable to workers in hospitals and private clinics. The two Orders contain simil: articles on non-ionizing radiation. The most important articles in the Safety Order for Other Group are abridged below:

Article 51

(1) Apparatus which may emit harmful non-ionizing radiation must be of robust material an construction and in a good state of repair. Such apparatus must furthermore be installet assembled or shielded in such a way that when it is in operation, physical damage as a resu of intentionall exposure to radiation can be avoided as far as possible.

(2) Where the danger of physicall damage cannot be avoided entirely when apparatus of this kin is in operation, the performance of work on or using that apparatus and the presence : persons in the vicinity of the apparatus must be such that physical damage as a result: radiation is avoided as far as possible.

(3) The Minister (of Social Affairs and Employment) may, depending on the physical properties the non-ionizing radiation, set levels above which such radiation is for the purposes of thi Article deemed harmful. The Minister may lay down rules concerning the room in which sut apparatus is situated, or within which radiation emitted by such apparatus is encountered.

Article 52(3) and (4c) (extraction; information to workers on hazards)

Air contaminated by gases, fumes or aerosols must be removed efficiently from place occupied by workers for purposes of their work. If it is not reasonably possible to prever exposure, the workers exposed must be fully informed of the hazards entailed in the exposure.

Article 53 (supply of clean air)

Where contaminated air is removed, there must be a guaranteed supply of clean air.

Article 62 (personal protection)

(1) If, despite compliance with other articles, the danger of physical damage persists for worker exposed to that danger, the prescribed personal protection equipment for eyes ${ }_{n}$ skin and othe body parts exposed to radiation must be fully available and care must be taken to ensure the those workers use it.

(2) The personal protection equipment referred to in (1) must be in a good state of repair and mus be stored tilily.

\subsubsection{Monitoring of compliance with the Occupational Health and Safety Act}

Compliance with the Occupational Health and Safety Act is monitored by the Labour Inspectorait Line management is responsible for enforcing the rules on safe working. The role of the Labou Inspectorate will be outlined in further detail below. In 1978 the Health Council produced a repal on acceptable levels of electromagnetic radiation in the wavelength range $100 \mathrm{~nm}$ to $1 \mathrm{mr}$ (micrometer radiation) for human eyes and $\operatorname{skin}^{[18]}$. Up to the middle of 1992 the Labou Inspectorate used the recommendations of this Health Council report as a guide in day-to-da 
practice for the maximum permissible exposure of persons to laser radiation. Since the middle of 1992 the Labour Inspectiorate has been guided by a concept of maximum acceptable levels of exposure to non-ionizing radiation published by the INIRC-IRPA in 1991. The definitive version of this report was published in $1992^{(14)}$. At the same time. NEN-EN 60825 was published (see Section 18.2.4). This standard has been recommended by the Labour Inspectorate of the Ministry of Social Affairs and Employment since mid-1994. The Netherlands were obliged to enforce this standard by mid-1995. Finally, in June 1993 a revised version of the earlier 1978 Health Council report appeared under the title "Optical radiation. Guide values for exposure to electromagnetic radiation of wavelengths between $100 \mathrm{~nm}$ and 1 millimetre, with reference to health ${ }^{\mathrm{mt} i \mathrm{in}}$. Although this report could potentially have been used again by the Labour Inspectorate for guidance ${ }^{7}$ its practical role has been limited due to the implementation of NEN-EN 60825 .

\subsubsection{Development of legislation on medicall lasers in the Netherlands}

The question for the national legislator faced with the existence of medical lasers is twofold. One, is there a need to develop legislation? If so, should legislation be incorporated into existing structures (laws) or should a new law be drafted? The legislator has not yet pronounced on the issue. The view of the Health Councill ${ }^{\mid 26}$ was that legislation on medical lasers is indeed needed and that it should as far as possible dovetail with existing legislation. The view of the Nationall Commission is that, given the evolution of legislation at the EU level since the publication of the Health Council's report in 1992, apart from the desirability of legislation there is a pressing need for the Dutch legislator too to draft legislation on medical devices, including medical lasers (see Section 18.2.2). For further consideration of this subject see the Health Council's report ${ }^{[26]}$.

\subsubsection{Periodic eye tests}

The Health Council's view ${ }^{[26]}$ is that periodic eye tests serve no useful purpose. In reaching this opinion the Health Council considered the fact that laser safety standards are primarily designed to prevent eye injury from lasers. When accidents occur despite the safety measures, the eye injury is in practice often so slight, says the Council, that eye abnormalities can only be identified in the first few days after an accident. After one or two weeks, the resulting changes in the pigmentation of retina and choroid are often no longer distinguishable from normal pigment abnormalities. Where slight laser damage to the eye is discernible this is best observed two to three days after the accident by an ophthalmologist. Given that there is limited provision for ophthalmic check-ups at the weekend, the Health Council recommends that the worker concerned should visit an ophthalmologist within 72 hours and request an eye test and confirmation of any damage, if possible by using photography or fluorescein angiography. Where lasers are used intramurally it must be part of the laser safety officer's duties to check that this has been done and to record the incident in writing (see Chapter 3). The Health Council also thought that the previously described method of visual fielld measurement should be replaced by automatic perimetry or an Amsler's chart. The National Commission agrees. The laser safety officer should act as coordinator in any incidents involving lasers

\subsection{Liability for errors involving medical laser equipment}

\subsubsection{Introduction}

Liability can arise under both public and private law $w^{9}$. An important element of public law is criminal law. One speaks of criminal liability, as defined in the Criminal Code. Private liability is defined in the Netherlands in the Civil Code. In addition to these forms of liability there is also liability under the Medical Discipline Act ${ }^{127}$. A second distinction which needs to be made is that between natural 
and llegal persons. Natural persons are actual human beings. Legal persons may be governed by either public or private law". Importantly, both natural and legal persons can be sued.

The next few sections examine the legal rules on criminal and civil liability and the provisions of the Medical Discipline Act. Recent EU developments on liability are also considered.

\title{
18.4:2 Criminall liability
}

Claims under the criminal law may be made against both natural and legal persons ${ }^{211}$. There mus be three conditions for criminal liability to exist. Firstly, a criminal act must have been committer which comes within the legal definition of an offence (Article 1 of the Criminal Code). Secondly, the criminal act must have been committed in contravention of the law, that is to say the conduct ir question must have been unlawful. Thirdly, the offender must be shown to have committed it:27] Since the judgment on defective anaesthesia equipment $t^{[3]}$ presents an obvious analogy with the use of medical laser equipment, we reproduce below Hubben's description of this case in Intermezzo 18. $1^{[2 \pi \mid]}$

\section{Intermezzo 18.1 Liability for emrors inwolving anesthesia equipment}

\begin{abstract}
A patient died in hospital during an operation. When it was time to bring the patient back to consciousness, he was given not the oxygen he should have received but a fatal dose of nitrous oxide. When the facts of the case wert investigated it was found that shortly beforehand, the two gas nipples on the anaesthesia machine had been replaced by the hospital's technical department. During replacement the nitrous oxide nipple had been mistakenty connected to the machine's oxygen port, and the oxygen nipple had been connected to the nitrous oxide port. The public prosecutor decided to bring criminal proceedings against both the hospital and the anaesthetist concernet for culpable homicide. The anaesthetist was given a six months suspended sentence. He was accused of having negligently failed to observe the effect in question and also of failing to ensure that the ventilation system had in axygen level meter and an alarm. The foundation, as the legal person managing the hospital, was fined 25000 guilders. In finding the hospital guilty the judge ruled that the hospital had not properly discharged its responsibitity for the general functioning of the hospital, including concern for the quality and reliability af equipment, "as expressed in the Order on Criteria for the Recognition of Hospitals's3 (see Section 18.3.2). According to Hubbers the judge was drawing in this case on the recogniltion criterion that hospitals thave a duty to take measures which ensure that "the safety (of patients and staff) is safeguarded as far as possible" (Article 11.2(b) ${ }^{[i 7}$. No appeal was lodged against either this judgment or that concerning the anaesthetist $\left.{ }^{22}\right)$.
\end{abstract}

It is apparent from this that both a hospital and its employees may be held criminally liable for events which occur as part of services provided in hospitals. Naturally this will also apply to services involving lasers.

\subsubsection{Clvil llability}

The Civil Code applies to anyone who is a part of the legal process in the Netherlands. It is specifically complemented in matters of health care by the Contract for Medical Treatment Aci, which has come into effect 1 Aprill $1995^{177}$. The purpose of the Act is to regulate the relationship between practitioner and patient, in view of the vulnerable position of the latter. Secondly, Articles 185 - 193 of the Civil Code incorporate an EU directive on liability for defective products ${ }^{229 !}$. The Civil Code thus regulates liability for errors caused by persons and by defective objects. The result of these rules on liability for persons and objects is thus that the patient has a "central address" for his claim, namely the hospital ${ }^{[4,37]}$. Under the new Act the difference between self-employed specialists and payroll specialists is no longer existing ${ }^{[4,37]}$.

Hospitals, specialists and other employees can insure themselves against liability claims. Current legal liability insurance for hospitals mostly provides "intramural cower", that is to say damage to a patient sustained within the precincts of the hospital establishment is covered, irrespective of who causes $i^{[35]}$. If a patient sues a hospital for damages and the claim is upheld, the hospital may try to offload liability on to its employees or an equipment manufacturer. The various rules which apply are described below. 
Liability under private law may arise from legal and de facto acts. In the former case, llability through negligence may be claimed, in the latter case, liability through unlawful act.

\section{Lilability through negligence}

in health care there is the concept of a special obligation linking patient and physician andior establishment - the contract for medical treatment, which is generally deemed to entail an obligation to provide a service ${ }^{[27]}$. The obligation to provide a service contrasts with the obligation to achieve a result, where the objective is the attainment of a specific outcome. If the debtor, e.g. a physician in the case of a contract for medical treatment, does not carry out his contract to provide an agreed service properly, the law speaks of fault. The fault which the debtor is deemed to have committed is, in legal language, that of negligence. The law states as a general principle that the debtor is liable in the event of negligence ${ }^{[25]}$. A debtor is deemed to have committed a fault if the fault is attributable to the debtor and it is attributable to the debtor by virtue of the law, a legal act or convention ${ }^{|25|}$.

In general, fault arising from an obligation to achieve a result is easier to prove than fault arising from an obligation to provide a service. In health care the difference between error and risk is important in deciding whether or not there has been an attributable fault. Appropriate application of an appropriate method does not necessarily achieve the intended result. Normal risks of medical acls carried out in accordance with proper professional medical standards do not lead to liability on the part of the physician. Only professional error can lead to attributable faults ${ }^{[27}$.

The physician is also responsible for the persons the uses in the performance of the contract. There is no requirement that such persons should be employed by the physician. They may be made available to him, as for example in the case of nursing staffi27!

\section{Liability through unlawfull act}

An unlawful act is basically a de facto act whereby one person unlawfully causes harm to another. The person causing the damage is obliged to make recompense for that damage. In contrast to the position with legal acts, the question of intent is not generally a factor in de facto acts such as unlawful acts. The obligation to make recompense is simply there, whether the person causing the damage intended to cause it or not. The Civill Code distinguishes three forms of unlawful act: infringement of a right, commission or omission of an act in contravention of a legal obligation, and commission or omission of an act in contravention of what is proper under the unwritten laws of sociall behaviour ${ }^{[25]}$.

There are five criteria which must be met in respect of liability through unlawful act: the existence of an unlawful act, fault, damage, causality and relativity. The last-named criterion says that there is only an obligation to make recompense if the standard violated includes protection against damage of the kind sustained by the injured party ${ }^{[271}$. In health care the burden of proving that an act committed or omitted can be regarded as an unlawful act used to lie with the victim, usually the patient ${ }^{|2|]}$. This situation has changed with the advent of the Contract for Medical Treatment Act. Why? In the old situation the patient could hold hospitalls and/or physicians liable for professional error on grounds of unlawful act or negligence. Where a given act was both negligent and unlawful, the plaintiff could invoke either. The consequences of the actions were virtually the same, though the procedures were different. An example of a difference is that persons assisting had secondary liability in the case of liability through unlawful act, but this was not the case with contractual liability ${ }^{[27]}$. This example shows that for the patient it was often hard to establish who is liable for a protessional error committed against him either under a contract or as an unlawful act. Therefore, in an attempt to simplify this situation, Article $1653 \mathrm{q}$ of the Contract for Medical Treatment Act sets out rules for the liability of hospitals and equivalent health care establishments in respect of all activities carried out by care personnel in such establishments. Under Article 1653q a "central address" must be provided for patients seeking to lodge claims. For these and other reasons the Article concerned construes the hospital's liability as a contractual liability: if it is not itself a party to the contract for treatment the hospital is deemed liable for faults in the performance of that contract for treatment, provided that treatment is given within the hospital precincts, as if it were 
itself a party to the contract. This is important in regard to liability for objects used in treatmen such as medicall lasers and other equipment ${ }^{(3)}$.

In its provisions based on the draft version of the Contract for Medical Treatment Act, Article? of the Civil Code correctly anticipated, on the matter of contractual liability, that the debtor is liabl for objects used in the performance of the obligation, "unless this is unreasonable in view of th content and import of the legal act from which the obligation derives, current conventions and th other circumstances of the case". In the memorandum of reply on this provision, which wa consistent with applicable law, reference was made in connection with this exception to th Contract for Medical Treatment. If a claim is brought against a physician or hospital in respeds defective equipment the Civil Code must allow for that claim to be rejected, where there seems greater possibility of liability on the part of the manufacturer ${ }^{[37]}$. This brings us to the area of liabilit for objects and, more specifically, the area of product liability.

\section{Liability in respect of objects}

As a general principle a hospital is liable for objects it uses such as defective equipment employe in the treatment of patients unless such liability would be unreasonable. The hospital itself mus prove that liability in a given case is unreasonable. It may be unreasonable to hold the hospit: liable if the product is inherently defective. In that case the issue is one of product liability and thi risk is borne by the manufacturer $r^{\mid 4,27 !}$.

In 1985 the EU Council of Ministers issued a Directive on liability for defective products (OJ Na: 210/29/EC of 7 October 1985). In 1990 this Directive was incorporated into Dutch law in Article: $185-193$ of the Civil Code ${ }^{[29,30]}$. The objective of these rules is to counter unfair competition an obstacles to the free movement of goods within the EU and to place consumer protection in thr member states on a more equal footing.

Article 185 of the Civil Code places a liability for risk on the manufacturer once a product marketer by him proves defective and thus causes damage ${ }^{|29|}$. A product is deemed to be marketed whei it has passed into the channels of distribution. This is usually when the product has been sald ank supplied or when it has been rented or leased. Six restrictive exceptions to liability for risk ant named in Article 185(6). A manufacturer can invoke these when defending a liability claim. If addition to the proviso that the product must have been placed on the market, the most importan provisos are that it must be possible to assume, in the circumstances, that the defect which causer the damage did not already exist when the product was first marketed by the manufacturer, but tha this defect developed later (e.g. as a result of improper maintenance). A second exception is th: so-called "state-of-the-art defence", namely that it was not possible, at the level of scientific ant technological knowledge current when the manufacturer first imarketed the product, to identify the existence of the defect ${ }^{[29]}$.

Article 186 defines what makes a product defective. The criterion is whether or not the produc: offers a llevel of safety which matches up to expectations. In principle the expectations are those of the general public, but where specific expectations of safety exist for specific products only ir specific (specialist) circles, these are the expectations considered ${ }^{12.51}$ "It would seem reasonable th apply the latter option to lasers.

Article 187 defines the concepts of "product" and "manufacturer". Under paragraph 1 "product" is taken to mean primarily moveable objects, together with electricity. Paragraph 2 defines "manufacturers" as manufacturers of an end product, the producer of a raw material or the manufacturer of a part, together with anyone who presents himself as the manufacturer by causing his name, logo or any other distinguishing mark to appear on the product. Paragraph 3 stipulates that, notwithstanding the liability of the manufacturer, anyone who imports a product into the El with a view to selling, renting, leasing or otherwise distributing it as part of his business activitie: is regarded as a manufacturer; his liability is the same as that of the manufacturer. Paragraph 4 stipulates that if it is not possible to ascertain who the manufacturer of a product is, every suppliel is regarded as the manufacture ${ }^{[29]}$.

In normal circumstances the hospital will be regarded in these rules as the consumer. However because of the broad definition of what constitutes a manufacturer ${ }^{\{27.29,30\}}$. Ankone (1990) believes it possible that a hospital too may count as a manufacturer within the meaning of this legislation 
Examples are an establishment which imports products direct from a non-EU country, a hospltal which does not declare the identity of a product or uses it under an own-brand name. One might also point to situations in which hospitals themselves manufacture and use products, e.g. laser fbres, tips and probes. But according to Pluyter and Veenendaal ${ }^{30}$ the hospital will not readily be regarded in practice as a manufacturer of medical equipment. Even if the hospital manufactures or assembles the equipment itself, they believe the establishment cannot be deemed liable for damage resulting from defects in these products because the proviso that the manufacturer must have marketed the product will not have been met. Different authors thus disagree on whether or not an establishment can be a manufacturer for the purposes of the law. In the absence of a clear statement on the subject, hospitals are advised to be reticent in decision-making towards homemanufacturing or adapting products or materials that are used in the context of medical laser applications.

Under Article 188 the burden of proof rests with the injured party. The idea here is that the patient can recover from the manufacturer the costs of damages sustained as a result of defective or nonfunctioning medical equipment, by demonstrating that he has suffered damage and that this damage was caused by a defect in the equipment.

A characteristic of the rules on liability for risk is that the patient expressly does not have to prove fault on the part of the manufacturer. It is enough to demonstrate damage caused by a defect in the product ${ }^{\left.20,22_{1} 30\right]}$. Proof here is easier than proof of negligence, where fault does have to be demonstrated. In both cases (in line with the concept of central liability) the hospital will be deemed liable by the patient, but ultimate responsibility in this case rests with the manufacturer ${ }^{\mid 37 !}$. The hospital cannot itself sue on grounds of product liability because it suffers no damage as a result of the product itself (Aiticle 190(1)). Hospitals are thus advised to back the patient in proceedings so that in any lawsuits responsibility is in fact passed to the manufacture $r^{[4]}$. In practice, in order to protect the patient, the judge may rule that the hospital is liable. Following this ruling the hospital may then try to pass on that liability to the manufacturer.

\subsubsection{Legal aspects of medical equipment supplied on approval, and the maintenance of medical equipment}

\section{Agreement between manufacturer or supplier and the hospital for the supply of equipment on approval}

The acquisition of medical equipment on approval is usually regarded by the law as a "trial purchase ${ }^{\text {"l }}$ or a tacit agreement ${ }^{30]}$. Other forms, e.g. hire purchase and leasing are also found. With a trial purchase it is assumed that a deferred purchase agreement has been concluded, subject to the purchaser approving the equipment within a given period of time. If the purchaser allows the agreed deadline to pass he cannot then reject the equipment (Article 45, Vol. 7 of the Civil Code). Whilst the deadline has not yet been reached and the purchase is thus not yet final, any risks for the equipment are borne by the seller (Article 46 of the Civil Code). The main law applying is the law of contract which says that parties may reach other agreements with each ofher. That law thus applies if parties have nol agreed otherwise. Pluyter and Veenendaal ${ }^{(30)}$ advise hospitals to get the terms and conditions of any supplies provided on approval recorded in writing.

As the representative of the legal person, the management of the establishment is authorized to conclude contracts with manufacturers. However, the statutes of the establishment may stipulate that other persons too may be authorized representatives. Certain officers may also be empowered to conclude (certain) contracts. If a contract is concluded on the hospital's behalf by a nonauthorized person the hospital is not bound by it and can have the contract declared null and void. The hospital may, however, be bound to a contract concluded without authority if it was reasonable for the other party to the contract (the supplier) to assume from the hospital's actions that authority had in fact been granted. For example, if a specialist has had long-standing agreements with suppliers for the provision of equipment on approval, the supplier can in a specific case reasonablly assume that the speciallist is authorized to order it. If he is not, the hospital is nevertheless bound 
to the contract. Clear written agreements in the hospital and between suppliers and hospital an thus advisable

\section{Liability for equilpment supplied on approval}

Central liability of hospitals for persons and objects applies equally to cases of damage sustaine during the treatment of patients with equipment installed in the hospital on approval unless th hospital can show that it is not reasonable to hold it liable. A second form of liability is that of th hospital towards the supplier. One can think here of e.g. damage to the equipment, failure to retun it or returning it too late, and improper use. For damage of this kind the supplier may hold th hospital liable ${ }^{\{30 !}$.

\section{Liability in the event of a maintenance agreement}

Here again reference can be made to the wording of the provisions on the central liability 0 hospitals. Where damage has been caused it is doubtful if a hospital can prove that the cause 0 the damage lies in defective equipment and that the cause of the equipment's defectiveness lie: in poor maintenance, if, moreover, all maintenance work is carried out by the manufacturer unde contract. In general a disaster can hardly ever be ascribed solely to defective maintenance, as is apparent from the judgment on defective anaesthesia equipment ${ }^{\text {s5 }}$. There are nearly alway: organizational factors involved too. Therefore, it will be necessary to prove not only poo maintenance but responsible administration as we If $^{[30]}$. It is fair to assume that hospitals will not be able to rely too much on the product liability rules. Even so a Dutch Committee on the Coordination of the Assessment of Hospital Insiruments ${ }^{(t) \mid}$ found in 1990 that following incorporation of the product liability rules into the Civil Code, manufacturers were increasingly inclined to dc maintenance work on medical equipment themselves. For both legal and economic reasons the Committee regarded this as undesirable in most instances. Therefore, the Committee prepared a checklist for the drawing up of maintenance agreements between manufacturer and hospital. The report published by the Committee also contains recommendations on purchasing terms, procurement policy and procedures, coordination amongst hospital departments and hospitals, and the quality control of medical instruments.

In the vast majority of cases of damage to patients as a result of treatment using medical lasers supplied on approval or previously purchased, it is the hospital which will be deemed liable. Given the difficulties of proof, it will make little difference whether the hospital has a maintenance agreement with the manufacturer or supplier or not.

\subsubsection{The Medical Discipline Act}

The Medical Discipline Act dates from 1928. The aim of this Act is to create a framework for testing and monitoring how well health professionals exercise their professions. The Act covers physicians, dentists, obstetricians and pharmacists. Under Article 10 of the Medical Discipline Act a complaint may be lodged with a medical disciplinary board by a directly interested party (the patient, close family members and others from his immediate circle who have been involved in the patient's illness by virtue of caring for him), by the management of an establishment where the practitioner is employed or accredited, and by the inspector of health ${ }^{[27}$.

The principal criteria against which the disciplinary boards must measure complaints are:

* Has confidence in the medical profession been undermined?

* Has there been negligence resulting in serious damage to a person in respect of whom medical, therapeutic or obstetric advice or care is sought or to whom such advice or care is given?

* Has a health professional demonstrated gross incompetence in the exercise of his profession? (Articles 1 - 3a of the Medical Discipline Act.) 
The Act may also be invoked against a practitioner who must be rregarded as unfit to practise because he is mentally or physically ill, too old or physically incapable, or who regularly abuses alcohol or drugs (Articles 4 and 4 a of the Medical Discipline Act) ${ }^{2 m}$ ? The Medical Discipline Act has been updated and incorporated into the Individual Health Care Professions Act. Chapter seven of this new Act (article 47-78) covers medical discipline law. This part of the Individual Health Care Professions Act, which will be enforced gradually, is anticipated to be applied to physicians starting in 1997. Until then the Medical Discipline Act will continue to be applied In addition to the professional groups already covered by the disciplinary rules of this Act, the new system will also cover, among other workers, nursing personnel and physiotherapists (Article 62). Under it, a complaint against a nurse can only be made by those with whom the nurse has a relationship of employment (the establishment employing him/her or the person who has given him/her an instruction, e.g. a physician) and the inspector of health. In the case of other practitioners a complaint may also be lodged directly by a directly interested party (Article 80):

A second change concerns the disciplinary criteria. In place of the present wording the new Act starts (Article 62) from the premise of inadequacy in the care which the practitioner ought to exercise vis a wis his patient. The second disciplinary criterion, which complements the first, concerns conduct incompatible with the exercise of good individual thealth care ${ }^{[27]}$. This criterion can also cover aspects of (inadequacies in) cooperation by fellow professionals which do not cause harm to the individual patient ${ }^{[21]}$. A third change concerns the possibility of imposing rules to cover unfitness on grounds of illness or incapacity and addiction. This subject has been transferred from the disciplinary rules to Chapter 8 (article 79-85) of the new Act.

Despite a number of improvements the Individual Health Care Professions Act will not be able to cope with all the problems that are associated with the implementation of the Medical Discipline Act. Due to the growing complexity of medical interventions it is increasingly the case, for example, that one cannot ascertain who or what (combination of) circumstances was responsible for the error ${ }^{[2]}$. This happens in the case of team work. If errors occur in such cases it is difficult to apply the Medical Discipline Act because only individual members of a team can be deemed liable under the Medical Discipline Act. This situation has not changed under the Individual Health Care Professions Act.

To sum up, then, in cases concerning the use of lasers in medicine, both the physician and nursing staff involved in treatment may be deemed liable for damages. That will not be the case with the laser safety officer.

\subsubsection{How the different forms of liability relate to each other}

The option of bringing criminal liability suits against natural and legal persons is exercised with the utmost reticence in health care. The possibilities of disciplinary action are usually explored first in the case of professions which are subject to the Medical Discipline Act. But criminal proceedings may always be brought in tandem or afterwards given that the physician is answerable to the criminal law just like any other citizen. But in practice, dual proceedings are rare. The Public Prosecutor and the Inspector of Health generally consult on the best course to follow. Preference is generally given to disciplinary action.

\subsection{Discussion}

Al the level of the EU, the development of medical device Directives can be regarded as an improvement over the current situation. Nevertheless, it can be concluded that the scope of the Directives is limited to safety in the vast majority of medical devices. Only in case of active implantable medical devices and class III medical devices, the Directives oblige manufacturers to produce data on efficacy. The requirements are not very strict in comparison with the pre-market requirements on documentation of the safety and efficacy of drugs. With respect to medicall lasers and all other devices in class 1, lla and Ilb, documentation of safety, expressed by a CE marking. 
suffices for marketing, notwithstanding that manufacturers have to include instructions for us checking and maintenance of devices. Clearly, the provisions of the Directive do not reach furthe Responsibilities during actual assistance using medical devices fall outside the scope of th Directive. Nor are there any criteria set for the qualifications and skill required of the use ${ }^{\text {"I. }}$. Th limits in the Directive's scopie is the result of a compromise between differing EU objectives. Th Directive is intended to promote both the free movement of medical devices and the protection. health ${ }^{[1 ; 16,3 y)}$. This situation stresses the mecessity that professionals take responsibility to assur the development and implementation of appropriate credentialing rules, supported by adequat education and training programs (see Chapter 3 and Chapter 20).

In the Netherlands, medical devices are essentially unregulated, despite the existence of a Medic Devices Act. Therefore, the incorporation of the EU Medical Device Directives in the Medic Devices Act can be considered as an improvement over the current situation, despite the fact th: the scope of the Directive is limited to safety in the vast majority of cases.

The development and implementation of medical device Directives in the EU results in a syster that differs in some respects with the regulatory system of the United States, where the Medic: Device Amendments Act was signed in 1976. One difference can be found with respect to th requirements for post-marketing surveillance of devices. In Europe, manufacturers have to registe to whom they sold devices and they should record any (safety) failures of the device. The user of the devices do not have to contribute to the registry. In contrast, in the US medical devic reporting is also aimed at the user of the device. Beside this, evaluation of data on devices afte they have entered the market is compulsory in the US Also, a 'tracking' requirement can b mentioned here, which is a registry of patients who have been treated using selected devices. I 1985 this 'tracking' irequirement was added to the Medical Device Amendments Act by the U Congress. In 1996 this requirement was actually enforced. Overall, the medical device regulaton system has been much milder in individual European countries than in the United states sinc 1976. Although this situation has changed to some extent with the implementation of the El medical device Directives, a regulatory gap will continue to exist. As a result it takes more time introduce a new product on the market in the US (for an implantable device this time is about fiv years) than in Europe. According to van Otterloo, a Member of the Statutory Committee on Publi Health of Dutch Parliament in a testimony at a public hearing on quality and efficiency in healt care, due to the relative absence of regulation in Europe. American producers of medical device! see golden opportunities for marketing devices in this continent ${ }^{[34 !}$. To solve part of th: shortcomings of the European Medical Device Directives Faro, in her testimony, pointed at thi possibility that manufacturers voluntarily set-up data-bases on the performance of the device a: part of a post-marketing surveillance. She explained that such databases, which are presently sel up in the field of implantable devices, for example, are in the interest of the industry because the! create a form of protection of already marketed products against competition of newcomers ${ }^{34 !}$.

Overall, although the implementation of the EU Medical Device Directives can be regarded as at improvement over the current situation, additional legislation would be beneficial. Ideally, this woult result in a decrease of the number of liability procedures due to inappropriate or incompetent us: of medical devices. 


\subsection{References}

1. Aarts ACM. wan Riet Paap KW Arbeidsomstandighedenwet. Nederlandse Staatswetten. Editie Sthuuman Jordens: 141 (vierde druk). WEJ Tjeenk Willimk Zwolle, 1990 (im Dutch):

2. ACGIH. Thresholdimit values for chemical substances and physical agents and biological exposure limits, 1992 . 1993 (second printing). ACGHH, Cincinnati; OH, US, 1992

3. Amonymous. Jurisprudentie. Arondissementsrechtbank te Leevwarden, 23 december 1987 . Tidschrift voor Gezondheidsrecht 1988(12)3:173-6 (in Dutch).

4. Ankoné A. Wat bestuurders maeten weten over aansprakellikheid. Het Ziekenhuis 1990 (14)586-8 (in Dutch).

5. Beshilt eisen voor erkenming van algemene zlekenhuzen. Staatscourant $1984(234)$, labtstelike gewijzigd op 20 november 1987 , Staatscourant 1987 (117) (in Dutch).

6. Beslut van 20 september 1990 tot waststelling van een algemene maatregel van bestuur als bedoeld in artikel 24 wan de Arbeidsomstandighedenwet (Veiligheidsbesluit Restgroepen). Staatscourant 1990 (491) (in Dutch).

7. de Bill, NPYM. De Europese Gemeenschappen na 1992: betekenis voor gezondheidsbescheming in Nederkand. Gezoncheidsraad, Rapport Nr A90104. Den Haag, 1990 (in Dutch).

8. CIBZ werkgroep 'Onderhowd/Produktaansprakelijkheid'. Instrumentatie, "veverancier en produlktaansprakelijkheid. KV90.2245. Publikatie NZI/NZR 90.713. Utrecht, 1990 (in Dutch).

9. Commissie Structuur en Financiering Gezondheidszorg (Commissie Dekker). Bereidheid tot verandering. Rapport, 's Gravenhage, 1987 (in Dutch).

10. Commission of the European Communities. Proposal for a Council Directive concerning medical devices, 91/C 237103 (put forward by the Commission on 30 Augus 1991). Officlal Jouinal of the Europoan Communities No. $C$ $237 / 3$ of 12 September 1991

11. Commission of the European Communities. Council Directive $93 / 42 / E$ EC of 14 June 1993 concerning medical devices. Official Journal of the European Communities No. L 169 of 12 July 1993.

12. Commission of the European Communities. Modified proposal for a Council directive concerning medical devices, $92 / 0.251 / 02$ (put forward by the Commission on 28 July 1992). Official Joumal of the European Communities No. C 251/40 of 28 September 1992

3. Duchene AS, Lakey JRA, Repacholi MH (Eds.). RPA Guidelines on Protection Against Non-lonizing Radiation. Mc Graw Hill, Highstown, NJ, US, 1992.

14. European Community. Proposal for a Cauncil Directive on the minimum heath and safety requirements regareting the exposure of workers to the risks arising from physical agients. CON(92) 560 final $-5 Y N$. 449. Brussels, 23 December 1992 .

15. Faro LMC. De kwaliteit van implantaten in juridiscth perspectief (thesis). Erasmus University Rotterdam, the Netherlands, 1986 (in Dutch).

16. Faro LMC. Medische hulpmiddelen in Europa. Tijdschrift voor Gezondheid en Politiek 1994(Mei): 11-3 (in Dutch).

17. Gewers LKMM. De WGBO. De wetgever en de rechten van de patient. Medisch Contact 1994(49):741-2 (in Dutch).

18. Gezondheidsraad, Advies inzake aanvaardbare niveaus voor elektromagnetische straling in het golflengtegebied tussen $100 \mathrm{~nm}$ en $1 \mathrm{~mm}$ (micrometerstraling). Gezondheidsraad, Rapport Nr 1978/06. Rijswijk, 1978 (in Dutch).

19. Gezondheidsraad. Optische straling. Gezondheidskundige advieswaarden voor blootstelling aan elektromagnetische straling in het galflengtegebied tussen 100 nanometer on 1 millmeter. Gezondheldsraad, Advies nr. 1993/09. Den Haag. 1993 (in Dutch).

20. van Gurp W. Produktaansprakelikheid. Een zaak van ziekenhuis en leverancler, ofwel wie passief bliff betaralt. Technologie in de Gezondheidszorg 1991(3);63 (in Dutch).

21. Hubben H. Het ziekenthuis als strafbare rechtspersoon (ilnaugurele rede). Uitgeversmaatschappil) De Tijdstroom, Lochem, 1989 (in Dutch).

22. International Electrotechnical Commission (IEC). Radiation Safety of Laser Products, Equipment Classification, Requirements and User's Guide. Draft prEN 60 825. CENELEC, Brussels, 1989.

23. International Electrotechnical Commission ( $E$ EC). Radiation Safety of Laser Products, Equipment Clasisification, and User's Guidle. Dooument WS 825-1. IEC, Genewa, 1993

24. International Electrotechnical Commission (IEC). Radiation Safety of Laser Products. Equipment Classilfication. Rquirements and User's Guide. Publication 825. IEC. Geneva, 11984.

25. Knottenbelt B, Torringa RA, Verheugt JWP. Inteiding in thet Nederlandse Recht (zesde druk). Gouda, Quint BV, Arnhem, 1990 (in Dutch).

26. Laserveilligheid in de gezondheid szorg. Toelsing van het rapport Laserveilligheid in het ziekenhuis" van de Nationale Commissie Laserveiligheid. Gezondheidsraad, Rapport Nr 1992106. Dan Haag, 1992 (in Dutch).

27. Leenen H.w. Handboek Gezondheidsrecht. Gezondheidszorg en recht (weede druk). Samsom H.D. Tjeerk Willink, Alphen aan den Rijn, 1991 (in Dutch)

28. Nederlands Normalisatie Instituut (NNI). Stralingsveiligheid van laserproducten, classilicatie van materieel, eisen en gebruikersrichtijnen. vederlandse Norm NEN EN 60825. NNI, Delft, 1992 (im Dutch). 


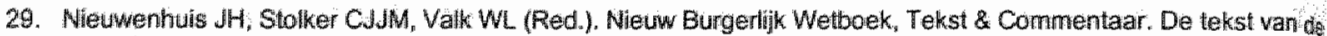
Boekeri 3,5 en 6, en de titels $1,7,9$ en 14 wan Boek 7 voorzien van commentaar. Uitgeverij Kluwer, Dewenter. 190 (in Dutch).

30. Pluyter $B$, Veenendaal WH. Jufidische aspecten wan onderhoud. Kinische Fysica 1991(3): 102-3 (in Dutch).

3月. Roscam-Abbing HDC. Patient en gezondheidszorg in het recht van de Europese Gemeenschap. Preadvie witgebracht ten behoeve wan de jalurvergadering wan de Vereniging woor Gezondheidsrecht op 16 april 1993 Verenging voor Gezondheidsrecht, 1993 (in Dutch).

32. SENTER. Nieuwstrief Medische Technologie 1993(19). SENTER, Den Haag. 1993 (un Dutch).

33. Shney DH. IRPA Gudelines on Protection Againsi Non-lonizing Radiation. Health Physics 1993(64):196-7.

34. Tweede Kamer der Staten General, Verslag vam een hoorztting over doelmatigheid en kwaliteit in de gezondheidszorg. Tweede Kamer, Vergaderjaar 1993-1994, 23400 XVI, mr. 80. Sdu, Den Haag, 1994 (in: Dutch)

35. Vaartjes R. Briewwisseling met Centraal Beheer Schadeverzekeringen NV, 1989 (in Dutch).

36. Veiligheidsbesluit Fabrieken en Werkplaatsen; Staatscourant 1938(792) (in Dutch).

37. Wijziging van het Burgerlijk Wetboek en enige andere weitten in verband met de opneming van bepalingen omtrent de overeenkomst tot het verrichten van handelingen op het gebied van de geneeskunst. Tweede Kamer. Vergaderjaer 1989-1990, 21561, nir 1,2,3. Sdu, Den Haag, 1990 (in Dutch). 


\section{Chapter 18 Notes}

1 After 1957 when the European Economic Community (EEC) was established, the European Coal and Steel Community (ECSC) and the European Atomic Energy Community (Euratom) were set up. After 1965 these were merged as the European Communities (EC) $)^{7 ?}$. As of November 1993, when all EC-countries had ratified the Maastricht Treaty, the EC is officially referred to as the European Union. The European Union is referred to in this report by the abbreviation "EU".

2 Under Article 30 the member states may not take measures on goods and products which will impose quantitative restrictions on imports or any measures having an equivalent effect. Examples are quotas and tariff measures. The normal effect of these is to protect one's own market $^{[15 !}$.

3 In the Netherlands, account will have to be taken, with the EU Directive on medical devices, of a 1992 proposal for legislative rules on medical experiments (Dutch Parliament Second Chamber, 1991-1992 session, 22485 , Nos. 1-2).

4 This is a declaration by the World Medical Association adopted in Helsinki in 1964. It was amended in 1975 (Tokyo) in 1983 (Venice) and in Hong Kong (1989)(3. Commission of the European Communities 1993). The declaration draws a distinction between experiments for therapeutic and non-therapeutic purposes ${ }^{\|27\|}$.

5 The ceiling level is the level which must not be exceeded; the threshold level is the level to be aimed for. The action levels, by definition between the threshold and ceiling levels, indicate when certain measures must be implemented.

6 It was decided in 1992 that the INIRC would in future function independently of the IRPA under the name International Committee on Non-lonizing Radiation Protection (ICNIRP) ${ }^{\text {(3)! }}$.

7 The Health Council report ${ }^{[19]}$ makes a comparison with the guide values formulated by the ACGIH and INIRC-IRPA. The Health Council considers that the recommendations of these bodies contain a generous safety margin which, in health terms, is unnecessary. Thus the Health Council's recommendations will differ somewhat from those in Annex 3 of the proposal for a special EU Directive on the protection of workers against optical radiation ${ }^{[14]}$ (see Section 18.2.6), since this draft Directive is based on the recommendations of the ACGIH and INIRCIRPA. Given that the standards applied by the Health Council are not stricter than those of the ACGIH and INIRC-IRPA this should nol cause any problems in EU context.

8 Public law is the law which governs the organization of the state and the relationship between citizen and authority. Examples are constitutional law, administrative law and criminall law. Private (or civil) law regulates reciprocal relations between citizens ${ }^{\mid 25\}}$.

9 Legal persons under public law are e.g. government bodies such as the State, provinces and municipalities. Examples of legal persons under private law are the limited company, association and foundation ${ }^{25]}$. Hospitals for example may be managed as a foundation by a legal person ${ }^{121 !}$. 


\section{Appendix 18.1 Translation of English terms for Dutch laws}

Civil Code

Contract for Medical Treatment Act

\section{Criminal Code}

General Act on Insurance Against Special Sickness Costs

Individual Health Care Professions Act

Medical Discipline Act

Occupational Health and Saffety Act

Order on Criteria for the Recognition of Hospitals

Safety Order for Factories and Workplaces

Safety Order for Other Groups

Sick Funds Act
Burgerlijk Wetboek

Wet Geneeskundige Behandelingsovereenkoms (WGBO)

Wetboek van Strafrecht

Algemene Wet Bijzondere Ziektekosten (AWBZ)

Wet Beroepen in de Individuele Gezondheidszorg (Wt BIG)

Medische Tuchtwet

Arbeidsomstandighedenwet (Arbowet)

Besluit eisen voor erkenning van ziekenhuizen

Veiligheidsbesluit

Fabrieken en Werkplaatsen (VBF, zie Arbo wet)

Veiligheidsbesluit Restgroepen (VBR, zie Arbowet)

Ziekenfondswet 


\title{
19 Strategies for successful evaluation and policy- making toward health care technology on the move: the case of medical lasers
}

\author{
H David Banta", ${ }^{*}$ Vondeling ${ }^{2}$ \\ The Netherlands Organization for Applied Scientific Research (TNO) \\ Center for Medical Technology (CMT) \\ P.O. Box 430 \\ 2300 AK Leiden, the Netherlands \\ ? Department of Health Economics \\ University of Limburg \\ P.O. Box 616 \\ 6200 MD Maastricht, the Netherlands
}

Originally published as: Strategies for successful evaluation and policy-making toward health care technology on the move: the case of medical lasers. Social Science and Medicine 1994(38)12:1663-74. Reprinted with permission of the publisher.

\section{Summary}

Evaluating new health care technology that is rapidly diffusing is one of the greatest challenges to researchers and policy-makers. If no evaluation is done until the technology is mature, evaluation will mot influence processes of diffusion. If evaluation is done early, it may be irrelevant when it is completed, because of developments in the technology and changing indications for its use. Nonetheless, early evaluation seems to be the only strategy possible to improve the integration of evaluation and diffusion.

These difficulties re illustrated by the case of lasers. Lasers are diffusing relatively rapidly into health care, and yet few laser applications have been well-evaluated. Looking back over the past 20 years or so, only one public body, the National Eye Institute of the US National Institutes of Health (NIH) seems to have tried to address the problem of laser evaluation. In the case of the Eye Institute, it has consistently identified new technologies for treatment of eye conditions and has mounted well-designed prospective evaluations aimed at influencing clinical practice. However, these evaluations have not been integrated with public policy-making, and therefore their influence has been relatively slow to develop.

in recent years, concerns about technology have brought more active attempts to develop public policies to affect diffusion. Excimer laser treatment of coronary artery disease, especially as dealt with in the Netherlands, illustrates how a strategy can be developed. Regulation has allowed diffusion to be constrained while evaluation is carried out. Results of the evaluation will guide subsequent diffusion. In the future, such results will probably be used in determining if the laser treatment should be included in the benefit package of health insurance.

A strategy for improving diffusion processes requires continuous monitoring of technological developments in health care to identify candidates for such early assessment. Since assessment resources are limited, setting priorities between candidates for assessment is necessary. Once priorities have been determined, an evaluative strategy can be formulated. As in the case of laser treatment of coronary artery disease, a mechanism for constraining diffusion until evaluations are completed is necessary. Once the studies are completed, policy-making must be done promptly. 
The problem of successful implementation of this strategy lies with the public bodies, which an often not prepared to develop an integrated strategy of diffusion based on assessment an economic appraisal. Developing such a strategy, which could involve slowing diffusion in som cases and speeding it up in others, seems to have clear benefits. The field of medical lasers, an the broader field of minimally invasive therapy, seem to offer good opportunities to implement surd a strategy.

Key-words: Lasers, Diabetic retinopathy, Excimer laser coronary angioplasty, Emergin technology, Technology assessment, Economic appraisal, Health policy.

\subsection{Introduction}

Ideally, continuous monitoring would yield reliable and up-to-date information concemin developments in health care technology. Decisions as to which technologies to evaluate could the be based on a comprehensive, credible and transparent priority-setting process. The selecte medical technologies would be well-evaluated on a small scale, as early in their life-cycle a possible. If results appeared to be useful, the technology could be tested in larger groups o patients in well-designed studies. Both during and after evaluation, appropriate policy measure could be constructed to guide the process of technological diffusion. Depending on the results 0 evaluation, policy measures could either stimulate, impede or prevent uncontrolled or additiona diffusion of the technology. In case diffusion was supported, monitoring published literature on thi particular technology, and perhaps the actual practice situation itself, could continue. circumstances changed substantially, for example because of a general widening of the indication: for the use of the technology, because of changes in the underlying disease, emerging technology or other factors, reassessment might be warranted. In this case, the results of such studies wouk have to be translated into policy measures that could result in additional diffusion or partia abandoning of the technology. In this model, evaluation or assessment can be thought of in term: of accompanying the life-cycle of the technology ${ }^{\text {ind }}$.

In the present climate toward health care technology in Europe, there are unutilized mechanism: for influencing processes of diffusion. Some technologies are directly regulated in some countries In other cases, the number of specialists is determined as part of health planning for the future Such policy measures can use the results of economic appraisal or other assessments to guid: decisions. Furthermore, almost all European counties now have explicit financial constraints or health care. Assessment can take the role of indicating which new technologies are worthy, or grounds of effectiveness or cost-effectiveness, to come into widespread use. The financia mechanism can be used directly in some countries, as in the Netherlands. Assessment can guide a decision to include a new technology in the benefit package of payment to specialists.

This of course is an ideal, seldom realized even partially in practice. In the real world, any technologies emerge, diffuse and disappear without monitoring or systematic evaluation. Polic measures are often absent or inadequate and in practice reassessment of technologies is hardly ever seen ${ }^{110}$. Also, due to increasing rates of technological change, the process outlined here has become more complex. Not anly does the number of new technologies increase more rapidly thar before, but the length of the life-cycle of the technology is decreasing because of new technologie: replacing older technologies. Therefore, the complexity of assessment reflects both the difficulties in selecting health care technologies for evaluation and increasing pressures to reduce the length of time it takes for evaluation, so the results can have value in decision-making in clinical practics and health policy.

At the same time, methods to support the process of assessment have become mort sophisticated. Methods of priority-setting between different technologies have been developed th a reasonably high level ${ }^{(22,34)}$. Clinical trial methods, especially methods of randomized clinicall triak: 
(RCTs), have been standardized and codified ${ }^{[22,34,49}$. Methods for economic evaluation of health care technologies are being improved and standardized ${ }^{123.275}$. Furthermore, there is increasing attention in Europe and the United States to unutilized or underutilized mechanisms for influencing the process of diffusion ${ }^{\text {rel }}$. Some technologies are directly regulated. Ih some countries, the numbers and types of specialists are determined as part of health planning. Such policy measures can use the results of economic appraisal or other assessments to guide decisions. Furthermore; amost all European countries now have explicit financial constraints on health care, and the DRG system has limited payments for health care in the US Medicare program. Assessments can play an important role as part of financial mechanisms. In the Netheriands, for example, assessment is used directly to influence appropriate diffusion and use. Assessments are used to guide a decision to include a new technology in the benefit package of payment to specialists ${ }^{\text {in! }}$.

Despite these developments, and improving support for evaluation in mast European countries, evaluating medical technology and relating its results to policy measures is still difficult for a number of reasons. One problem is the timing of the assessment. If the technology is assessed too early, the evaluation may be of little benefit both for the researchers and policy-makers. The technology may not be mature, new uses appear continually, technological change may be impeded. If the technology is assessed too late, the evaluation may be of little use to decisionmakers. This problem has been called Buxton's law of technological evaluation: "It's always too early, until fortunately, it's suddenly to late ${ }^{\text {inj }}$. Although reassessment gives a partial answer to this dilemma, it can also lead to an inefficient use of scarce assessment resources. Another problem is how can appropriate policy measures be constructed, both during and after the period of evaluation? As outlined earlier, both phases are important because if uncontrolled diffusion occurs during evaluation, it will not be possible to implement effective policy measures after evaluation. These problems will be dealt with in this paper, which will attempt to make a contribution to the development of a strategy for selecting, evaluating, and guiding the diffusion of rapidly developing health care technology that comes close to the ideal picture outlined above.

The paper focuses on a representative health care technology, the case of medical lasers. medical lasers are diffusing relatively rapidly in Europe and North America ${ }^{(\theta)}$. They are part of the new field of minimally invasive therapy, which has the potential to change the organization of health care dramatically while increasing the cost-effectiveness of health care ${ }^{[5]}$.

The second section of the paper provides a brief introduction to medical lasers. It will discuss how the subject was selected for evaluation in the Netherlands and, later, in the European Commission (EC) project.

The third section addresses the quality and timing of initial evaluations of medical laser applications in Europe and the United States. As only a few laser applications have been well-evaluated, the impediments to evaluation are summarized and analyzed. Then we focus on the history of the evaluation of two specific laser applications, argon laser treatment of diabetic retinopathy and excimer laser coronary laser angioplasty.

We selected these cases for three reasons:

1. On the basis of the quality of the evidence on effectiveness and cost-effectiveness,

2. because these cases largely differ with respect to the stage in the life-cycle when initial assessment was performed, and,

3. Because of similarities in the underlying diseases.

Argon laser treatment of diabetic retinopathy, introduced in 1965, was selected for evaluation by the National Eye Institute of the US Institutes of health in 1970. A well-organized RCT was followed by formal cost-analyses and cost-effectiveness analyses. Today, laser treatment of diabetic retinopathy is one of the oldest, most mature and best-evaluated medical laser applications. Its evaluation started at a point when the technology was already relatively mature. This contrasts with the excimer laser case. 
Excimer laser coronary angioplasty (ELCA), introduced in 1988 , was selected for evaluation in the US as early as 1989 in the context of Food and Drug Administration (FDA) regulations for marketing of new medical devices. One year later, in 1990, the procedure was selected for evaluation in the Netherlands in the context of the Investigational medicine Program of the Dutch health Insurance Council. The effects and costs of ELCA are being compared to the effects and costs of percutaneous transluminal coronary angioplasty (PTCA).

The underlying diseases in these cases, diabetes mellitus and coronary artery disease, are systemic chronic diseases. There is no definitive cure or preventive measure of either. The technologies are designed therefore to deal with symptoms. They are classic 'half-way' technologies ${ }^{[(0)]}$. The technology in these cases may be very helpful in the short-run, but it does not affect the underlying problem.

The final section of the paper discusses the contrasts and similarities in the cases and the implications of different approaches to the selection. (initial) evaluation, diffusion process. Some steps towards a strategy for evaluating and guiding the diffusion of rapidly developing health care technologies is then formulated. In the context of this strattegy, the need for future assessments of these cases is briefly discussed.

\subsection{Identification and evaluation of medical lasers in the COMAC-HSR project}

The first functional laser, using ruby as a medium, was built by Theodore Maiman and described in $1960^{[48)}$. Maiman also coined the acronyme Light Amplification by the Stimulated Emission of Radiation. One year later, in 1961, lasers were first used in medicine. Since then, lasers have gradually found applications in virtually every specialty of medicine and new types and applications of lasers are appearing frequent ${ }^{[i 9 !}$.

In 1985, the Dutch Steering Committee for Future Health Scenarios (STG) supported a study to identify future and emerging technology and to do prospective assessment on some selected technologies ${ }^{[7]}$. A rather informal survey letter was sent to experts in Europe and the United States inviting ideas about coming applications of health care technology that would be significant - in terms of clinical outcome, institutional effects, economic effects, social or ethical implications, or otherwise. Approximately 200 letters were received. Most respondents gave information on one or more technology, including estimates of the time until it would be availlable and of its (potential) importance. After the letters were analyzed "literature searches supplemented the information received. Some intervilews with selected experts were also carried out.

The Committee supervising the study was asked to rate 30 apparently important technologies on a scale of 0 (no impact) to 5 (very high impact) in several categories: health needs, health status

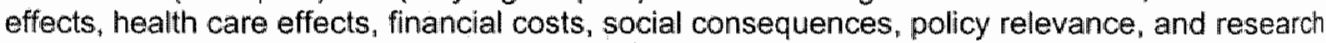
and development consequences. The answers were combined by simple addition. The result was that 5 technologies were selected as highest priority. Medical lasers was one of these. A prospective assessment of lasers in coronary artery disease was carried out to examine possible futures $^{[6]}$. In 1988, the European Commission (EC) COMAC-HSR support for studies in economic appraisal gave the possibility of going more deeply into this subject. The entire field of medical lasers, with the exception of clearly experimental areas such as photodynamic therapy, was surveyed with the help of the medical literature, an expert committee, and interviews with selected experts. This study, completed in 1991, resulted in its own publication ${ }^{\left[{ }^{[i]}\right.}$. At the same time, British collaborators of the COMAC-HSR project published a study on priority-setting of laser applications specifically for economic evaluation ${ }^{[57]}$. In the Netherlands, the Health Council commissioned a literature survey of laser applications in gynecology $y^{\text {(t)3 }}$. In 1991, the subject was broadened 10 examine the field of minimally invasive therapy, focusing on barriers to diffusion. As part of this 
study, supported by the FAST Program of the European Commission and the Swedish Council on Technology Assessment in Health Care (SBU), diffusion of selected laser applications was studied in depth ${ }^{\mid 5 !}$.

In the COMAC-HSR project the effectiveness and cost-effectiveness of 43 laser applications in more-or-less common use were examined. Judgments were made in each case of the state of development and evaluation. This work, which has been updated for the purpose of the thesis and presented in summary in Chapter 11 (see Table 11.1) shows that two categories of laser applications can be distinguished. The first category comprises laser applications that have not yet been subjected to systematic evaluation. The second category lists those laser applications that have been subject to evaluation in well-designed studies such as randomized controlled trials (RCTs). Evaluation of laser applications in both categories will be discussed.

Table 11.1 shows that evaluation of laser technology is generally poor. Clinical data is at least limited in about one third of the applications listed in the Table. About $40 \%$ of the applications judged to be established by clinical experience are potentially cost-effective, contrasting with only $7 \%$ of applications that may not potentially be cost-effective. This increases the importance of determining the factors that impede evaluation of laser applications.

Table 19.1 shows some common reasons explaining why evaluation of laser applications has not been done.

Evaluation of benefit may be difficult for a number of reasons. If benefit seems obvious, researchers may consider it unethical to enroll patients in randomized trials either because the control group would receive placebo treatment or conventional treatment. Also, no alternative treatment may be available. In addition, rare conditions may cause organizational difficulties if multi-institutional studies are required. There may be lack of knowledge of the natural history and genesis of the disease. Finally, evaluation of benefits (and risks) in chronic disease may be difficult because long-term follow-up may be necessary. This may be particularly true in the case of cancer.

Technological factors such as the lack of availability of reasonable alternatives, or very rapid developments, can make evaluations difficult.

Industrial factors impede evaluation if industry promotes the technology without relying on good evaluations.

Patient and physician factors include reluctance of patients and physicians to participate in evaluative studies. Physicians may be conservative. Some specialties have limited traditions of evaluation. Physicians may believe strongly in personal experience. Finally, competition between specialties may hinder evaluation. 


\begin{tabular}{|c|c|}
\hline Speclalty/Application & Explanatory Factors \\
\hline \multicolumn{2}{|l|}{ Dermatology } \\
\hline Portwine stains & $\begin{array}{l}\text { No alternative treatment, resistance to idea of placebo } \\
\text { trials, difficulty to determine end-point }\end{array}$ \\
\hline Other applications & Benefit obvious \\
\hline \multicolumn{2}{|l|}{ Otolaryngology } \\
\hline Pharyngeal pouch & $\begin{array}{l}\text { Rare condition makes evaluation difficult "benefit } \\
\text { obvious }\end{array}$ \\
\hline Carcinoma of larynx & $\begin{array}{l}\text { Surgeon resistance, long-term follow-up necessary, } \\
\text { emotionall factors in dealing with cancer }\end{array}$ \\
\hline \multicolumn{2}{|l|}{ Pulmonology } \\
\hline Palliation of advanced lung cancer & $\begin{array}{l}\text { Lack of good alternatives, certain mortality makes } \\
\text { patients and physicians reluctant to participate in } \\
\text { studies }\end{array}$ \\
\hline \multicolumn{2}{|l|}{ Gastroenterology } \\
\hline $\begin{array}{l}\text { Hemostasis of intestinal vascular } \\
\text { malformation }\end{array}$ & $\begin{array}{l}\text { Benefit obvious, evaluation difficult because the } \\
\text { condition is rare }\end{array}$ \\
\hline $\begin{array}{l}\text { Palliation of carcinoma of } \\
\text { esophagus and collon }\end{array}$ & $\begin{array}{l}\text { Conservatism in cancer therapy, competition between } \\
\text { specialties, lack of good alternatives. Certain mortality } \\
\text { makes patients and physicians reluctant to participale } \\
\text { in evaluative studies }\end{array}$ \\
\hline \multicolumn{2}{|l|}{ Urology } \\
\hline $\begin{array}{l}\text { Cancer of the bladder, prostate, } \\
\text { upper urinary tract }\end{array}$ & $\begin{array}{l}\text { Difficult to evaluate benefits (and risks) because long- } \\
\text { term follow-up is necessary }\end{array}$ \\
\hline Benign prostate hypertrophy & Industry promotes technology without good evaluation \\
\hline \multicolumn{2}{|l|}{ Gynecology } \\
\hline Endometriosis & $\begin{array}{l}\text { Lack of knowledge of genesis and course of problem, } \\
\text { several alternatives }\end{array}$ \\
\hline $\mathrm{CIN}$ & Physician conservatism, changing concept of etiology \\
\hline VIN + VAIN & Rare condition makes evaluative study difficult. \\
\hline
\end{tabular}

Table 19.1 illustrates that impediments to evaluation are many. There are few reasons to become involved in evaluation. Besides these factors, policies may not encourage evaluation. There is a lack of funding to support evaluation, other rewards (such as promotion) are often lacking, and the lack of availability of payment or financial constraints on new services can also hinder evaluation.

Many of these difficulties are valid. However, despite the barriers to evaluation, a few laser applications have been well-evaluated. Several of these will be discussed. 


\subsection{Laser treatments that have been well-evaluated}

Laser treatment of UGI-bleeding is an example of laser intervention in gastroenterology. Laser treatment as well as heater probe treatment have shown to save resources as compared to surgical treatment $^{[53]}$. This shows that laser is not the only intervention used through the endoscope. Besides the application of lasers or heater probes to the bleeding site, injection of such substances as epinephrine (adrenalin) and polidocanol can be used. The endoscopic intervention, introduced about 1970, has been tested in more than 25 randomized controlled trials, and has been shown to save lives compared with watchful waiting and surgical intervention where necessary ${ }^{113,55]}$ This treatment is now considered standard. Because the effectiveness of injection is comparable to that of lasers and heater probes, and because it is easier to administer and relatively cheap, of all endoscopic therapies, injection for UGl bleeding is considered the most cost-effective. Although systematic, direct comparison of different endoscopic methods is largely lacking, laser treatment of UGI bleeding is the first laser treatment in medicine that is both well-evaluated and is being replaced by other modalities of treatment. The replacement by modalities that are likely to be more cost-effective has been rapid, probably due to the support of a large number of RCTs in gastroenterology, in turn probably because of the traditions of that specialty. This process has taken place without any governmental intervention.

In gynecology, laser treatment of cervical intraepithelial neoplasia (CIN) can be treated by laser, but also by an alternative, cryotherapy (freezing). A few randomized clinical trials have shown either laser or cryotherapy to be equally effective to the surgical treatment, but in this case the less invasive therapy has not become standard. Surgical intervention is still common ${ }^{\text {[6.2 }}$. The main explanation in this case is probably conservatism of the medical specialists, especially in the face of a pre-cancerous condition.

Most ophthalmological laser applications are well-evaluated. The maturity of the field is surely related to a policy of planning clinical trials carried out at the national Eye Institute of the US National Institutes of Health ${ }^{[45]}$. The results of these studies have shown that the applications are effective. Of all treatments examined in the COMAC-HSR project, only treatment of diabetic retinopathy was considered to be proven cost-effective. We will now focus on this particular case. Subsequently, the evaluation of excimer laser coronary angloplasty will be contrasted with treatment of diabetic retinopathy.

\subsection{Argon laser treatment of diabetic retinopathy}

For information on this case, the reader is referred to Chapter 6 and in particular to Chapter 16 . in short, argon laser treatment of diabetic retinopathy (DR) is the best evaluated case in the field of laser therapy. A well-organized randomized controlled trial was followed by formal cost analyses and cost-effectiveness analyses. Laser treatment of DR proved to be cost-effective in a situation where there was no satisfactory treatment previously. The case illustrates how evaluation can influence the life-cycle of a technology. Aside from the decision of the NIH to evaluate laser ireatment, there were no policy interventions dealing with the laser treatment in either America or Europe. In 1991, the World health organization (EURO) recommended that national screening programs for diabetic retinopathy be considered ${ }^{[1]}$. Sweden has now decided to implement such a mational screening strategy for diabetic retinopathy. Other countries will probably follow. 


\subsection{Excimer laser coronary angioplasty in the United States and in the Netherlands}

Cardiovascular disease is the greatest cause of death in industrialized countries. Coronary artery disease accounts for the greatest part of these deaths, primarily resulting from narrowing of coronary arteries, leading to failure or death of myocardial (heart) tissue. A number of interventions have been developed to increase circulation of blood to the heart. The standard procedure is now coronary artery bypass grafting (CABG). An established alternative is percutaneous transluminal coronary arigioplasty (PTCA), or balloon angioplasty.

PTCA is much less invasive than CABG and it has an initial success rate in opening coronary arteries of more than 90 per cent, but it also has persistent shortcomings: a rate of abrupt closure of around 7 per cent and a 6 month restenosis rate of between 25 and 35 per cent. The rate of restenosis has provided the rationale for the development of new technologies ${ }^{[5 . \mid}$.

The laser has been used in a number of systems to open blocked coronary arteries. Among the avallable applications, excimer laser coronary angioplasty (ELCA) has received the greatest attention.

Early investigations with the excimer laser demonstrated that it could make very precise craters in cadaver tissue. Grundfest and Litvack first used the excimer laser on cadaver tissue; this work was reported worldwide in the press in $1984^{\mid 19 !}$. In 1988, Litvack and colleagues performed the first excimer laser coronary angioplasty, employing a xenon-chloride laser and following the procedure with a balloon angioplasty ${ }^{\text {(a) }}$. A non-randomized clinical trial ultimately involving 13 centers in the United States was then initiated ${ }^{[33]}$. It was expected that ELCA could cause less vessel wall trauma and would therefore be associated with a lower incidence of restenosis ${ }^{[38]}$. At the same time, other countries acquired ELCA systems. For example, the Academic Medical Center (AMC) in Amsterdam acquired an ELCA system from Advanced Interventional Systems (AIS) in California in 1989. The first procedure in Amsterdam was carried out in February 1990.

During the early diffusion of the ELCA system, the technology changed relatively rapidly. In particular, the earliest catheter was $1.6 \mathrm{~mm}$ in diameter and was rather rigid. By late 1989 , more flexible catheters with a diameter of 1.3 and $2.0 \mathrm{~mm}$ were available, resulting in a higher percentage of initially successful procedures.

Despite the hopes for a higher success rate with ELCA, studies have not demonstrated results superior to PTCA. In fact, the restenosis rate appears to be about 35 per cent, whether ELCI is used as a stand-alone procedure or in conjunction with PTCA ${ }^{|54|}$. However, initial results were promising in treatment of long lesions. As a result, in January 1991, a US Food and Drug Administration advisory panel recommended that the FDA should approve the excimer laser coronary angioplasty system manufactured by Advanced interventional Systems (AIS) as safe and effective for marketing for lesions $20 \mathrm{~mm}$ or longer ${ }^{311}$. The reason for this cautious approach was that alternative (FDA approved) laser systems for peripheral angioplasty, had lost physician support because laser heat could damage healthy tissue. It should be noticed, however, that the lasers referred to, the argon and the Nd:YAG laser, were thermall laser systems, whereas an Excimer laser (predominantly) ablates tissue ${ }^{[36]}$. The FDA concluded that the technology should be limited to teaching and research facilities until there was more clinical consensus on the ${ }^{\text {subjeat }}$. In 1991, 44 systems were sold to teaching and research iacilities! Therefore, before definite FDA approval was obtained, a total of sixty systems had already been sold.

The expense of the ELCA system is about US $\$ 245000$. Single use catheters sell for US\$600. 1000 each $^{[301}$. Approximately 1.3 catheters is required per patient procedure. Profitability is therefore related to the number of catheters sold. It is, then, no surprise that clinical laser angioplasty systems are designed such that the manufactured laser catheter can only be coupled 
to that manufacturer's laser\%?. It is also worth noting that Litvack and other pioneers in the field were among the founders of Advanced International Systems ${ }^{[47]}$.

AIS has invested US $\$ 12-15$ million in the devellopment of ELCA ${ }^{4 n !}$ and had the capability to manufacture about 70 lasers in 1992. The company signed an agreement with Voluntary Hospitals of America (VHA) Supply Company. As part of the agreement, the alliance will help AllS identify UHA hospitals that might be candidates for the laser. Als is targeting hospitals with a caseload of about 400 coronary balloon angioplasty procedures annually ${ }^{(33)}$. Ail estimates that there are well in excess of 200 hospitals that meets its criteria ${ }^{301}$. At the same time. Als is also seaking FDA clearance to sell its laser to treat clogged bypass grafts, ostial lesions, and total occlusions ${ }^{|333|}$. Since the AIS system came on the market Spectranetics and Summit Technology have also introduced excimer laser systems for angioplasty ${ }^{[35}$. Spectranetics has received an approval recommendiation for its excimer laser from a panel of consultants to FDA ${ }^{[333}$. Another US company, Eclipse Surgical Technologies, Inc., has submitted results from its clinical studies of its holmium laser to FDA for review ${ }^{[33]}$.

The Dutch situation has been different. Advanced cardiological procedures are regulated in the Netherlands under Article 18 of the Hospital Facilities Act. In addition, the Investigational Medicine Fund, administered by the Dutch Health Insurance Council, supports evaluations of new and emerging technologies that may be important to Dutch health care ${ }^{1401}$.

The regulation that allowed control of ELCA in the Netherlands, Article 18 of the Hospital Provisions Act, gives the Dutch government the authority to guide the diffusion of high technology services. Guidance is achieved by licensing hospitals to perform specific procedures, based (ideally) on the results of a needs assessment. This assessment is translated into a judgment on the maximum annual treatment capacity required for the procedure. This treatment capacity can be distributed over a number of hospitals in different parts of the country, assuring availability of care to all patients.

In 1983, both heart catheterization and heart surgery were regulated under Article 18 (Planning Decisions). The planning decision on heart catheterization was published in $1987^{15 / 1}$. In this document, diagnostic and therapeutic heart catheterizations were distinguished. The latter category included PTCA and coronary laser angioplasty. The planning decision included a definition of the maximum annual number of therapeutic catheterizations to be performed and implied that therapeutic catheterizations should be restricted to centers that perform heart surgery.

Heart surgery (e.g., coronary artery bypass grafting) has been subject to licensing since 1983. In 1987, 13 centers applied for and received a license for heart surgery. In early 1989, a planning decision on heart surgery was published ${ }^{[52]}$. For 1990, a total (indicative) number of 10800 procedures was allocated over the 13 licensed centers.

Thus, as early as 1989, one year after its introduction in the US and one year before its introduction in the Netherlands, the diffusion of ELCA was already limited to a potential number of 13 centers in the Netherlands. The diffusion of the main alternative to ELCA, PTCA, is also regulated. The early regulation is explained by use of other lasers in coronary disease beginning about $1986^{[19]}$. The STG study of the future of lasers in cardiology mentioned above ${ }^{|\theta|}$ may have played a part in government awareness. There was no explicit need to cover laser procedures in 1987 because the numbers were low, but policy makers judged that it would not be harmful to have this category of procedures covered (van den Wijngaard, Ministry of Health, personal communication).

In 1990 the first of the centers in Europe to buy an ELCA system, the Academical Medical Center in Amsterdam, submitted a proposal to the Investigational Medicine Fund to carry out a randomized clinical trial comparing PTCA and ELCA. The proposal had to be revised because of rapidly developing information, especially as it developed in the US ELCA registry, and because of rapidly changing technology. In April 1990, a detailed analysis of ELCA results from the United States was 
presented in Maastricht: the Netherlands ${ }^{|3 y|}$. Availability of various new sizes of catheters and technological improvements in the structure of the catheters resulted in a higher percentage of ELCA procedures as a stand-alone procedure and a predicted improvement in treatment results. However, preliminary data indicated a restenosis rate of 35 per cent after ELCl, comparable to that of PTCA ${ }^{(54)}$ In its comments on the AMC proposal, the Investigational Medicine Committee (IMC) specifically referred to these results and stated that the proposal should be based on the latesi availlable knowledge. Furthermore, the Committee commented that ELCA was immature, and that the proposed economic evaluation was therefore premature. US data indicated that ELCA might have applications in those lesions not well-suited for PTCA, especially with long lesions. Therefore, the revised AMC proposal only included patients with long stenoses. Since the number of patients with such lesions was limited, the revised proposal included a cooperation with a second university hospital, the academic hospital of Erasmus University in Rotterdam. The revised protocol was resubmitted to the Investigational Medicine Committee in December 1990 and approved in 1991. During the trial, another Dutch center and a center in the United States would join the study. In practice. ELCA was followed by PTCA in $98 \%$ of the procedures and therefore the trial in fact compared combined ELCA and PTCA, and PTCA alone. The results showed that the combined procedure provides no extra benefit compared to PTCA alone, neither initially nor after a follow-up period of $B$ months. As ELCA is associated with considerable additional costs, the procedure is not cost-effective compared to PTCA as a stand-alone procedure ${ }^{(2)}$. Subsequently, it was decided not to include ELCA in the health insurance benefit package ${ }^{\text {i3al }}$, essentially discouraging its diffusion. In summary, the regulation of laser angioplasty made a strategy of basing diffusion on evaluation feasible in the Netherlands.

\subsection{Discussion}

The case of the laser illustrates many of the issues and problems in evaluating innovative applications of devices in health care. The laser was identified by the National Eye Institute of the US Institutes of Health as a priority for evaluation in 1970. Subsequently, a number of other bodies, including the study on economic appraisal funded by COMAC-HSR, have identified applications of medical lasers as a priority.

The field of medical lasers is still developing rapidly. A good example is the field of Photodynamic Therapy (PDT). In 1988, at the start of the COMAC-HSR project, the field was clearly experimental, and therefore it was not extensively covered in the project. In 1992, however, the first RCT of PDT in patients with lung cancer was funded by the Investigational Medicine Program of the Dutch Health Insurance Executive Council. Considering on-going developments in this field $d^{\text {the process }}$ of priority-setting needs to be repeated every 3 or 4 years.

ideally, the process of priority-setting, and the expense and difficulties of organizing studies, could be shared among countries. This is illustrated by the fact that the National Health Technology Advisory Panel of Australia has identified the laser as apriority in 1985, and a detailed assessment was published in $1987^{(4)}$. These different activities were not coordinated in any way.

Considering the attention to medical lasers, the number and quality of existing evaluations is disappointing. A variety of impediments to evaluation heip explain this situation. We do not minimize the difficulties in evaluating rapidly-changing technology. Many of these difficulties are valid. In addition "an RCT is not always the most practical design for evaluating a technology ${ }^{12 !}$. For example $e_{n}$ in the case of treatment of portwine stains, it is probably not possible to organize controlled trials because there is no good alternative. The laser produces obvious cosmetic benefit. In the Netherlands, the Investigational Medicine Committee approved a non-randomized design for study of portwine stains in 1992. 
Many of the difficulties could be addressed by policy bodies. For example, the DRS-trial was organized by the National Eye Institute of the US National Institutes of Health. In general, public bodies funding clinicall trials could become much more active identifying important studies to fund. This would involve applying explicit methods of priority-setting. This could also include synthesizing results of important studies. In addition, such public bodies need to take steps to assure that the results of their assessments are used by policy bodies and clinicians in making decisions.

Evaluation has played a part in the diffusion of both of the cases presented in this paper, but both cases can be criticized. In the case of treatment of diabetic retinopathy, the trial was initiated in 1972,7 years after its introduction. By then, it was in rather widespread use. The evaluation was able to deal with a mature technology. The results of the evaluation determined the subsequent diffusion, but the trial could have been initiated perhaps 5 years earlier. After trial results were available, their dissemination was perhaps not optimal ${ }^{[59}$. In any case, there was limited follow-up by health policy bodies. In the netherlands, for example, there is still no specific payment for laser photocoagulation ${ }^{\| 611}$. It was 13 years after the first publication of the DRS trial before a costeffectiveness analysis was published. That analysis, to some extent, raised the issue of screening for diabetic retinopathy. Thus, years were lost during which many cases of blindness could have been prevented.

Besides implementing formal payment policies, other formall policies could have played a constructive role in encouraging appropriate technological change. Argon laser treatment could have been stimulated by means of government funds to support capital investments after the technology was shown to be cost-effective. Individuals and institutions could have been certified for carrying out this and related (laser) procedures. Governments could have supported training programs for the laser. Concerted efforts could have improved lay knowledige of health practices. so that people would have had a better basis for choice. The lay media could have been actively involved in such efforts. In many countries, these suggestions could still be useful.

In summary, although this case is the best-evaluated laser application in health care, due to the time-lags between introduction, evaluation and policy-making, the overall process of diffusion is suboptimal.

Since the publication of the DRS, argon laser therapy has become the standard therapy for treatment of diabetic retinopathy. The technology has evolved gradually since then but has not undergone dramatic changes. However, as patients for the DRS were included in the period 19721975 , it could be worthwhile to assess changes in the technique of delivering therapy. If distinctly different techniques can be discerned in the international literature of argon laser treatment of different types of lesions, a re-evaluation of effectiveness could be justified. With respect to the underlying disease, studies indicated that the prevalence of diabetes is increasing, while no cure is in sight. The advent of new technology, in this case the smaller and cheaper diode laser, does not seriously question the status of the argon laser (see also Chapter 6). Therefore, there does not seem to be a need for a large-scale short-term reassessment of this technology.

The ELCA system represents the more common problem today. With the growth in visibility of technology assessment, policy-makers would like to test the technology before it comes on the market. However, devices seldom reach final form before testing in humans ${ }^{[4]}$. Improvements continue during the clinical testing phase. This may make it difficult to organize clinical trials that are meaningful when they are completed. Furthermore, who is to carry out the testing? The inventor will inevitably be enthusiastic and his/her claims must be looked at skeptically. Clinical investigators also often show undue enthusiasm. They are enthusiastic by nature, and they must justify their work to the world, including hospital boards and the broader scientific community. In addition, increasingly the inventors and clinical investigators have invested in the company that is making the device. Their objectivity must therefore be questioned even more than in the past. Negative results are seldom published and do not lead to promotion ${ }^{|17\rangle}$ "Therefore, another type of investigator, with a specific interest in evaluation, is needed ${ }^{\mid 44 !}$ 
In the case of ELCA, organizing evaluations of the technology during its early development and diffusion have been difficult and protocols have had to be revised several times. Undoubtedly, the story of this case is not over. However the contrast between the United States and the Netherlands is instructive. In the United States, rapid diffusion of laser angioplasty systems has occurred, despitte limited evidence of increased benefit. Industry has profited from this development, as have many entrepreneurs "but patients may not, and in some cases have undoubtedly been harmed. In the Netherlands, on the other hand, regulation has prevented widespread diffusion of these devices until (and if) they were demonstrated to be cost-effective.

In the Netherlands, the expectation that results of the evaluation of ELCA would become available in thee years time provided an argument for the Dutch government to not provide any additional licenses during the period of evaluation. This strategy has been highlighted by the government as a method of major importance to control diffusion ${ }^{\{58]}$. Given the rapidly evolving nature of the field, close monitoring and new trials will probably be necessary.

In summary, due to rapid international diffusion of trial results and follow-up of the early results, the case of ELCA in the Netherlands illustrates a reasonable process of introduction, evaluation and policy-making, and integration of these steps.

\subsection{Towards a strategy for timely evaluation}

In general, especially in cases of half-way technology such as the laser, the costs of delaying diffusion must be put against the costs of rapid diffusion of experimental (and possibly useless) devices. This is a difficult balance to find. However, the benefit from unrestrained diffusion of experimental devices is questionable, to say the least. It is surely not desirable to stop diffusion indefinitely. It does seem wise, however, to restrain diffusion until an application is shown to be effective.

A model might involve free purchase of new devices by recognized experimental centers. Industry donation of devices to such centers should also be encouraged, as should industry support for studies. These early studies, which will probably involve clinical innovators involved in the development, will usually only be uncontrolled observations. If these studies are promising, larger data collections can be undertaken, involving several centers (with or without controls). If these studies are promising, randomized controlled trials can be organized involving as many centers as necessary and supported by a central authority for such studies, such as the National institutes of Health $(\mathrm{NHH})$ in the United States or the Investigational Medicine Fund in the Netherlands. Integrating economic analyses into these trials is also a constructive step ${ }^{i 25}$. During evaluation. adoption of the device by centers that are not involved in the evaluation must be discouraged. Appropriate policy-making must follow promptly after completion of a study. Continuous monitoring of the field and allowing possibilities for reassessment of selected technologies complement the strategy.

\subsection{Conclusions}

It is possible to identify and monitor new technological developments, as shown by the case of lasers. As far as evaluation and diffusion are concerned, the main lesson in the two cases is that evaluation can, and should, be integrated much more closely into the process of diffusion. By identifying new technologies early in their life-cycle, data collection can also be mounted early. Diffusion can be restrained by regulation or by refusal to pay for unproven services outside of the context of controlled trials or other designs when appropriate. Controlled diffusion, continuous monitoring and repetitive priority-setting complete the iterative or repetitive character of the process. The problem of successful implementation of this strategy lies with the public bodies, 
which are not prepared to develop an integrated strategy of diffusion based on technology assessment. Developing such a strategy, which would involve slowing diffusion in some cases and speeding it up in others, seems to have clear benefits. The field of medical lasers, and the broader field of minimally invasive therapy, seem to offer good opportunities to implement such a strategy.

\subsection{References}

1. Anonymous. Screening for diabetic retinopathy in Europe (editorial) Biabetic Nedicine 1991(8):197-8.

2. Appelman YEA, Piek JJ, Strikwerda S, Tijssen JGP, de Feyter PJ, Davio GK, Serruys PW, Nargolis JR, Koelemay MJ. Montauban van Swijndregt EWJ. Koolen JJ. Randomised trial of excimer laser angioplasty versus balloon angioplasty for treatment of obstructive coronary artery disease. Lancel $1996(347): 79-84$.

3. Aron-Rosa D. Ophthalmolagy - effectiveness. In: Banta HD, Schoul (Eds). Lasers in Health Care. Effectiveness, Cost-Effectiveness and Policy Implications. Academic Publishing. Frederiksberg, Denmark, 1991, $p .90-6$.

4. Australian Institute of Health and Welfare. Australian Center for Medical Laser Technology, Lasers in mediche. Australian Institute of Health and Welfare. Health Care Technology/Series Number 8, Canberra, Australlia, 1993.

5. Banta HD (Ed.). Minimally invasive therapy in five European countries. Diffuslon, effiectiveness, and costeffectiveness. Health Policy Monographs: Vol, 3. Elsevier, Amsterdar, London, New York, Tokio, 1993.

6. Banta $H_{D}$, Gelijns A (Eds.). Anticipating and assessing health care technology, Vol. 4. Health care applications of lasers: the future treatment of coronary artery disease. Report of the Scenario Commission on Futurie Health Care Technology. Martinus Nijhoff Publishers, a member of the Kluwer Academic Publishers Group, Dordrecht, Boston, Lancaster, 1988.

7. Banta HD, Gelijns A. Anticipating and assessing heath care technology, Vol. 1, General considerations and policy conclusions. Report of the Scenario Commission on Future Health Care Technology. Martinus Nilhoff Publishers, a member of the Kluwer Academic Publishers Group, Dordrecht, Boston, Lancaster, 1987.

3. Banta HD, Luce B. Health care technology and its assessment: an international perspectiwe. Oxford University Piness, Oxford 1993.

9. Banta HD, Schou 1 (Eds). Lasers in Health Care. Effectiveness, Cost-Effectiveness and Pollcy Implications. Academic Publishing, Frederiksberg. Denmark, 1991

10. Banta HD, Thacker SB. The case for reassessment of health care technology, once is not enough. Journal of the American Medical Association 1990(264):235-40.

11. Borst-Eilers. E. Assessing hospital technology in the Netherlands. British Medical Journal 1993(306):226.

12. Bouter L. Methods of effect measurement. Which designs ane acceptable? Tijdschrift voor Sociale Gezondheldszorg 1992/70):490-3 (in Dutch).

13. Bown S. Gastroenterology - effectiveness. In: Banta HD, Schou I (Eds.). Lasers in Health Care. Effectiveness, CostEffectiveness and Policy Implications. Academic Publishing, Frederiksberg, Denmark, 1991"p. 122-34.

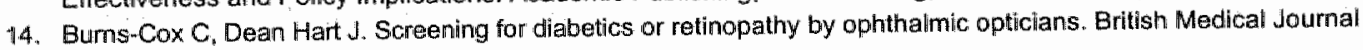
$1985(290): 1052-4$

15. Buxton M. Problems in the economic appraisal of new health technology: the evaluation of heart transplants in the U.K. In: Drummond MF (Ed.). Economic appraisal of health technology in the European Community. Oxford Medical Publications, Oxford, 1987.

16. Cantrill H. The diabetic refinopathy study and the early treatment diabetic retinopathy study. International Opinthalmology Clinics $1984(24): 13-29$.

17 Chatmers 1. Undermeporting research is sclentific misconduct. Joumal of the American Medical Assiociation $1990(263): 1405-8$

18. Cross $F$. Bowker $T$. The excimer laser-tissue interactions and early clinical results. In: Abela $G$ (Ed.) Lasers in cardiovascular medicine and surgery: fundamentals and techniques. Kuwer Academic Publishers, London, 1990, p. $45-58$.

19. Cumberland DC, Oakley GDG, Smith GH, Tayler DI, Starkey IR, Fleming JS, Goith Ju, Dawis J. Percutaneous laserassisted cononany angioplasty (latter). The Lancet $1986(8500), 214$.

20. Diabetic Retinopathy (DRS) Research Group. Preliminary report on the effects of photocoagulation therapy American Journall of Ophthaimology $1976(81)$ ) $383-96$.

21. Diabelic Retinopathy Study (DRS) Research Group. Photocoagulation treatment of PDR, cllnical applications of diabetic retinopathy study (DRS) findings. DRS Repart Number B. Ophthalmology $1981(88): 583-600$.

22. Donaldson $M$ and Sax H (Eds.). Setting priorities for health technology assessment, a model process. Nationa Academy Press, Washington, DC, 1992.

23. Drummond $M$. Brandf $A$, Luce $B$. Rovira J. Standardizing economic evaluation methodologies in health care international Journal of Technology Assessment in Health Care 1993(9)1:26-36.

24. Drummond $M$, Dawies $L$, Ferris $F$. Assessing the costs of medical research: the Diabetic Retinopathy Study. Report to the National Eye Institute. Health Senvices Management Center, Birmingham. England, 1990 
26. Drummond M; Stoddar GL. Econonic analysis and chnical trials. Controlied Clinical Trials 1984(5):115-20.

26. Drummond M. Ophthalmology - costeffectiveness. In Banta HD. Schou I (Eds). Lasers in Heaith Care. Effoctveness, Cost-Effectweness and Pollcy Implicatons. Academic Publishing, Frederiksberg;, Denmark, 1901 , p. $97-8$.

27. Diwmmond M. Principtes of econtomic appraisal in health Care. Oxford University Press, London, 1980.

28. Early Treatment Diabetic Retinopathy Study (ETDRS) Research Group. Early photocoagulation for diatetto retinopathy. Eary treatment diabetic retinopathy study report number 9. Ophthalmology 1991909 (Supplement): $766-85$

29. Early Treatment Dlabetic Retinopathy Study (ETDRS) Research Group. Photacoagulation for diabetic mactiar edema. Eearty treatment diabetic retinopathy study report number 1. Archives of Ophthalmology $1985(103) \% 1786$ $1806:$

30. ECRI. Exclmer laser for heart nears FDA OK Health Technology Trends 1992(4)2:1.

31. ECRI. FDA Panel okays excimer laser. Technology for Cardiology $1991(7), 3-4$.

32. ECRl. Hospitais technology budget climb. In: Heallh Technology Checklist. Health Techrology Trends $1992(4)$ : Supplement.

33. ECRI. VHA canves out market for Excimer laser. Health Technology Trends 1992(4)1:1.

34. Edty D. Selecting technologies for assessment. International Joumal of Technology Assessment in Health Care $1989(5): 485-5011$.

35. Gelijins A. Innovation in clinical praclice. The dynamics of technology development. National Academy Press, Washington, DC, 1991.

36. Grundfest WS, Segalowitz $\|_{1}$ Laudenslager J. The physical and biologicall basis for laser angioplasty. In: Litwack F (Ed.). Coronary Laser Angloplasty. Volume in Series on Interventional Cardiology. Blackwell Scientific Publications, Oxford, London, Edimburgh, Melbourne, Paris, Berlin, Vienna, 1992, p. 1-25.

37. Grundfest W, Litvack $F$, Margolis J, Rothbaum D, King $S$, Unterraker W, Goldenberg T, Laudenslager J. Percutaneous coronary excimer laser anglography: initial results of a multi-center trial (abstract). Laser and Balloon Angioplasty and Restenosis. Book of Abstracts, Workshop, 27-28 April 1990, Maastricht, the Netherlands.

38. Haassie K, Kersch K. Coronary excimer laser angioplasty. In: White $C_{1}$ Ramee $S$ (Eds.). Interventional cardiology: clinical application of new technologies. Raven Press, New York, 1991.

39. Health Insurance Execultive Council. Annual report of Investigational Medicine 1995. Investigational Medicine Cominitee, Health Insurance Executive Council, Amstelveen, the Netherlands (in Dutch).

40. Hulsjes HJ. Inwestigational medicine underway. Tijdschrift voor Sociale Gezondheidszorg1992(70):484-6 (in Dutch).

41. Isner J, Rosenfield C. White A, Ramee S, Kearny $M_{1}$ Pieczek A, Langevin Jr. E, Razvî S. In vivo assessment of vascular pathology resulting from laser irradiation. Analysis of 23 patients studied by directional atherectomy immediately after laser angioplasty. Circulation 1991(85)6:2185-96.

42. Javitt $J$, Canner $M_{8}$ Frank $R$, Steinwachs $D M_{n}$ Sommer A. Detecting and treating retinopathy in patients with $y p e$ 1 diabetes mellitus, a health policy model. Ophthalmology( 1991$) 97: 483-95$.

43. Jawith J, Canner M, Sommer A. Cost-effectiveness of current approaches to the control of retinopathy in Type 1 diabetics. Ophthalmology(1989)96:255-64.

44. King S. Role of new technology in balloon angioplasty. Circulation 1991(84):2574-9.

45. Kupfer C. Clinical trials concerning the effectiveness of lasers, the case of ophthalmalogy. In: Banta HD, Schou (Eds.) Lasmers in Health Care, Effectiveness, Cost-Effectiveness and Policy Implicationis. Academic Publishing. Frederiksberg, Denmark. 1994, p. 63-8.

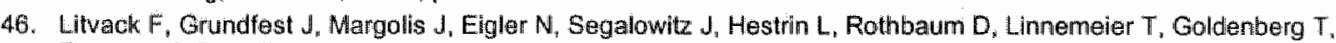
Forrester J. Complications during excimer laser angioplasty are similar to those during PTCA (abstract). Circulation $1989(80) 4$ (Supplement 11):254.

47. Litvack F. Proface. In: Litwack F (Ed.). Coronary laser angioplasty. Series in Interventional Cardiology. Blackwnll Scientific Publications, Oxford, London, Edimburgh, Melbourne, Paris, Berhin, Vienna, 1992, p. xv-xvii..

48. Maiman T. Stimulated optical radiation in ruby. Nature 1960(187):493-4.

49. Mainert CM. Towards more definitive clinical trials. Controlled Clinical Trials 1980(1)3:249-61.

50. Phelps P. Parente S. Priority-setting in medical technology and medical practice assessment. Medical Carre $1990(28) 2: 703-23$.

51. Planning Decision Heart Catheterization. Ministry of Health, Rijswijk, the Netherlands. Staatscourant 1987(152) (in Outch).

52. Planning Decision Heart Surgery. Ministry of Health, Rijswijk, the Netherlands. Staatscourant 1989 (154) (in Dutchi).

53. Rees M. The costing of a controlled trial of the use of laser and thermal probe for the treatment of bleeding peptic ulcer. Gastroenterological Unit, St. James Hospital, Blalham, London, 1987.

54. Rienks $R$, Borst $C$. Treatment of artherosclerotic cardiovascular disease - effectiveness. In: Banta HD, Schou (Eds). Lasers in Health Care. Effectiveness, Cost-Effectiveness and Policy Implications. Academic Publishing. Frederiksberg, Denmark, 1991, p. 155-74. 
15 . Sacks HS, Chalmers TC, Blum AL; Bermier J, Pagano D. Endoscopic hemostasis: an effective therapy fior bleeding peptic ulcers. Joumal of the American Medical Assciation 1990(264);494\%9.

50i. Sculpher MJ, Buxton MJ, Ferguson BA, Humphreys JE, Altman JBF, Splegellialter DJ, Kirby AJ. Screening for diabetic retinopathy: a relative cost-effectiveness analysis of altemative modalities and strategies. Brunel University. London, 1992 (unpublished).

57. Sculpher MJ, Buxton MJ. Report of phase I of the medical laser technology assessment. HERG Report Number 9. Health Economics. Research Group, Brunel Univensity, Uxbridge, London, 1991.

30. Secretary of State. Top Clinical Care 1991-1994, Letter of the Ministry of Health of the Netherlands to Parlianent. Dutch Parliament, 1991-1992, Meeting Year 21944, nr. 5 , sDu Publishers, the Hague, 1992 (in Dutch).

59. Stross JK, Harlan WR. The dissemination of new medical information. Joumal of the American Medical Association $1979(241): 2622-4$

60. Thomas $L$. The lives of a cell. The Viking Press, New York, 1974.

61. Vondeling H. Exaluation of angon laser treatment of diabetic retinopathy and its diffusion in the Netherlands. Health Policy 1993(23):97-112.

62. de Wit GA, Trimbos J. Gynecology - effectiveness. In: Banta HD, Schou I (Eds). Lasers in Health Care. Effectiveness, Cost-Effectiveness and Policy Implications. Acadernic Publishing, Frederiksberg, Denmark, 19g1, p. 189-200.

63. de Wit GA, Vondeling H. Banta HD. Lasers in gynecology. A a litterature survey. Health Council, Report no. A90/07, the Hague, the Netherlands, 1990 (in Dutch). 


\section{Discussion and recommendations}

\subsection{Introduction}

In the thity-six years since the introduction of lasers in medicine, its use has rapidly expanded. According to Judy (1995), three factors have contributed to this process ${ }^{[76]}$. These factors are: 1 ) the increased understanding of laser-tissue interaction, 2) the rapidly increasing number of new types of lasers and 3) the availability of the combination of optical fiber and lens technologies and endoscopic technologies for delivery of the laser radiation to the often remote internal treatment site. Assessment of medical laser applications has been carried out on a limited scale since the inception of methods and techniques for medical technology assessment in the mid-seventies. Although the capacity for medical technology assessment is slowly increasing, there has been a discrepancy between both processes. The consequences of this discrepancy are discussed in the first part of this chapter, integrating the information that has been presented. In the last part of the chapter the boundaries of the thesis are briefly discussed, followed by a final statement and a set of recommendations.

\subsection{Safety, effectiveness and cost-effectiveness}

\subsubsection{Safety and effectiveness of the use of lasers in medicine}

In the thirty-six years since the introduction of lasers in medicine, the scientific basis for their use has steadily been growing. This pertains in particular to the safety of the use of medical lasers but much less to aspects of effectiveness. It can be concluded that lasers can be safely applied in welltrained, experienced hands. However, systematic evaluation of the effectiveness of laser applications "g. by RCTs (when appropriate), is still largely lacking. As a consequence, most laser applications have been established by clinical experience as reflected in case-reports and caseseries, in exceptional cases including historical controls. As a result in relatively few applications is there sufficient evidence to support wider use of lasers. For most applications the situation is characterized by the mere fact that a laser can be used, which does not necessarily imply that the laser is indeed the best treatment option for a specific indication. If effectiveness is evaluated, it is usually restricted to clinical outcomes. Issues of description and valuation of health-related quality of life are hardly ever dealt with in the laser field. This is a serious omission, considering the fact that lasers are used for example in prevention of blindness and in palliative treatment of cancer, where important trade-offs may exist between length and quality of life.

There are relatively large differences in the quality of evidence between individual specialties. The main message here is that ophthallmology is the only specialty where the safety and effectiveness of a variety of major indications for the use of lasers has been convincingly demonstrated by largescale multicenter RCTs. The evaluation of ophthalmological laser applications could serve as a model for evaluation of laser applications in other specialties ${ }^{p *}$.ent. It should be added that selected laser applications in other specialties, in particular in gastroenterology, and to some extent in gynecology, have been thoroughly evaluated by RCTs as well. However, these trials were usually monocentric, relatively small and with limited followup. Meta-analysis has proven to be very helpful in interpreting the combined results of these trials. However, the length of time that would have been required to achieve a convincing conclusion in these cases would have been reduced considerably when either a small(er) number of large-scale trials had been organized early in the life-cycle of the interventions or when the technique of cumulative meta-analysis had been applied earlier ${ }^{[36 !}$. In all other specialties, the quality of evidence is considerably weaker, with scattered exceptions for specific indications. Those who are interested in a particular specialty or a particular laser application are referred to Part III. 


\subsubsection{Safety and effectiveness of the use of lasers in medicine compared to other minimally invasive procedures}

Conclusive evidence on the effectiveness of many procedures in the field of minimally invasive therapy outside the laser field is available in just a few cases. In this respect the field of lasers is not very different from the field of minimally invasive therapy as a whole. There is a great potential left for clinical evaluation. The few small RCTs that have so far been published comparing lasers with other minimally invasive procedures have demonstrated that lasers offer no significant clinical advantages (see Part II).

\subsubsection{Cost-effectiveness of the use of lasers in medicine compared to conventional 'open' surgery or otherwise conventional treatment}

The quality of evidence on economic evaluation of laser application versus conventional treatment is rather disappointing, both quantitative and qualitative, as tentatively assessed by a checklist developed by Drummond Stoddart and Torrance $(1987)^{[50]}$. In the health economics literature no examples can be found of economic evaluations carried out prospectivelly alongside clinical trials comparing laser treatment and conventional, invasive procedures.

Regarding the published evidence in individual specialties, it is only in exceptional cases. particularly in ophthalmology and gastroenterology, that results of RCTs have been used as an input for retrospective cost-effectiveness analyses ${ }^{\text {17:99]. In }}$ one particular case it could be demonstrated in the United States" context that argon laser treatment of diabetic retinopathy was not just cost-effective, but cost-saving from a societal perspective ${ }^{[75]}$. This is a quite exceptional situation. More details on the case of argon laser treatment of diabetic retinopathy can be found in Chapter 16. In general, the quality of the evidence on economic evaluation of ophthalmological laser applications is unsurpassed in the laser field.

Just as retrospective economic evaluations based on results of RCTs, prospective or retrospective cost-studies based on case-series are uncommon. The evidence suggests that the majority of laser applications is potentially cost-effective compared to conventional treatment options. Unfortunately, methodological and other shortcomings are abundant in these studies. Far a survey of these shortcomings the reader is referred to Chapter 11 . Some key points will be reiterated. At the start of an analysis its perspective should be clarified. However whenever analyses are being done the perspective is often unclear, although usually the analyses seem to be carried out from the perspective of providers and insurers, as in most of the publications attention is exclusively focused on direct health care costs based on fees. Other perspectives than that of the insurers or providers could be adopted, for example a societal perspective or the perspective of the patients. Of these, a societal perspective is most comprehensive and reflects the public interest ${ }^{\mid 35 !}$. Closely related to the choice of perspective and equally important is whether or not uncertainty is dealt with adequately. However, sensitivity analysis is often completely lacking in studies evaluating laser applications. Although no exception compared with other studies, this is a serious omission. For example, when an economic evaluation of a medical laser application would be carried out in an academic setting with a relatively high intensity of use of the device, sensitivity analysis should be applied to demonstrate the impact of lower intensities of use, which may be expected in community hospitals. This of course has implications for the generalizability of the conclusions of the analysis. Although the intensity of use of lasers is rather low, impressive differences in intensity of use of the device in university hospitals and community hospitals exist. Obviously, the probability of costeffective use of lasers in medicine is higher when, for the appropriate indications, annual patient throughput is maximized. The easiest way to achieve this is to share lasers between specialties and to regulate laser facilities. 


\subsubsection{Cost-effectiveness of the use of lasers in medicine compared to the cost- effectiveness of other minimally invasive procedures}

Although laser applications are usually potentially cost-effective compared to conventional procedures, this picture often changes dramatically when laser applications are compared to other minimally invasive procedures. While considering that conclusive evidence on the effectiveness of many procedures in the field of minimally invasive therapy is lacking, and that convincing evidence on the cost-effectiveness of minimally invasive procedures is lacking in all but a few exceptional cases ${ }^{[15]}$, the most general conclusion is that, within these constraints, the costeffectiveness of laser treatment is often at least questionable compared to other minimally invasive procedures. Of course, this conclusion does not include laser applications that have no competition from other minimally invasive procedures, such as treatment of portwine stains. This contrasts with many other situations, where laser treatment can not be regarded in isolation of other minimally invasive treatment modalities ${ }_{n}$ which are jointly integrated in treatment strategies. In such circumstances, one needs to compare two or more alternative treatment strategies, of which one relies to some extent on the use of lasers. An example of such a study is the one by Appelman et al. in 1996, comparing Excimer Laser Coronary Angioplasty (ELCA) followed by Percutaneous Transluminal Coronary Angioplasty (PTCA) with PTCA alone ${ }^{[13}$. Only a few of such studies are underway. Even when these studies are published, in many cases a judgement of the costeffectiveness of either laser treatment as a stand-alone therapy or as part of an integrated strategy is likely to be outdated soon due to rapid technical developments in the field of minimally invasive therapy as a whole. Of course, an evaluation could be repeated in that case.

\subsubsection{Comparison between the 1990 consensus judgments and the 1996 assessment}

The findings on the safety, effectiveness and cost-effectiveness of laser applications as reported in this thesis, can be regarded as an update of the results of a project on lasers that was supported during the period 1988-1991 by the COMAC-HSR of Directorate General XII of the European Commission (EC). As part of this project, a judgment on the safety, effectiveness and costeffectiveness of 35 selected applications was achieved in a consensus-meeting with international experts in the field in October 1990, using an extensive literature review, closed in June 1990, as a major input for discussion ${ }^{[19]}$. In this exercise, lasers were compared to conventional treatment options. Comparing the judgments of laser-experts in late 1990 and the judgments made for this thesis on the same indications in 1996, it is striking that there are hardly any differences between the judgments then and now. A tentative explanation for this similarity is a potential positive bias of the laser-experts who may have been overly optimistic in their judgment of the evidence as collected and presented in 1990 . It may be that the additional evidence produced in the period of six years between 1990 and 1996 bridges the gap between the overly optimistic judgments in 1990 and the perhaps less optimistic and hopefully less subject to bias judgment based on the 1996 assessment.

As indicated by the checklist developed by Drummond and colleagues and as emphasized throughout in this thesis, the choice of competing alternatives is essential, both in a clinical evaluation and in a clinical evaluation combined with an economic evaluation ${ }^{150 \mid}$. In this sense, the 1990 exercise is now to some extent outdated because in one category of cases the more relevant comparison has become the one between laser treatment and other minimally invasive treatment alternatives- or strategies. In these cases the evidence on the effectiveness of the minimally invasive procedures to be compared is sufficient to warrant comparison in an RCT. Likewise, the evidence demonstrating advantages of the minimally invasive procedure over the conventional procedure must have been conclusively shown. In another category, the evidence on individual minimally invasive procedures may be promising, but the advantages may not have been conclusively demonstrated compared to the conventional procedure, which is then included as one treatment arm in the RCT. Although this complicates the issue due to the more or less arbitrary 
conclusion on the effectiveness of individual minimally invasive treatment alternatives, strong: patient preferences for the minimaily invasive procedure and rapid technological developments: this notion is expressed in the evaluation programs that are presently ongoing in a number of countries in the European Union. Sometimes a clear choilce is made exclusively to evaluate alternative MIT procedures in an RCT, in other cases both (one or more) MIT procedures are compared to the conventional procedure (see paragraph 20.5).

\subsubsection{Comparison of the quality of the evaluation of medical lasers with the quality of evaluation of other devices, procedures and drugs}

The quality of the findings on the issues of safety, effectiveness and cost-effectiveness of lasers and minimally invasive therapy as presented in this thesis may be representative for the quality of evaluation of new medical devices in general. Compared to the evaluation of procedures the findings with lasers are difficult to judge. One could argue that lasers, although devices, find clinical applications as part of new procedures and that therefore one should compare the findings in the laser field to other categories of procedures. Procedures usually find their way in clinical practice without systematic evaluation. Compared to the quality of evaluation of minimally invasive therapy in general, the findings with lasers are judged to be fairly representative. However, the findings with lasers are judged to clearly represent a lower quality of evaluation than is the case in the evaluation of pharmaceuticals, which is closely related to the relatively strict regulatory requirements for registration and/or reimbursement of drugs (see paragraph 20.5). As a result, economic evaluation of pharmaceuticals is likely to be based on better medical evidence than evaluations of medical equipment or surgical procedures, merely because clinical trials of pharmaceutical are more often carried out ${ }^{[49 !}$. This does not necessarily imply that pharmacoeconomic evaluation by definition is of a higher quality than the economic evaluation of devices and procedures. In an article on this subject, Mason and Drummond (1995) cited findings of authors that found no apparent relationship between the quality of conduct of RCTs and their respective economic analysis. The author's analysis of 147 studies, including both pharmaceuticals, devices and procedures, revealed many methodological problems. In this sense the limited quality of economic evaluation of lasers may be representative for the entire field of economic evaluation, which is related in particular to the lack of appropriate reporting standards ${ }^{[66]}$.

\subsection{Diffusion}

\subsubsection{Diffusion of medical lasers}

Lasers have diffused widely in health care across the Western world, starting from the United States in 1961. The United States is the country where lasers have become most widespread. This pattern is visible for many other technologies as well, including PTCA and CABG (see Chapter 15) and for example CTs, MRIs and Radiation Therapy Units ${ }^{[85]}$. In Europe, innovative countries include in particular Germany and to some extent the United Kingdom and France where, for example: laparoscopic cholecystectomy was developed. In Asia, Japan has achieved outstanding technical innovation results, e.g. in endoscopes (see paragraph 20.4).

In Europe, it has been suggested that there is a north-south divide in the uptake of medical lasers $^{1091}$. Stocking (1991), in a discussion on the diffusion of three medical technologies in EC countries and Sweden, suggested that the Northern European countries develop and take up medical innovations early on. Southern European countries, though, while starting late, may leapfrog a stage and catch up fast ${ }^{1101}$. In general, national wealth, but in particular health care expenditures, are positively correlated with medical technology diffusion ${ }^{[B 5]}$.

Of the individual types of lasers, the argon laser, the Nd:YAG laser, the Q-switched Nd:YAG laser and the $\mathrm{CO}_{2}$ laser are most widespread. In different countries, the relative importance of each of these lasers varies, probably depending on the presence of a laser industry and local opinion 
leaders: Perhaps for this reason, the Nd:YAG laser is prominent in Germany Support for this hypothetical causal relationship has been provided in the case of ESWL in Germany and CTS, MRI: and Linear Accelerators (LAs) in the United States and in Japan in a study by Lazaro and Fitch (1995) In some countries, for example the Netherlands, the argon laser has been documented to be the imost widespread type of laser and the type of laser that is used most frequently in patient treatment, predominantly in ophthalmology. Perhaps this pattern is most common in all countries with medical lasers.

Ophthalmology and dermatology were among the first specialties where lasers were used. In the course of time, the number of types of medical lasers has increased, as has the number of specialties that employ these devices. Not only the year of introduction but also the rate of diffusion differs markedly between different types of lasers within and between countries. Taking all types of lasers together, usually an initial period characterized by a low rate of diffusion is followed by a period characterized by an increased rate, reflecting the first part of the classical s-shaped diffusion curve. In the Europe and in the United States, investment in new lasers is positively correlated with the size of a hospital. This is a common finding in medical technology diffusion research ${ }^{[103]}$. In the Netherlands, the status of being a university hospital is also positively correlated with investing in lasers as well as with its size. Sharing laser systems between different specialties is rather uncommon "although of the most important types of lasers in particular the Nd:YAG laser offers good opportunities for sharing. In both Europe and the United States there is evidence that the intensity of use of lasers is rather low. This combination of findings indicates that sharing could be the easiest way to use the presently available lasers more intensively. In selected countries, e.g. the Netherlands and Australia ${ }_{n}$ private clinics have become more important as a laser market. This may be caused to some extent by a variety of health care cost containment policies and coincides with a trend to introduce and market new medical laser systems in a variety of industrialized countries with short time-lags. A paper on the international diffusion of medical lasers is available at the author, using Excimer laser treatment of nearsightedness as a case ${ }^{\| 149 !}$.

\subsubsection{Diffusion of medical lasers in the context of the diffusion of minimally invasive therapy}

As part of the project on the diffusion of selected cases of minimally invasive therapy, two cases were included that focused primarily on lasers: Nd:YAG laser treatment of bladder tumours and Nd:YAG laser palliation of colon cancer (see Chapter 14 and 15). These laser applications diffused hardly or slowly in the health care system. For both cases combined this could be attributed to a combination of factors including budgetary pressures on hospitals, financial incentives on hospitals (which make shorter stays disadvantageous), a lack of evidence on (cost)-effectiveness and competition between specialties. The impact of one of the prominent factors impeding the diffusion of lasers and MIT, financial constraints on hospitals, was highlighted in a report by the Parliamentary Office of Science and Technolagy in the United Kingdom ${ }^{[96]}$. The report states that laser equipment would more than double the BP $30000-40000$ cost of the basic equipment needed for minimally invasive therapy. The report continues that most minimally invasive procedures can be performed perfectly adequately without lasers, although in selected cases lasers may offer specific advantages. Thus, if one has to choose which equipment to invest in, hospitals may be inclined to buy in first instance the equipment typically required in minimally inwasive therapy. Financial constraints could thus contribute to a lower rate of diffusion of lasers than other forms of minimally invasive therapy. Some findings may support this tentative conclusion. The first of these is that of the remaining eight cases of MIT in a study sponsored by EC-FAST, where lasers play at best a subordinate role, three diffused rapidly: ESWL, laparoscopiC cholecystectomy, and catheter treatment of coronary artery disease. However, of these ESWL is. extremely expensive. The second finding is that when lasers are concurrently marketed with a laparoscopic procedure, such as in the (exceptional) case of laparoscopic cholecystectomy, the laser diffuses slower than the laparoscopic procedure it is supposed to support. In this case this 
was due to the fact that it was demonstrated that electrocautery, which is much cheaper than a laser, could be used just as safely and effectively as part of the procedure (see Chapter 2). It should be noticed, however, that the case is atypical in a sense that laparoscopic cholecystectony was probably the fastest diffusing procedure in the field of MIT ${ }^{\text {it }}$. Some authors have interpreted these findings as a sign of 'the technological imperative' at work ${ }^{[59]}$ (see Chapter 1). Our analysis of the factors influencing the diffusion of laparoscopic cholecystectomy was confirmed by an analysis by Dirksen and colleagues (1994), who updated our findings specifically for the Netherlands ${ }^{|t| 6 \mid}$. The case of laparascopic cholecystectomy and the case of ESWL best demonstrate that in exceptional circumstances firancial and other constraints on diffusion can be overturned by stimulating factors. In all other cases studied in the EC-FAST project, budgetary pressures impeded the diffusion process (see also paragraph 20.5).

\subsubsection{The appropriateness of the diffusilon of medical lasers in light of the findings on safety, effectiveness and cost-effectiveness}

For the most part, the widespread use of lasers cannot presently be defended on grounds of proven effectiveness or cost-effectiveness (see Part II). Of all specialties ${ }_{n}$ diffusion of lasers seems most appropriate in ophthalmology. In most other specialties, but in particular in specialties where other well-evaluated minimally invasive therapies play an important role as well (e.g. in gastroenterology and gynecology), the adoption and use of lasers seems to be warranted for a limited and decreasing number of indications. At the same time, there is a continuous stream of new and sometimes promising laser applications, that for the most part diffuse in international health care systems without systematic evaluation. Of the more recently introduced types of lasers, the diode laser may have the highest potential for widespread diffusion (see paragraph 20.6). Timely economic evaluation should accompany this process.

\subsubsection{Generalizability}

Of all forms of endoscopic procedures, laparoscopic cholecystectomy has received most attention of diffusion researchers, predominantly because of its extremely rapid diffusion. However, taking all medical technology diffusion studies together, several authors think that those devices which require relatively large capital expenditures and regulatory approval, have received most attention of diffusion researchers. This pertains to new imaging techniques such as CT scanning and MRI, but also to new therapeutic devices such as ESWL machines ${ }^{[59,61 !}$. Similarly, studies focusing on hospital investment decision-making in new medical technology may have focused predominantly on big ticket technologies (e.g. exemplified by a study of Dutrée in the Netherlands ${ }^{[53]}$. Therefore, the conclusions that were drawn based on the EC-FAST project may pertain predominantly to "medium-ticket" technologles, including laser applications, which largely escape specific governmental regulation. Few studies have been done in this probably undervalued field of diffusion research.

\subsubsection{Methods for studying diffusion}

The study on the diffusion of medical lasers in the Netherlands was done by telephone surveys using key informants. We consider this as a reliable and economical method to collect rich data. The high response rate obtained with this method compares favourably with the response rale in postal surveys, as demonstrated for example in the report by Sterenborg and Müller (1994), who studied the diffusion of lasers at the level of the European Community ${ }^{1099}$ (see Chapter 12 and 13 ). Of course, the Netherlands is a small country, allowing more easy data collection than in the European Community, with its many different languages. But independent of the scale of the studies, telephone surveys generally yield a higher response rate than postal surveys. 
The study on the diffusion of selected cases of minimally invasive therapy in the Netherlands: was done by a combination of literature review, interviewing physicians using a semi-structured questionnaire, and by drafting a report which was used as part of the input for ameeting on the subject of Dutch experts and policy-makers. The countries that were part of the EC-FAST study did not all follow a similar approach, although the semi-structured questionnaire was used in each country in each case. In some countries, for example in the Netherlands, the study was done by a team of researchers, whereas in other countries the study was done by one individual researcher. This method resulted in a varying quality per country-report ${ }^{153}$. As a result, when the individual country-reports were summarized to provide a general picture, the conclusions thad to be formulated in rather general terms (see Chapter 15). This pattern, although quite common in international diffusion studies ${ }^{[1: 10]}$ can be avoided when more detailed methodological guidelines (and perhaps more resources) would be available.

\subsection{Implications of lasers \& minimally invasive therapy}

This paragraph features a discussion of the implications of lasers and minimally invasive therapy for patients, health care providers, industry and society, respectively. Then the implications of lasers and minimally invasive therapy are discussed for the organization of care and for the quality of care. These subjects are closelly related to a final subject the need for improved communication on patient management between different providers of care.

\subsubsection{Implications of lasers and MIT for patients}

Perhaps the most important implication of lasers and MIT is for patients. For patients, assuming that a procedure is reasonably evaluated and carried out by an experienced surgeon, these procedures offer great advantages. These include a lower mortality rate, reduced postoperative pain, a decreased risk of wound-related complications, and the obvious cosmetic advantages. The patients recover faster and the period of post-operative disability is reduced. This allows patients to return to work or normal activities early ${ }^{[18,96]}$.

\subsubsection{Implications of lasers and MIT for health care providers}

Lasers and MIT have important implications for physicians and nurses, who need special training. Physicians need (especially) to be trained in the use of endascopes and the associated instruments. Anesthesiologists need to be trained in the use of regional and local anesthesia, and need to be oriented to avoid general anesthesia wherever possible. Nurses need to monitor the status of the patient after the therapy, but also to promote early ambulation and early eating ${ }^{\mid 18 !}$.

\subsubsection{Implications of lasers and MIT for industry}

The development of lasers, but in particular the development of minimally invasive therapy has had a stimulating impact on the medical device industry. The laser industry, being only modestly profitable, has been briefly documented in Chapter 13 . It can be added that the worldwide revenues of surgical lasers in 1994 were estimated to amount US\$203 million ${ }^{[8]}$. Over $55 \%$ of surgical lasers were sold in the US. Europe was the second largest market, followed by the Pacific Rim countries. The developing countries in the rest of the world were slow to adopt laser technology, because of the cost and training difficulties ${ }^{(\theta)}$.

The industry producing instruments for MIT is highly diverse. Profitability is particularly high in the cardiovascular market, and in the large-sized market for diagnostic imaging equipment such as CT- 
scanners and MRI equipment ${ }^{2}$. It has been estimated that the market for endoscopes in the United States will grow from US\$ 352 milion in 1995 to $\$ 425$ million by the year $2002^{\text {ls9. }}$. According to analysts, the economics of the health care industry will lead to market concentration in a few glant manufacturers, in an analogous pattern to the pharmaceutical industry/9].

In the field of minimally irvasive therapy, the market structure has been documented relatively well in the case of aparoscopic cholecystectomy and related surgical procedures. In 1993, Gelijns and Fendrick reported that more than 20 new companies have been founded to supply the tools of minimally invasive therapy ${ }^{1631}$. Other companies had already vested interests in this area, e.g. the European company Stortz and Wolf and the Japanese company Olympus " producing laparoscopes, other endoscopes and associated equipment such as insufflators, trocars and cannulas, and tissue manipulation devices. New companies included, among others, Steris, producing sterilizers, Circon and Stryker, producing cameras and monitors, and Valleylab, Bircher and Conmed, producing electrocautery systems. The European manufacturers produced predominantly reusable instruments, whereas the United States based companies, at least initially, produced disposable instruments. Examples are US Surgical and Ethicon, producing disposable trocars, cannulas and tissue manipulation devices. The increasing numbers of producers resulted in increasing competition and decreasing prices ${ }^{\mid 63 !}$. Because the market for laparoscopic cholecystectomy in the United States was commercially most interesting and most easy to access; both European and US-based companies tried to serve this market first. In addition to producing the devices, industry built commercial training centers that provided hands-on experience in animals and simultaneously introduced surgeons to procedure-related products in order to meet the burgeoning demand for laparoscopic cholecystectomy in the United States ${ }^{[63]}$.

An illustration of the European context is offered by the development of the market for trocars in the UK. Trocars are the instruments used to make the incisions through which the ports are passed for laparoscopic surgery. The UK market for such products increased from BP 3.3 million in 1991 to BP 6.3 million in 1992, coinciding with the growth in laparoscopic cholecystectomy. This rapid growth was followed by a more gradual but steady upward trend to around BP 9.7 million in 1995 . At least part of this more recent tail-off was accounted for by the increasing proportion of reusable trocars ${ }^{1963}$. In the case of laparascopic cholecystectomy in the Netherlands, more than a third of the 134 hospitals providing the procedure in the two years after the introduction of this procedure in this country used instruments on loan from the industry ${ }^{[104]}$. These hospitals may have purchased the equipment after deciding to regularly offer laparoscopic cholecystectomy to patients. Overall, according to Gelijns and Fendrick (1993) the possibilities for the industry were somewhat better in the United States than in Europe ${ }^{[63]}$.

\subsubsection{Implications of lasers and MIT for the society}

For the broad society, lasers and MIT are potentially quite valuable, as it will mostly improve the cost-effectiveness of health care. Early return to work or normal activities is beneficial to the broad society and for individuals ${ }^{\mid \text {e日 }}$ "

\subsubsection{Implications of lasers and MIT for the organization of care}

As laser treatment and MIT are done more and more without hospital admission, it will foster changes in the organization of care both in and outside the hospital ${ }^{[181}$. For example, in the Netherlands the number of day care admissions in hospitals increased from 464000 in 1991 to 593 000 in 1994 (a $28 \%$ increase), while the number of hospital admissions increased from 1548000 to $160 \% 000$ in this period (an increase of $3.4 \%)^{\mid 14 !}$.

As a result of this trend some hospitals have organized special units or departments to facilitate day surgery for a variety of laser-related and other minimally invasive procedures of different 
specialties ${ }^{152.77}$. After-care is increasingly given in the home. This requires a different type of community care, involving, for example, nurses and general practitioners. MIT then, is a force encouraging integration of the health care system. As MIT becomes more and more the norm, the procedures will become more complex and patients in day surgery will be sicker. This will require more attention to patient selection and after-care ${ }^{|t| t \mid}$.

\subsubsection{Implications of MIT for the quality of care}

A routine system of on-going monitoring of quality of care of MIT is extremely important because of the lack of supervision of the patient after discharge. The starting paint for such a system is making the goals of care explicit. Decision rules can be formulated based on these goals. The data system should include information on the patient's condition at the time of pre-assessment, information about the operation (or therapy), information on nursing after the procedure, information about home-care (follow-up), including information on complications, and information on satisfaction of complaints of the patient. Ideally, the professionals involved in the care would then use this information to improve their own care. Evaluation and learning, of course, feed back into the standard-setting process ${ }^{[19]}$.

\subsubsection{Implications of lasers and MIT for communication}

The development of lasers and MIT require new forms of communication between patients, physicians, other health care providers, and management. The main reason is that lasers and MIT have reduced stays in hospital, partly because the patient recovers at home. Therefore, the most important communication is that for the patient to provide feed-back on the results of the procedure after he or she goes home. Besides the hospital, which should know the outcome of therapy, general practitioners and community nurses should also be part of the communication system when they become involved in the care before the procedure and care in the home after the procedure $\mathrm{i}^{[18]}$.

\subsection{Policies towards lasers and MIT}

\subsubsection{Introduction}

The most important general health policy for the last decade in Europe has been to control rising costs of health care by limited budgets on the hospital sector. The increasing technological possibilities and the limited budget for health care has brought the realization to policy-makers that diffusion of new and promising procedures must be guided. Health policies are aimed primarily at slowing diffusion ${ }^{1+184}$. Perhaps as a result of the generall orientation of health policies, policy-makers have been slow to recognize and realize the potential of lasers and MIT ${ }^{193}$. On the contrary, interest in this area seems mainly to have been fuelled by concerns of inappropriate use. As we have seen in Chapter 17, questions on laser applications were asked for the first time in Dutch Parliament in 1993 when Excimer laser treatment of myopla was advertised in a High School magazine. In 1995, the Parliamentary Office of Science and Technology in the United Kingdom published a report on the implications of MIT as a result of concerns arising in particular from well publicised cases where patients suffered following MIT. In the UK, parliamentary interest focused on the extent of use and safety of MIT, and the level of training required by surgeons using MIT. The report covered other 
issues as well; including costs and cost-effectiveness and logistical implications of MiTisa). Besides these issues, several others can be distinguished. In order to systematically discuss the policy implications of lasers and MIT, a framework published by Banta, Schersten and Jonsson (1993) will be used in a slightly modified form ${ }^{\text {ita }}$. The following subjects are included: policies towards

- research and development;

- experiments, including priority setting and evaluation of safety, effectiveness and costeffectiveness of lasers and MIT;

- publication:

- education, training certification and quality assurance;

- planning, budgets, investment controls and payment;

- dissemination and implementation;

- consumer ínformation.

\subsubsection{Policies towards research and development}

Ordinarily, one thinks of health care technology as the result of processes of basic and applied research in the health field. Governments in Europe and North America invest large sums in healthrelated research for the purpose of improving the scientific and technological base of health care $^{[107]}$. In fact, development of MIT, and in particular the laser has followed a quite different path. The laser has not developed primarily as a health care tool. Government research programs dealing with health have paid little attention to lasers. In the eighties, developments have probably been spurred more by the US Strategic Defense Initiative (SDI) than any other factor, since the ultimate success of the SDI was judged to partly depend on advanced lasers. Although SDI was cancelled, the US Department of Defense is still investing huge amounts in research and development in this area. This resulted, among other products, in laser weapons designed specifically for blinding (see Chapter 1) ${ }^{\prime}$. In Europe, in France, the laser indiustry and the country's laser institutes have been strongly oriented towards military projects, which has hampered the transfer of research results into industry ${ }^{\mid 3{ }^{30 \mid}}$, including the medical device industry.

In 1993, Gelijns and Fendrick described and briefly analysed the innovation processes of the three cases of minimally invasive therapy that diffused most rapidly: catheter treatment of coronary artery disease (in casu PTCA), ESWL, and laparoscopic cholecystectomy ${ }^{\mid 631}$. It was shown that the R\&D in case of PTCA and laparoscopic cholecystectomy was a collaborative enterprise of clinicians and industry, without governmental support. An exception was the development of ESWL. In this case the Germany-based firm Dornier was active in the air- and spacecraft industry and had no previous experience in the medical field. Originally, this firm had a research grant from the Ministry of Defense to study the interaction between shock waves and tissue in animals. When the firm had successfully organized collaborative efforts with leading urologists in Germany, the German government financially supported the associated R\&D needed for the development of ESWL in humans ${ }^{\left|\mathrm{sa}_{2}: 10\right|}$. Likewise, as we have seen in Chapter 15 , the Danish and French government supported their national industries to produce its own ESWL machine, which can be interpreted as a form of 'technology-nationalism'. A similar phenomenon has been observed in the case of lasers. In Germany, when Lambda Physic was bought by a US company in 1981, the industry feared il could become dependent on the US for laser equipment. The German Ministry of Research and Technology (BBWFT) then declared lasers as a key technology, increased it financial support from DM 9 million to DM 27 million in 1986, and set up the Technologiezentrum im Verein Deutscher Ingenieure (VDI-TZ) in Düsseldorf to manage research across the country. By 1988, Germany had reached a leading position on the world laser market ${ }^{\text {f9el }}$. It should be added that although this

1 in Vienna, in Octaber 1995, delegates of gowernments at the United Nations Convention on Conventional Weapons prohibited the production, transfer and use of these weapons. This was judged as an unexpected outcome of the Vienne conference and is important because the decision applies to a weapon before its effects have been witnessed at the battlefiel $\mathrm{d}^{\text {that }}$. 
particular analysis covered civilian applications of lasers, its focus was in particular on industrial lasers.

For other cases of MIT, government funding for research and development is likely to have been minimal. Within the health care area, public policies deal primarily with basic research ${ }^{161}$, and therefore have little direct applicability to lasers and MIT. Applied research has traditionally been the sphere of private companies. In the United States in 1988, the total government investment in thealth R\&D was US\$ 9.7 billion, whereas the industry investment was US\$ 8.3 billion ${ }^{[90 !}$. In the European Community, average governmental spending on $R \& D$ in the health field represented about $4.5 \%$ of all R\&D expenditures in $1990^{[44]}$. No estimates were provided for industrial investments. In England, in 1993 the estimated overall expenditure on health research and development was around BP 2 billion, of which $60 \%$ (1.2 billion) was accounted for by the industry ${ }^{[40]}$. In the Netherlands, the Ministry of Health spent Dfl. 150.5 million on research and development in the health field, representing $3.1 \%$ of total governmental R\&D expenditures in $1993^{[44]}$. Again, no estimates were available for industrial investments in the health sector. Although comparative data of the United States and (individual countries in) Europe are largely lacking with respect to the relative contribution of governmental and private investments in $R \& D$ in the health field, the generalization that industry spends more on applied research than governments seems almost certain to hold. Industry investment in an area depends on a number of complex factors. One is profitability. If the company generates substantial profits, it may invest part of those profits in further development. In general, the medical device industry is a high profit area, and its investment in research and development is therefore relatively generous ${ }^{[\theta !]}$. The laser area, however, has not been very profitable, so health care developments of lasers tend to be slow in most applications (see paragraph 20,4.3). As the laser has gradually become accepted as an important tool in different areas of health care, funding for laser related services has increased, increasing profitability for industry. This may have happened with the Nd:YAG laser "given its rapid diffusion in the late elighties/early nineties, and may presently happen with the diode laser. An option for governments and those who pay for health care might be to allow increased funding for laser services, which would make the laser field more profitable and could have the rellated effect of increasing research and development. This may be usefull in selected cases of MIT as well, although it should be taken into account, as described earlier, that the field of MIT seems more profitable than the field of lasers. Another option, of course, would be for governments to invest directly in research and development. However, since at this stage of applied research and dewelopment collaboration between industry and clinical experts is necessary, direct government funding might not be efficient.

\subsubsection{Evalluation policies}

Technology assessments depend on reliable experimental data, especially on safety, efficacy (or effectiveness) and cost-effectiveness. The most reliable source of such data is randomized trials (RCTs). Despite the growth in the field of technology assessment, investments in assessment are still small.

In the United States the total investment in health care technology assessment in 1984 was estimated to be no more than $\$ 1.3$ billion, of which US\$ 1.1 billion was invested in clinical trials, mostly for pharmaceuticals. Spending direct to technology assessment was less than US\$50 million, about 0.5 per cent of health R\&D Funds $s^{\left[7^{7}\right]}$.

in the Netherlands too, investments in medical technology assessment are small. The most important fund for Medical Technology Assessment is the Investigational Medicine Fund, which annually spends Dfl 36 million (about US $\$ 22.5$ million; 1 US $\$$ equals about 1.6 Dfl), almost exclusively to clinical trials while virtually excluding pharmaceuticals. In this country, the total health care costs amounted 59.4 billion Dutch guilders (more than US $\$ 33.9$ billion) in 1995 . The funds 
for MTA therefore represent less than $0.07 \%$ per cent of national health expenditures in the Netherlands:

In 1993, as indicated in paragraph 20.5.1, the expenditures on health related R\&D by the NHS in the Unted Kingdom amounted 0.8 billion BP. Of these resources, about $20 \%$ was spent by the Medical Research Council, one of the main sponsors of MTA-studies. The expenditures for MTA represented approximately $0: 2 \%$ of total NHS expenditure in $1993^{(40)}$. Of the scarce resources spent for evaluation, the number of RCTs covering laser applications or applications of MIT has been quite limited on an international scale. In recent years, however, this has changed in both the Netherlands and in the United Kingdom (see Intermezzo 20.1).

In many countries, investment in technology assessment is slowly increasing ${ }^{[24]}$. Despite this encouraging trend, the problem of limited funds for supporting studies in the field of medical technology assessment will probably not be solved: It is possible to set priorities for available funds, however. An explicit policy for stimulating and funding RCTs of important technologies can be quite beneficial. An early example of such a policy is provided by the National Eye Institute (NEI) of the National Institutes of Health in the US. The 1988 investment of the National Eye Institute of US\$ 30 million, a relatively large sum in comparison with other components of $\mathrm{NIH}$, helps explain the good documentation of ophthalmological conditions. For a number of years, the NEI has funded clinical trials of technologies important or potentially important in oplithalmology. In this thesis, the quality and implications of several of these trials were described (see Chapter 6). The potentials off a policy for improving care, saving resources, and preventing costly errors are trully enormous. This potential is increasingly recognized. For a specific discussion on priority-setting, see paragraph 20.6.4.

Intermezzo 20.1 Evaluation of laser applications and MIT in the Netherlands and in the United Kingdiom

In the Netherlands, the cost-effectiveness of four laser applications has been evaluated in the context of the investigational Medicine Fund since its inception in 1988. The first of these. Excimer Laser Coronary Angioplasiy (ELCA) followed by Percutaneous Transluminal Coronary Angioplasty (PTCA) versus PTCA alone in patients with long coronary artery esions demonstrated that there were no significant differences in effectiveness of either mathad and that ELCA was associated with considerable additional costs ${ }^{\text {ty }}$. The second study, dye-laser treatment of portwine stains, has been completed but its results are not yet published in peer-reviewed joumals ${ }^{\text {sal }}$. The third study, evaluation of external radiotherapy, external radiotherapy preceded by endobronchial brachytherapy, and extemal radiotherapy preceded by Photodynamic Therapy (PDT) in palliation of patients with non-small cell isng cancer (NSCLC), was terninated in 1994 before patient enrolnent was completed because of excess early mortally in the grour of patients who were treated with the combination of external radiotherapy preceded by enobronchial brachytherapy ${ }^{[67}$. A founth study, evaluation of laser treatrment of pigment naewi, started in late 1995. Likewise, a number of studies in the field of MT have been funded by the Investigational Medicine Program. An example of a study that has been finished is the evaluation of hyperthermia as a supplement to radiotherapy in treatment of non-surgical and radiation resistant tumours. Examples of studies that are in progress are: prophylactic sclerotherapy of esophagreal varices, non-sugical treatment of patients with bile duct stones, transurethral microwave thermotherapy (TUMT) of benigm prostate hypertrophy (BPH), cost-utility analysis of continual positive pressure treatment at home of petients with sheeping disorders, evaluation of the use of stents after percutaneous transilininal coronary angiopla sity (PTCA), evaluallon of non-invasive diagnosis and non-surgical treatment of extrauterine gravidity, and laparoscopic sungeny versus conventional surgical treatment of groin hernia ${ }^{\text {in? }}$.

In the Urited Kingdom, basically three sources of public funds exist for medical technology assessmentross. These are the Standing Group on Health Technology (SGHT) of the National Health Sanvice (NHS) Research and Development Directorate (RDD), the Department of Heatth Policy Research Program (OHPRP), and the Medical Research Council (MRC). An example of a finished study in the laser field funded by the DHRPP is a literailure neview by the Health Economics Research Group of Bninal University rwal. Other experimental studies are underway. For example, in lete 1995 the Standing Group on Health Technology or the Mational Health Service (NHS) Research and Devalopment Directorate (RDD) called for research proposals on the comparative effects of treatment for convical cancer. The four treatment options that were listed are laser treatment, diathermy, conventional surgery (by knife), and cryosurgery. In the field of MTT, the Standing Group on Health Technology of the National Heatin Service (NHS) Research and Development Directorate (RDD) awaits a report on laparoscopic versus (nowing laparoscopic cholecystectomy. A trial comparing the efficacy and cost-effectiveness transcervical endometrial ablation versus hysterectomy for menorhagia was funded in 1995 . Furthermore, six proposals were considered late 1995 to assess the cost-effectiveness of new and established treatments of berign prostatic hyperplasia (BPH) and conventional versus laparoscopic gynecological surgery. The Medical Research Council (MRC) funded trials comparing hysteroscopic endometral resection and hysterectomy in treatment of menormagia, two trials focusing on laparoscopic hemia nepair, and trials assessing laparoscopic surgery for colorectal and gastric cancer. respectively. The Depantment of Health Policy Research Program (DHPRP) commissioned several systematic 
Mterature reviews in 1995. Subjects include laparoscopic chotecystectomy cancer of the gastromestinal tract (esophageal, stomach and calon), haparoscopic appendicectomy, prostatic disease (including benign prostatic hyperplasia and prostate cancer) and thoracoscopy (for cancer of the lungl. Finally, the Standing Group on Health Technology (SGHT) or the National Healh Service (NMS) Research and Development Dinectorate (RDD) awaited resubmission of a systematic rewew of coronany artery bypass grafting (CABG) wersus Percutaneous Transtuminal Coronary Angloptasty (PTCA) wersus medical management of heart disease in late $1995^{\text {resl. }}$

\section{The legal basis for experiments (evaluation) of devices, procedures and drugs}

In paragraph 20.2 .6 it was tentatively concluded that the quality of the findings on the issues of safety, effectiveness and cost-effectiveness of lasers may be representative for the quality of evaluation of new medical devices and procedures in general. It was also argued that the findings with lasers can be judged to represent a lower quality of evaluation than is the case in the evaluation of pharmaceuticals. This is likely to be closely related to regulatory requirements for registration and/or reimbursement of drugs. Sometimes the requirements for reimbursement of drugs even extend to an economic evaluation of alternative regimens, e.g. in Australia, where guidelines were developed for economic evaluation of pharmaceuticals were released as early as 1.1990, revised and implemented in 1992, and revised again in 1995 $5^{[82]}$. An increasing number of countries have followed this example (see paragraph 20.6.2). Profiting from this pro-evaluation climate, the field of pharmacoeconomics has shown more progress than economic evaluation of devices or procedures. A subjective indication for this process is the increase of pharmacoeconomic studies and the successful launch of a new journal 'PharmacoEconomics' in $1992^{\left|s^{2}\right| l}$. Perhaps a spin-off of these developments can be expected both in terms of clinical trial design and in the evaluation of devices and procedures. This has not (yet) clearly become manifest. A sign of progress could be, for example, the establishment of a journal carrying the title "Medical Device Economics" or (in terms of coverage even better) "Medical Device and (Medical) Procedures Economics'. Due to a limited legal basis for evaluation of medical devices and medical procedures, increasing assessment activities in general and increasing assessment activities in particular of lasers and MIT, will not have as profound an influence as can be witnessed in the field of evaluation of drugs. Within this constraint, this general picture differs depending on the continent. In Europe, as noted in Chapter 19, the Medical Device Directives are essentially restricted to technical safety. In the United States, the Safe Medical Devices Act (SMDA) of 1990 has come into effect April 11, 1996. The implications of this Act have been translated by the US Food and Drug Administration (FDA) in the form of medical device reporting regulations for health care facilities and manufacturers. Failure to comply may result in civil monetary penalties and increased liabillity exposures $^{[\{d]}$. This development will stimulate evaluation of medical devices and device-dependent procedures in the United States. In the slipstream of these events the quality of economic. evaluations may improve as well. In Europe, such incentives can probably not be expected in the short-term, although Concept-Directives on liability for medical procedures and privacy protection of citizens have been published. A more detailed account of policies towards evaluation of safety can be found in Chapter 19.

\subsubsection{Publication policies}

Research on lasers and MIT is just as research of any other technologies subject to a phenomenon called publication bias. This can be described as any tendency on the parts of investigators, reviewers and editors to submit or accept manuscripts for publication based on the direction or strength of the study findings $s^{\{33 !}$. In general, clinical research, e.g. (randomized) clinical trials, is more likely to be submitted to journals and subsequently to be reported in print when the analysis of the results allows to conclude that there are statistically significant differences between treatments ('positive' findings). Instead, studies should be accepted or rejected on the basis of whether they have been well conceptualized and completely executed ${ }^{[35 \mid}$. Although both investigators, reviewers and editors are subject to publication bias, a detailed study in the United States has shown that investigators are the primary group responsible for failure to publish ${ }^{[42 i}$ Failure to publish an adiequate account of a well-designed clinical trial has been called a form of 
scientific misconduct that can lead those who care for patients to make inappropriate treatment decisions $^{\text {t5s }}$. Likewise publication blas can create a serious problem for anyone conducting reviews of evidence about the safety, effectiveness and/or cost-effectiveness of health care interventions: If the reviewers do not have available for consideration all relevant evidence related to a given treatment, the possibility exists that the conclusions they reach will be wrong. This could happen when publication bias would result in selection bias in the data collection methods of reviewers, for example when meta-analysis is undertaken ${ }^{[4 / 2]}$. Many suggestions have been made to awoid publication bias. Perhaps one of the most effective measures to promote change in this direction is to create registers of clinical trials prior to initiation. This is becoming increasingly common, both in the United States and in Europe $\mathrm{e}^{\mid 35,42,43 !}$.

Has publication bias played a role in arriving at the conclusions in this thesis? I think so but we of course do not know to what extent. Research shows that the majority of the ratios of published to unpublished trials fall between 10:1 and $1: 1^{\mid * 31}$. This problem could therefore be big or small in this specific case. In general, publication bias on the part of editors could, hypothetically "be more likely in journals edited by laser-enthusiasts and innovators in the field of minimally invasive surgery than in general medical joumals because the editors of the first category of journals may be more likely to have a private interest in positive findings. Examples of this category of journals are "Lasers in Medical Science', 'Lasers in Surgery and Medicine' and 'Minimally Invasive Therapy \& Allied Technologies'. It is emphasized that there are no specific indications of an increased likelihood of publication bias caused by editorial policies of these specialized joumals.

\section{Publication bias and publication policies towards economic evaluations}

The first general medical journal that published guidelines for the conduct and reporting of economic evaluations was the New England Joumal of Medicine in $1991^{[\text {"1] }}$. The background of the development of these guidelines was that published pharmacoeconomic findings that are distinctly unfavourable to the sponsoring firm's product are rare. The authors hypothesized that this was the result of the marketing considerations of pharmaceutical companies underlying their interest in economic evaluation. This would result in a systematic distortion of the balance of completed and published results. In addition to these guidelines, the editors of this journal published a specific policy on cost-effectiveness analyses in $1994^{179}$. The editors decided to exclude from consideration articles presenting cost-effectiveness analysis if any of the authors had a personal conflict of interest. Examples are authors who receive a direct salary from the sponsoring company or a competing company, authors who have an equity interest in, an ongoing consultancy with, or membership on the scientific advisory board of such a company, or a related patent pending. This policy was defended by referring to the fact that financial arrangements create an incentive for bias and that cost-effectiveness analysis is more susceptible to many forms of bias than clinical research. According to Drummond ${ }^{[49]}$ it is unclear whether there is more under-reporting of negalive economic than of negative clinical study results. In his opinion it is not necessarily the case that economic appraisals are more likely subject to publication bias than clinical trials. For example, clinical trials embody many implicit value judgernents in their selection and reporting of endpoints. In contrast, economic appraisals incorporate value judgements explicitly.

Although publication blas is a serious theme in both clinical research and in cost-effectiveness analysis, the role of publication bias in economic evaluations of laser applications and applications of MIT may be relatively small compared to pharmacoeconomic evaluations. Cost-effectiveness research in this field has so far almost exclusively been performed by independent researchers with a neutral source of funding. The medical device industry is not nearly as active in the field of economic evaluation as the pharmaceutical industry. A measure to reduce the potential for publication bias in this field would be to create specific registries of all clinical trials alongside which economic evaluations are carried out. Such a registry has been created in the United Kingdom. 


\subsubsection{Policies towards education, training, certification and quality assurance}

\section{Education and training}

As noticed in Chapter 1 and 2, as a consequence of the complexity of lasers, its safe and effective use requires considerable education and training for physicians, nurses, (laser) safety officers and technicians. Chapter 3 and 19 have shown that the situation in the US and the EU is very unsatisfactory in this respect because most education in laser technology is fragmented and because any practitioner may legally purchase a laser and begin to use it in clinical practice without the need for demonstrating that he or she has acquired appropriate expertise. In the EU, the subject of education and training is not included in any of the Medical Device Directives. This situation has resulted in a number of cases of malpractice and, of course, to the awareness that lasers should only be used by those physicians who are fully trained and who can rely on a welltrained supporting staff. To achieve this, the different needs of these professionals should be reflected in specific training programs, which have been discussed in Chapter 3 . A number of nongovernmental organizations are involved in these laser training programs: the European Laser Association (ELA), national laser societies and safety boards, laser centers affiliated with hospitals and universities, laser specialty societies, manufacturers, hospitals and independent groups of laser surgeons.

In analogy with laser-using physicians, physicians who are not fully qualified for minimally invasive therapy risk causing accidental injury to adjacent organs or tissue, which can in extreme circumstances lead to death but more usually results in increased complication rates: Since the introduction of MIT, a steady trickle of stories appeared in the media of patients sustaining serious injuries as a result of such therapy. This is not surprising since the approach for physicians wanting to try out a new MIT was characterized at one stage as: 'see one, watch a video, do one ${ }^{(96)}$. This has changed and a number of initiatives can be listed to ensure proper training for surgeons wishing to perform MIT. Just as in the case of lasers, the development of training programs for MIT has been the responsibility of non-governmental organizations, in particular medical societies. An example is the United Kingdom, where in May 1994 the Senate of the Royal Surgical Colleges published new training requirements that covered not just MIT but all new and existing techniques across all surgical specialties. Within this overall framework, the various specialist professional bodies have drawn up precise details of the course of each of the various surgical specialties. For example, guidelines for training in laparoscopic surgery were published in December 1994 by the Senate of the Royal Surgical Colleges. The Colleges' guidelines make it clear that only appropriately trained surgeons should conduct laparoscopic procedures, and that cholecystectomy is the only such procedure that may be routinely undertaken. Surgeons are advised not to perform other laparoscopic operations without careful auditing or participating in controlled trials ${ }^{96 \mid}$. In the United Kingdom, the government has played a stimulating role as well. In that country the government announced in September 1993 that funds would be made available to set up special MIT training centers to provide the facilities for surgeons to acquire the new skills. In late 1995, 4 centers had been established, two in England, one in Scotland, and one in Wales ${ }^{|96|}$

\section{Certification}

Training programs are usually accompanied by some form of certification. Basically, two types of certification can be distinguished: procedure-specific and specialty-specific. With procedurespecific systems, the types of operation that a surgeon is allowed to perform are specified in detail, either being listed on their certificates (so-called credentialing) or decided (with reference to the surgeons's qualifications and experience) by a hospital committee (privileging). In the alternative (specialty-specific) approach, certificates, issued by medical societies, merely indicate the specialty in which a physician may practice ${ }^{[96]}$. In practice, these terms are sometimes used as synonyms, in particular in the US ${ }^{\mid 92 !}$.

In the case of lasers, a formal system of accreditation for medical practitioners wishing to work with these devices was proposed by laser users in the United Kingdom as early as $1989^{\mid 321}$. Critics 
emphasized that the existing situation offered adequate control and monitoring of specialists within their discipline and that, therefore, further controls were unnecessary and unwelcome ${ }^{\text {is }}$.

Just as in the field of lasers, credentialing proposals have been made in case of MIT, again in the United Kingdom ${ }^{i 6 i}$ (see next section). In general, credentialing for surgeons is important for a facility's legall protection, as well as its overall safety program. Critics, howewer, while referring to the US, stated that credentialing is a bureaucratic nightmare ${ }^{909}$. The choice between procedure specific or specialty-specific certification is, just as the potential next step choilce between credentialing and privileging, often subject to question and controversy. In fact, certification, and in particular credentialing is probably the most complicated issue facing laser-or MIT equipped facilities $_{i}$ and no one solution will work for every facility ${ }^{[55,96]}$. Some possibilities and solutions in case of lasers and MIT will be outlined.

\section{Certification: procedure specific or specialty-specific?}

In the United States, physicians are licensed to legally practice medicine. With a medical license from a given State, a physician can practice medicine in that State, in whatever specialty area he or she chooses. Some physicians, in addition to having general medicall training, may have received training in a particular (sub)specialty area. Such training is not required for medical licensure, but physicians who have specialty training may be eligible to become certified by a specialty board. Thus, certification is specialty or subspecialty specific in the US ${ }^{|92|}$. One of the main disadvantages of this system is that it does not rigorously evaluate clinical skills of physicians. For this reason, the American College of Physicians (ACP) started a Clinical Privileges Pilot Project in the mid-eighties ${ }^{|92|}$. As part of this project the ACP developed guidelines defining minimum skills, education and training that physicians need to perform competently eight specific medical procedures. These guidelines included, among other procedures, flexible fiberoptic sigmoidoscopy, colonoscopy, esophagogastroduodenoscopy, and endoscopic retrograde cholangiopancreatography (ERCP). These guidelines became official policy of the ACP, and preceded a project to develop guidelines for a number of other procedures in all subspecialties of internal medicine. Guidelines for granting physicians privileges to perform specific procedures have also been developed by ather organizations. For example, the American Association of Urology published guidelines for the training of physicians in the use of ESWL in 1987. To obtain a certificate of training for this procedure, a physician was required to have had 5 days of training and to have performed 15 procedures. The Association also started approving potential sites where lithotripsy training could take place. In addition, in 1987 a task force of the American College of Cardiology proposed training standards for performing coronary angioplasty. The guidelines stated that the physician training to perform angioplasty must complete a fourth year of residency training and a minimum of 125 coronary angioplasty procedures, including 75 as primary operations. The task force also called for a certificate of added experience and qualification in advanced cardiac catheterization procedures (such as angioplasty) ${ }^{|92|}$.

In summary, in the United States a partial shift has been realized resulting in a change from a (sub) specialty credentialing system (board-certification) to a procedure-specific privileging system, starting with guidelines for privileging for procedures that are now labelied as minimally invasive diagnostic or - therapeutical.

How about lasers? This situation is not clear. Presumably functioning in the 'old' system of (sub)specialty certification, the American Board of Laser Surgery was one of the 69 specially boards that, at least until 4987, had not been recognized by the American Board of Medical Specialties or the Advisory Board for Osteopathic Specialists. All of the forty specialty boards recognized either by the American Board of Medical Specialties or by the Advisory Board for Osteopathic Specialists require physicians to complete a specified amount of training and a certain set of requirements and to pass an examination ${ }^{\natural 2 !}$. This suggests that, at least in the early days, it was relatively easy for physicians to start calling themselves "laser specialists " or "laser surgeons" or anything like that. Since then, with an increasing role of the American Society of Laser Surgery 
in Medicine, standards of education have increased, but the issues of training, credentialing and Borard certification are still lively debated in the Society ${ }^{(23.94)}$. A relevant development in the United States which may have (re)encouraged these discussions is that the Safe Medical Devices Act (SMDA) of 1990 has come into effect April 11, 1996 (see paragraph 20.52).

In Europe, neither the subject of training and education nor the subject of certification is dealt with in any of the EU Medical Device Directives. Because the EU Medical Device Directives are superficial in the area of functional requirements and do not relate to appropriate use, little impact on clinical practice will result ${ }^{174}$. In this situation every member country has to decide its own way. which is difficult. The German certification program has been discussed in Chapter 3 because of its interesting training aspects ${ }^{125}$. This seems a (sub) specialty-specific system. In the United Kingdom, there is no consensus whether to use procedure-specific or specialty specific systems of certification. The medical societies (Colleges) favour a specialty-specific approach, while a report published by the Department of Health favoured a system of procedure-specific privileging. This discussion focuses for the most part on the issue of MIT ${ }^{[9]}$ (see previous section).

In the Netherlands, efforts to develop accreditation processes for hospitals and certification procedures for professionals are almost all in an early stage of development ${ }^{\text {its }}$. Certification systems for physicians have been developed exclusively for services regulated under Article 18 of the Hospital Provisions Act ${ }^{[17]}$. The Dutch Chief Medical Inspectorate for Public Health has started activities that will result in publication of an issue of the Medical Inspectorate Bulletin specifically on laser safety. The issue will include laser-and specialty specific recommendations ${ }^{\text {is? }}$. These recommendations, of course, could in the future be used as part of a training process resulting in certification of laser users.

\section{Quality assurance}

Quality of care can be defined by a number of different dimensions, such as access to care, range and level of health care services, and consumer satisfaction. In this section, the focus is on quality, quality assessment and quality assurance at the level of the clinic, and relations of this quality to technology assessment. From the standpoint of technology assessment, the main purpose of quality assurance is to see that the care provided has maximum benefit and minimal risk, that is, outcomes as good as possible ${ }^{[17]}$.

In the United States, clinical practice guidelines are being actively developed by physicians organisations, insurance companies, government agencies, and others. Eddy (1990) defined practice guidelines as 'recommendations issued for the purpose of influencing health interventions ${ }^{\prime 155}$. The National Institutes of Health $(\mathrm{NIH})$ manages a consensus development program. The goal of these conferences is to seek consensus on the safety, efficacy, and appropriate conditions for use of various health care technologies ${ }^{[t]}$. With respect to lasers and MIT consensus statements include e.g. therapeutic endoscopy and bleeding ulcers (1989) and gallstones and laparoscopic cholecystectomy (1992).

In the United Kingdom, an important role for improvement of outcomes is granted to a system of clinical audit, which is defined by the Royal College of Surgeons (RCS) as the interdisciplinary process by which clinical staff collectively review, evaluate and improve their practice, with the common aim of improving standards ${ }^{\text {ig6l. }}$. Clinical audit programs are relevant for MIT because they can identify problems with specific procedures. Where a particular surgical procedure is shown to be less safe, effective or cost-effective than an alternative, improving the consistency of clinical practice is one of the key abjectives of the UK Department of Health Research and Development Directorate (RDD). Although professional bodies support this development and progress is being made, several problems have been defined, including the quality of information collected on surgical procedures and the quality of the clinical outcome indicators used ${ }^{196 !}$. These problems have been recognized in the United States and other countries as wellial. 
In the Netherlands; as described in Chapter 19, there have been legislative changes redefining the legal status of professionals practising medicine. As part of this process, the Individual Health Care Professions Act defined a category af procedures that can only be performed by a limited category of registered professionals, predominantly physicians. Minimally invasive procedures included in this list are: endoscopies, catheterizations ${ }_{i}$ and lithotripsy ${ }^{4{ }^{4} b_{l}}$. It seems reasonable to interpret these regulations such that endoscopy-or catheter-assoclated procedures, for example including a laser, are also included in this list. In the Netherlands, there is a general agreement concerning the importance of setting standards or criteria for quality of care. As a result, guidelines for medical practice have been increasingly developed in the Netherlands during the last 10-15 years. Two organizations are actively involved in this process, the National Organization for Quality Assurance in Hospitals (CBO), and the Netherlands Society of General Practitioners (NHG). Subsequently, these guidelines can be used as the basis for audit procedures. Of the final reports published by $\mathrm{CBO}$, which are actually the result of consensus conferences, at least one has a direct link with laser treatment, which is the report on diabetic retinopathy. Laser treatment of diabetic retinopathy is recommended as well in the guideline on Diabetes Mellitus type II issued by the NHG for general practitioners $^{(17)}$.

\subsubsection{Policies towards planning, budgets, investment controls and payment policies}

Collective constraints on the adoption and use of health care technology can be characterized as either direct or indirect. Direct constraints come in the form of prohibitions against the adoption or use of a technology, or detailed specification of the circumstances under which it may be adopted. For example, standards may require that only hospitalls with open heart surgery may do cardiac catheterization or that physicians involved in laser use participate in a special approved training program. Indirect constraints are most often financial. For example, a government or an insurance fund may decide about the budget or fees to be permitted. Decisions about fees include whether to reimburse for the use of the technology at all, and, if so; how much. Fees can be coupled with conditions, for example, that the use of the technology will be reimbursed only for patients with specified symptoms or only if the work is done by certain specialists. Personnel policies offer another form of indirect constraint. Through controls over the number of health professions students, the kind of training they receive, and the kinds of posts available for them when they graduate "governments can influence the climate for a new technology.

In the past, governments have tended to emphasize direct controls. In the Netherlands, where medical technology is relativelly heavily regulated, under Article 18 of the Hospital Provisions Act, the government has the authority to forbid certain services of a specific nature without a special license. More than 12 technologies are directly regulated by this program, including interventional cardiology (e.g. PTCA and implantation of a defibrillator) as the most prominent example of MIT. Sometimes, as in the case of computed tomography (CT) scanners and cardiac angiography, the regulation is lifted when the technology comes to be considered a standard procedure ${ }^{[17]}$. Such direct controls, often called "planning," are usually aimed at large items of expenditure. Groot (1988), as cited in the report by Banta, Oortwijn and van Beekum (1995) estimated that the technologies covered by Article 18 made up only $3.3 \%$ of all health care expenditures ${ }^{66 !}$. More recent estimates are that Article 18 covers about $2.5 \%$ of health care expenditures, but approximately 16 per cent of the budgets of university hospitals that provide the technologies covered by the program ${ }^{[17}$.

As probably can be expected, this form of direct regulation has little application to lasers and MIT. As indicated in paragraph 20.5 , the most frequently used types of lasers cost approximately US\$50 000 - \$100 000 and the basic equipment for MIT may require about half the capital outlay. Regulatory programs do not generally deal directly with such a relatively small investment. Apparently, no country directly regulates the purchase of lasers or the provision of services with lasers or minimally invasive therapy. Direct regulation can also deal with quality, and this form of 
regulation may be more applicable to lasers and MIT. For example, hospitals can be accredited by government or special independent commissions. One part of accreditation should deal with appropriate training and experience of medical and technical staff( (see paragraph 20.6).

An alternative to direct regulation is indirect regulation using payment in this model, the technology is not forbidden, but payment is used to regulate adoption and use. for example, services are not paid for until the technology has been accepted by those who pay for care, the government, sick funds, or insurance companies. In the Netherlands, for example, a technology is not paid for uniess it is part of the benefit package ${ }^{[17]}$. Likewise, in the United States third-party payers may not pay for the cost of using a laser for a certain procedure if the laser is still considered investigational by the FDA. In theory, this mechanism could control lasers and MIT. The policy could be for example, that only physicians who have had appropriate training could be paid for a certain procedure. However, again contrasting with visible and expensive technologies such as heart transplants, lasers and MIT largely escape such controls. One reason is that minimally invasive procedures and laser applications, which are often only an adjunct to a procedure, can be billed as conventional procedures until those paying for health care make an explicit decision. A separate paper on the application of criteria for reimbursement to the case of Dye laser treatment of portwine stains and Excimer laser treatment of shortsightedness in the United States and in the Netherlands is available at the author ${ }^{[1 \text { el }}$. The system is not perfect. Meyers (1990) stated that the absence of a consistent and explicit policy for reimbursement for new technologies results in an escalation of charges and provides incentives to conceal innovations ${ }^{\mid 87 !}$. Two perverse behaviors may result: "upcoding', (or CPT-4 creep in the United States setting), where a code representing a more expensive medical procedure or service is used in place of the code that would be most applicable, and, "fee unbundling' using multiple (CPT-4) codes to describe a procedure rather than one primary code.

Irrespective of the timing and criteria for reimbursement or ways of 'fragmented billing' for a particular procedure, every payment system has positive and negative incentives for adoption and use of medical techmology. In the EC-FAST project on MIT it was concluded that for the physician and the hospital, implications of lasers and MIT largely depend on the nature of the health care system, especially the payment system ${ }^{[4]}$. To express this, the title of the report to the EC was called "The budget is blind: the case of minimally invasive therapy in Europe ${ }^{1211}$. This was intended to draw attention to the fact that a budget system by itself does not assure that investments are wise. The most common situation is that the service or person with the greatest power in the system wins out. An alternative is based on the fact that the medical profession is generally conservative, and tends to continue to use the same treatments. Both of these methods are likely to discourage the adoption of lasers and MIT. An obviously superior method that was promoted as part of the project would be to base decisions on technology assessment. This would imply an active policy for supporting technology assessments whose purpose would be to help those who must make decisions about competing technology. The background of this recommendation was that hospitals, insurance companies, unemployment insurance programs, and so forth, tend to define cost-effectiveness form their own perspective. This means that the incentives to move toward lasers and MIT may not be clear. For example, early discharge may not be in the financial interest of the hospital under the budget system. The same applies to the necessary investment in new medical devices, e.g. endoscopes and ancillary equipment. Likewise, there may be adverse financial incentives to physicians. Innovators are not paid more, and MIT may actually reduce their incomes through the requirement for extra training and the extra time it takes to start a new procedure. Early return to work or normal activities is beneficial to the broad society and for individuals, but may not be of interest to health care insurers. In particular when indications for treatment expand greatly, which has happened for example in the case of laparoscopic cholecystectomy, expenditures for care have actually risen. Therefore, as was emphasized in the EC-FAST project, the implications of MIT for society are mainly positive if the diffusion and use of the new procedures can be guided. 
The background of the existence of budget-financed health services is the need to control health care costs. In a study on health care cost containment in the European Community. Abel-Smith (1992) documented that this is a common way to control supply which, combined with other measures; is fairly successful in achieving this goall. However, if countries choose to limit expenditures and to control incentives for new technologies such as lasers and MIT there will be a relatively slow investment in potentially cost-effective medical technology. In an extreme form, there may be a straightforward tension between cost-containment and the underutilization of effective health services, e.g. in case of screening programs for diabetic retinopathy ${ }^{[27]}$.

Another implication of such a policy is limited funds for industrial innovation in the national industry. Such countries as the United States, Japan, and Germany, which are very innovative in the medical devices area also have rather open health care systems that invest rather readily in new services and machines. Thus, payment policy is also a form of pollicy toward innovation and industrial development. it is a trade-off, and the overall results are far from clear. On one side, an open, innovative health care system that brings the fruits of modern technology quickly to health services can result in an active industry, producing jobs and exports, but at the same time this may produce some harm in the population due to the premature use of new technologies. On the other side, a more closed system with less investment produces less innovation, less latrogenic disease, fewer benefits from new advances, and a climate not encouraging for industry.

\subsubsection{Policies towards dissemination and implementation}

Perhaps the United Kingdom is most advanced in formulating and implementing a policy on the dissemination and implementation of research findings. In 1994, the National Health Service (NHS) Center for Reviews and Dissemination (CRD) was established ${ }^{[1+2]}$. Besides providing the NHS with information on the effectiveness of treatments and the delivery and organization of health care, the CRD should promote implementation as well. To support these activities, a research program focusing on the most effective and cost-effective ways of implementing research findings in the health service in the United Kingdom was formulated in $1995^{[4.89]}$. In the Netherlands, as part of a strategy aimed at implementation of research findings, issuing a new bulletin, an Appropriate Care Bulletin, is contemplated ${ }^{|89|}$. Policies towards dissemination and implementation receive more attention. "Using the evidence" has been a primary theme of the Second International Conference on the Scientific Basis of Health Services, which was organized in late 1997 in the Netherlands.

\subsubsection{Policies towards consumer information}

A particularly important target group for dissemination is the lay public. In Chapter 14 and 15, we have seen that media reporting, fostering both patient demand and physician interest, has played an important stimulating role in the diffusion of ESWL, laparoscopic cholecystectomy, PTCA and arthroscopic knee surgery. In general, the public continues to be infatuated with lasers and "laser surgery". The public, at least in the United States, seems to continue to equate any new, high tech, or minimally invasive procedure or treatment with lasers ${ }^{[8]}$. Usually, consumers are informed on advances in medical technology by the mass media, e.g. newspapers, radio and TV. It is wellknown that the mass media are often uncritical in their reporting, in a sense that they are biased in favour of high-technology medicine ${ }^{(29)}$. Furthermore, the mass media usually pay little attention to broader issues, such as effects on quality of life, economic implications and organizational aspects of technology ${ }^{[1]}$. Although the mass media are often criticized for these reasons it should be added that the right of the media in bringing the news to the public is an essential ingredient of a democracy ${ }^{[2]}$.

Altman (1993), a Niew York Times journalist, states that, just as the Founding Fathers wanted a strong press to challenge the awful power of government, journalism has a responsibility to challenge the life-and-death action of doctors, particularly when so much of medical education and 
research comes from the public purse ${ }^{2 !}$. The author also pointed out that widespread editorial policies of medical joumals, such as the Ingelfinger rule (rejection of a manuscript when it has been published elsewhere) and the system of peer review have a chilling effect on the free flow of information, thus curtaling the role of mass media in informing the public ${ }^{(3)}$ The role of mass media in informing consumers can thus be regarded as the result of two contradictive forces. Notwithstanding these competitive pressures the mass media seem often to simply promote new medical technology, which of course may be reflected in the opinion of the public on medical advances.

In addition to joumalist coverage of new medical technology, explicit promotion of specific tectunologies, either by companies or physician groups, is common in the media: For example, laser treatment of nearsightedness has been actively promoted by physician groups. This practice of media promotion was criticized because of inflated claims of safety and effectiveness of the procedure, as discussed in Chapter 17. So, although mass media may be effective in informing the public on the mere existence of lasers and MIT in general, either by reporting or promotion, it is not likely that in this way the consumer gets full information on its benefits, risks and costs. Therefore, policies should be aimed at developing a more critical consumer of health care. Educated consumers can reject ineffective and cost-ineffective care and demand beneficial care, including new innovations. Only an educated consumer can relate to a physician as an equal partner ${ }^{17 !}$. Another motive to educate consumers is that there is a trend towards more predictive medicine, in which understanding of risks, probability and epidemiologicall arguments is often required for making personal decisions ${ }^{[1+6 !}$.

To strengthen the role of consumers in health care, several initiatives can be supported by policymakers. As an example, three initiatives in the Netherlands are briefly described, which all receive governmental support ${ }^{[17]}$. Firstly, a Federation of Patients and Consumers was founded in 1993. This organization, among other activities, publishes its own journal describing developments in health care from a consumer point of view. Another organization that involves the public in questions concerning health care technology is the Foundation for Public Education on Science and Technology (PWT). This is an independent institution which main goal it is to inform the public on issues related to science and technology. Examples of public controversies about medical hazards are the risks of BSE, passive smoking and cancer, and the interpretation of studies on long-term use of contraceptives ${ }^{[16 j}$. A third publicly-oriented organization in the Netherlands is the Platform for Science and Ethics, implemented by the Rathenau Institute since 1994. The main purpose of the Platform is to organize debates on specific subjects to stimulate public knowledge and action ${ }^{[17 !}$.

Despite these initiatives, methods to educate consumers have not been worked out and evaluated. Recent work in this field has shown that an implication of improving public understanding of science requires a willingness to work towards a scientist's better understanding of the public ${ }^{1464}$. Furthermore, lay people are usually only interested in information on health care technology when they need this information personally. Perhaps due to the complexity of the issue, the initial activities to strengthen the role of consumers in the Netherlands have not all been very successful ${ }^{[7]}$. In late 1995, matters were taken a step further with the announcement of a number of additional stimulating policy measures for consumer information by the Ministry of Health ${ }^{(\theta) y}$.

Besides stimulating initiatives to educate consumers, as mentioned above, the policy may include issuing an Appropriate Care Bulletin, which is aimed at educating health care professionals. One could consider education of professionals as complementary to education of consumers. When patients are successfully educated to ask, for example, about the clinical trial evidence of one proposed treatment over another - is one any better or worse than another? - then, of course, physicians should be trained to not consider their patients hostile when they ask these questions ${ }^{1334}$.

A different example of the ways in which patients can be adequately informed about treatment options has been developed as a result of the activities of the Patient Outcomes Research Team 
(PORT) on benign prostate hyperplasia (BPH) in the United States ${ }^{[11}$. There are many different treatment options for $\mathrm{BPH}$ : expectant management called watchful waiting", transurethral resection of the prostate (TURP), open prostatectomy, laser surgery, etc, each with their own benefits, risks and costs (see also Chapter 9). Two of the most frequently applied treatment options are watchful waiting and TURP. The PORT Team found out that individual men strongly differ in the way they review the impact of $\mathrm{BPH}$ on their lives. Similarly, these men were found to differ in their concerns about the most important risks of TURP, impotence and incontinence. Therefore; in order to learn What the patient wants - to make the normatively correct treatment choice from the perspective of the individual - individual patients were asked to participate in a decision process that disentangled. the patient's preferences from those of others, in particular, the physician. The result of this process would be shared decisionmaking (SDM) on the choice of treatment by both the physician and the patient: To achieve this, the PORT team created an interactive video disc-based patient education program designed to help patilents malke an informed choice between watchful waiting and TURP ${ }^{[12 t]}$. Evaluation of the SDM program in an RCT suggested that the patient's treatment choice reflected their preferences ${ }^{23,120 \%}$. The example demonstrates that educational interventions can be effective in involving patients more actively in decisions about their care.

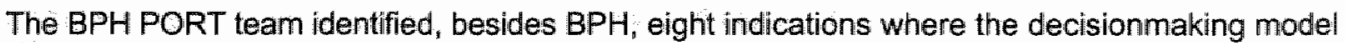
used in their own research could be useful as well. Among these indications there are five where lasers or minimally invasive therapy play a role: noncancerous condition(s) of the uterus, angina pectoris, peripheral vascular disease, gallstones and herniated disc $\mathrm{i}^{1211}$.

\subsection{The future}

Future developments can be classified into two broad themes: technological developments in the field of lasers and MIT and their associated implications for health care delivery in heall th care technology assessment.

\subsubsection{Lasers: technical developments}

The technical improvements of diode lasers, in particular the $805 \mathrm{~nm}$ GaAlAs laser, are perhaps most impressive of all existing types of lasers. At the same time, its clinical utility has become rather promising. The main attractions of diode lasers are that they are of small size, compact, easily portable, (energy)-efficient, relatively quiet, and very simple to use. They have a stable power output, expected long life, no installation costs and are virtually maintenance free. Furthermore, the predicted future costs are low. This laser may have the potential to replace in particular many $\mathrm{Nd}$ :YAG laser applications and applications of the argon laser in ophthalmology ${ }^{(5)}$ Besides the diode laser, portable Nd:YAG-, $\mathrm{CO}_{2}-$ and argon lasers have become available ${ }^{8.121}$ Increasingly, two or more lasers are combined in one product by manufacturers. For example. Coherent has produced a combined Ho:YAG- and Nd:YAG laser. Furthermore, innovative optical fiber technology enables more lasers to be used in minimally invasive therapy ${ }^{[8]}$.

Rapid technological development can be witnessed too in case of the Free Electron Laser (FEL). This is a unique laser which is tunable over a large part of the mid-infrared part of the spectrum. Its tunability can open a wide range of applications in medicine ${ }^{|62,25|}$. According to Ossoff and colleagues (1994)[95] the FEL enables the surgeon to understand and test ablation models across the spectrum and using a constant laser pulse structure. The FEL is also predicted to lead to modifications of surgicall lasers and laser delivery systems, which will make the lasers more effective and initiate innovative surgical procedures ${ }^{[95]}$. The FEL is a huge, extremely costly machine that will need more extra facilities than any other type of lasers has needed. For these partly contrasting reasons the diode laser and the Free Electron Laser perhaps deserve extra attention in the near future (see paragraph 20.8). Other new types of lasers continue to be developed and no doubt some of these may find applications in medicine. An indication for the high rate of this process is that one section of the abstract book of each annual meeting of the American 
Society for Laser Medicine and Surgery is specially devoted to new devices. Besides carving niches for new indications these new types of laser may challenge the position of the older types. For example, the neodymium-doped potassium gadolinium tungstate crystal (Nd:KGW), and the ytterbium-doped strontium fluorapatite crystal (Yb:S-FAP) are candidates that may challenge the position of Nd:YAG lasers in the (near) future ${ }^{[70.97]}$. Of the three major "classic" types of lasers, the position of the $\mathrm{CO}_{2}$ laser may be the least challenged. An example of booming interest in these lasers discussed in this thesis is treatment of snoring (see Chapter 7 ). Recently, the development of pulsed and scanned $\mathrm{CO}_{2}$ lasers has initiated a revolution in aesthetic skin surgery in the US (laser facial surfacing) ${ }^{[4]}$. Complementary" very high power $\mathrm{CO}_{2}$ lasers $(1000 \mathrm{~W}$ ) may have potential for the treatment of patients with end stage coronary artery disease in a procedure called transmyocardial laser revascularization ${ }^{[28]}$. Many other technical developments are anticipated, warranting continuous monitoring by laser safety committees and laser-using physicians.

\subsubsection{Minimally invasive therapy: technical developments}

In 1995, the United Kingdom Parliamentary Office of Science and Technology published a report on minimally invasive therapy and its implications ${ }^{[96]}$. In this report four types of significant recent developments were distinguished: in imaging systems, robotics, surgical instruments and microengineering. The combination of developments in separate fields, according to the authors, may offer most potential for future clinical applications. Developments in surgical instruments and microengineering may be most important in the context of this thesis. The report anticipates that surgical instruments for MIT will become more sophisticated. Among the most difficult procedures are those involving needle and thread (sufuring, ligation, etc.) and these are likely to be replaced by tissue welding techniques using microwaves or radiofrequency heat energy in the near future. Laser tissue welding could probably play a role here as well. Other advances include high-speed drills and ultrasound systems which can be used for tissue maceration outside the body, and allow the removal of large organs or tissues through small holes through the body. Advances in microrengineering have resulted in the construction of microscopic electric motors less than $1 \mathrm{~mm}$ in size. Such motors could power tiny surgical instruments such as forceps and scissors be introduced into body cavities or organs to conduct operations that are not possible using existing lechniques ${ }^{\mid 96)}$

\subsubsection{Implications for health care}

Technological development in the field of lasers and MIT will have implications both on the number of procedures performed with these techniques and on the future organization of care.

\section{Development in numbers of procedures of lasers and MIT}

With respect to lasers perhaps the strongest growth in numbers of procedures can be expected in the specialty with most proven effective and cost-effective applications and with the least competition of other minimally invasive procedures. This specialty is ophthalmology. As indicated above, other specialties where substantial growth in the number of procedures using lasers is anticipated include dermatology and otolaryngology. Just as with lasers, it is estimated that the use of minimally invasive therapy will increase. For example in the United Kingdom even the most conservative estimates suggest that minimally invasive approaches will account for at least $40 \%$ of all annual operations - at least 2 million patients per year - by the turn of the Millennium ${ }^{|0| t \mid}$.

Impact of lasers and MIT on the organization of care

Lasers and MIT will reduce the need for hospital beds and will change patterns of specialization and practice. In the United Kingdom it has been estimated that due to increases in day-care surgery more than $40 \%$ of all 125.000 hospital beds in use in 1993 may not be needed by the year 2002, which would be primarily due to the application of MIT. For some reasons this may be an overestimation $^{965}$. Firstly, conventional procedures also tend to improve, allowing reduced lengths 
of hospital stay. This has resuited in a situation where an increasing number of the planned operations to be replaced by minimally invasive procedures are carried out as a day-case. Secondly, it may occasionally be necessary to shift from a minimally invasive procedure to a

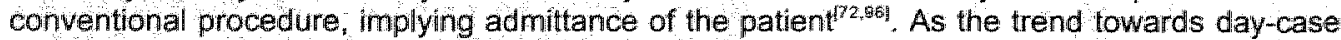
conventional surgery may have been stimulated by the advent of MIT, both trends provide a cumulative stimulus for the reduction of beds.

Specialties will change as a result of MIT. But how and to what extent is rather speculative: According to Banta, Schersten and Jonsson (1993) general surgery has gradually lost much of its work through technological changes and shifts to other specialties. As the volume of surgery falls, and as some procedures are done by other specialists, surgeons may find themselves in a difficult position. In the long run, the authors state, these developments seem sure to change pattems of specialization and practice ${ }^{116]}$. However, in the first of a more recent review of trends, Treacy et al. (1995) suggested that the field of minimal-access surgery will widen, including to, for example, coronary revascularization, endoluminal graft repair of aortic aneurysms, and solitary axillary node biopsy in place of axillary clearance for breast cancer ${ }^{[24 \mid}$. Organ and colleagues (1996) expect minimally invasive surgery to expand with the addition of splenectomy, lymph node biopsy for hematologic disorders, adrenalectomy, repair of perforated ulcers, staging of abdominal malignant neoplasms, and diagnostic laparoscopy combined with ultrasonography ${ }^{[941}$. These developments may perhaps slow the trend described by Banta, Schersten and Jonsson in 1993.

\subsubsection{Lasers, MIT and MTA}

In the future, it can be expected that more laser applications and applications in other fields of MIT will be evaluated and that these evaluations will be planned earlier in their life cycle. The selection of these applications will be justifiable from both a societal and scientific point of view, and the quality of these evaluations will improve, contributing to more rational policy-making towards medical technology.

Considering the increasing capacity and efforts to evaluate new medical technologies and the growing potential for evidence-based medicine and evidence-based health policy-making it can be anticipated that less medical technologies will diffuse in health care systems without guidance from policy-makers. However, as markets are global, this may only be realized in countries where technology assessment is carried out on a considerable scale. Since the inception of medical technology assessment in the United States in 1975, many other countries have established either national or regional MTA-programs. Examples of national programs are those in Australia (since 1982). Sweden (1987), France (1990), the United Kingdom (1990), and Canada (1990). In addition, both in Canada and Spain regional programs have been established ${ }^{[24]}$. This progress has expressed itself on an international level as well, with the establishment of for example INAHTA and EUR-ASSESS ${ }^{\mid 122 !}$. These developments can be translated to the (arbitrarily) four stages in the process of medical technology assessment: (identification and) priority-setting, testing, synthesis, and dissemination (and implementation). Another field is the study of the diffusion of medical technology. Progress in each of these areas is briefly discussed in order to support the predictions on the intensity, timing and quality of evaluation of lasers and MIT in the future.

\section{Priority-setting}

The necessity of priority-setting in each area of medical technology can be illustraled by data provided by the World Bank in its 1993 report 'Investing in Health"114]. Around US\$ 71 billion is spent each year on medical equipment worldwide. Clearly, according to the Bank, the ability of the medical equipment industry to develop new health care technologies has vastly exceeded the capacity of researchers to evaluate the clinicall value and cost-effectiveness of such innovations. In 1993, it was estimated that about 6000 distinct types of medical devices and more than 750000 brands, models and sizes, produced by perhaps 12000 manufacturers worldwide, are on the market $^{[114]}$. 
To meet this challenge, health care technology assessment organizations in many countries have become involved in priority-setting processes. Formal methods of prionty-setting have been

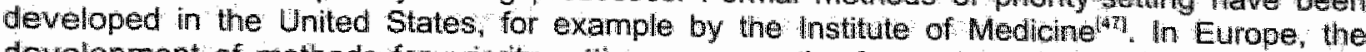
development of methods for priority-setting was one the four substantive tasks of the EURASSESS project; an EU-subsidized collaborative project of several European HCTA organizations $^{[122]}$. In the United Kingdiom, the rials and systematic reviews which are currently carried out (see Intermezzo 20:1) are the result of a priority setting exercise using the following criteria: safety, potential benefit to patients, costeffectiveness and policy implications $s^{\text {iabl. With }}$ respect to safety, individual MIT procedures ranked higher on the priority score when the procedure requires a fundamental change in surgery (e.g. some types of hernia repair), when the procedure irvolves high complication rates (e.g. surgery of the colon) and when the procedure is applied to dissect tumours, because this may be associated with the tumour "seeding' at the sites of entry. With respect to benefit to patients, individual MIT procedures ranked higher on the priority score when the scope for benefits are less clear-cut; for example in cases where conventional operations are considered simple and quick (e.g. appendectomy). With respect to cost-effectiveness; individual MIT procedures ranked higher on the priority score when the procedure is more likely to offer significant cost savings compared to alternative treatments (e.g. trials to compare the treatment of gastro-esophageal reflux disease using laparoscopic surgery as opposed to current drug-based treatments involving prolonged courses of relatively expensive proton pump inhibitors). In the UK, there is general agreement on the need for further evaluation of new MIT procedures, and on the main priorities identified ${ }^{[6 ! !}$.

In the Netherlands, there has been a lively debate on methods for priority setting in medica: technology assessment $t^{4,31,93 \mid}$. In this country it is recognized that besides new medical technologies, existing technologies should be included in the process of priority-setting as well. In 1993, the Dutch Health Insurance Executive Council published a priority list of 126 current provisions in need of evaluation ${ }^{(59)}$. Basically, three criteria were used: uncertainty with respect to the safety, effectiveness and cost-effectiveness of medical technology ${ }_{n}$ the cost per treatment and the total annual cost of treatment, and the potential to change clinical practice as a result of evaluation studies. Among the first 30 subjects there are several applications of MIT, including MRI versus diagnostic arthroscopy of the knee (number 5). PTCA (number 19), diagnostic laparoscopy (number 28) and laparoscopic cholecystectomy (number 30). The only laser application included is the use of soft-lasers in physiotherapy in treatment of musculoskeletal disease (number 20 ). Other countries have produced similar lists, sometimes including both old and new medical technology. An example of the latter approach is provided by the Swedish Council on Technology Assessment in Health Care (SBU), in its report 'medical technologies in need of assessment $t^{\text {tht? }}$. Lasers were one of the subjects included in the report.

The quality of the priority-setting process is, besides its dependency on the availability of appropriate methods, to a large extent dependent on 'early-warning' systems of new medical technology. These systems too need international collaboration and coordination. As many new medical laser applications and new forms of minimally invasive therapy can be expected in the future, this seems one of the areas that will no doubt yield good candidates for evaluation. Furthermore, as existing forms of minimally invasive therapy have demonstrated to be highly competitive with laser applications, in particular cases (priority-setting based) reassessment may already be appropriate. Even other recent cases of MIT, for example laparoscopic cholecystectomy, may already require reassessment due to an unwarranted widening of indications ('technology creep') ${ }^{24 j}$. In general, of course, existing technologies should be included in the process of priority-setting as well.

\section{Testing}

This step includes stimulating, requiring, funding or conducting studies and collection of data. Testing is the step that in an ideal system follows after the process of 'early-warning' and priority setting. In case of new medical technologies such as lasers and MIT testing and subsequent policy-making may have the highest impact on the adoption and use of the technology when it is performed early in its life cycle. Permitted well functioning systems of identification, priority-setting 
and coordination of testing, this step can theoretically be performed without much unnecessary duplication. Unfortunately, duplication is rather common, e.g. five organizations united in INAHTA assessed excimer laser treatment of nearsightedness ${ }^{[73}$. Testing early in the life cycle of the technology could include primary data collection in studies designed as RCTs in selected cases, paying appropriate attention to issues of quality of life, costs and other relevant aspects. In Chapter 18 it has been demonistrated that in principle this is a feasible approach for laser applications, butt that such trials have hardly ever been organized. In the recent past, more attention has been paid to the fileld of MIT, where the call for (early) RCTs increases ${ }^{[94: 1241}$. This approach to testing receives increasing support, not only by clinicians but also by governments, e.g., in the Netherlands. However, although the number of RCTs rapidly increases it will not be possible nor appropriate to mount large-scale randomized trials for any medical innovation. Neither will it be possible (nor appropriate) to add an economic evaluation to any $\mathrm{RCT}$ that is being organized.

To ease this problem Sculpher et al. (1995) suggest a comprehensive strategy for economic evaluation that can be viewed analogous to the stages of clinical studies (phase I, II, III and phase IV) studies ${ }^{[105]}$. The authors emphasize that the potential of economic evaluation in particular in the earlier stages of testing has not yet been fully realized. If this were the case the need for largescale studies including an economic evaluation would probably be reduced. In particular decision analytical modelling techniques, which are relatively inexpensive, could play a role in 'phase II' economic evaluations ${ }^{(105)}$ : Economic evaluation alongside RCTs could, as part of such a strategy. be limited to selected phase III studies ${ }^{[5.1}$. These ideas come close to what has been proposed in the Netherlands as "constructive technology assessment" (CTA ${ }^{[17]}$. In CTA, technologies that might have great potential or might be associated with societal problems are identified early in their lifecycle. The attempt is then to intervene in the processes of development and the diffusion of the technology. Perhaps 'constructive' or 'very early' assessments will be encountered more often in the future.

Presently, methods and techniques for testing are developing rapidly. This pertains in particular to the specific field of health-related quality of life measurement and the field of economic evaluation. These subjects are discussed more in detail in Chapter 4 . In the field of health-related quality of life measurement perhaps the World Health Organization's project to develop an international, multicultural quality of life instrument (WHOQOL) holds most promise for the future use of these measures on a globall scale ${ }^{1133}$ In the field of economic evaluation one can point at many international attempts to harmonize economic appraisal of health technology. For example, on the European level researchers have united in the HARMET initiative for this purpose ${ }^{[1021}$ "These and other projects are expected to guide the future of economic evaluation, which will as a result increase in quality and usefulness for policy-making. Complementary to this development it seems fair to expect that applications of utility theory in the economic evaluation of health care will become less subject to controversy ${ }^{[26]}$. This may stimulate improvement of the quality of cost-utility analyses. Such studies have been shown to be very difficulit to perform in a technically adequate way, which presently limits their use in policy-making ${ }^{[6] !}$.

\section{Synthesis}

Synthesis involves a critical analysis of the results of testing (available data from preclinical to clinical experience, epidemiological studies, and experiments) and all other available and relevant information. Synthesis of the information generated during the testing phase of the assessment process is necessary to provide a reasonable basis for decisions made on the technology. Because policy makers and others, e.g. clinicians, are not trained in study design and interpretation, presenting the raw data may be of little use. For this reason, synthesis often takes the form of judgments or recommendations. As without synthesis there is no assessment, this step has sometimes been considered as the most important step in the assessment process ${ }^{[109]}$. Many organizations in the field of medical technology assessment have a long-standing tradition in synthesis activities, e.g. the (former) Office of Technology Assessment (OTA) and the National Center for Health Services Research (NCHSR) in the United States and the Health Council in the Netherlands. 
Although various methods of qualitative syrthesis, including e.g. ilterature revlews, have produced and still do produce useful results in many fields, including lasers and minimally invasive therapy (see Chapter 2), a formal, quantitative method for this activity has long been lacking. A major improvement in synthesis activities has been achieved with the development of the Cochrane Collaboration, which started in the United Kingdom in 1992 and which activities have since spread to many other countries, including the Netherlands. The Cochrane Collaboration was developed in response to Cochrane's call for systematic, up-to-date reviews of all relevant RCTs of health care. The task of the Collaboration is therefore defined as to prepare, maintain, and disseminate systematic, up-to-date reviews of RCTs in health care, and, when RCTs are not available, reviews of the most reliable evidence from other sources. The reviews are stored and disseminated by means of the Cochrane Database of Systematic Reviews, which is an electronic database ${ }^{[34]}$. When RCTs serve as the input for a systematic review, the synthesis technique that is applied is called meta-analysis. In the field of lasers and minimally invasive therapy, we have encountered two such meta-analyses, focusing on treatment of upper gastrointestinal bleeding and hemorrhoids, respectively. In the future, the activities of the Cochrane Collaboration are likely to become more important in the field of lasers and minimally invasive therapy.

In the United Kingdom, in York, the National Health Service (NHS) Center for Reviews and Dissemination (CRD) was founded in 1994'112]. As part of its activities, the CRD developed the Database of Abstracts of Effectiveness (DARE). DARE is a collection of abstracts of good quality research reviews about the effectiveness of health care interventions and organization of health services. This database is wider in scope than that of the Cochrane Collaboration. Another major improvement, although not strictly aimed at synthesis, is the CRD's development and maintenance of a database of critical abstracts of economic evaluations of health care. This database was developed in order to make easily accessible information about the cost-effectiveness of health technologies, and to provide a critical appraisal of these studies. In order to assess the quality of these studies the CRD developed a manual for writing structured abstracts. The manual, described by Mason and Drummond (1995) and by Vanoli et al. (1996) is a sophisticated tool for assessing economic evaluations, and thus will contribute to the quality of any future reviews or syntheses in the field of economic evaiuation ${ }^{[86.117]}$. Other areas of synthesis are still relatively undeveloped and need to be validated. For example, the development of methods how to synthesize the results of the many prospective, but uncontralled studies needs more attention ${ }^{(17)}$.

\section{Dissemination and implementation}

Since MTA is policy research, it can only be considered successful when it has led to changes in policy, and hopefully in health care and health outcomes of the population. A major tool for making impact is dissemination of the results of medical technology assessment. The dissemination of health care technology assessment information used to be done in a rather passive way. The main wehicles were the medical scientific literature and scientific conferences. Because this presented a number of problems many organizations have become involved in more active dissemination of MTA and other relevant information ${ }^{17 \%}$. One way that is becoming more popular is the use of electronic media, in particular Internet facilities. Internationally, for example, the Cochrane Collaboration, besides stimulating improvements in synthesising evidence, stimulates active dissemination of information in particular through its use of electronic media. The plans of the Collaboration include an online distribution of the complete database. Smaller, specialized databases derived from the main database will also be compiled and published on floppy disk ${ }^{(34)}$ The International Network of Agencies for Health Technology Assessment (INAHTA), which was founded in 1993, plays a role in active dissemination of the results of ongoing and completed reports of its members. These reports have been abstracted and made available in hard copy. The abstracts will be made available on-line as wellin!

An example in the United States is the initiative of the National Library of Medicine (NLM) "in collaboration with the Agency for Health Care Policy and Research (AHCPR), the National Institutes of Health (NIH) and the Public Health Service, to launch Health Service/Technology Assessment Text (HSTAT) in April 1994. This free, electronic service provides computer access to lots of information, e.g. the full text of AHCPR clinical practice guidelines. In the field of MIT the 
Agency has produced guidelines for example in treatment of benign prostate hyperplasia. The use of these facilities is rapidly increasing ${ }^{|5|}$. In Europe, for example, the Dutch Health Insurance Executive Council created its own homepage in mid-1996, which guides the user to e.g. the complete list of pharmaceuticals that are covered in the benefit packagle ${ }^{[66]}$.

Although (active) dissemination contributes to the avallability of information from medicall technology assessment, it has become apparent that it is not having the desired effect on health care practice. In other words, merely disseminating information often does not lead to behavioral change. This has led to an increasingly emphasis on implementation. Implementation means processes that aim to improve clinician's compliance with MTA recommendations. Changes in knowledge and attitudes should turn into changes in clinical practice ${ }^{[17]}$. While this is still a relatively new concept, it seems certain to guide the field of MTA in the future.

Recent activities provide an indication for this process. In the US, for example, the Agency of Health Care Policy and Research (AHCPR) introduced a new generation of medical effectiveness research, known as PORT II. PORT II (Patient Outcomes Research Teams) projects are pragmatic, methodologically sophisticated, multidisciplinary and focus on patient outcomes for common clinical problems. However, they are intended to differ from the original PORTs by their individualized research strategies and their expected impact on clinical practice, patient outcomes and health care policy ${ }^{[\text {iै] }}$. On the European level, EUR-ASSESS developed a report on implementation that has become available in $1997^{\mid 17 !}$. In the Netherlands, the Health Insurance Executive Council has proposed a program on implementation into practice of results of studies funded by the Investigational Medicine Program, but this is not yet formally approved ${ }^{[7]}$. In the United Kingdom, the NHS Center for Reviews and Dissemination (CRD), uses a range of methods to promote dissemination and implementation, such as bulletins, workshops, and continuing medical education. The CRD also disseminates information to members of the public through patient leaflets and other media ${ }^{1112 !}$. Perhaps it is in these final steps of medical technology assessment where most progress will be made in the future.

In the laser field many traditional ways are used to disseminate information to professionals. In the United States, the Society for Laser Medicine and Surgery (ASLMS) organizes annual meetings since 1980 and its journal 'Lasers in Surgery and Medicine' was inaugurated that same year. In 1996, the International Society for Podiatric Laser Surgery joined its membership with ASLMS, which had grown to a membership of 3,000 early that year ${ }^{[83,84 \mid}$. When members of the public write a specified request to the ASLMS, the society provides written information about various laser procedures as well as a list of its members who are board certified by the American Board of Medical Specialties ${ }^{[7 !}$.

In Europe, the European Laser Association (ELA) was founded in the early eighties, which organizes biannual meetings since $1982^{|60|}$. Starting in the mid-eighties the COMAC-BME concerted action program into the development of medical laser applications, focusing on the safety of laser applications in general and in particular on treatment of tumours and cardiovascular applications, offered a good opportunity to create a European collaborative network of professionals ${ }^{[106]}$. In conjunction with this development, national laser societies were founded which, since 1988, became associated with the European Laser Association. In mid 1994 the ELA had a membership of about 600 professionals living in 12 different countries ${ }^{60 \mid}$. The ELA does not direct specific activities to the lay public.

The first society on mimimally invasive therapy was founded in the United Kingdom in late $1989^{1423 !}$. In 1991 the Society inaugurated its own journal, ' Minimally Invasive Therapy" which merged in 1995 with the Journal of Endoscopic Surgery and Allied Technologies to become the journal with the title "Minimally Invasive Therapy and Allied Technologies" in 1996. This society does not direct specific activities to the lay public. Thus $\mathbf{s}_{\mathrm{y}}$ both in the field of lasers and minimally invasive therapy the process of dissemination of results is organized but narrow in scope. Implementation of the findings, for example by developing (procedure-specific) guidelines, seems not (yet) to be part of the activities of these societies. 


\section{Diffusion}

Users of an assessment of medical technology include every organization involved in health care

The goal is that MTA should guide adoption and use (diffusion) of technology. However, diffusion of medical technology is extremely dynamic an complex and it is not well understood. In the future. implementation of technology assessment will require more understanding of diffusion ${ }^{1 \% 1}$. Some siteps in this direction have been made in the recent past. In the late eighties and early nineties the European Community, via its committee COMAC-HSR in DG XII (Science, Research and Development), sponsored a project focusing on the diffusion of heart and liver transplantation, selected prenatal screening tests, and ESWL and PCN in renal stone treatment. These studies were aimed at a description and analysis of the diffusion process of these technologies in 11 countries of the European Community and in Sweden ${ }^{30,20,000 i}$

In the field of lasers and MIT one could of course add the studies that form the basis of this thesis: the COMAC-HSR project (Health Services Research) on lasers and the EC-FAST project (Forecasting and Assessment of Science and Technology) on MIT. The EC-FAST project on MIT built to some extent on the laser project and, to a lesser extent, on the European project on the diffusion of medical technolagy described above, in particular on the study focusing on the diffusion of ESWL and PCN in renal stone treatment. In addition, the European Community sponsored a study on the safety of medical lasers which, among other subjects, included a study on the diffusion of medical lasers in the European Community ${ }^{1693}$. This study was sponsored in the context of the COMAC-BME program (Biomedical Engineering) of the EC in the period mid 1988-1991. These were all descriptive studies. At present, however, there are no European research initiatives for the methodology of medical technology diffusion research. In 1994, the International Society of Technology Assessment in Health Care (ISTAHC) announced a two-year fellowship to assess the international diversity in the diffusion (including the adoption and use) of one or more health technologies. Although a thorough analysis of factors that underlie or mediate any variation of cross-national use of the technology is required, the fellowship is not primarily aimed at tackling methodological issues ${ }^{[1]}$.

Perhaps a more standardized, uniform methodology in (multi-national) research efforts on the diffusion of medical technology could result in an overall increase in the quality of information and thereby in an increase of the utility of this information for policy-making. What now often happens is that different scholars, each with a unique theoretical model and with distinctive methodological tools, come to different conclusions on the factors that drive the diffusion process of a particular medical technology ${ }^{101 !}$. If the research community would be convinced that such a standardizing or harmonizing project would produce useful results then a number of subjects could be identified that could be included, e.g. the principles for development of diffusion questionnaires, criteria for the choice between a postal survey or a telephone survey strategles for the choice of key informants, strategies to abtain as high a response as possible "guidelines for data-analysis (among other factors taking missing values into account), and reporting of results. Furthermore, a standardization or harmonization procedure could include criteria for the selection of researchers (or country-rapporteurs as they are sometimes called in international studies). In some published studies country-rapporteurs had played an important part in the policy decisions affecting the spread of the technology. Obviously, their point of view may be prejudiced ${ }^{(110)}$.

A good formal starting point for setting a research agenda could be based on the (fourth edition of the) standard work 'Diffusion of innovations" by Prof. Everett Rogers ${ }^{101 / 3}$, combined with an up to date review of the statistical aspects of medical technology diffusion studies. An example of the latter is the study by Trajtenberg and Yitzhaki (1989) who proposed the Gini's expected mean difference as a measure for and base of comparison of the speed of diffusion of a wide variety of medical and other technologies. The utility of this approach was demonstrated using the diffusion of CT scanners in the United States as an example ${ }^{[145}$. The line of enquiry suggested by these authors may prove fruitful in studying the diffusion of medical technology. However, no such studies seem (yet) to have been done. 


\subsection{Boundaries of the thesis}

This thesis has primarily been aimed at the evaluation of a selection of about 60 therapeutically relevant medical laser applications in six specialties. A context was provided by paying attention to the field of minimally invasive therapy, in particular to the diffusion of a selection of 10 therapeutic cases. If a new project were to be organized, widening the scope to lasers in health care would be preferable, including for example laser applications in physiotherapy and in dentistry. Complementary to the choice for therapeutic applications, a project focusing on diagnostic applications of lasers and other minimally invasive techniques could be organized. This would be highly interesting, as it has been recognized that the introduction of minimally invasive techniques have revolutionised diagnostic and surgical procedures alike ${ }^{\mid 96 !}$.

\subsection{Final statement}

This thesis has presented the case of lasers in medicine. Lasers have rapidly diffused into medical practice during the last two decades, and in this sense, can be considered an example of the "technologization" of medicine often referred to in the literature. The question is " does the diffusion of medical lasers represent an advance or not? The health care system exists to promote a healthier popullation, so ideally, one would wish for reasonable evidence that medical lasers have produced health benefits while not causing undue harm. Unfortunately, for the most part, laser diffusion cannot be justified on the basis of available evidence concerning efficacy and safety. Furthermore, lasers are expensive. Perhaps in the long-run laser use will be cost-effective in many areas, either as stand-alone therapy or as part of minimally inwasive treatment strategies, but whether this will be true or not cannot be seen from research done to date.

The question then is, what can societies do to rationalize the diffusion of such technologies as the laser. The thesis has laid out a number of policy opportunities. One of these, and an important focus for this thesis, is to develop medical technology assessment (MTA) as a strong tool to assist policy "administrative, and clinical decisions. While MTA has developed in the Netherlands and in ather countries, especially countries in Europe, it is still not an important factor in most decisions in health care. In the future, it can be anticipaled that demands for more cost-effective health care will promote more assessments and more use of such assessments as an important guide to decision-making.

\subsection{Recommendations}

The first set of recommendations is addressed to clinical researchers, researchers in health economics, researchers on diffusion of medical technology and to those funding clinical research and technology assessment (not necessarily in order of prionity).

1 Lasers and other minimally invasive procedures in treatment of tumours have great potential and further research could lead to benefits for a large number of patients.

2 Further research is needed on the role of lasers compared to other types of minimally invasive therapy or as part of minimally invasive treatment strategies using endoscopes, laparoscopes, and implanted fibers. In particular, the additional costs and benefits of the laser in treatment of urological and gynecologicall conditions need to be established.

3 Meta-analyses should be carried out in particular fields where a sufficient number of RCTs have been dione, e.g. in gynecology, urology and, where necessary, in ophthalmology. 
4. Registries should be created including all randomized controlled trials prior to initiation. A separate registry could list the RCTs alongside which an economic evaluation is cartied out.

5 Complementary, the methodology of creating systematic reviews from sources other than RCTs needs further development.

6 A small set of core guidelines for economic evaluation should be actively promoted and taught to researchers, in particular to clinicians and other non-economists assessors.

7 Further multidisciplinary research is needed on the description, analysis and modelling of the diffusion of medical technology.

The second set of recommendations is addressed to industry and to technical workers involved in laser development and development of equipment for minimally invasive therapy

The following areas are high priority for development:

1 Treatment monitoring systems

The goal of research in this area is to enable the laser surgeon to monitor the laser-tissue interaction as part of the procedure in order to increase selectivity of tissue destruction. The advantage of sensitive monitoring systems is that the desired effect can be maximized, and that unwanted effects can be minimized.

2 Delivery systems

Further development is needed for steering systems and delivery systems for distributing the laser light within the target organ. Two main groups of technologies are needed:

- multifiber systems for interstitial treatment;

- modified delivery systems to optimize laser light distribution within body cavities (e.g. , hollow organs or blood vessels).

3 Laser systems and accessories with minimal use of disposable materials

Development and assessment of durable materials is needed. Such an assessment needs to include the perspectives of manufacturers, users, and society. The research can focus on:

- better quality of endurable materials;

- design of accessories to optimize sterilization;

- reliability of sterilization (especially with respect to AIDS);

- consequences of non-disposable accessories for working routines of health care staff;

- less polluting disposables.

Cost-effectiveness analysis can be used to guide technical development in this area.

4 New lasers

Two laser systems under development have considerable potential in the near future:

- the diode laser;

- the Free Electron Laser.

There is a need for research into potentiall laser-tissue interactions and possible new applications of these lasers. 
The third sel of recommendations is mainly addressed to national governments and national and regional health systems and health care payers, but they could also be implemented in some cases by policy bodies such as medical societies.

1 Guidelines

Therapeutic guidelines should be established for those laser applications and applications of minimally invasive therapy where there is convincing evidence of safety and effectiveness. If avallable, information on cost-effectiveness should be incorporated in these guidelines.

2 Training and safety requirements

Training and safety requirements should be worked out and could be coupled to reimbursement systems as a means of "steering" practice, in countries where the reimbursement system makes such coupling feasible.

\section{Knowledge centers}

For the sake of all interested parties (doctors, hospital administrators, government agencies, and industry) clearing houses (data banks) for exchange of available information need to be established on a national and/or international level. The clearing house should be user oriented and should accumulate information on technical developments, safefy, clinical applications, and evidence of effectiveness and cost-effectiveness of lasers and minimally invasive procedures

4 Medical laser centers and centers for minimally invasive therapy

Health care authorities (and financers) should actively support the establishment of regional laser centers and centers for minimally invasive therapy that could act as reference centers for medical laser applications and applications of minimally invasive therapy in general (combining basic research, clinical research, treatment of patients, and possibly also technical research). These centers may generate, collect and disseminate knowledge on specific procedures and set up multicenter trials involving other centers.

5 Reimbursement and funds

In a number of countries the health care financing system (global budgets, reimbursement system) actualty blocks the development of appropriate, cost-effective use of lasers and minimally invasive treatment techniques in clinical practice. There is a need for proper reimbursement of laser applications and applications of minimally invasive therapy which are proven to be cost-effective in order to stimulate diffusion of such procedures. Secondly, specific funds could be set up for capital investment in cases where the technology appears to be cost-effective.

6. Technology assessment

Technology assessments (effectiveness, cost-effectiveness, life-cycle studies) in the field of lasers and minimally invasive therapy are valuable as a tool for health policy decision making. Many applications of lasers are still in the phase of clinical experiment or of unproven effectiveness. The results of such studies can be used to encourage appropriate diffusion and application of laser systems and minimally invasive procedures, respectively.

7 Consumer information

A concerted effort could be made to improve lay knowledge of health practices, so that people will have a better basis for choice. The lay media could be actively involved in such efforts.

8 Implementation

International arganizations, such as the EC and WHO, should support and encourage the implementation of these recommendations in individual countries. Exchange of information developed by technology assessments is a particular priority. 
The fourth set of recommendations is addressed to hospital boards; hospital administrators and clinicians and addresses the adoption of lasers and minimally invasive treatment techniques.

1 Hospitals should use technology assessment to guide investment decisions in new medical technology

\section{The adoption of lasers}

Due to the overall limited evidence on effectiveness and cost-effectiveness of lasers and due to a relatively low intensity of use, sharing of existing laser systems between specialties and a purchasing strategy based on the size of a hospital and the characteristics of the adherent patient population seem the most straightforward ways to prevent both underuse of installed systems and unwarranted adoption and use of new laser systems. See Chapter 3 for more detailled, tentative guidelines by ECRI for the adloption of laser systems.

3 The adoption of minimally invasive treatment techniques

Although no specific recommendations have been published for the adoption of minimally invasive therapy, it is recommended to take the same factors into account for this type of technology as those formulated in the case of lasers.

\subsection{References}

1. Abel-Smith BA. Cost containment and new pritorities in health care. A study of the European Community. Avebury, Ashgate Publishing LId., Aldershot, Brookfield US, Hong Kong, Singapore, Sidney, 1992.

2. Altman LA. Bringing the news to the public: the role of the media. In: Warren KS, Mosteller F (Eds.). Doing more good than harm: the evaluation of health care interventions. Annals of the New York Academy of sciences $1993(703): 200-8$.

3. Altman LA. The Ingelfinger rule, embargoes, and journal peer newiew - part 1 and part 2. Lancet 1996(347):4382$86 ; 1459-63$

4. Ament AJHA, Mater P. Top-down approach in the context of technology assessment: evaluation of the priority list of the Health Insurance Executive Council. Tijdschrift voor Sociale Gezondheidszorg 1995(73):401-5 (in Dutch).

5. Amin Z. Diode lasers: experimental and clinical review. (Invited review) Lasers in Surgery and Medicine 1995(10): 157-63.

16. Anoniymous. Agency of Health Care Policy and Research (AMCPR). AHCPR invites suggestions for outcomes/effectiveness research topics. AHCPR, Research Actiwities 1996(193):11.

7. Anonymous. American Society for Laser Medicine and Surgery, Inc.. News and Announcements. Lasers in Surgery and Medicine (1996)18:215.

8. Anonymous. Frost \& Sullivan/Market Intelligence Reports. World surglical laser market, Journal of Medicol Engineering \& Technology 1995(19)5:187

9. Anonymous. Frost \& Sullvan/Market Intelligence Reports. US endoscope market. Journal of Medical Engineering \& Technology $1996(20) 292$

10. Anonymous. Intemet "surfers" discover the ease of accessing AHCPR-supported guidelines. AHCPR News and Notes 1995(189):25.

11. Anonymous. ISTAHC Announces fhe first international fellowship for technology assessment in health care. International Journal of Technology Assessment in Health Care 1994(10)2 335.

12. Anonymous. Laser endoscope for a range of applications. Journal of Medical Engineering 8 Techinology $1995(19) 1: 39$

13. Appelman YEA, Plek لU, Strikwerda S, Tijssen JGP, de Fayter PJ, David GK, Serruys PW, Margolis JR, Koelemay MJ. Montaubam van Swijndregt EW.J, Koolen J.J. Randomised trial of excimer laser angloplasty versus balloon angioplasty for treatment of obstructive coronary artery disease. Lancet 1996(347):79-84.

14. Appendices of the Financial Oversight on Care 1996. Sdu Publishers, the Hague, the Netherlands, 1995 (in Dutch)

15. Banta HD (Ed.). Minimally inwasive therapy in five European countries. Diffusion, effectiweness and cost effectiveness. Heath Policy Monographs Vol 3. Elsevier. Amsterdam, London, Niew York "Tokyo, 1993

16. Banta HD, Behney $C$, Willems JS. Towards rational technolagy in medicine. New York, Springer Verlag. 1981.

17. Banta HD, Oortwijn WJ, wan Beekum WT. The organization of health care technology assessment in the Netherlands. Rathenau Institute, the Hague, the Netherlands, 1995. 


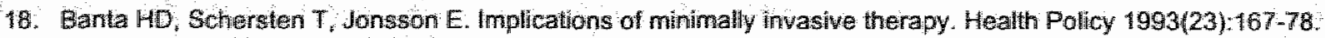

19. Banta $\mathrm{HD}$, schou $\mathrm{T}$, Vondeling $\mathrm{H}$, de Wht A. Economic appraisal of laser applications in health cane. Repont of a project. Lasers in Medical Science 1992(7),9-21.

20. Banta HD. Industral unvollwement with medical tasers. In: Banta HD. Schou 1 (Eds.). Lasers in Health Care. Effectiveness, Cost-Effectiveness and Policy Implications. Academic Publishing. Frederiksberg. Denmark., 1991. p. $43-9$.

21. Banta HD. The budget is blind the case of minimally Invasiwe therapy in Europe. The Netherlands Organization for Applied Sclentific Research (TNO), Institute of Ageing and Vascular Research. Center for Medical Technology. Leiden, the Netherlands, 1991.

22. Banta HD. The cost-effectweness of 10 selected applications in minimally invasive therapy. Health Policy $1993(23) \div 135-51$

23. Barry MS, Fowler FJ Mully AG, Henderson JV, Wennberg JE. Patient reactions to a program designed to facilitate patient participation in treatment decisions for benign prostatic hyperplasia. Medical Care 1995(33)8:77 1-82.

24. Battista R, Banta HD, Jonsson E, Hodge M, Gelband H Lessons from the eight countries. In: Banta HD, Gelband $H$, Johnson $E$, Baltista $R$ Eds.). Health care technology and its assessment in eight countries. OTA-B-P-H-140. Washington, DC: US Government Printing Office, February 1995.

25. Berlien HP. German accreditation rules in laser medicine. In: European Community Medical Laser Concerted Action Program. Proceedings of the first plenary workshop on safety and leser-tlssue interaction. London, Bailliere Tindall, 1989, p. 385-95.

26. Bleichrodt $\mathrm{H}$. Applications of utility theory in the economic evaluation of health care (thesis). Erasmus University Rotterdam, the Netherlands, 1996.

27. Bloom BS, Fendrick AM. The tension between cost containment and the underutilzation of effective health care services. International Journal of Technology Assessment in Health Care 1996(12)1:1-8.

28. Bann D. High-power lasers help the ischemic heart. Lancet 1996(348):118.

29. Bos MA. Health care technology in the Netherlands. In: Banta HD, Gelband H, Johnson E, Battista R (Eds.). Health care techmollogy and its assessment in eight countries. OTA-B-P-H-140. Washington, DC: US Government Printing Office. February 1995.

30. Bas MA. The diffusion of heart and liver transplantations across Europe. In: Stocking B (Ed.). A study of the diffusion of medical technology in Europe. King's Fund Center for Health Services Development, London, England, 1991.

31. Bouter $L M$, Oortwin WJ, Vondeling $H$. Societal criteria for health care research. (lm)possibilities fior rational funding and selection of research. Tijdschrift voor Sociale Gezondheidszorg 1996(74)6:235-42 (in Dutch).

32. Carruth JAS. Training in Great Britain. In: European Community Medical Laser Concerted Action Program. Proceedings of the first plenary workshop on safety and laser-tissue interaction. London. Bailliere Tindall, 1989, p. $373-4$

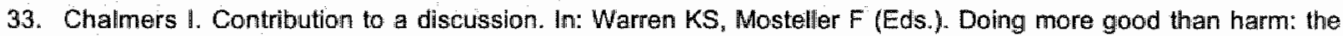
ewaluation of health care interventions. Annals of the New York Academy of Sciences 1993(703):236.

34. Chalmers I. The Cochrane Collaboration: preparing, maintaining and disseminating systematic reviews of the effects of health care. In: Warren KS, Mosteller F (Edls.). Doing more good than harm. The evaluation of health care interventions. Annals of the New York Academy of Sciences 1993(703):156-65.

35. Chaimers I. Underreporting research is scientific misconduct. Joumal of the American Medical Association 1990(263)10:1405-8.

36. Chalmers TC, Lau J. Randomized controlled trials and meta-anallyses in gastroenterology: major achievements and

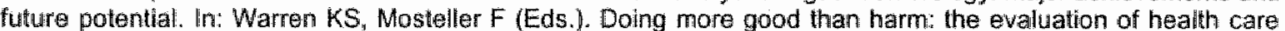
interventions. Annass of the Now York Academy of Sciemces 1993(703):96-106.

37. Cochrame AL. Effectiveness and efficiency: random reflections on thealth services. The Nuffield Provincial Hosplals Trust, London, 1972.

38. Coupland RM. The effect of weapons on health. Lancet 1996(347):450-1.

39. Davidoff AJ, Powe. The role of perspective in defining economic measures for the evaluation of medicat technology. International Joumal of Technology Assessment in Health Care $1996(12) 1: 9-2 \%$.

40. Department of Health. Reseanch for Health. Department of Health, Research and Development Division, London, United Kingdom, 1993.

41. Department of Heaith. Methods to promote the implementation of research findings in the NHS - priorities for evaluation. Report to the NHS central research and development committee. Department of Health, United Kingdom, London, October 1995.

42. Dickersin $K$, Min YI. Publication bias: the problem that won't go away. Im: Warren KS, Mostelier F (Eds.). Doing more good than harm: the evaluation of health care interventions. Annals of the New York Academy of Sciences $1993(703): 135-48$

43. Dickersin $K$. The existence of publication bias and risk factors for its occurrence. The Journal of the American Medical Association 1990(263)10:1385-9.

44. Dijk A van, Frankfort $J$, Horn T, Vos K. Science and technology policy in the Netherlands, DSWO Press, University of Leiden, the Netherlands, 1993 (in Dutch) 
45, Dijksterhuis WHK, Wever LJS. Yearbook Health Care 1996. Oversight of developments in policy and legislation. Bohn, Saftev; wan Loghum, Houtten, the Netherlands, 1996 (in Dutch).

46. Dirksen CD, Ament AJHA, Go PMNYH, Hupkens-van der Elst H. The diffusion af endoscoplc surgery. Economisch Statistisicive Berichten (ESB) 1994(79):82 5 (in Dulch):

47. Donaldison MS, Sox HC (Eds). Setting priorittes for health technology assessment: a model process. National Academy Press, Washington, 492.

48. Dover IS, Arndt KA. Dermatology. Journall of the Anerican Medical Association 1996(275):1901-2.

49. Drummond M. Economic evaluation of phamaceuticais. Science or marketing? Phamaco Economics $1992(1) 11: 8$ 13.

50. Drummond MF, Stoddart GL, Torrance GW. Methods for the economic evaluation of health care programs. Oxford University Press, Oxford, New York Toronto, 1987.

51. Drummond MF, Stoddart GL. Economic analysis and clinical trials. Controlled Clinical Trials 1984(5)115-28.

52. Duijn FW van. Day surgery offers advantages for all partes. Het Zekenhuis 1994(2):80-2 (in Dutch).

53. Dutré MA. Introduction and spread of expensive medical technology in the Netherlands, a model (thesis). Erasmas University Rotterdam, the Netherlands, 1991 (in Dutch).

54. ECRI. ECRI offers help with new SMDA regulations. ECRI News 1996(4) $1: 1,2$.

55. Eddy D. Practice policies, what are they? Joumal of the American Madical Association 1990(263):877-80.

56. Eggink GJ, Kaptein C, van Kemper RJ, van der Meulen FW, Teirlinck CJPM, Vaarties SR (Eds.). Dutch Nationall Committee on Laser Safety. Laser Safety in Health Care (second ediltion). The Netherlands Onganization for Applied Scientific Reseiarch (TNO), Leiden, the Netherlands, 1993 (in Dutch).

57. Eisenberg JM. Why a joumal of Pharmacoeconomics? PharmacoEconomics 1992(1)1:2-4.

58. Ekkelboom $\mathrm{J}$. The light in medicine. Interview with Prof. Dr. Ir. M.. van Gemert. AMC Magazine 1995(November):5-7 (in Dutchi).

59. Escarce $J \mathrm{~J}$, Bloom BS, Hillman AL, Shea JA, Schwartz JS. Diffusion of laparoscopic cholecystectomy among general surgeons in the United States. Medical Care 1995(33):256-71.

60. Europeian Laser Association. Newsletter, June 1994

61. Fendrick AM, Escarce JU, McLane C, Shea JA, Schwartz US. Hospital adoption of Iaparoscopic cholecystectomy. Mendical Care 1994(32):1058-63.

62. Gannot $J$, Wayant $R$. Free electron laser radiation transmitting flexible waveguide (abstract). Lasers in Surgery and Medicine 1996(18)Supplement 8:15.

63. Gelijns $A C$, Fendrick AM. The dynamics of innovation in minimally invasive therapy. Health Policy 1993(23):153-66.

64. Gerard K. A review of cost-utitity studies: assessing their policy-making relevance. HERU Discussion Paper $11 / 91$. Health Economics Research Unit, University of Aberdeen, 1991.

65. Gray F, Mitteiman H. Implementation and management of a laser program. in: Apfelberg DB (Ed.). Evaluation and installation of surgical laser systems. Springer Verlag. New York, 1987, p.54-68.

66. Groot LMJ. Medical technology in the Netherlands. In: Stocking B (Ed.). Expensive health technologles, regulatory and administrative mechanisms in Europe. Oxford Medical Publications, Oxford, 1988.

67. Health Insurance Executive Council. 1995 Annual report of Investigational Medicine. Investigational Medicine Committee. Health Insurance Executive Council, Amstelveen "the Netherlands, 1996 (in Dutch).

68. Health Insurance Executive Council. Health Insurance Executive Council on interngt. Raadzaam $1996(14) 5: 14$ (in Dutcin).

69. Health Insurance Executive Council. Cost-effectivenessanalyis of current provisions. Health insurance Executive Council, Advise N. 597, Amstelveen, the Nethertands, 1993 (in Dutch).

70. Hill P. New crystal host raises efficiency of neodymilum laser. Opto \& Laser Europe 1996 (28):32.

71. Hilman AL. Elsenberg JM, Pauly M, Bloom BS, Glick $H$, Kinosian B, Schwantz JS. Avolding bias in tho conduct and reporting of costeffectiveness research sponsored by pharmaceutical companies. New England Journall of Medicine 1991(324):1362-5.

72. Hirsch $N_{\text {, Halley }} \mathrm{D}$. The evolution of laparoscopic surgery in routine health care. International Journal of Technology Assessment in Health Care 1995(14)4:779-85.

73. Intemational Network for Agencies in Health Care technology Assessment (INAHTA). INAHTA Registry (Binding *2). INAHTA Secretariat, Ottawa, Cianada, May 1996.

74. Institurte of Medicine. Assessing medical technologies. National Acaderny Press. Washington. DC. 1985.

75. Javitt JC. Canner MK, Summer A. Cost-effectiveness of current approaches to the control of retinopathy in type I diabetics. Ophthaimology 1989(96):255-64.

76. Judy MM. Biomedical lasers. In Bronzino JD (Ed). The Biomedical Engineering Handbook, CRC Presss, Inc "United States, $1995, p \cdot 1333-45$.

77. Kaag M. Driebergen $P$, de Jong D. Short stay surgeny causes many worries to organization (Project in VU-hospital shows). Het Zlekenhuis 1994(3):123-4 (in Dutch).

78. Kaiser-Kupfer MI, Ethics in cinical research. In: Banta HD, Schou l (Eds.). Lasers in Health Care. Effectiveness, Cost-Effectiveness and Poilicy-Implications. Academic Publishing. Frederiksberg. Denmark, 1991, p. 69-71. 
79. Kassire JP Angell N. The joumal's policy on cost-effectiveniess analyses fediloraly. New England Joumal of Medicine 1994(331):669 70

80 Kurchberger 8 . The diffusion of wo technologies for renal stone treatment across Europe. In: Stocking B (Ed.). A study of the diffusion of medical technology in Europe. King"s Fund Center for Health Services Development, London: England, 199:,

81. Kupfer C. Cinical trials concerning the effectiveness of lasers. The case of ophthalmotogy. In:Banta HD, Schou! (Eds.). Lasers in Health Care. Effectiveness, Cost-Effectiveness and Policy-Implications. Academic Publishing. Frederiksberg. Denmark, 1991, 103-8.

82. Langtey PC. The November 1995 revised Australian guidelines for the economic ewaluation of pharmaceuticals. PhaimacoEconomics $1996(4), 341-52$

83. Lanzafame RJ!. 'OZ's reprise" (presudential column). Lasers in Surgery and Medicine 1996(18):109-10.

84. Lanzafame Ru. 'Quitoxic numinations' (presidential column). Lasers in Surgen and Meducine 1996(18):210-11.

95. Lazaro $P$, Fitch $K$. Thie distribution of "big ticket' medical technologies in the OECD. International Journal of Technology Assessment in Heatth Care 1995(11)3:552-70.

86. Mason J, Drummond H. Reporting guidelines for economic studies. Health Economics 1995(4)85-94.

87. Meyers AD. Laser reimbursement: who pays for progress? Lasers in Surgery and Medicine 1990(10):396-400.

8. Ministry of Healh. Care in the Netherlands: Summary of the Financial Owersight on Care 1995, Ministry of Health, Rijswik, the Netherlands, 1995 (in Dutch).

89: Ministry of Health. Public Health policy 1995. Dutch Parliament, Second Chamber, Meeting-Year 1995-1996, 24 126 ni 9. Sdu Publishers, the Hague, the Netherlands, 1995 (in Dutch).

90. National Institutes of Health. NIH data book 1989. Basic data relating to the National Institutes of Health. NHH, Bethesda, MD, 1989 .

91. Office of Technology Assesisment. Federal policies and the medical devices industry. Washington, DC, US Government Printing Office. 1984.

92. Office of Technology Assessment. The quality of medical care: Information for consumers. OTA-H-386. Washington, DC. US Government Printing Office, 1988.

93. Oortwin WJ, Ament AJHA, Vondeling H. Application of societal criteria in selecting research proposals: useful and feasible? Tijdschrift woor Socialle Giezondheidszong 1994(72):99-101 (in Dutch).

94. Organ $\mathrm{CH}_{\mathrm{t}}$ Bold RJ. Surgery. Journal of the American Medicial Association 1996(275):1855-7.

95. Ossoff RH, Coleman JA, Courey MS, Duncavage JA, Werkhaven JA. Clinical application of lasers in otolaryngology - head and neck surgery. Lasers in Surgery and Medicine 1994(15):217-48.

96. Parliamentary Office of Sicience and Technology. Minimal access ('keyhole') surgery and its implications. Parliamentary Office of Sicience and Technology, House of Commons, London, 1995.

97. Peach M. Ytterbium-doped crystal will deliver 100mW of green light. Opto \& Laser Europe 1996 (28):35-6.

98. Putz T. France needs tempting by Europe's laser suppliers. Opto \& Laser Europe 1995(21):23-7.

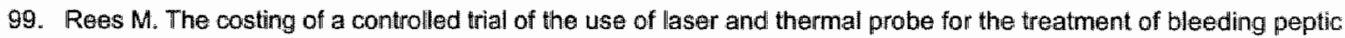
ulcer. Gastroenterological Unit, St James Hospitall, Balham, London, 1987.

100. Reid $M$. The diffusion of four prenatal screiening tests across Europe. In: Stocking $B$ (Ed.). A study of the diffusion of medical technology in Europe. King's. Fund Center for Health Services Development, London, England, 1991 .

101. Rogers EM. Diffusion of Innovations (fourth edition). The Free Press, New York, London, Toronto, Sidney, Tokyo, Singapore, 1995.

102. Rovira J. Standardizing oconomic appraisal of health technology in the European Communily. Sacial Science and Medicine 1994(38)12:1675-8.

103. Russell LB. Technology in hospitals; Medical advances and their diffusion. Brookings Institution, Washington, DC, 1979.

104. Schol FPG. Laparascopic cholecystectomy in the Netherlands (thesis). University of Limburg, Maastricht, the Netherlands, 1995.

105. Sculpher $M_{i}$ Drummond $M$, Buxton $M$. Economic evaluation in health care research and development: undertake it early and often. HERG Discussion Paper No 12. Health Economics Research Giroup, Brunell University, Uxbridge, Londion, England, 1995.

106. Sculpher M. Final report: Phase II Medical Laser Technology Assessment. HERG Research Report Nio 18. Health Economics Research Group, Bunel University, Uxbridge, London, England, 1994.

407. Shepherd DS, Dunch JS. Intermational comparison of resource aliocation in health sciences: an analysis of expenditures on biomedical reseanch in 19 industrialized countries (unpublished). Manuscript supported by the Fogarty International Center, National Institutes of Health, Public Health Service. US Department of Health and Human Services, Bethesda, MD, 1985.

108. Steering Committee on Future Health Scenarios. Anticipating and assessing health care technology. Volume 1. General considerations, and policy conclusions. Martinus Nijhoff Publishers, A member of the Klluwer Academic Publishers Group, Dordrecht, Boston, Lancaster, 1987. 
109: Sterenborg HMCM. Muller GJ. Eunopean Community Concented Action Program into the development of medical laser apphications (final concept). Working Group Laser Safety, 1994.

110. Stocking B. Factors affecting the diffusion of the kinds of innowative medical tecthology in European Community countries and Sweden. In: Stocking B (Series editor). A study of the difusion of medical technology in Europe. King's Fund Center for Health Services Development London, England, 1991, p.92-136; $1.95-134$ p. 129-68.

111. The Swedish Council on Technology Assessment in Health Care (SBU), Medical technologies in need of assessment. SBU, Stockhom; 1989.

112. The University of York. NHS Center for Reviews and Dissemination. Promoting the application of researchbased knowledge in health care (leaflel). The Unwersity of York, May 1994

113. The WHOQOL Group. The World Health Organization Quality of Life Assessment (WHOQOL): position paper from the World Health Organization. Social Science and Medicine 1995(41)1403-9.

114. The World Bank. World Development Report 1993. Investing in Health. World development indicators. Oxford University Press, Oxford. New York, Toronto. Delhi, Bombay, Calcutta Madras, Karachi, Kuala Lumpur, Singapore, Hong Kong. Tokyo, Nairobi, Dar es Salaam, Cape Town, Melbourne, Auckland, 1993.

115. Trajtenberg $M$, Yilzhaki $S$. The diffusion of innowations; a methodological reappraisal. journal of Business \& Economic Statistics 1989(7)1:35-47.

116. Turney 3 . Public understanding of science. Lancet 1996(347) $1087-90$.

117. Vanoli A, Drummond MF. Sheldon TA. The NHS economic evaluation database and manual. CRD Report 7. NHS Center for Reviews and Dissemination, York, United Kingdom, 1996.

118. Vondelling $\mathrm{H}_{3}$ Banta $\mathrm{HD}$. Cosmetic or medically necessany? Reimbursement of Dyemaser tratment of portwime: stains and Excimer laser treatment of nearsightedness in the United States and in the Netheriands (unpublished). Vrije Uniwersiteit Amsterdam, tDepartment of Epidemiology and Biostatistics, Amsiterdam, the Netherlands 1996.

119. Vondeling $H$. The international diffusion of Excimer laser treatment of nearsightedness (unpublished). Vrije Universiteit Amsterdam, Department of Epidemiology and Blostatistics, Amsterdam, 1994.

120. Wagner EH, Barrett P, Barry MJ, Barlow W, Fowler FJ. The effect of a shared decisionmaking program on rates of surgery for benign prostatic hyperplasia. Medical Care 1995(33)8:765-70.

121. Wennberg \&E, Barry MJ, Fowler FJ, Mulley A. Outcomes Research, PORTs, and Health Care Reform. In: Warren KS, Mosteller $F$ (Eds.). Doing more good than harm: the evaluation of health care interventions. The New York Academy of Sciences, 1993(703):52:62.

122. Werkö L, Banta D. Report from the EUR ASSESS Project. International Journal of Technology Assessment in Health Care 1995(11):797-9.

123. Wickham JAE. An introduction into minimally invasive therapy. Health Policy 1993(23):7-15.

124. Treacy PJ, Johnson AG. Is the laparoscopic bubble bursting? Lancet 1995 (346)Supplement:23. 
$\therefore \quad \therefore \quad \vdots$

क.

$\ldots$

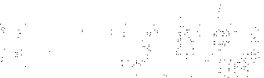

. 


\section{Samenvatting}

Dit proefschrift belicht een aantal aspecten van het gebruik van lasers in de geneeskunde. Lasers zijn fascinerende instrumenten die in verschillende segmenten van de samenleving gemakkelijk ingang hebben gevonden. Ook in de geneeskunde zijn de verwachtingen van lasers altijd hooggespannen geweest. Lasers hebben een imago van 'high-technology' en regelmatig worden aansprekende resultaten gemeld in de media en in de medische vakbladen. Anderzijds vormt het toenemend gebruik van lasers en aanverwante technieken een uiting van de 'vertechnologisering' van de gezondheidszorg en draagt het gebruik van deze en andiere kostbare medische technologie aanzienlijk bil aan de stijgende kosten van gezondheidszorg. Dit zijn zorgwekkende ontwikkelingen, waardoor steeds vaker de vraag wordt gesteld of de sneile introductie van nieuwe medische technologie ook werkellijk een verbetering betekent: Met behulp van methoden en technieken die schuligaan achter de naam Medical Technology Assessment (MTA), een vorm van multidisciplinair beleidsondersteunend onderzoek, is getracht deze vraag te beantwoorden.

Het proefschrift is opgebouwd uit vijf delen. Het eerste deel bevat algemene informatie en schetst het theoretisch kader. Reeds in het begin van deze eeuw werd de theoretische basis voor de ontwikkeling van lasers door Albert Einstein gelegd. Het zou tot 1960 duren voordat de eerste laser werd gebouwd, waarna in 1961 de eerste medische experimenten werden uitgevoerd. Reeds een jaar later leidden deze experimenten tot de eerste medische toepassing. Sindsdien heeft het gebruik van lasers in de geneeskunde een hoge vlucht genomen. Laserlicht is een speciaal soort licht. Volgens de kenners verhoudt laserlicht zich tot gewoon licht zoals muziek zich verhoudt tot lawaai. De ontwikkeling van medische lasers vormt een onderdeel van een technologische revolutie in de geneeskunde die bekend is geworden onder de verzamelnaam minimaal-invasieve therapie. Met name door het beschikbaar komen van endoscopen, catheters en nieuwe beeldvormende technieken kunnen een groot aantal aandoeningen tegenwoordig op een ellegante, weinig belastende manier behandeld worden. Een bekend voorbeeld hiervan zijn "kijkoperaties" in de knie.

ledere vorm van gezondheidszorg ontleend zịn bestaansrecht aan de verbetering van de gezondheid van de bevolking. Dus idealiter zou men wensen dat met enige zekerheid valt vast te stellen dat medische lasertoepassingen bijdragen aan de gezondheid en het welzijn van de bevolking zonder onnodig letsel en leed te veroorzaken. In deel 2 wordt onderzocht in hoeverre dit het geval is bij circa zestig lasertoepassingen in een zestal specialismen; dermatologie, oogheelkunde, keel-, neus- en oorheelkunde, gastro-enterologie, urologie en gynaecologie.

Geconcludeerd wordt dat, op enkele uitzonderingen na, het gebruik van medische lasers niet gerechtvaardigd kan worden door de beschikbare evidentile over de veiligheid en werkzaamheid van de verschillende toepassingen. De uitzonderingen worden met name gevonden in de oogheelkunde, en in mindere mate in de gynaecologie en de gastro-enterologie.

Naast overwegingen van veiligheid en werkzaamheid dient rekening te worden gehouden met de kosten van lasierbehandeling. Leveren de toepassingen 'vallue for money'? Door een gebrek aan deugdelijke informatie kan slechts argon laser behandeling van netvliesafwijkingen bil patiënten met suikerziekte als doelmatig worden aangemerkt. Een groot aantal lasertoepassingen zou echter, indien de evidentie over de werkzaamheid overtuigend zou zijn, tevens potentieel doelmatig kunnen zijn. Dit hangt nauw samen met de omstandigheid dat lasers voor een aanzienlijk aantal indicaties een alternatief vormen voor een conventionele chirurgische ingreep. In de gevallen waarin lasers voor een bepaalde indicatie moeten concurreren met een andere vorm van minimaalinvasieve therapie zijn de perspectieven voor doelmatig gebruik veelal minder gunstig.

In deel 3 wordt de verspreiding van medische lasers en verwante minimaal-invasieve technieken beschreven en geanalyseerd. Behalve in Nederland werden hiertoe door een internationale projectgroep gegevens verzameld in Denemarken, Zweden, Duitsland, het Verenigd Koninkrijk en Frankrijk. De belangrijkste remmende factoren voor de verspreiding van deze behandelingen zijn:

1) het beperkte budget van instellingen, 2) gebrek aan evidentie over de werkzaamheid, 3) 
conservatisme van specialisten. De belangrijkste stimulerende factoren zijn: 1) de vraag van patiênten naar een minimaal-invasieve behandeling, 2) aandacht in de media, en 3) aanwezigheid van vooraanstaande; invloedrijke specialisten die de betreffende minimaal-invasieve techniek propageren. Het in de praktijk aangetroffen patroon van verspreiding wordt gezien als de resultante van met name deze fwee tegengestelde combinaties van factoren. Zowel in Nederland als elders in Europa biljkt het verspreidingspatroon slechts in geringe mate samenhang te vertonen met de kwaliteit: van de evidentie over veiligheid, werkzaamheid en doelmatigheid.

Deel 4 behandelt in detail de evaluatie en verspreiding van een tweetal lasertoepassingen in de oogheelkunde. Door het National Eye Institute, éen van de National Institutes of Health in de Verenigde Staten, is op overtuigende wijze aangetoond dat argon laser behandeling van netvliesafwijkingen bij patienten met suikerziekte in een groot deel van de gevallen biindheid kan voorkomen. Vanuit maatschappelijk perspectief is de screening van patiënten met suikerziekte op netvliesafwijkingen, en vervolgens behandeling met argon lasers, zeer doelmatig. In ons land had men eerder en op grotere schaal kunnen beginnen met het aanbiedlen van deze behandeling dan in de praktijk heeft plaatsgevonden. In contrast, excimer laser behandeling van bijziendheid is een controversiële behandeling die zich in korte tijd verspreid heeft. De rol van alle betrokkenen wordt kritisch tegen het licht gehouden, waarbij de 'bijziendheid' van de Nederlandse overheid niet aan de aandacht ontsinapt.

In deel 5 worden beleidsvraagstukken ten aanzien van de veiligheid, evaluatie en de verspreiding van laserapparatuur behandeld. Europese regelgeving wordt steeds belangrijker voor het veilig gebruik van medische hulpmiddelen. Evaluatie van medische lasertoepassingen in een vroeg stadium van de levenscyclus lijkt een goede strategie ter voorkoming van ongecontroleerde verspreiding. Alleen de meest relevante lasertoepassingen kunnen voor evaluatie in aanmerking te komen, aangezien de capaciteit voor evaluatie-onderzoek sterk achterblijft bij het aanbod van nieuwe medische technologie. Tot slot worden de bevindingen in een breder kader geplaatst. De vraag is dam: hoe kunnen landen zoals Nederland, met ons onvolprezen poldermodel zo verstandig mogelijk omgaan met medische lasers en andere nieuwe medische technologie? Hiervoor worden verschillende suggesties gedaan. Eén van deze suggesties, en een belangrijke bovendien in het kader van deze studie, is de verdere ontwikkelling van Medical Technology Assessment als een krachtig hulpmiddell ter ondersteuning van besluitvorming over medische technologie. Hoewel Medical Technology Assessment sterk in opkomst is in Nederland en elders. wordt er nog betrekkelijk weinig gebruik gemaakt van de informatie die dergelijke studies opleveren. In de toekomst mag worden verwacht dat de noodzaak van een meer doelmatige toepassing van medische technologie ertoe zal leiden dat een groter aantal van dit type studies zal worden verricht en dat de resultaten van deze studies van toenemend belang zullen worden bij hell formuleren van beleid. 


\section{Dankwoord}

In essentie is het onmogelijk om in woorden mijn dank uit te drukken aan hen die interesse getoond hebben voor dit werk. Dit voorop gesteld

Tijdens mijn studie aan de Rijksumversiteit Groningen heb ik enige jaren blologie gecombineerd met sociologie. Hierdoor werd mijn interesse gewekt voor een zeer breed thema, gemakshalve te omschrijven als de interactie tussen wetenschap, technologie en samenleving. Met name de boeiende discussies in de themagroep Technology Assessment van drs. $M$. van Delden aan het Sociologisch Instituut hebben hieraan bijgedragen. In eerste instantie dacht ik na mijn studie iets met milieu-effectrapportage te gaan doen, maar toen de gelegenheid kwam om me aan de Rijks. universiteit Limburg verder te bekwamen in de medische variant van Technology Assessment ben ilk daar enthousiast op ingegaan. Voor deze mogelijkheid ben ik Prof. H.D. Banta, Prof.dr. F.F.H. Rutten en Prof.dr. E.K.A van Doorslaer dank verschuldigd. Beste David, Frans en Eddy, jullie enthousiasme voor het vak is ook het mijne geworden. Destijds voelde het alsof ik twee vliegen in éen klap had geslagen: interessant werk en de mogelijkheid te verhuizen naar de stad waar mijn vriendin woonde.

Prof. H.D. Banta heeft als dagelijks begeleider een essentiële rol vervuld bij de totstandkoming van dit proefschrift. Door in eerste instantie bij Felici en mij in Maastricht in huis te komen wonen strekte de begeleiding zich soms uit tot de avonduren! Dear David, I would like to thank you very much indeed for your continuous support, both as supervisor and as a friend. Even after you left the University of Limburg we continued to work as though we were next-door neighbors. The international experience you made me part of widened my scope and created a natural framework for this thesis. I am sure we will cantinue to discuss matters that are relevant for our personal and professional lives and perhaps do some work in the meantime. I guess you will never retirel Ellen, je weet dat ik je hier natuurlijk in éen adem naast David noem. Dank je wel voor de interesse die je toonde voor mijn werk.

Eenmaal in de Vakgroep Economie van de Gezondheidszorg opgenomen heeft dr. G. Haan me in korte tijd de eerste beginselen van economische evaluatie bijgebracht. Mijn dank hiervoor Ger. Na enigszins wegwijs gemaakt te zijn in het vak moest ik uiteraard de boer op voor het verzamelen van data. Graag wil ik prof.dr. E.M.H. Mathus-Vliegen (AMC) en prof.dr. T.A. Boon (AZU) bedanken voor hun grote gastvrijheid. Beste Lisbeth, beste Tom, jullie hebben me geleerd wat het betekent ais onderzoeker te werken in een ziekenhuisomgeving. Prof.dr. C.J.H. van de Velde en prof.dr. J. Kievit will ik bedanken voor de mogelijkheid een deel van mijn onderzoek in het AZL uit te voeren. Drs. A. Witteveen, beste Anda, je assistentie heeft me enorm geholpen bij het verwerken van een grote hoeveelheid data. Prof.dr. W. van Rossum, beste Wouter, bedankt voor de gelegenheid als gast-onderzoeker in de Vakgroep Organisatie, Technologie en Innovatie van de Faculteit Bedriffskunde aan de Rijksuniversiteit Groningen te verblijven. Dr. W.T. van Beekum, beste Taeke, jij hebt destijds voor me geregeld dat ik een extern secretariaat bij de Gezondheidsraad kon doen, mijn dank hiervoor. Dr. N.A.J. Mul (beste Nico) en prof.dr. P.T.V.M. de Jong (beste Paulus), hartelijk dank voor de begeleiding bij het voeren van het secretariaat van de Commissie 'Laserveiligheid' van de Gezondheidsraad. Al snel volgde het lidmaatschap van de Nationale Commissie Laserveiligheid (NCL), bestaande uit drs. G.J. Eggink, Ph.J. Ham ing., drs. C. Kaptein, drs. R.J. ("WP") van Kempen, dr. F.W. van der Meulen, drs. V.L.B. Oei-Lim, mevr. P. Siliakus, P.GJ. Sterrenburg ing., ir. C.J.P.M. Teirlinck, dr. S.R. Vaartjes, ing. L.C.E. Vlaskamp en R.A. Zandbergen ing. Met plezier denk ik terug aan de goede sfeer waarin onze vaak intensieve, leerzame discussies plaatsvonden. Uiteindelijk leidde ons werk tot het uitbrengen van een fraai rapport. Beste Gert, Phillip, Cees, Ruud, Freek, Vivian, Paula, Pieter, Carool, Ron, Charles en Rob, hartelijk dank voor de prettige samenwerking. In mijn bijdrage aan de NCL begaf ik me op juridisch terrein. Ik had dat niet verantwoord kunnen doen zonder steun van een aantal adviseurs, waarvan ik met name prof. mr. F.C.M. van Wijmen en mr. N.P.J.M. de Bijl will noemen. Beste Frans en Nicole, bedankt hilervoor. 
Drs. H. Rosendal, beste Henk, je was een enthousiaste, creatieve doctoraalstudent die, eenmaal werkzaam bij het Ministerie van VWS, belangstelling bleef houden voor het lasergebeuren. In je functie als MTA-onderzoeker bil TNO wens ik je veel succes met het schrijven van je proefschrift. Drs. G.A. de Wit, beste Ardine, na een korte periode in de vakgroep Economie van de Gezondheidsszorg vertrok je naar de Erasmus Universiteit. Via allerlei projecten van David, ik denk wooral aan de EEG-projecten, hadden we daarna nog regelmatig contact. Je vrolijkheid en optimisme maakten het altijd prettig samenwerken. Veel succes bij het RIVM! Voorts dank ik alle nog niet genoemde mede-auteurs (drs. M.A. Bos, P.H. Dignum ing., drs. H.M.J. Haerkens en dr. I. Schou) voor hun aandeel in de artikelen die deel uitmaken van dit proefschrift.

In de periode 1992-1996 werd de Vakgroep Economie van de Gezondheidszorg geleid door prof.dr. R.M. Leidl. Beste Reiner, bedankt voor de gelegenheid die je me hebt geboden mijn onderzoek voort te zetten, in de laatste periode gecombineerd met een betrekking als toegevoegd docent: Mijn oud-collega's in de Vakgroep Economie van de Gezondheidszorg will ik bedanken voor hum blijvende belangsteling voor mijn werk. We kunnen terugkijken op een enerverende tijd. Vooruitkijkend doet het me plezier dat er op korte termijn nog enkele promoties te verwachten zijn, onder meer van mijn oud-kamergenoot drs. H.B.G. Starmans. Beste Bert, wie had kunnen vermoeden dat we ons boekje vrijwel tegelijkertijd af zouden hebben?

De leden van de beoordelingscommissie, prof.dr. J.A. Knottnerus (voorzitter), prof.dr. M.J.C. van Gemert, prof.dr. F. Hendrikse, prof.dr. Ph.E.V.A. Van Kerrebroeck en prof.dr. W. van Rossum ben ik bijzonder erkentelijk voor hun bereidheid om, ondanks hun drukke werkzaamheden, dit werk op zijn merites the beoordelen. Prof.dr. M.J.C. van Gemert, beste Martin, mede met jouw steun hoop ik dat het proefschrift nog een staartje krijgt in de vorm van enkele hernieuwde publikaties in Lasers in Medical Science. Prof.dr. J.A.M. Maarse, beste Hans, mijn dank voor het vervullen van de rol van officiële promotor aan het eind van mijn onderzoek.

In de fase van de afronding van het manuscript wil ik mevr. E.H.E. Brounts-Hendrickx hartelijk danken voor de accurate wijze waarop zij de referenties in het manuscript heeft venwerkt. Beste Emily, je dochter moet die laserbehandeling nog maar eens heroverwegen! J.P.M.A. van Emmerik ing. heeft zijn onvolprezen talenten ruimschoots aangewend om ook dit proefschrift van een fraaie lay-out te voorzien. Daarnaast verzorgde je ook soepel de computerondersteuning van het onderzoek wat ik in de vakgroep heb uitgevoerd. Beste Jan "je maakt het me lastig je op gepaste wijze te bedanken. Wanneer gaan we nou eens de stad in? Mevr. I. Jeuken, beste Itilla, geweldig hoor, zo'n fraai omsiagontwerp. Dhr. T. Peters (Comma-s Ontwerpers), beste Theo, dank je wel voor de belettering van het omslag. Je typering van mij als 'Wurmus Libris' doet het nog steeds goed. Dhr." T.R. Leliveld (Datawyse) wil ik bedanken voor de wijze waarop het eindproduct tot stand is gekomen. Beste Ruud, ik was onder de indruk van het grote scala en het fraaie uiterlijk van je producties.

Dr. H.J. Waalkens, dirs. A.L.M. Huppertz, beste Herman, beste Anke, ik dank jullie voor het enthousiasme waarmee jullie de taak van paranimf hebben aanvaard.

Mijn huidige collega's in de Vakgroep Epidemiologie en Biostatistiek en het Instituut voor Extramuraal Geneeskundig Onderzoek van de Vrije Universiteit Amsterdam wil ik hartelijk danken woor hun belangstelling voor mijn werk. Ik bedank ook de collega"s in het Onderzoekscentrum 1e$2 e$ lijn van het AZVU en collega's in verschillende afdelingen van het AZVU voor hun interesse. Met drs. W.J. Oortwijn, drs. R.C.W. Hutubessy en drs. A.H.E. Koolman heb ik het meest intensief samengewerkt. Beste Wija . Raymond en Xander, het was een genoegen samen met jullie in de afgelopen jaren het gedachtengoed over MTA te verspreiden in de VU en het AZVU. Dit boekje vormt naar ik aanneem een afdoende verklaring voor mijn overwerk!

Mijn vrienden en vriendinnen bedank ik voor de relativering het plezier en de flitsende ontspannende en inspirerende activiteiten die we samen hebben ondernomen. 
Dil proefschrift was nooit tot stand gekomen zonder de inbreng van mijn ouders en mijn opa. De "discussiecultuur' die jullie creëerden maakte me al vroeg politiek bewust. Ook gaven jullie me met een rotsvast vertrouwen in mijn kunnen de zekerheid die $\mathrm{k}$ zo hard nodig had.

Karla, in het voonwoord van je doctoraalscriptie Verplegingswetenschap staat dal je hoopte mij te kunnen stimuleren bij het schriven van mijn proefschrift zoals ik jou had gestimuleerd bij het schrijven van je scriptie. Terugkijkend is dat slechts het topje van de spreekwoordelijke ijsberg geweest. Soms hangen we van gezondheidszorg aan elkaar! Dank je wel, vooral voor je flexibele opstelling als ik weer eens met tijd woekerde. 


\section{Curriculum Vitae}

Hindrik Vondeling was born June 4, 1960 in Groningen, the Netherlands. He attended High School (WO-B) in Oosterwolde at the Scholengemeenschap Ooststelingwerf in 1972-1978. From 19781988 he studied Eiology. Geology and Sociology at the University of Groningen. In 1983 he acquired a B.Sc. degree in Biology, complemented in 1984 with a B.Sc. degree in Blology whth Geology as a second major. In 1988 he graduated in Blology (M.Sc.) with a major in Phystology 8. Behaviour and subsidiaries in Environmental Sciences and Public Administration. in 1988-1993 he was a Research Fellow with the Department of Health Economics of the University of Limburg. Maastricht, supervised by Prof. H.D. Banta. In 1993 he combined an appointment as Lecturer at the Department of Health Economics of the University of Limburg and a research position in Medical Technology Assessment at the Department of Epidemology and Biostatistics and the Institute for Research in Extramural Medicine of the Vrije Universiteit Amsterdam. Since 1994 he is exclusively employed at the Vrije Universitel Amsterdam. His main research interests include economic evaluation of heatth care programmes, priority-setting in MTA, and diffusion of medical technology. 